\title{
Hanford Tank 241-C-106: Residual Waste Contaminant Release Model and Supporting Data
}

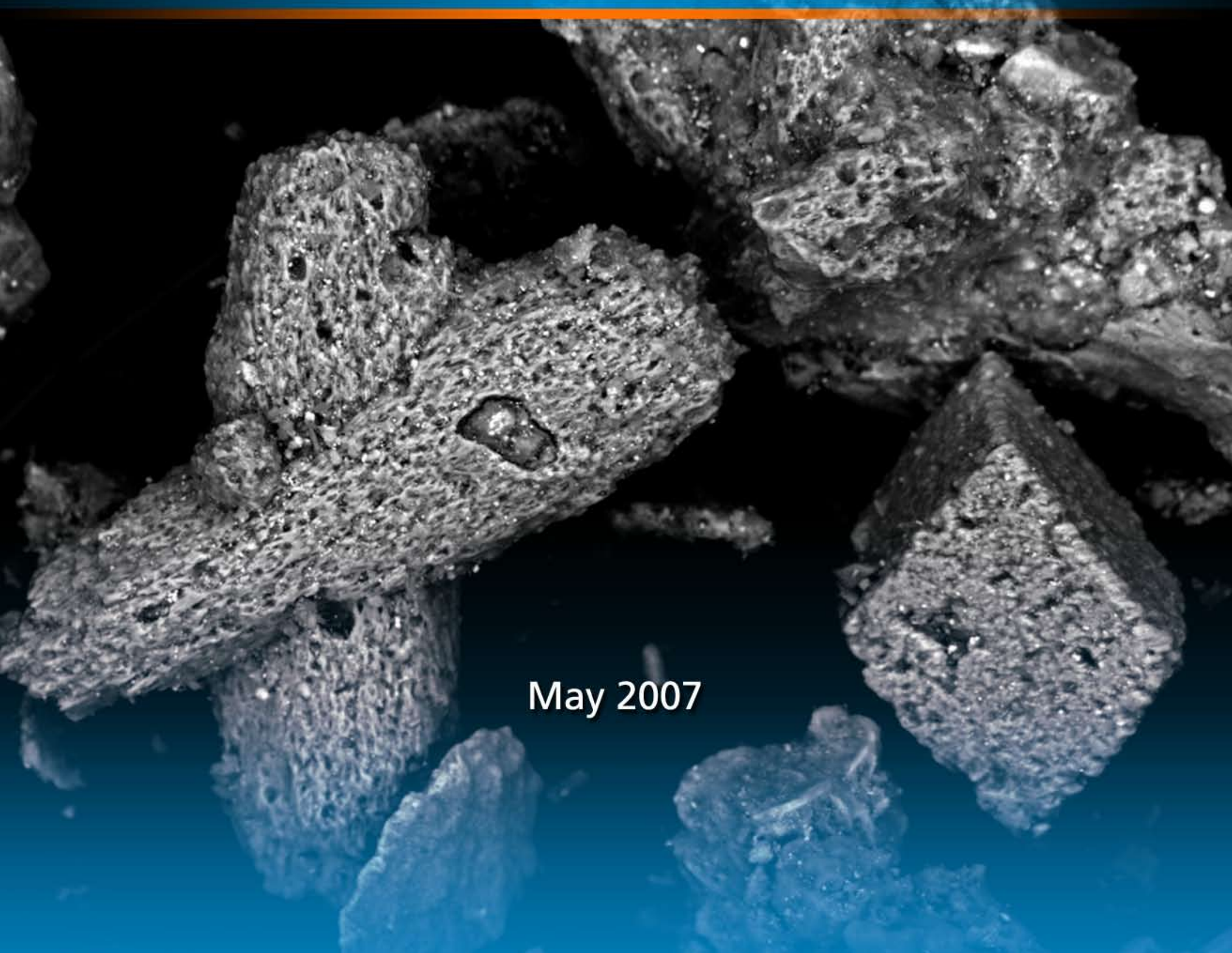

Prepared for CH2M HILL Hanford Group, Inc.

and the U. S. Department of Energy under Contract DE-AC05-76RL01830 


\section{DISCLAIMER}

This report was prepared as an account of work sponsored by an agency of the United States Government. Neither the United States Government nor any agency thereof, nor Battelle Memorial Institute, nor any of their employees, makes any warranty, express or implied, or assumes any legal liability or responsibility for the accuracy, completeness, or usefulness of any information, apparatus, product, or process disclosed, or represents that its use would not infringe privately owned rights. Reference herein to any specific commercial product, process, or service by trade name, trademark, manufacturer, or otherwise does not necessarily constitute or imply its endorsement, recommendation, or favoring by the United States Government or any agency thereof, or Battelle Memorial Institute. The views and opinions of authors expressed herein do not necessarily state or reflect those of the United States Government or any agency thereof.

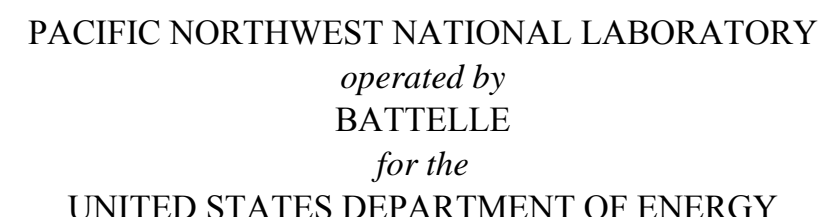

under Contract DE-AC05-76RL01830

Printed in the United States of America

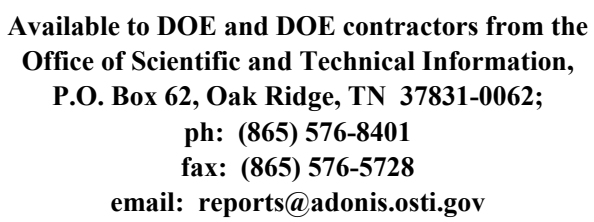

Available to the public from the National Technical Information Service, U.S. Department of Commerce, 5285 Port Royal Rd., Springfield, VA 22161 ph: (800) 553-6847 fax: (703) 605-6900

email: orders@ntis.fedworld.gov

online ordering: http://www.ntis.gov/ordering.htm

The cover image of tank $C-106$ residual material is from a scanning electron microscope. See page 3.40 for discussion.

This document was printed on recycled paper. 


\title{
Hanford Tank 241-C-106: Residual Waste Contaminant Release Model and Supporting Data
}

\author{
W. J. Deutsch \\ K. J. Cantrell \\ K. M. Krupka \\ C. F. Brown \\ M. J. Lindberg \\ H. T. Schaef
}

May 2005

Prepared for

CH2M HILL Hanford Group, Inc. and the U.S. Department of Energy under Contract DE-AC05-76RL01830

Pacific Northwest National Laboratory Richland, Washington 99352 


\section{Summary}

This report was revised in May 2007 to correct values in Section 3.4.1.7, second paragraph, last sentence; ${ }^{90} \mathrm{Sr}$ values in Tables 3.22 and 3.32; and ${ }^{99} \mathrm{Tc}$ values Table 4.3 and in Chapter 5 . In addition, the tables in Appendix F were updated to reflect corrections to the ${ }^{90} \mathrm{Sr}$ values. The rest of the text remains unchanged from the original report issued in May 2005.

The CH2M HILL Hanford Group, Inc. (CH2M HILL) is producing risk/performance assessments to support the closure of single-shell tanks at the U.S. Department of Energy's Hanford Site. As part of this effort, staff at Pacific Northwest National Laboratory were asked to develop release models for contaminants of concern that are present in residual sludge remaining in tank 241-C-106 (C-106) after final retrieval of waste from the tank. The primary contaminants of concern in the sludge are ${ }^{99} \mathrm{Tc},{ }^{238} \mathrm{U},{ }^{129} \mathrm{I}$, and $\mathrm{Cr}$ because of their potential mobility in the environment and the long half-lives of the radionuclides. A key result from this work is that high percentages $(>90 \%)$ of these primary contaminants are not readily leachable from the residual waste. This minimizes their future release rates to the environment, and is similar to ${ }^{99} \mathrm{Tc}$ results found in related studies of sludges from tanks AY-102, C-203, and C-204.

Developing release models consists of laboratory testing to produce contaminant release data and a conceptual source release model. After development, the release model can be incorporated into a fate and transport model as part of a long-term risk assessment for the closed tank.

Initial laboratory tests (Tier 1) were conducted to characterize the sludge and identify water-leachable constituents. Based on the results of Tier 1 tests, additional analyses were performed to augment the characterization of the material and determine the controlling mechanism(s) for release of contaminants. Tier 2 tests consisted of X-ray diffraction (XRD) and scanning electron microscopy/energy dispersive spectrometry (SEM/EDS) analyses of the solids to identify reactive phases, and selective extractions to quantify the release of contaminants from particular solid phases.

The laboratory results of sludge and liquid testing were used to develop source term models that describe the release of contaminants as infiltrating water contacts the solids in the future. These models simulate the geochemical system in the tank sludge and take into account interactions between the solution phase and the contaminant-containing solids. The release models are simplifications of the complex geochemical interactions occurring; however, they adequately represent the release of the primary contaminants of concern from the sludge as measured in the laboratory tests.

Because of the highly complex chemical nature of tank C-106 residual sludge, clear and quantitative phase associations of the contaminants of concern with the phases known to exist in the sludge are difficult to specify. Although the various characterization methods employed in this study have revealed a number of important observations and have provided valuable data for constructing a scientifically defensible release model, many questions remain. Because a thorough understanding of all the important phase associations for the contaminants of concern cannot be developed at this time, an empirically based release model has been developed. Although less satisfying from a mechanistic point of view, this approach provides a release model that can be used now and is conservative in nature. Later work may provide a better understanding of the phase associations with the contaminants of concern and the release 
mechanisms from these phases. In this case, a less conservative, but more scientifically defensible release model could be developed.

The major conclusions from this work are summarized in the following items:

- The total concentrations of the primary contaminants of concern are relatively low in the residual $\mathrm{C}-106$ sludge. The average ${ }^{99} \mathrm{Tc}$ concentration is $1.2 \mu \mathrm{g} / \mathrm{g}$ and the ${ }^{238} \mathrm{U}$ concentration is $247 \mu \mathrm{g} / \mathrm{g}$. The ${ }^{129}$ I concentration had an average value of $0.62 \mu \mathrm{g} / \mathrm{g}(110 \mathrm{pCi} / \mathrm{g})$. The $\mathrm{Cr}$ concentration in the sludge was near the instrument quantification limit, and was estimated to be $897 \mu \mathrm{g} / \mathrm{g}$.

- Only 2 to $3.5 \%$ of the ${ }^{99} \mathrm{Tc}$ in the residual sludge was water leachable and only 1.3 to $4 \%$ of the ${ }^{238} \mathrm{U}$ was leachable. The water leachability of ${ }^{129}$ I from the sludge was found to be much less than $1 \%$. The $\mathrm{Cr}$ concentrations in solutions from the water leach tests were below the estimated quantitation limit; however the semi-quantitative results show leachabilities from 1 to $7 \%$.

- Mechanistic release models of contaminants from the sludge could not be developed from the available data because of the complexity of the geochemical system. Empirical release models based on measured total sludge concentrations and maximum solution concentrations in water leaching tests should be used for risk/performance assessment modeling. These models are expected to provide conservatively high release rates into fresh water percolating through the residual waste.

- The concentrations of major metals and anions in the sludge were:

$$
\begin{array}{ll}
\circ & \mathrm{Al}-131,483 \mu \mathrm{g} / \mathrm{g} \\
\circ & \mathrm{Mn}-117,767 \mu \mathrm{g} / \mathrm{g} \\
\circ & \mathrm{Fe}-43,777 \mu \mathrm{g} / \mathrm{g} \\
\circ & \mathrm{Ca}-38,221 \mu \mathrm{g} / \mathrm{g}
\end{array}
$$

$$
\begin{array}{ll}
\circ & \mathrm{Na}-60,400 \mu \mathrm{g} / \mathrm{g} \\
\circ & \text { oxalate }-63,900 \mu \mathrm{g} / \mathrm{g} \\
\circ & \text { carbonate }-39,500 \mu \mathrm{g} / \mathrm{g}
\end{array}
$$

- The oxalic acid treatment of sludge in the tank during retrieval removed much of the water-leachable constituents from the solid. Leachable amounts of $\mathrm{Al}$ and Fe were barely detectable, while about $6 \%$ of the $\mathrm{Ca}, 40 \%$ of the $\mathrm{Mn}$ and $50 \%$ of the $\mathrm{Na}$ were water leachable. The primary water leachable anions were oxalate and carbonate.

- Several of the metals and contaminants could not be leached from the sludge even under the condition of aggressive leaching with concentrated nitric acid. It was found that $56 \%$ of the ${ }^{99} \mathrm{Tc}$ could not be leached under these conditions and $40 \%$ of the $\mathrm{Cr}$ was recalcitrant. $\mathrm{Fe}$ and $\mathrm{Al}$ were also relatively immobile with $65 \%$ and $41 \%$ not leachable, respectively.

- The XRD results indicate that the unleached sludge samples contain detectable quantities of the following crystalline phases:

$$
\begin{array}{ll}
\circ & \text { lindbergite }\left[\mathrm{MnC}_{2} \mathrm{O}_{4} \cdot 2 \mathrm{H}_{2} \mathrm{O}\right] \\
\circ & \text { gibbsite }\left[\mathrm{Al}(\mathrm{OH})_{3}\right] \\
\circ & \text { dawsonite }\left[\mathrm{NaAlCO}_{3}(\mathrm{OH})_{2}\right] \\
\circ & \text { hematite }\left(\mathrm{Fe}_{2} \mathrm{O}_{3}\right)
\end{array}
$$
○ böhmite $[\mathrm{AlO}(\mathrm{OH})]$
- rhodochrosite $\left(\mathrm{MnCO}_{3}\right)$
- whewellite (Ca oxalate monohydrate, $\mathrm{CaC}_{2} \mathrm{O}_{4} \cdot \mathrm{H}_{2} \mathrm{O}$ )


Testing of sludge from tank C-106 has shown the need for future development of analytical techniques and release models. These developments include the following:

- Improved selective extraction methods to identify the solid phases containing trace levels of contaminants

- Testing of contaminant release from sludge under environmental conditions other than fresh water infiltration (e.g., cement grout/sludge system) 


\section{Acknowledgments}

The authors wish to acknowledge M. Connelly, F.J. Anderson, and T.E. Jones at CH2M HILL Hanford Group, Inc. (Richland, Washington) for providing project funding and technical guidance. We greatly appreciate the technical reviews provided by F.M. Mann (CH2M HILL), M.I. Wood (Fluor Hanford, Inc., Richland, Washington), R.J. Serne, and W. Um (both of PNNL). The authors would also like to thank B.W. Arey (PNNL) for conducting the SEM/EDS analyses of the sludge samples and S.R. Baum, K.M. Geiszler, I.V. Kutnyakov, and R.D. Orr (all of PNNL) for completing the chemical and radiochemical analyses of the solution samples from our studies. We are also particularly grateful to L.F. Morasch (PNNL) for completing the editorial review and K.R. Neiderhiser (PNNL) for final formatting of this technical report. 


\section{Acronyms and Abbreviations}

$\begin{array}{ll}\text { AEA } & \text { alpha energy analysis } \\ \text { ASTM } & \text { American Society for Testing and Materials } \\ \text { BSE } & \text { backscattered electron } \\ \text { CCV } & \text { continuing calibration verification } \\ \text { CH2M HILL } & \text { CH2M HILL Hanford Group, Inc. } \\ \text { DDI } & \text { double deionized (water) } \\ \text { DOE } & \text { U.S. Department of Energy } \\ \text { DUP } & \text { duplicate sample } \\ \text { EDS } & \text { energy-dispersive X-ray spectrometry } \\ \text { EPA } & \text { U.S. Environmental Protection Agency } \\ \text { EQL } & \text { estimated quantitation limit } \\ \text { GEA } & \text { gamma energy analysis } \\ \text { HASQARD } & \text { Hanford Analytical Services Quality Assurance Requirements Document } \\ \text { HF } & \text { hydrofluoric } \\ \text { ICP-MS } & \text { inductively coupled plasma-mass spectroscopy (spectrometer) } \\ \text { ICP-OES } & \text { inductively coupled plasma-optical emission spectroscopy (same as ICP-AES) } \\ \text { ICDD } & \text { International Center for Diffraction Data, Newtown Square, Pennsylvania } \\ \text { JCPDS } & \text { Joint Committee on Powder Diffraction Standards } \\ \text { LEPS } & \text { Low Energy Photon System } \\ \text { LSC } & \text { liquid scintillation counting } \\ \text { NIST } & \text { National Institute of Standards and Technology } \\ \text { PDFTM } & \text { powder diffraction file } \\ \text { pe } & \text { redox potential (-log a } \text { e-) } \\ \text { PNNL } & \text { Pacific Northwest National Laboratory } \\ \text { QA } & \text { quality assurance } \\ \text { RPL } & \text { Radiochemical Processing Laboratory } \\ \text { SBMS } & \text { Standards-Based Management System } \\ \text { SE } & \text { secondary electron } \\ \text { SEM } & \text { scanning electron microscopy (or microscope) } \\ \text { SI } & \text { Saturation Index } \\ \text { SRM } & \text { Standard Reference Material } \\ \text { TEM } & \text { transmission electron microscopy (or microscope) } \\ \text { TRU } & \text { transuranic (waste) } \\ \text { XRD } & \text { X-ray powder diffractometry analysis (commonly called X-ray diffraction) } \\ & \end{array}$




\section{Units of Measure}

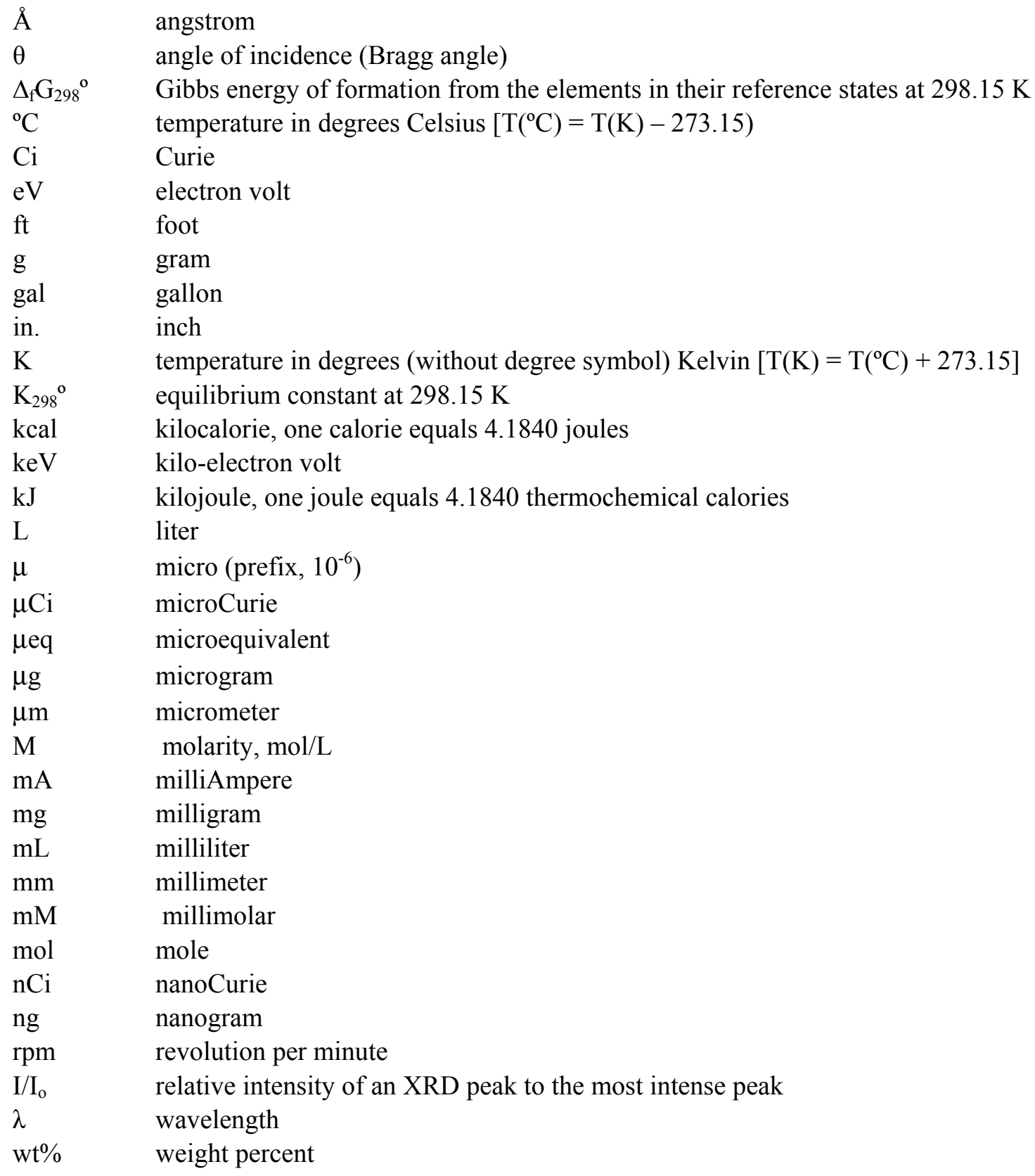




\section{Contents}

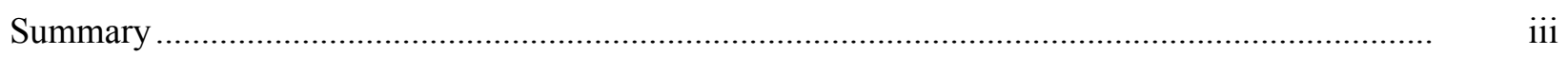

Acknowledgments..................................................................................................................... vii

Acronyms and Abbreviations …………………………………………………………..... ix

Units of Measure

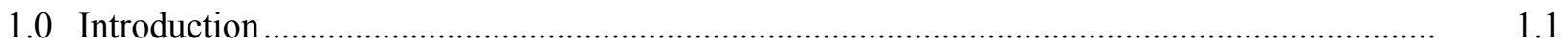

1.1 Scope

1.2 C-106 Tank Description ...........................................................................................

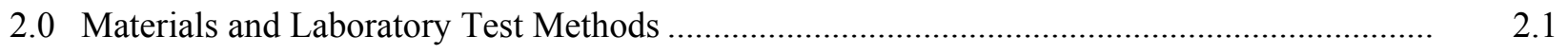

2.1 Tank C-106 Samples ……………………………………………………………....

2.2 Sludge Composition by Fusion and Acid Digestions......................................................... 2.1

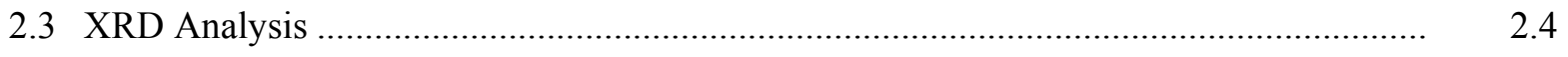

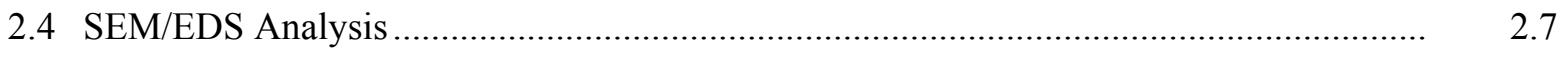

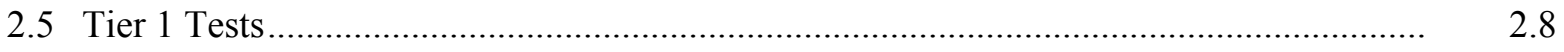

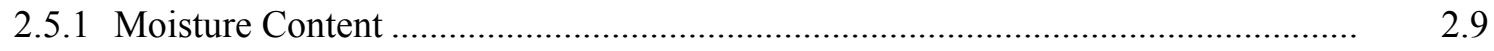

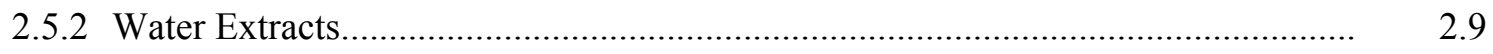

2.5.3 Periodic Replenishment Tests........................................................................ 2.9

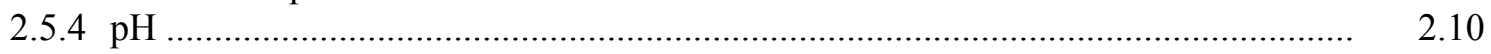

2.5.5 Anion Analysis .......................................................................................... 2.10

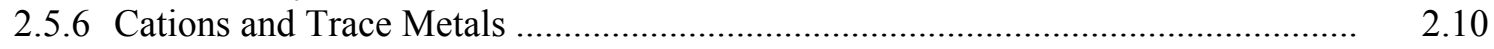

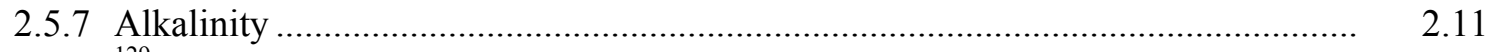

2.5.8 ${ }^{129} \mathrm{I}$ Extraction and Analysis ......................................................................... 2.11

2.5.9 Radioanalysis ………………………………………………………….... 2.12

2.6 Selective Extraction Tests ........................................................................................... 2.13

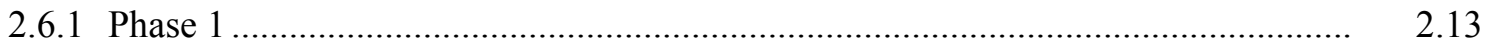

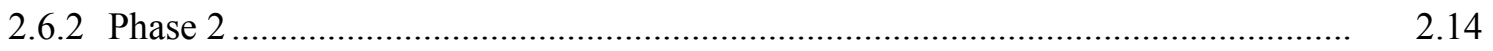

3.0 Laboratory Results ...........................................................................................................

3.1 Sludge Composition ...............................................................................................

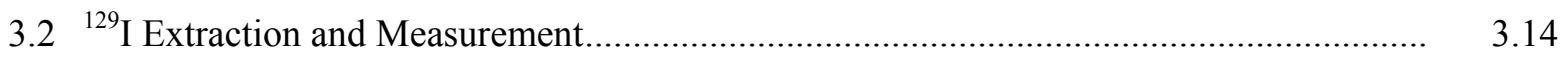




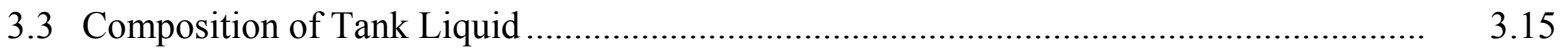

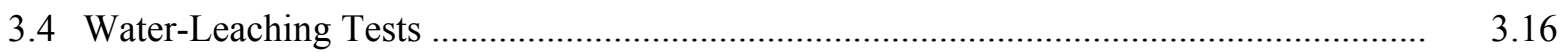

3.4.1 Single-Contact Test Results ..................................................................... 3.16

3.4.2 Periodic Replenishment Test Results.......................................................... $\quad 3.20$

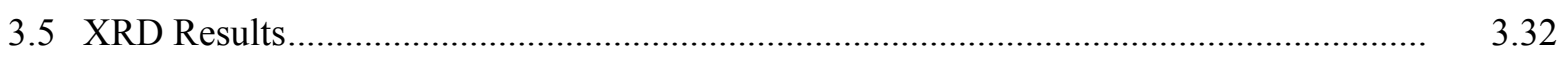

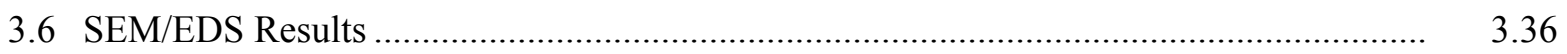

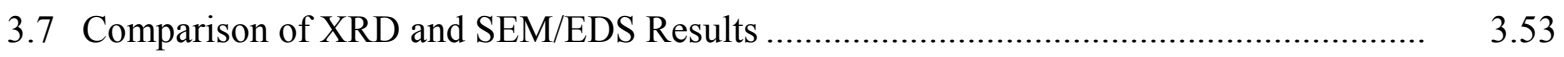

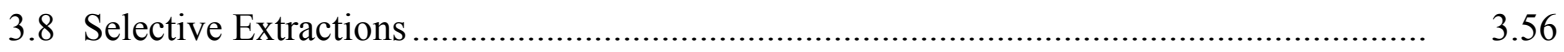

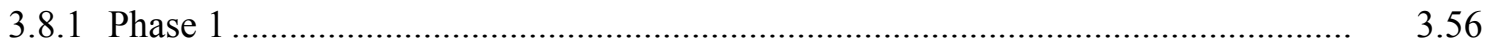

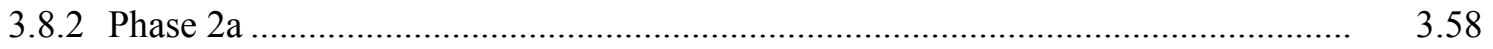

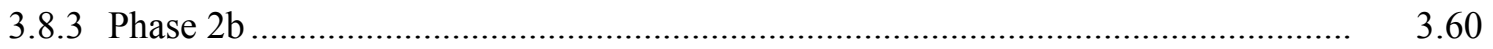

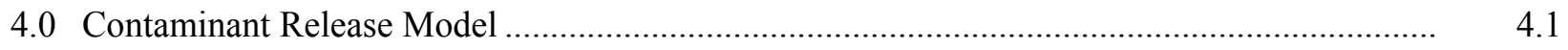

4.1 Conceptual Model of Chemical Transformations of Tank C-106 Sludge Resulting from the Retrieval Process ....................................................................................... 4.2

4.2 Technetium, Uranium, Iodine, and Chromium Release Models .................................... 4.6

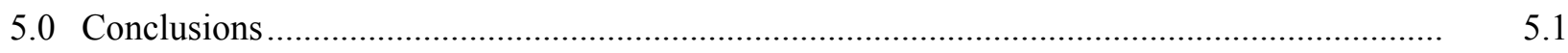

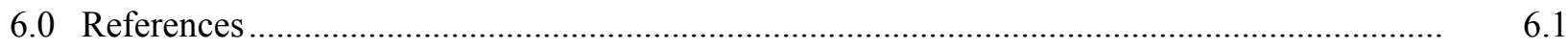

Appendix A - X-Ray Diffraction Patterns for Unleached, Water-Leached, and HF-Extracted Sludge Samples from Tank C-106 ................................................................... A. A

Appendix B - SEM Micrographs and EDS Spectra for Unleached Tank C-106 Sludge ................ B. $\quad$ B.1

Appendix C - SEM Micrographs and EDS Spectra for Water-Leached Sludge from Tank C-106

Appendix D - SEM Micrographs and EDS Spectra for HF Sequential Extract of Tank C-106 Sludge............................................................................................ D.

Appendix E - SEMS/EDS Element Distribution Maps for Samples of Tank C-106 Sludge ............ E.1

Appendix F - Solution Concentrations of Tank C-106 Residual Liquid and Water Contact Tests with Residual Sludge

Appendix G - Chemical Equilibrium Modeling Calculations ............................................... G. 


\section{Figures}

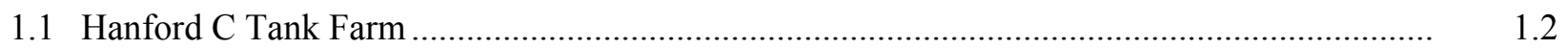

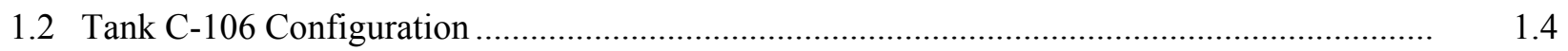

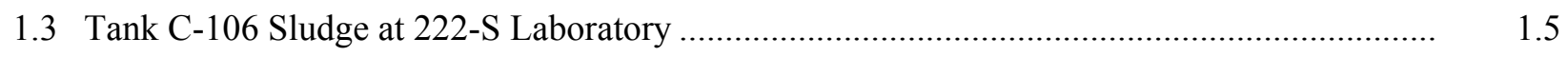

2.1 Tank C-106 Field Primary Solid Composite Sludge Sample 404 ….................................... 2.2

2.2 Tank C-106 Field Duplicate Solid Composite Sludge Sample 405 ......................................... 2.2

2.3 Exploded Schematic View of the XRD Sample Holder....................................................... 2.5

2.4 XRD Pattern for Collodion Film Measured in the Absence of any Sludge Material................ $\quad 2.6$

2.5 Phase 1 Selective Extraction Sequence Used to Determine Phase Associations of ${ }^{99} \mathrm{Tc}$, ${ }^{238} \mathrm{U}$, and $\mathrm{Cr}$ in Tank C-106 Sludge.

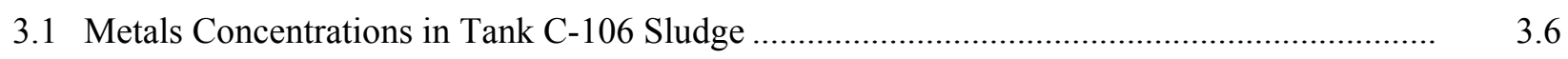

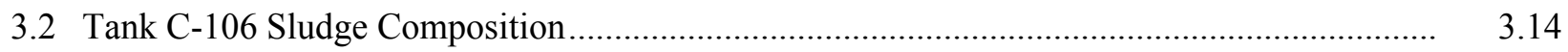

3.3 Background-Subtracted XRD Pattern for the Unleached Sludge Samples 404 and 405 from Tank C-106 Shown with Matching PDF Database Patterns......

3.4 Background-Subtracted XRD Pattern for the 1-Month Water-Leached Sludge Samples 404 and 405 from Tank C-106 Shown with Matching PDF Database Patterns

3.5 Background-Subtracted XRD Pattern for the 82-Day Water-Leached Sludge Samples 404 and 405 from Tank C-106 Shown with Matching PDF Database Patterns

3.6 Background-Subtracted XRD Pattern for the HF-Extracted Sludge Samples 404 and 405 from Tank C-106 Shown with Matching PDF Database Patterns.....

3.7 Comparison of Background-Subtracted XRD Pattern for Unleached, 1-Month and 82-Day Water-Leached, and HF-Extracted Sludge Sample 405 from Tank C-106 Shown with Matching PDF Database Patterns

3.8 Low Magnification SEM Micrographs of Particles Present in the Unleached and 1-Month Water-Leached Tank C-106 Sludge Samples

3.9 Low Magnification SEM Micrographs of Particles Present in the 82-Day WaterLeached and HF Stage 1 Extract Tank C-106 Sludge Samples

3.10 Unique Morphologies of Large Particles Common in all Tank C-106 Samples Inspected by SEM/EDS. 
3.11 Additional Examples of Large Particles with Unique Morphologies Shown in

Figure 3.10 and Consisting of $\mathrm{Mn}, \mathrm{Al}, \mathrm{Fe}, \mathrm{Na}, \mathrm{P}, \mathrm{Si}, \mathrm{Ca}, \mathrm{O}$, and Possibly $\mathrm{C}$ and $\mathrm{H}$....

3.12 Typical EDS Spectra for Particles Shown in Figure 3.10 from Unleached and HF Extract Samples

3.13 Representative SEM Micrograph Images of Particles Identified in Unleached Sludge Samples 404 and 405 from Tank C-106

3.14 Representative SEM Micrograph Images of Particles Identified in Unleached Sludge Samples 404 and 405 from Tank C-106

3.15 Representative SEM Micrograph Images of Particles Identified in 1-Month WaterLeached Sludge Samples 404 and 405 from Tank C-106

3.16 SEM Micrograph Images of Particles Identified in 1-Month Water-Leached and HF-Extracted Sludge Samples 404 and 405 from Tank C-106.

3.17 SEM Micrographs of Fe Oxide Particles in Unleached, 1-Month and 82-Day Water-Leached, and HF-Extracted Sludge Samples 404 and 405 from Tank C-106

4.1 Source Release Model Development for Long-Term Risk/Performance Assessments

4.2 Conceptual Model of Chemical Transformations for Tank C-106 Sludge Resulting from Tank Retrieval Process and Water Leaching Relevant to Contaminant Release Modeling.......

\section{Tables}

1.1 Tank C-106 Waste Transfer History through 1979

2.1 Tank C-106 Samples Provided by 222-S Laboratory to PNNL

2.2 Digestion Factors for Samples of Tank C-106 Residual Sludge Solids Used for the EPA Acid Digestion and $\mathrm{KOH}-\mathrm{KNO}_{3}$ Fusion Treatments.

2.3 Digestion Factors for Samples of C-106 Sludge Solids Used for the Modified $\mathrm{KOH}-\mathrm{KNO}_{3}$ Water Fusion Treatment to Measure ${ }^{129} \mathrm{I}$

3.1 Moisture Contents of Tank C-106 Sludge Samples 404 and 405.

3.2 Concentrations of Elements Measured by ICP-OES per Gram of Dry Sludge

3.3 Concentrations of Elements Measured by ICP-OES per Gram of Dry Sludge

3.4 Concentrations of Elements Measured by ICP-OES per Gram of Dry Sludge 
3.5 Concentrations of Elements Determined from ICP-MS Analysis per Gram of Dry Sludge ..

3.6 Concentrations of Elements Determined from ICP-MS Analysis per Gram of Dry Sludge .. $\quad 3.8$

3.7 Concentrations of ${ }^{99} \mathrm{Tc}$ and ${ }^{238} \mathrm{U}$, Measured by ICP-MS per Gram of Dry Sludge.................

$\quad 3.8 \quad{ }^{137} \mathrm{Cs}$ and ${ }^{60} \mathrm{Co}$ Concentrations in Dry Sludge as Measured by GEA .................................. 3.10

3.9 ICP-MS Analysis for Actinides per Gram of Dry Sludge ................................................ 3.10

3.10 Total Beta and Total Alpha Activities and ${ }^{90} \mathrm{Sr}$ Concentrations per Gram of Dry Sludge ..... $\quad 3.11$

3.11 Summary of Average Concentrations for Tank C-106 Residual Sludge............................. 3.12

3.12 Summary of ${ }^{129}$ I Concentrations for Modified $\mathrm{KOH}-\mathrm{KNO}_{3}$ Water Fusion Extracts for Tank C-106

3.13 Sample 403 - Tank C-106 Liquid Sample Composition................................................. 3.15

3.14 Water Extract pH and Alkalinity Values Corrected to Grams of Dry Sludge ..................... 3.16

$3.15{ }^{99} \mathrm{Tc}$ and ${ }^{238} \mathrm{U}$ Concentrations in Tank C-106 Sludge from Single-Contact WaterLeach Tests

3.16 Water-Leachable Percentages of ${ }^{99} \mathrm{Tc}$ and ${ }^{238} \mathrm{U}$ in C-106 Sludge Samples Compared with Fusion Results

3.17 Summary of ${ }^{129}$ I Concentrations from the One Day Single-Contact Water-Leach Tests

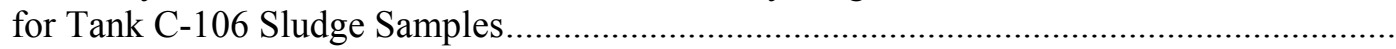

3.18 Water-Leachable Average Metal Concentrations in Tank C-106 Single-Contact

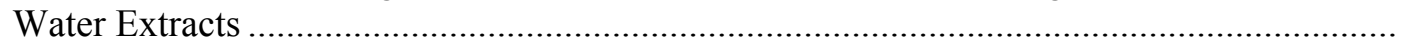

3.19 Water-Leachable Metals in Tank C-106 Single-Contact Water Extract Compared with Fusion Results

3.20 Average Anion Concentrations in Tank C-106 Single-Contact Water Extract.....

3.21 GEA for Tank C-106 Single-Contact Water Extractions and Direct Solids.

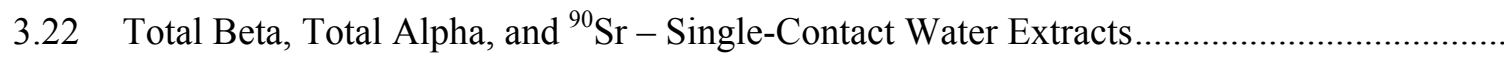

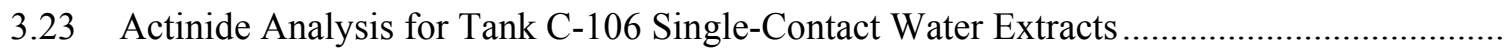

3.24 Water-Leachable Percentage for Actinides in C-106 Single-Contact Water Extracts Compared to Fusion Analysis. 
3.25 Contact Times, Average pH Values, and Alkalinities for Periodic Replenishment Tests on Tank C-106 Sludge Samples

$3.26{ }^{99} \mathrm{Tc}$ and ${ }^{238} \mathrm{U}$ Concentrations in Tank C-106 Sludge Samples - Periodic Water Replenishment Tests.

3.27 Water-Leachable Percentages of ${ }^{99} \mathrm{Tc}$ and ${ }^{238} \mathrm{U}$ in Tank C-106 Sludge Samples Periodic Water Replenishment Tests Relative to Fusion Results.

3.28 Water-Leachable Average Metals in Tank C-106 Sludge Samples - Periodic Water Replenishment Tests

3.29 Percent Water-Leachable Average Metal Concentrations in Tank C-106 Sludge Samples - Periodic Water Replenishment Tests

3.30 Average Anion Concentrations in Tank C-106 Sludge Samples - Periodic Water Replenishment Tests

3.31 GEA for Tank C-106 Periodic Water Replenishment Tests

3.32 Total Beta, Total Alpha, and ${ }^{90} \mathrm{Sr}$ Analysis for Tank C-106 Periodic Water Replenishment Tests.

3.33 Actinide Analysis for Tank C-106 Periodic Water Replenishment Tests

3.34 Water-Leachable Percentage for Actinides in Tank C-106 Periodic Water Replenishment Tests Compared with Fusion Analysis

3.35 Comparison of Characterization Data for Tank C-106 Sludge Prior to Treatment by Oxalic Acid

3.36 Comparison of XRD and SEM/EDS Characterization Results from This Study to Those Reported by Bechtold et al. (2003) for Tank C-106 Sludge treated with Oxalic Acid.

3.37 Phase 1 Selective Extraction Results for Sludge Samples 404 and 405 from Tank C-106

3.38 Phase 1 Selective Extraction Results for Sludge Samples 404 and 405 from Tank C-106

3.39 Phase 2a Selective Extraction Results for C-106 Sludge Samples 404 and 405

3.40 Phase 2a Selective Extraction Results for Primary and Duplicate Sludge Samples from Tank C-106

3.41 Phase 2b Selective Extraction Results for Sludge Samples 404 and 405 from Tank C-106 
3.42 Phase 2b Selective Extraction Results for Sludge Samples 404 and 405 from

Tank C-106

4.1 Three Major Sludge Components and Major Contaminants of Concern in Tank C-106 Sludge Before and After 1 M Oxalic Acid Treatment...

4.2 Contact Times and Average $\mathrm{pH}$ Values, Oxalate and Mn Concentrations, and Oxalate/Mn Ratios, for the Periodic Replenishment Test on Tank C-106

Sludge Samples

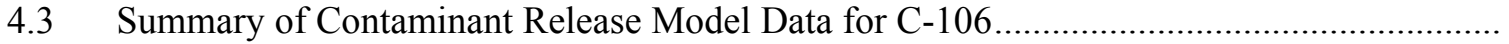




\subsection{Introduction}

This report describes the development of release models for contaminants of concern that may be present in residual sludge in Hanford tank 241-C-106 (C-106) after closure. These release models are necessary components of the risk assessments being conducted as part of the closure process. From the perspective of long-term risk to the environment, the primary contaminants of concern are ${ }^{99} \mathrm{Tc},{ }^{238} \mathrm{U},{ }^{129} \mathrm{I}$, and $\mathrm{Cr}$ because of their mobility in the environment and long half-lives for the radionuclides. Sludge samples and a sample of the liquid from tank C-106 were collected after final sludge retrieval to characterize the geochemistry of the reactive phases and to quantify the release of primary contaminants into water that may contact residual sludge after tank closure.

The remainder of this section describes the scope of work for laboratory testing and release model development as well as background information on this tank. The samples and laboratory testing procedures for this project are described in Section 2 of this report, and the results are provided in Section 3. Release models are discussed in Section 4 and general conclusions in Section 5. Cited references are listed in Section 6, and supporting material is included in the appendices.

\subsection{Scope}

Initial (Tier 1) laboratory tests were conducted to characterize the sludge and identify water-leachable constituents. The Tier 1 tests consisted primarily of fusion and acid digestions (which measured element concentrations in the solid) and water leaching of contaminants from the sludge to evaluate their mobility in infiltrating water. Based on the results of Tier 1 tests, additional analyses were performed to augment the characterization of the material and determine the controlling mechanism(s) for release of contaminants. Tier 2 tests consisted of analyses of the solids using X-ray diffraction (XRD) and scanning electron microscopy/energy dispersive spectrometry (SEM/EDS) to identify reactive phases, and selective extractions in order to quantify the release of contaminants from particular solid phases.

The laboratory results of residual sludge and liquid testing were used to develop source term models that describe the release of contaminants as infiltrating water contacts the solids in the future. These models simulate the geochemical system in the tank sludge and take into account interactions between the solution phase and the contaminant-containing solids. The release models are simplifications of the complex geochemical interactions occurring between the phases; however, they adequately represent the release of the key contaminants ${ }^{99} \mathrm{Tc},{ }^{238} \mathrm{U},{ }^{129} \mathrm{I}$, and $\mathrm{Cr}$ from the sludge as measured in laboratory tests.

\subsection{C-106 Tank Description}

Tank C-106 is a single-shell underground waste tank located in the C Tank Farm in the 200 East Area of the Hanford Site (Figure 1.1). It was constructed between 1943 and 1944 and put into service during September 1947. This tank is $22.8 \mathrm{~m}$ (75 ft) in diameter and has a capacity of 2,006,268 L (530,000 gal) when filled to a depth of $5.2 \mathrm{~m}(17 \mathrm{ft})$. Figure 1.2 is a diagram showing the configuration of tank C-106. 


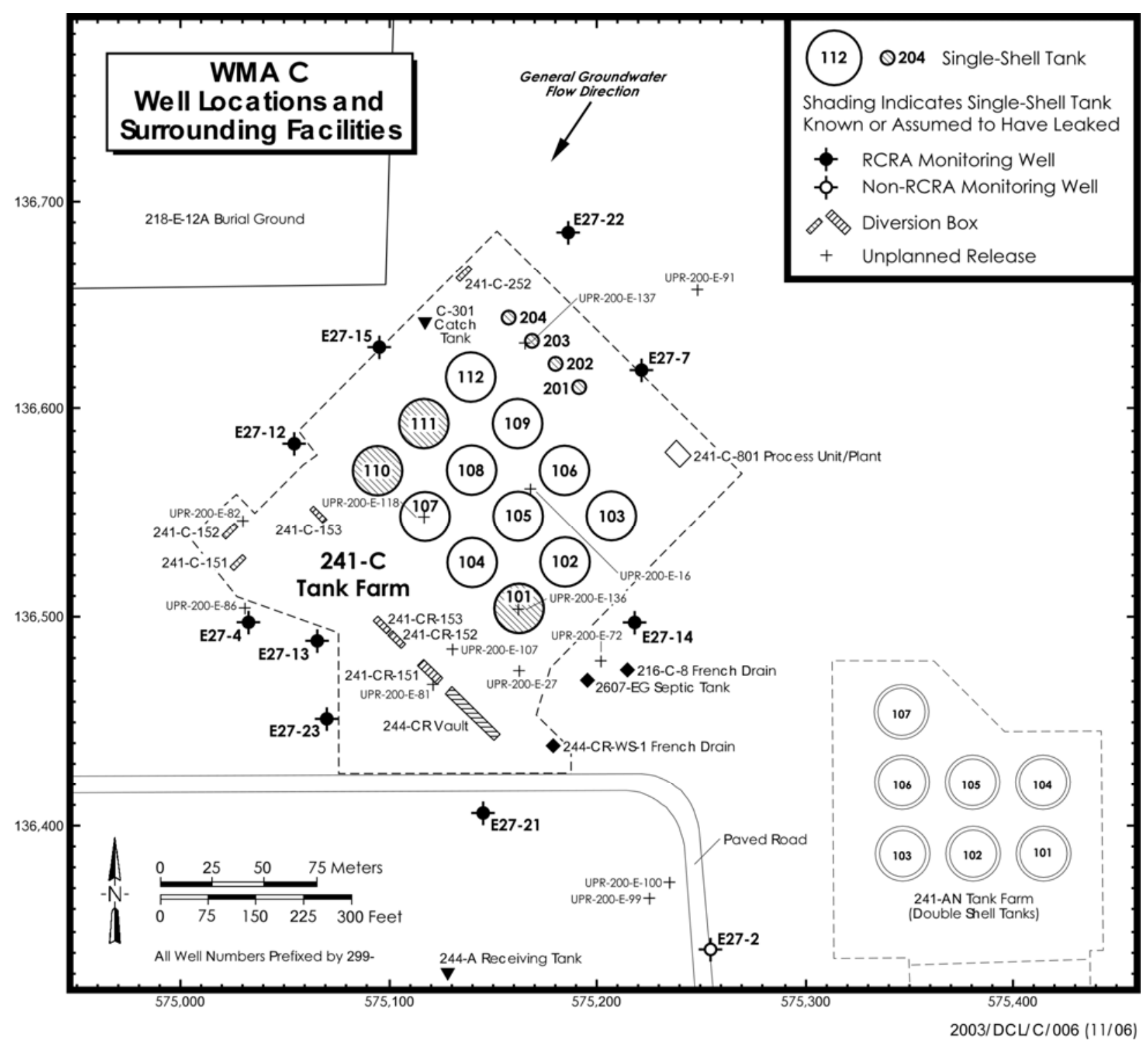

Figure 1.1. Hanford C Tank Farm 
Table 1.1 shows the history of waste transfers into and removals from tank C-106 through March 1979 when it was removed from service. This table is from Schreiber et al. (1996, Table 2-3) who obtained the information from Anderson (1990) and Agnew et al. (1995). The transfer history shows that a large variety of waste has been stored in this tank throughout its operational period. The tank was placed on the High-Heat Load Watch List in January 1991, and active ventilation was used to cool the tank by evaporation. The heat was produced by decay of radionuclides in the waste, principally ${ }^{90} \mathrm{Sr}$. Periodic water additions were made to replace evaporated moisture. In 1995, the Environmental Assessment (DOE 1995) stated that approximately 22,712 L (6,000 gal) of water were added to the tank each month for cooling purposes. As of May 31, 1996, the tank contained an estimated 866,859 L $(229,000$ gal) of waste $(745,726 \mathrm{~L}$ [197,000 gal] of sludge plus 121,133 L [32,000 gal] of supernatant liquid) classified as non-complexed (Hanlon 1996; Schreiber et al. 1996). From November 1998 through September 1999, the contents of tank C-106 were sluiced to tank 241-AY-102 using supernatant from tank 241-AY-102 as the sluicing fluid. Approximately, 97\% of the waste was removed, which left approximately $68,137 \mathrm{~L}(18,000$ gal) in the tank.

In late 2003, sludge from tank C-106 was further removed using a $0.9 \mathrm{M}$ oxalic acid solution to dissolve and suspend the solids and pump out as much as possible. The goal was to lower the sludge volume from about 68,137 to $10,599 \mathrm{~L}\left(18,000\right.$ to $2,800 \mathrm{gal}$ [360 $\left.\left.\mathrm{ft}^{3}\right]\right)$. This goal was achieved by several additions and removals of the oxalic acid solution. After the final removal, the sludge was rinsed with water to remove as much of the acid solution as possible. Approximately 151,416 L (40,000 gal) or $35.5 \mathrm{~cm}$ (14 in.) of water was added to measure the sludge volume, and then as much liquid as possible was removed. A $0.5 \mathrm{M} \mathrm{NaOH}$ solution was added to neutralize the residual waste, and then removed. The residual liquid in the tank was sampled for analysis through Riser 14 (Figure 1.2), and multiple sludge samples were acquired using a clamshell device by CH2M HILL in January 2004. All samples were delivered to the Hanford 222-S Laboratory for processing and characterization. Figure 1.3 is a picture of the sludge at the 222-S Laboratory. Subsamples were sent to PNNL for testing and release model development. 

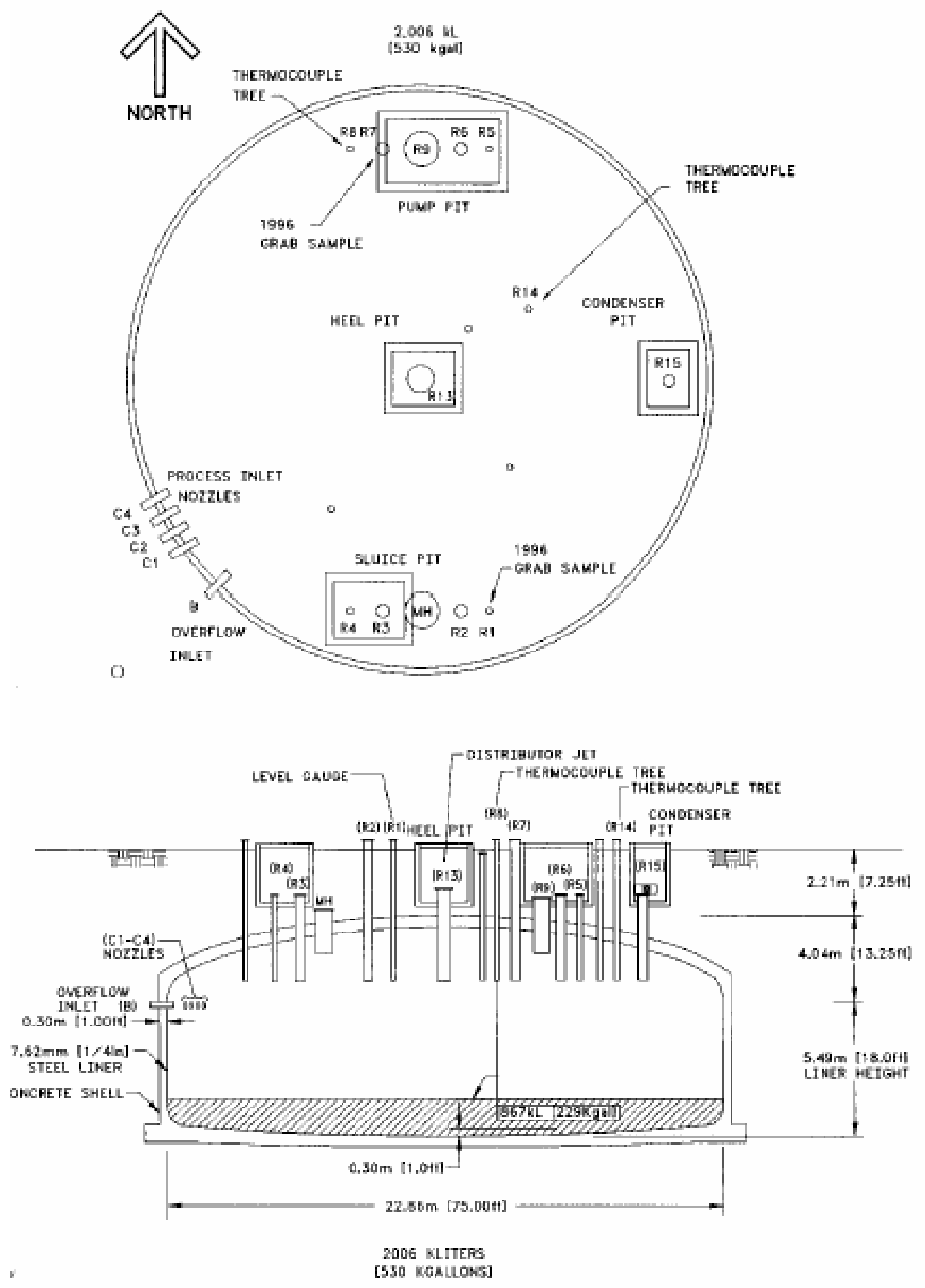

Figure 1.2. Tank C-106 Configuration (Conner 1996) 


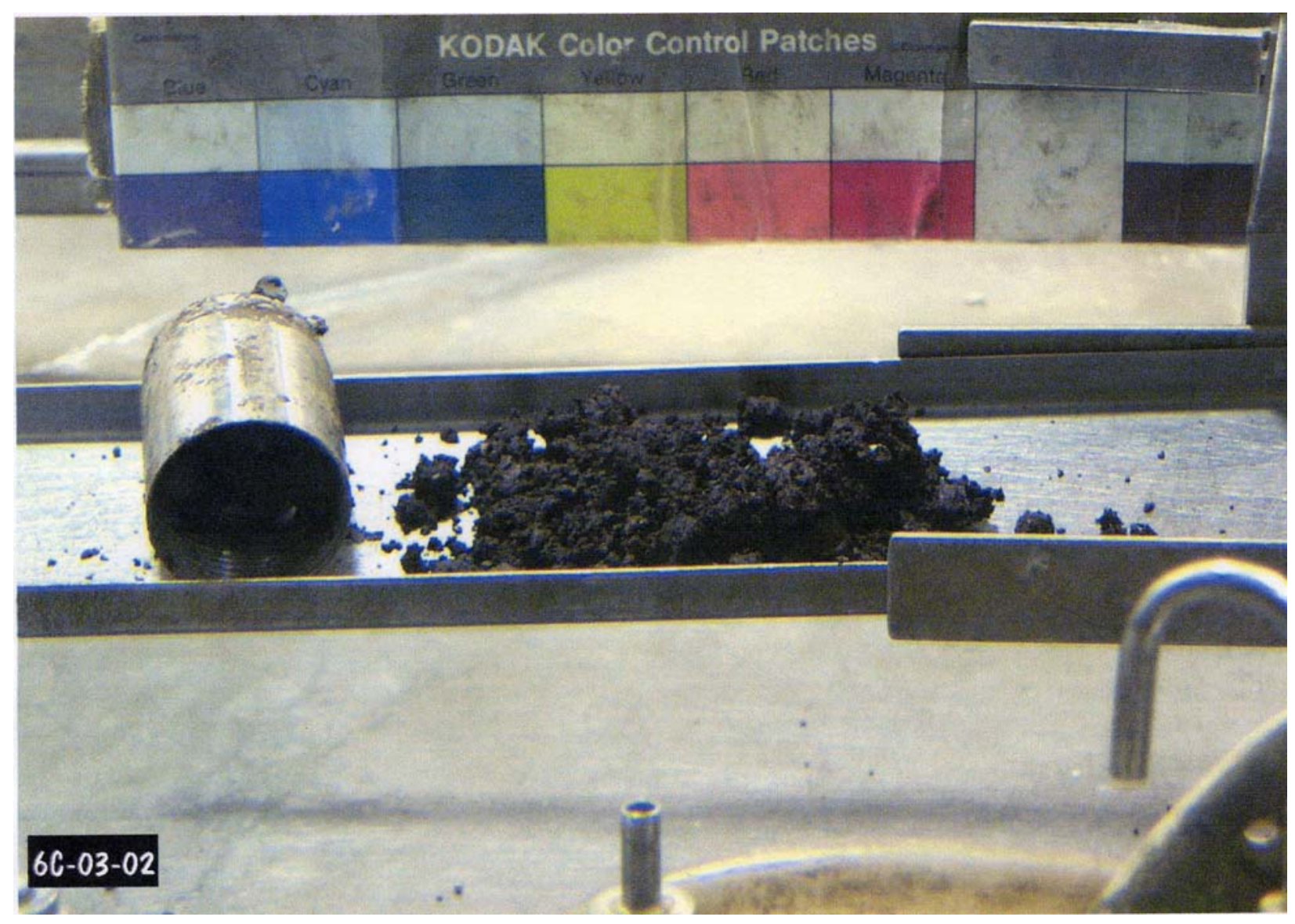

Figure 1.3. Tank C-106 Sludge at 222-S Laboratory 
Table 1.1. Tank C-106 Waste Transfer History through 1979 (from Schreiber et al. 1996)

\begin{tabular}{|c|c|c|c|c|}
\hline \multirow[b]{2}{*}{ Transfer Location } & \multirow[b]{2}{*}{ Waste Type } & \multirow[b]{2}{*}{ Time } & \multicolumn{2}{|c|}{ "Estimated Waste Volume ${ }^{(a)}$} \\
\hline & & & $\mathbf{I n}^{(\mathbf{b})}$ & Out $^{(\mathbf{b})}$ \\
\hline $241-\mathrm{C}-105$ & Metal waste supernate & 1947 & $\begin{array}{c}530,000 \mathrm{gal} \\
\end{array}$ & NA \\
\hline $\begin{array}{c}\text { 241-BY-102, 241-C-104, } \\
\text { 241-C-105, 241-C-202, } \\
241-\mathrm{C}-203,241-\mathrm{C}-204\end{array}$ & Metal waste & 1953 & $1,387,000 \mathrm{gal}$ & NA \\
\hline Miscellaneous & Flush water & 1953 & $1,828,000 \mathrm{gal}$ & NA \\
\hline U-Plant & $\begin{array}{l}\text { Sluicing metal waste for } \\
\text { uranium recovery }\end{array}$ & $1953-1954$ & 538,000 gal & $3,680,000 \mathrm{gal}$ \\
\hline Miscellaneous & Flush water & 1957 & $164,000 \mathrm{gal}$ & NA \\
\hline 241-C-109, 241-C-112 & $\begin{array}{l}\text { Uranium recovery waste } \\
\text { for ferrocyanide } \\
\text { scavenging }\end{array}$ & 1957 & NA & 550,000 gal \\
\hline 241-A-101, 241-A-102 & $\begin{array}{l}\text { Organic wash waste and } \\
\text { high-level PUREX waste }\end{array}$ & 1957 & $481,000 \mathrm{gal}$ & NA \\
\hline $\begin{array}{l}\text { 241-BY-103, } \\
\text { 241-BY-110 }\end{array}$ & Supernatant & $1957-1958$ & NA & $463,000 \mathrm{gal}$ \\
\hline PUREX & Cladding waste & 1958,1960 & $420,000 \mathrm{gal}$ & NA \\
\hline 241-B-101, 241-B-107 & Supernatant & 1963 & NA & $353,000 \mathrm{gal}$ \\
\hline $241-\mathrm{A}-102$ & PUREX waste & $1963-1964$ & 427,000 gal & 99,000 gal \\
\hline CR Vault & Decontamination waste & 1965 & $36,000 \mathrm{gal}$ & NA \\
\hline 241-C-105 & Supernatant & 1968-1969 & NA & $637,000 \mathrm{gal}$ \\
\hline $\begin{array}{c}\text { 244-AR Vault, } \\
\text { 241-A-106 }\end{array}$ & Washed PUREX waste & $1969-1970$ & $1,019,000 \mathrm{gal}$ & NA \\
\hline $\begin{array}{c}\text { 241-A-102, 241-C-103, } \\
\text { 241-C-105 }\end{array}$ & $\begin{array}{l}\text { PUREX and low-level } \\
\text { B-Plant supernate }\end{array}$ & $1970-1971$ & $638,000 \mathrm{gal}$ & $1,446,000 \mathrm{gal}$ \\
\hline Miscellaneous & Flush water & 1971 & 27,000 gal & NA \\
\hline $\begin{array}{l}\text { 244-AR Vault, } \\
\text { 241-A-106 }\end{array}$ & Washed PUREX waste & $1971-1972$ & 151,000 gal & NA \\
\hline 241-AX-103 & Supernatant & 1974 & NA & $221,000 \mathrm{gal}$ \\
\hline Miscellaneous & Flush Water & $1974-1975$ & 26,000 gal & NA \\
\hline 241-C-103, 241-C-104 & Supernatant & $1974-1976$ & NA & $2,392,000 \mathrm{gal}$ \\
\hline B-Plant & Low-level B-Plant waste & 1974-1976 & $2,937,000 \mathrm{gal}$ & NA \\
\hline 241-A-102 & $\begin{array}{l}\text { Strontium recovery } \\
\text { waste from B-Plant }\end{array}$ & 1976-1977 & 247,000 gal & $362,000 \mathrm{gal}$ \\
\hline 241-AZ-101 & Supernatant & 1978 & NA & $85,000 \mathrm{gal}$ \\
\hline 241-A-102 & $\begin{array}{l}\text { Complexed and } \\
\text { evaporator waste }\end{array}$ & $1978-1979$ & 365,000 gal & 445,000 gal \\
\hline $\begin{array}{l}\text { (a) Waste volumes do no } \\
\text { (b) Volumes are given in } \\
\text { NA = Not applicable. }\end{array}$ & clude unknown transfers & transfers o & $\begin{array}{l}\text { condenser. } \\
\text { e gallons by }\end{array}$ & \\
\hline
\end{tabular}




\subsection{Materials and Laboratory Test Methods}

Sludge and liquid samples from tank C-106 were collected by CH2M HILL during post retrieval activities in January 2004. This section provides a description of the samples and the various tests used to characterize the material, measure contaminant release, and identify controlling solids.

\subsection{Tank C-106 Samples}

On January 26, 2004, after neutralization of tank waste with $\mathrm{NaOH}$ and during pumping of residual fluid from tank C-106, a sample of the liquid was collected and transported to the Hanford 222-S Laboratory for storage and analysis. On January 29, 2004, nine clamshell samples of residual sludge were collected from the tank and sent to the 222-S Laboratory. On January 30 the sludge samples were extruded in the 11A Hot Cell. Part of the extruded samples was combined to produce the Field Primary Solid Composite. The remaining sludge was combined to produce the Field Duplicate Solid Composite. On April 13, 2004, samples of the sludge and liquid were shipped to the PNNL Radiochemical Processing Laboratory (RPL). Table 2.1 lists the samples received by PNNL.

Testing of sludge samples to develop contaminant release models was conducted on Field Primary Solid Composite S04T000404 (primary 404, Figure 2.1) and Field Duplicate Solid Composite S04T000405 (duplicate 405, Figure 2.2). Liquid sample S04T000403 (403) was also analyzed to determine the porewater concentrations of contaminants in the sludge.

Table 2.1. Tank C-106 Samples Provided by 222-S Laboratory to PNNL

\begin{tabular}{||l|c|c|c||}
\hline \multicolumn{1}{|c|}{ Sample } & $\begin{array}{c}\text { Jar Size } \\
(\mathbf{m L})\end{array}$ & $\begin{array}{c}\text { Labcore } \\
\text { Number }\end{array}$ & $\begin{array}{c}\text { Net Weight/Volume of } \\
\text { Material (g) }\end{array}$ \\
\hline \hline Field Primary Solid Composite & 60 & S04T000109 & 20.4 \\
\hline Field Primary Solid Composite & 60 & S04T000404 & 20.6 \\
\hline Field Duplicate Solid Composite & 60 & S04T000405 & 20.1 \\
\hline 6C-03-11 (Liquid) & 60 & S04T000403 & 61.6 \\
\hline
\end{tabular}

\subsection{Sludge Composition by Fusion and Acid Digestions}

The bulk compositions of the sludge solids were determined using PNNL internal procedure AGG-ESL-001 ${ }^{(\mathrm{a})}$ and a modified version of U.S. Environmental Protection Agency (EPA) Method 3050B (EPA 1996a). These methods were used to measure the element composition of the sludge, but are not appropriate for the anion concentrations due to the acids used in the analyses. The anion compositions were measured separately in solutions obtained by water leaching of the solids (see Section 2.5.5).

(a) Lindberg MJ. 2003. "Solubilization of Metals from Solids Using a KOH-KNO 3 Fusion." AGG-ESL-001 (Rev. 0), unpublished PNNL Technical Procedure, Pacific Northwest National Laboratory, Richland, Washington. 


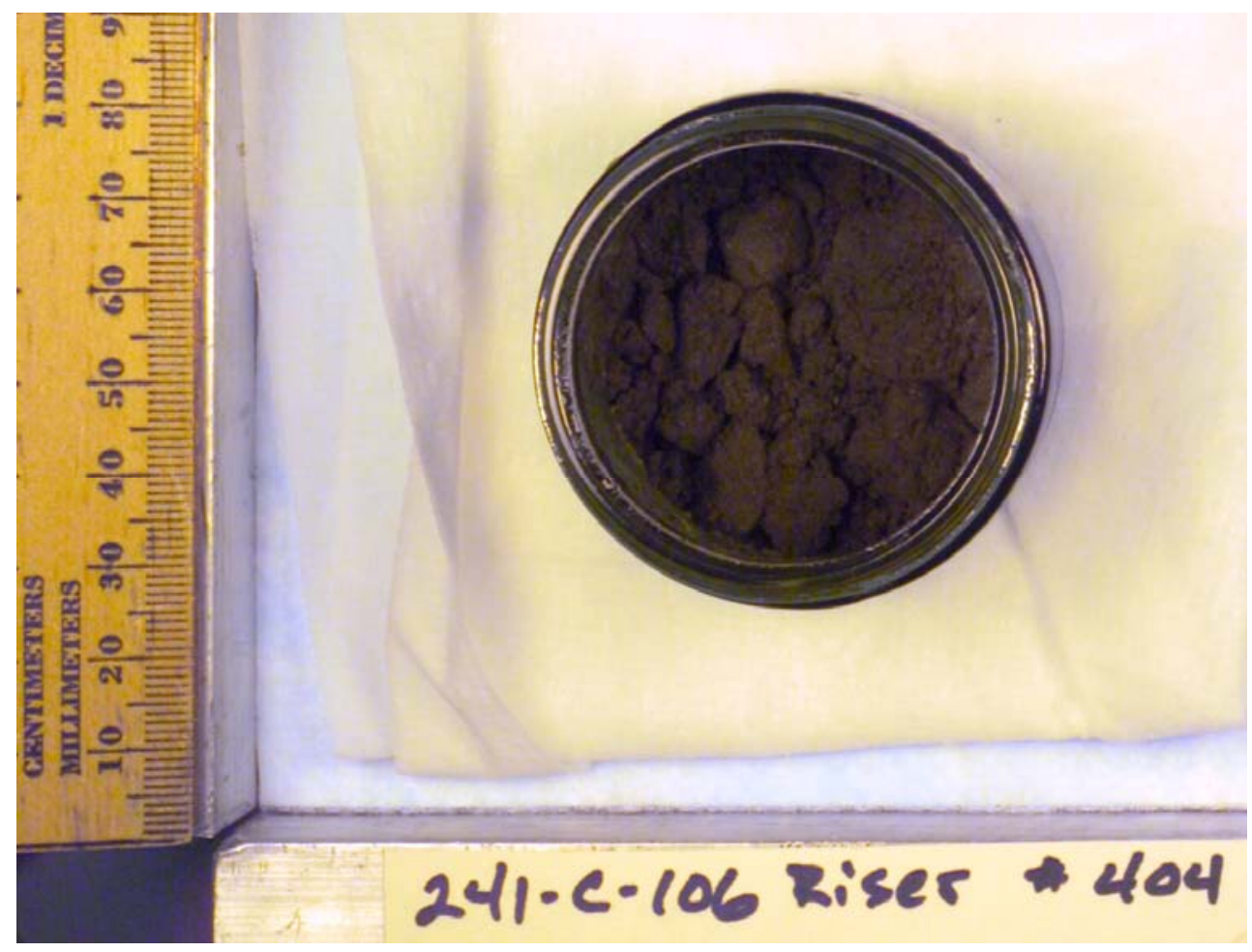

Figure 2.1. Tank C-106 Field Primary Solid Composite Sludge Sample 404

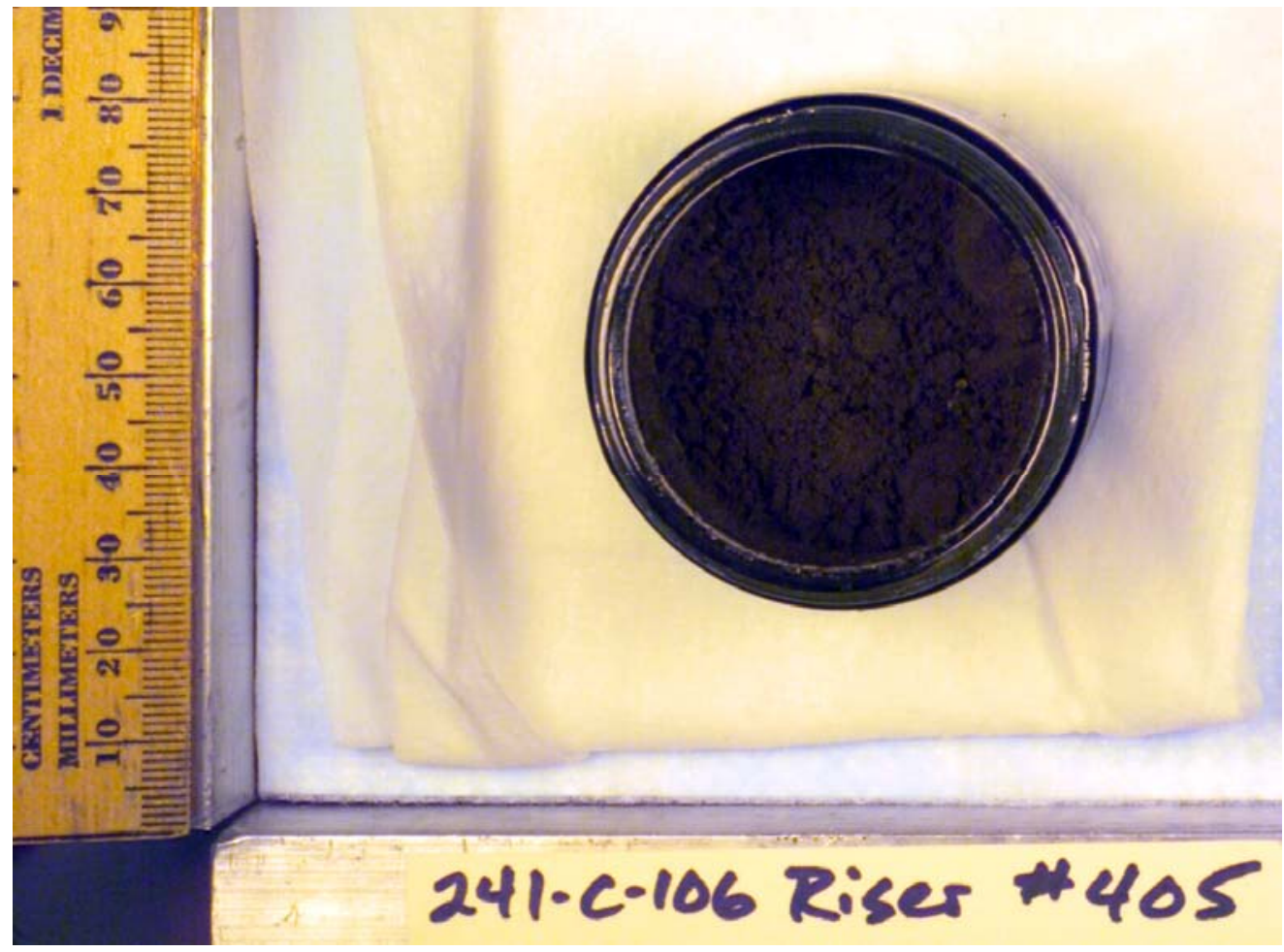

Figure 2.2. Tank C-106 Field Duplicate Solid Composite Sludge Sample 405

2.2 
The potassium hydroxide $(\mathrm{KOH})$-potassium nitrate $\left(\mathrm{KNO}_{3}\right)$ fusion-dissolution procedure is the most commonly used method for solubilization of Hanford tank sludge samples for chemical analysis by inductively coupled plasma-mass spectroscopy (ICP-MS) and other methods (De Lorenzo et al. 1994; Simpson 1994; Fiskum et al. 2000; Smith et al. 2001). Benefits of this procedure include effective metathesizing of insoluble salts such as $\mathrm{SrSO}_{4}, \mathrm{PuPO}_{4}, \mathrm{PuF}_{3}$, and $\mathrm{ThF}_{3}$ into acid soluble hydroxides; fusion completed at relatively low temperature $\left(550^{\circ} \mathrm{C}\right)$ compared to other fluxing agents, such as $1100^{\circ} \mathrm{C}$ for the $\mathrm{LiBO}_{2}$ (lithium metaborate) fluxing agent; and use of nickel or zirconium crucibles, as opposed to the more costly platinum crucibles, for the fusion. ${ }^{(a)}$

The $\mathrm{KOH}-\mathrm{KNO}_{3}$ fusion-dissolution procedure consists of chemical analyses of a solution resulting from water and acid dissolutions of a solid that has been fused at a high temperature with a caustic fluxing agent. In this procedure, $0.3 \mathrm{~g}$ of the tank waste sludge material was mixed with $10 \mathrm{~mL}$ of a $20 \% \mathrm{KOH}$ and $2 \% \mathrm{KNO}_{3}$ solution as a fluxing agent in a zirconium crucible. The crucible was then placed on a hot plate and allowed to evaporate to dryness, after which it was covered and transferred to a muffle furnace preheated to $550^{\circ} \mathrm{C}$. Fusion was accomplished by heating the sample-flux mixture for 60 minutes at $550^{\circ} \mathrm{C}$. After 60 minutes, the crucible was removed from the furnace and allowed to cool to ambient room temperature. The fused solid was then dissolved in double deionized (DDI) water. The resulting solution was transferred to a $100-\mathrm{mL}$ volumetric flask to which $1 \mathrm{~mL}$ of $1.0 \mathrm{M}$ hydroxylamine $\mathrm{HCl}$ was added. Ten $\mathrm{mL}$ of an 8-M nitric acid $\left(\mathrm{HNO}_{3}\right)$ solution was added to the crucible to try to dissolve any remaining residual solid. The acid wash solution was also added to the volumetric flask. The crucible was then triple-rinsed with DDI water, and these washes were also added to the volumetric flask. The resulting solution was diluted up to a total volume of $100 \mathrm{~mL}$ with DDI water. Prior to chemical analysis, the final $100-\mathrm{mL}$ solution was passed through a Whatman 41 filter with final filtration through a $0.45-\mu \mathrm{m}$ pore-size syringe filter to remove any particles that did not dissolve in the $\mathrm{HNO}_{3}$ solution. The insoluble fractions were not characterized because of the difficulty in removing them from the syringe filter media.

Chemical analyses of an acid digestion of the sludge solids were also completed for comparison with the $\mathrm{KOH}-\mathrm{KNO}_{3}$ fusion procedure. For the acid digestion, $0.3 \mathrm{~g}$ of tank sludge was digested following the basic procedure described in U.S. Environmental Protection Agency (EPA) Method 3050B (EPA 1996a) with the following exceptions: 1) all reagent volumes were reduced by $50 \%$ because safety considerations necessitated smaller sample masses due to the high radioactivity levels associated with these sludge solids, and 2) $\mathrm{HCl}$ was not added during the digestion because it interferes with analysis of the resulting solutions by ICP-MS. If $\mathrm{HCl}$ is used, an $\mathrm{ArCl}^{+}$species is formed during ICP-MS analysis, which creates a spectral interference that impedes analysis of certain analytes. Throughout the remainder of this report, this treatment of sludge solids will be referred to as "acid digestion."

For the acid digestion procedure, $300 \mathrm{mg}$ of the sample was placed in a $50 \mathrm{~mL}$ Griffin beaker, $8 \mathrm{M}$ $\mathrm{HNO}_{3}$ was added to the sample, the beaker was covered with a watch glass, and the unit was heated to $95^{\circ} \mathrm{C}$. Successive additions of concentrated $\mathrm{HNO}_{3}$ and heating are performed until no reaction with the sample was noted. The sample was then allowed to digest for two hours at $95^{\circ} \mathrm{C}$. The sample was removed and allowed to cool, $30 \% \mathrm{H}_{2} \mathrm{O}_{2}$ was added to the sample, and the temperature was increased to $95^{\circ} \mathrm{C}$. Successive addition of $\mathrm{H}_{2} \mathrm{O}_{2}$ and heating are performed until no reaction was noticed. The sample was allowed to digest for 2 hours at $95^{\circ} \mathrm{C}$. After cooling, the solution was filtered through a Whatman 41

(a) Personal communication from WI Winters (CH2M HILL) to the authors, December 22, 2003. 
filter to remove any insoluble particles and brought to a final volume of $50 \mathrm{~mL}$ with DDI water. Samples were filtered through a $0.45-\mu \mathrm{m}$ pore-size syringe filter prior to analysis. The limited quantity of the insoluble fractions and the inability to remove this material from the filter media precluded their characterization.

Table 2.2 lists the digestion factors (wet solid-to-solution ratios) for the samples of C-106 sludge solids used for the $\mathrm{KOH}-\mathrm{KNO}_{3}$ fusion treatments and acid digestion. These factors were calculated from the wet weight of sludge material divided by the volume of extracting solution. The digestion factors were then multiplied by the percent solids, as determined from moisture content analysis, to convert to a dry weight basis. All EPA acid-digestion and fused-sample solutions were filtered using $0.45-\mu \mathrm{m}$ poresize syringe filters prior to analysis. The dissolved concentrations and the total beta and total alpha activities for the filtered solutions were then analyzed by a combination of methods, including ICP-MS, inductively coupled plasma-optical emission spectroscopy (ICP-OES), and several radiochemical analytical techniques. These analytical methods are described in Lindberg and Deutsch (2003).

Table 2.2. Digestion Factors for Samples of Tank C-106 Residual Sludge Solids Used for the EPA Acid Digestion and $\mathrm{KOH}-\mathrm{KNO}_{3}$ Fusion Treatments

\begin{tabular}{|c|c|c|}
\hline Treatment & Sample Number & $\begin{array}{l}\text { Dry Weight Corrected } \\
\text { Digestion Factor }(\mathrm{g} / \mathrm{L})\end{array}$ \\
\hline \multirow{4}{*}{$\mathrm{KOH}-\mathrm{KNO}_{3}$ fusion } & 404 & 2.0880 \\
\hline & 404 DUP & 1.8505 \\
\hline & 405 & 2.5348 \\
\hline & 405 DUP & 2.1843 \\
\hline \multirow{4}{*}{ EPA acid digestion } & 404 & 4.0515 \\
\hline & 404 DUP & 3.4368 \\
\hline & 405 & 4.6821 \\
\hline & 405 DUP & 3.8920 \\
\hline
\end{tabular}

\subsection{XRD Analysis}

Crystalline phases present in the unleached (as-received), 1-month and 82-day water-leached, and hydrofluoric (HF)-extracted sludge samples 404 and 405 from tank C-106 were characterized by standard powder XRD techniques. The water leach and selective extraction tests are described in Sections 2.5 and 2.6 , respectively. Because the sludge materials were highly radioactive, dispersible powders, it was necessary to prepare the XRD mounts of these samples inside a fumehood regulated for handling radioactive materials. Sludge samples were prepared for XRD analysis by placing milligram quantities of each sample into a mixture of water and collodion solution. The collodion solution consists of $2 \%$ nitrocellulose dissolved in amyl acetate, and is an X-ray amorphous, viscous binder commonly used to make random powder mounts for XRD when only a limited amount of sample is available. Apparent shifts in $d$-spacings (i.e., measured $2 \theta$ angles) were observed in some of the XRD patterns for the water leached and HF-extracted sludge samples. To determine if these shifts were due to a minor misalignment of the mounted sample or variations in the composition of one or more of the crystalline solids detected by XRD, mounts of the 82-day water leached samples were also prepared with the addition of trace 
quantities of reference-material corundum powder $\left(\alpha-\mathrm{Al}_{2} \mathrm{O}_{3}\right.$, alumina) [National Institute of Standards and Technology Standard Reference Material (NIST SRM) 676] to each sample slurry. The corundum particles in each sample provided an internal $2 \theta$ standard for each XRD pattern. The measured patterns for the sludge samples analyzed with corundum confirmed that the observed peak shifts were due to slight misalignments of the mounted samples. Based on these results, $2 \theta$ corrections were estimated using $\mathrm{JADE}^{\circledR} \mathrm{XRD}$ processing software and applied to the XRD patterns for the water-leached and HF-extract sludge samples.

Using a pipette, each slurry was transferred onto a circular-shaped platform (1-cm diameter) and placed on top of the post located on the base inside a disposable XRD specimen holder (Figure 2.3). This specimen holder was designed specifically for safe handling of dispersible powders containing highly radioactive or hazardous materials (Strachan et al. 2003). After allowing samples to air dry overnight, the holder was assembled and a piece of Kapton ${ }^{\circledR}$ film was placed between the cap and the retainer. The holder was sealed with wicking glue and removed from the fumehood.

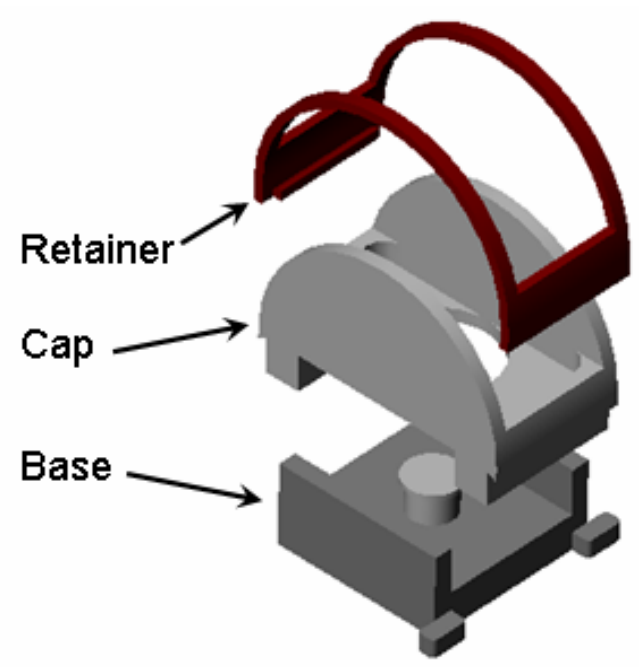

Figure 2.3. Exploded Schematic View of the XRD Sample Holder (Kapton ${ }^{\circledR}$ film not shown)

Each sample was analyzed using a Scintag XRD unit equipped with a Pelter thermoelectricallycooled detector and a copper X-ray tube. The diffractometer was operated at $45 \mathrm{kV}$ and $40 \mathrm{~mA}$. Individual scans were obtained from 2 to $65^{\circ} 2 \theta$ with a dwell time of 4 and 14 seconds. Scans were collected electronically and processed using the $\mathrm{JADE}^{\circledR}$ XRD pattern-processing software.

Krupka et al. (2004) prepared and analyzed by XRD a sample consisting of only a dry film of the collodion solution so that its contribution relative to the background signals of the XRD patterns for the sludge samples could be quantified. The resulting XRD pattern for the collodion solution film is shown in Figure 2.4. The most obvious feature of this diffraction pattern is the broad peak positioned between $10^{\circ}$ and $30^{\circ} 2 \theta$. The symmetry of this peak is characteristic of those resulting from the XRD of amorphous (noncrystalline) material. Although subtracting the collodion background from sludge XRD patterns allows for better phase matching, this process may eliminate minor reflections and inconspicuous features of a pattern. Therefore, each as-measured XRD pattern was examined before and after background subtraction to ensure that the integrity of the pattern was maintained. For background subtraction, 
the $\mathrm{JADE}^{\circledR}$ software provides the user with control over the selection of background-subtraction points. This process allows a better fit to $2 \theta$ regions under broad reflections, such as those resulting from amorphous materials. On average, 30 to 40 background points were selected from each XRD pattern, and a cubic-spline curve was then fit through each set of points. Adjustments to this curve were made by selecting additional background points in regions of a pattern that were difficult to fit. Once a wellmatched curve was fitted to a pattern, the background was subtracted from each as-measured XRD pattern, resulting in a smooth tracing.

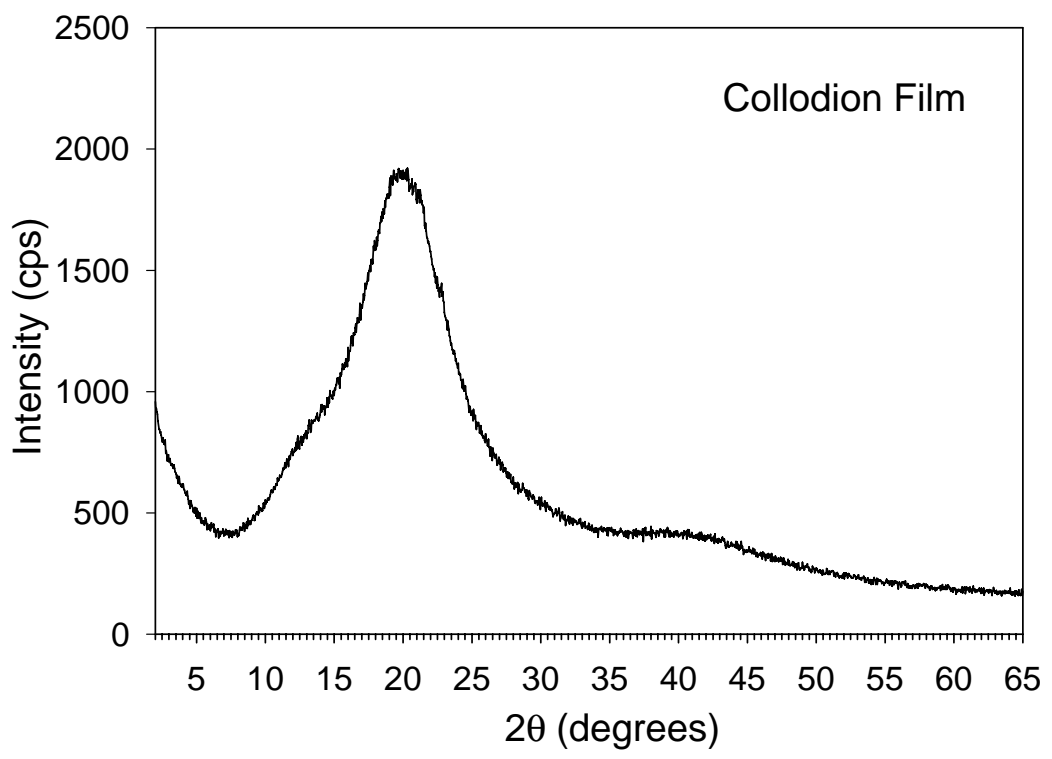

Figure 2.4. XRD Pattern for Collodion Film Measured in the Absence of any Sludge Material (from Krupka et al. 2004)

Identification of the mineral phases in the background-subtracted patterns was based on a comparison of the XRD patterns measured for the sludge samples with the mineral powder diffraction files (PDFTM) published by the Joint Committee on Powder Diffraction Standards (JCPDS) International Center for Diffraction Data (ICDD). As a rule of thumb, a crystalline phase must be present at greater than $5 \mathrm{wt} \%$ of the total sample mass (greater than $1 \mathrm{wt} \%$ under optimum conditions) to be readily detected by XRD. In general, the measured peak intensities depend on several factors, including the combined mass of each crystalline phase in the sample. Due to the physical characteristics of these tank sludge samples such as high radioactivity, high dispersibility, and variable moisture content, the mass of tank sludge combined with the collodion solution for each XRD mount could not be controlled or easily determined. Dissimilarities in mineral segregation (settling) resulting from the different densities of minerals mixed with the collodion solution and associated effects on relative peak intensities also influence the overall pattern intensity. The combined effect of these factors could have some effect on the characteristic mineral peak intensities, which precluded quantitative comparisons of peak intensities for equivalent reflections in background-subtracted XRD patterns for different sludge samples. 


\subsection{SEM/EDS Analysis}

The morphologies, sizes, surface textures, and compositions of phases present in the unleached (as-received), 1-month and 82-day water leached, and HF-extracted sludge samples 404 and 405 from tank C-106 were characterized by SEM/EDS. Two or three mounts were prepared of each sample to compensate for the possibility that one or more less-than-optimum mounts of a sample might occur, thus improving the likelihood of obtaining representative SEM images of each sample. The mounts used for SEM/EDS consisted of double-sided carbon tape attached to standard aluminum mounting stubs. For each mount, small aliquots of each sludge sample were placed on the exposed upper surface of the carbon tape using a micro spatula. Each mount was then coated with carbon using a vacuum sputter-coater to improve the conductivity of the samples and thus the quality of the SEM images and EDS signals.

A JEOL JSM-840 SEM was used for high-resolution imaging of micrometer/submicrometer-sized particles from the sludge samples. The SEM system is equipped with an Oxford Links ISIS 300 EDS that was used for qualitative element analysis. Operating conditions consisted of 10 to $20 \mathrm{keV}$ for SEM imaging and $20 \mathrm{keV}, 100$ live seconds ${ }^{(\mathrm{a})}$ for the EDS analyses. The EDS analyses of particles are limited to elements with atomic weights heavier than boron. Photomicrographs of high-resolution secondary electron (SE) images and backscattered electron (BSE) images were obtained as digital images and stored in electronic format. To help identify particles that contain elements with large atomic numbers, such as uranium, the SEM was typically operated in the BSE mode. Secondary electrons are low-energy electrons ejected from the probed specimen as a result of inelastic collisions with beam electrons, whereas backscattered electrons are primary electrons emitted as a result of elastic collisions. Backscattered electron emission intensity is a function of the element's atomic number - the larger the atomic number, the brighter the signal. Backscattered electron images are obtained in exactly the same way as secondary electron images.

The SEM micrographs included in the main body of this report (Section 3.6) were selected because they show typical morphologies, sizes, and surface textures of particles in the sludge subsample mounts. The entire area of each SEM mount was examined by SEM at low magnification (typically 50 to 100x) to identify those particles and surface features that were typical or unusual for the sample. During this examination, SEM micrographs were recorded at low magnification (e.g., 100x) for typically two to four areas of the mount to show a general perspective of the sizes, types, and distributions of particles that make up the SEM mount. Within these imaged regions, additional SEM micrographs were recorded of several particles at greater magnifications to provide a more detailed representation of the particles' characteristics, and selected points on these particles then analyzed by EDS. Depending on the perceived importance of such particles, regions on these particles were sometimes analyzed by SEM and EDS at even greater magnifications.

All of the SEM micrographs and EDS spectra determined for sludge samples 404 and 405 from tank C-106 are shown in Appendices B (unleached [as-received] sludge), C (1-month and 82-day water leached sludge), and D (HF Stage I extracted). The name of each digital image file, sample identification

(a) Live time is when (real time less dead time) the EDS system is available to detect incoming X-ray photons. Dead time is the portion of the total analyzing time that is actually spent processing or measuring X-rays. While each X-ray pulse is being measured, the system cannot measure another X-ray that may enter the detector and is, therefore, said to be "dead." 
number, and a size scale bar are given, respectively, at the bottom left, center, and right of each SEM micrograph in this report. Micrographs labeled BSE near the digital image file name indicate that the micrograph was collected with BSE. Areas outlined by a white or black dotted-line squares or particles indicated by arrows in a micrograph designate sample material that is imaged at higher magnification and typically shown in the next figure of the series for that subsample.

Areas labeled "eds" in SEM micrographs in this report indicate areas of particles for which EDS spectra were recorded. Compositions determined by EDS are qualitative and have large uncertainties resulting from alignment artifacts caused by the variable sample and detector configurations that exist when different particles are imaged by SEM.

After the studies and analyses of tank C-106 sludge had been completed and presented in draft form, the instrument hardware and software for the JEOL JSM-840 SEM used for the SEM/EDS analyses described above were upgraded to INCA Energy EDS System ${ }^{(a)}$ to automate the collection of EDS spectra over multi-micrometer-sized areas of an SEM-imaged sample. This upgrade permits the mapping of the spatial distributions over user-selected areas and/or lines of the relative concentrations of any userspecified element detectable by EDS. The INCAEnergy EDS System was demonstrated by using it to generate element distribution maps for a limited number of imaged areas of existing SEM mounts of unleached, 82-day water leached, and HF-extracted sludge samples. A few sets of element distribution maps are shown and discussed in Section 3.6, and the remainder of the generated element maps are shown in Appendix E.

\subsection{Tier 1 Tests}

Sludge samples were analyzed in a tiered approach similar to the one developed to investigate contaminant fate and transport issues associated with past single-shell tank leaks in the vadose zone. Such an approach allows for initial (Tier 1) screening of samples using relatively inexpensive analytical techniques. This is followed by an analysis of the data to determine the need for further testing (Tier 2). The Tier 1 tests are described in this section, and the Tier 2 tests are described in Sections 2.6 through 2.8. In addition to the analysis of the sludge samples, the sample of the liquid collected near the termination of retrieval activities was analyzed using many of the Tier 1 methods described in this section.

All laboratory activities were conducted in accordance with the requirements of Title 10, Code of Federal Regulations, Part 830.120 "Quality Assurance” and the Hanford Analytical Services Quality Assurance Requirements Document (HASQARD, DOE 1998). These requirements were implemented using PNNL's internal quality assurance (QA) plan. ${ }^{(b)}$ PNNL's QA Plan is based on the requirements of U.S. DOE Order 414.1A, the HASQARD, relevant elements of NQA-1, as well as recognized industry standards (e.g., EPA, ASTM, American National Standards Institute).

(a) Oxford Instruments, Concord, Massachusetts.

(b) PNNL. 2001. Conducting Analytical Work in Support of Regulatory Programs. Internal unpublished procedure, Pacific Northwest National Laboratory, Richland, Washington. 


\subsubsection{Moisture Content}

The moisture contents of the tank waste samples were measured to calculate dry weight concentrations for constituents in the waste. Dry weight concentrations provide a consistent measurement unit for comparison purposes that eliminates the effect of variable water content on sample concentrations.

Gravimetric water content of the waste material was determined using ASTM procedure D2216-98, Standard Test Method for Laboratory Determination of Water (Moisture) Content of Soil and Rock by Mass (ASTM 1998) with the following minor exceptions: 1) the volume of sample recommended was decreased due to radiological concerns and 2) the sample was dried at a lower oven temperature, $105^{\circ} \mathrm{C}$, for a longer period of time to prevent dehydration of the solids.

Sludge samples were placed in tarred containers, weighed, and dried in an oven until a constant weight was achieved, usually requiring 24 to 48 hours. The container was then removed from the oven, sealed, cooled, and weighed. All weighings were performed using a calibrated balance. The gravimetric water content is computed as the percentage change in soil weight before and after oven drying (i.e., [\{wet weight - dry weight $\} /$ dry weight $]$ ).

\subsubsection{Water Extracts}

Water-soluble inorganic constituents were determined using a DDI water extraction method. The extract was prepared by adding $30 \mathrm{~mL}$ of DDI water to 0.2 to $0.4 \mathrm{~g}$ of the residual sludge sample contained in a $50 \mathrm{~mL}$ polypropylene centrifuge tube. The centrifuge tube was sealed and briefly shaken by hand, and then placed on a mechanical orbital shaker for three time periods: 1 day, 2 weeks, and 1 month. After shaking for the predetermined time, the tube was placed in a centrifuge and spun at 4,000 rpm for 20 minutes. The supernatant was carefully decanted, filtered through $0.45-\mu \mathrm{m}$ pore size membrane, and analyzed. For this test, the first stage of the Periodic Replenishment test (Section 2.5.3) was used as the 1-day leach sample. The 2-week and 1-month leach tests used the same sludge sample. After 2 weeks, a $5 \mathrm{~mL}$ aliquot was removed and analyzed. The remaining $25 \mathrm{~mL}$ was allowed to shake for 2 more weeks and represents the 1-month leach sample. The 2-week and 1-month leach sample results were corrected for a $30 \mathrm{~mL}$ final volume. More details of the test method can be found in ASTM Procedure D3987-85, Standard Test Method for Shake Extraction of Solid Waste with Water (ASTM 1999).

\subsubsection{Periodic Replenishment Tests}

Periodic replenishment tests were conducted on samples of residual sludge from tank C-106. In this test, the water-leachate solution was periodically removed and replaced with an equal volume of fresh solution. This test was conducted to evaluate whether the solution concentration might be limited by the solubility of one or more solid phases. For these tests, the samples were contacted with DDI water a total of five times. The contact periods ranged from 2 to 3 days, the length of time between replenishment of water-leachate solutions. The sludge samples were prepared and handled in the same manner as the single-contact water extracts for each repetitive step. After the fifth stage, samples were replenished with fresh solutions and placed back on the shaker for evaluation of long-term solubilities. The contact time for one of these long-term samples was 43 days (Stage 6A). For the other long-term test the contact time 
was 82 days (Stage 6B). After these long-term tests, the samples were centrifuged and the supernatant carefully decanted and filtered through $0.45-\mu \mathrm{m}$ pore size membranes prior to analysis for the same constituents as the shorter-term tests.

\subsection{4 pH}

The $\mathrm{pH}$ values of the solutions were measured using a solid-state $\mathrm{pH}$ electrode and a $\mathrm{pH}$ meter calibrated with buffers bracketing the expected range. This measurement is similar to EPA Method 9040B (EPA 1995).

\subsubsection{Anion Analysis}

Anion analysis was performed using an ion chromatograph. Fluoride, acetate, formate, chloride, nitrite, bromide, nitrate, carbonate, sulfate, oxalate, and phosphate were separated on a Dionex AS17 column with a gradient elution technique from $1 \mathrm{mM}$ to $35 \mathrm{mM} \mathrm{NaOH}$ and measured using a conductivity detector. This methodology is similar to EPA Method 9056A (EPA 1994b) with the exception of using gradient elution with $\mathrm{NaOH}$.

\subsubsection{Cations and Trace Metals}

Major cation analysis (including $\mathrm{Al}, \mathrm{Ca}, \mathrm{Fe}, \mathrm{K}, \mathrm{Mg}, \mathrm{Mn}, \mathrm{Na}$, and $\mathrm{Si}$ ) was performed by ICP-OES EPA Method 6010B (EPA 1996b). Trace metals analysis (including Ag, As, Cd, Cr, Mo, Pb, Se, ${ }^{99} \mathrm{Tc}$, ${ }^{238} \mathrm{U},{ }^{239} \mathrm{Pu},{ }^{241} \mathrm{Am}$, and ${ }^{237} \mathrm{~Np}$ ) was performed by ICP-MS. This method is similar to EPA Method 6020 (EPA 1994a). For both ICP-OES and ICP-MS, high-purity calibration standards were used to generate calibration curves and to verify continuing calibration during the analysis. Multiple dilutions of selected samples (ranging from $3 \mathrm{x}$ to $100 \mathrm{x}$ ) were made and analyzed to investigate and correct for matrix interferences.

ICP-MS data is reported as total element concentration in terms of the specific isotope measured. The instrument software converts the concentration of an isotope of an element to the total concentration of the element based on the distribution of isotopes in the natural environment. For example, the total $\mathrm{Cr}$ concentration is reported from the raw count rates for both ${ }^{52} \mathrm{Cr}$ and ${ }^{53} \mathrm{Cr}$ based on taking the raw counts and dividing by the fraction of ${ }^{52} \mathrm{Cr}$ and ${ }^{53} \mathrm{Cr}$ found in nature to yield estimates of total $\mathrm{Cr}$ in the sample.

Standard ICP-MS operating software does not allow the direct input of the various element isotope concentrations as percent relative abundance. Under most circumstances, such as when only naturally occurring isotopes are present, the lack of correction for percent abundances creates no analytical quantification issues. However, care must be taken to interpret data under circumstances where the products of nuclear fission could be present. In this case, the greatest impact to the quantification of element data using standard calibration methods would be an overestimation of the concentration of an element in the presence of a fission product. For instance, Mo has seven natural isotopes with relative abundances ranging from 9.25 to $24.13 \%$. When the ICP-MS is calibrated, the counts per second measured at a specific mass are assigned a concentration by the operator. Unfortunately, the current manufacturer-supplied software does not account for the relative percent abundance of the seven Mo isotopes, nor will it allow the operator to assign different standard concentrations for the isotopes. In other words, for a $1 \mathrm{ng} / \mathrm{mL}$ calibration standard, $1 \mathrm{ng} / \mathrm{mL}$ must be assigned as the concentration for all 
seven Mo isotopes rather than $1 \mathrm{ng} / \mathrm{mL}$ multiplied by the percent abundance of the natural isotope. As mentioned in the preceding paragraph, this is not an issue when dealing only with natural systems, which will always have the relative abundance breakdown of 9.25 to $24.13 \%$ for the seven Mo isotopes.

However, if ${ }^{95} \mathrm{Mo},{ }^{97} \mathrm{Mo},{ }^{98} \mathrm{Mo}$, or ${ }^{100} \mathrm{Mo}$ fission products are present in the samples being analyzed, the actual fission product contribution to the total isotope concentration will be overestimated by a factor of 100 divided by the percent relative abundance of the natural isotope.

For future studies of tank sludge, the new Perkin Elmer Elan DRC II ICP-MS's software will be available. The Elan software allows the operator to assign different concentration values for each isotope of a respective element. This feature will enable user calibration based on the percent relative abundance of the natural isotopes. Measurement in this manner will permit the determination of individual masses without corrections for natural abundance. As the new ICP-MS is brought online, this technique will be tested with the objective that all subsequent calibration will be the direct quantification of total isotope concentrations. This will aid in the interpretation and possible quantification of fission products present in tank sludge.

\subsubsection{Alkalinity}

The sample alkalinity was measured by standard titration. A volume of standardized sulfuric acid $\left(\mathrm{H}_{2} \mathrm{SO}_{4}\right)$ was added to the sample to an endpoint of $\mathrm{pH} 8.3$ and then an endpoint of $\mathrm{pH} 4.5$. The volume of $\mathrm{H}_{2} \mathrm{SO}_{4}$ needed to achieve each endpoint is used to calculate the phenolphthalein $\left(\mathrm{OH}^{-}+\mathrm{CO}_{3}{ }^{2-}\right)$ and total $\left(\mathrm{OH}^{-}+\mathrm{HCO}_{3}{ }^{-}+\mathrm{CO}_{3}{ }^{2-}\right)$ alkalinity as calcium carbonate $\left(\mathrm{CaCO}_{3}\right)$. The alkalinity procedure is similar to Standard Method 2320 B (Clesceri et al. 1998).

\subsection{8 $\quad{ }^{129} I$ Extraction and Analysis}

From a long-term risk standpoint, ${ }^{129}$ I is a key potential contaminant in residual Hanford tank waste. For this reason, its presence in the waste material and mobility in infiltrating water is of interest. Although ${ }^{129} \mathrm{I}$ is generally considered mobile as a dissolved constituent in water, small partition coefficients $(0.2$ to $1 \mathrm{~mL} / \mathrm{g})$ are typically calculated when its uptake is measured on Hanford sediments (Cantrell et al. 2003; Um et al. 2004). Therefore, it is imperative to identify an extraction method that will enable quantitative measurement of total iodine in solid samples such as tank waste. Previous research (Brown et al. 2004) has shown the potential applicability of water leaches and $\mathrm{KOH}: \mathrm{KNO}_{3}$ water fusions for the removal of iodide from solid samples spiked with ${ }^{129} \mathrm{I}$. The results from Brown et al. (2004) have led to the modification of the accepted PNNL internal procedure, to determine the ${ }^{129} \mathrm{I}$ concentration in sludge solids. In the case of ${ }^{129} \mathrm{I}$, the $\mathrm{KOH}-\mathrm{KNO}_{3}$ fusion-dissolution procedure (Section 2.2) was modified (steps 10 through 14 were omitted) to prevent volatilization of iodine. In this modified procedure, $0.3 \mathrm{~g}$ of the tank waste sludge material was mixed with $10 \mathrm{~mL}$ of a $20 \% \mathrm{KOH}$ and $2 \% \mathrm{KNO}_{3}$ solution as a fluxing agent in a $\mathrm{Zr}$ crucible. Several process spike samples, which were used to determine extraction efficiencies, were prepared by adding $0.05 \mu \mathrm{g}{ }^{129} \mathrm{I}$ to a crucible containing $\mathrm{KOH}-$ $\mathrm{KNO}_{3}$ solution (blank spike) or sample and $\mathrm{KOH}-\mathrm{KNO}_{3}$ solution (matrix spike). The crucible was then placed in a $95^{\circ} \mathrm{C}$ oven and allowed to evaporate to dryness, after which it was covered and transferred to a muffle furnace preheated to $550^{\circ} \mathrm{C}$. Fusion was accomplished by heating the sample-flux mixture for 60 minutes at $550^{\circ} \mathrm{C}$. After 60 minutes, the crucible was removed from the furnace and allowed to cool to 
ambient room temperature. The fused solid was then dissolved in DDI water. The resulting solution was transferred to a 50-mL centrifuge tube. The crucible was then triple-rinsed with DDI water, and these wash solutions were also added to the centrifuge tube. Up to $25 \mathrm{~mL}$ of 1:1 concentrated sulfuric acid:1 M sodium bisulfite was added to the centrifuge tube to dissolve any remaining solids that may have transferred from the crucible during solution transfer. The contents of the centrifuge were swirled to facilitate mixing/dissolution. Once dissolution was complete, the resulting solution was diluted to a total volume of $50 \mathrm{~mL}$ with DDI water (the final solution volume in the centrifuge tube was determined gravimetrically and corrected for solution density).

Table 2.3 lists the digestion factors (wet solid-to-solution ratios) for the samples of tank C-106 sludge solids used for the modified $\mathrm{KOH}-\mathrm{KNO}_{3}$ water fusion treatments to measure ${ }^{129} \mathrm{I}$. These factors were calculated from the wet weight of sludge material divided by the volume of extracting solution. The digestion factors were then multiplied by the percent solids, as determined from moisture content analysis, to convert to a dry weight basis. The fused sludge samples, as well as the samples from the oneday, single-contact water leach tests (Section 2.5.2), were analyzed for dissolved ${ }^{129}$ I concentrations using either a VG Elemental PQS ICP-MS or a Perkin Elmer Elan DRC II ICP-MS. Spectrasol CFA-C from Spectrasol, Inc. (Warwick, NY) was diluted in DDI water $(18 \mathrm{M} \Omega-\mathrm{cm})$ to create a $1 \%$ working solution.

Table 2.3. Digestion Factors for Samples of C-106 Sludge Solids Used for the Modified $\mathrm{KOH}-\mathrm{KNO}_{3}$ Water Fusion Treatment to Measure ${ }^{129} \mathrm{I}$

\begin{tabular}{|c|c|c|}
\hline Treatment & Sample Number & $\begin{array}{l}\text { Dry Weight Corrected } \\
\text { Digestion Factor }(\mathrm{g} / \mathrm{L})\end{array}$ \\
\hline \multirow{4}{*}{$\mathrm{KOH}-\mathrm{KNO}_{3}$ fusion } & 404 C-106 (Primary) & 3.888 \\
\hline & 404 C-106 DUP ${ }^{(\mathrm{a})}$ (Primary) & 3.414 \\
\hline & 405 C-106 (Duplicate) & 4.834 \\
\hline & 405 C-106 DUP ${ }^{(\mathrm{a})}$ (Duplicate) & 5.406 \\
\hline Matrix spike & 405 C-106 (Duplicate) MS & 6.627 \\
\hline
\end{tabular}

Calibration standards were prepared by diluting a $1 \mathrm{mg} / \mathrm{L}{ }^{129} \mathrm{I}$ certified stock standard (NIST, Gaithersburg, MD) into appropriate volumes of the $1 \%$ Spectrasol CFA-C solution containing $5 \mathrm{ng} / \mathrm{mL}$

${ }^{121} \mathrm{Sb}$ as the internal standard. Internal standards are chosen based on their proximity (atomic mass) to an element of interest and are used to verify instrument performance and correct for instrument drift. An independent calibration check standard was prepared from a $1 \mathrm{mg} / \mathrm{L}^{129} \mathrm{I}$ certified stock standard (Amersham, Piscataway, NJ) in 1\% Spectrasol CFA-C. One percent Spectrasol CFA-C was used to prepare instrument blanks and was used as the rinse solution throughout the run.

\subsubsection{Radioanalysis}

In addition to the radionuclides listed in Section 2.5.6 that were analyzed in solution by ICP-MS, shortlived radionuclides were analyzed by conventional counting methods as described in the following sections.

\subsubsection{Gamma Energy Analysis}

All samples for gamma energy analysis (GEA) were analyzed using 60\% efficient intrinsicgermanium gamma detectors. All germanium counters were efficiency calibrated for distinct geometries 
using mixed gamma standards traceable to the NIST. Direct solids, acid extracts, and water extracts were analyzed for gamma energy. Spectral analysis was conducted using libraries containing most mixedfission products, activation products, and natural decay products. Control samples were run throughout the analysis to ensure correct operation of the detectors. The controls contained isotopes with photo peaks spanning the full detector range and were monitored for peak position, counting rate, and full-width half-maximum. Details are found in PNNL internal procedure RRL-001. ${ }^{\text {(a.) }}$

\subsubsection{2 $\quad{ }^{90} \mathrm{Sr}$ Analyses}

Aliquots of filtered acid extracts, fusions, and water extracts were diluted in $8 \mathrm{M} \mathrm{HNO}_{3}$ and submitted for strontium separation and analysis by internal PNNL procedure PNL-RRL-003.2. ${ }^{\text {(b) }}$ A 0.1 to $5 \mathrm{~mL}$ aliquot of sample was spiked with ${ }^{85} \mathrm{Sr}$ tracer and passed through a SrSpec ${ }^{\circledR}$ column (Eichrom Technologies, Chicago) to capture Sr. The columns were washed with 10 column volumes $(20 \mathrm{~mL})$ of $8 \mathrm{M} \mathrm{HNO}_{3}$. The strontium was eluted from the SrSpec column into glass liquid scintillation vials using $15 \mathrm{~mL}$ of deionized water. The vials were placed under a heat lamp overnight to evaporate the water to dryness. A $15 \mathrm{~mL}$ Optifluor ${ }^{\circledR}$ scintillation cocktail was added to each vial. Gamma spectroscopy was used to determine the chemical yield from the added ${ }^{85} \mathrm{Sr}$ tracer. The samples were then analyzed by liquid scintillation counting (LSC) to determine the amount of ${ }^{90} \mathrm{Sr}$ originally present in the sludge sample. A matrix spike, a blank spike, a duplicate, and blanks were run with each sample set to determine the efficiency of the separation procedure as well as the purity of reagents.

\subsubsection{Gross Alpha and Beta Analysis}

Gross alpha and beta measurement were made on both the water and acid extracts. For each extract, $0.100 \mathrm{~mL}$ sample volume was placed in a $20-\mathrm{mL}$ liquid scintillation vial containing $15 \mathrm{~mL}$ of scintillation cocktail. ${ }^{(\mathrm{c})}$ The samples were then mixed and counted on a Wallace model 1415 liquid scintillation counter as prescribed in internal PNNL procedure AGG-RRL-002. ${ }^{\text {(d) }}$

\subsection{Selective Extraction Tests}

\subsubsection{Phase 1}

In order to identify the relative amount of potentially mobile contaminants $\left({ }^{99} \mathrm{Tc},{ }^{238} \mathrm{U}\right.$, and $\left.\mathrm{Cr}\right)$ that may be sequestered within the three metal oxyhydroxide solids ( $\mathrm{Mn}, \mathrm{Al}$, and $\mathrm{Fe}$ ), the following sequence of selective extractions was conducted. The standard $0.3 \mathrm{~g}$ of residual sludge per $30 \mathrm{~mL}$ of solution was

(a) RRL-001, Gamma Energy Analysis, Operation, and Instrument Verification using Genie2000 Support Software, unpublished PNNL Technical Procedure, Pacific Northwest National Laboratory, Richland, Washington.

(b) PNL-RRL-003.2. 2000. Tc99 and Sr90 Analysis using Eichrom TEVA-spec and Sr-spec resin. Technical Procedure, Pacific Northwest National Laboratory, Richland, Washington.

(c) The scintillation cocktail used is Packard Optifluor, which is based on the high flash-point solvent LAB (Linear Alkylbenzene) (http://las.perkinelmer.com/catalog/Product.aspx?ProductId=6013199).

(d) AGG-RRL-002, Liquid Scintillation Counting and Instrument Verification using the 1400 DSA ${ }^{T M}$ Support Software, unpublished PNNL Technical Procedure, Pacific Northwest National Laboratory, Richland, Washington. 
changed to $0.1 \mathrm{~g}$ per $30 \mathrm{~mL}$ of solution for this set of extractions. For the manganese oxyhydroxide extractions, a much higher solution-to-solid ratio was required (2000:1). This series of selective extractions was done in duplicate for both samples 404 and 405 . The entire sequence of extractions is shown schematically in Figure 2.5.

Selective Extraction Stage 1. $30 \mathrm{~mL}$ of DDI water was added to between 0.1 to $0.14 \mathrm{~g}$ of sample 404 or 405 sludge material. The samples were placed on a shaker table for 24 hours, then removed and centrifuged. The supernatant was removed and stored for later analysis. This step was performed to remove readily soluble components (i.e., salts) from the sludge.

Selective Extraction Stage 2. $200 \mathrm{~mL}$ of $0.1 \mathrm{M} \mathrm{NH}_{2} \mathrm{OH}-\mathrm{HCl}(\mathrm{pH}$ 3.6) was added to each sample and placed on the shaker table for 2 hours. The samples were removed and centrifuged. The supernatant was then removed and stored for later analysis. This step removes the Mn oxyhydroxides and associated contaminants from the sludge.

Selective Extraction Stage 3 and 4. Two successive extraction steps consisting of a $0.01 \mathrm{M} \mathrm{HF}$ acid/0.01 M sodium fluoride buffer ( $\mathrm{pH}$ approximately 3.2 ) were performed on the solids. The contact time on the shaker table was 2 hours. The samples were centrifuged and the supernatant removed after each stage and stored for analysis. These steps were performed to remove $\mathrm{Al}$ oxyhydroxides and associated contaminants.

Selective Extraction Stage 5. The remaining solids were transferred from the centrifuge cones to $50 \mathrm{~mL}$ Griffin beakers and contacted with an $8 \mathrm{~N} \mathrm{HNO}_{3}$ solution. The solids and solutions were heated to $95^{\circ} \mathrm{C}$. Over the subsequent 2 hours, two $1-\mathrm{mL}$ aliquots of concentrated $\mathrm{HNO}_{3}$ were added to the slurries. The samples were allowed to cool and brought to a $30-\mathrm{mL}$ final volume. The solution was filtered through a $0.45-\mu \mathrm{m}$ syringe filter and the filtrate stored for later analysis.

The solutions from each stage were analyzed for ${ }^{99} \mathrm{Tc},{ }^{238} \mathrm{U}, \mathrm{Cr}, \mathrm{Mn}, \mathrm{Al}$, and $\mathrm{Fe}$. The $\mathrm{pH}$ was also measured on the extract solutions prior to and following each extraction. Extra samples were run so that the solids after the hydroxyl amine hydrochloride and the second fluoride buffer stages could be saved for analysis by XRD and SEM/EDS.

\subsubsection{Phase 2}

A second series of extractions was conducted to compare the HF/NaF buffer solution extraction with a hot concentrated $\mathrm{NaOH}$ extraction method for selective removal of $\mathrm{Al}$ oxyhydroxides. This set of extractions was conducted on both samples 404 and 405 in duplicate. Phase 2a was the HF/NaF buffer solution extraction, and the Phase $2 \mathrm{~b}$ was the $\mathrm{NaOH}$ extraction method. 


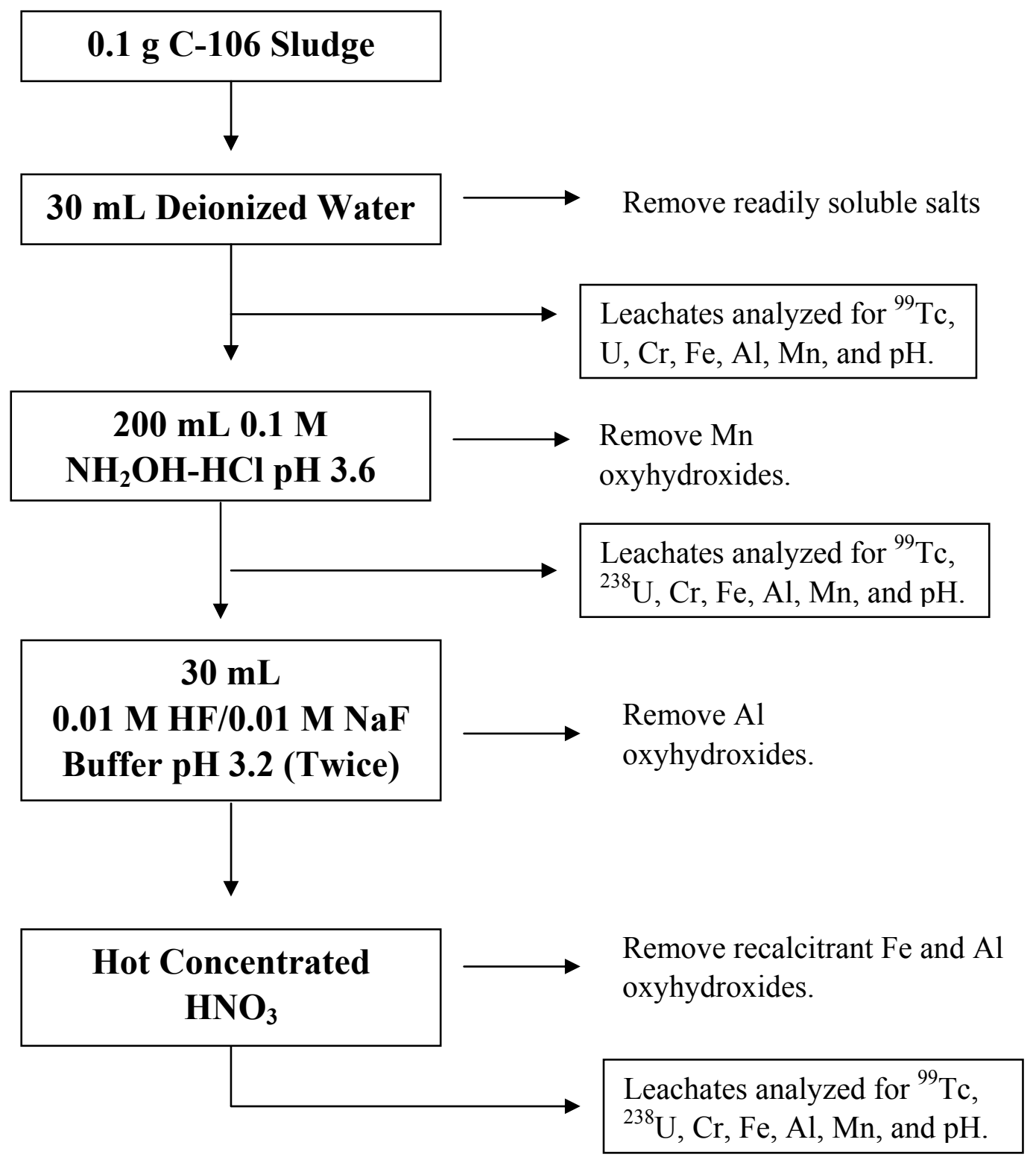

Figure 2.5. Phase 1 Selective Extraction Sequence Used to Determine Phase Associations of ${ }^{99} \mathrm{Tc},{ }^{238} \mathrm{U}$, and $\mathrm{Cr}$ in Tank C-106 Sludge 


\subsubsection{Phase 2a}

Selective Extraction Stage 1. $30 \mathrm{~mL}$ DDI water was added to 0.3 to $0.33 \mathrm{~g}$ of sludge samples 404 and 405. The samples were placed on a shaker table and extracted for 24 hours. The samples were centrifuged, and the supernatant was discarded. This step removed the readily soluble constituents.

Selective Extraction Stage 2 and 3. Two successive extractions consisting of $0.01 \mathrm{M} \mathrm{HF}$ acid/0.01 M NaF buffer ( $\mathrm{pH}$ approximately 3.2) were performed on the solids. The contact time on the shaker table was 2 hours for each extraction. The slurry was then centrifuged and the supernatant removed after each stage and stored for analysis. These steps were intended to selectively remove Al oxyhydroxide.

Each of the extract solutions was analyzed for ${ }^{99} \mathrm{Tc},{ }^{238} \mathrm{U}, \mathrm{Mn}, \mathrm{Cr}, \mathrm{Al}$, and Fe.

\subsubsection{Phase 2b}

Selective Extraction Stage 1. $30 \mathrm{~mL}$ DDI water was added to 0.1 to $0.12 \mathrm{~g}$ of sludge samples 404 or 405 to remove readily soluble constituents. The samples were placed on a shaker table and extracted for 24 hours. The samples were centrifuged, and the supernatant was discarded. This step removed the readily soluble constituents.

Selective Extraction Stage 2. The sludge materials were rinsed into $6 \mathrm{~mL}$ distillation vessels with DDI water and placed in a drying oven. The solids were allowed to come to dryness and $5 \mathrm{ml}$ of $3 \mathrm{M} \mathrm{NaOH}$ was added to each vessel. Cooling columns were attached and the sludge samples were heated to $95^{\circ} \mathrm{C}$ for 5 hours. The resulting solution was removed and brought to a final volume of $10 \mathrm{~mL}$ and stored for later analysis.

Selective Extraction Stage 3. Five $\mathrm{mL}$ of fresh $3 \mathrm{M} \mathrm{NaOH}$ was added to each vessel and heated to $95^{\circ} \mathrm{C}$ for 11 days. The solution was removed and brought to a final volume of $10 \mathrm{~mL}$ and stored for later analysis.

The solutions from each stage were analyzed for ${ }^{238} \mathrm{U}, \mathrm{Cr}, \mathrm{Mn}, \mathrm{Al}$, and Fe. 


\subsection{Laboratory Results}

This section provides the results of the tests conducted on sludge and liquid samples from tank C-106. The discussion begins with sludge composition by fusion and acid digestions in Section 3.1. Section 3.2 presents ${ }^{129} \mathrm{I}$ extraction and measurement results, and Section 3.3 provides the results of the analysis of the liquid sample collected during final retrieval operations. Section 3.4 contains the results of batch waterleaching tests, including single-contact and periodic replenishment methods. X-ray diffraction and SEM/EDS analyses are described in Sections 3.5 and 3.6, while Section 3.7 discusses selective extractions.

\subsection{Sludge Composition}

An important component of calculating contaminant release rates is an accurate measurement of the total concentration of contaminants in the source material. As described in Section 2.2, the total metal and radionuclide concentrations of the sludge samples were measured using two methods (fusion and acid digestions). The results of these analyses are described in this section. The anionic (nonmetal) composition of the sludge was estimated by water extraction as part of the Tier 1 analyses (Section 2.5.2). The results are discussed in Section 3.4.1.

An insoluble solid representing a very small fraction of the original mass of the sludge samples was observed at the end of the fusion preparations and acid digestions for both samples 404 and 405 . The residuals of both preparations consisted of very fine rust-colored particles. Because the insoluble fractions are not a significant amount of the total masses of the sludge, their presence is not expected to have a large impact on the measured total element compositions of the sludge.

Concentrations listed in parentheses in the tables are defined as less than the estimated quantitation limit (EQL) but greater than a zero instrument signal. These values are reported for informational purposes only. They may reflect actual concentrations that are real but have larger associated uncertainties than values above the EQL or they may reflect values that were calculated from the instrument's background signal and are not representative of actual sludge composition. The EQL of an element is determined by analyzing a suite of continuing calibration verification (CCV) standards at the beginning and end of each analytical run. The lowest CCV standard that is within $\pm 10 \%$ of its certified value is multiplied by the dilution factor for the sample to determine the EQL for the element for the particular analytical run. The EQL may vary with each analysis depending on sample matrix, dilution factors, and instrument performance. 
Concentrations listed as less than $(<)$ values in the tables refer to instrument measurements that are less than zero. In these instances, the reported analyte concentration is assigned a value of " $<\mathrm{EQL}$ " using the EQL value appropriate for that particular analyte and set of analytical conditions.

The following discussion of element concentrations of the sludge is organized in terms of the analytical method used to measure concentrations in the solution extract. These methods were ICP-OES, ICP-MS, GEA, wet chemical separations and ICP-MS (actinides), wet chemical separations and liquid scintillation $\left({ }^{90} \mathrm{Sr}\right)$, and gross alpha/beta analysis. For the tables, the solution concentrations have been converted from a per-liter basis to a dry sludge mass basis. Each table provides results from the fusion and acid digestion methods. Table 3.1 lists the moisture contents (relative to total sludge mass) of the $\mathrm{C}-106$ sludge samples used for the fusion and acid digestions. These values are used with the digestion factors (Table 2.2) to convert the solution analyses of the extracts from the treatments to dry weight solid concentrations. The moisture contents of the sludge samples ranged from 42.2 to $58.9 \%$, suggesting that the samples were completely water saturated.

Tables 3.2 through 3.4 contain the results of ICP-OES analyses of the sludges. Because K-containing compounds were used as the fluxing agent for the fusion technique, $\mathrm{K}$ concentrations are not reported in Table 3.3 for the fusion analysis. The metals detected above their respective EQLs were Al, Mn, Na, Fe, $\mathrm{Ca}, \mathrm{Pb}, \mathrm{Mg}, \mathrm{Ni}$, and $\mathrm{Ba}$. Figure 3.1 shows the concentrations of the major metals in the sludge based on fusion analyses of the primary and duplicate samples. The elements present in highest concentrations in the samples with their maximum values in units of $\mu \mathrm{g} / \mathrm{g}$ are $\mathrm{Al}\left(1.49 \times 10^{5}\right), \mathrm{Mn}\left(1.23 \times 10^{5}\right), \mathrm{Na}(6.17 \mathrm{x}$ $\left.10^{4}\right), \mathrm{Fe}\left(4.7 \times 10^{4}\right)$, and $\mathrm{Ca}\left(3.95 \times 10^{4}\right)$. For the metals, there is some variability in concentrations between the fusion method and the EPA acid digestion technique. The fusion method gave higher concentrations of the major metals by 10 to $40 \%$ compared to the concentrations measured by acid digestion.

Table 3.1. Moisture Contents of Tank C-106 Sludge Samples 404 and 405 [(wet wt - dry wt)/dry wt]

\begin{tabular}{||l|c||}
\hline \multicolumn{1}{|c|}{ Sample Number } & Moisture Content \\
\hline \hline 404 & $58.86 \%$ \\
\hline 404 DUP & $56.11 \%$ \\
\hline 405 & $42.19 \%$ \\
\hline 405 DUP & $44.88 \%$ \\
\hline DUP = Duplicate sample. \\
\hline
\end{tabular}


Table 3.2. Concentrations of Elements Measured by ICP-OES per Gram of Dry Sludge

\begin{tabular}{|c|c|c|c|c|c|c|c|c|c|c|}
\hline \multirow{2}{*}{ Sample Number } & Al & As & B & Ba & Be & $\mathbf{B i}$ & Ca & Cd & Co & $\mathbf{C r}$ \\
\hline & \multirow{2}{*}{\multicolumn{10}{|c|}{ 1 }} \\
\hline \multicolumn{9}{|c|}{ KOH-KNO ${ }_{3}$ Fusions } & & \\
\hline 404 & $1.32 \mathrm{E}+05$ & $(6.03 \mathrm{E}+02)$ & $(7.15 \mathrm{E}+02)$ & $1.49 \mathrm{E}+03$ & $(6.47 \mathrm{E}+01)$ & $(3.5 \mathrm{E}+02)$ & $3.75 \mathrm{E}+04$ & $(2.74 \mathrm{E}+02)$ & $(1.0 \mathrm{E}+02)$ & $(8.61 \mathrm{E}+02)$ \\
\hline 404 DUP & $1.49 \mathrm{E}+05$ & $<6.76 \mathrm{E}+03$ & $(3.26 \mathrm{E}+02)$ & $(6.47 \mathrm{E}+02)$ & $(5.77 \mathrm{E}+01)$ & $(1.9 \mathrm{E}+02)$ & $3.95 \mathrm{E}+04$ & $(2.33 \mathrm{E}+02)$ & $<1.35 \mathrm{E}+03$ & $(8.20 \mathrm{E}+02)$ \\
\hline 405 & $1.23 \mathrm{E}+05$ & $<4.93 \mathrm{E}+03$ & $(2.64 \mathrm{E}+02)$ & $(5.87 \mathrm{E}+02)$ & $(3.87 \mathrm{E}+01)$ & $<4.93 \mathrm{E}+03$ & $3.75 \mathrm{E}+04$ & $(2.41 \mathrm{E}+02)$ & $<9.86 \mathrm{E}+02$ & $(9.77 \mathrm{E}+02)$ \\
\hline 405 DUP & $1.23 \mathrm{E}+05$ & $<5.72 \mathrm{E}+03$ & $(2.22 \mathrm{E}+02)$ & $1.39 \mathrm{E}+03$ & $(4.14 \mathrm{E}+01)$ & $<5.72 \mathrm{E}+03$ & $3.84 \mathrm{E}+04$ & $(2.41 \mathrm{E}+02)$ & $<1.14 \mathrm{E}+03$ & $(9.27 \mathrm{E}+02)$ \\
\hline 404 Avg & $1.40 \mathrm{E}+05$ & $<3.68 \mathrm{E}+03$ & $(5.20 \mathrm{E}+02)$ & $1.07 \mathrm{E}+03$ & $(6.12 \mathrm{E}+01)$ & $(2.7 \mathrm{E}+02)$ & $3.85 \mathrm{E}+04$ & $(2.54 \mathrm{E}+02)$ & $<7.27 \mathrm{E}+02$ & $(8.41 \mathrm{E}+02)$ \\
\hline $405 \mathrm{Avg}$ & $1.23 \mathrm{E}+05$ & $<5.33 \mathrm{E}+03$ & $(2.43 \mathrm{E}+02)$ & $9.89 \mathrm{E}+02$ & $(4.01 \mathrm{E}+01)$ & $<5.33 \mathrm{E}+03$ & $3.79 \mathrm{E}+04$ & $(2.41 \mathrm{E}+02)$ & $<1.07 \mathrm{E}+03$ & $(9.52 \mathrm{E}+02)$ \\
\hline \multicolumn{11}{|c|}{$\begin{array}{l}\text { EPA Acid Digestion } \\
\end{array}$} \\
\hline 404 & $8.21 \mathrm{E}+04$ & $(1.03 \mathrm{E}+02)$ & $(1.76 \mathrm{E}+02)$ & $1.03 \mathrm{E}+03$ & $(1.81 \mathrm{E}+01)$ & $<3.09 \mathrm{E}+03$ & $2.73 \mathrm{E}+04$ & $(2.35 \mathrm{E}+02)$ & $<6.17 \mathrm{E}+02$ & $(7.55 \mathrm{E}+02)$ \\
\hline 404 DUP & $7.80 \mathrm{E}+04$ & $<3.64 \mathrm{E}+03$ & $(9.50 \mathrm{E}+01)$ & $9.22 \mathrm{E}+02$ & $(2.28 \mathrm{E}+01)$ & $<3.64 \mathrm{E}+03$ & $1.07 \mathrm{E}+05$ & $(2.01 \mathrm{E}+02)$ & $<7.27 \mathrm{E}+02$ & $(6.47 \mathrm{E}+02)$ \\
\hline 405 & $8.84 \mathrm{E}+04$ & $<2.67 \mathrm{E}+03$ & $(6.47 \mathrm{E}+01)$ & $9.06 \mathrm{E}+02$ & $(1.52 \mathrm{E}+01)$ & $<2.67 \mathrm{E}+03$ & $2.49 \mathrm{E}+04$ & $(2.41 \mathrm{E}+02)$ & $<5.34 \mathrm{E}+02$ & $7.36 \mathrm{E}+02$ \\
\hline 405 DUP & $7.83 \mathrm{E}+04$ & $<3.21 \mathrm{E}+03$ & $(1.66 \mathrm{E}+02)$ & $7.97 \mathrm{E}+02$ & $(1.64 \mathrm{E}+01)$ & $<3.21 \mathrm{E}+03$ & $2.69 \mathrm{E}+04$ & $(2.41 \mathrm{E}+02)$ & $<6.42 \mathrm{E}+02$ & $(7.72 \mathrm{E}+02)$ \\
\hline 404 Avg & $8.01 \mathrm{E}+04$ & $<1.87 \mathrm{E}+03$ & $(1.35 \mathrm{E}+02)$ & $9.76 \mathrm{E}+02$ & $(2.05 \mathrm{E}+01)$ & $<3.36 \mathrm{E}+03$ & $6.71 \mathrm{E}+04$ & $(2.18 \mathrm{E}+02)$ & $<6.72 \mathrm{E}+02$ & $(7.01 \mathrm{E}+02)$ \\
\hline $405 \mathrm{Avg}$ & $8.33 \mathrm{E}+04$ & $<2.94 \mathrm{E}+03$ & $(1.15 \mathrm{E}+02)$ & $8.52 \mathrm{E}+02$ & $(1.58 \mathrm{E}+01)$ & $<2.94 \mathrm{E}+03$ & $2.59 \mathrm{E}+04$ & $(2.41 \mathrm{E}+02)$ & $<5.88 \mathrm{E}+02$ & $(7.54 \mathrm{E}+02)$ \\
\hline \multicolumn{11}{|c|}{$\begin{array}{l}\text { Avg = Average. } \\
\text { DUP = Duplicate. } \\
\text { EPA = U.S. Environmental Protection Agency. } \\
\text { ICP-OES = Inductively coupled plasma-optical emission spectroscopy. } \\
\mathrm{KNO}_{3}=\text { Potassium nitrate. } \\
\mathrm{KOH}=\text { Potassium hydroxide. }\end{array}$} \\
\hline
\end{tabular}


Table 3.3. Concentrations of Elements Measured by ICP-OES per Gram of Dry Sludge

\begin{tabular}{|c|c|c|c|c|c|c|c|c|c|c|}
\hline \multirow{2}{*}{ Sample Number } & $\mathrm{Cu}$ & $\mathbf{F e}$ & $\mathbf{K}$ & $\mathbf{L i}$ & $\mathbf{M g}$ & Mn & Mo & $\mathbf{N i}$ & $\mathbf{P}$ & $\mathbf{P b}$ \\
\hline & \multirow{2}{*}{\multicolumn{10}{|c|}{ 1 }} \\
\hline \multicolumn{9}{|c|}{$\begin{array}{ll}\mathrm{KOH}-\mathrm{KNO}_{3} \text { Fusions } \\
\end{array}$} & & \\
\hline 404 & $(8.51 \mathrm{E}+02)$ & $4.7 \mathrm{E}+04$ & NR & $<1.20 \mathrm{E}+05$ & $3.09 \mathrm{E}+03$ & $1.20 \mathrm{E}+05$ & $(3.46 \mathrm{E}+01)$ & $1.92 \mathrm{E}+03$ & $(7.82 \mathrm{E}+03)$ & $5.01 \mathrm{E}+03$ \\
\hline 404 DUP & $(5.96 \mathrm{E}+02)$ & $4.0 \mathrm{E}+04$ & NR & $<1.35 \mathrm{E}+05$ & $(2.25 \mathrm{E}+03)$ & $1.07 \mathrm{E}+05$ & $(2.14 \mathrm{E}+02)$ & $2.17 \mathrm{E}+03$ & $(7.22 \mathrm{E}+03)$ & $4.61 \mathrm{E}+03$ \\
\hline 405 & $(6.13 \mathrm{E}+02)$ & $4.4 \mathrm{E}+04$ & NR & $<9.86 \mathrm{E}+04$ & $2.21 \mathrm{E}+03$ & $1.21 \mathrm{E}+05$ & $<9.86 \mathrm{E}+02$ & $1.44 \mathrm{E}+03$ & $(7.85 \mathrm{E}+03)$ & $4.65 \mathrm{E}+03$ \\
\hline 405 DUP & $(6.92 \mathrm{E}+02)$ & $4.5 \mathrm{E}+04$ & NR & $<1.14 \mathrm{E}+05$ & $2.38 \mathrm{E}+03$ & $1.23 \mathrm{E}+05$ & $(7.43 \mathrm{E}+01)$ & $1.32 \mathrm{E}+03$ & $(7.88 \mathrm{E}+03)$ & $5.09 \mathrm{E}+03$ \\
\hline 404 Avg & $(7.23 \mathrm{E}+02)$ & $4.33 \mathrm{E}+04$ & NR & $<1.27 \mathrm{E}+05$ & $2.67 \mathrm{E}+03$ & $1.14 \mathrm{E}+05$ & $(1.24 \mathrm{E}+02)$ & $2.04 \mathrm{E}+03$ & $(7.52 \mathrm{E}+03)$ & $4.81 \mathrm{E}+03$ \\
\hline 405 Avg & $(6.53 \mathrm{E}+02)$ & $4.43 \mathrm{E}+04$ & NR & $<1.07 \mathrm{E}+05$ & $2.30 \mathrm{E}+03$ & $1.22 \mathrm{E}+05$ & $<5.30 \mathrm{E}+02$ & $1.38 \mathrm{E}+03$ & $(7.86 \mathrm{E}+03)$ & $4.87 \mathrm{E}+03$ \\
\hline \multicolumn{11}{|c|}{ EPA Acid Digestion } \\
\hline 404 & $(5.39 \mathrm{E}+02)$ & $3.80 \mathrm{E}+04$ & $(7.98 \mathrm{E}+02)$ & $<6.17 \mathrm{E}+04$ & $1.84 \mathrm{E}+03$ & $1.11 \mathrm{E}+05$ & $<6.17 \mathrm{E}+02$ & $5.57 \mathrm{E}+03$ & $(6.78 \mathrm{E}+03)$ & $5.0 \mathrm{E}+03$ \\
\hline 404 DUP & $(5.25 \mathrm{E}+02)$ & $3.41 \mathrm{E}+04$ & $(3.90 \mathrm{E}+03)$ & $<7.27 \mathrm{E}+04$ & $7.25 \mathrm{E}+03$ & $1.00 \mathrm{E}+05$ & $(3.63 \mathrm{E}+02)$ & $5.03 \mathrm{E}+03$ & $1.43 \mathrm{E}+04$ & $4.60 \mathrm{E}+03$ \\
\hline 405 & $(4.58 \mathrm{E}+02)$ & $3.79 \mathrm{E}+04$ & $<1.33 \mathrm{E}+04$ & $<5.34 \mathrm{E}+04$ & $1.75 \mathrm{E}+03$ & $1.09 \mathrm{E}+05$ & $<5.34 \mathrm{E}+02$ & $5.51 \mathrm{E}+03$ & $(6.11 \mathrm{E}+03)$ & $4.79 \mathrm{E}+03$ \\
\hline 405 DUP & $(4.3 \mathrm{E}+02)$ & $3.67 \mathrm{E}+04$ & $<1.61 \mathrm{E}+04$ & $<6.42 \mathrm{E}+04$ & $1.81 \mathrm{E}+03$ & $1.12 \mathrm{E}+05$ & $<6.42 \mathrm{E}+02$ & $5.38 \mathrm{E}+03$ & $(5.82 \mathrm{E}+03)$ & $4.91 \mathrm{E}+03$ \\
\hline 404 Avg & $(5.32 \mathrm{E}+02)$ & $3.61 \mathrm{E}+04$ & $2.35 \mathrm{E}+03$ & $<6.72 \mathrm{E}+04$ & $4.54 \mathrm{E}+03$ & $1.06 \mathrm{E}+05$ & $(4.90 \mathrm{E}+02)$ & $5.30 \mathrm{E}+03$ & $(1.05 \mathrm{E}+04)$ & $4.78 \mathrm{E}+03$ \\
\hline $405 \mathrm{Avg}$ & $(4.44 \mathrm{E}+02)$ & $3.73 \mathrm{E}+04$ & $1.47 \mathrm{E}+04$ & $<5.88 \mathrm{E}+04$ & $1.78 \mathrm{E}+03$ & $1.11 \mathrm{E}+05$ & $<5.88 \mathrm{E}+02$ & $5.45 \mathrm{E}+03$ & $(5.96 \mathrm{E}+03)$ & $4.85 \mathrm{E}+03$ \\
\hline \multicolumn{11}{|c|}{$\begin{array}{l}\text { Avg = Average. } \\
\mathrm{DUP}=\text { Duplicate sample. } \\
\mathrm{EPA}=\text { U.S. Environmental Protection Agency. } \\
\text { ICP-OES = Inductively coupled plasma-optical emission spectroscopy. } \\
\mathrm{KNO}_{3}=\text { Potassium nitrate. } \\
\mathrm{KOH}=\text { Potassium hydroxide. } \\
\mathrm{NR}=\text { Not Reported }-\mathrm{K} \text { is a major component of the fluxing agent using in the fusion analysi }\end{array}$} \\
\hline
\end{tabular}


Table 3.4. Concentrations of Elements Measured by ICP-OES per Gram of Dry Sludge

\begin{tabular}{|c|c|c|c|c|c|c|c|c|c|c|}
\hline \multirow{2}{*}{ Sample Number } & Se & $\mathrm{Sr}$ & Tl & $\mathbf{V}$ & Zn & $\mathbf{N a}$ & Si & $\mathbf{S}$ & Ti & $\mathbf{Z r}$ \\
\hline & \multicolumn{10}{|c|}{ " } \\
\hline \multicolumn{11}{|c|}{ KOH-KNO ${ }_{3}$ Fusions } \\
\hline 404 & $<1.20 \mathrm{E}+04$ & $(2.65 \mathrm{E}+02)$ & $<1.20 \mathrm{E}+07$ & $(3.49 \mathrm{E}+02)$ & $(2.38 \mathrm{E}+03)$ & $6.13 \mathrm{E}+04$ & $(1.93 \mathrm{E}+04)$ & $<2.39 \mathrm{E}+05$ & $(1.82 \mathrm{E}+02)$ & $(7.39 \mathrm{E}+01)$ \\
\hline 404 DUP & $<1.35 \mathrm{E}+04$ & $(2.10 \mathrm{E}+02)$ & $<1.35 \mathrm{E}+07$ & $(4.22 \mathrm{E}+02)$ & $(3.52 \mathrm{E}+03)$ & $6.17 \mathrm{E}+04$ & $(1.86 \mathrm{E}+04)$ & $<2.70 \mathrm{E}+05$ & $(1.17 \mathrm{E}+02)$ & $(7.28 \mathrm{E}+01)$ \\
\hline 405 & $<9.86 \mathrm{E}+03$ & $(2.80 \mathrm{E}+02)$ & $<9.86 \mathrm{E}+06$ & $(2.45 \mathrm{E}+02)$ & $(2.20 \mathrm{E}+03)$ & $5.77 \mathrm{E}+04$ & $(1.89 \mathrm{E}+04)$ & $<1.97 \mathrm{E}+05$ & $(2.02 \mathrm{E}+02)$ & $(4.24 \mathrm{E}+01)$ \\
\hline 405 DUP & $<1.14 \mathrm{E}+04$ & $(2.70 \mathrm{E}+02)$ & $<1.14 \mathrm{E}+07$ & $(2.32 \mathrm{E}+02)$ & $(2.35 \mathrm{E}+03)$ & $6.09 \mathrm{E}+04$ & $(1.95 \mathrm{E}+04)$ & $<2.29 \mathrm{E}+05$ & $(1.42 \mathrm{E}+02)$ & $(2.32 \mathrm{E}+01)$ \\
\hline 404 Avg & $<1.27 \mathrm{E}+04$ & $(2.38 \mathrm{E}+02)$ & $<1.27 \mathrm{E}+07$ & $(3.85 \mathrm{E}+02)$ & $(2.95 \mathrm{E}+03)$ & $6.15 \mathrm{E}+04$ & $(1.90 \mathrm{E}+04)$ & $<2.55 \mathrm{E}+05$ & $(1.49 \mathrm{E}+02)$ & $(7.33 \mathrm{E}+01)$ \\
\hline 405 Avg & $<1.07 \mathrm{E}+04$ & $(2.75 \mathrm{E}+02)$ & $<1.07 \mathrm{E}+07$ & $(2.39 \mathrm{E}+02)$ & $(2.27 \mathrm{E}+03)$ & $5.93 \mathrm{E}+04$ & $(1.92 \mathrm{E}+04)$ & $<2.13 \mathrm{E}+05$ & $(1.72 \mathrm{E}+02)$ & $(3.28 \mathrm{E}+01)$ \\
\hline \multicolumn{11}{|c|}{$\begin{array}{c}\text { EPA Acid Digestion } \\
\end{array}$} \\
\hline 404 & $<6.17 \mathrm{E}+03$ & $(4.11 \mathrm{E}+02)$ & $<6.17 \mathrm{E}+06$ & $(1.55 \mathrm{E}+02)$ & $(1.52 \mathrm{E}+03)$ & $4.39 \mathrm{E}+04$ & $(2.04 \mathrm{E}+03)$ & $<1.23 \mathrm{E}+05$ & $(4.71 \mathrm{E}+01)$ & $(1.13 \mathrm{E}+02)$ \\
\hline 404 DUP & $<7.27 \mathrm{E}+03$ & $7.75 \mathrm{E}+02$ & $<7.27 \mathrm{E}+06$ & $(1.81 \mathrm{E}+02)$ & $5.08 \mathrm{E}+04$ & $4.96 \mathrm{E}+04$ & $(1.28 \mathrm{E}+04)$ & $(4.44 \mathrm{E}+04)$ & $(4.89 \mathrm{E}+01)$ & $(5.27 \mathrm{E}+01)$ \\
\hline 405 & $<5.34 \mathrm{E}+03$ & $(3.90 \mathrm{E}+02)$ & $<5.34 \mathrm{E}+06$ & $(1.18 \mathrm{E}+02)$ & $(1.49 \mathrm{E}+03)$ & $5.00 \mathrm{E}+04$ & $(1.96 \mathrm{E}+03)$ & $(8.6 \mathrm{E}+01)$ & $(5.50 \mathrm{E}+01)$ & $(5.66 \mathrm{E}+01)$ \\
\hline 405 DUP & $<6.42 \mathrm{E}+03$ & $(3.97 \mathrm{E}+02)$ & $<6.42 \mathrm{E}+06$ & $(2.10 \mathrm{E}+02)$ & $(1.65 \mathrm{E}+03)$ & $4.34 \mathrm{E}+04$ & $(2.78 \mathrm{E}+03)$ & $<1.28 \mathrm{E}+05$ & $(4.33 \mathrm{E}+01)$ & $<6.42 \mathrm{E}+02$ \\
\hline 404 Avg & $<6.72 \mathrm{E}+03$ & $(5.93 \mathrm{E}+02)$ & $<6.72 \mathrm{E}+06$ & $(1.68 \mathrm{E}+02)$ & $(2.62 \mathrm{E}+04)$ & $4.67 \mathrm{E}+04$ & $(7.42 \mathrm{E}+03)$ & $<8.39 \mathrm{E}+04$ & $(4.80 \mathrm{E}+01)$ & $(8.26 \mathrm{E}+01)$ \\
\hline 405 Avg & $<5.88 \mathrm{E}+03$ & $(3.94 \mathrm{E}+02)$ & $<5.88 \mathrm{E}+06$ & $(1.64 \mathrm{E}+02)$ & $(1.57 \mathrm{E}+03)$ & $4.67 \mathrm{E}+04$ & $(2.37 \mathrm{E}+03)$ & $<6.43 \mathrm{E}+04$ & $(4.92 \mathrm{E}+01)$ & $(3.49 \mathrm{E}+02)$ \\
\hline \multicolumn{11}{|c|}{$\begin{array}{l}\text { Avg }=\text { Average. } \\
\text { DUP }=\text { Duplicate sample. } \\
\text { EPA }=\text { U.S. Environmental Protection Agency. } \\
\text { ICP-OES = Inductively coupled plasma-optical emission spectroscopy. } \\
\mathrm{KNO}_{3}=\text { Potassium nitrate. } \\
\mathrm{KOH}=\text { Potassium hydroxide. }\end{array}$} \\
\hline
\end{tabular}




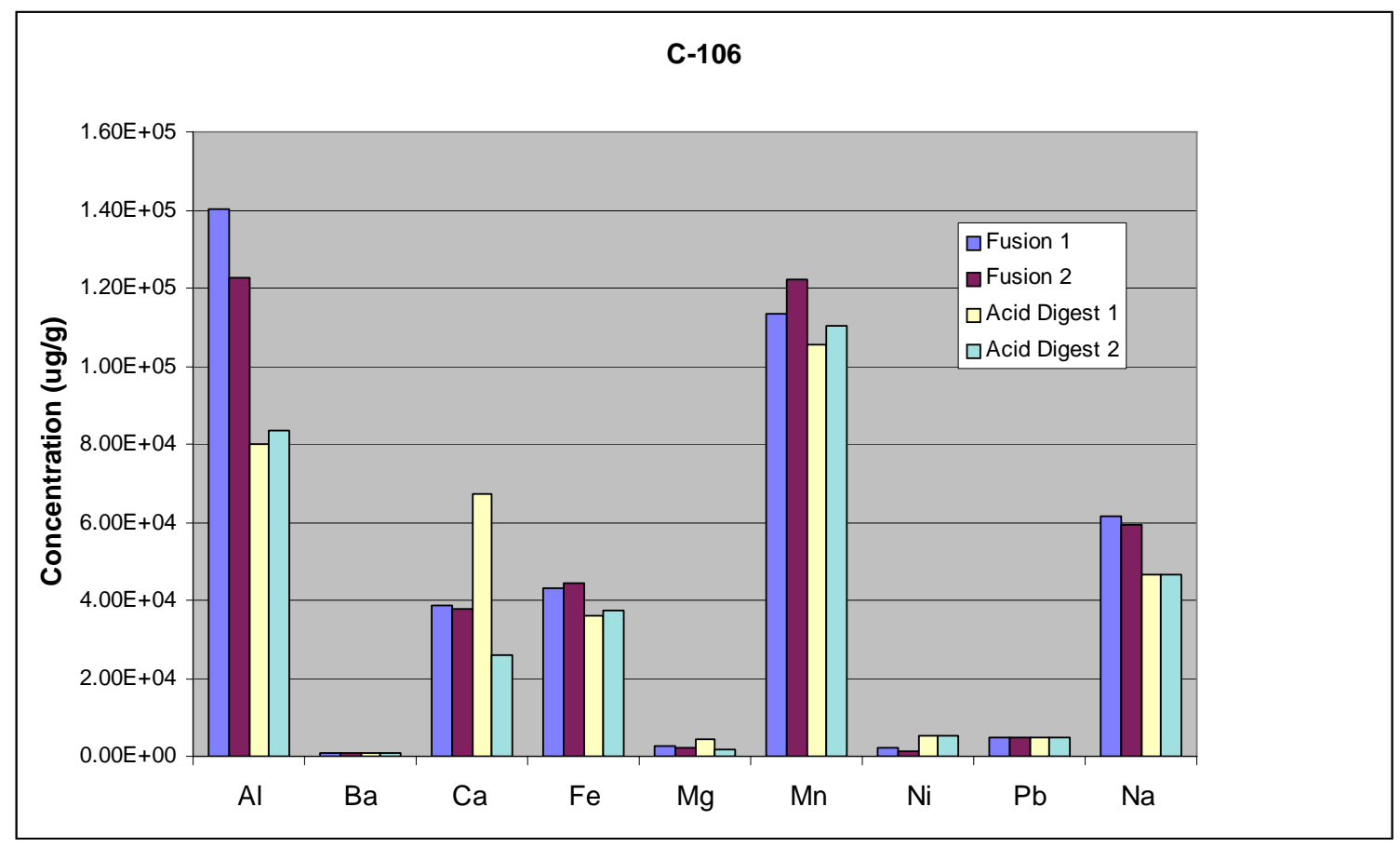

Figure 3.1. Metals Concentrations in Tank C-106 Sludge

The element concentrations in Tables 3.5 and 3.6 were derived from the ICP-MS analyses, which can obtain lower detection limits than ICP-OES for some metals and allow for measurement of trace metals. The elements listed in Tables 3.5 and 3.6 with concentrations greater than their EQLs are $\mathrm{Pb}, \mathrm{Cr}, \mathrm{Cd}$, and $\mathrm{Ru}$. The average $\mathrm{Pb}$ concentrations (based on ${ }^{208} \mathrm{~Pb}$ and ${ }^{208} \mathrm{~Pb}$ ) in the sludge measured by the fusion method were $4,560 \mu \mathrm{g} / \mathrm{g}$ and by EPA acid digestion $4,610 \mu \mathrm{g} / \mathrm{g}$, which are within $10 \%$ of the ICP-OES respective values of $4,840 \mu \mathrm{g} / \mathrm{g}$ and $4,820 \mu \mathrm{g} / \mathrm{g}$. The average $\mathrm{Cr}$ concentrations (based on ${ }^{52} \mathrm{Cr}$ and ${ }^{53} \mathrm{Cr}$ ) in the C-106 sludge measured by the fusion method were $699 \mu \mathrm{g} / \mathrm{g}$ and by EPA acid digestion $624 \mu \mathrm{g} / \mathrm{g}$. The average $\mathrm{Cd}$ concentrations (based on ${ }^{111} \mathrm{Cd}$ and ${ }^{114} \mathrm{Cd}$ ) measured by the fusion method were $290 \mu \mathrm{g} / \mathrm{g}$ and by EPA acid digestion $295 \mu \mathrm{g} / \mathrm{g}$. The average Ru concentrations (based on ${ }^{101} \mathrm{Ru},{ }^{102} \mathrm{Ru}$, and ${ }^{104} \mathrm{Ru}$ ) measured by the fusion method were $270 \mu \mathrm{g} / \mathrm{g}$ and by EPA acid digestion $163 \mu \mathrm{g} / \mathrm{g}$.

The sludge concentrations of ${ }^{99} \mathrm{Tc}$ and ${ }^{238} \mathrm{U}$ measured by ICP-MS are listed in Table 3.7. The ${ }^{99} \mathrm{Tc}$ concentration measured by the fusion method is in the range of 0.875 to $1.42 \mu \mathrm{g} / \mathrm{g}$ with an average value of $1.16 \mu \mathrm{g} / \mathrm{g}$. These values are similar to the range of 1.05 to $1.22 \mu \mathrm{g} / \mathrm{g}$ measured by the EPA acid digestion method with an average value of $1.14 \mu \mathrm{g} / \mathrm{g}$. The ${ }^{238} \mathrm{U}$ sludge concentration measured by the fusion method was in the range of 232 to $271 \mu \mathrm{g} / \mathrm{g}$ with an average value of $247 \mu \mathrm{g} / \mathrm{g}$. The ${ }^{238} \mathrm{U}$ sludge concentration measured by the acid digestion method was in the range of 297 to $327 \mu \mathrm{g} / \mathrm{g}$ with an average value of $311 \mu \mathrm{g} / \mathrm{g}$. 
Table 3.5. Concentrations of Elements Determined from ICP-MS Analysis per Gram of Dry Sludge

\begin{tabular}{|c|c|c|c|c|c|c|c|}
\hline \multirow{3}{*}{ Sample Number } & \multicolumn{2}{|c|}{$\mathrm{Cr}$ - total based on } & \multirow{2}{*}{\begin{tabular}{|c|} 
As - total based on \\
${ }^{75} \mathrm{As}$ \\
\end{tabular}} & \multirow{2}{*}{\begin{tabular}{|c|} 
Se - total based on \\
${ }^{82} \mathrm{Se}$ \\
\end{tabular}} & \multicolumn{3}{|c|}{ Mo - total based on } \\
\hline & ${ }^{52} \mathrm{Cr}$ & ${ }^{53} \mathrm{Cr}$ & & & ${ }^{95} \mathrm{Mo}$ & ${ }^{97} \mathrm{Mo}$ & ${ }^{98} \mathrm{Mo}$ \\
\hline & \multicolumn{7}{|c|}{ (2) } \\
\hline \multicolumn{8}{|c|}{ KOH-KNO ${ }_{3}$ Fusions } \\
\hline 404 & $6.69 \mathrm{E}+02$ & $7.91 \mathrm{E}+02$ & $(4.15 \mathrm{E}+01)$ & $<2.39 \mathrm{E}+03$ & $(5.60 \mathrm{E}+01)$ & $(3.39 \mathrm{E}+01)$ & $(2.00 \mathrm{E}+01)$ \\
\hline 404 DUP & $3.75 \mathrm{E}+02$ & $4.24 \mathrm{E}+02$ & $(3.71 \mathrm{E}+01)$ & $<2.70 \mathrm{E}+03$ & $(4.53 E+01)$ & $(2.83 \mathrm{E}+01)$ & $(1.61 \mathrm{E}+01)$ \\
\hline 405 & $8.13 \mathrm{E}+02$ & $8.65 \mathrm{E}+02$ & $(2.69 \mathrm{E}+01)$ & $(3.71 \mathrm{E}+00)$ & $(4.86 \mathrm{E}+01)$ & $(2.61 \mathrm{E}+01)$ & $(1.19 \mathrm{E}+01)$ \\
\hline 405 DUP & $8.01 \mathrm{E}+02$ & $8.52 \mathrm{E}+02$ & $(5.67 \mathrm{E}+01)$ & $<2.29 \mathrm{E}+03$ & $(5.00 \mathrm{E}+01)$ & $(2.71 \mathrm{E}+01)$ & $(1.51 \mathrm{E}+01)$ \\
\hline 404 Avg & $5.22 \mathrm{E}+02$ & $6.07 \mathrm{E}+02$ & $(3.93 \mathrm{E}+01)$ & $<2.55 \mathrm{E}+03$ & $(5.07 \mathrm{E}+01)$ & $(3.11 \mathrm{E}+01)$ & $(1.81 \mathrm{E}+01)$ \\
\hline 405 Avg & $8.07 \mathrm{E}+02$ & $8.58 \mathrm{E}+02$ & $(4.18 \mathrm{E}+01)$ & $(1.15 \mathrm{E}+03)$ & $(4.93 \mathrm{E}+01)$ & $(2.66 \mathrm{E}+01)$ & $(1.35 \mathrm{E}+01)$ \\
\hline \multicolumn{8}{|c|}{ EPA Acid Digestion } \\
\hline 404 & $6.84 \mathrm{E}+02$ & $7.12 \mathrm{E}+02$ & $(6.90 \mathrm{E}+01)$ & $<6.17 \mathrm{E}+03$ & $(5.17 \mathrm{E}+01)$ & $(4.87 \mathrm{E}+01)$ & $(2.28 \mathrm{E}+01)$ \\
\hline 404 DUP & $(5.94 \mathrm{E}+02)$ & $6.33 \mathrm{E}+02$ & $(1.11 \mathrm{E}+02)$ & $(1.28 \mathrm{E}+01)$ & $(4.62 \mathrm{E}+01)$ & $(6.18 \mathrm{E}+01)$ & $(2.47 \mathrm{E}+01)$ \\
\hline 405 & $6.17 \mathrm{E}+02$ & $6.46 \mathrm{E}+02$ & $(9.45 \mathrm{E}+01)$ & $(3.11 \mathrm{E}+01)$ & $(5.17 \mathrm{E}+01)$ & $(5.41 \mathrm{E}+01)$ & $(1.82 \mathrm{E}+01)$ \\
\hline 405 DUP & $(5.45 \mathrm{E}+02)$ & $5.58 \mathrm{E}+02$ & $(8.79 \mathrm{E}+01)$ & $<6.42 \mathrm{E}+03$ & $(4.64 \mathrm{E}+01)$ & $(4.96 \mathrm{E}+01)$ & $(2.08 \mathrm{E}+01)$ \\
\hline 404 Avg & $6.39 \mathrm{E}+02$ & $6.73 \mathrm{E}+02$ & $(9.01 \mathrm{E}+01)$ & $<3.09 \mathrm{E}+03$ & $(4.90 \mathrm{E}+01)$ & $(5.53 \mathrm{E}+01)$ & $(2.38 \mathrm{E}+01)$ \\
\hline $405 \mathrm{Avg}$ & $5.81 \mathrm{E}+02$ & $6.02 \mathrm{E}+02$ & $(9.12 \mathrm{E}+01)$ & $(3.23 \mathrm{E}+03)$ & $(4.90 \mathrm{E}+01)$ & $(5.19 \mathrm{E}+01)$ & $(1.95 \mathrm{E}+01)$ \\
\hline \multicolumn{8}{|c|}{$\begin{array}{l}\text { Avg = Average. } \\
\text { DUP = Duplicate sample. } \\
\text { EPA = U.S. Environmental Protection Agency. } \\
\text { ICP-OES = Inductively coupled plasma-optical emission spectroscopy. } \\
\mathrm{KNO}_{3}=\text { Potassium nitrate. } \\
\mathrm{KOH}=\text { Potassium hydroxide. } \\
\text { (a) The indicated isotope is the suggested isotope for use to quantify the total conce }\end{array}$} \\
\hline
\end{tabular}


Table 3.6. Concentrations of Elements Determined from ICP-MS Analysis per Gram of Dry Sludge

\begin{tabular}{|c|c|c|c|c|c|c|c|c|c|}
\hline \multirow{3}{*}{ Sample Number } & \multicolumn{3}{|c|}{ Ru -total based on } & \multicolumn{2}{|c|}{ Ag - total based on } & \multicolumn{2}{|c|}{ Cd - total based on } & \multicolumn{2}{|c|}{$\mathrm{Pb}$ - total based on } \\
\hline & ${ }^{101} \mathbf{R u}$ & ${ }^{102} \mathrm{Ru}$ & ${ }^{104} \mathrm{Ru}$ & ${ }^{107} \mathrm{Ag}$ & ${ }^{109} \mathrm{Ag}$ & ${ }^{111} \mathbf{C d}$ & ${ }^{114} \mathrm{Cd}$ & ${ }^{206} \mathrm{~Pb}$ & ${ }^{208} \mathrm{~Pb}$ \\
\hline & \multicolumn{9}{|c|}{ KOH-KNO ${ }_{3}$ Fusions } \\
\hline 404 & $4.39 \mathrm{E}+02$ & $2.08 \mathrm{E}+02$ & $2.05 \mathrm{E}+02$ & $(2.24 \mathrm{E}+01)$ & $(2.12 \mathrm{E}+01)$ & $3.06 \mathrm{E}+02$ & $2.85 \mathrm{E}+02$ & $4.40 \mathrm{E}+03$ & $4.93 \mathrm{E}+03$ \\
\hline 404 DUP & $3.81 \mathrm{E}+02$ & $1.92 \mathrm{E}+02$ & $1.70 \mathrm{E}+02$ & $(2.43 \mathrm{E}+01)$ & $(2.37 \mathrm{E}+01)$ & $2.67 \mathrm{E}+02$ & $2.57 \mathrm{E}+02$ & $3.98 \mathrm{E}+03$ & $4.45 \mathrm{E}+03$ \\
\hline 405 & $4.23 \mathrm{E}+02$ & $2.01 \mathrm{E}+02$ & $1.96 \mathrm{E}+02$ & $(1.90 \mathrm{E}+01)$ & $(1.97 \mathrm{E}+01)$ & $3.10 \mathrm{E}+02$ & $2.92 \mathrm{E}+02$ & $4.56 \mathrm{E}+03$ & $5.10 \mathrm{E}+03$ \\
\hline 405 DUP & $4.15 \mathrm{E}+02$ & $2.03 \mathrm{E}+02$ & $1.96 \mathrm{E}+02$ & $(2.28 \mathrm{E}+01)$ & $(2.28 \mathrm{E}+01)$ & $2.94 \mathrm{E}+02$ & $2.90 \mathrm{E}+02$ & $4.26 \mathrm{E}+03$ & $4.77 \mathrm{E}+03$ \\
\hline 404 Avg & $4.10 \mathrm{E}+02$ & $2.00 \mathrm{E}+02$ & $1.87 \mathrm{E}+02$ & $(2.34 \mathrm{E}+01)$ & $(2.24 \mathrm{E}+01)$ & $2.87 \mathrm{E}+02$ & $2.71 \mathrm{E}+02$ & $4.19 \mathrm{E}+03$ & $4.69 \mathrm{E}+03$ \\
\hline $405 \mathrm{Avg}$ & $4.19 \mathrm{E}+02$ & $2.02 \mathrm{E}+02$ & $1.96 \mathrm{E}+02$ & $(2.09 \mathrm{E}+01)$ & $(2.12 \mathrm{E}+01)$ & $3.02 \mathrm{E}+02$ & $2.91 \mathrm{E}+02$ & $4.41 \mathrm{E}+03$ & $4.94 \mathrm{E}+03$ \\
\hline \multicolumn{10}{|c|}{$\begin{array}{l}\text { EPA Acid Digestion } \\
\end{array}$} \\
\hline 404 & $(2.38 \mathrm{E}+02)$ & $1.28 \mathrm{E}+02$ & $1.29 \mathrm{E}+02$ & $1.29 \mathrm{E}+03$ & $1.33 \mathrm{E}+03$ & $3.12 \mathrm{E}+02$ & $2.86 \mathrm{E}+02$ & $4.58 \mathrm{E}+03$ & $5.14 \mathrm{E}+03$ \\
\hline 404 DUP & $(2.35 \mathrm{E}+02)$ & $(1.21 \mathrm{E}+02)$ & $(1.23 \mathrm{E}+02)$ & $1.09 \mathrm{E}+03$ & $1.10 \mathrm{E}+03$ & $2.85 \mathrm{E}+02$ & $2.64 \mathrm{E}+02$ & $4.12 \mathrm{E}+03$ & $4.60 \mathrm{E}+03$ \\
\hline 405 & $(2.53 \mathrm{E}+02)$ & $1.25 \mathrm{E}+02$ & $1.32 \mathrm{E}+02$ & $1.32 \mathrm{E}+03$ & $1.33 \mathrm{E}+03$ & $2.98 \mathrm{E}+02$ & $2.83 \mathrm{E}+02$ & $4.35 \mathrm{E}+03$ & $4.86 \mathrm{E}+03$ \\
\hline 405 DUP & $(2.34 \mathrm{E}+02)$ & $(1.23 \mathrm{E}+02)$ & $(1.21 \mathrm{E}+02)$ & $1.07 \mathrm{E}+03$ & $1.08 \mathrm{E}+03$ & $3.45 \mathrm{E}+02$ & $2.88 \mathrm{E}+02$ & $4.40 \mathrm{E}+03$ & $4.85 \mathrm{E}+03$ \\
\hline 404 Avg & $(2.37 \mathrm{E}+02)$ & $(1.24 \mathrm{E}+02)$ & $(1.26 \mathrm{E}+02)$ & $1.19 \mathrm{E}+03$ & $1.22 \mathrm{E}+03$ & $2.99 \mathrm{E}+02$ & $2.75 \mathrm{E}+02$ & $4.35 \mathrm{E}+03$ & $4.87 \mathrm{E}+03$ \\
\hline 405 Avg & $(2.43 E+02)$ & $1.24 \mathrm{E}+02$ & $1.26 \mathrm{E}+02$ & $1.19 \mathrm{E}+03$ & $1.21 \mathrm{E}+03$ & $3.22 \mathrm{E}+02$ & $2.85 \mathrm{E}+02$ & $4.37 \mathrm{E}+03$ & $4.85 \mathrm{E}+03$ \\
\hline
\end{tabular}


Table 3.7. Concentrations of ${ }^{99} \mathrm{Tc}$ and ${ }^{238} \mathrm{U}$, Measured by ICP-MS per Gram of Dry Sludge

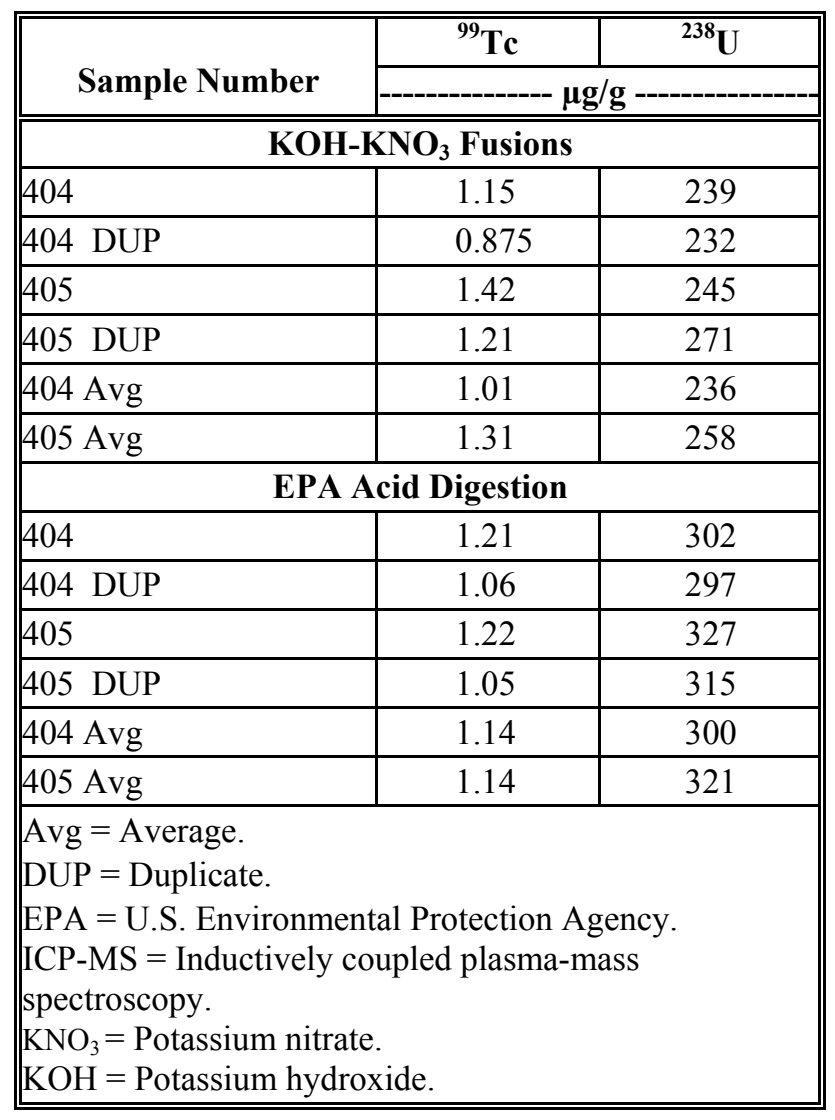

The ${ }^{60} \mathrm{Co}$ and ${ }^{137} \mathrm{Cs}$ concentrations in C-106 sludge measured by GEA are listed in Table 3.8. Data are presented for the untreated sludge and for the fusion and acid digestion extracts. For ${ }^{60} \mathrm{Co}$, the values are similar for the three analyses and range from 0.3301 to $0.4521 \mu \mathrm{Ci} / \mathrm{g}\left(2.9 \times 10^{-4}\right.$ to $\left.3.99 \times 10^{-4} \mu \mathrm{g} / \mathrm{g}\right)$. For ${ }^{137} \mathrm{Cs}$, the values are also similar for the three analyses and range from 106 to $173 \mu \mathrm{Ci} / \mathrm{g}$ ( 1.2 to $2.0 \mu \mathrm{g} / \mathrm{g}$ ). The highest concentrations for both isotopes are associated with the direct readings and the lowest readings are generally found for the acid extracts.

Table 3.9 lists the concentrations of the transuranic (TRU) isotopes ${ }^{239} \mathrm{Pu},{ }^{237} \mathrm{~Np}$, and ${ }^{241} \mathrm{Am}$ in the sludge samples. The average ${ }^{239} \mathrm{Pu}$ concentration in 404 sludge measured by the fusion method is $29.5 \mu \mathrm{g} / \mathrm{g}(1.83 \mu \mathrm{Ci} / \mathrm{g})$ and the average value by the EPA acid digestion method is $27.3 \mu \mathrm{g} / \mathrm{g}(1.70 \mu \mathrm{Ci} / \mathrm{g})$. The ${ }^{237} \mathrm{~Np}$ concentration in 404 sludge measured by the fusion method is $8.84 \mu \mathrm{g} / \mathrm{g}\left(6.28 \times 10^{-3} \mu \mathrm{Ci} / \mathrm{g}\right)$ and the average value by the EPA acid digestion method is $8.71 \mu \mathrm{g} / \mathrm{g}\left(6.18 \times 10^{-3} \mu \mathrm{Ci} / \mathrm{g}\right)$. The average ${ }^{241} \mathrm{Am}$ concentration in 404 sludge measured by the fusion method is $1.78 \mu \mathrm{g} / \mathrm{g}(6.05 \mu \mathrm{Ci} / \mathrm{g})$, and the average value by the EPA acid digestion method is $2.03 \mu \mathrm{g} / \mathrm{g}(6.89 \mu \mathrm{Ci} / \mathrm{g})$. 
Table 3.8. ${ }^{137} \mathrm{Cs}$ and ${ }^{60} \mathrm{Co}$ Concentrations in Dry Sludge as Measured by GEA

\begin{tabular}{|c|c|c|c|c|}
\hline \multirow[b]{2}{*}{ Sample Number } & 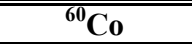 & $\bar{~}{ }^{60} \mathrm{Co}$ & ${ }^{137} \mathrm{Cs}$ & ${ }^{137} \mathrm{Cs}$ \\
\hline & 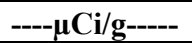 & 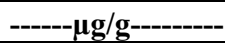 & $---\mu \mathrm{Ci} / \mathrm{g}----$ & 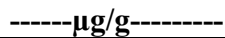 \\
\hline \multicolumn{5}{|c|}{ Untreated (Raw) Solid } \\
\hline 404 & $4.521 \mathrm{E}-01$ & $4.110 \mathrm{E}-04$ & $1.583 \mathrm{E}+02$ & $1.820 \mathrm{E}+00$ \\
\hline 404 DUP & $4.312 \mathrm{E}-01$ & $3.920 \mathrm{E}-04$ & $1.545 \mathrm{E}+02$ & $1.776 \mathrm{E}+00$ \\
\hline 405 & $4.287 \mathrm{E}-01$ & $3.897 \mathrm{E}-04$ & $1.697 \mathrm{E}+02$ & $1.951 \mathrm{E}+00$ \\
\hline 405 DUP & $4.128 \mathrm{E}-01$ & $3.753 \mathrm{E}-04$ & $1.733 \mathrm{E}+02$ & $1.992 \mathrm{E}+00$ \\
\hline 404 Avg & $4.417 \mathrm{E}-01$ & $4.015 \mathrm{E}-04$ & $1.564 \mathrm{E}+02$ & $1.798 \mathrm{E}+00$ \\
\hline 405 Avg & $4.208 \mathrm{E}-01$ & $3.825 \mathrm{E}-04$ & $1.715 \mathrm{E}+02$ & $1.971 \mathrm{E}+00$ \\
\hline \multicolumn{5}{|c|}{ 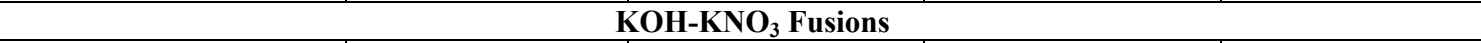 } \\
\hline 404 & $3.413 \mathrm{E}-01$ & $3.103 \mathrm{E}-04$ & $1.411 \mathrm{E}+02$ & $1.622 \mathrm{E}+00$ \\
\hline 404 DUP & 4.483E-01 & $4.075 \mathrm{E}-04$ & $1.566 \mathrm{E}+02$ & $1.799 \mathrm{E}+00$ \\
\hline 405 & $4.186 \mathrm{E}-01$ & $3.805 \mathrm{E}-04$ & $1.599 \mathrm{E}+02$ & $1.837 \mathrm{E}+00$ \\
\hline 405 DUP & $4.108 \mathrm{E}-01$ & $3.735 \mathrm{E}-04$ & $1.610 \mathrm{E}+02$ & $1.851 \mathrm{E}+00$ \\
\hline 404 Avg & $3.948 \mathrm{E}-01$ & $3.589 \mathrm{E}-04$ & $1.488 \mathrm{E}+02$ & $1.711 \mathrm{E}+00$ \\
\hline 405 Avg & $4.147 \mathrm{E}-01$ & 3.770E-04 & $1.604 \mathrm{E}+02$ & $1.844 \mathrm{E}+00$ \\
\hline \multicolumn{5}{|c|}{ EPA Acid Digestion } \\
\hline 404 & 3.899E-01 & $3.544 \mathrm{E}-04$ & $1.06 \mathrm{E}+02$ & $1.224 \mathrm{E}+00$ \\
\hline 404 DUP & $3.301 \mathrm{E}-01$ & $3.001 \mathrm{E}-04$ & $1.18 \mathrm{E}+02$ & $1.351 \mathrm{E}+00$ \\
\hline 405 & $3.726 \mathrm{E}-01$ & $3.387 \mathrm{E}-04$ & $1.32 \mathrm{E}+02$ & $1.514 \mathrm{E}+00$ \\
\hline 405 DUP & 4.069E-01 & $3.699 \mathrm{E}-04$ & $1.283 \mathrm{E}+02$ & $1.474 \mathrm{E}+00$ \\
\hline 404 Avg & $3.600 \mathrm{E}-01$ & $3.273 \mathrm{E}-04$ & $1.120 \mathrm{E}+02$ & $1.287 \mathrm{E}+00$ \\
\hline 405 Avg & $3.897 \mathrm{E}-01$ & $3.543 \mathrm{E}-04$ & $1.300 \mathrm{E}+02$ & $1.494 \mathrm{E}+00$ \\
\hline \multicolumn{5}{|c|}{$\begin{array}{l}\text { Avg = Average. } \\
\text { DUP = Duplicate. } \\
\text { EPA = U.S. Environmental Protection Agency. } \\
\text { GEA = Gamma energy analysis. } \\
\mathrm{KNO}_{3}=\text { Potassium nitrate. } \\
\mathrm{KOH}=\text { Potassium hydroxide. }\end{array}$} \\
\hline
\end{tabular}

Table 3.9. ICP-MS Analysis for Actinides per Gram of Dry Sludge

\begin{tabular}{|c|c|c|c|c|c|c|}
\hline \multirow[b]{2}{*}{ Sample Number } & ${ }^{239} \mathrm{Pu}$ & ${ }^{239} \mathrm{Pu}$ & ${ }^{237} \mathrm{~Np}$ & ${ }^{237} \mathrm{~Np}$ & ${ }^{241} \mathrm{Am}$ & ${ }^{241} \mathrm{Am}$ \\
\hline & $\mu \mathrm{Ci} / \mathrm{g}$ & $\mu \mathrm{g} / \mathrm{g}$ & $\mu \mathrm{Ci} / \mathrm{g}$ & $\mu \mathrm{g} / \mathrm{g}$ & $\mu \mathrm{Ci} / \mathrm{g}$ & $\mu \mathrm{g} / \mathrm{g}$ \\
\hline \multicolumn{7}{|c|}{ KOH-KNO 3 Fusions } \\
\hline 404 & 1.82 & 29.36 & $6.64 \mathrm{E}-03$ & 9.35 & 6.22 & 1.83 \\
\hline 404 DUP & 1.84 & 29.72 & $5.92 \mathrm{E}-03$ & 8.34 & 5.88 & 1.73 \\
\hline 405 & 1.85 & 29.82 & $7.11 \mathrm{E}-03$ & 10.01 & 6.30 & 1.85 \\
\hline 405 DUP & 1.88 & 30.31 & $6.65 \mathrm{E}-03$ & 9.36 & 6.43 & 1.89 \\
\hline 404 Avg & 1.83 & 29.54 & $6.28 \mathrm{E}-03$ & 8.84 & 6.05 & 1.78 \\
\hline 405 Avg & 1.86 & 30.06 & $6.88 \mathrm{E}-03$ & 9.69 & 6.36 & 1.87 \\
\hline \multicolumn{7}{|c|}{ EPA Acid Digestion } \\
\hline 404 & 1.85 & 29.80 & $6.62 \mathrm{E}-03$ & 9.32 & 7.12 & 2.09 \\
\hline 404 DUP & 1.54 & 24.88 & $5.75 \mathrm{E}-03$ & 8.09 & 6.65 & 1.96 \\
\hline 405 & 1.78 & 28.78 & $6.44 \mathrm{E}-03$ & 9.07 & 7.10 & 2.09 \\
\hline 405 DUP & 1.69 & 27.30 & $6.85 \mathrm{E}-03$ & 9.64 & 6.90 & 2.03 \\
\hline 404 Avg & 1.70 & 27.34 & $6.18 \mathrm{E}-03$ & 8.71 & 6.89 & 2.03 \\
\hline 405 Avg & 1.74 & 28.04 & $6.64 \mathrm{E}-03$ & 9.36 & 7.00 & 2.06 \\
\hline \multicolumn{7}{|c|}{$\begin{array}{l}\text { Avg = Average. } \\
\text { DUP = Duplicate. } \\
\text { EPA = U.S. Environmental Protection Agency. } \\
\text { ICP-MS = Inductively coupled plasma-mass spectroscopy. } \\
\mathrm{KNO}_{3}=\text { Potassium nitrate. } \\
\mathrm{KOH}^{=} \text {Potassium hydroxide. }\end{array}$} \\
\hline
\end{tabular}


The average ${ }^{239} \mathrm{Pu}$ concentration in 405 sludge measured by the fusion method is $30.1 \mu \mathrm{g} / \mathrm{g}$ $(1.86 \mu \mathrm{Ci} / \mathrm{g})$, and the average value by the EPA acid digestion method is $28.0 \mu \mathrm{g} / \mathrm{g}(1.74 \mu \mathrm{Ci} / \mathrm{g})$. The ${ }^{237} \mathrm{~Np}$ concentration in 405 sludge measured by the fusion method is $9.69 \mu \mathrm{g} / \mathrm{g}\left(6.88 \times 10^{-3} \mu \mathrm{Ci} / \mathrm{g}\right)$, and the average value by the EPA acid digestion method is $9.36 \mu \mathrm{g} / \mathrm{g}\left(6.64 \times 10^{-3} \mu \mathrm{Ci} / \mathrm{g}\right)$. The ${ }^{241} \mathrm{Am}$ concentration in 405 sludge measured by the fusion method is $1.87 \mu \mathrm{g} / \mathrm{g}(6.36 \mu \mathrm{Ci} / \mathrm{g})$, and the average value by the EPA acid digestion method is $2.06 \mu \mathrm{g} / \mathrm{g}(7.00 \mu \mathrm{Ci} / \mathrm{g})$.

DOE defines TRU waste as radioactive waste that at the time of assay contains more than $100 \mathrm{nCi} / \mathrm{g}$ of alpha-emitting isotopes with atomic numbers greater than 92 and half-lives greater than 20 years (DOE Order 5820.2A). ${ }^{239} \mathrm{Pu},{ }^{237} \mathrm{~Np}$, and ${ }^{241} \mathrm{Am}$ are transuranic isotopes with half-lives greater than 20 years. The sum of the averages for sludge samples 404 and 405 for ${ }^{239} \mathrm{Pu},{ }^{237} \mathrm{~Np}$, and ${ }^{241} \mathrm{Am}$ isotopes measured by the fusion method is $8,057 \mathrm{nCi} / \mathrm{g}$ and by the $\mathrm{EPA}$ acid digestion method $8,670 \mathrm{nCi} / \mathrm{g}$. Based on the definition, this would classify the sludge itself in C-106 as TRU waste; however, the final waste form will be a mixture of residual sludge and a tank filling grout, which will lower the concentrations of contaminants an unspecified amount.

Table 3.10 lists the gross beta, gross alpha, and ${ }^{90} \mathrm{Sr}$ concentrations in sludge samples from tank C-106. The average gross beta values for the primary (404) and duplicate (405) samples measured by fusion extraction were 5,426 and $6,729 \mu \mathrm{Ci} / \mathrm{g}$; by EPA acid digestion the average values were 8,064 and $8,312 \mu \mathrm{Ci} / \mathrm{g}$. The average gross alpha values for this sludge measured by fusion extraction were 959 and $1,364 \mu \mathrm{Ci} / \mathrm{g}$ and by EPA acid digestion were 3,083 and $3,099 \mu \mathrm{Ci} / \mathrm{g}$. The average ${ }^{90} \mathrm{Sr}$ value measured by fusion extraction was $3,620 \mu \mathrm{Ci} / \mathrm{g}$, and the average by EPA acid digestion was $6,080 \mu \mathrm{Ci} / \mathrm{g}$.

Table 3.10. Total Beta and Total Alpha Activities and ${ }^{90} \mathrm{Sr}$ Concentrations per Gram of Dry Sludge

\begin{tabular}{|c|c|c|c|c|}
\hline \multirow[b]{2}{*}{ Sample Number } & \multirow{2}{*}{\multicolumn{2}{|c|}{\begin{tabular}{l|r} 
Total Beta & Total Alpha \\
- & \\
-
\end{tabular}}} & \multicolumn{2}{|c|}{${ }^{90} \mathrm{Sr}$} \\
\hline & & & ------- $\mu \mathrm{Ci} / \mathrm{g}$------- & ------- $\mu \mathrm{g} / \mathrm{g}-------$ \\
\hline \multicolumn{5}{|c|}{$\begin{array}{r}\mathrm{KOH}-\mathrm{KNO}_{3} \text { Fusions } \\
\end{array}$} \\
\hline 404 & $5.949 \mathrm{E}+03$ & $1.118 \mathrm{E}+03$ & $3.51 \mathrm{E}+03$ & $2.51 \mathrm{E}+01$ \\
\hline 404 DUP & $4.903 \mathrm{E}+03$ & $7.988 \mathrm{E}+02$ & $2.75 \mathrm{E}+03$ & $1.97 \mathrm{E}+01$ \\
\hline 405 & $7.011 \mathrm{E}+03$ & $1.482 \mathrm{E}+03$ & $4.16 \mathrm{E}+03$ & $2.974 \mathrm{E}+01$ \\
\hline 405 DUP & $6.447 \mathrm{E}+03$ & $1.245 \mathrm{E}+03$ & $4.02 \mathrm{E}+03$ & $2.87 \mathrm{E}+01$ \\
\hline 404 Avg & $5.426 \mathrm{E}+03$ & $9.586 \mathrm{E}+02$ & $3.13 \mathrm{E}+03$ & $2.24 \mathrm{E}+01$ \\
\hline $405 \mathrm{Avg}$ & $6.729 \mathrm{E}+03$ & $1.364 \mathrm{E}+03$ & $4.09 \mathrm{E}+03$ & $2.92 \mathrm{E}+01$ \\
\hline \multicolumn{5}{|c|}{ EPA Acid Digestion } \\
\hline 404 & $8.823 \mathrm{E}+03$ & $2.81 \mathrm{E}+03$ & $6.15 \mathrm{E}+03$ & $4.39 \mathrm{E}+01$ \\
\hline 404 DUP & $7.304 \mathrm{E}+03$ & $3.39 \mathrm{E}+03$ & $5.71 \mathrm{E}+03$ & $4.08 \mathrm{E}+01$ \\
\hline 405 & $8.680 \mathrm{E}+03$ & $2.52 \mathrm{E}+03$ & $6.22 \mathrm{E}+03$ & $4.44 \mathrm{E}+01$ \\
\hline 405 DUP & $7.944 \mathrm{E}+03$ & $3.64 \mathrm{E}+03$ & $6.24 \mathrm{E}+03$ & $4.46 \mathrm{E}+01$ \\
\hline 404 Avg & $8.064 \mathrm{E}+03$ & $3.099 \mathrm{E}+03$ & $5.93 \mathrm{E}+03$ & $4.24 \mathrm{E}+01$ \\
\hline 405 Avg & $8.312 \mathrm{E}+03$ & $3.083 \mathrm{E}+03$ & $6.23 \mathrm{E}+03$ & $4.45 \mathrm{E}+01$ \\
\hline \multicolumn{5}{|c|}{$\begin{array}{l}\text { Avg = Average. } \\
\text { DUP = Duplicate. } \\
\mathrm{EPA}=\text { U.S. Environmental Protection Agency. } \\
\mathrm{KNO}_{3}=\text { Potassium nitrate. } \\
\mathrm{KOH}^{=} \text {Potassium hydroxide. }\end{array}$} \\
\hline
\end{tabular}


Table 3.11 provides a summary of the major components of the sludge as measured for the metals by fusion and acid digestion analysis. For completeness, the anion analyses, as measured by the periodic replenishment water leaching tests (Section 3.4.2.5) have been included in this table. Figure 3.2 shows the components of the tank C-106 sludge that were measured at concentrations greater than $1,000 \mu \mathrm{g} / \mathrm{g}$ by the fusion method for the metals and represents the sum of the period replenishment water-leaching experiments for the anions (Section 3.4.1.7). The predominant metals are $\mathrm{Al}(13.1 \mathrm{wt} \%), \mathrm{Mn}(11.8 \mathrm{wt} \%)$, $\mathrm{Na}(6.0 \mathrm{wt} \%)$, and $\mathrm{Fe}(4.4 \mathrm{wt} \%)$. The predominant anions are oxalate $(6.4 \mathrm{wt} \%)$ and carbonate $(4.0 \mathrm{wt} \%)$.

Table 3.11. Summary of Average Concentrations for Tank C-106 Residual Sludge

\begin{tabular}{|c|c|c|}
\hline \multirow[b]{2}{*}{ Analyte } & \multicolumn{2}{|c|}{ C-106 $(\mu \mathrm{g} / \mathrm{g})$} \\
\hline & Fusion & $\begin{array}{c}\text { EPA Acid } \\
\text { Digestion }\end{array}$ \\
\hline$\overline{\mathrm{Al}}$ & (131,483 & (281,699 \\
\hline $\mathrm{Ba}$ & 1,028 & 914 \\
\hline $\mathrm{Ca}$ & 38,221 & 46,490 \\
\hline $\mathrm{Cr}$ & (896) & (727) \\
\hline $\mathrm{Fe}$ & 43,777 & 36,663 \\
\hline $\bar{K}$ & $\mathrm{~N} / \mathrm{A}$ & 8,526 \\
\hline $\mathrm{Mg}$ & 2,485 & 3,162 \\
\hline $\mathrm{Mn}$ & 117,767 & 108,069 \\
\hline $\mathrm{Na}$ & 60,400 & 46,720 \\
\hline $\mathrm{Ni}$ & 1,712 & 5,373 \\
\hline $\mathrm{Pb}$ & 4,841 & 4,814 \\
\hline $\mathrm{Si}$ & $(19,086)$ & $(4,895)$ \\
\hline $\mathrm{Sr}$ & $(256)$ & (493) \\
\hline${ }^{99} \mathrm{Tc}$ & 1.16 & 1.14 \\
\hline \multirow[t]{2}{*}{${ }^{238} \mathrm{U}$} & 247 & 310 \\
\hline & \multicolumn{2}{|c|}{ Water Leach } \\
\hline $\mathrm{F}^{-}$ & \multicolumn{2}{|c|}{33} \\
\hline Formate & \multicolumn{2}{|c|}{$<65$} \\
\hline $\mathrm{Cl}^{-}$ & \multicolumn{2}{|c|}{87} \\
\hline $\mathrm{NO}_{2}^{-}$ & \multicolumn{2}{|c|}{$<73$} \\
\hline $\mathrm{NO}_{3}^{-}$ & \multicolumn{2}{|c|}{$<70$} \\
\hline $\mathrm{CO}_{3}{ }^{2-}$ & \multicolumn{2}{|c|}{39,500} \\
\hline $\mathrm{SO}_{4}^{2-}$ & \multicolumn{2}{|c|}{$<66$} \\
\hline Oxalate & \multicolumn{2}{|c|}{63,900} \\
\hline $\mathrm{PO}_{4}^{3-}$ & \multicolumn{2}{|c|}{$<91$} \\
\hline
\end{tabular}

The Hanford 222-S Laboratory also conducted extensive characterization of the residual sludge samples collected from tank C-106. Results of these analyses are reported in the Analytical Results for Tank 241-C-106 Solid Clam Shell Samples Supporting Closure Action (McKinney 2004). Results by the laboratories are similar for most analytical methods (ICP/MS, IC, alpha energy analysis, gamma energy analysis, ${ }^{99} \mathrm{Tc}$, percent water). Differences were observed for some analyses/analytes (ICP/OES and $\left.{ }^{129} \mathrm{I}\right)$. The differences in the measured concentrations for the two laboratories are due to a variety of factors. 
Sample heterogeneity is a primary factor in measurement variability for this material. Given the wide variety of sludge solid phases with their variable particle sizes and densities, it is very difficult to mix the material uniformly and produce exact replicate samples. Also, very small amounts (tens of grams) of material were available producing a greater likelihood that duplicate samples collected separately from the tank could have significantly different compositions. Even if the samples were identical, analytical precision and accuracy in the laboratory affects the ability to reproduce the same measurement (most analytical instruments are calibrated within the specification of $\pm 10 \%$ ). Therefore, there will be some variability when the same sample is measured several times on an instrument.

Another factor leading to variability in the tank C-106 results between the two laboratories is the use of different extraction/digestion techniques for the sludge. The 222-S Laboratory used an acid digestion technique to prepare liquid aliquots for the analysis of nonradioactive components of the sludge. The PNNL laboratory used both the fusion technique and an acid digestion method that differs somewhat from the method used by 222-S Laboratory. As shown in Table 3.11, the two methods used to dissolve the sludge resulted in different concentrations for the PNNL analyses, and, thus, it is not surprising that there are differences between the laboratories. For the major metals, these differences are most likely due to the ability of the fusion method to more completely dissolve the silicate minerals and more recalcitrant oxide and hydroxide phases. The digestion methods used by both laboratories produced a solid residue that could not be analyzed. The likely variability in the compositions of these residues adds to the variability in the reported sludge compositions. For these reasons, it is expected that some differences in reported concentrations of the sludges will occur and should be considered a natural outcome of the characterization process of these materials. Chemists from the two laboratories are comparing preparation and analytical techniques for potential improvements in the analysis of Hanford tank waste.

In the case of ${ }^{129} \mathrm{I}$, discrepancies in the reported concentrations from the two laboratories exceeded a ratio that could easily be explained by sample heterogeneity. The PNNL results provided in the following section are approximately 3 times greater than the analytical detection limit reported by chemists from the 222-S Laboratory. An analytical detection limit was reported for ${ }^{129} \mathrm{I}$ by the $222-\mathrm{S}$ Laboratory staff because it was not detected in the C-106 sludge samples analyzed at the laboratory. The discrepancy in the ${ }^{129} \mathrm{I}$ data from the two laboratories could be an artifact of two things: sample preparation and analytical techniques. Both laboratories performed a $\mathrm{KOH}: \mathrm{KNO}_{3}$ fusion/water extraction to remove ${ }^{129} \mathrm{I}$ from the sludge. However, due to the presence of oxidized metals and residual solids in the extracted samples, researchers at PNNL added a mixture of concentrated $\mathrm{H}_{2} \mathrm{SO}_{4}$ and $1 \mathrm{M} \mathrm{NaHSO}_{3}$ to the sample. This additional step resulted in complete sample dissolution while preventing oxidation of the dissolved species.

The way in which researchers at the two laboratories analyzed the extracted samples differed considerably. Scientists at PNNL performed dilutions on the fully dissolved samples and analyzed them for ${ }^{127} \mathrm{I}$ and ${ }^{129} \mathrm{I}$ using ICP-MS. Chemists at the 222-S Laboratory took the $\mathrm{KOH}: \mathrm{KNO}_{3}$ fusion/water extracted samples through a multi-step liquid:liquid extraction/purification process, followed by precipitation as AgI. The AgI precipitate was eventually counted (X-rays) using a Low Energy Photon Spectrometry (LEPS) detector. Although the liquid:liquid procedure utilized by researchers at the 222-S Laboratory is a common technique, care must be taken throughout the various steps to ensure 
volatilization of iodine does not occur. Given the thoroughness of the PNNL extraction procedure and the straightforward analytical technique, it would be prudent and conservative to use the ${ }^{129}$ I values provided in this report (Section 3.2) for risk assessment calculations.

\section{2 $\quad{ }^{129}$ I Extraction and Measurement}

Table 3.12 contains results of the ${ }^{129} \mathrm{I}$ analysis of the modified $\mathrm{KOH}: \mathrm{KNO}_{3}$ water fusion of tank C-106 sludge material. The data are reported as $\mathrm{pCi}^{129} \mathrm{I}$ per gram of sludge (calculated on a dry weight basis). The italicized data reported for sample 405 C-106 matrix spike indicate that the reported value was corrected for the ${ }^{129}$ I spike contribution. ICP-MS analysis of ${ }^{129}$ I was better than $\pm 10 \%$ of certified reference standards, with the linear operating range extending three orders of magnitude ( 0.01 to $10 \mathrm{ng} / \mathrm{mL}$ ). The measured values are in the range of 73.4 to $143 \mathrm{pCi} / \mathrm{g}$ for sludge samples 404 and 405 .

The ${ }^{129}$ I concentrations in extracts from the standard $\mathrm{KOH}: \mathrm{KNO}_{3}$ fusion method were greater than the EQL (see Section 3.1 for a discussion of EQL calculation). The instrument EQL for this analysis was $0.88 \mathrm{pCi} / \mathrm{L}$, which resulted in dilution-corrected sample EQLs ranging from $13.3 \mathrm{pCi} / \mathrm{g}$ to $22.6 \mathrm{pCi} / \mathrm{g}$. The modified $\mathrm{KOH}: \mathrm{KNO}_{3}$ water fusion method was successful at dissolving the entire sludge sample tested. Additionally, the ${ }^{129}$ I recovery of the blank spike sample analyzed as part of this dataset was excellent at $98.7 \%$. Therefore, the fusion data reported in Table 3.12 represent the total ${ }^{129}$ I present in tank C-106 sludge.

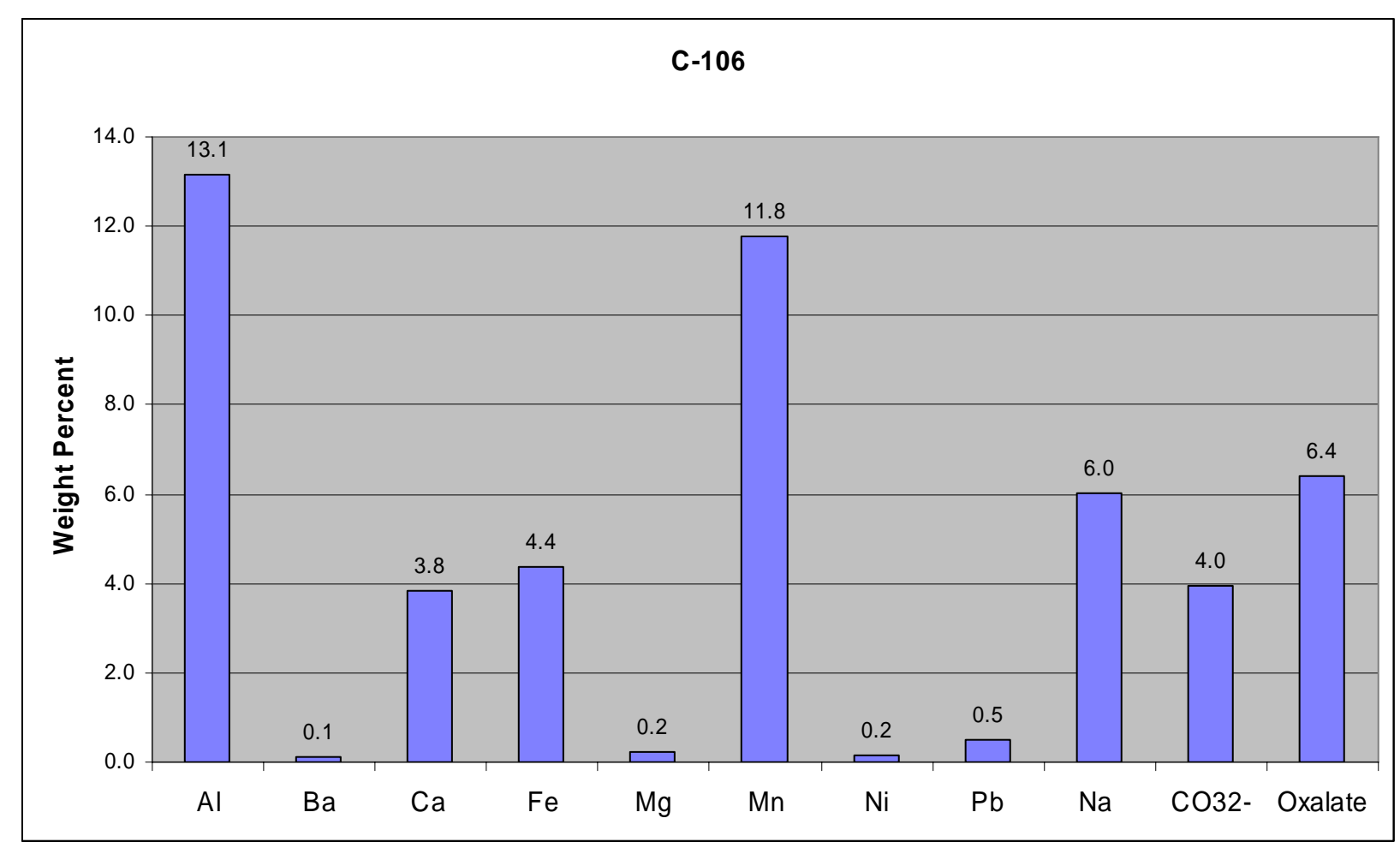

Figure 3.2. Tank C-106 Sludge Composition 
Table 3.12. Summary of ${ }^{129} \mathrm{I}$ Concentrations for Modified $\mathrm{KOH}-\mathrm{KNO}_{3}$ Water Fusion Extracts for Tank C-106

\begin{tabular}{||l|c||}
\hline \multicolumn{1}{|c|}{ Sample Number } & ${ }^{\mathbf{1 2 9}} \mathbf{I ~ p C i / g}$ \\
\hline \hline 404 C-106 & 105 \\
\hline 404 C-106 DUP & 112 \\
\hline 405 C-106 & 143 \\
\hline 405 C-106 DUP & 116 \\
\hline 405 C-106 Matrix Spike & 73.4 \\
\hline Italicized data means spike contribution has been removed. \\
DUP = Duplicate sample. \\
\hline
\end{tabular}

\subsection{Composition of Tank Liquid}

Sample 403 is a sample of the liquid in the tank collected near the cessation of final pumping during retrieval. It is representative of any supernatant and porewater in the residual sludge remaining in the tank. The composition of this liquid is provided in Table 3.13. The solution $\mathrm{pH}$ is 12.9 because of the addition of $0.5 \mathrm{M} \mathrm{NaOH}$ to neutralize remaining oxalic acid in the tank. The major cations in solution are $\mathrm{Na}(10,200 \mathrm{mg} / \mathrm{L} \mathrm{avg})$ and $\mathrm{Al}(121 \mathrm{mg} / \mathrm{L} \mathrm{avg})$; while the major anions are carbonate $(9,930 \mathrm{mg} / \mathrm{L} \mathrm{avg})$ and oxalate $(1,345 \mathrm{mg} / \mathrm{L}$ avg).

Table 3.13. Sample 403 - Tank C-106 Liquid Sample Composition

\begin{tabular}{|c|c|c|}
\hline \multirow[b]{2}{*}{ Analyte } & \multicolumn{2}{|c|}{ Concentration $\left(\mathrm{mg} / \mathrm{L}^{(\mathrm{a})}\right)$} \\
\hline & Primary & Duplicate \\
\hline pH (standard units) & 12.9 & \\
\hline \multicolumn{3}{|c|}{ Metals } \\
\hline $\mathrm{Al}$ & 126.0 & 116 \\
\hline $\mathrm{Ba}$ & 3.5 & $(0.09)$ \\
\hline $\mathrm{Ca}$ & 21.9 & $(4.1)$ \\
\hline $\mathrm{Cr}$ & $(0.28)$ & $(0.34)$ \\
\hline $\mathrm{Fe}$ & $(1.9)$ & $(0.6)$ \\
\hline $\mathrm{K}$ & $(14)$ & $(12)$ \\
\hline $\mathrm{Mg}$ & $(0.9)$ & $(0.24)$ \\
\hline $\mathrm{Mn}$ & $(0.16)$ & $(0.1)$ \\
\hline $\mathrm{Na}$ & 10,300 & 10,100 \\
\hline $\mathrm{Ni}$ & 3.9 & 3.0 \\
\hline $\mathrm{Pb}$ & $(0.16)$ & $(0.13)$ \\
\hline $\mathrm{Si}$ & $(158)$ & $(141)$ \\
\hline \multicolumn{3}{|c|}{ Radionuclides } \\
\hline${ }^{99} \mathrm{Tc}$ & 0.0003 & $(0.00012)$ \\
\hline${ }^{238} \mathrm{U}$ & 1.24 & 1.03 \\
\hline${ }^{137} \mathrm{Cs}(\mu \mathrm{Ci} / \mathrm{L})$ & 505 & 442 \\
\hline Total Alpha $(\mu \mathrm{Ci} / \mathrm{L})$ & 30.3 & 30.2 \\
\hline Total Beta $(\mu \mathrm{Ci} / \mathrm{L})$ & 470 & 469 \\
\hline \multicolumn{3}{|c|}{ Anions } \\
\hline Oxalate & 1,340 & 1,350 \\
\hline $\mathrm{CO}_{3}^{2-}$ & 10,020 & 9,840 \\
\hline $\mathrm{Cl}^{-}$ & 20.5 & 20.5 \\
\hline $\mathrm{NO}_{3}{ }^{-}$ & 9.43 & 9.41 \\
\hline $\mathrm{F}^{-}$ & 1.38 & $<1.17$ \\
\hline $\mathrm{SO}_{4}{ }^{2-}$ & 27.6 & 27.6 \\
\hline $\mathrm{PO}_{4}^{3-}$ & 75.1 & 74.6 \\
\hline
\end{tabular}




\subsection{Water-Leaching Tests}

The results of the water-leaching tests of sludge samples are discussed in this section. These include results from the single-contact and periodic replenishment tests. Concentration values in this section are given in terms of $\mu \mathrm{g}$ or $\mu \mathrm{Ci}$ per gram of dry sludge. Dissolved concentrations in extracts from these tests are provided on a per liter of solution basis in Appendix F.

\subsubsection{Single-Contact Test Results}

The single-contact water-leach tests were run in duplicate with contact times of 1 day, 2 weeks, and 1 month. DDI water was used as the leaching solution. The results of the solution analyses of the water extracts are described in this section.

\subsubsection{Digestion Factors and Moisture Contents - Single-Contact Water Extracts}

The digestion factors for the 404 and 405 sludge samples used for the water extracts varied from 7 to $13.5 \mathrm{~g} / \mathrm{L}$. These digestion factors are the ratios of wet weight of sludge to $30 \mathrm{~mL}$ DDI water used to dissolve the soluble portion of the solid. The digestion factors were then multiplied by the percent solids, as determined from moisture content analysis, to convert to a dry weight basis. The variability is a function of the mass of sludge used, which ranged from approximately 0.2 to $0.4 \mathrm{~g}$. The moisture contents averaged about $50.5 \%$ for the 404 and 405 samples (Table 3.1).

\subsubsection{Water Extract pH and Alkalinity - Single-Contact Water Extracts}

The average $\mathrm{pH}$ values of the duplicate water extracts of the sludge samples are listed in Table 3.14. The effluent $\mathrm{pH}$ values for sludge sample 404 were 7.03 and 7.17, and the values for 405 were 6.71 and 7.41. The $\mathrm{pH}$ values increase slightly between the 1-day and 1-month tests.

Table 3.14. Water Extract $\mathrm{pH}$ and Alkalinity Values Corrected to Grams of Dry Sludge

\begin{tabular}{||l|c|c||}
\hline \multicolumn{1}{|c|}{ Sample Number } & pH & $\begin{array}{c}\text { Total Alkalinity as } \\
\mathbf{C a C O}_{\mathbf{3}} \mathbf{~ m g / g}\end{array}$ \\
\hline 4041 Day Avg & 7.03 & 42.7 \\
\hline 4042 Week Avg & INS & INS \\
\hline 4041 Month Avg & 7.17 & 15.52 \\
\hline \hline 4051 Day Avg & 6.71 & 59.6 \\
\hline 4052 Week Avg & INS & INS \\
\hline 4051 Month Avg & 7.41 & 21.38 \\
\hline $\begin{array}{l}\text { Avg = Average. } \\
\text { INS = Instrument malfunction. }\end{array}$ \\
\hline
\end{tabular}


The total alkalinities of the water extracts reported in units of $\mathrm{CaCO}_{3} \mathrm{mg} / \mathrm{g}$ sludge are also shown in Table 3.14. The values for sludge sample 404 were 15.52 (for the month-long test) and $42.7 \mathrm{mg} / \mathrm{g}$ (for the day-long test), and the values for sample 405 after 1 month and 1 day were 21.38 and $59.6 \mathrm{mg} / \mathrm{g}$, respectively. The alkalinities decrease by about a factor of three between the 1-day and 1-month tests. This suggests the precipitation of a carbonate mineral.

\subsubsection{3 $\quad{ }^{99} \mathrm{Tc}$ and ${ }^{238} \mathrm{U}$ - Single-Contact Water Extracts}

${ }^{99} \mathrm{Tc}$ and ${ }^{238} \mathrm{U}$ are two of the more important potential long-term risk constituents in tank sludge because of their long half-lives and high mobility once dissolved in water. Table 3.15 lists the concentrations of these two radionuclides in units of $\mu \mathrm{g}$ leached/g-solid and $\mu \mathrm{Ci}$ leached/g-solid for the singlecontact water extracts. Table 3.16 lists the percentage of the total ${ }^{99} \mathrm{Tc}$ and ${ }^{238} \mathrm{U}$ leached from the sludge by each water-leach test versus the total available concentration given by the fusion analysis. The waterleachable amount of ${ }^{99} \mathrm{Tc}$ in sample 404 ranges from 0.0180 to $0.0339 \mu \mathrm{g} / \mathrm{g}$, which is 1.78 to $3.35 \%$ of the total ${ }^{99} \mathrm{Tc}$ in the sludge. For sample 405 , the water-leachable ${ }^{99} \mathrm{Tc}$ ranges from 0.0161 to $0.0349 \mu \mathrm{g} / \mathrm{g}$, which is 1.22 to $2.66 \%$ of the available ${ }^{99} \mathrm{Tc}$. The lowest amount of ${ }^{99} \mathrm{Tc}$ leached in the one-day contact test, and similar amounts leach at contact times of 2 weeks and 1 month. That is, a small amount of ${ }^{99} \mathrm{Tc}$ leaches within 1 day but the leaching rate decreases such that the total amount leached after 30 days is still small. The recalcitrant nature of some of the ${ }^{99} \mathrm{Tc}$ in other Hanford tank sludge has been documented in similar water-leach studies of sludges from tanks AY-102, C-203, and C-204 (Lindberg and Deutsch 2003; Krupka et al. 2004).

Table 3.15. ${ }^{99} \mathrm{Tc}$ and ${ }^{238} \mathrm{U}$ Concentrations in Tank C-106 Sludge from Single-Contact Water-Leach Tests

\begin{tabular}{|c|c|c|c|c|}
\hline \multirow[b]{2}{*}{ Sample Number } & 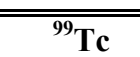 & ${ }^{2{ }^{238} \mathrm{U}}$ & $\bar{~}$ & "238$U$ \\
\hline & \multicolumn{2}{|c|}{ - } & \multicolumn{2}{|c|}{ - } \\
\hline 4041 Day Avg & 0.018 & (6.41 & 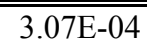 & 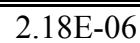 \\
\hline 4042 Week Avg & 0.0339 & 3.67 & $5.76 \mathrm{E}-04$ & $1.25 \mathrm{E}-06$ \\
\hline 4041 Month Avg & 0.0313 & 3.10 & $5.32 \mathrm{E}-04$ & $1.05 \mathrm{E}-06$ \\
\hline 4051 Day Avg & 0.0162 & 7.66 & 2.73E-04 & $2.61 \mathrm{E}-06$ \\
\hline 4052 Week Avg & 0.0349 & 3.99 & $5.94 \mathrm{E}-04$ & $1.36 \mathrm{E}-06$ \\
\hline 4051 Month Avg & 0.0326 & 3.70 & $5.54 \mathrm{E}-04$ & $1.26 \mathrm{E}-06$ \\
\hline
\end{tabular}

Table 3.16. Water-Leachable Percentages of ${ }^{99} \mathrm{Tc}$ and ${ }^{238} \mathrm{U}$ in C-106 Sludge Samples Compared with Fusion Results

\begin{tabular}{||l|l|c||}
\hline \multirow{2}{*}{ Sample Number } & ${ }^{{ }^{99}} \mathbf{T c}$ & ${ }^{\mathbf{2 3 8}} \mathbf{U}$ \\
\cline { 2 - 3 } & \multicolumn{2}{|c||}{ Percent Water Leachable } \\
\hline \hline 404 1 Day Avg & $1.78 \%$ & $2.72 \%$ \\
\hline 4042 Week Avg & $3.35 \%$ & $1.56 \%$ \\
\hline 4041 Month Avg & $3.09 \%$ & $1.31 \%$ \\
\hline \hline 4051 Day Avg & $1.22 \%$ & $2.97 \%$ \\
\hline 405 2 Week Avg & $2.66 \%$ & $1.55 \%$ \\
\hline 405 1 Month Avg & $2.48 \%$ & $1.43 \%$ \\
\hline Avg = Average.
\end{tabular}


The water-leachable amount of ${ }^{238} \mathrm{U}$ in sample 404 ranges from 3.10 to $6.41 \mu \mathrm{g} / \mathrm{g}$, which is 1.31 to $2.72 \%$ of the total ${ }^{238} \mathrm{U}$ in the sludge. For sample 405 , the water-leachable ${ }^{238} \mathrm{U}$ ranges from 3.70 to $7.66 \mu \mathrm{g} / \mathrm{g}$, which is 1.43 to $2.97 \%$ of the available ${ }^{238} \mathrm{U}$. The water leachability of ${ }^{238} \mathrm{U}$ appears to decrease with contact time; however, this decrease is small and not considered to be statistically significant.

\subsubsection{4 $\quad{ }^{129}$ I Concentrations - Single-Contact Water Extracts}

Table 3.17 contains results of the ${ }^{129} \mathrm{I}$ analysis of the one-day water extracts of the C-106 sludge samples. The data are reported as $\mathrm{pCi}^{129} \mathrm{I}$ per gram of sludge (calculated on a dry weight basis). ICP-MS measurement uncertainties for ${ }^{129}$ I were smaller than $\pm 10 \%$ of certified reference standards, with the linear operating range extending 3 orders of magnitude $(0.01$ to $10 \mathrm{ng} / \mathrm{mL})$. The water-leachable ${ }^{129} \mathrm{I}$ concentrations in extracts from these tests were less than the EQL (see Section 3.1 for a discussion of EQL calculation) for the analysis. The instrument EQL for this analysis was $1.8 \mathrm{pCi} / \mathrm{L}$, which resulted in dilution-corrected sample EQLs ranging from 2.2 to $3.2 \mathrm{pCi} / \mathrm{g}$ for the water extracts. Although the water leachable ${ }^{129}$ I results were less than the EQL of the analysis, it is apparent from a comparison with the total ${ }^{129} \mathrm{I}$ in the sludge (73.4 to $143 \mathrm{pCi} / \mathrm{g}$, Section 3.2) that very little (less than $3 \%$ ) of the ${ }^{129} \mathrm{I}$ in $\mathrm{C}-106$ residual sludge samples was leachable in this one-day, water contact test.

\subsubsection{Selected Metal Concentrations - Single-Contact Water Extracts}

Metals detected at measurable concentrations in one or more samples in the water extracts or the fusion analyses are listed in Table 3.18. A discussion of the meaning of the less-than values $(<)$ and the values in parentheses are provided in Section 3.1. The fusion analyses showed that $\mathrm{Al}, \mathrm{Mn}, \mathrm{Na}, \mathrm{Fe}$, and $\mathrm{Ca}$ are the dominant metals present in the tank C-106 sludge (Figure 3.1 and Figure 3.2). The water extracts show that $\mathrm{Na}$ and $\mathrm{Mn}$ are the primary water-soluble constituents with much smaller amounts of $\mathrm{Ba}, \mathrm{Ca}, \mathrm{Mg}, \mathrm{Ni}$, and (perhaps) Si also being leachable. Very little $\mathrm{Al}$ or Fe are water leachable from the sludge. (Note that the concentrations measured in the water leachates are the sums of the water-leachable amounts and the initial dissolved pore water concentrations.)

Table 3.17. Summary of ${ }^{129}$ I Concentrations from the One Day Single-Contact Water-Leach Tests for Tank C-106 Sludge Samples

\begin{tabular}{||l|c||}
\hline \multicolumn{1}{|c|}{ Sample Number } & $\begin{array}{c}{ }^{\mathbf{1 2 9}} \mathbf{I} \\
\mathbf{p C i} / \mathbf{g}\end{array}$ \\
\hline \hline 404 1 Day & $(1.28)$ \\
\hline 4041 Day DUP & $(1.44)$ \\
\hline \hline 405 1 Day & $(1.28)$ \\
\hline 405 1 Day DUP & $(1.59)$ \\
\hline DUP = Duplicate. \\
\hline
\end{tabular}


Table 3.18. Water-Leachable Average Metal Concentrations in Tank C-106 Single-Contact Water Extracts

\begin{tabular}{|c|c|c|c|c|c|c|c|c|c|c|c|c|c|}
\hline \multirow{2}{*}{$\begin{array}{c}\text { Sample } \\
\text { Number }\end{array}$} & Al & Ba & Ca & $\mathrm{Cr}$ & Fe & $\mathbf{K}$ & Mg & Mn & $\mathbf{N a}$ & $\mathbf{N i}$ & $\mathbf{P b}$ & $\mathbf{S i}$ & Sr \\
\hline & \multicolumn{13}{|c|}{ 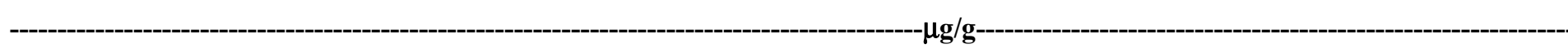 } \\
\hline $\begin{array}{l}4041 \text { Day } \\
\text { Avg }\end{array}$ & 83.2 & 35.1 & 329 & $(1.79)$ & $(24.2)$ & $(85.4)$ & 152 & 8,870 & 10,700 & 183 & $(2.63)$ & (357) & (4.23) \\
\hline $\begin{array}{l}4042 \text { Week } \\
\text { Avg }\end{array}$ & $(27.3)$ & 9.81 & 69.5 & $(2.89)$ & $(4.36)$ & (77.6) & 193 & 10,400 & 11,900 & 121 & $(0.89)$ & (281) & (5.37) \\
\hline $\begin{array}{l}4041 \\
\text { Month Avg }\end{array}$ & (10.1) & 15.3 & 70.4 & 2.51 & (1.09) & (77.9) & 176 & 8,680 & 10,400 & 96.4 & $(0.39)$ & 314 & 5.04 \\
\hline $\begin{array}{l}4051 \text { Day } \\
\text { Avg }\end{array}$ & 126 & 91.1 & 739 & $(32.7)$ & (58.6) & (141) & 247 & 15,500 & 9,890 & 298 & (10) & (524) & (8.74) \\
\hline $\begin{array}{l}4052 \text { Week } \\
\text { Avg }\end{array}$ & (34.7) & 18.6 & 115 & $(4.38)$ & $(4.75)$ & (121) & 235 & 15,000 & 10,900 & 154 & (1.66) & (364) & $(8.59)$ \\
\hline $\begin{array}{l}4051 \\
\text { Month Avg }\end{array}$ & (12.0) & 23.7 & 112 & 3.74 & (2.16 & $(89.2)$ & 211 & 12,700 & 9,600 & 121 & $(1.20)$ & 451 & 7.94 \\
\hline
\end{tabular}


Table 3.19 lists the leachable percentages of metals present in the single-contact water extracts. These values show that $16.2 \%$ to $19.3 \%$ of the total $\mathrm{Na}$ is water-leachable and $4.7 \%$ to $21.6 \%$ of the $\mathrm{Ni}$ is leachable. About $7 \%$ to $12 \%$ of the $\mathrm{Mn}$ and $\mathrm{Mg}$ are dissolved in water. Other elements that have leachabilities greater than $1 \%$ in one or more of the tests are $\mathrm{Ba}, \mathrm{Ca}, \mathrm{Si}$, and $\mathrm{Sr}$. Elements with lower water leachabilities are $\mathrm{Al}, \mathrm{Cr}, \mathrm{Fe}$, and $\mathrm{Pb}$.

\subsubsection{Anion Concentrations - Single-Contact Water Extracts}

The anion concentrations in water extracts from the tank C-106 sludge samples are listed in Table 3.20. The primary leachable anions are carbonate and oxalate, with much smaller quantities of chloride and fluoride leaching. The presence of high concentrations of oxalate $(25,911$ to $39,211 \mu \mathrm{g} / \mathrm{g})$ in the water extracts shows that the sludge has the capacity to act as a reductant for more oxidized species. However, its presence in the sludge in contact with air suggests the oxalate is not readily oxidized in this environment.

\subsubsection{Radioanalytical Results - Single-Contact Water Extracts}

The results of the GEA analysis for ${ }^{60} \mathrm{Co}$ and ${ }^{137} \mathrm{Cs}$ in the water extractions are listed in Table 3.21. For the tank C-106 sludge samples, ${ }^{60} \mathrm{Co}$ was not detected in any of the leachates and ${ }^{137} \mathrm{Cs}$ was measured at values of 2.03 and $2.65 \mu \mathrm{g} / \mathrm{g}$ in the one-day water leach.

Total beta and total alpha levels for the one-day, water-leach experiments are listed in Table 3.22. Comparing these results with the fusion analysis levels (Table 3.10) shows that about $2 \%$ of the total beta is water leachable from tank C-106 sludge. Approximately $0.02 \%$ of the total alpha is water leachable from the sludge. Table 3.22 also lists the water-leachable concentrations of ${ }^{90} \mathrm{Sr}$. Comparing these levels to the fusion analysis values (Table 3.10) shows that $1.0 \%$ of the ${ }^{90} \mathrm{Sr}$ is water leachable from tank C-106 sludge.

Table 3.23 lists the water-leachable concentrations of ${ }^{239} \mathrm{Pu},{ }^{237} \mathrm{~Np}$, and ${ }^{241} \mathrm{Am}$ in terms of the original sludge compositions. The percent water leachabilities for these actinides are listed in Table 3.24. The water leachability of ${ }^{239} \mathrm{Pu}$ and ${ }^{241} \mathrm{Am}$ are much less than $1 \%$. The leachability of ${ }^{237} \mathrm{~Np}$ is in the range of 7.78 to $9.8 \%$ for the 1-day and 1-month contact tests. No actinide measurements were made on the 2-week water leach test solutions.

\subsubsection{Periodic Replenishment Test Results}

The periodic replenishment tests were conducted by contacting each sludge sub-sample with sequential 30-mL quantities of fresh DDI water. As shown in Table 3.25, the sludge was contacted six times with fresh water. The first five contacts were of short duration ( 1 or 4 days each), which was assumed sufficient for leaching contaminants of concern from the sludge. To test this assumption, an 
Table 3.19. Water-Leachable Metals in Tank C-106 Single-Contact Water Extract Compared with Fusion Results (\%)

\begin{tabular}{|c|c|c|c|c|c|c|c|c|c|c|c|c|c|}
\hline \multirow{2}{*}{\begin{tabular}{|c|} 
Sample \\
Number
\end{tabular}} & $\mathbf{A l}$ & $\mathbf{B a}$ & 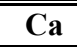 & $\mathbf{C r}$ & $\mathbf{F e}$ & $\mathbf{K}$ & Mg & Mn & $\mathbf{N a}$ & Ni & $\mathbf{P b}$ & Si & Sr \\
\hline & \multicolumn{13}{|c|}{ } \\
\hline $\begin{array}{l}4041 \text { Day } \\
\text { Avg }\end{array}$ & 0.06 & 3.29 & 0.86 & $(0.21)$ & $(0.06)$ & N/A & 5.69 & 7.81 & 17.33 & 8.96 & $(0.05)$ & (1.88) & (1.78) \\
\hline $\begin{array}{l}4042 \text { Week } \\
\text { Avg E }\end{array}$ & $(0.02)$ & 0.92 & 0.18 & $(0.34)$ & $(0.01)$ & N/A & 7.22 & 9.18 & 19.31 & 5.91 & $(0.02)$ & $(1.48)$ & $(2.26)$ \\
\hline $\begin{array}{l}4041 \text { Month } \\
\text { Avg }\end{array}$ & $(0.0)$ & 1.44 & 0.18 & $(0.30)$ & $(0.00)$ & N/A & 6.57 & 7.64 & 16.85 & 4.71 & $(0.01)$ & 1.66 & 2.12 \\
\hline $\begin{array}{l}4051 \text { Day } \\
\text { Avg }\end{array}$ & 0.10 & 9.20 & 1.95 & $(3.44)$ & $(0.13)$ & N/A & 10.73 & 12.73 & 16.69 & 21.64 & $(0.21)$ & $(2.73)$ & $(3.18)$ \\
\hline $\begin{array}{l}4052 \text { Week } \\
\text { Avg E }\end{array}$ & $(0.03)$ & 1.88 & 0.30 & $(0.46)$ & $(0.01)$ & N/A & 10.21 & 12.29 & 18.43 & 11.14 & $(0.03)$ & (1.89) & (3.12) \\
\hline $\begin{array}{l}4051 \text { Month } \\
\text { Avg }\end{array}$ & $(0.01)$ & 2.40 & 0.30 & 0.39 & $(0.00)$ & N/A & 9.18 & 10.39 & 16.20 & 8.79 & $(0.02)$ & 2.35 & 2.89 \\
\hline
\end{tabular}

Table 3.20. Average Anion Concentrations in Tank C-106 Single-Contact Water Extract

\begin{tabular}{|c|c|c|c|c|c|c|c|c|c|c|}
\hline \multirow[b]{2}{*}{ Sample Number } & Fluoride & Acetate & Formate & Chloride & Nitrite & Nitrate & Carbonate & Sulfate & Oxalate & Phosphate \\
\hline & \multicolumn{10}{|c|}{ 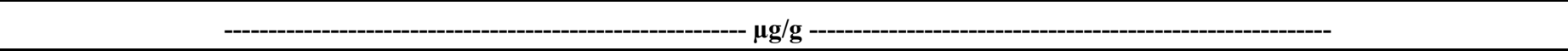 } \\
\hline 4041 Day Avg & 35.3 & $<173$ & $<51.1$ & 82.6 & $<57$ & $<55$ & 20,829 & $<52$ & 25,911 & $<84$ \\
\hline 4042 Week Avg & INS & INS & INS & INS & INS & INS & INS & INS & INS & INS \\
\hline 4041 Month Avg & $<129$ & $<1,508$ & $<445$ & 519 & $<496$ & $<475$ & $<55,026$ & $<450$ & 34,350 & $<0.72$ \\
\hline 4051 Day Avg & 34.4 & $<285$ & $<84$ & 99 & $<94$ & $<90$ & 12,633 & $<85$ & 39,211 & $<105$ \\
\hline 4052 Week Avg & INS & INS & INS & INS & INS & INS & INS & INS & INS & INS \\
\hline 4051 Month Avg & 191 & $<2,176$ & $<642$ & 410 & $<716$ & $<686$ & $<79,404$ & $<650$ & 37,946 & $<0.96$ \\
\hline
\end{tabular}


Table 3.21. GEA for Tank C-106 Single-Contact Water Extractions and Direct Solids

\begin{tabular}{|c|c|c|c|c|}
\hline & ${ }^{60} \mathrm{Co}$ & ${ }^{60} \mathrm{Co}$ & ${ }^{137} \mathrm{Cs}$ & ${ }^{137} \mathrm{Cs}$ \\
\hline Sample Number & $---\mu \mathrm{Ci} / \mathrm{g}-----$ & ----- $\mu g / g--------$ & $---\mu \mathrm{Ci} / \mathrm{g}-----$ & 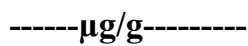 \\
\hline 4041 Day Avg & $<1.77 \mathrm{E}-02$ & $<1.61 \mathrm{E}-05$ & $2.65 \mathrm{E}+00$ & $3.044 \mathrm{E}-02$ \\
\hline 4051 Day Avg & $<2.92 \mathrm{E}-02$ & $<2.65 \mathrm{E}-05$ & $2.03 \mathrm{E}+00$ & $2.329 \mathrm{E}-02$ \\
\hline
\end{tabular}

Table 3.22. Total Beta, Total Alpha, and ${ }^{90} \mathrm{Sr}-$ Single-Contact Water Extracts

\begin{tabular}{|c|c|c|c|c|}
\hline \multirow[b]{2}{*}{ Sample Number } & Total Beta & Total Alpha & \multicolumn{2}{|c|}{${ }^{{ }^{90} \mathrm{Sr}}$} \\
\hline & \multicolumn{2}{|c|}{------- $\mu \mathrm{Ci} / \mathrm{g}-------$} & $--\mu \mathrm{Ci} / \mathrm{g}--$ & $--\mu g / g--$ \\
\hline (4041 Day Avg & $9.97 \mathrm{E}+01$ & 1.68E-01 & $2.37 \mathrm{E}+01$ & 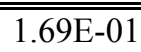 \\
\hline 4051 Day Avg & $1.75 \mathrm{E}+02$ & $2.87 \mathrm{E}-01$ & $4.29 \mathrm{E}+01$ & $3.06 \mathrm{E}-01$ \\
\hline
\end{tabular}

Table 3.23. Actinide Analysis for Tank C-106 Single-Contact Water Extracts

\begin{tabular}{|c|c|c|c|c|c|c|}
\hline \multirow[b]{2}{*}{ Sample Number } & \multicolumn{2}{|c|}{${ }^{239} \mathbf{P u}$} & \multicolumn{2}{|c|}{${ }^{2237} \mathrm{~Np}$} & \multicolumn{2}{|c|}{${ }^{241} \mathrm{Am}$} \\
\hline & $--\mu \mathrm{Ci} / \mathrm{g}--$ & $--\mu \mathrm{g} / \mathrm{g}--$ & $--\mu \mathrm{Ci} / \mathrm{g}--$ & $--\mu \mathrm{g} / \mathrm{g}--$ & $--\mu \mathrm{Ci} / \mathrm{g}--$ & $--\mu g / g--$ \\
\hline 4041 Day & $(3.81 \mathrm{E}-04)$ & (6.14E-03) & $5.33 \mathrm{E}-04$ & $7.51 \mathrm{E}-01$ & $2.57 \mathrm{E}-03$ & $7.55 \mathrm{E}-04$ \\
\hline 4041 Month & $9.10 \mathrm{E}-05$ & $1.47 \mathrm{E}-03$ & $4.88 \mathrm{E}-04$ & $6.88 \mathrm{E}-01$ & $4.88 \mathrm{E}-04$ & $1.66 \mathrm{E}-03$ \\
\hline 4051 Day & $(1.18 \mathrm{E}-02)$ & $6.74 \mathrm{E}-04$ & $9.50 \mathrm{E}-01$ & $<1.92 \mathrm{E}-03$ & $<5.65 \mathrm{E}-04$ & $(1.18 \mathrm{E}-02)$ \\
\hline 4051 Month & $2.33 \mathrm{E}-03$ & $6.74 \mathrm{E}-04$ & $9.49 \mathrm{E}-01$ & 7.38E-04 & $2.51 \mathrm{E}-03$ & $2.33 \mathrm{E}-03$ \\
\hline
\end{tabular}

Table 3.24. Water-Leachable Percentage for Actinides in C-106 Single-Contact Water Extracts Compared to Fusion Analysis

\begin{tabular}{|c|c|c|c|}
\hline Sample Number & ${ }^{239} \mathrm{Pu}$ & ${ }^{237} \mathbf{N p}$ & ${ }^{241} \mathrm{Am}$ \\
\hline \multicolumn{4}{|c|}{ \% Water Leachable } \\
\hline 4041 Day & $(0.02 \%)$ & $8.49 \%$ & $0.04 \%$ \\
\hline 4041 Month & $(0.005 \%)$ & $7.78 \%$ & $0.09 \%$ \\
\hline 4051 Day & $(0.04 \%)$ & $9.80 \%$ & $0.03 \%$ \\
\hline 4051 Month & $(0.008 \%)$ & $9.79 \%$ & $0.13 \%$ \\
\hline
\end{tabular}

extended period of leaching was conducted in which the sludge, after the first five stages of leaching, was contacted with water for 43 days (Stage 6A) or 82 days (Stage 6B). The objective was to evaluate the long-term leaching characteristics of contaminants from the sludge. Very little of the primary contaminants of concern, ${ }^{99} \mathrm{Tc}$ and ${ }^{238} \mathrm{U}$, were leachable during these tests. Over $90 \%$ of the ${ }^{99} \mathrm{Tc}$ and ${ }^{238} \mathrm{U}$ remained in the sludge and was not leachable during the six stages of water leaching. 


\subsubsection{Digestion Factors and Moisture Contents - Periodic Replenishment Water Tests}

The digestion factors for the 404 and 405 sludge samples used for the replenishment tests varied from $4.5 \mathrm{~g} / \mathrm{L}$ to $8.1 \mathrm{~g} / \mathrm{L}$. These digestion factors are the ratios of wet weight of sludge to the amount of DDI water $(30 \mathrm{~mL})$ used to dissolve the soluble portion of the solid. The digestion factors were then multiplied by the percent solids, as determined from moisture content analysis, to convert concentrations to a dry weight basis. The variability in digestion factors is a function of the mass of sludge used, which ranged from approximately 0.2 to $0.38 \mathrm{~g}$.

The average moisture contents [(wet wt - dry wt)/dry wt] were $57.5 \%$ for the 404 samples and $43.5 \%$ for the 405 samples.

\subsubsection{Water Extract pH and Alkalinity - Periodic Replenishment Water Tests}

Table 3.25 lists the $\mathrm{pH}$ values and alkalinities for the leachates from the replenishment tests. The leachate $\mathrm{pH}$ values for sample 404 during the first five stages are in the range 6.87 to 7.03 and then increase to an average of 7.5 in Stage 6. There is a similar trend in $\mathrm{pH}$ for sample 405, although the initial $\mathrm{pH}$ values are lower in the range of 6.68 to 6.71. The increase in $\mathrm{pH}$ at Stages $6 \mathrm{~A}$ and $6 \mathrm{~B}$ may reflect equilibration with a carbonate mineral. The alkalinity values decrease from values of 42.71 and $59.58 \mathrm{mg}$ $\mathrm{CaCO}_{3}$ per gram of sludge to about 13 and $16 \mathrm{mg} \mathrm{CaCO}$ per gram of sludge in samples 404 and 405 , respectively.

Table 3.25. Contact Times, Average $\mathrm{pH}$ Values, and Alkalinities for Periodic Replenishment Tests on Tank C-106 Sludge Samples

\begin{tabular}{||c|c|c|c||}
\hline \multicolumn{1}{||c|}{ Contact Stage } & $\begin{array}{c}\text { Contact Duration } \\
\text { (days) }\end{array}$ & $\mathbf{p H}$ & $\begin{array}{c}\text { Alkalinity as mg/g } \\
\mathbf{C a C O}_{\mathbf{3}}\end{array}$ \\
\hline \multicolumn{5}{|c|}{ Sample 404 } \\
\hline 1 & 1 & 7.03 & 42.71 \\
\hline 2 & 1 & 6.87 & $<31.62$ \\
\hline 3 & 4 & 6.93 & $<31.62$ \\
\hline 4 & 1 & 6.75 & $<31.62$ \\
\hline 5 & 1 & 6.73 & $<31.62$ \\
\hline $6 \mathrm{~A}$ & 43 & 7.43 & 12.43 \\
\hline $6 \mathrm{~B}$ & 82 & 7.62 & 13.89 \\
\hline \multicolumn{5}{|c|}{ Sample $\mathbf{4 0 5}$} & 59.58 \\
\hline 1 & 1 & 6.71 & 53.59 \\
\hline 2 & 1 & 6.70 & $<52.09$ \\
\hline 3 & 4 & 6.79 & $<52.09$ \\
\hline 4 & 1 & 6.68 & $<52.09$ \\
\hline 5 & 1 & 6.70 & 15.04 \\
\hline $6 \mathrm{~A}$ & 43 & 7.50 & 16.99 \\
\hline $6 \mathrm{~B}$ & 82 & 7.70 & \\
\hline
\end{tabular}




\subsubsection{3 $\quad{ }^{99}$ Tc and ${ }^{238} \mathrm{U}-$ Periodic Replenishment Tests}

Table 3.26 lists the amount of ${ }^{99} \mathrm{Tc}$ and ${ }^{238} \mathrm{U}$ leached from the two sludge samples during the replenishment tests, and Table 3.27 lists the percentages of the available radionuclides that were leached. As was found in the single-contact water leach tests (Table 3.16), very little of the ${ }^{99} \mathrm{Tc}$ or ${ }^{238} \mathrm{U}$ was water leachable. Only $2.3 \%$ of the ${ }^{99} \mathrm{Tc}$ is leachable in the first five contact stages for sample 404, while an additional 3.57\% and 3.73\% were leachable during Stages 6A and 6B, respectively. Similar amounts of ${ }^{99} \mathrm{Tc}$ were leachable throughout the six stages of leaching sample 405.

Approximately $4 \%$ of the ${ }^{238} \mathrm{U}$ was water leachable throughout the six contact stages for sample 404, with similar amounts leaching from sample 405. These results show the recalcitrant nature of the residual ${ }^{99} \mathrm{Tc}$ and ${ }^{238} \mathrm{U}$ in the sludge in tank $\mathrm{C}-106$ after the oxalic acid sluicing campaign.

Table 3.26. ${ }^{99} \mathrm{Tc}$ and ${ }^{238} \mathrm{U}$ Concentrations in Tank C-106 Sludge Samples - Periodic Water Replenishment Tests

\begin{tabular}{|c|c|c|c|c|c|}
\hline \multirow[b]{2}{*}{ Contact Stage } & \multirow{2}{*}{$\begin{array}{c}\text { Contact Duration } \\
\text { (days) }\end{array}$} & ${ }^{99} \mathrm{Tc}$ & ${ }^{238} \mathbf{U}$ & ${ }^{99} \mathrm{Tc}$ & ${ }^{238} \mathbf{U}$ \\
\hline & & \multicolumn{2}{|c|}{ - } & \multicolumn{2}{|c|}{--------- $\mu \mathrm{Ci} / \mathrm{g}$-------- } \\
\hline \multicolumn{6}{|c|}{ Sample 404} \\
\hline 1 & 1 & $1.98 \mathrm{E}-02$ & $7.03 \mathrm{E}+00$ & $3.36 \mathrm{E}-04$ & $2.39 \mathrm{E}-06$ \\
\hline 2 & 1 & $(2.31 \mathrm{E}-03)$ & $1.31 \mathrm{E}+00$ & $(3.93 \mathrm{E}-05)$ & $4.46 \mathrm{E}-07$ \\
\hline 3 & 4 & $3.56 \mathrm{E}-03$ & $5.56 \mathrm{E}-01$ & $6.06 \mathrm{E}-05$ & $1.89 \mathrm{E}-07$ \\
\hline 4 & 1 & $(2.51 \mathrm{E}-03)$ & $2.28 \mathrm{E}-01$ & $(4.27 \mathrm{E}-05)$ & $7.75 \mathrm{E}-08$ \\
\hline 5 & 1 & $<3.79 \mathrm{E}-03$ & $9.25 \mathrm{E}-02$ & $<6.45 \mathrm{E}-05$ & $3.14 \mathrm{E}-08$ \\
\hline $6 \mathrm{~A}$ & 43 & $3.62 \mathrm{E}-02$ & $2.04 \mathrm{E}-01$ & $6.15 \mathrm{E}-04$ & $6.92 \mathrm{E}-08$ \\
\hline $6 \mathrm{~B}$ & 82 & $3.77 \mathrm{E}-02$ & $1.66 \mathrm{E}-01$ & $6.41 \mathrm{E}-04$ & $5.63 \mathrm{E}-08$ \\
\hline \multicolumn{6}{|c|}{ Sample 405} \\
\hline 1 & 1 & $1.61 \mathrm{E}-02$ & $7.66 \mathrm{E}+00$ & $2.73 \mathrm{E}-04$ & $2.61 \mathrm{E}-06$ \\
\hline 2 & 1 & $(3.60 \mathrm{E}-03)$ & $1.68 \mathrm{E}+00$ & $(6.12 \mathrm{E}-05)$ & $5.71 \mathrm{E}-07$ \\
\hline 3 & 4 & (5.29E-03) & $6.65 \mathrm{E}-01$ & $(9.00 \mathrm{E}-05)$ & $2.26 \mathrm{E}-07$ \\
\hline 4 & 1 & $<4.20 \mathrm{E}-03$ & $1.89 \mathrm{E}-01$ & $<7.13 \mathrm{E}-05$ & $6.44 \mathrm{E}-08$ \\
\hline 5 & 1 & $<6.25 \mathrm{E}-03$ & $8.28 \mathrm{E}-02$ & $<1.06 \mathrm{E}-04$ & $2.81 \mathrm{E}-08$ \\
\hline $6 \mathrm{~A}$ & 43 & $3.62 \mathrm{E}-02$ & $2.45 \mathrm{E}-01$ & $6.16 \mathrm{E}-04$ & $8.35 \mathrm{E}-08$ \\
\hline $6 \mathrm{~B}$ & 82 & $4.63 \mathrm{E}-02$ & $2.74 \mathrm{E}-01$ & $7.87 \mathrm{E}-04$ & $9.31 \mathrm{E}-08$ \\
\hline
\end{tabular}

\subsubsection{Selected Metal Concentrations - Periodic Replenishment Water Extracts}

Table 3.28 lists the concentrations of metals leached during the periodic replenishment tests, and Table 3.29 lists the percentage of the total available metals that were leached during each stage of the test. The leachable metals from the two sludge samples are $\mathrm{Ba}, \mathrm{Ca}, \mathrm{Mg}, \mathrm{Mn}, \mathrm{Na}$, and $\mathrm{Ni}$. Non-leachable metals throughout the periodic replenishment tests were $\mathrm{Al}, \mathrm{Cr}, \mathrm{Fe}, \mathrm{Pb}, \mathrm{Si}$, and $\mathrm{Sr}$. 
Table 3.27. Water-Leachable Percentages of ${ }^{99} \mathrm{Tc}$ and ${ }^{238} \mathrm{U}$ in Tank C-106 Sludge Samples - Periodic Water Replenishment Tests Relative to Fusion Results

\begin{tabular}{|c|c|c|c|}
\hline \multirow[b]{2}{*}{ Contact Stage } & \multirow[b]{2}{*}{$\begin{array}{c}\text { Contact Duration } \\
\text { (days) }\end{array}$} & ${ }^{99} \mathrm{Tc}$ & 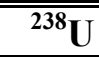 \\
\hline & & \multicolumn{2}{|c|}{$\%$ Water Leachable } \\
\hline \multicolumn{4}{|c|}{ Sample 404} \\
\hline 1 & 1 & 1.95 & 2.98 \\
\hline 2 & 1 & $(0.23)$ & 0.56 \\
\hline 3 & 4 & 0.35 & 0.24 \\
\hline 4 & 1 & $(0.25)$ & 0.10 \\
\hline 5 & 1 & $<0.37$ & 0.04 \\
\hline $6 \mathrm{~A}$ & 43 & 3.57 & 0.09 \\
\hline $6 \mathrm{~B}$ & 82 & 3.73 & 0.07 \\
\hline \multicolumn{4}{|c|}{ Sample 405} \\
\hline 1 & 1 & 1.22 & 2.97 \\
\hline 2 & 1 & $(0.27)$ & 0.65 \\
\hline 3 & 4 & $(0.40)$ & 0.26 \\
\hline 4 & 1 & $<0.32$ & 0.07 \\
\hline 5 & 1 & $<0.48$ & 0.03 \\
\hline $6 \mathrm{~A}$ & 43 & 2.76 & 0.10 \\
\hline $6 \mathrm{~B}$ & 82 & 3.53 & 0.11 \\
\hline
\end{tabular}

There are two trends observable in the metals that are leachable in this test. The amount leachable, as a percentage of the total metal in the sludge, during the first five stages of leaching for the metals $\mathrm{Ba}, \mathrm{Ca}$, and $\mathrm{Ni}$, is approximately constant. The percent water leachable ranges for these metals in sample 404 are $\mathrm{Ba}(2.13-4.34 \%), \mathrm{Ca}(0.92-1.69 \%)$, and $\mathrm{Ni}(9.30-10.83 \%)$. The percent water leachable ranges for these metals in sample 405 are $\mathrm{Ba}(5.24-14.38 \%), \mathrm{Ca}(1.54-2.09 \%)$, and $\mathrm{Ni}(15.97-26.88 \%)$. For the metals $\mathrm{Mg}, \mathrm{Mn}$, and $\mathrm{Na}$, there is a strong, uniform, decreasing trend of leachability for the first five stages of this test. For example, in sample 404 the Mn leachability decreases from 8.57 to $1.62 \%$ and the $\mathrm{Na}$ leachability decreases from 19.01 to $1.48 \%$ for stages 1 and 5. Constant levels of leachability, such as those observed for $\mathrm{Ba}, \mathrm{Ca}$, and $\mathrm{Ni}$, suggest equilibrium control on solution concentration by a single mineral for each metal. The decreasing leachability trend observed for $\mathrm{Mg}, \mathrm{Mn}$, and $\mathrm{Na}$ suggests the dissolution and removal from the sludge of a soluble mineral containing the metal.

At Stages 6A (43-day contact) and 6B (82-day contact), the leachable percentages of $\mathrm{Ba}, \mathrm{Mg}, \mathrm{Mn}$, and $\mathrm{Ni}$ decrease from values observed in the first five stages of leaching, whereas the value for $\mathrm{Ca}$ is fairly constant. The leachability of $\mathrm{Na}$ during stages $6 \mathrm{~A}$ and $6 \mathrm{~B}$ increases relative the values for Stages 3 through 5, but decreases compared to Stages 1 and 2. Sr leachability at measurable amounts above the EQL only occurs in stages 6A and 6B. 
Table 3.28. Water-Leachable Average Metals in Tank C-106 Sludge Samples - Periodic Water Replenishment Tests

\begin{tabular}{|c|c|c|c|c|c|c|c|c|c|c|c|c|c|}
\hline \multirow{2}{*}{$\begin{array}{l}\text { Contact } \\
\text { Stage }\end{array}$} & Al & Ba & $\mathbf{C a}$ & $\mathrm{Cr}$ & Fe & $\mathbf{K}$ & Mg & Mn & $\mathbf{N a}$ & $\mathbf{N i}$ & $\mathbf{P b}$ & Si & Sr \\
\hline & \multicolumn{13}{|c|}{ 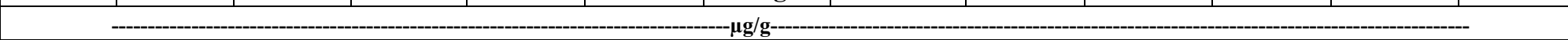 } \\
\hline \multicolumn{14}{|c|}{ Sample 404} \\
\hline 1 & $9.13 \mathrm{E}+01$ & $3.85 \mathrm{E}+01$ & $3.61 \mathrm{E}+02$ & $(1.97 \mathrm{E}+00)$ & $(2.66 \mathrm{E}+01)$ & \begin{tabular}{|l|}
$(9.37 \mathrm{E}+01)$ \\
\end{tabular} & $1.67 \mathrm{E}+02$ & $9.73 \mathrm{E}+03$ & $1.17 \mathrm{E}+04$ & $2.01 \mathrm{E}+02$ & $(2.88 \mathrm{E}+00)$ & $(3.91 \mathrm{E}+02)$ & $(4.64 \mathrm{E}+00)$ \\
\hline 2 & $8.16 \mathrm{E}+01$ & $3.45 \mathrm{E}+01$ & $3.97 \mathrm{E}+02$ & $<1.97 \mathrm{E}+01$ & $(2.17 \mathrm{E}+01)$ & $<4.12 \mathrm{E}+02$ & $1.59 \mathrm{E}+02$ & $9.45 \mathrm{E}+03$ & $7.41 \mathrm{E}+03$ & $1.89 \mathrm{E}+02$ & $(4.52 \mathrm{E}+00)$ & $(3.52 \mathrm{E}+02)$ & $(5.48 \mathrm{E}+00)$ \\
\hline 3 & $(6.68 \mathrm{E}+01)$ & $3.42 \mathrm{E}+01$ & $3.54 \mathrm{E}+02$ & $(3.95 \mathrm{E}+01)$ & $(2.06 \mathrm{E}+01)$ & $(4.25 \mathrm{E}+02)$ & $1.57 \mathrm{E}+02$ & $9.02 \mathrm{E}+03$ & $2.12 \mathrm{E}+03$ & $1.90 \mathrm{E}+02$ & $(4.19 \mathrm{E}+00)$ & $(3.19 \mathrm{E}+02)$ & $(5.86 \mathrm{E}+00)$ \\
\hline 4 & $6.41 \mathrm{E}+01$ & $4.48 \mathrm{E}+01$ & $3.55 \mathrm{E}+02$ & $<2.10 \mathrm{E}+01$ & $(2.50 \mathrm{E}+01)$ & $<5.11 \mathrm{E}+01$ & $1.50 \mathrm{E}+02$ & $7.88 \mathrm{E}+03$ & $1.90 \mathrm{E}+03$ & $1.95 \mathrm{E}+02$ & $(2.46 \mathrm{E}+00)$ & $(3.34 \mathrm{E}+02)$ & $(6.46 \mathrm{E}+00)$ \\
\hline 5 & $6.18 \mathrm{E}+01$ & $(4.62 \mathrm{E}+01)$ & $3.62 \mathrm{E}+02$ & $<2.10 \mathrm{E}+01$ & $\begin{array}{l}(2.24 \mathrm{E}+01) \\
\end{array}$ & $(2.51 \mathrm{E}+01)$ & $1.28 \mathrm{E}+02$ & $6.39 \mathrm{E}+03$ & $1.63 \mathrm{E}+03$ & $1.94 \mathrm{E}+02$ & $<1.54 \mathrm{E}+00$ & $(3.38 \mathrm{E}+02)$ & $(7.03 \mathrm{E}+00)$ \\
\hline $6 \mathrm{~A}$ & $(4.47 \mathrm{E}+00)$ & $1.52 \mathrm{E}+01$ & $2.52 \mathrm{E}+02$ & $(8.78 \mathrm{E}-01)$ & \begin{tabular}{|l|}
$(4.57 \mathrm{E}-01)$ \\
\end{tabular} & $(2.04 \mathrm{E}+01)$ & $4.14 \mathrm{E}+01$ & $1.54 \mathrm{E}+03$ & $3.32 \mathrm{E}+03$ & $7.96 \mathrm{E}+01$ & $<1.63 \mathrm{E}+01$ & $(2.47 \mathrm{E}+02)$ & $1.11 \mathrm{E}+01$ \\
\hline $6 \mathrm{~B}$ & $(7.05 \mathrm{E}+01)$ & $1.99 \mathrm{E}+01$ & $(4.30 \mathrm{E}+02)$ & $(1.19 \mathrm{E}+00)$ & $1.13 \mathrm{E}+00$ & $(8.65 \mathrm{E}+01)$ & $8.38 \mathrm{E}+01$ & $1.81 \mathrm{E}+03$ & $4.02 \mathrm{E}+03$ & $7.96 \mathrm{E}+01$ & $<1.53 \mathrm{E}+01$ & $(2.23 \mathrm{E}+02)$ & $1.71 \mathrm{E}+01$ \\
\hline \multicolumn{14}{|c|}{$\begin{array}{lc} & \text { Sample 405 } \\
\end{array}$} \\
\hline 1 & $1.26 \mathrm{E}+02$ & $9.11 \mathrm{E}+01$ & $7.39 \mathrm{E}+02$ & $(3.27 \mathrm{E}+01)$ & $(5.86 \mathrm{E}+01)$ & $(1.41 \mathrm{E}+02)$ & $2.47 \mathrm{E}+02$ & $1.55 \mathrm{E}+04$ & $9.89 \mathrm{E}+03$ & $2.98 \mathrm{E}+02$ & $(1.00 \mathrm{E}+01)$ & $(5.24 \mathrm{E}+02)$ & $(8.74 \mathrm{E}+00)$ \\
\hline 2 & $1.10 \mathrm{E}+02$ & $5.19 \mathrm{E}+01$ & $7.00 \mathrm{E}+02$ & $<3.52 \mathrm{E}+01$ & $(4.91 \mathrm{E}+01)$ & $(6.90 \mathrm{E}+02)$ & $1.95 \mathrm{E}+02$ & $1.17 \mathrm{E}+04$ & $2.05 \mathrm{E}+03$ & $3.71 \mathrm{E}+02$ & $<6.13 \mathrm{E}+01$ & $(3.85 \mathrm{E}+02)$ & $(9.58 \mathrm{E}+00)$ \\
\hline 3 & $1.11 \mathrm{E}+02$ & $6.50 \mathrm{E}+01$ & $5.84 \mathrm{E}+02$ & $<6.51 \mathrm{E}+01$ & $(3.25 \mathrm{E}+01)$ & $(8.10 \mathrm{E}+01)$ & $1.40 \mathrm{E}+02$ & $7.45 \mathrm{E}+03$ & $1.98 \mathrm{E}+03$ & $3.59 \mathrm{E}+02$ & $(3.09 \mathrm{E}+00)$ & $(4.62 \mathrm{E}+02)$ & $(1.11 \mathrm{E}+01)$ \\
\hline 4 & $1.09 \mathrm{E}+02$ & $8.54 \mathrm{E}+01$ & $7.32 \mathrm{E}+02$ & $<6.51 \mathrm{E}+01$ & $(1.89 \mathrm{E}+01)$ & $<1.30 \mathrm{E}+03$ & $(5.16 \mathrm{E}+01)$ & $2.71 \mathrm{E}+03$ & $1.34 \mathrm{E}+03$ & $3.22 \mathrm{E}+02$ & $(3.35 \mathrm{E}+00)$ & $(3.14 \mathrm{E}+02)$ & $(1.19 \mathrm{E}+01)$ \\
\hline 5 & $1.13 \mathrm{E}+02$ & $1.42 \mathrm{E}+02$ & $7.94 \mathrm{E}+02$ & $(2.91 \mathrm{E}+00)$ & $(1.54 \mathrm{E}+01)$ & $(1.40 \mathrm{E}+02)$ & $(3.98 \mathrm{E}+01)$ & $1.39 \mathrm{E}+03$ & $1.26 \mathrm{E}+03$ & $2.20 \mathrm{E}+02$ & $(1.04 \mathrm{E}+01)$ & $(2.69 \mathrm{E}+02)$ & $(1.30 \mathrm{E}+01)$ \\
\hline $6 \mathrm{~A}$ & $(1.36 \mathrm{E}+01)$ & $1.99 \mathrm{E}+01$ & $2.92 \mathrm{E}+02$ & $(1.11 \mathrm{E}+00)$ & $(9.84 \mathrm{E}-01)$ & $(3.47 \mathrm{E}+01)$ & $3.35 \mathrm{E}+01$ & $9.24 \mathrm{E}+02$ & $4.80 \mathrm{E}+03$ & $3.87 \mathrm{E}+01$ & $<2.45 \mathrm{E}+01$ & $(2.49 \mathrm{E}+02)$ & $2.47 \mathrm{E}+01$ \\
\hline $6 \mathrm{~B}$ & $(1.32 \mathrm{E}+02)$ & $3.45 \mathrm{E}+01$ & $(7.83 \mathrm{E}+02)$ & $(1.76 \mathrm{E}+00)$ & $(1.50 \mathrm{E}+00)$ & $(2.17 \mathrm{E}+01)$ & $(9.40 \mathrm{E}+01)$ & $1.37 \mathrm{E}+03$ & $5.90 \mathrm{E}+03$ & $3.68 \mathrm{E}+01$ & $(1.20 \mathrm{E}+00)$ & $(2.92 \mathrm{E}+02)$ & $(3.71 \mathrm{E}+01)$ \\
\hline
\end{tabular}


Table 3.29. Percent Water-Leachable Average Metal Concentrations in Tank C-106 Sludge Samples - Periodic Water Replenishment Tests

\begin{tabular}{|c|c|c|c|c|c|c|c|c|c|c|c|c|c|}
\hline \multirow{2}{*}{$\begin{array}{c}\text { Contact } \\
\text { Stage } \\
\end{array}$} & Al & Ba & $\mathbf{C a}$ & $\mathbf{C r}$ & $\mathbf{F e}$ & $\mathbf{K}$ & Mg & Mn & $\mathbf{N a}$ & $\mathbf{N i}$ & $\mathbf{P b}$ & $\mathbf{S i}$ & $\mathrm{Sr}$ \\
\hline & \multicolumn{13}{|c|}{ - } \\
\hline \multicolumn{14}{|c|}{$\begin{array}{lc} & \text { Sample } 404\end{array}$} \\
\hline 1 & 0.07 & 3.61 & 0.94 & $(0.23)$ & $(0.06)$ & N/A & 6.24 & 8.57 & 19.01 & 9.83 & $(0.06)$ & $(2.06)$ & $(1.95)$ \\
\hline 2 & 0.06 & 3.24 & 1.03 & $<2.34$ & $(0.05)$ & N/A & 5.94 & 8.32 & 12.04 & 9.22 & $(0.09)$ & $(1.86)$ & $(2.30)$ \\
\hline 3 & $(0.05)$ & 3.21 & 0.92 & $(4.70)$ & $(0.05)$ & N/A & 5.88 & 7.94 & 3.45 & 9.30 & $(0.09)$ & $(1.68)$ & $(2.47)$ \\
\hline 4 & 0.05 & 4.20 & 0.92 & $<2.49$ & $(0.06)$ & N/A & 5.63 & 6.94 & 3.09 & 9.53 & $(0.05)$ & $(1.76)$ & $(2.72)$ \\
\hline 5 & 0.04 & $(4.34)$ & 0.94 & $<2.49$ & $(0.05)$ & N/A & 4.78 & 5.63 & 2.66 & 9.47 & $<0.03$ & $\begin{array}{l}(1.78) \\
\end{array}$ & \begin{tabular}{|l|}
$(2.96)$ \\
\end{tabular} \\
\hline $6 \mathrm{~A}$ & $(0.003)$ & 1.43 & 0.65 & $(0.10)$ & $(0.001)$ & N/A & 1.55 & 1.36 & 5.40 & 3.89 & $<0.34$ & $(1.30)$ & 4.67 \\
\hline $6 \mathrm{~B}$ & $(0.05)$ & 1.87 & $(1.12)$ & $(0.14)$ & 0.003 & N/A & 3.14 & 1.59 & 6.54 & 3.89 & $<0.32$ & $(1.18)$ & 7.18 \\
\hline \multicolumn{14}{|c|}{ Sample 405} \\
\hline 1 & 0.10 & 9.20 & 1.95 & (3.44) & $(0.13)$ & N/A & 10.73 & 12.73 & 16.69 & 21.64 & $(0.21)$ & (2.73) & (3.18) \\
\hline 2 & 0.09 & 5.24 & 1.84 & $<3.70$ & $(0.11)$ & N/A & 8.47 & 9.56 & 3.46 & 26.88 & $<1.26$ & $(2.00)$ & (3.48) \\
\hline 3 & 0.09 & 6.57 & 1.54 & $<6.84$ & $(0.07)$ & N/A & 6.10 & 6.11 & 3.34 & 26.06 & $(0.06)$ & $(2.40)$ & $(4.04)$ \\
\hline 4 & 0.09 & 8.63 & 1.93 & $<6.84$ & $(0.04)$ & N/A & $(2.25)$ & 2.22 & 2.26 & 23.35 & $(0.07)$ & (1.64) & $(4.34)$ \\
\hline 5 & 0.09 & 14.38 & 2.09 & $(0.31)$ & $(0.03)$ & N/A & $(1.73)$ & 1.14 & 2.13 & 15.97 & $(0.21)$ & $(1.40)$ & $(4.73)$ \\
\hline $6 \mathrm{~A}$ & $(0.01)$ & 2.01 & 0.77 & $(0.12)$ & $(0.002)$ & N/A & 1.46 & 0.76 & 8.10 & 2.81 & $<0.50$ & $(1.30)$ & $\begin{array}{l}8.97 \\
\end{array}$ \\
\hline $6 \mathrm{~B}$ & $(0.11)$ & 3.49 & $(2.06)$ & $(0.18)$ & $(0.003)$ & N/A & $(4.09)$ & 1.12 & 9.96 & 2.67 & $(0.02)$ & $(1.52)$ & $(13.49)$ \\
\hline
\end{tabular}


Table 3.30. Average Anion Concentrations in Tank C-106 Sludge Samples - Periodic Water Replenishment Tests

\begin{tabular}{|c|c|c|c|c|c|c|c|c|c|c|}
\hline \multirow[b]{2}{*}{ Contact Stage } & Fluoride & Acetate & Formate & Chloride & Nitrite & Nitrate & Carbonate & Sulfate & Oxalate & Phosphate \\
\hline & & & & & & & & & & \\
\hline \multicolumn{11}{|c|}{$\begin{array}{l}\text { Sample } 404 \\
\end{array}$} \\
\hline 1 & $3.53 \mathrm{E}+01$ & $<1.73 \mathrm{E}+02$ & $<5.11 \mathrm{E}+01$ & $8.26 \mathrm{E}+01$ & $<5.71 \mathrm{E}+01$ & $<5.48 \mathrm{E}+01$ & $2.08 \mathrm{E}+04$ & $<5.18 \mathrm{E}+01$ & $2.59 \mathrm{E}+04$ & $<8.38 \mathrm{E}+01$ \\
\hline 2 & $<1.48 \mathrm{E}+01$ & $<1.73 \mathrm{E}+02$ & $<5.11 \mathrm{E}+01$ & $4.04 \mathrm{E}+01$ & $<5.71 \mathrm{E}+01$ & $<5.48 \mathrm{E}+01$ & $<6.32 \mathrm{E}+03$ & $<5.18 \mathrm{E}+01$ & $1.71 \mathrm{E}+04$ & $<6.39 \mathrm{E}+01$ \\
\hline 3 & $<1.48 \mathrm{E}+01$ & $<1.73 \mathrm{E}+02$ & $<5.11 \mathrm{E}+01$ & $3.20 \mathrm{E}+01$ & $<5.71 \mathrm{E}+01$ & $<5.48 \mathrm{E}+01$ & $<6.32 \mathrm{E}+03$ & $<5.18 \mathrm{E}+01$ & $1.24 \mathrm{E}+04$ & $<6.39 \mathrm{E}+01$ \\
\hline 4 & $<1.48 \mathrm{E}+01$ & $<1.73 \mathrm{E}+02$ & $<5.11 \mathrm{E}+01$ & $<3.16 \mathrm{E}+01$ & $<5.71 \mathrm{E}+01$ & $<5.48 \mathrm{E}+01$ & $<6.32 \mathrm{E}+03$ & $<5.18 \mathrm{E}+01$ & $6.80 \mathrm{E}+03$ & $<6.39 \mathrm{E}+01$ \\
\hline 5 & $<1.48 \mathrm{E}+01$ & $<1.73 \mathrm{E}+02$ & $<5.11 \mathrm{E}+01$ & $<2.99 \mathrm{E}+01$ & $<5.71 \mathrm{E}+01$ & $<5.48 \mathrm{E}+01$ & $<6.32 \mathrm{E}+03$ & $<5.18 \mathrm{E}+01$ & $4.01 \mathrm{E}+03$ & $<6.39 \mathrm{E}+01$ \\
\hline $6 \mathrm{~A}$ & $<1.53 \mathrm{E}+01$ & $3.14 \mathrm{E}+02$ & $1.06 \mathrm{E}+02$ & $<3.07 \mathrm{E}+01$ & $1.05 \mathrm{E}+02$ & $1.43 \mathrm{E}+02$ & $<6.53 \mathrm{E}+03$ & $<5.34 \mathrm{E}+01$ & $4.01 \mathrm{E}+03$ & $2.06 \mathrm{E}+02$ \\
\hline $6 \mathrm{~B}$ & $<1.43 \mathrm{E}+01$ & $5.68 \mathrm{E}+02$ & $2.50 \mathrm{E}+02$ & $<2.88 \mathrm{E}+01$ & $1.33 \mathrm{E}+02$ & $1.34 \mathrm{E}+02$ & $<6.12 \mathrm{E}+03$ & $<5.01 \mathrm{E}+01$ & $4.37 \mathrm{E}+03$ & $<6.18 \mathrm{E}+01$ \\
\hline \multicolumn{11}{|c|}{ Sample 405} \\
\hline 1 & $3.44 \mathrm{E}+01$ & $<2.85 \mathrm{E}+02$ & $<8.42 \mathrm{E}+01$ & $9.87 \mathrm{E}+01$ & $<9.40 \mathrm{E}+01$ & $<9.02 \mathrm{E}+01$ & $1.26 \mathrm{E}+04$ & $<8.53 \mathrm{E}+01$ & $3.92 \mathrm{E}+04$ & $<1.05 \mathrm{E}+02$ \\
\hline 2 & $<2.44 \mathrm{E}+01$ & $<2.85 \mathrm{E}+02$ & $<8.42 \mathrm{E}+01$ & $6.18 \mathrm{E}+01$ & $<9.40 \mathrm{E}+01$ & $<9.02 \mathrm{E}+01$ & $<1.04 \mathrm{E}+04$ & $<8.53 \mathrm{E}+01$ & $2.29 \mathrm{E}+04$ & $<1.05 \mathrm{E}+02$ \\
\hline 3 & $<2.44 \mathrm{E}+01$ & $<2.85 \mathrm{E}+02$ & $<8.42 \mathrm{E}+01$ & $<5.00 \mathrm{E}+01$ & $<9.40 \mathrm{E}+01$ & $<9.02 \mathrm{E}+01$ & $<1.04 \mathrm{E}+04$ & $<8.53 \mathrm{E}+01$ & $1.49 \mathrm{E}+04$ & $<1.05 \mathrm{E}+02$ \\
\hline 4 & $<2.44 \mathrm{E}+01$ & $<2.85 \mathrm{E}+02$ & $<8.42 \mathrm{E}+01$ & $<4.92 \mathrm{E}+01$ & $<9.40 \mathrm{E}+01$ & $<9.02 \mathrm{E}+01$ & $<1.04 \mathrm{E}+04$ & $<8.53 \mathrm{E}+01$ & $6.12 \mathrm{E}+03$ & $<1.05 \mathrm{E}+02$ \\
\hline 5 & $<2.44 \mathrm{E}+01$ & $<2.85 \mathrm{E}+02$ & $<8.42 \mathrm{E}+01$ & $<5.82 \mathrm{E}+01$ & $<9.40 \mathrm{E}+01$ & $<9.02 \mathrm{E}+01$ & $<1.04 \mathrm{E}+04$ & $<8.53 \mathrm{E}+01$ & $2.65 \mathrm{E}+03$ & $<1.05 \mathrm{E}+02$ \\
\hline $6 \mathrm{~A}$ & $<2.29 \mathrm{E}+01$ & $3.43 \mathrm{E}+02$ & $1.82 \mathrm{E}+02$ & $<4.60 \mathrm{E}+01$ & $1.44 \mathrm{E}+02$ & $2.25 \mathrm{E}+02$ & $<9.79 \mathrm{E}+03$ & $1.04 \mathrm{E}+02$ & $3.60 \mathrm{E}+03$ & $1.90 \mathrm{E}+02$ \\
\hline $6 \mathrm{~B}$ & $<2.59 \mathrm{E}+01$ & $7.73 \mathrm{E}+02$ & $3.66 \mathrm{E}+02$ & $<5.19 \mathrm{E}+01$ & $1.79 \mathrm{E}+02$ & $2.24 \mathrm{E}+02$ & $<1.11 \mathrm{E}+04$ & $<9.04 \mathrm{E}+01$ & $3.56 \mathrm{E}+03$ & $<1.12 \mathrm{E}+02$ \\
\hline
\end{tabular}


$\mathrm{Cr}$ water leachability from the sludge is potentially important because it can be a major contributor to noncarcinogenic risk and the Hazard Index calculation. Cr was not detected above its EQL in any of the periodic water replenishment tests or the single-contact water leach tests (Table 3.19), except for the sample 405 single contact 1 -month test where $0.39 \%$ of the $\mathrm{Cr}$ was water leachable. $\mathrm{Cr}$ in the sludge (estimated at $<900 \mu \mathrm{g} / \mathrm{g}$, Table 3.11 ) has very low water leachability.

\subsubsection{Anion Concentrations - Replenishment Water Extracts}

The leachable anion concentrations at the six stages of the periodic replenishment tests are listed in Table 3.30. The primary leachable anions at Stage 1 were oxalate and carbonate, with much less leachable quantities of chloride and fluoride. Oxalate continues to leach from the sludge samples in measurable quantities at all stages of the test; however, the amount of carbonate leached is below the detection limit after stage 1. (The detection limit for carbonate is high at a level of about $6,300 \mu \mathrm{g} / \mathrm{g}$ in sample 404 and $10,000 \mu \mathrm{g} / \mathrm{g}$ in sample 405.) The amount of oxalate leaching from the sludge decreases fairly uniformly throughout the six stages of leaching. The amount leached from sample 404 in Stage 1 was $25,900 \mu \mathrm{g} / \mathrm{g}$ and in Stage $6 \mathrm{~B}$, it was $4,370 \mu \mathrm{g} / \mathrm{g}$. The amount leached from sample 405 in Stage 1 was $39,200 \mu \mathrm{g} / \mathrm{g}$ and in Stage $6 \mathrm{~B}$, it was $3,560 \mu \mathrm{g} / \mathrm{g}$.

\subsubsection{Radioanalytical Results - Periodic Replenishment Water Tests}

The results of the GEA analysis of the periodic replenishment water tests for ${ }^{60} \mathrm{Co}$ and ${ }^{137} \mathrm{Cs}$ are listed in Table 3.31. ${ }^{60} \mathrm{Co}$ was not detected in any of the test samples. The water leachable concentrations of ${ }^{137} \mathrm{Cs}$ for the first contacts were 2.65 and $2.03 \mu \mathrm{Ci} / \mathrm{g}$ for sludge samples 404 and 405 , respectively. This represents about $2 \%$ of the total ${ }^{137} \mathrm{Cs}$ in the sludge samples (Table 3.8). The subsequent concentrations in each leachate decrease by over a factor of ten compared to the initial level. This shows that a small amount of the ${ }^{137} \mathrm{Cs}$ is very leachable, but the remaining majority is relatively recalcitrant to water leaching.

Table 3.31. GEA for Tank C-106 Periodic Water Replenishment Tests

\begin{tabular}{|c|c|c|c|c|c|}
\hline \multirow[b]{2}{*}{ Contact Stage } & \multirow{2}{*}{$\begin{array}{c}\begin{array}{c}\text { Contact Duration } \\
\text { (days) }\end{array} \\
\end{array}$} & ${ }^{60} \mathrm{Co}$ & ${ }^{60} \mathrm{Co}$ & ${ }^{137} \mathrm{Cs}$ & ${ }^{137} \mathrm{Cs}$ \\
\hline & & ---- $\mu \mathrm{Ci} / \mathrm{g}-----$ & $--\mu \mathrm{g} / \mathrm{g}-$ & $----\mu \mathrm{Ci} / \mathrm{g}--$ & ------ $\mu \mathrm{g} / \mathrm{g}---$ \\
\hline \multicolumn{6}{|c|}{ Sample 404} \\
\hline 1 & 1 & $<0.0177$ & $<1.61 \mathrm{E}-05$ & 2.65 & $3.044 \mathrm{E}-02$ \\
\hline 2 & 1 & $<0.0177$ & $<1.61 \mathrm{E}-05$ & 0.128 & $1.471 \mathrm{E}-03$ \\
\hline 3 & 4 & $<0.0177$ & $<1.61 \mathrm{E}-05$ & 0.0389 & 4.473E-04 \\
\hline 4 & 1 & $<0.0177$ & $<1.61 \mathrm{E}-05$ & 0.0244 & $2.806 \mathrm{E}-04$ \\
\hline 5 & 1 & $<0.0177$ & $<1.61 \mathrm{E}-05$ & 0.0244 & $2.806 \mathrm{E}-04$ \\
\hline $6 \mathrm{~A}$ & 43 & NA & NA & NA & NA \\
\hline $6 \mathrm{~B}$ & 82 & $\mathrm{NA}$ & NA & NA & NA \\
\hline \multicolumn{6}{|c|}{ Sample 405} \\
\hline 1 & 1 & $<0.0292$ & $<2.65 \mathrm{E}-05$ & 2.03 & $2.329 \mathrm{E}-02$ \\
\hline 2 & 1 & $<0.0292$ & $<2.65 \mathrm{E}-05$ & 0.0402 & $4.623 \mathrm{E}-04$ \\
\hline 3 & 4 & $<0.0292$ & $<2.65 \mathrm{E}-05$ & 0.0402 & $4.623 \mathrm{E}-04$ \\
\hline 4 & 1 & $<0.0292$ & $<2.65 \mathrm{E}-05$ & 0.0402 & $4.623 \mathrm{E}-04$ \\
\hline 5 & 1 & $<0.0292$ & $<2.65 \mathrm{E}-05$ & 0.0402 & $4.623 \mathrm{E}-04$ \\
\hline $6 \mathrm{~A}$ & 43 & NA & NA & NA & NA \\
\hline $6 \mathrm{~B}$ & 82 & NA & $\mathrm{NA}$ & NA & NA \\
\hline
\end{tabular}


Total beta, total alpha and ${ }^{90} \mathrm{Sr}$ levels for the periodic replenishment tests are listed in Table 3.32. These results show that, for each of these constituents, the values start out relatively low in the first extraction and then increase by a factor of two of more by the fifth extraction. For example, the total beta in sample 404 starts at $99.7 \mu \mathrm{Ci} / \mathrm{g}$ and increases to $200 \mu \mathrm{Ci} / \mathrm{g}$ by Stage 5, and total alpha in sample 405 increases from 0.287 to $0.843 \mu \mathrm{Ci} / \mathrm{g}$ over the five stages.

The ${ }^{239} \mathrm{Pu},{ }^{237} \mathrm{~Np}$, and ${ }^{241} \mathrm{Am}$ leachable concentrations for the replenishment tests are listed in Table 3.33, and the leachable percentages are shown in Table 3.34. ${ }^{239} \mathrm{Pu}$ was not measured above its EQL during any of the stages of leaching. The estimated extent of ${ }^{239} \mathrm{Pu}$ leaching was in the range of 0.01 to $0.04 \%$ of the total ${ }^{239} \mathrm{Pu}$ in the sludge. Measurable quantities of leachable ${ }^{237} \mathrm{~Np}$ were measured at most stages of the test. The highest amounts $(8.5 \%$ and 9.8\%) were leached during Stage 1 with decreasing percentages to Stage 5 values of approximately $0.1 \%$. The leachability of ${ }^{237} \mathrm{~Np}$ increased to about $1 \%$ for the longerterm contact Stages 6A and 6B. ${ }^{241} \mathrm{Am}$ was only measured above its EQL during the first stage of leaching of sample 404 . The percentage leached for this stage was $0.04 \%$. Similar low percentages of leaching were estimated for ${ }^{241} \mathrm{Am}$ for the other stages.

Table 3.32. Total Beta, Total Alpha, and ${ }^{90} \mathrm{Sr}$ Analysis for Tank C-106 Periodic Water Replenishment Tests

\begin{tabular}{|c|c|c|c|c|}
\hline \multirow[b]{2}{*}{ Contact Stage } & \multirow{2}{*}{\multicolumn{2}{|c|}{\begin{tabular}{l|r} 
Total Beta & Total Alpha \\
\end{tabular}}} & \multicolumn{2}{|c|}{ 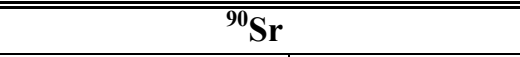 } \\
\hline & & & $-{ }_{----} \mu \mathrm{Ci} / \mathrm{g}-{ }_{-}$ & ----- $\mu \mathrm{g} / \mathrm{g}-----$ \\
\hline \multicolumn{5}{|c|}{ Sample 404} \\
\hline 1 & 99.7 & 0.168 & $2.37 \mathrm{E}+01$ & $1.69 \mathrm{E}-01$ \\
\hline 2 & 160 & 0.380 & $3.88 \mathrm{E}+01$ & $2.77 \mathrm{E}-01$ \\
\hline 3 & 202 & 0.576 & $5.30 \mathrm{E}+01$ & 3.79E-01 \\
\hline 4 & 192 & 0.517 & $4.82 \mathrm{E}+01$ & $3.44 \mathrm{E}-01$ \\
\hline 5 & 200 & 0.465 & $4.79 \mathrm{E}+01$ & $3.42 \mathrm{E}-01$ \\
\hline $6 a$ & NA & NA & NA & NA \\
\hline $6 \mathrm{~b}$ & NA & NA & NA & NA \\
\hline \multicolumn{5}{|c|}{ Sample 405} \\
\hline 1 & 175 & 0.287 & $4.29 \mathrm{E}+01$ & 3.06E-01 \\
\hline 2 & 262 & 0.627 & $7.08 \mathrm{E}+01$ & $3.98 \mathrm{E}-01$ \\
\hline 3 & 317 & 0.869 & $7.80 \mathrm{E}+01$ & $5.05 \mathrm{E}-01$ \\
\hline 4 & 312 & 0.719 & $7.61 \mathrm{E}+01$ & $5.15 \mathrm{E}-01$ \\
\hline 5 & 341 & 0.843 & $9.05 \mathrm{E}+01$ & $5.57 \mathrm{E}-01$ \\
\hline $6 a$ & NA & NA & NA & NA \\
\hline $6 \mathrm{~b}$ & NA & NA & NA & NA \\
\hline
\end{tabular}


Table 3.33. Actinide Analysis for Tank C-106 Periodic Water Replenishment Tests

\begin{tabular}{|c|c|c|c|c|c|c|}
\hline \multirow[b]{2}{*}{ Contact Stage } & \multicolumn{2}{|c|}{${ }^{239} \mathrm{Pu}$} & \multicolumn{2}{|c|}{${ }^{237} \mathbf{N p}$} & \multicolumn{2}{|c|}{${ }^{241} \mathrm{Am}$} \\
\hline & $--\mu \mathrm{Ci} / \mathrm{g}--$ & $--\mu \mathrm{g} / \mathrm{g}--$ & $--\mu \mathrm{Ci} / \mathrm{g}--$ & $--\mu g / g--$ & $--\mu \mathrm{Ci} / \mathrm{g}--$ & $--\mu g / g--$ \\
\hline \multicolumn{7}{|c|}{ Sample 404} \\
\hline 1 & (3.81E-04) & (6.14E-03) & $5.93 \mathrm{E}-05$ & $8.35 \mathrm{E}-02$ & $2.57 \mathrm{E}-03$ & $7.55 \mathrm{E}-04$ \\
\hline 2 & $(1.32 \mathrm{E}-04)$ & $(2.13 \mathrm{E}-03)$ & $2.53 \mathrm{E}-05$ & $3.57 \mathrm{E}-02$ & $(9.86 \mathrm{E}-04)$ & $(2.90 \mathrm{E}-04)$ \\
\hline 3 & (1.05E-04) & (1.69E-03) & $1.75 \mathrm{E}-05$ & $2.47 \mathrm{E}-02$ & $<2.15 \mathrm{E}-03$ & $<6.32 \mathrm{E}-04$ \\
\hline 4 & (1.40E-04) & $(2.25 \mathrm{E}-03)$ & $1.01 \mathrm{E}-05$ & $1.43 \mathrm{E}-02$ & $<2.15 \mathrm{E}-03$ & $<6.32 \mathrm{E}-04$ \\
\hline 5 & (1.01E-04) & (1.63E-03) & $1.03 \mathrm{E}-05$ & $1.45 \mathrm{E}-02$ & $<2.15 \mathrm{E}-03$ & $<6.32 \mathrm{E}-04$ \\
\hline $6 \mathrm{~A}$ & (1.30E-04) & (2.09E-03) & $7.37 \mathrm{E}-05$ & $1.04 \mathrm{E}-01$ & $<2.22 \mathrm{E}-03$ & $<6.53 \mathrm{E}-04$ \\
\hline $6 \mathrm{~B}$ & $(5.31 \mathrm{E}-05)$ & (8.57E-04) & $6.93 \mathrm{E}-05$ & $9.76 \mathrm{E}-02$ & $<2.08 \mathrm{E}-03$ & $<6.12 \mathrm{E}-04$ \\
\hline \multicolumn{7}{|c|}{ Sample 405} \\
\hline 1 & $(7.31 \mathrm{E}-04)$ & $(1.18 \mathrm{E}-02)$ & $8.07 \mathrm{E}-05$ & 1.14E-01 & $(1.92 \mathrm{E}-03)$ & $(5.65 \mathrm{E}-04)$ \\
\hline 2 & (5.02E-04) & (8.09E-03) & $3.14 \mathrm{E}-05$ & $4.42 \mathrm{E}-02$ & $(2.81 \mathrm{E}-03)$ & $(8.27 \mathrm{E}-04)$ \\
\hline 3 & $(4.63 \mathrm{E}-04)$ & $(7.47 \mathrm{E}-03)$ & $1.88 \mathrm{E}-05$ & $2.65 \mathrm{E}-02$ & $<3.54 \mathrm{E}-03$ & $<1.04 \mathrm{E}-03$ \\
\hline 4 & (2.57E-04) & $(4.15 E-03)$ & $9.49 \mathrm{E}-06$ & $1.34 \mathrm{E}-02$ & $<3.54 \mathrm{E}-03$ & $<1.04 \mathrm{E}-03$ \\
\hline 5 & $(1.65 \mathrm{E}-04)$ & $(2.66 \mathrm{E}-03)$ & $(6.92 \mathrm{E}-06)$ & $(9.75 \mathrm{E}-03)$ & $<3.54 \mathrm{E}-03$ & $<1.04 \mathrm{E}-03$ \\
\hline $6 \mathrm{~A}$ & $(2.60 \mathrm{E}-04)$ & (4.20E-03) & $7.85 \mathrm{E}-05$ & $1.11 \mathrm{E}-01$ & $<3.76 \mathrm{E}-03$ & $<1.11 \mathrm{E}-03$ \\
\hline $6 \mathrm{~B}$ & $(1.27 \mathrm{E}-04)$ & $(2.06 \mathrm{E}-03)$ & $8.24 \mathrm{E}-05$ & $1.16 \mathrm{E}-01$ & $<3.33 \mathrm{E}-03$ & $<9.79 \mathrm{E}-04$ \\
\hline
\end{tabular}

Table 3.34. Water-Leachable Percentage for Actinides in Tank C-106 Periodic Water Replenishment Tests Compared with Fusion Analysis

\begin{tabular}{|c|c|c|c|}
\hline \multirow[b]{2}{*}{ Contact Stage } & 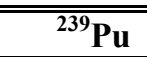 & 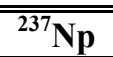 & 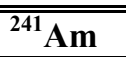 \\
\hline & \multicolumn{3}{|c|}{ 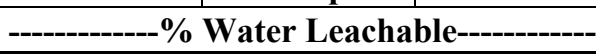 } \\
\hline \multicolumn{4}{|c|}{ Sample 404} \\
\hline 1 & $(0.02)$ & 8.5 & 0.04 \\
\hline 2 & $(0.01)$ & 3.4 & $(0.02)$ \\
\hline 3 & $(0.01)$ & 0.2 & $<0.04$ \\
\hline 4 & $(0.01)$ & 0.1 & $<0.04$ \\
\hline 5 & $(0.01)$ & 0.1 & $<0.04$ \\
\hline $6 \mathrm{~A}$ & $(0.01)$ & 1.0 & $<0.04$ \\
\hline $6 \mathrm{~B}$ & $(0.003)$ & 1.0 & $<0.03$ \\
\hline \multicolumn{4}{|c|}{ Sample 405} \\
\hline 1 & $(0.04)$ & 9.8 & $(0.03)$ \\
\hline 2 & $(0.03)$ & 3.9 & $(0.04)$ \\
\hline 3 & $(0.02)$ & 0.3 & $<0.06$ \\
\hline 4 & $(0.01)$ & 0.1 & $<0.06$ \\
\hline 5 & $(0.01)$ & $(0.07)$ & $<0.06$ \\
\hline $6 \mathrm{~A}$ & $(0.01)$ & 1.1 & $<0.06$ \\
\hline $6 \mathrm{~B}$ & $(0.01)$ & 1.0 & $<0.05$ \\
\hline
\end{tabular}




\subsection{XRD Results}

This section discusses the crystalline solids identified in the XRD patterns for the unleached, 1-month and 82-day water-leached, and HF Stage 1 sludge samples 404 and 405. The as-measured and background-subtracted XRD patterns for these samples are found in Appendix A. Phase identification was based on a comparison of the peak reflections and intensities observed in each pattern to the mineral powder diffraction files (PDFTM) published by the Joint Committee on Powder Diffraction Standards (JCPDS) International Center for Diffraction Data (ICDD). Phase identification from the XRD patterns was refined in an iterative fashion by considering phases with particle compositions that were determined by SEM/EDS (see Section 3.6) as present in the sludge samples. The XRD patterns in Appendix A show greater detail than those plotted in this section because they do not include the schematic PDF-XRD database patterns (as plotted in this section) used for phase identification.

Each pattern in this section and Appendix $\mathrm{A}$ is shown as a function of degrees $2 \theta$ based on $\mathrm{Cu}_{\mathrm{K} \alpha}$ radiation $(\lambda=1.5406 \AA)$. The vertical axis in each pattern represents the intensity or relative intensity of the XRD peaks. The XRD patterns included in this section show, for comparison purposes, one or more schematic database (PDF) patterns considered for phase identification. The height of each line in the schematic PDF patterns represents the relative intensity of an XRD peak (i.e., the most intense [the highest $]$ peak has a relative intensity $\left[\mathrm{I} / \mathrm{I}_{\mathrm{o}}\right]$ of $100 \%$ ). Quantitative analyses of the relative masses of individual phases present in each solid sample were not estimated using these XRD patterns due to the factors discussed at the end of Section 2.3. Also, as noted previously in Section 2.3, a crystalline phase typically must be present at greater than $5 \mathrm{wt} \%$ of the total sample mass (greater than $1 \mathrm{wt} \%$ under optimum conditions) to be readily detected by XRD.

The background subtracted XRD patterns for the unleached, 1-month and 82-day water leached, and HF-extracted sludge samples 404 and 405 from tank C-106 are plotted with the PDF patterns used for phase identification in Figure 3.3 through Figure 3.6, respectively. The XRD results indicate that the unleached, 1-month and 82-day water-leached, and HF-extracted sludge samples contain detectable quantities of the following crystalline phases based on the indicated PDF database patterns:

- gibbsite - $\mathrm{Al}(\mathrm{OH})_{3}(\mathrm{PDF} 33-0018)$

- dawsonite - $\mathrm{NaAlCO}_{3}(\mathrm{OH})_{2}(\mathrm{PDF} 45-1359)$

- hematite $-\mathrm{Fe}_{2} \mathrm{O}_{3}$ (PDF 86-0550)

- böhmite - $\mathrm{AlO}(\mathrm{OH})(\mathrm{PDF} 83-1505)$

- rhodochrosite - $\mathrm{MnCO}_{3}$ (PDF 83-1763)

- whewellite - Ca oxalate monohydrate, $\mathrm{CaC}_{2} \mathrm{O}_{4} \cdot \mathrm{H}_{2} \mathrm{O}$ (PDF 20-0231)

The XRD patterns were also consistent with the possible presence of an Ag-Hg phase (PDF 43-1465). Its identification, however, was based on a match to a single reflection at $37.153^{\circ} 2 \theta$, which is insufficient for a conclusive identification of any phase by XRD. However, the search of the XRD patterns for the possible presence of Ag-Hg phases was instigated by the discovery of Ag-Hg particles by SEM/EDS analysis of the sludge samples (see Section 3.6). It was not possible to further validate its presence in these samples by XRD. 
The XRD patterns also show that lindbergite (Mn oxalate dihydrate, $\mathrm{MnC}_{2} \mathrm{O}_{4} \cdot 2 \mathrm{H}_{2} \mathrm{O}$ ) (PDF 25-0544) is also present in the unleached sludge samples 404 and 405 from tank $\mathrm{C}-106$. The major reflections for lindbergite (PDF 25-0544), including the split peak at $18.4^{\circ} \theta$ that is due to the overlap of the $100 \%$ peaks for gibbsite and lindbergite (see red arrow in Figure 3.3), are absent in the XRD patterns for the 1-month and 82-day water-leached and HF-extracted sludge samples. The split peak at $18.4^{\circ} \theta$ is more apparent in the XRD patterns for the unleached samples in Appendix A.

There were no unassigned reflections in the background-subtracted XRD patterns for the samples of unleached, 1-month and 82-day water-leached, and HF-extracted sludge samples 404 and 405 . This suggests that all crystalline phases present at greater than $5-10 \mathrm{wt} \%$ in these sludge samples were identified by XRD. Based qualitatively on the observed relative heights for peaks in the background subtracted XRD patterns (Figure 3.3 through Figure 3.6), gibbsite, dawsonite, and rhodochrosite appear to be the most dominant crystalline phases present in the unleached, 1-month and 82-day water-leached, and HF-extracted sludge samples 404 and 405.

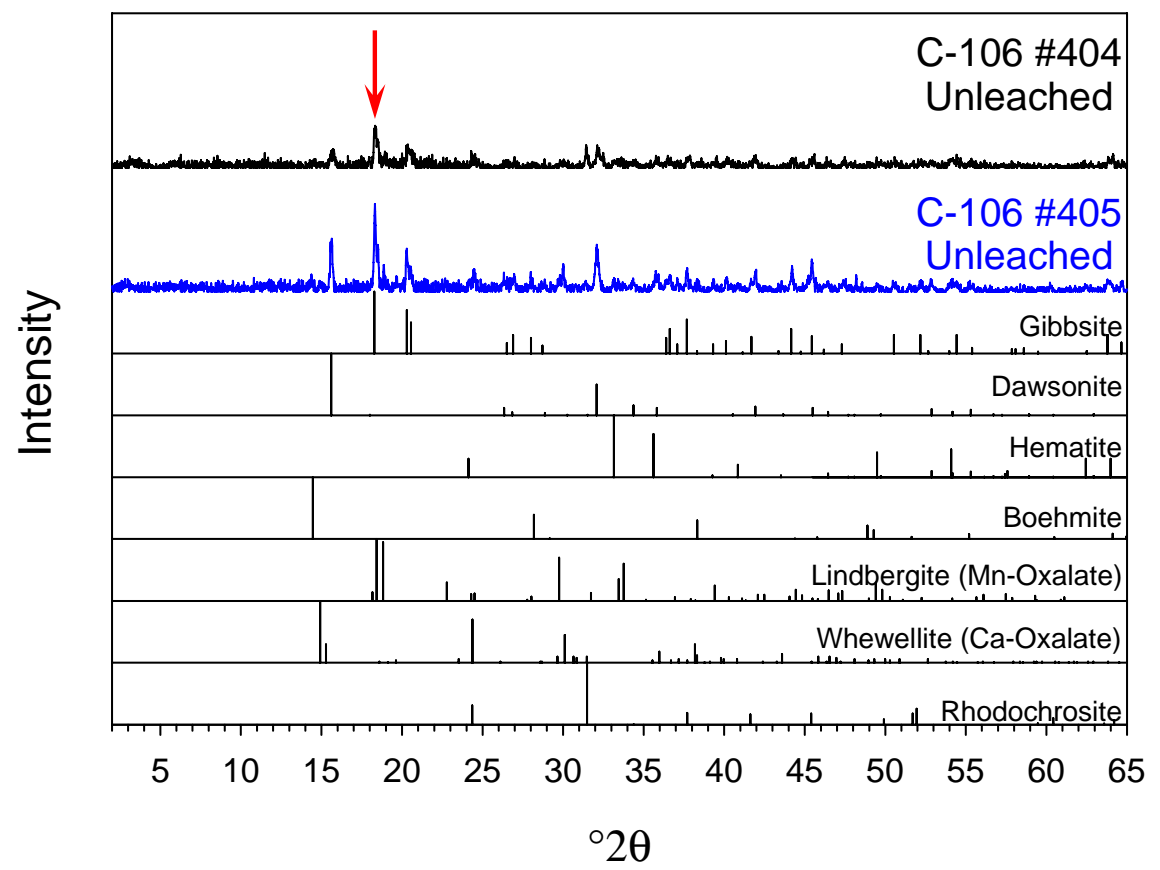

Figure 3.3. Background-Subtracted XRD Pattern for the Unleached Sludge Samples 404 and 405 from Tank C-106 Shown with Matching PDF Database Patterns 


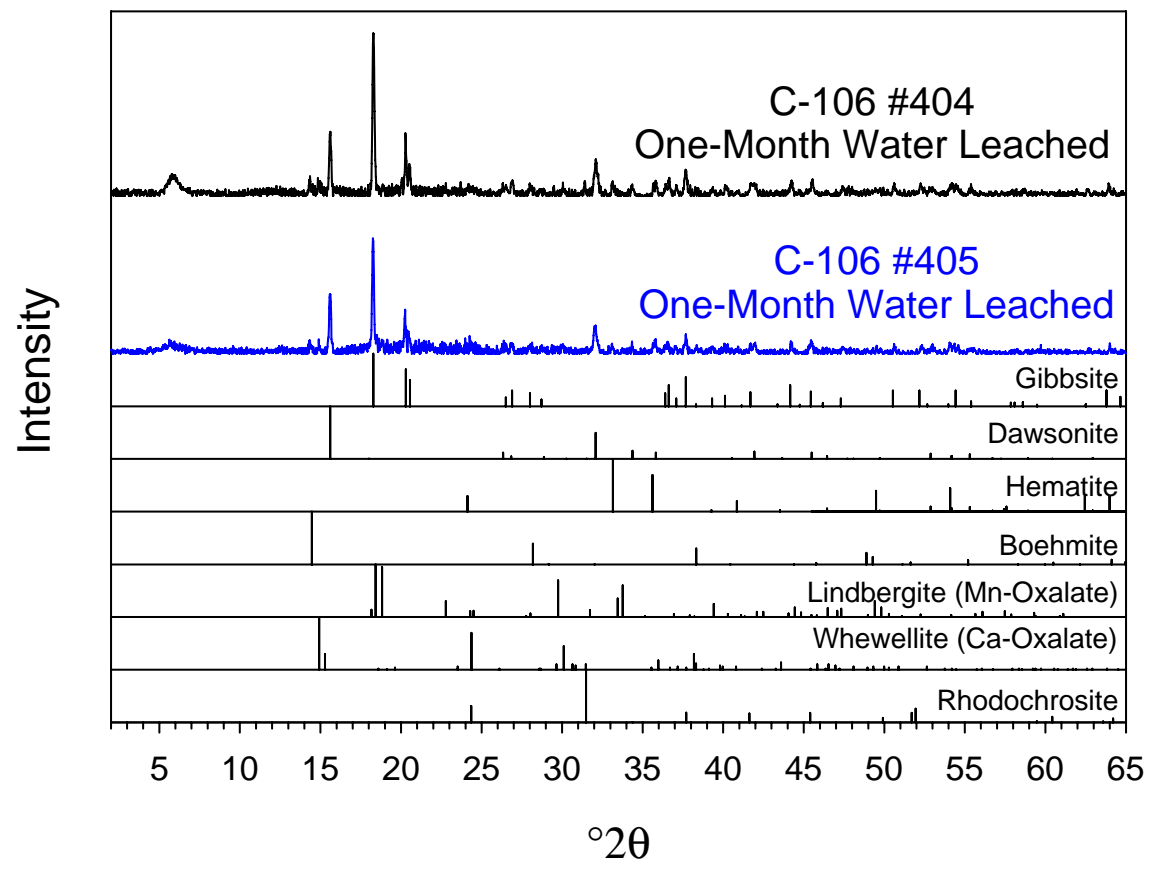

Figure 3.4. Background-Subtracted XRD Pattern for the 1-Month Water-Leached Sludge Samples 404 and 405 from Tank C-106 Shown with Matching PDF Database Patterns

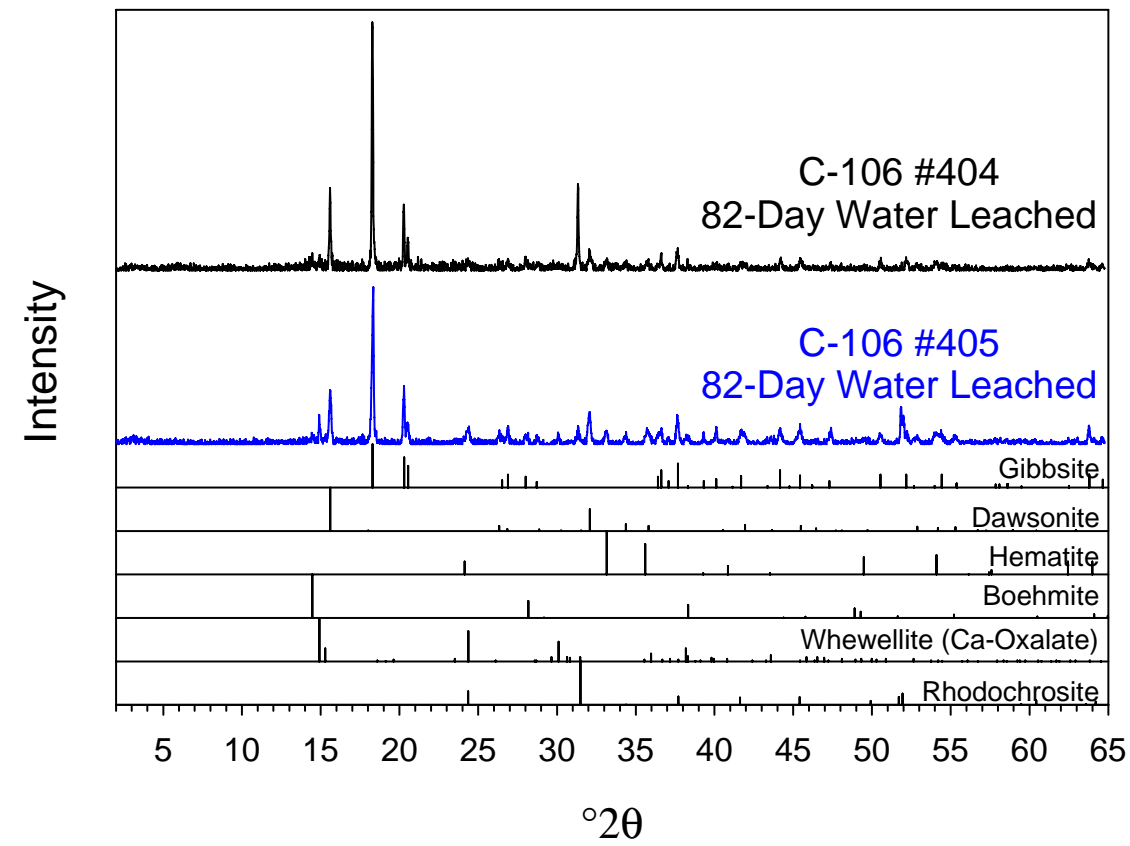

Figure 3.5. Background-Subtracted XRD Pattern for the 82-Day Water-Leached Sludge Samples 404 and 405 from Tank C-106 Shown with Matching PDF Database Patterns 


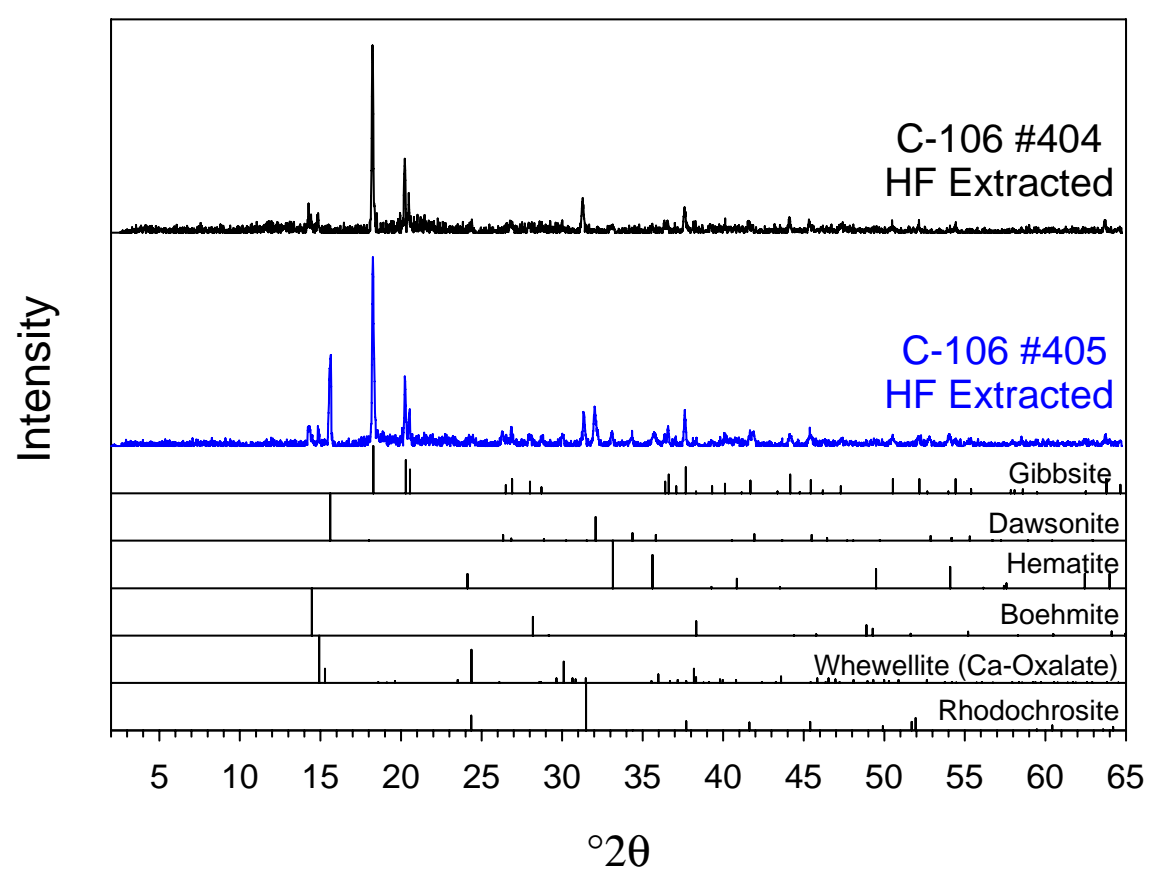

Figure 3.6. Background-Subtracted XRD Pattern for the HF-Extracted Sludge Samples 404 and 405 from Tank C-106 Shown with Matching PDF Database Patterns

The background-subtracted XRD patterns for the unleached, water leached, and HF-extracted sludge samples 404 and 405 from tank C-106 are generally very similar to each other. For example, Figure 3.7 shows a comparison of the XRD patterns for the unleached, 1-month and 82-day water-leached, and HFextracted sample 405. Besides the presence of reflections for lindbergite (Mn oxalate dihydrate, $\mathrm{MnC}_{2} \mathrm{O}_{4} \cdot 2 \mathrm{H}_{2} \mathrm{O}$ ) in the unleached tank C-106 samples, only two other minor differences were observed between the XRD patterns for the four samples of tank C-106 sludge. One obvious difference is the low peak intensities (i.e., peak heights) observed in the background-subtracted XRD patterns for the unleached samples relative to those in the patterns for the water-leached and HF-extracted samples. If the same amount of solid sludge material was used for each of the four sample types, then it can be assumed from the low peak intensities that the amount of amorphous relative to crystalline material is greater in the unleached samples than in the water leached and HF-extracted sludge samples 404 and 405 from tank C-106. Because of the hazardous nature of these sludge samples, it is not possible to control the exact amount of material used for each XRD mount. However, when these XRD mounts are prepared, a concerted effort is made to follow the same procedure and use the same amount of sludge material as closely as possible. 


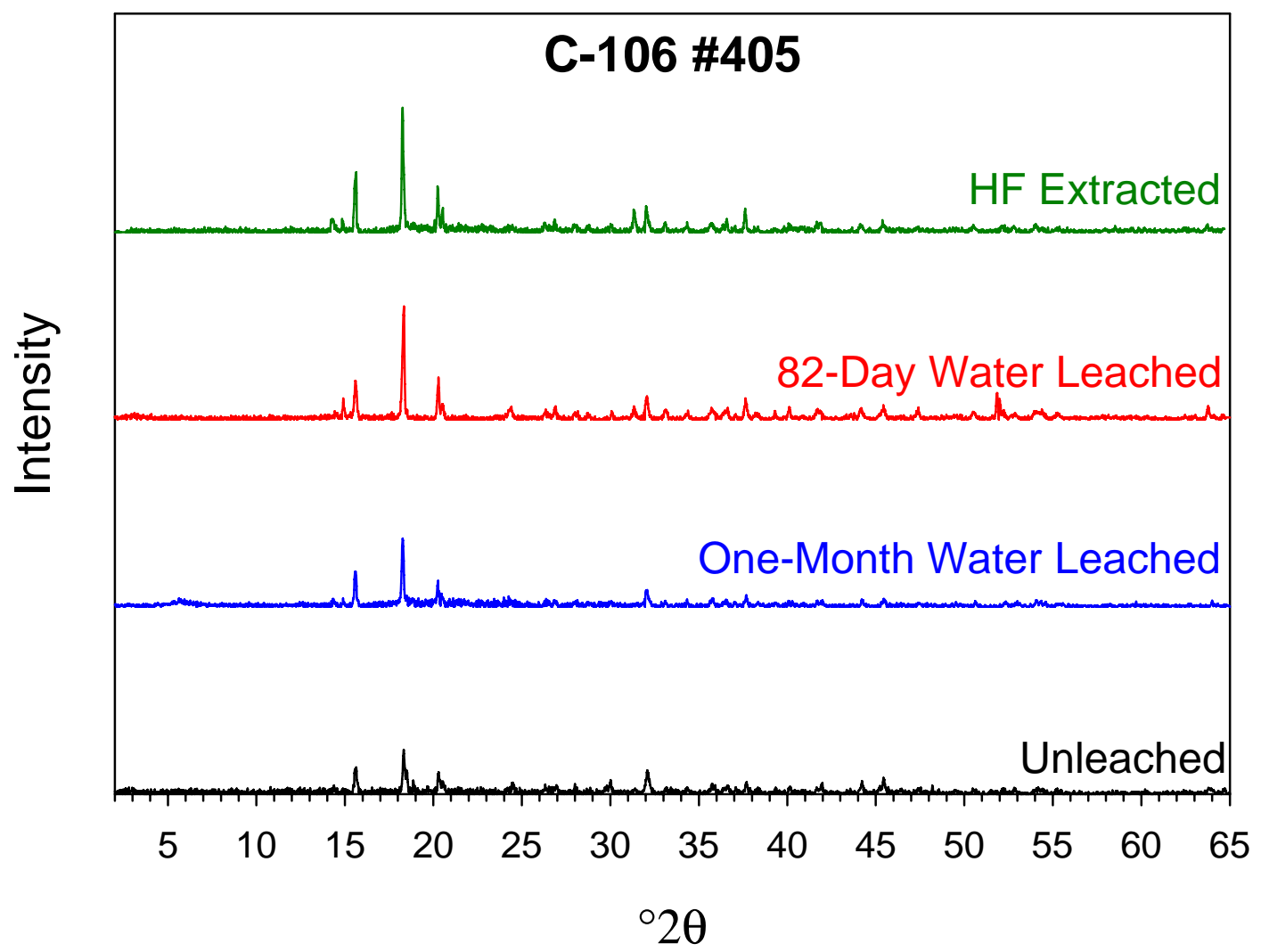

Figure 3.7. Comparison of Background-Subtracted XRD Pattern for Unleached, 1-Month and 82-Day Water-Leached, and HF-Extracted Sludge Sample 405 from Tank C-106 Shown with Matching PDF Database Patterns

The other difference is the presence of a small, broad peak at approximately $5.9^{\circ} \theta(15 \AA)$ that only exists in the patterns for the 1-month water-leached sludge samples 404 and 405 from tank C-106 (Figure 3.4). This peak is likely due to reflections from the Kapton ${ }^{\circledR}$ film used in the specimen holder described in Section 2.3, and has occasionally been observed in other patterns where samples were mounted in this type of specimen holder.

Other minor differences include the absence of major reflections for rhodochrosite, such as the major peak at $31.36^{\circ} 2 \theta$, in the XRD pattern for the 1-month water-leached 405 sample, and for dawsonite in the XRD pattern for the HF-extracted 404 sample. Because these phases were identified in the other patterns for sludge samples 404 and 405, it is likely that rhodochrosite and dawsonite were also present in these two particular samples but at levels near or below the limits of detection by XRD.

\subsection{SEM/EDS Results}

This section discusses the results of the SEM/EDS analyses for the unleached (as-received), 1-month and 82-day water-leached, and HF-extracted sludge samples 404 and 405 from tank C-106. The SEM micrographs presented in this section show representative morphologies, sizes, and surface textures of 
particles in each of these samples. After the studies and analyses of tank C-106 sludge had been completed and presented in draft form, the instrument hardware and software for the JEOL JSM-840 SEM were upgraded to the INCAEnergy EDS System to automate the collection of EDS spectra over multi-micrometer-sized areas of an SEM-imaged sample. This new capability was demonstrated using existing SEM mounts of the tank C-106 sludge samples. All of the SEM micrographs and EDS spectra determined for sludge samples 404 and 405 prior to the installation of the INCAEnergy EDS System are shown without discussion or interpretation in Appendices B (unleached [as received] sludge, C (1-month and 82-day water-leached sludge), and D (HF Stage 1 extracted). Three sets of SEM micrographs and element distribution maps collected with the INCAEnergy EDS System are presented at the end of this section. The remainder of the SEM micrographs and element distribution maps determined using this system upgrade is shown in Appendix E.

Each micrograph included in this section before Figure 3.18 shows the name of the SEM digital image file, sample identification number, and a size scale bar, respectively, at the bottom left, center, and right of each image. Micrographs labeled with "BSE" near the digital image file name indicate that the micrograph was collected with backscattered electrons. Areas labeled by "eds" identify locations of particles for which EDS spectra were recorded and presented in the appendices. The micrographs presented in this section are typically reproduced at reduced size to conserve page space. To get a more detailed view of these micrographs, the reader is referred to Appendices B through $\mathrm{D}$, where the micrographs are shown at a larger size.

Figure 3.8 and Figure 3.9 show SEM micrographs obtained at relatively low magnification of material present in the unleached (top row in Figure 3.8), 1-month water-leached (bottom row in Figure 3.8), 82-day water-leached (top row in Figure 3.9), and HF-extracted (bottom row in Figure 3.9) sludge samples. The SEM micrographs for the sludge samples 404 and 405 are presented in the left and right columns, respectively, in both Figure 3.8 and Figure 3.9. The sludge particles range in size from a few hundred micrometers to submicrometer in size, and occur often as aggregates of one or more compositionally distinct phases. Generally, there are no obvious major differences visible in the morphologies and distributions of particles in the SEM images for the same treatment (or lack of) of 404 and 405 samples, or between the unleached, water-leached, or HF extract samples. Although very subjective, it appears that with extended water leaching, especially after HF extraction, the samples may possibly contain fewer particle aggregates and an increased relative mass of dark gray particles having multifaceted, blocky shapes (see particles labeled A in the SEM micrograph in the top left of Figure 3.9). The surfaces of these blocky dark gray particles are also typically free of coatings or other sorbed particles, and do not show any obvious dissolution features regardless of their treatment. As discussed below, these multi-faceted, blocky dark gray particles primarily contain $\mathrm{Al}-\mathrm{O} \pm \mathrm{H}$ or $\mathrm{Al}-\mathrm{Na}-\mathrm{O}-\mathrm{C} \pm \mathrm{H}$.

Figure 3.10 shows several large (tens of micrometers or larger on edge) particles that have the same "composition" as determined by EDS but at least four or five distinguishing morphologies. These particle shapes were common in all tank C-106 samples inspected by SEM, and can be seen in Figure 3.8 and Figure 3.9. The EDS analyses of these particles indicate the presence of $\mathrm{Mn}, \mathrm{Al}, \mathrm{Fe}, \mathrm{Na}, \mathrm{P}, \mathrm{Si}$, O, and possibly $\mathrm{C}$ and $\mathrm{H}$ (listed generally in decreasing order of peak heights of metal elements in the EDS spectra). The particles include morphologies that are oblong and rounded (micrograph A), orthorhombic (90 ${ }^{\circ}$ angles) (micrograph $\mathrm{B}$ ), rhombohedral (oblique angles) (micrograph $\mathrm{C}$ ), and pyramidal rhombic 


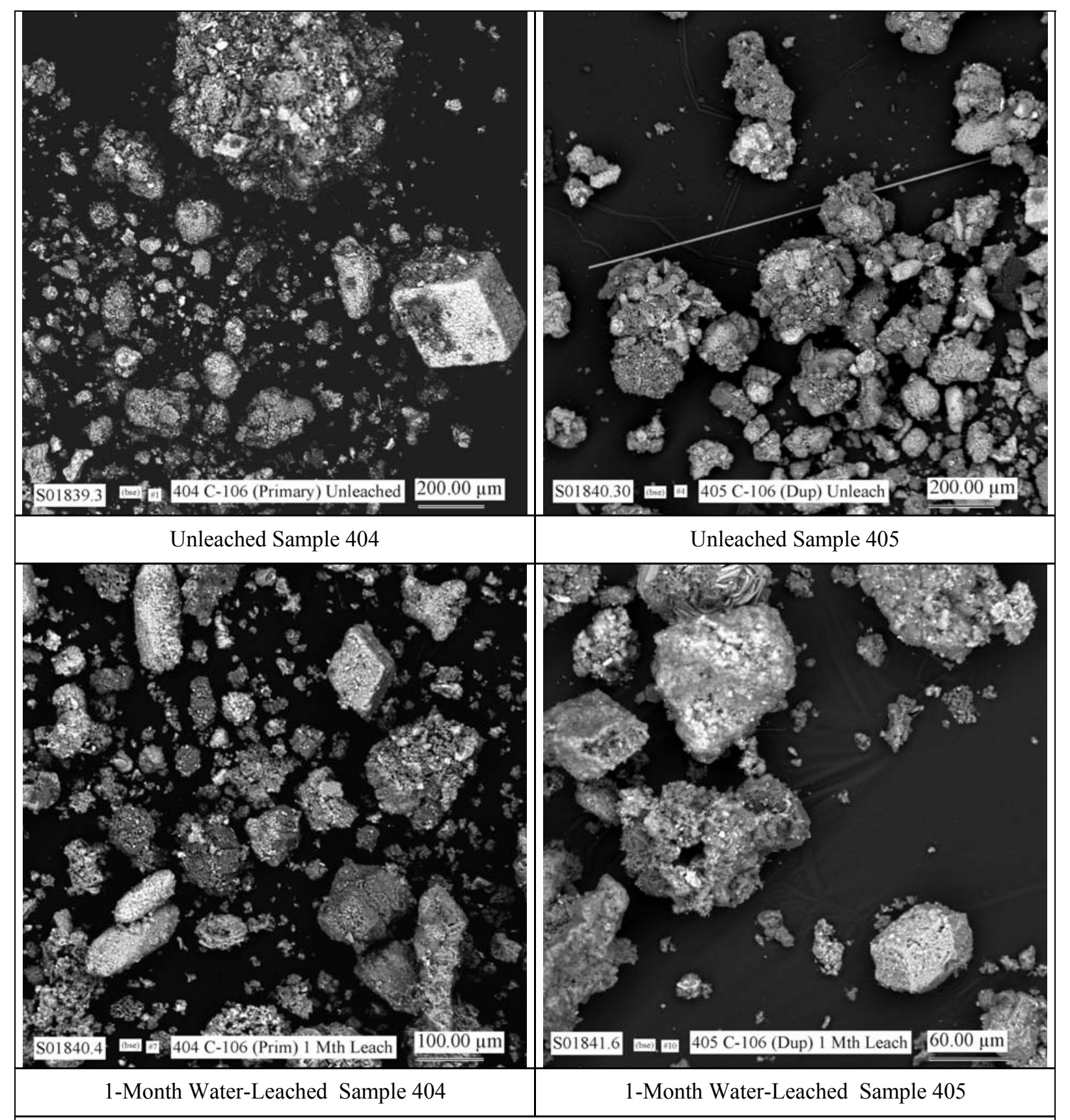

Figure 3.8. Low Magnification SEM Micrographs of Particles Present in the Unleached (top row) and 1-Month Water-Leached (bottom row) Tank C-106 Sludge Samples 


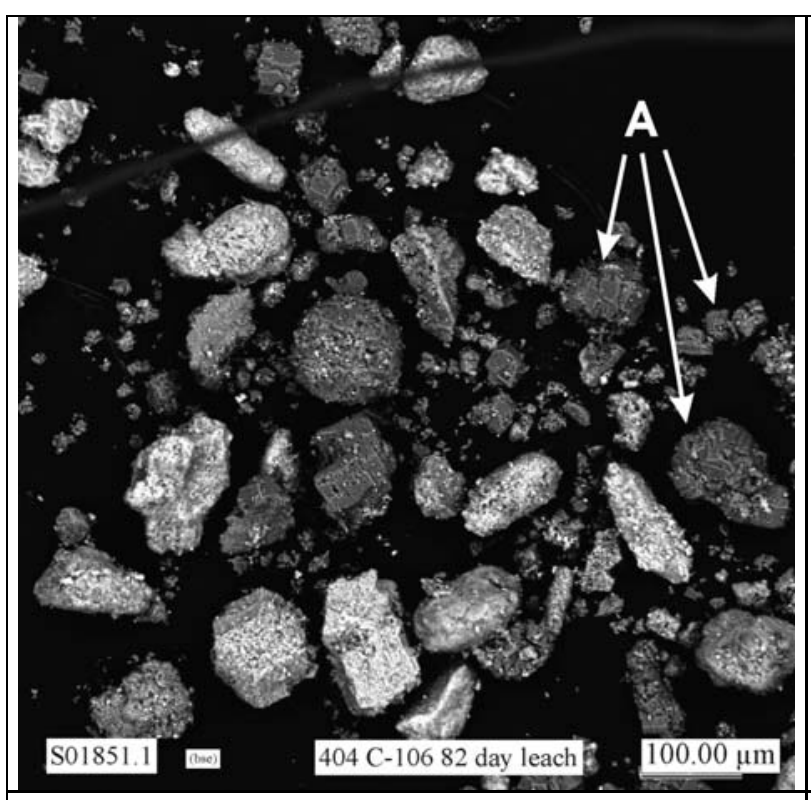

82-Day Water-Leached Sample 404

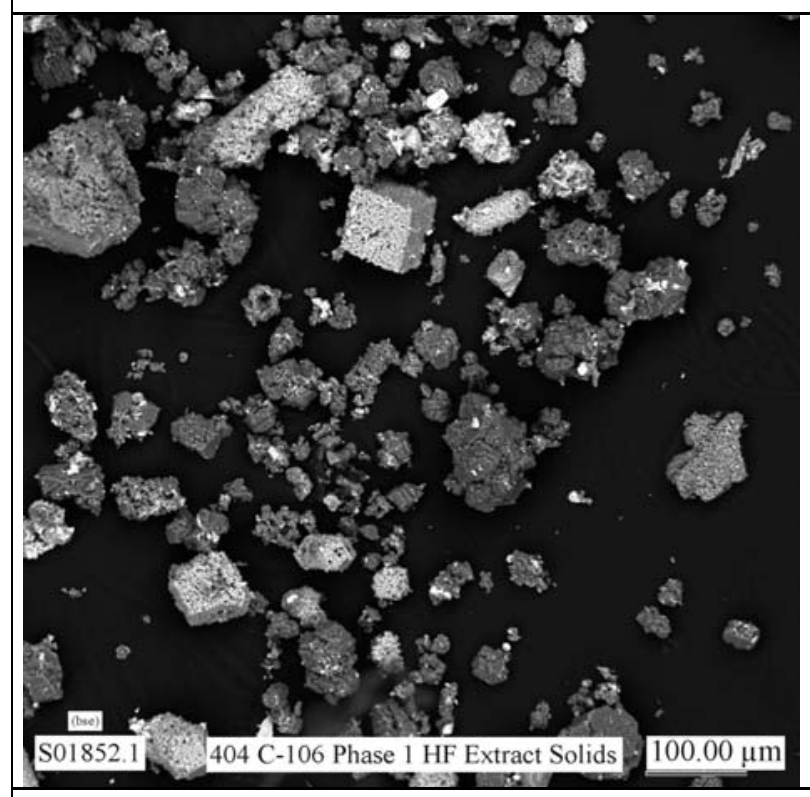

HF Stage 1 Extract of Sample 404

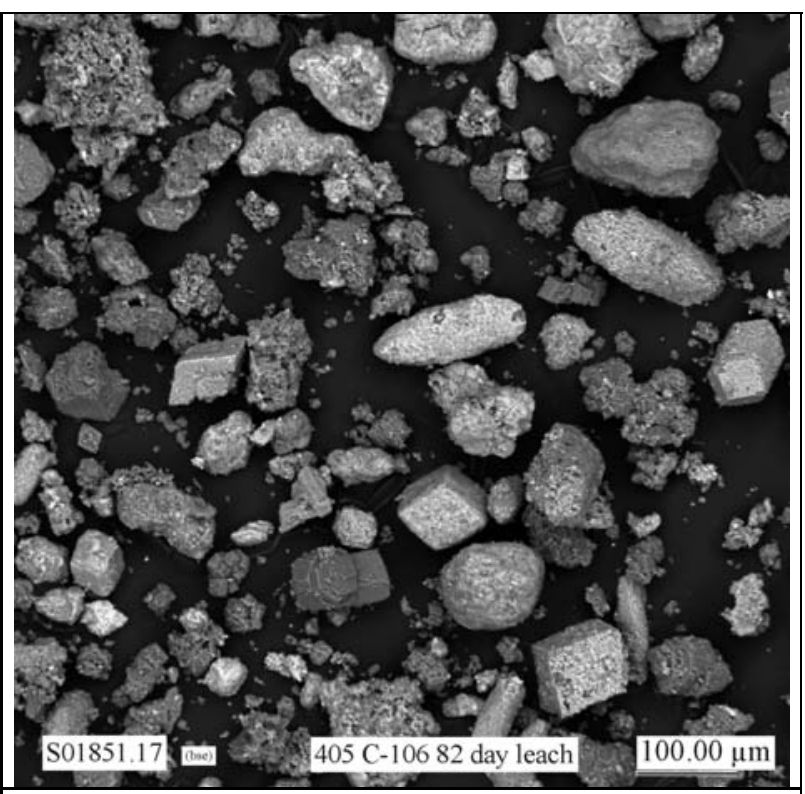

82-Day Water-Leached Sample 405

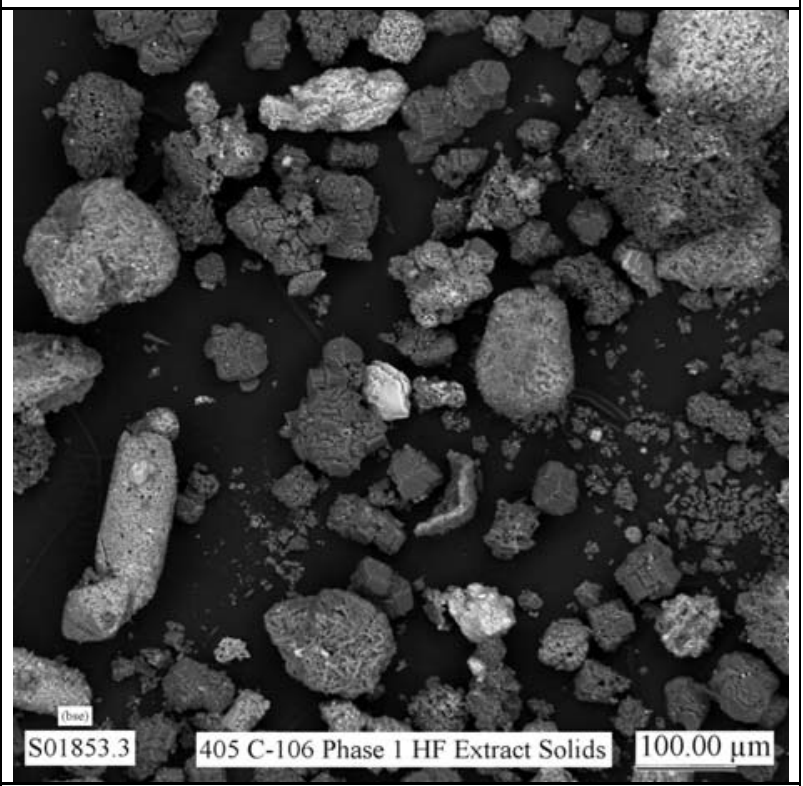

HF Stage 1 Extract of Sample 405

Figure 3.9. Low Magnification SEM Micrographs of Particles Present in the 82-Day WaterLeached (top row) and HF Stage 1 Extract (bottom row) Tank C-106 Sludge Samples 


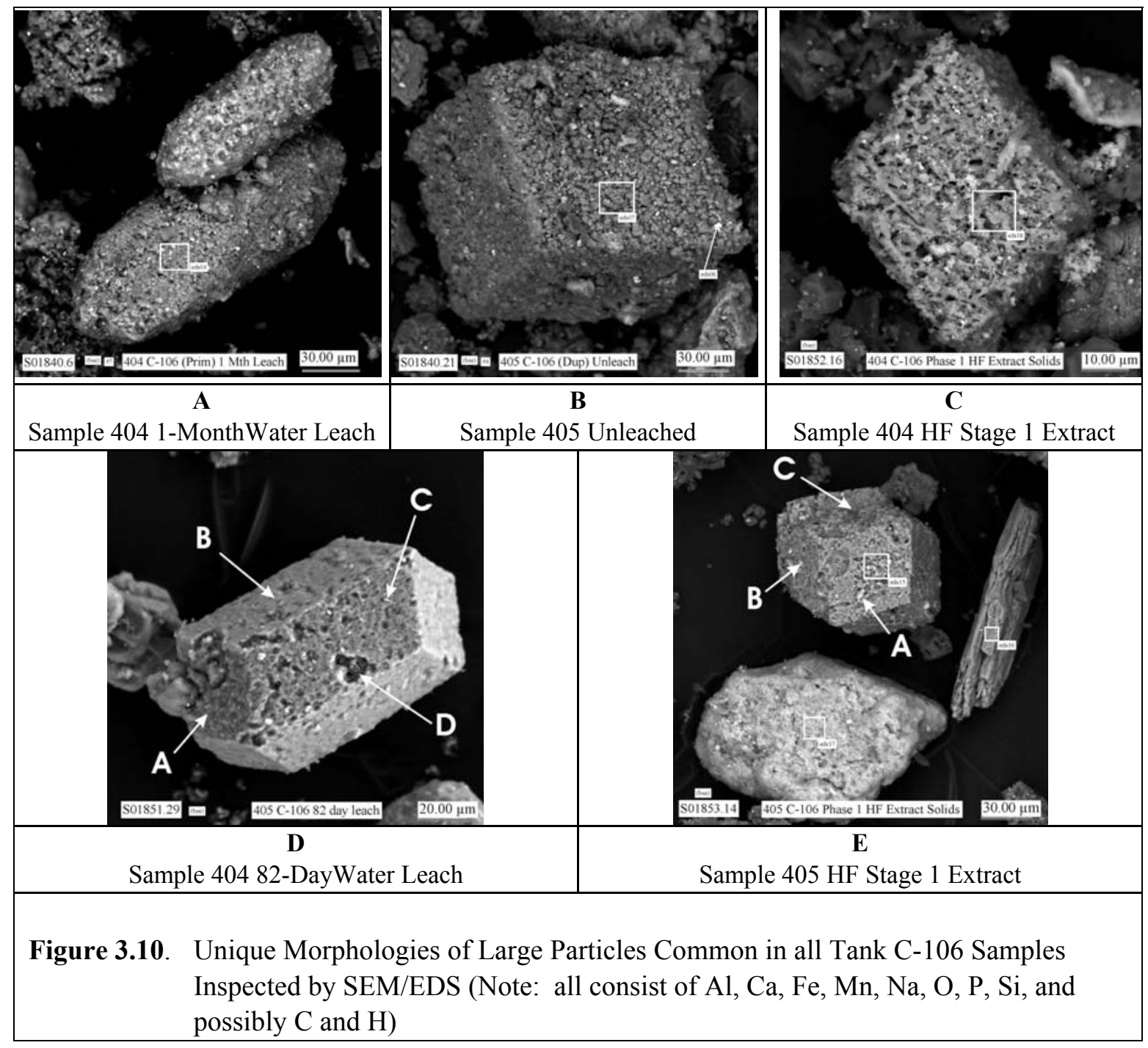

(micrograph D) in Figure 3.10. Another particle shape having this composition and common in these samples is shown at the top of micrograph $\mathrm{E}$ in Figure 3.10. The particle shape in micrograph D is likely an elongated form of that in micrograph $\mathrm{E}$, where the particle faces labeled A, B, and C in micrograph D are equivalent, respectively, to those in micrograph $\mathrm{E}$.

It is not known if the differently shaped particles in Figure 3.10 are related crystallographically to each other, or if these particles consist entirely of a single phase (or phase assemblage) or are just coated with a phase(s) consisting of $\mathrm{Mn}, \mathrm{Al}, \mathrm{Fe}, \mathrm{Na}, \mathrm{P}, \mathrm{Si}, \mathrm{O}$, and possibly $\mathrm{C}$ and $\mathrm{H}$. Given the distinctive geometric shapes of these particles, some phase at the core of these particles is likely controlling the external morphologies of these grains. The SEM images in micrographs A and B in Figure 3.11 suggest that these particles may have a coating of this bulk composition. This conclusion is further supported in other SEM images that show surface cavities containing "darker" particles (usually Al-rich) that are different in texture and composition from the surface material, such as the areas labeled D in 
micrograph D in Figure 3.10 and B in micrograph C in Figure 3.11. The surface textures of the particles in micrographs $\mathrm{C}$ and $\mathrm{D}$ in Figure 3.11 also suggest that the material at the surfaces of these particles consists of more than one phase. The EDS spectra (Figure 3.12) for the HF extract samples typically indicate the particle surfaces have become deficient in $\mathrm{Al}, \mathrm{Na}, \mathrm{P}, \mathrm{Si}$, and $\mathrm{Ca}$ relative to $\mathrm{Mn}$ and $\mathrm{Fe}$, when compared to the compositions of the unleached samples. These particles exist primarily as large isolated grains, and are rarely observed to be intergrown, except for a few rare instances such as those shown in micrographs $C$ (particles labeled A) and D (particles labeled C) in Figure 3.11. Unfortunately, the results of our studies did not provide any additional data that would allow further identification of these particles or explain the conditions of their existence.

Figure 3.13 through Figure 3.17 show examples of other particles typically present in the unleached, 1-month and 82-day water-leached, and HF-extracted Stage 1 sludge samples 404 and 405 . The EDS analyses indicate the following compositions for particles in these samples (" \pm " symbol indicates the following element was possibly present):

- Mn-Al-Fe-Na-P-Si-Ca-O $\pm \mathrm{C} \pm \mathrm{H}$ - see Figure 3.10, Figure 3.11, Figure 3.13(B), Figure 3.15(A) and (D), Figure 3.16(A)

- $\mathrm{Al}-\mathrm{O} \pm \mathrm{H}-$ see Figure 3.13(A) and (B), Figure 3.15(A)

- $\mathrm{Ag}-\mathrm{Hg} \pm \mathrm{O} \pm \mathrm{H}-$ see Figure 3.13(A), Figure 3.15(A) and (B)

- $\mathrm{Ca}-\mathrm{O} \pm \mathrm{C} \pm \mathrm{H}-$ see Figure 3.13(C) and Figure 3.16(B)

- $\mathrm{Mn}-\mathrm{O}-\mathrm{C} \pm \mathrm{H}-$ possibly two different phases based on morphology, see Figure 3.14(A) and (B)

- Al-Na-O-C $\pm \mathrm{H}$ - see Figure 3.13(C) and (D), Figure 3.15 (B), (C), and (D)

- $\mathrm{Mn}-\mathrm{O}-\mathrm{P} \pm \mathrm{Al} \pm \mathrm{C} \pm \mathrm{H}$ - see Figure 3.14(C) and Figure 3.15(D)

- $\mathrm{Si}-\mathrm{Al}-\mathrm{Na}-\mathrm{O} \pm \mathrm{C} \pm \mathrm{H}$ - see Figure 3.15(A)

- $\mathrm{Fe}-\mathrm{Cr}-\mathrm{O} \pm \mathrm{C} \pm \mathrm{H}$ - see Figure 3.16(A)

- Fe-Mn- $\pm \mathrm{C} \pm \mathrm{H}$ - see Figure 3.16(D) (rare morphology) and Figure 3.17

- Rare earth element (REE)-rich - see Figure 3.16(C) (rare but present in most samples)

- $\mathrm{Ca}-\mathrm{Si}-\mathrm{Al}-\mathrm{O} \pm \mathrm{C} \pm \mathrm{H}$ - see Figure 3.13(C) (rare)

The SEM/EDS analyses did not indicate the presence of I, Tc, or $U$ in any of particles present in the unleached, 1-month and 82-day water-leached, and HF-extracted sludge samples 404 and 405 from tank C-106. This was due to their low concentrations in the sludge and the high detection limits for this analytical method.

Some unusual needle-like particles were observed in some micrographs (e.g., Figure 3.8 [upper right], Figure 3.11[D], and Figure 3.13[B]), especially for samples of the unleached tank C-106 sludge. These needle-like particles had clean shafts, and when not broken, terminated in a fine, sharp point at one end. They ranged up to approximately $1 \mathrm{~mm}$ in length. The EDS analyses indicated that these needle-like particles consisted primarily of $\mathrm{O}$ and $\mathrm{Si}$ and with lesser amounts of $\mathrm{Ca}, \mathrm{Na}, \mathrm{Al}$, and possibly $\mathrm{Mg}$ (listed in estimated order of abundance and common components in manufactured glass products). These needle-like particles are probably from fibrous glass wool that was used in the laboratory to prevent dispersion of these radioactive powders during the drying of wet sludge samples. They are not present in the residual waste in the tank. 


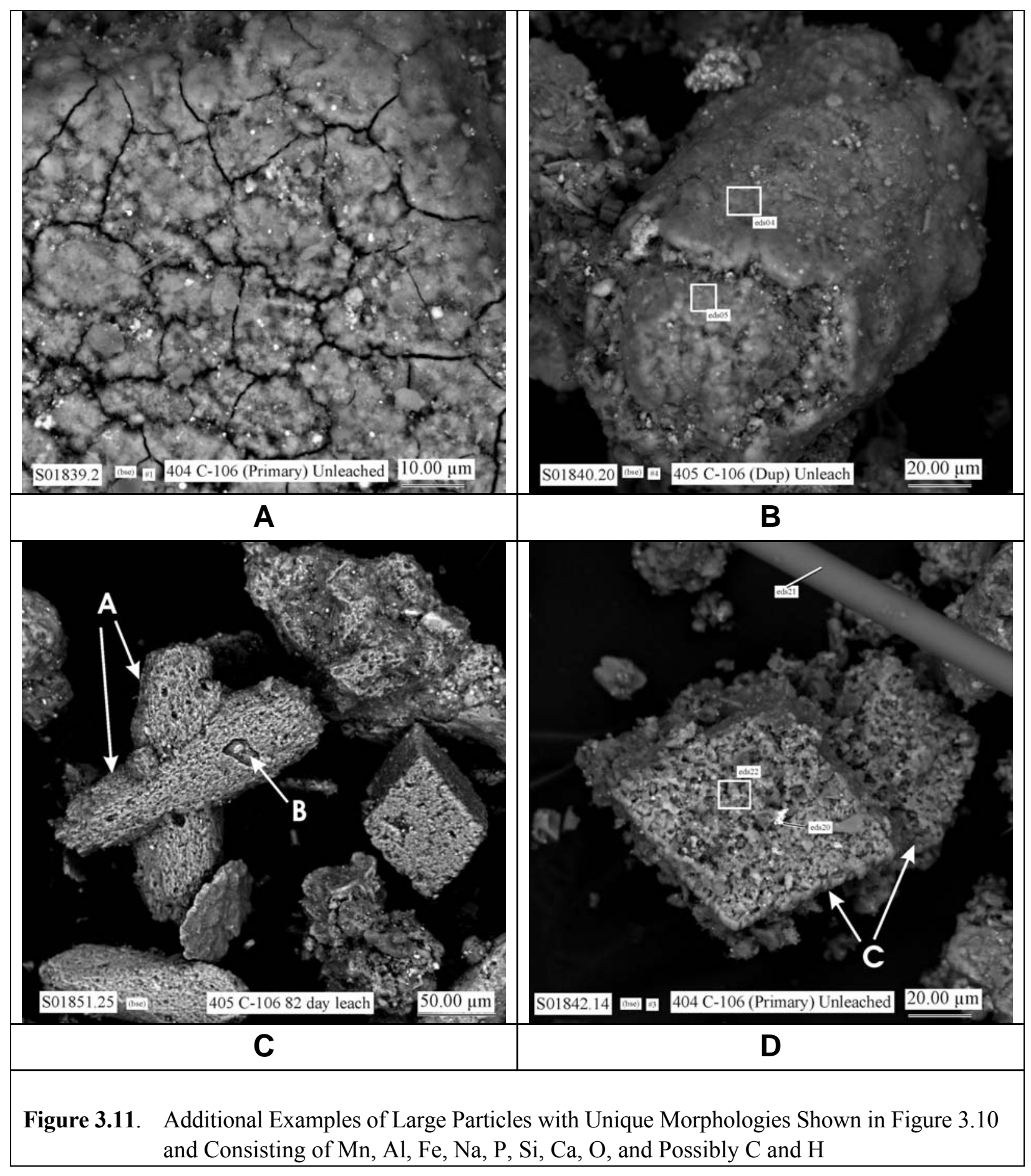




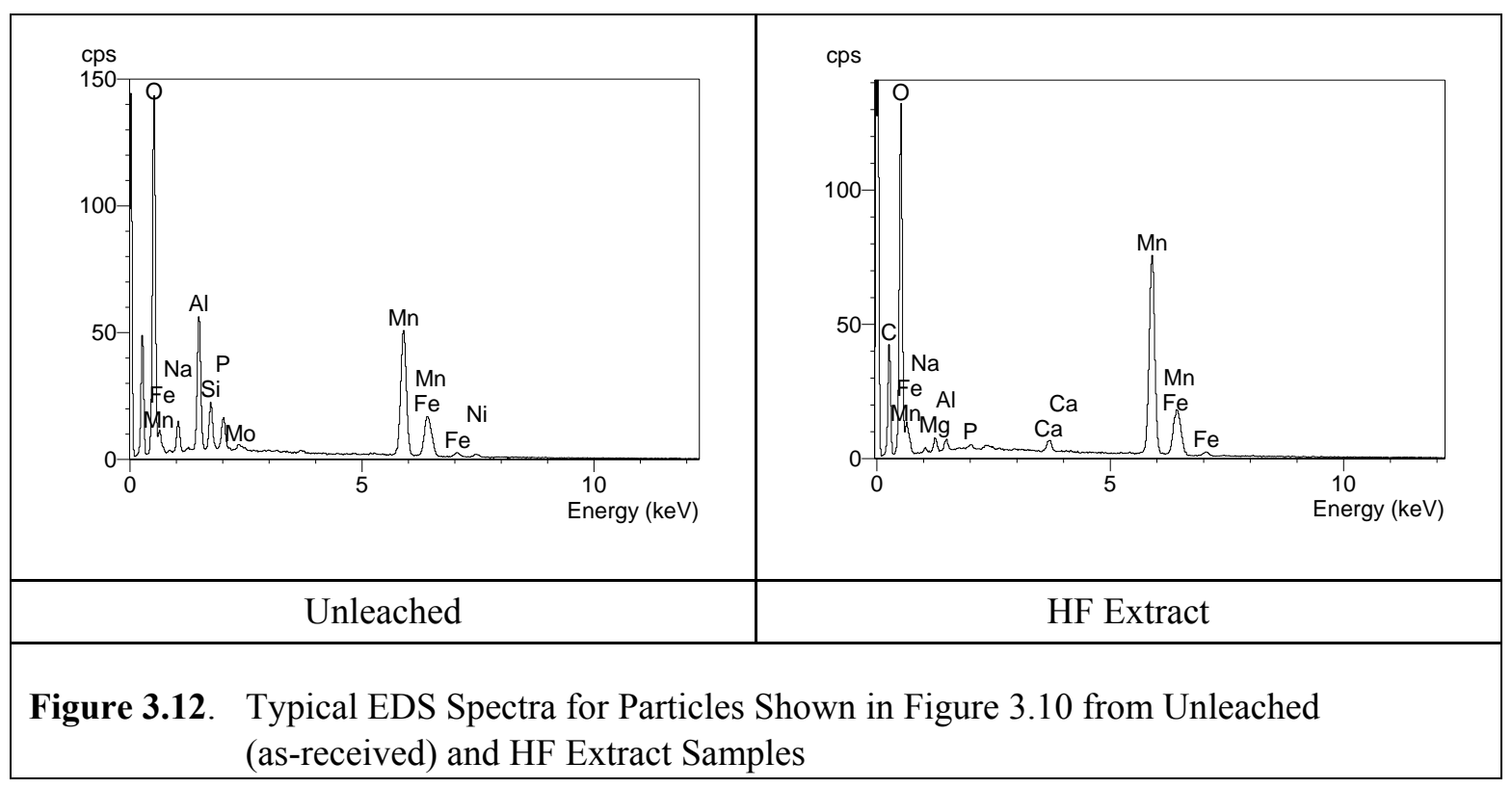

As noted in Section 3.1, Mn is a major component of sludge samples 404 and 405 from tank C-106. This is supported by the SEM/EDS analyses which show that four phases, two of which are common, in these samples contain $\mathrm{Mn}$ as the main element component. These include the phases consisting of MnAl-Fe-Na-P-Si-Ca- $\pm \mathrm{C} \pm \mathrm{H}$, Mn-O-C $\pm \mathrm{H}$, and Mn- $\mathrm{O}-\mathrm{P} \pm \mathrm{Al} \pm \mathrm{C} \pm \mathrm{H}$. Based on morphology, the SEM analyses suggest that there may possibly be two different phases consisting of Mn-O-C $\pm \mathrm{H}$. These phases include the blocky rhombohedral-like particles shown in Figure 3.14(A), and the cracked, blade-like particles in Figure 3.14(B). The latter form of particles was only observed in the unleached sludge samples 404 and 405.

The bright particle aggregates shown in Figure 3.13(A) and Figure 3.15(A) contained primarily Ag, $\mathrm{Hg}, \mathrm{O}$, and possibly $\mathrm{C}$. These $\mathrm{Ag}-\mathrm{Hg}$ particle aggregates were common in all of the unleached, 1-month and 82-day water-leached, and HF-extracted sludge samples 404 and 405. Although some of these aggregates were several tens of micrometers in size, many micrometer-to-submicrometer sized $\mathrm{Ag}-\mathrm{Hg}$ particles also existed, such as the bright (white) "specks" observable in most of the micrographs shown in Figure 3.13 through Figure 3.16.

The SEM/EDS analyses also indicated the presence of numerous particles and particle aggregates containing $\mathrm{Al}$ oxide/hydroxide ${ }^{(a)}$ in the unleached, 1-month and 82-day water-leached, and HF-extracted tank C-106 sludge samples. These multi-faceted, blocky particles appear dark gray in the SEM micrographs and were exceptionally common in the samples, especially the HF-extracted materials.

(a) Note that hydrogen $(\mathrm{H})$ cannot be detected by EDS analysis and is, therefore, listed as possibly present in the compositions shown in the SEM micrographs. 


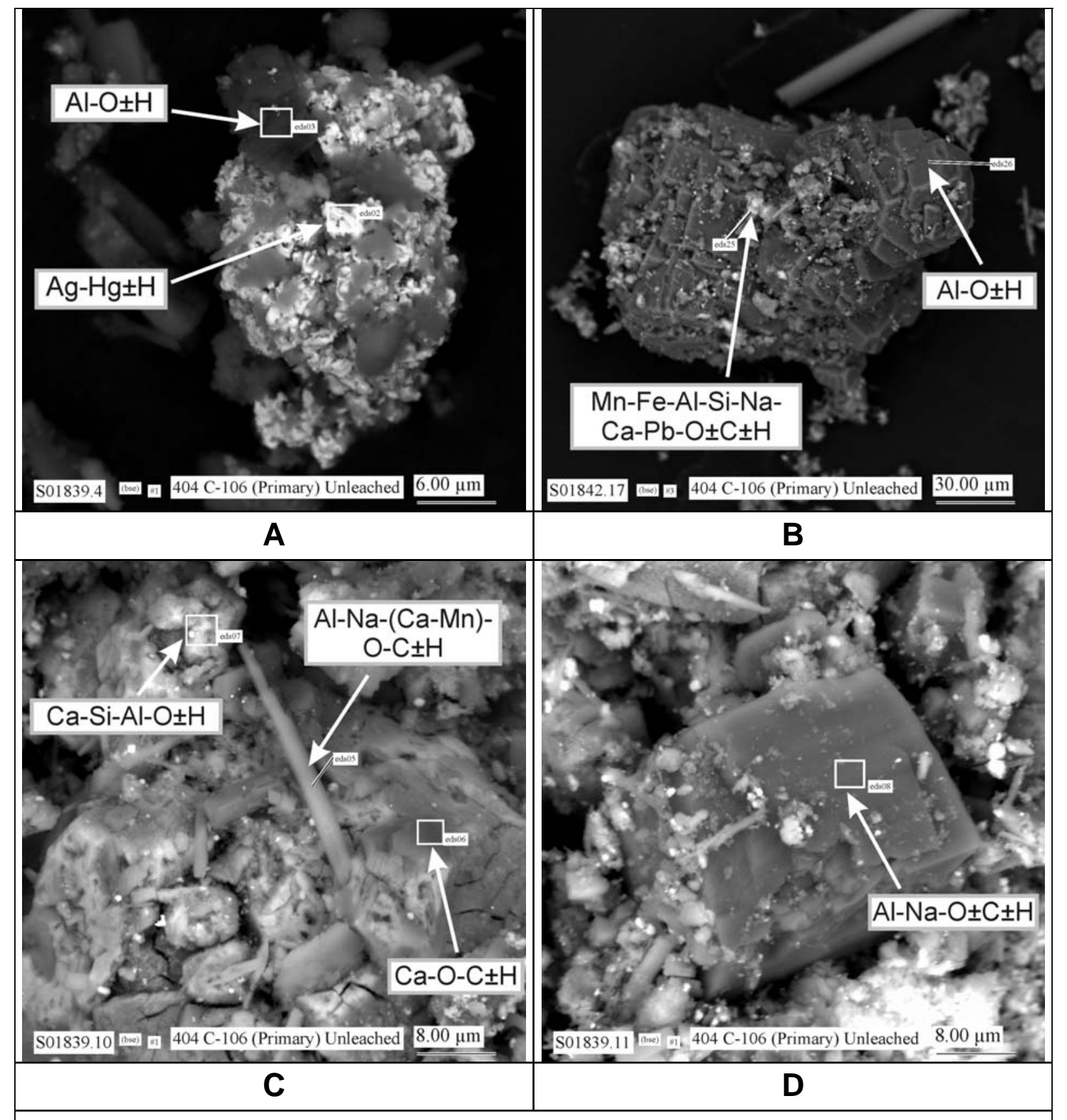

Figure 3.13. Representative SEM Micrograph Images of Particles Identified in Unleached Sludge Samples 404 and 405 from Tank C-106 


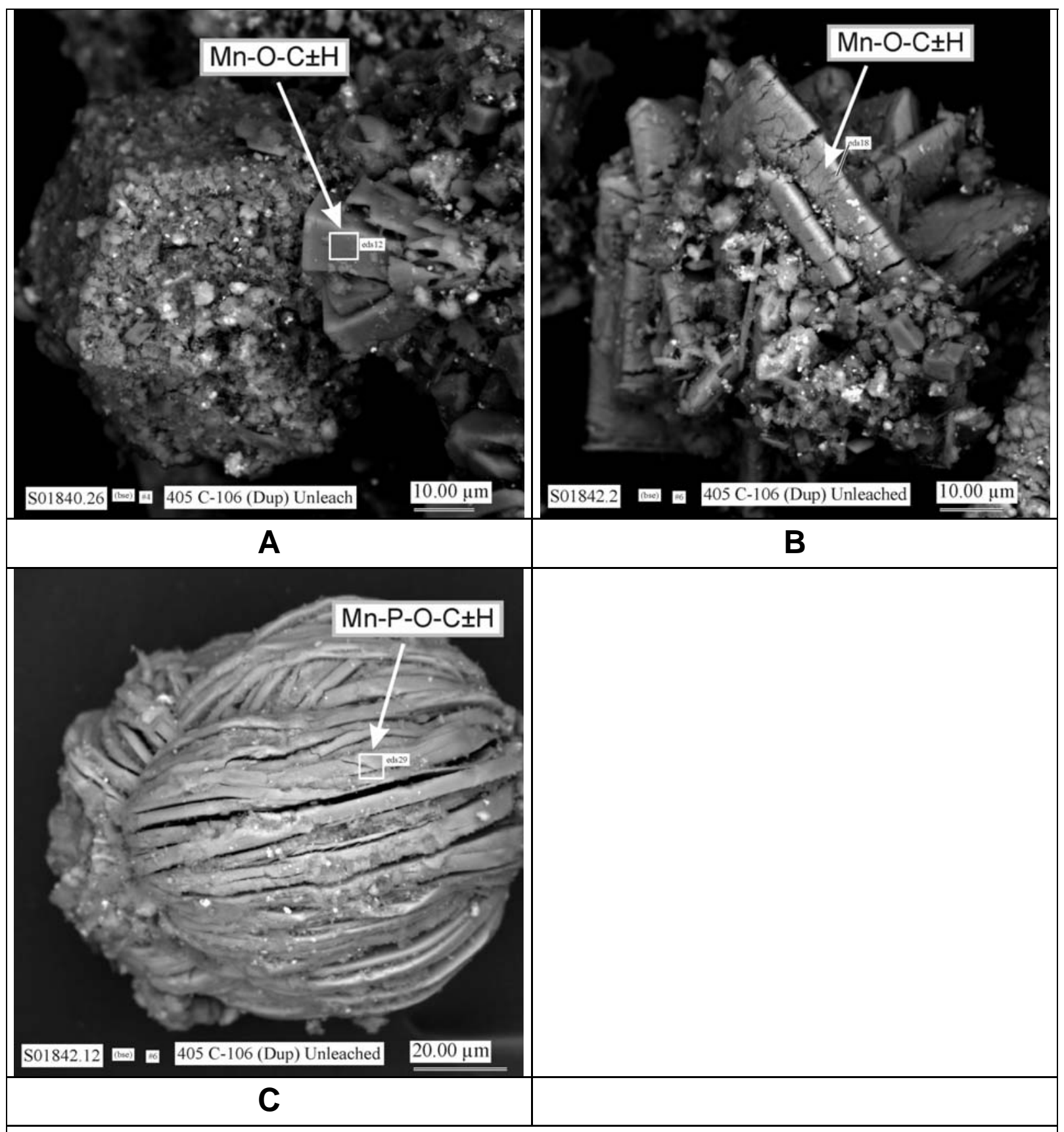

Figure 3.14. Representative SEM Micrograph Images of Particles Identified in Unleached Sludge Samples 404 and 405 from Tank C-106 


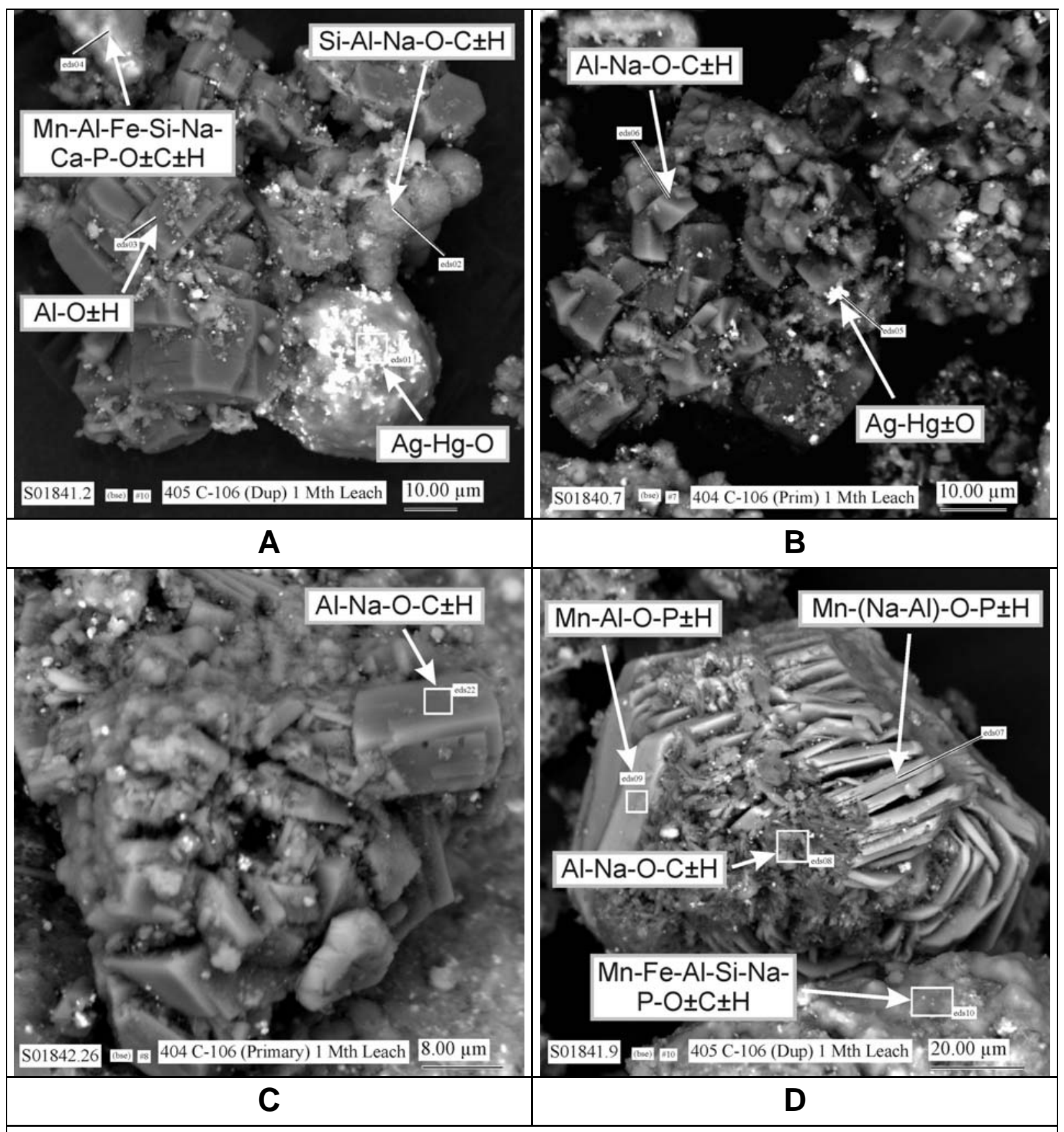

Figure 3.15. Representative SEM Micrograph Images of Particles Identified in 1-Month WaterLeached Sludge Samples 404 and 405 from Tank C-106 


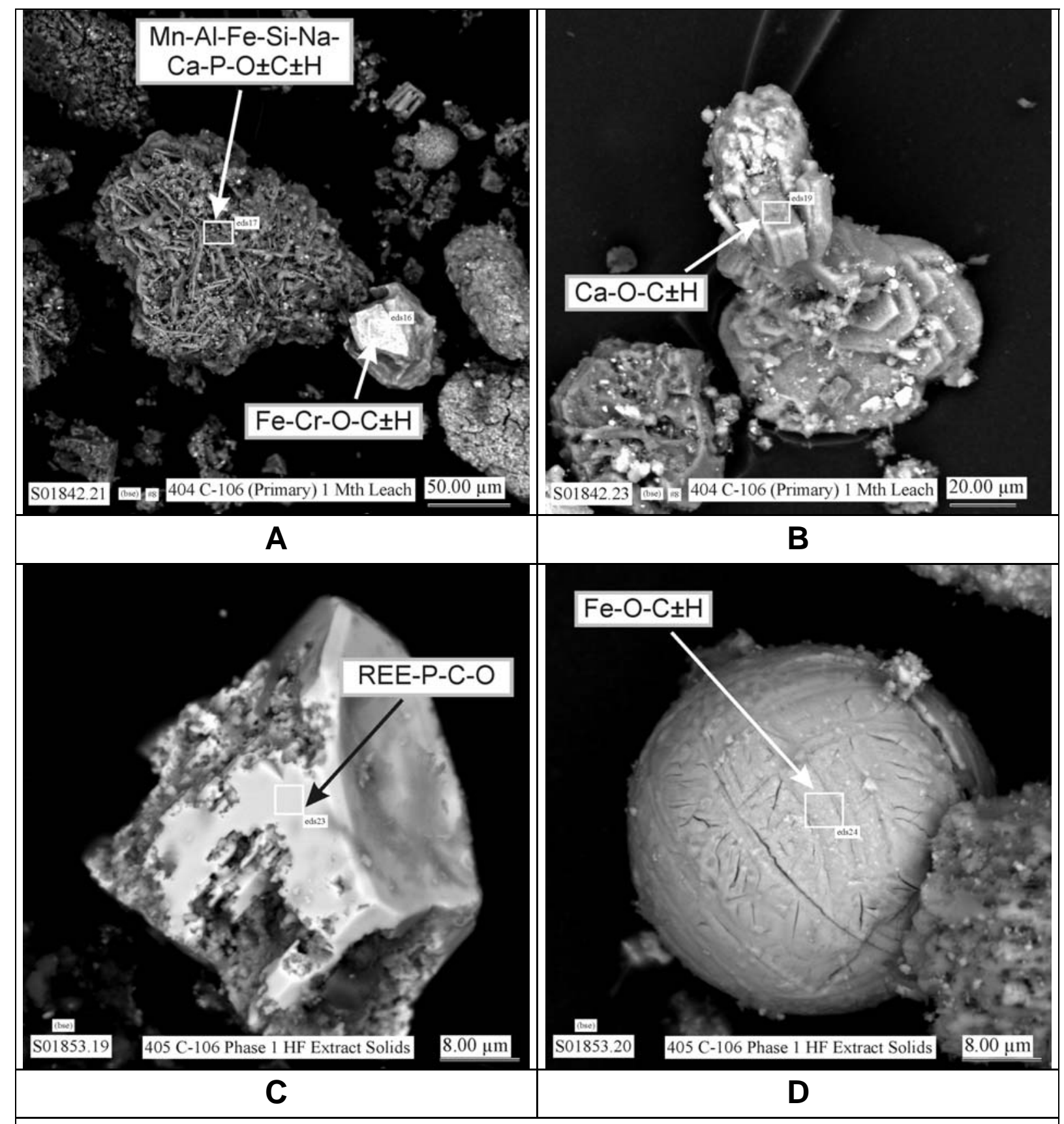

Figure 3.16. SEM Micrograph Images of Particles Identified in 1-Month Water-Leached and HF-Extracted Sludge Samples 404 and 405 from Tank C-106 
Although less common than the particle compositions discussed in the preceding paragraphs, some relatively large (tens of micrometers) particles consisting primarily of $\mathrm{Fe}, \mathrm{O}$, possibly $\mathrm{H}$, and often with Mn occur in the unleached, 1-month and 82-day water-leached, and HF-extracted tank C-106 sludge samples. When $\mathrm{Cr}$ and $\mathrm{Ni}$ were detected by EDS, these elements were always associated with these $\mathrm{Fe}$ oxides. Examples of these particles are shown in Figure 3.17. The surfaces of these particles do not exhibit any crystal faces, and except for being heavily pitted, were generally nondescript. Micrograph $\mathrm{C}$ at the bottom of Figure 3.17 is a higher magnification SEM image of the surface of an Fe oxide particle shown in Figure 3.17(B) from a sample of the HF extract of tank C-106 sludge. This micrograph shows the complex submicrometer layering and particle structure present in the Fe oxides that exist in these tank C-106 sludge samples. Detailed EDS analyses of the necklace-like structures in the lower left and right corners of micrograph $\mathrm{C}$ were not possible due their submicrometer size. However, based on the blackand-white contrast of these structures compared to the other material in this micrograph, these necklacelike structures possibly contained Fe and/or Mn as primary components. The conditions that produce these structures are not known. The cavities and complex submicrometer layering and particle structure present in the Fe oxides (see micrograph $\mathrm{C}$ in Figure 3.17) suggest that some solid phases, which may contain constituents of importance to sluicing and long-term stability of residual sludges and their risk assessment, can become encapsulated during precipitation of these Fe oxides and thus isolated from tank waste or sluicing solutions. Except when exposed at the surfaces of the Fe oxides, the dissolution of these sequestered phase(s) will likely be delayed and controlled ultimately by the low solubility of the Fe-oxide host phase.

Three sets of SEM micrographs and element distribution maps collected with the newly installed INCAEnergy EDS System are presented in Figures 3.18, 3.19, and 3.20. As noted previously, the capabilities of the INCAEnergy EDS System were demonstrated by completing a limited number of SEM/EDS analyses of SEM mounts of unleached, 82-day water-leached, and HF-extracted sludge samples. Figures 3.18 and 3.19 are low magnification BSE SEM micrographs and element distribution maps for particles in unleached and 82 day water leached sludge from Tank C-106, respectively. SEM micrographs and element distribution maps for the areas indicated by the white dotted rectangles in Figures 3.18 and 3.19 are shown at higher magnification in Appendix E. The concentration of each listed element is directly proportional to the regions of brightness (i.e., brighter the area, the higher the concentration of the selected element) in the corresponding distribution map. The element associations indicated by the distribution maps in Figures 3.18 and 3.19 and Appendix E are consistent with the EDS analyses discussed previously in this section. However, the element distribution maps provide a detailed visualization of the spatial distributions of the selected elements within the imaged particle assemblage, which are not apparent from numerous spot EDS analyses for different particles. Element distribution maps prepared using the INCAEnergy EDS System did not indicate any regions in any particles in the unleached, 82 day water-leached, and HF-extracted sludge samples with detectable concentrations for ${ }^{129} \mathrm{I}$, ${ }^{99} \mathrm{Tc}$, or ${ }^{238} \mathrm{U}$. The element distribution maps however did indicate the presence of some particles, which typically also contained $\mathrm{Ag}$ and $\mathrm{Hg}$, with detectable quantities of $\mathrm{Cu}$ and/or $\mathrm{Pb}$. Although these two metals were sporadically detected in a few particles of tank C-106 sludge by spot EDS analyses, particles enriched in these metals had not been previously identified. 


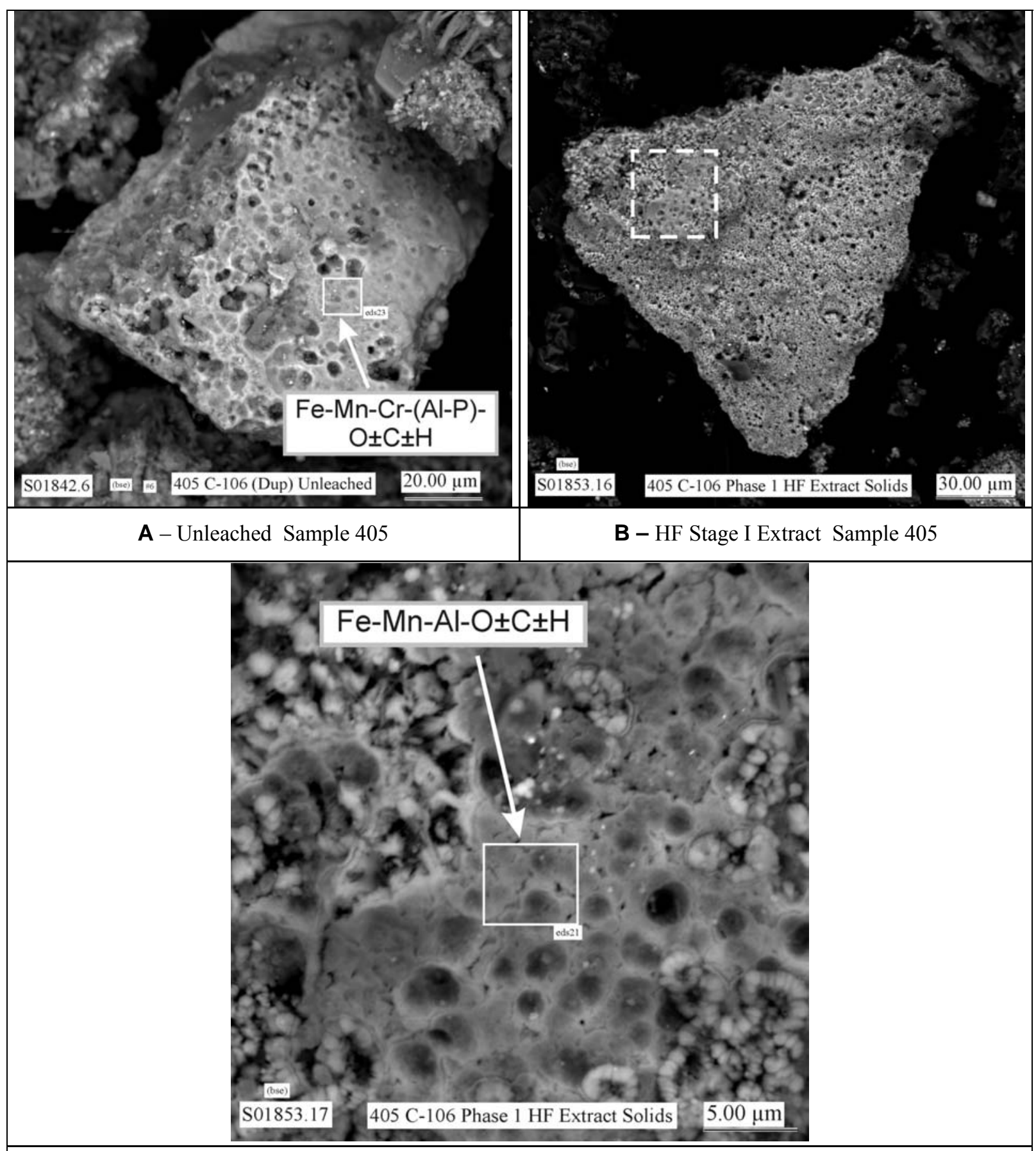

C - SEM micrograph showing at higher magnification the area in micrograph B (above) indicated by the white, dashed-line square

Figure 3.17. SEM Micrographs of Fe Oxide Particles in Unleached, 1-Month and 82-Day WaterLeached, and HF-Extracted Sludge Samples 404 and 405 from Tank C-106 

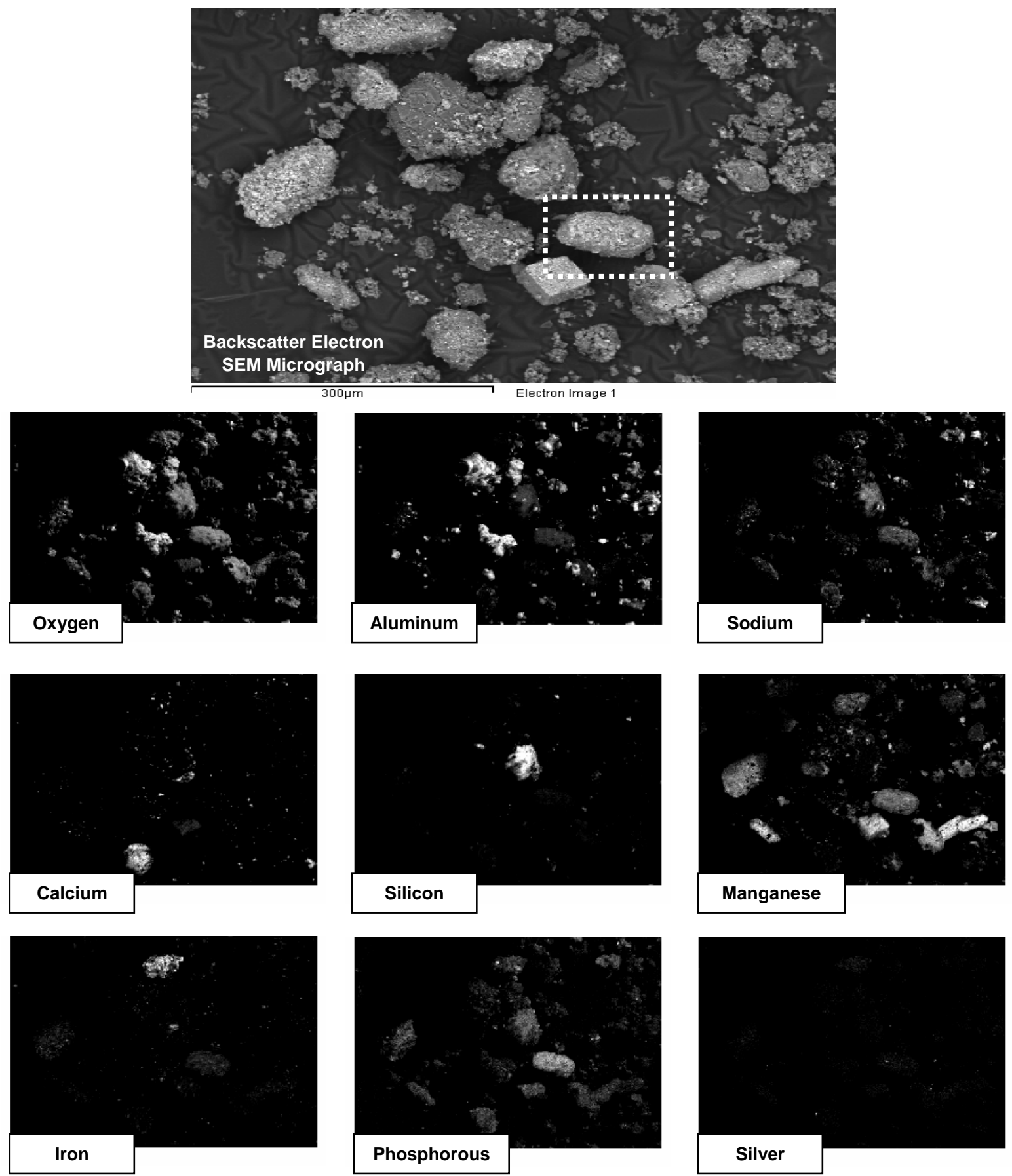

Figure 3.18. Low Magnification Backscatter-Electron SEM Micrograph and Element Distribution Maps for Particles in Unleached Sludge from Tank C-106 

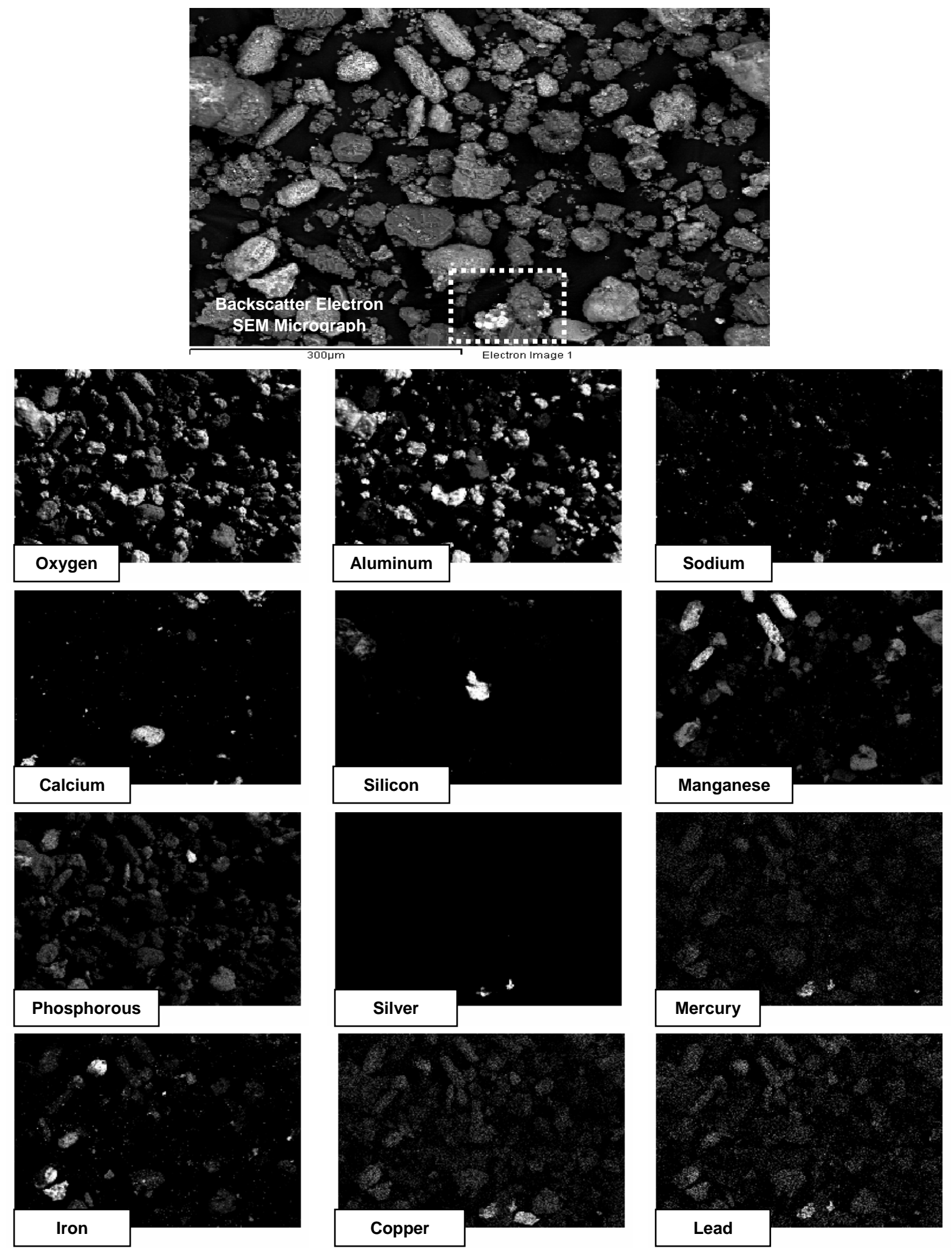

Figure 3.19. Low Magnification Backscatter-Electron SEM Micrograph and Element Distribution Maps for Particles in 82-Day Water-Leached Sludge from Tank C-106 

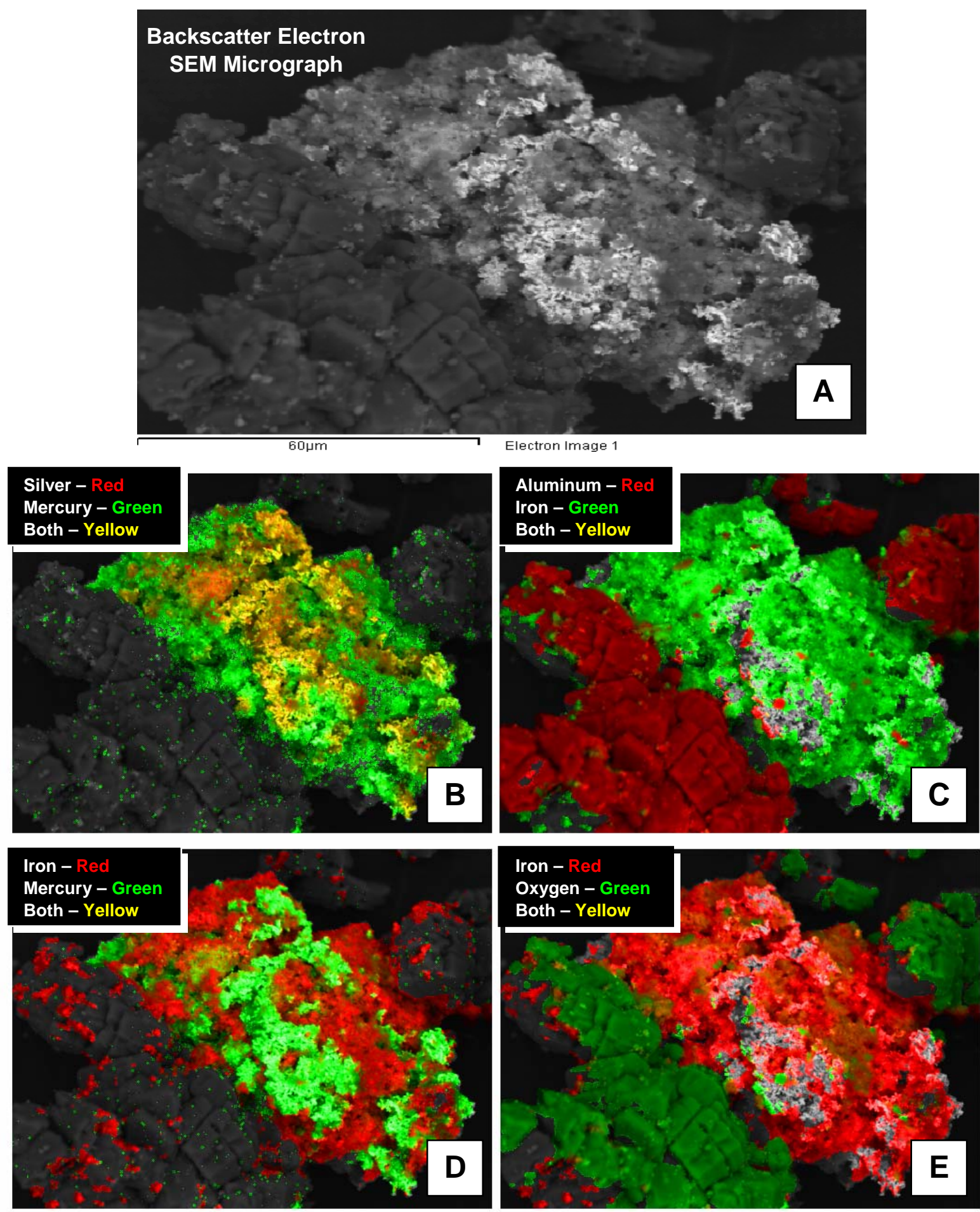

Figure 3.20. Backscatter-Electron SEM Image (A) and Colorized Element Maps (B Through E) for a Particle Aggregate from the HF Extract of Tank C-106 Sludge 
Figure 3.20 shows examples of colorized element maps (Figure 3.20B to Figure 3.20E) for an SEMimaged particle aggregate (Figure 3.20A) from an HF-extracted sample of C-106 residual sludge. The colors in the element maps denote the presence of the indicated, user-specified elements, where the "red areas" signifies the presence of one specified element, the "green areas" for the presence a second element, and the "yellow areas" for the presence both of the selected elements. The INCAEnergy software has the capability to create colorized maps for up to three elements selected by the user. The maps in Figure 3.20 show the relationship between the presence of Ag, $\mathrm{Hg}$, and $\mathrm{Fe}$ (Figure 3.20B and Figure 3.20D) and of $\mathrm{Fe}$ to $\mathrm{Al}$ and $\mathrm{O}$ (Figure 3.20C and Figure 3.20E, respectively) for this particle aggregate. These elements correspond to the presence of phases \{e.g., the Ag-Hg solid; hematite $\left(\mathrm{Fe}_{2} \mathrm{O}_{3}\right)$; and gibbsite $\left[\mathrm{Al}(\mathrm{OH})_{3}\right]$, böhmite $[\mathrm{AlO}(\mathrm{OH})]$, and/or dawsonite $\left.\left[\mathrm{NaAlCO}_{3}(\mathrm{OH})_{2}\right]\right\}$ determined by bulk $\mathrm{X}$-ray diffraction analysis (XRD) (see Section 3.5). These results also show for the first time that there might be two $\mathrm{Hg}$-containing solids where one is enriched in $\mathrm{Ag}$, and the possible coexistence of $\mathrm{Fe}$ with the $\mathrm{Hg}$-rich/Ag-poor solid. Some of the very small colored areas, such as the small green and red points in the southwest areas of Figure 3.20B and Figure 3.20D, respectively, are false positives resulting from overlaps in the corresponding energies of the EDS peaks.

\subsection{Comparison of XRD and SEM/EDS Results}

The XRD and SEM/EDS results are consistent with each other and with the results reported by Lumetta et al. (1996) and Bechtold et al. (2003). Lumetta et al. (1996) used transmission electron microscopy (TEM) to identify the solids present in samples of an as-received sample of C-106 sludge (obtained several years ago when most of the contents of tank C-106 were sluiced into tank AY-102) and a sludge leached by $\mathrm{NaOH}$. In the as-received tank $\mathrm{C}-106$ sludge, they identified only the presence of amorphous $\mathrm{Al}(\mathrm{OH})_{3}$ and amorphous aluminosilicates. In the caustic leached residue, Lumetta et al. (1996) found amorphous aluminosilicates, $\mathrm{FeO}(\mathrm{OH}), \mathrm{Ag}_{2} \mathrm{O}$, and $\mathrm{ZrO}_{2}$. Bechtold et al. (2003) used XRD and SEM/EDS to characterize a baseline sample of C-106 sludge and a baseline sample leached with $1 \mathrm{M}$ oxalic acid. They identified dawsonite, hematite, cancrinite $\left[\mathrm{Na}_{6} \mathrm{Ca}_{1.5} \mathrm{Al}_{6} \mathrm{Si}_{6} \mathrm{O}_{24}\left(\mathrm{CO}_{3}\right)_{1.6}\right]$, and possibly sidorenkite $\left(\mathrm{Na}_{2} \mathrm{MnPO}_{4} \mathrm{CO}_{3}\right)$ in the baseline $\mathrm{C}-106$ sample. In residue from baseline $\mathrm{C}-106$ sludge treated with $1 \mathrm{M}$ oxalic acid, Bechtold et al. (2003) reported the presence of hematite, gibbsite, böhmite, possibly "Mn(II) oxalate" $\left(\mathrm{MnC}_{2} \mathrm{O}_{4} \cdot 2 \mathrm{H}_{2} \mathrm{O}\right)$, and traces of Nd-rich and $\mathrm{Pb}$-rich particulates.

Table 3.35 lists the characterization data available for tank C-106 sludge prior to treatment by oxalic acid from Bechtold et al. (2003) and for unleached AY-102 sludge from Krupka et al. (2004). As noted in Section 1.2, the contents of tank C-106 were sluiced to tank AY-102 using supernatant from tank AY-102 as the sluicing fluid, and therefore should be similar to tank C-106 sludge prior to its treatment by oxalic acid. 
Table 3.35. Comparison of Characterization Data for Tank C-106 Sludge Prior to Treatment by Oxalic Acid

\begin{tabular}{|c|c|c|c|}
\hline Type of Sample & \multicolumn{2}{|c|}{$\begin{array}{c}\text { XRD and SEM/EDS } \\
\text { Analyses by Krupka et al. (2004) } \\
\end{array}$} & $\begin{array}{c}\text { Characterization } \\
\text { Results by } \\
\text { Bechtold et al. (2003) } \\
\end{array}$ \\
\hline \multirow{10}{*}{$\begin{array}{l}\text { C-106 sludge prior } \\
\text { to treatment by } \\
\text { oxalic acid }\end{array}$} & \multicolumn{2}{|c|}{$\begin{array}{l}\text { Unleached AY-102 as a Surrogate for C-106 Sludge } \\
\text { Prior to Treatment by Oxalic Acid }\end{array}$} & $\begin{array}{l}\text { "Baseline Sample" of C-106 } \\
\text { Sludge Prior to Treatment by } \\
\text { Oxalic Acid }\end{array}$ \\
\hline & $\begin{array}{l}\text { Dawsonite } \\
{\left[\mathrm{NaAlCO}_{3}(\mathrm{OH})_{2}\right]}\end{array}$ & $\begin{array}{l}\text { Lath-shaped, } \mathrm{Na} \pm \mathrm{Al} \pm \mathrm{Fe} \\
\text { particles }\end{array}$ & Dawsonite \\
\hline & Hematite $\left(\mathrm{Fe}_{2} \mathrm{O}_{3}\right)$ & $\begin{array}{l}\text { Globular aggregates of } \mathrm{Fe} \\
\pm \mathrm{Na} \pm \mathrm{Mn} \text { particles }\end{array}$ & Hematite \\
\hline & Gibbsite $\left[\mathrm{Al}(\mathrm{OH})_{3}\right]$ & & Gibbsite \\
\hline & & $\mathrm{Na}, \mathrm{Al}$,Si-rich particles & $\begin{array}{l}\text { Cancrinite } \\
{\left[\mathrm{Na}_{6} \mathrm{Ca}_{1.5} \mathrm{Al}_{6} \mathrm{Si}_{6} \mathrm{O}_{24}\left(\mathrm{CO}_{3}\right)_{1.6}\right]}\end{array}$ \\
\hline & & & Sidorenkite $\left(\mathrm{Na}_{2} \mathrm{MnPO}_{4} \mathrm{CO}_{3}\right)$ \\
\hline & & Na-rich particles & \\
\hline & & Ag-containing particles & \\
\hline & & $\begin{array}{l}\text { U,Na-containing crusty } \\
\text { particles }\end{array}$ & \\
\hline & & $\begin{array}{l}\text { Large carbon-rich (graphite?) } \\
\text { particles }\end{array}$ & \\
\hline
\end{tabular}

Table 3.36 compares the XRD (Section 0) and SEM/EDS (Section 3.6) results from this study to those reported by Bechtold et al. (2003) for tank C-106 sludge treated with $1 \mathrm{M}$ oxalic acid. The XRD and SEM/EDS results from this study overall are consistent with each other and with phases identified by Bechtold et al. (2003). Several phases, however, observed by SEM/EDS were not detected by XRD in this study or do not correspond to any of phases identified by Bechtold et al. (2003). Some of the phases that were not identified by XRD may be amorphous or present at concentrations too low for detection by XRD.

As discussed in the Section 3.6, the phase that consists of Mn-Al-Fe-Na-P-Si-Ca-O $\pm \mathrm{C} \pm \mathrm{H}$ was present on many of the particles in all of the unleached, water-leached, and HF-extracted samples 404 and 405 of sludge from tank C-106. The identity of this phase is not known and does not correspond to any of the solids identified by this XRD analyses or those of Bechtold et al. (2003). The texture of these particles, as characterized by SEM, does not provide any definitive information to evaluate if this material is amorphous or crystalline. Moreover, the results of HF extractions suggest that this phase may consist of more than one phase. Without additional characterization by techniques, such as TEM, it can be presumed at this time that the Mn-Al-Fe-Na-P-Si-Ca-O $\pm \mathrm{C} \pm \mathrm{H}$ phase is possibly amorphous or consists of a mixture of two or more of the phases identified by XRD. 
Table 3.36. Comparison of XRD and SEM/EDS Characterization Results from This Study to Those Reported by Bechtold et al. (2003) for Tank C-106 Sludge treated with Oxalic Acid

\begin{tabular}{|c|c|c|c|}
\hline Type of Sample & $\begin{array}{l}\text { XRD Analyses } \\
\text { From This Study }\end{array}$ & $\begin{array}{l}\text { SEM/EDS Analyses } \\
\text { From This Study }\end{array}$ & $\begin{array}{c}\text { Characterization } \\
\text { Results by } \\
\text { Bechtold et al. (2003) }\end{array}$ \\
\hline \multirow{14}{*}{$\begin{array}{l}\text { C-106 sludge treated } \\
\text { by oxalic acid } \\
\text { (prior to water } \\
\text { leaches and HF } \\
\text { extraction) }\end{array}$} & & $\begin{array}{l}\text { Mn-Al-Fe-Na-P-Si-Ca- } \\
\mathrm{O} \pm \mathrm{C} \pm \mathrm{H}\end{array}$ & \\
\hline & Gibbsite & \multirow{2}{*}{$\mathrm{Al}-\mathrm{O} \pm \mathrm{H}$} & Gibbsite \\
\hline & Böhmite & & Böhmite \\
\hline & Dawsonite & Al-Na-O-C $\pm \mathrm{H}$ & \\
\hline & Hematite & $\begin{array}{l}\mathrm{Fe}-\mathrm{Cr}-\mathrm{O} \pm \mathrm{C} \pm \mathrm{H} \\
\mathrm{Fe}-\mathrm{Mn}-\mathrm{O} \pm \mathrm{C} \pm \mathrm{H}\end{array}$ & Hematite \\
\hline & Rhodochrosite & \multirow{2}{*}{$\begin{array}{l}\text { Mn-O-C } \pm \mathrm{H} \text { (possibly two } \\
\text { different phases based on } \\
\text { morphology) }\end{array}$} & \multirow[b]{2}{*}{ "Mn(II) oxalate"?? } \\
\hline & Lindbergite $\left(\mathrm{MnC}_{2} \mathrm{O}_{4} \cdot 2 \mathrm{H}_{2} \mathrm{O}\right)$ & & \\
\hline & $\begin{array}{l}\text { Whewellite } \\
\left(\mathrm{CaC}_{2} \mathrm{O}_{4} \cdot \mathrm{H}_{2} \mathrm{O}\right)\end{array}$ & $\mathrm{Ca}-\mathrm{O} \pm \mathrm{C} \pm \mathrm{H}$ & \\
\hline & Possible Ag-Hg phase & $\begin{array}{l}\text { Possibly } 1 \text { or } 2 \text { phases with } \\
\mathrm{Ag}-\mathrm{Hg} \pm \mathrm{Fe} \pm \mathrm{Pb} \pm \mathrm{Cu} \pm \mathrm{O} \pm \mathrm{H}\end{array}$ & \\
\hline & & $\mathrm{Mn}-\mathrm{O}-\mathrm{P} \pm \mathrm{Al} \pm \mathrm{C} \pm \mathrm{H}$ & \\
\hline & & $\mathrm{Si}-\mathrm{Al}-\mathrm{Na}-\mathrm{O} \pm \mathrm{C} \pm \mathrm{H}$ & \\
\hline & & REE-rich oxide & Nd-rich particules \\
\hline & & $\mathrm{Ca}-\mathrm{Si}-\mathrm{Al}-\mathrm{O} \pm \mathrm{C} \pm \mathrm{H}$ & \\
\hline & & $\mathrm{Pb}$-containing phase & Pb-rich particules \\
\hline $\begin{array}{l}\text { Oxalic acid-treated } \\
\text { C-106 sludge after } \\
\text { water leaches and } \\
\text { HF extraction }\end{array}$ & $\begin{array}{l}\text { Same as XRD results above } \\
\text { except for the absence of } \\
\text { lindbergite }\end{array}$ & Same as SEM/EDS results & $\begin{array}{l}\text { No data available, since } \\
\text { similar water leach and HF } \\
\text { extraction studies were not } \\
\text { part of the studies by } \\
\text { Bechtold et al. (2003) }\end{array}$ \\
\hline
\end{tabular}

There are several similarities in the phases identified in sludge from tanks C-106 and AY-102 that has not been leached by oxalic acid with respect to those observed in the tank C-106 sludge tested in this study. For example, gibbsite, dawsonite, and hematite were common to both types of sludge. The phase determined by SEM/EDS to contain Si-Al-Na-O-C $\pm \mathrm{H}$ [see ball-shaped crystals in the upper right of Figure 3.15(A)] possibly corresponds to cancrinite $\left[\mathrm{Na}_{6} \mathrm{Ca}_{1.5} \mathrm{Al}_{6} \mathrm{Si}_{6} \mathrm{O}_{24}\left(\mathrm{CO}_{3}\right)_{1.6}\right]$ that Bechtold et al. (2003) identified in the baseline $\mathrm{C}-106$ sample prior to oxalic acid treatment. The ball-shaped crystals in the upper right of Figure 3.15(A) look like balls of twine, which is similar to the crystal habit identified by others as being the mineral cancrinite (Bickmore et al. 2001; Bredt et al. 2003; Buck and McNamara 2004). Although highly speculative, the $\mathrm{Ag}-\mathrm{Hg}$ and $\mathrm{Mn}-\mathrm{O}-\mathrm{P} \pm \mathrm{Al} \pm \mathrm{C} \pm \mathrm{H}$ phases determined by SEM/EDS in this study might be related, respectively, to the Ag-containing phase observed in unleached AY-102 sludge by Krupka et al. (2004) and to sidorenkite $\left(\mathrm{Na}_{2} \mathrm{MnPO}_{4} \mathrm{CO}_{3}\right)$ reported for baseline tank C-106 sludge by Bechtold et al. (2003) (see Table 3.35). 


\subsection{Selective Extractions}

\subsubsection{Phase 1}

Phase 1 selective extraction results for samples 404 and 405 are shown in Table 3.37. Data for ${ }^{99} \mathrm{Tc}$, ${ }^{238} \mathrm{U}, \mathrm{Cr}, \mathrm{Fe}, \mathrm{Al}$, and $\mathrm{Mn}$ are tabulated on the basis of mass of contaminant released per gram of dry sludge. The total contaminant concentrations used in the calculations were those determined by fusion analysis. The results displayed in parentheses are less than the lowest instrument EQL but greater than a zero instrument signal. These values may reflect real concentrations but have larger associated uncertainties. Values with a $<$ sign are instrument values that are less than zero. The number indicated is the EQL. To facilitate easier evaluation of these data, the values are presented as a percentage of the total (fusion) concentrations in Table 3.38. Also included in this table are totals determined by summation of the results from each individual extraction step.

Table 3.37. Phase 1 Selective Extraction Results for Sludge Samples 404 and 405 from Tank C-106 (in $\mu \mathrm{g} / \mathrm{g}$ )

\begin{tabular}{|c|c|c|c|c|c|c|c|}
\hline $\begin{array}{c}\text { Sample } \\
\text { Number }\end{array}$ & Extractant & $\begin{array}{c}{ }^{99} \mathrm{Tc} \\
(\mu \mathrm{g} / \mathrm{g})\end{array}$ & $\begin{array}{c}{ }^{238} \mathrm{U} \\
(\mu \mathrm{g} / \mathrm{g}) \\
\end{array}$ & $\begin{array}{c}\mathrm{Cr} \\
(\mu \mathrm{g} / \mathrm{g}) \\
\end{array}$ & $\begin{array}{c}\mathbf{F e} \\
(\mu \mathrm{g} / \mathrm{g}) \\
\end{array}$ & $\begin{array}{c}\mathrm{Al} \\
(\mu \mathrm{g} / \mathrm{g}) \\
\end{array}$ & $\begin{array}{c}\text { Mn } \\
(\mu \mathrm{g} / \mathrm{g}) \\
\end{array}$ \\
\hline 404 & Deionized Water & $1.86 \mathrm{E}-02$ & $6.03 \mathrm{E}+00$ & $(2.62 \mathrm{E}+00)$ & $(2.64 \mathrm{E}+01)$ & $7.46 \mathrm{E}+01$ & $8.79 \mathrm{E}+03$ \\
\hline 404 DUP & Deionized Water & $1.75 \mathrm{E}-02$ & $6.78 \mathrm{E}+00$ & $(9.66 \mathrm{E}-01)$ & $(2.20 \mathrm{E}+01)$ & $9.18 \mathrm{E}+01$ & $8.94 \mathrm{E}+03$ \\
\hline 404 & $\mathrm{NH}_{2} \mathrm{OH}-\mathrm{HCl}$ & $2.25 \mathrm{E}-01$ & $1.25 \mathrm{E}+02$ & $8.63 \mathrm{E}+01$ & $3.37 \mathrm{E}+03$ & $1.06 \mathrm{E}+04$ & $6.24 \mathrm{E}+04$ \\
\hline 404 DUP & $\mathrm{NH}_{2} \mathrm{OH}-\mathrm{HCl}$ & $2.24 \mathrm{E}-01$ & $1.58 \mathrm{E}+02$ & $9.82 \mathrm{E}+01$ & $4.10 \mathrm{E}+03$ & $1.36 \mathrm{E}+04$ & $8.76 \mathrm{E}+04$ \\
\hline 404 & $0.01 \mathrm{HF} / 0.01 \mathrm{KF}$ & $4.92 \mathrm{E}-02$ & $6.71 \mathrm{E}+01$ & $6.03 \mathrm{E}+01$ & $2.57 \mathrm{E}+03$ & $1.91 \mathrm{E}+04$ & $6.86 \mathrm{E}+03$ \\
\hline 404 DUP & $0.01 \mathrm{HF} / 0.01 \mathrm{KF}$ & $6.10 \mathrm{E}-02$ & $7.76 \mathrm{E}+01$ & $8.74 \mathrm{E}+01$ & $3.03 \mathrm{E}+03$ & $2.36 \mathrm{E}+04$ & $5.92 \mathrm{E}+03$ \\
\hline 404 & $0.01 \mathrm{HF} / 0.01 \mathrm{KF}$ & $5.36 \mathrm{E}-02$ & $1.29 \mathrm{E}+01$ & $5.85 \mathrm{E}+01$ & $1.80 \mathrm{E}+03$ & $7.96 \mathrm{E}+03$ & $2.47 \mathrm{E}+03$ \\
\hline 404 DUP & $0.01 \mathrm{HF} / 0.01 \mathrm{KF}$ & $6.84 \mathrm{E}-02$ & $1.78 \mathrm{E}+01$ & $1.17 \mathrm{E}+02$ & $3.58 \mathrm{E}+03$ & $8.53 \mathrm{E}+03$ & $7.04 \mathrm{E}+03$ \\
\hline 404 & Hot Conc. $\mathrm{HNO}_{3}$ & $3.65 \mathrm{E}-01$ & $2.46 \mathrm{E}+01$ & $4.70 \mathrm{E}+02$ & $2.30 \mathrm{E}+04$ & $7.34 \mathrm{E}+04$ & $3.23 \mathrm{E}+04$ \\
\hline 404 DUP & Hot Conc. $\mathrm{HNO}_{3}$ & 4.12E-01 & $1.95 \mathrm{E}+01$ & $3.09 \mathrm{E}+02$ & $1.68 \mathrm{E}+04$ & $5.85 \mathrm{E}+04$ & $2.23 \mathrm{E}+04$ \\
\hline Total & Fusion & $1.01 \mathrm{E}+00$ & $2.36 \mathrm{E}+02$ & $8.41 \mathrm{E}+02$ & $4.33 \mathrm{E}+04$ & $1.40 \mathrm{E}+05$ & $1.14 \mathrm{E}+05$ \\
\hline 405 & Deionized Water & $1.92 \mathrm{E}-02$ & $7.37 \mathrm{E}+00$ & $(4.23 \mathrm{E}+00)$ & $(8.22 \mathrm{E}+01)$ & $1.38 \mathrm{E}+02$ & $1.64 \mathrm{E}+04$ \\
\hline 405 DUP & Deionized Water & $1.29 \mathrm{E}-02$ & $7.95 \mathrm{E}+00$ & $<6.12 \mathrm{E}+01$ & $(3.50 \mathrm{E}+01)$ & $1.15 \mathrm{E}+02$ & $1.47 \mathrm{E}+04$ \\
\hline 405 & $\mathrm{NH}_{2} \mathrm{OH}-\mathrm{HCl}$ & $2.05 \mathrm{E}-01$ & $1.25 \mathrm{E}+02$ & $9.86 \mathrm{E}+01$ & $3.83 \mathrm{E}+03$ & $1.22 \mathrm{E}+04$ & $8.05 \mathrm{E}+04$ \\
\hline 405 DUP & $\mathrm{NH}_{2} \mathrm{OH}-\mathrm{HCl}$ & $1.44 \mathrm{E}-01$ & $9.66 \mathrm{E}+01$ & $6.96 \mathrm{E}+01$ & $2.99 \mathrm{E}+03$ & $9.63 \mathrm{E}+03$ & $6.87 \mathrm{E}+04$ \\
\hline 405 & $0.01 \mathrm{HF} / 0.01 \mathrm{KF}$ & $5.24 \mathrm{E}-02$ & $7.56 \mathrm{E}+01$ & $8.05 \mathrm{E}+01$ & $2.81 \mathrm{E}+03$ & $2.10 \mathrm{E}+04$ & $7.43 \mathrm{E}+03$ \\
\hline 405 DUP & $0.01 \mathrm{HF} / 0.01 \mathrm{KF}$ & $4.73 \mathrm{E}-02$ & $5.98 \mathrm{E}+01$ & $5.25 \mathrm{E}+01$ & $2.18 \mathrm{E}+03$ & $1.76 \mathrm{E}+04$ & $9.66 \mathrm{E}+03$ \\
\hline 405 & $0.01 \mathrm{HF} / 0.01 \mathrm{KF}$ & $5.84 \mathrm{E}-02$ & $1.56 \mathrm{E}+01$ & $8.37 \mathrm{E}+01$ & $2.59 \mathrm{E}+03$ & $6.55 \mathrm{E}+03$ & $2.57 \mathrm{E}+03$ \\
\hline 405 DUP & $0.01 \mathrm{HF} / 0.01 \mathrm{KF}$ & $4.40 \mathrm{E}-02$ & $1.63 \mathrm{E}+01$ & $6.48 \mathrm{E}+01$ & $2.07 \mathrm{E}+03$ & $8.15 \mathrm{E}+03$ & $2.27 \mathrm{E}+03$ \\
\hline 405 & Hot Conc. $\mathrm{HNO}_{3}$ & $2.40 \mathrm{E}-01$ & $1.42 \mathrm{E}+01$ & $2.18 \mathrm{E}+02$ & $1.05 \mathrm{E}+04$ & $3.26 \mathrm{E}+04$ & $7.72 \mathrm{E}+03$ \\
\hline 405 DUP & Hot Conc. $\mathrm{HNO}_{3}$ & $5.67 \mathrm{E}-01$ & $2.12 \mathrm{E}+01$ & $7.19 \mathrm{E}+02$ & $3.29 \mathrm{E}+04$ & $9.63 \mathrm{E}+04$ & $4.94 \mathrm{E}+04$ \\
\hline Total & Fusion & $1.31 \mathrm{E}+00$ & $2.58 \mathrm{E}+02$ & $8.07 \mathrm{E}+02$ & $4.33 \mathrm{E}+04$ & $1.23 \mathrm{E}+05$ & $1.22 \mathrm{E}+05$ \\
\hline
\end{tabular}


Table 3.38. Phase 1 Selective Extraction Results for Sludge Samples 404 and 405 from Tank C-106 (in percentages)

\begin{tabular}{|c|c|c|c|c|c|c|c|}
\hline \multirow[b]{2}{*}{ Sample Number } & \multirow[b]{2}{*}{ Extractant } & 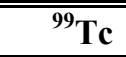 & 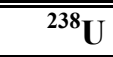 & $\mathbf{C r}$ & $\mathbf{F e}$ & $\mathbf{A l}$ & Mn \\
\hline & & \multicolumn{6}{|c|}{ \% Leached-1- } \\
\hline 404 & Deionized Water & 1.8 & 2.6 & 0.3 & 0.1 & 0.1 & 7.7 \\
\hline 404 DUP & Deionized Water & 1.7 & 2.9 & 0.1 & 0.1 & 0.1 & 7.8 \\
\hline 404 & $\mathrm{NH}_{2} \mathrm{OH}-\mathrm{HCl}$ & 22.3 & 53.0 & 10.3 & 7.8 & 7.6 & 54.7 \\
\hline 404 DUP & $\mathrm{NH}_{2} \mathrm{OH}-\mathrm{HCl}$ & 22.2 & 66.8 & 11.7 & 9.5 & 9.7 & 76.8 \\
\hline 404 & $0.01 \mathrm{HF} / 0.01 \mathrm{KF}$ & 4.9 & 28.4 & 7.2 & 5.9 & 13.7 & 6.0 \\
\hline 404 DUP & $0.01 \mathrm{HF} / 0.01 \mathrm{KF}$ & 6.0 & 32.9 & 10.4 & 7.0 & 16.9 & 5.2 \\
\hline 404 & $0.01 \mathrm{HF} / 0.01 \mathrm{KF}$ & 5.3 & 5.4 & 7.0 & 4.2 & 5.7 & 2.2 \\
\hline 404 DUP & $0.01 \mathrm{HF} / 0.01 \mathrm{KF}$ & 6.8 & 7.6 & 14.0 & 8.3 & 6.1 & 6.2 \\
\hline 404 & Hot Conc. $\mathrm{HNO}_{3}$ & 36.1 & 10.4 & 55.9 & 53.2 & 52.4 & 28.3 \\
\hline 404 DUP & Hot Conc. $\mathrm{HNO}_{3}$ & 40.8 & 8.3 & 36.8 & 38.7 & 41.8 & 19.5 \\
\hline 404 & Total & 70.4 & 999.8 & 80.6 & 71.1 & 79.5 & 999.0 \\
\hline 404 DUP & Total & 77.6 & 118.5 & 72.9 & 63.5 & 74.5 & 115.6 \\
\hline 405 & Deionized Water & 1.5 & 2.9 & 0.5 & 0.2 & 0.1 & 13.4 \\
\hline 405 DUP & Deionized Water & 1.0 & 3.1 & 0.0 & 0.1 & 0.1 & 12.0 \\
\hline 405 & $\mathrm{NH}_{2} \mathrm{OH}-\mathrm{HCl}$ & 15.6 & 48.3 & 12.2 & 8.6 & 9.9 & 65.9 \\
\hline 405 DUP & $\mathrm{NH}_{2} \mathrm{OH}-\mathrm{HCl}$ & 11.0 & 37.4 & 8.6 & 6.8 & 7.8 & 56.3 \\
\hline 405 & $0.01 \mathrm{HF} / 0.01 \mathrm{NaF}$ & 4.0 & 29.3 & 10.0 & 6.4 & 17.1 & 6.1 \\
\hline 405 DUP & $0.01 \mathrm{HF} / 0.01 \mathrm{NaF}$ & 3.6 & 23.2 & 6.5 & 4.9 & 14.3 & 7.9 \\
\hline 405 & $0.01 \mathrm{HF} / 0.01 \mathrm{NaF}$ & 4.5 & 6.0 & 10.4 & 5.8 & 5.3 & 2.1 \\
\hline 405 DUP & $0.01 \mathrm{HF} / 0.01 \mathrm{NaF}$ & 3.4 & 6.3 & 8.0 & 4.7 & 6.6 & 1.9 \\
\hline 405 & Hot Conc. $\mathrm{HNO}_{3}$ & 18.3 & 5.5 & 27.0 & 23.8 & 26.5 & 6.3 \\
\hline 405 DUP & Hot Conc. $\mathrm{HNO}_{3}$ & 43.3 & 8.2 & 89.1 & 74.2 & 78.3 & 40.5 \\
\hline 405 & Total & 43.9 & 92.0 & 60.1 & 444.8 & 58.9 & 93.9 \\
\hline 405 DUP & Total & 62.2 & 78.2 & 112.2 & 90.7 & 107.2 & 118.6 \\
\hline
\end{tabular}

The most notable result for the deionized water leaches is the fact that a relatively large amount of Mn (8-13\%) dissolved. Chemical equilibrium calculations conducted using the average concentration of the sample and duplicate for the sample 404 water leach solutions indicate that the water leachate solutions are near equilibrium (somewhat oversaturated) with respect to manganese oxalate $(\mathrm{SI}=0.43$, where $\mathrm{SI}=\log \left(\mathrm{Q} / \mathrm{K}_{\mathrm{sp}}, \mathrm{Q}\right.$ is the ion activity product for the solid and $\mathrm{K}_{\mathrm{sp}}$ is the solubility constant for the solid, see Appendix G for details). Manganese oxalate has been identified by XRD in samples 404 and 405 (see Section 0). Chemical equilibrium modeling also indicates that during the $0.9 \mathrm{M}$ oxalic acid treatment used in tank C-106, manganese (Mn(III) and Mn(IV)) oxyhydroxide phases would be reduced to $\mathrm{Mn}^{2+}$ (aq) ion, which would subsequently precipitate as manganese oxalate (see Appendix G).

Batrakov et al. (1998) have demonstrated that reductive dissolution of manganese dioxide to $\mathrm{Mn}^{2+}$ by oxalic acid is rapid under acidic conditions. They found that the maximum dissolution rate occurred at 
$\mathrm{pH}$ 1.6. The rate decreased to near zero above $\mathrm{pH}$ 4.5. Tank C-106 sludge also contained sidorenkite $\left(\mathrm{Na}_{3} \mathrm{MnPO}_{4} \mathrm{CO}_{3}\right)$ prior to oxalic acid treatment. It is expected that sidorenkite would readily dissolve under the acidic conditions of the oxalic acid treatment step. When sidorenkite dissolves in this process, the $\mathrm{Mn}^{2+}$ released to solution would also precipitate as manganese oxalate.

The hydroxylamine hydrochloride $\left(\mathrm{NH}_{2} \mathrm{OH}-\mathrm{HCl}\right)$ extraction step was designed to selectively dissolve manganese oxyhydroxides in the sludge; however, it is clear from the previous discussion that it is likely that, as a result of the $0.9 \mathrm{M}$ oxalic acid treatment step, a significant fraction of the Mn occurring as oxyhydroxides or sidorenkite in the tank sludge would have been transformed to Mn oxalate. The hydroxylamine hydrochloride extraction solution has a $\mathrm{pH}$ of approximately 3.6. This low $\mathrm{pH}$ would tend to protonate oxalate and result in dissolution of Mn-oxalate. As a result, the hydroxylamine hydrochloride extraction would also be effective at dissolving Mn oxalate in addition to manganese oxyhydroxides. The amount of Mn solids dissolved during this selective extraction step ranged from 55 to $77 \%$. Along with the $\mathrm{Mn}$, a large fraction of the ${ }^{238} \mathrm{U}$ was released (37 to 67\%). A fair amount of ${ }^{99} \mathrm{Tc}$ was also released (11 to $22 \%$ ). Lower quantities of $\mathrm{Cr}$ (9 to $12 \%), \mathrm{Fe}$ (7-10\%), and $\mathrm{Al}(8-10 \%)$ were released.

The $0.01 \mathrm{HF} / 0.01 \mathrm{NaF}$ extraction steps ( $\mathrm{pH} 3.2$ ) were intended to selectively release aluminum oxyhydroxide phases; however, during both extraction stages only between 19 and $23 \%$ of the aluminum was released. The $0.01 \mathrm{HF} / 0.01 \mathrm{NaF}$ extraction steps were more effective at releasing uranium. Between 30 and $41 \%$ of the uranium was released during the two $0.01 \mathrm{HF} / 0.01 \mathrm{NaF}$ extraction steps. Smaller amounts of ${ }^{99} \mathrm{Tc}(7-13 \%), \mathrm{Fe}(10-15 \%), \mathrm{Mn}(8-11 \%)$, and $\mathrm{Cr}$ (14-24\%) were also released.

Results of the final hot concentrated $\mathrm{HNO}_{3}$ extraction indicate that a significant amount of the $\mathrm{Fe}, \mathrm{Al}$, $\mathrm{Mn},{ }^{99} \mathrm{Tc},{ }^{238} \mathrm{U}$, and $\mathrm{Cr}$, remained immobile during the earlier mildly acidic extraction steps. The amounts of constituents released by the hot concentrated $\mathrm{HNO}_{3}$ extractions ranged from $24-74 \%$ for $\mathrm{Fe}, 27-78 \%$ for $\mathrm{Al}, 6-40 \%$ for $\mathrm{Mn}, 18-43 \%$ for ${ }^{99} \mathrm{Tc}, 6-10 \%$ for ${ }^{238} \mathrm{U}$, and $27-89 \%$ for $\mathrm{Cr}$. The sum of all the selective extractions indicate that significant fractions of ${ }^{99} \mathrm{Tc}, \mathrm{Cr}, \mathrm{Fe}$, and $\mathrm{Al}$, were frequently not dissolved even after the hot concentrated $\mathrm{HNO}_{3}$ extraction (up to $56 \%$ for ${ }^{99} \mathrm{Tc}$, up to $40 \%$ for $\mathrm{Cr}$, up to $65 \%$ for $\mathrm{Fe}$ and up to $41 \%$ for $\mathrm{Al})$.

Some general conclusions and observations can be made regarding these selective extraction results. $\mathrm{Cr}$ was found to exhibit a fairly strong correlation with Fe. This was also observed in the SEM/EDS analyses (Section 3.6). The ${ }^{238} \mathrm{U}$ appears to be most highly correlated with the release of $\mathrm{Mn}$. Some association of ${ }^{99} \mathrm{Tc}$ with the release of $\mathrm{Mn}$ may occur; however, the correlation is not as strong as the correlation with $\mathrm{Fe}$. In general, the selective extraction methods were less selective than anticipated for the $\mathrm{C}-106$ residual sludge samples. One possible reason for this result may be cation substitution among the various $\mathrm{Fe}, \mathrm{Al}, \mathrm{Mn}$ oxyhydroxides. The formation of the secondary $\mathrm{Mn}$ phases [Mn-oxalate and rhodochrosite $\left(\mathrm{MnCO}_{3}\right)$ ] during the sludge removal process may have also contributed to the problem by co-precipitating some of the trace constituents that were initially released by sludge dissolution reactions.

\subsubsection{Phase 2a}

Phase 2a selective extraction results for samples 404 and 405 are shown in Table 3.39. Data for ${ }^{99} \mathrm{Tc}$, ${ }^{238} \mathrm{U}, \mathrm{Cr}, \mathrm{Fe}, \mathrm{Al}$, and $\mathrm{Mn}$ are tabulated on the basis of mass of contaminant released per gram of dry 
Table 3.39. Phase 2a Selective Extraction Results for C-106 Sludge Samples 404 and 405 (in $\mu \mathrm{g} / \mathrm{g}$ )

\begin{tabular}{|c|c|c|c|c|c|c|c|}
\hline $\begin{array}{c}\text { Sample } \\
\text { Number }\end{array}$ & Extractant & $\begin{array}{l}{ }^{99} \mathrm{Tc} \\
(\mu \mathrm{g} / \mathrm{g})\end{array}$ & $\begin{array}{c}{ }^{238} \mathrm{U} \\
(\mu \mathrm{g} / \mathrm{g})\end{array}$ & $\begin{array}{c}\mathrm{Cr} \\
(\mu \mathrm{g} / \mathrm{g})\end{array}$ & $\begin{array}{c}\mathrm{Fe} \\
(\mu \mathrm{g} / \mathrm{g})\end{array}$ & $\begin{array}{c}\text { Al } \\
(\mu \mathrm{g} / \mathrm{g})\end{array}$ & $\begin{array}{c}\text { Mn } \\
(\mu \mathrm{g} / \mathrm{g})\end{array}$ \\
\hline 404 & $0.01 \mathrm{HF} / 0.01 \mathrm{KF}$ & $3.58 \mathrm{E}-02$ & $5.97 \mathrm{E}+01$ & $1.54 \mathrm{E}+01$ & $7.11 \mathrm{E}+02$ & $6.47 \mathrm{E}+03$ & $1.47 \mathrm{E}+04$ \\
\hline 404 DUP & $0.01 \mathrm{HF} / 0.01 \mathrm{KF}$ & $3.51 \mathrm{E}-02$ & $3.04 \mathrm{E}+01$ & $1.11 \mathrm{E}+01$ & $(4.34 \mathrm{E}+00)$ & $7.12 \mathrm{E}+03$ & $1.70 \mathrm{E}+04$ \\
\hline 404 & $0.01 \mathrm{HF} / 0.01 \mathrm{KF}$ & $3.90 \mathrm{E}-02$ & $5.50 \mathrm{E}+01$ & $1.98 \mathrm{E}+01$ & $4.18 \mathrm{E}+01$ & $7.66 \mathrm{E}+03$ & $1.63 \mathrm{E}+04$ \\
\hline 404 DUP & $0.01 \mathrm{HF} / 0.01 \mathrm{KF}$ & $3.74 \mathrm{E}-02$ & $5.45 \mathrm{E}+01$ & $1.92 \mathrm{E}+01$ & $1.29 \mathrm{E}+01$ & $7.33 \mathrm{E}+03$ & $1.62 \mathrm{E}+04$ \\
\hline Total & Fusion & $1.01 \mathrm{E}+00$ & $2.36 \mathrm{E}+02$ & $8.41 \mathrm{E}+02$ & $4.33 \mathrm{E}+04$ & $1.40 \mathrm{E}+05$ & $1.14 \mathrm{E}+05$ \\
\hline 405 & $0.01 \mathrm{HF} / 0.01 \mathrm{KF}$ & $3.33 \mathrm{E}-02$ & $3.99 \mathrm{E}+01$ & $8.36 \mathrm{E}+00$ & $1.30 \mathrm{E}+01$ & $6.58 \mathrm{E}+03$ & $1.43 \mathrm{E}+04$ \\
\hline 405 DUP & $0.01 \mathrm{HF} / 0.01 \mathrm{KF}$ & $3.39 \mathrm{E}-02$ & $4.50 \mathrm{E}+01$ & $1.04 \mathrm{E}+01$ & $1.28 \mathrm{E}+01$ & $7.35 \mathrm{E}+03$ & $1.53 \mathrm{E}+04$ \\
\hline 405 & $0.01 \mathrm{HF} / 0.01 \mathrm{KF}$ & $3.42 \mathrm{E}-02$ & $5.55 \mathrm{E}+01$ & $1.58 \mathrm{E}+01$ & $1.31 \mathrm{E}+01$ & $6.82 \mathrm{E}+03$ & $1.47 \mathrm{E}+04$ \\
\hline 405 DUP & $0.01 \mathrm{HF} / 0.01 \mathrm{KF}$ & $2.82 \mathrm{E}-02$ & $4.22 \mathrm{E}+01$ & $2.39 \mathrm{E}+01$ & $1.19 \mathrm{E}+03$ & $5.65 \mathrm{E}+03$ & $8.29 \mathrm{E}+03$ \\
\hline Total & Fusion & $1.31 \mathrm{E}+00$ & $2.58 \mathrm{E}+02$ & $8.07 \mathrm{E}+02$ & $4.33 \mathrm{E}+04$ & $1.23 \mathrm{E}+05$ & $1.22 \mathrm{E}+05$ \\
\hline
\end{tabular}

sludge. The total contaminant concentrations used in the calculations were those determined by fusion analysis. To facilitate easier evaluation of these data, the results are presented as a percentage of the total (fusion) concentrations in Table 3.40. Also included in this table are totals determined by summation of the results from both extraction steps. These selective extractions were designed to selectively remove $\mathrm{Al}$ oxyhydroxides. The results in Table 3.40 indicate that this extraction did not work as intended on sludge from tank C-106. Only about 10 to $11 \%$ of the total $\mathrm{Al}$ was removed with this method. Much higher

Table 3.40. Phase 2a Selective Extraction Results for Primary (404) and Duplicate (405) Sludge Samples from Tank C-106 (in percentages)

\begin{tabular}{|c|c|c|c|c|c|c|c|}
\hline Sample Number & Extractant & $\begin{array}{l}{ }^{99} \mathrm{Tc} \\
(\%)\end{array}$ & $\begin{array}{l}{ }^{238} \mathrm{U} \\
(\%)\end{array}$ & $\begin{array}{c}\mathrm{Cr} \\
(\%) \\
\end{array}$ & $\begin{array}{c}\mathrm{Fe} \\
(\%) \\
\end{array}$ & $\begin{array}{c}\text { Al } \\
(\mathbf{\%}) \\
\end{array}$ & $\begin{array}{l}\text { Mn } \\
\mathbf{( \% )} \\
\end{array}$ \\
\hline 404 & $0.01 \mathrm{HF} / 0.01 \mathrm{KF}$ & 3.5 & 25.3 & 1.8 & 1.6 & 4.6 & 12.9 \\
\hline 404 DUP & $0.01 \mathrm{HF} / 0.01 \mathrm{KF}$ & 3.5 & 12.9 & 1.3 & 0.0 & 5.1 & 14.9 \\
\hline 404 & $0.01 \mathrm{HF} / 0.01 \mathrm{KF}$ & 3.9 & 23.3 & 2.4 & 0.1 & 5.5 & 14.3 \\
\hline 404 DUP & $0.01 \mathrm{HF} / 0.01 \mathrm{KF}$ & 3.7 & 23.1 & 2.3 & 0.0 & 5.2 & 14.2 \\
\hline 404 & Total & 7.4 & 48.6 & 4.2 & 1.7 & 10.1 & 27.2 \\
\hline 404 DUP & Total & 7.2 & 36.0 & 3.6 & 0.0 & 10.3 & 29.1 \\
\hline 405 & $0.01 \mathrm{HF} / 0.01 \mathrm{NaF}$ & 2.5 & 15.5 & 1.0 & 0.0 & 5.3 & 11.7 \\
\hline 405 DUP & $0.01 \mathrm{HF} / 0.01 \mathrm{NaF}$ & 2.6 & 17.4 & 1.3 & 0.0 & 6.0 & 12.5 \\
\hline 405 & $0.01 \mathrm{HF} / 0.01 \mathrm{NaF}$ & 2.6 & 21.5 & 2.0 & 0.0 & 5.5 & 12.0 \\
\hline 405 DUP & $0.01 \mathrm{HF} / 0.01 \mathrm{NaF}$ & 2.2 & 16.4 & 3.0 & 2.7 & 4.6 & 6.8 \\
\hline 405 & Total & 5.2 & 37.0 & 3.0 & 0.1 & 10.9 & 23.8 \\
\hline 405 DUP & Total & 4.7 & 33.8 & 4.3 & 2.8 & 10.6 & 19.3 \\
\hline
\end{tabular}


percentages of $\mathrm{Mn}$ and ${ }^{238} \mathrm{U}$ were extracted with this method. The reason for the lack of intended specificity for this extraction is due primarily to an unexpected increase in $\mathrm{pH}$ that occurred during the extraction. The final $\mathrm{pH}$ values measured in the extracts ranged from 4.39 to 6.64 . The initial $\mathrm{pH}$ of the $0.01 \mathrm{HF} / 0.01 \mathrm{NaF}$ buffer was approximately 3.2. The reason for neutralization of the $\mathrm{pH}$ appears to be excessive proton consumption through dissolution of $\mathrm{Al}(\mathrm{OH})_{3}, \mathrm{Mn}$ oxyhydroxides, and carbonate phases. In other words, the buffer concentration used was not high enough to effectively dissolve all the $\mathrm{Al}(\mathrm{OH})_{3}$ and other proton-consuming phases in the sludge. The high fluoride concentrations may have been responsible for the relatively high concentrations of ${ }^{238} \mathrm{U}$ that were solubilized in these extractions. Fluoride is a fairly strong complexing agent for U(VI) (Grenthe et al. 1992).

\subsubsection{Phase 2b}

Phase 2 b selective extraction results for tank C-106 samples 404 and 405 are shown in Table 3.41. Data for ${ }^{238} \mathrm{U}, \mathrm{Cr}, \mathrm{Fe}, \mathrm{Al}$, and $\mathrm{Mn}$ are tabulated on the basis of mass of contaminant released per gram of dry sludge. The total contaminant concentrations used in the calculations were those determined by fusion analysis. To facilitate easier evaluation of these data, the results are presented as a percentage of the total (fusion) concentrations in Table 3.42. Also included in Table 3.42 are totals determined by summation of the results from both extraction steps. The $3 \mathrm{M} \mathrm{NaOH}$ extraction was also designed to selectively extract $\mathrm{Al}$ oxyhydroxides. This method was not particularly effective at solubilizing $\mathrm{Al}$ from C-106 residual sludge. Only about 11 to $13 \%$ of the total Al was extracted. The method does appear to be more selective than the previous methods used with respect to Fe and Mn. Very little Fe and Mn were extracted; however 7.4 to $11.3 \%$ of the ${ }^{238} \mathrm{U}$ was extracted. Interestingly, 3 to $4 \%$ of the $\mathrm{Cr}$ was mobilized during the second extraction step. (Note: the first extraction (E1) was conducted for 24 hours, whereas the second extraction (E2) was allowed to continue for 11 days.)

Table 3.41. Phase $2 b$ Selective Extraction Results for Sludge Samples 404 and 405 from Tank C-106

\begin{tabular}{|c|c|c|c|c|c|c|}
\hline $\begin{array}{c}\text { Sample } \\
\text { Number }\end{array}$ & Extractant & $\begin{array}{c}{ }^{238} \mathrm{U} \\
(\mu \mathrm{g} / \mathrm{g})\end{array}$ & $\begin{array}{c}\mathbf{C r} \\
(\mu \mathrm{g} / \mathrm{g})\end{array}$ & $\begin{array}{c}\mathbf{F e} \\
(\mu \mathrm{g} / \mathrm{g})\end{array}$ & $\begin{array}{c}\text { Al } \\
(\mu \mathrm{g} / \mathrm{g})\end{array}$ & $\underset{(\mu \mathrm{g} / \mathrm{g})}{\operatorname{Mn}}$ \\
\hline 404 & $3 \mathrm{M} \mathrm{NaOH}(\mathrm{E} 1)$ & $1.61 \mathrm{E}+01$ & $(1.07 \mathrm{E}+00)$ & $8.38 \mathrm{E}+00$ & $1.27 \mathrm{E}+04$ & $6.07 \mathrm{E}+00$ \\
\hline 404 DUP & $3 \mathrm{M} \mathrm{NaOH}(\mathrm{E} 1)$ & $1.94 \mathrm{E}+01$ & $(7.03 \mathrm{E}-01)$ & $8.98 \mathrm{E}+00$ & $1.41 \mathrm{E}+04$ & $5.93 \mathrm{E}+00$ \\
\hline 404 & $3 \mathrm{M} \mathrm{NaOH}(\mathrm{E} 2)$ & $4.29 \mathrm{E}+00$ & $2.51 \mathrm{E}+01$ & $2.04 \mathrm{E}+01$ & $2.49 \mathrm{E}+03$ & $(4.73 \mathrm{E}-01)$ \\
\hline 404 DUP & $3 \mathrm{M} \mathrm{NaOH}(\mathrm{E} 2)$ & $7.26 \mathrm{E}+00$ & $3.49 \mathrm{E}+01$ & $2.73 \mathrm{E}+01$ & $3.65 \mathrm{E}+03$ & $2.35 \mathrm{E}+00$ \\
\hline Total & Fusion & $2.36 \mathrm{E}+02$ & $8.41 \mathrm{E}+02$ & $4.33 \mathrm{E}+04$ & $1.40 \mathrm{E}+05$ & $1.14 \mathrm{E}+05$ \\
\hline 405 & $3 \mathrm{M} \mathrm{NaOH}(\mathrm{E} 1)$ & NA & $(1.19 \mathrm{E}+00)$ & $7.35 \mathrm{E}+00$ & $1.18 \mathrm{E}+04$ & $(1.71 \mathrm{E}-01)$ \\
\hline 405 DUP & $3 \mathrm{M} \mathrm{NaOH}(\mathrm{E} 1)$ & $1.46 \mathrm{E}+01$ & $(5.46 \mathrm{E}-01)$ & $1.41 \mathrm{E}+01$ & $1.26 \mathrm{E}+04$ & $8.26 \mathrm{E}+00$ \\
\hline 405 & $3 \mathrm{M} \mathrm{NaOH}(\mathrm{E} 2)$ & $4.23 \mathrm{E}+00$ & $3.03 \mathrm{E}+01$ & $2.11 \mathrm{E}+01$ & $2.52 \mathrm{E}+03$ & $(4.41 \mathrm{E}-01)$ \\
\hline 405 DUP & $3 \mathrm{M} \mathrm{NaOH}(\mathrm{E} 2)$ & $4.39 \mathrm{E}+00$ & $2.54 \mathrm{E}+01$ & $2.05 \mathrm{E}+01$ & $2.97 \mathrm{E}+03$ & (4.77E-01) \\
\hline Total & Fusion & $2.58 \mathrm{E}+02$ & $8.07 \mathrm{E}+02$ & $4.33 \mathrm{E}+04$ & $1.23 \mathrm{E}+05$ & $1.22 \mathrm{E}+05$ \\
\hline
\end{tabular}


Table 3.42. Phase $2 \mathrm{~b}$ Selective Extraction Results for Sludge Samples 404 and 405 from Tank C-106 (in percentages)

\begin{tabular}{|c|c|c|c|c|c|c|}
\hline Sample Number & Extractant & $\begin{array}{l}{ }^{238} \mathrm{U} \\
(\%)\end{array}$ & $\begin{array}{c}\mathrm{Cr} \\
(\%)\end{array}$ & $\begin{array}{c}\mathrm{Fe} \\
(\%)\end{array}$ & $\begin{array}{c}\mathrm{Al} \\
(\mathbf{\%})\end{array}$ & $\begin{array}{l}\text { Mn } \\
(\%)\end{array}$ \\
\hline 404 & $3 \mathrm{M} \mathrm{NaOH}(\mathrm{E} 1)$ & 6.8 & $(0.1)$ & 0.0 & 9.1 & 0.0 \\
\hline 404 DUP & $3 \mathrm{M} \mathrm{NaOH}(\mathrm{E} 1)$ & 8.2 & $(0.1)$ & 0.0 & 10.1 & 0.0 \\
\hline 404 & $3 \mathrm{M} \mathrm{NaOH}(\mathrm{E} 2)$ & 1.8 & 3.0 & 0.0 & 1.8 & 0.0 \\
\hline 404 DUP & $3 \mathrm{M} \mathrm{NaOH}(\mathrm{E} 2)$ & 3.1 & 4.2 & 0.1 & 2.6 & 0.0 \\
\hline 404 & Total & 8.6 & 3.1 & 0.1 & 10.8 & 0.0 \\
\hline 404 DUP & Total & 11.3 & 4.2 & 0.1 & 12.7 & 0.0 \\
\hline 405 & $3 \mathrm{M} \mathrm{NaOH}(\mathrm{E} 1)$ & NA & $(0.1)$ & 0.0 & 9.6 & 0.0 \\
\hline 405 DUP & $3 \mathrm{M} \mathrm{NaOH}(\mathrm{E} 1)$ & 5.7 & $(0.1)$ & 0.0 & 10.2 & 0.0 \\
\hline 405 & $3 \mathrm{M} \mathrm{NaOH}(\mathrm{E} 2)$ & 1.6 & 3.7 & 0.0 & 2.0 & 0.0 \\
\hline 405 DUP & $3 \mathrm{M} \mathrm{NaOH}(\mathrm{E} 2)$ & 1.7 & 3.1 & 0.0 & 2.4 & 0.0 \\
\hline 405 & Total & NA & 3.9 & 0.1 & 11.7 & 0.0 \\
\hline 405 DUP & Total & 7.4 & 3.2 & 0.1 & 12.6 & 0.0 \\
\hline
\end{tabular}




\subsection{Contaminant Release Model}

The primary objective of this project is to develop source release models for contaminants of concern present in residual waste upon closure of Hanford single-shell tanks. As shown in Figure 4.1, developing these models consists of laboratory testing to produce contaminant release data and a conceptual source release model. After development, the release model can be incorporated into a fate and transport model as part of a long-term risk/performance assessment for the closed tank. This section describes the conceptual release models developed for ${ }^{238} \mathrm{U},{ }^{99} \mathrm{Tc}, \mathrm{Cr}$, and ${ }^{129} \mathrm{I}$ from the laboratory data collected during testing of residual sludge from tank C-106.

Data collected and analyses conducted as part of this study indicated that contaminant release from tank C-106 residual sludge will be geochemically complex. Contaminant release from the sludge is expected to involve mineral dissolution and precipitation, reduction-oxidation reactions, solution phase complexation, and surface adsorption. Some of these reactions can be modeled as equilibrium reactions whereas others will be rate controlled. Although significant insight was gained in this study regarding the contaminant release mechanisms that will be important for tank C-106 sludge, it was not possible to adequately characterize the phase associations of the contaminants of concern in sufficient detail to produce a mechanistically rigorous geochemical release model. This could change with additional work; however, in the meantime, a release model based on empirical solubilities has been developed. The approach used is inherently conservative and will over-estimate contaminant release. Further characterization work could allow the development of a more mechanistically rigorous geochemical release model, if warranted.

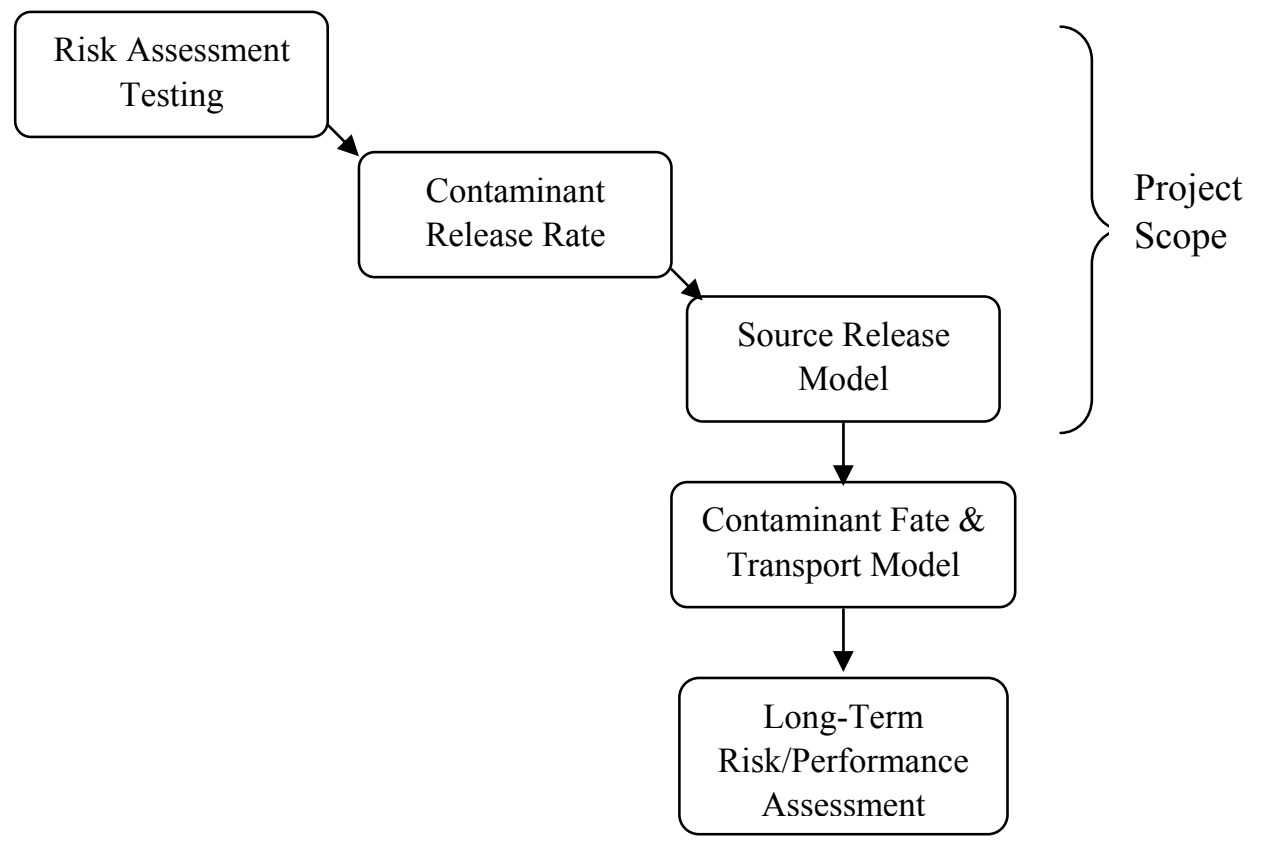

Figure 4.1. Source Release Model Development for Long-Term Risk/Performance Assessments 
The contaminant release model that has been developed for residual sludge in tank C-106 is based on empirical solubilities of the contaminants of concern. Empirical solubilities can be influenced by many geochemical conditions (e.g., $\mathrm{pH}, \mathrm{Eh}$, ionic strength, presence/concentration of complexing species) that are potentially dynamic throughout the history of the evolving sludge environment. In addition, available data indicate that portions of the contaminants of concern are incorporated into other slightly soluble phases. The dissolution of these relatively insoluble phases likely control the long-term release of the contaminants of concern in the residual sludge. For these reasons, the release models described in this section are expected to be conservative only to tank conditions that are currently expected at closure and will need to be modified as additional information becomes available regarding anticipated tank conditions (such as the addition of grout).

Before discussing the release models for the four individual contaminants of concern $\left({ }^{238} \mathrm{U},{ }^{99} \mathrm{Tc}, \mathrm{Cr}\right.$, and ${ }^{129} \mathrm{I}$ ), a conceptual model of the chemical transformations that have occurred in the tank as a result of chemical treatments used in the sludge removal process will be described. This has important implications with regard to how contaminants would be released from tank C-106 residual sludge.

\subsection{Conceptual Model of Chemical Transformations of Tank C-106 Sludge Resulting from the Retrieval Process}

A chemical treatment was used as part of the sludge retrieval process for tank C-106. In this process, a solution of $0.9 \mathrm{M}$ oxalic acid was added to the tank to dissolve and suspend sludge solids. Then, as much of the resulting suspension as possible was pumped out. This was repeated 4 to 6 times. After the final oxalic acid removal step, a single water rinse was conducted to remove as much of the acid solution as possible. The final step in the process was the addition of a $0.5 \mathrm{M} \mathrm{NaOH}$ solution to neutralize the remaining acidity in the sludge. As much of this neutralizing solution as possible was removed through pumping. It is estimated that approximately $75 \%$ of the sludge volume was removed throughout the entire retrieval process. Based on the results presented in this report (the average of the fusion results for samples 404 and 405) and previous analyses of untreated tank C-106 sludge (Bechtold et al. 2003, Table 7.1), it is apparent that the $0.9 \mathrm{M}$ oxalic acid treatment process has significantly altered the composition of the residual sludge. For example, Table 4.1 shows the weight percent of three major sludge components (Al, Fe, and $\mathrm{Mn}$ ) before and after treatment. Also included in this table are concentrations of three major contaminants of concern $\left({ }^{99} \mathrm{Tc},{ }^{238} \mathrm{U}\right.$, and $\left.\mathrm{Cr}\right)$. These results indicate that as a

Table 4.1. Three Major Sludge Components (Weight Percent) and Major Contaminants of Concern in Tank C-106 Sludge Before and After 1 M Oxalic Acid Treatment

\begin{tabular}{|c|c|c|c|c|c|c|}
\hline Sample & Al \% & $\mathrm{Fe} \%$ & Mn \% & $\begin{array}{c}{ }^{99} \mathrm{Tc} \\
(\mu \mathrm{g} / \mathrm{g})\end{array}$ & $\begin{array}{c}{ }^{238} \mathrm{U} \\
(\mu \mathrm{g} / \mathrm{g})\end{array}$ & $\begin{array}{c}\mathrm{Cr} \\
(\mu \mathrm{g} / \mathrm{g})\end{array}$ \\
\hline C-106 Before & 3.7 & 6.5 & 6.5 & 1.3 & $81^{\text {(a) }}$ & 2,100 \\
\hline 404/405 Avg & 13.2 & 4.4 & 11.8 & 1.2 & 247 & 897 \\
\hline
\end{tabular}


result of the oxalic acid treatment of tank C-106 sludge, $\mathrm{Al}, \mathrm{Mn}$, and ${ }^{238} \mathrm{U}$ have become enriched in the residual sludge. Fe and $\mathrm{Cr}$ have been depleted. Based on these results, and the fact that approximately $75 \%$ of the sludge was removed by the oxalic acid process, it would appear that very little, if any, of the Al was removed by this process.

A conceptual model has been developed to describe the chemical transformations that occurred in tank C-106 sludge during the sludge retrieval process and subsequent transformations that are expected to occur during future exposure to infiltrating natural recharge water. This model is illustrated schematically in Figure 4.2. Prior to the oxalic acid treatment, gibbsite $\left[\mathrm{Al}(\mathrm{OH})_{3}\right]$, hematite $\left[\mathrm{Fe}_{2} \mathrm{O}_{3}\right]$, dawsonite $\left[\mathrm{NaAlCO}_{3}(\mathrm{OH})_{2}\right]$, cancrinite $\left[\mathrm{Na}_{6} \mathrm{Ca}_{1.5} \mathrm{Al}_{6} \mathrm{Si}_{6} \mathrm{O}_{24}\left(\mathrm{CO}_{3}\right)_{1.6}\right]$, and sidorenkite $\left(\mathrm{NaMnPO}_{4} \mathrm{CO}_{3}\right]$ were identified by XRD and SEM/EDS (Bechtold et al. 2003). It is possible that poorly crystalline/isomorphically substituted Mn and Fe oxyhydroxides also occurred in the sludge, but could not be identified by XRD. During the oxalic acid treatment of the sludge, significant quantities of the $\mathrm{Fe}$ and $\mathrm{Mn}$ oxyhydroxides were likely to have dissolved through reductive dissolution (e.g., Batrakov et al. 1998; Suter et al 1991; Zhang et al. 1985). As the dissolved Mn(II) concentration increased, Mn(II) oxalate would precipitate as a result of its low solubility and the high oxalate concentration in the solution phase. Based on the $\mathrm{NH}_{2} \mathrm{OH}-\mathrm{HCl}$ selective extraction results, it would appear that some of the ${ }^{99} \mathrm{Tc},{ }^{238} \mathrm{U}$, and (possibly) Fe, released during the reductive dissolution of the Mn-oxyhydroxides, co-precipitated with the Mn-oxalate.

After the sludge was treated multiple times with the oxalic acid solution, the residual sludge was rinsed with water and then treated with a $\mathrm{NaOH}$ solution. The final $\mathrm{pH}$ of the solution in contact with the residual sludge was 12.9. After the base neutralization step, the sludge contained significant amounts of carbonate (see Table 3.12). The source of this carbonate is not clear; however, it is not likely that carbonates existing within the original sludge would have survived the oxalic acid treatments. After the base neutralization step, the sludge could have absorbed carbon dioxide from the head space in the tanks. Carbon dioxide could have also entered the tanks along with the $\mathrm{NaOH}$ solution used for the neutralization step. Carbon dioxide contamination of base can easily occur through exposure to air, both in solution or solid $\mathrm{NaOH}$ used to make up the $\mathrm{NaOH}$ solution. In any case, rhodochrosite $\left(\mathrm{MnCO}_{3}\right)$ was identified by XRD in the residual sludge (Figure 3.14). Rhodochrosite has a lower solubility constant than Mn-oxalate and would likely form at the expense of Mn-oxalate as the carbonate concentrations increased in the sludge.

Although both XRD and SEM/EDS data (Sections 3.6 and 3.7) support the existence of Mn-oxalate and/or rhodochrosite in the residual sludge, data in Table 3.12 suggest that a significant amount of the residual $\mathrm{Mn}$ in the sludge is still in the form of Mn oxyhydroxides. For example, if we assume that all the oxalate occurs in the residual sludge as Mn-oxalate and all the carbonate exists as rhodochrosite, $35 \%$ of the Mn would still remain unaccounted for. It is assumed that at least this much Mn occurs as Mn oxyhydroxides in the residual sludge. In addition to Mn-oxalate and rhodochrosite, gibbsite $\left[\mathrm{Al}(\mathrm{OH})_{3}\right]$, hematite $\left[\mathrm{Fe}_{2} \mathrm{O}_{3}\right]$, dawsonite $\left[\mathrm{NaAlCO}_{3}(\mathrm{OH})_{2}\right]$, boehmite $[\mathrm{AlO}(\mathrm{OH})]$, and whewellite [Ca-oxalate] were identified by XRD and SEM/EDS in the residual sludge. 


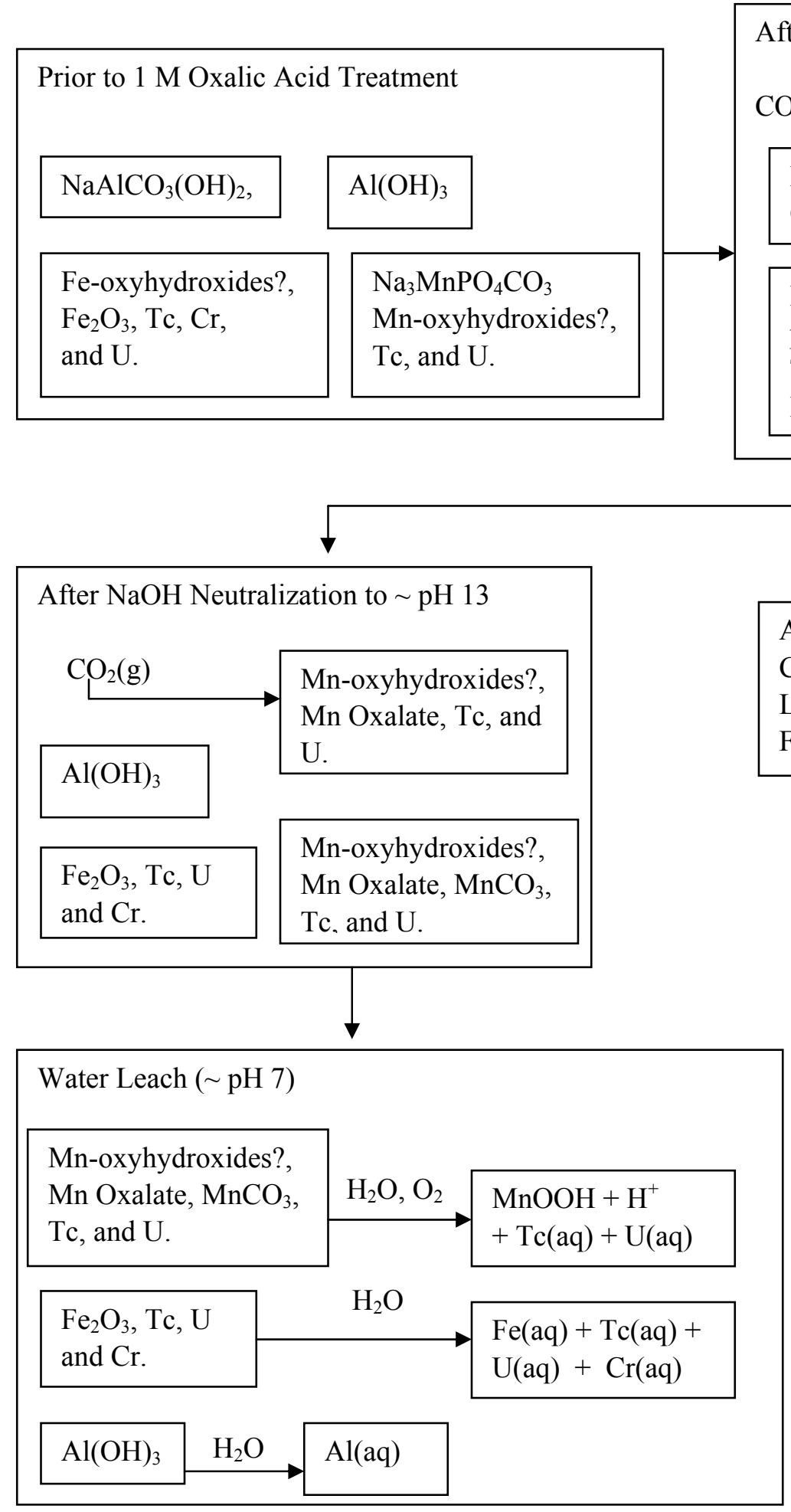

After $1 \mathrm{M}$ Oxalic Acid Treatment

$\mathrm{CO}_{2}$ outgases from sludge.

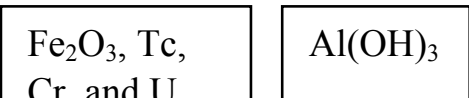
$\mathrm{Cr}$, and $\mathrm{U}$.

Fractional $\mathrm{Mn}$ reduced and precipitated as Mn Oxalate, Tc, and U co-precipitated, Mn-oxyhydroxides?

Approximately $75 \%$ of Sludge Components Removed with Liquids (Preferential Removal of $\mathrm{Fe})$.

Figure 4.2. Conceptual Model of Chemical Transformations for Tank C-106 Sludge Resulting from Tank Retrieval Process and Water Leaching Relevant to Contaminant Release Modeling 
As part of this study, sequential water leaches were conducted. Results of the water leach experiments indicated unexpectedly low $\mathrm{pH}$ values. For example, the tank $\mathrm{C}-106$ liquid had a $\mathrm{pH}$ of 12.9 (Table 3.13). Assuming a simple 100:1 water to sludge ratio for the sequential leach experiments and a $50 \%$ moisture content for the sludge (Table 3.1 ), a pH of approximately 10.6 could be expected for the sequential leach final solutions. The $\mathrm{pH}$ values measured during these experiments along with $\mathrm{Mn}$ and oxalate concentrations, and oxalate/Mn ratios are listed in Table 4.2. As can be seen, the measured $\mathrm{pH}$ values in the extracts of these experiments are in the range of 6.7 to 7.7. After analysis of all the analytical data for these extracts, the only plausible source of acidity that could explain the depressed $\mathrm{pH}$ values in these experiments is oxidation of $\mathrm{Mn}(\mathrm{II})$ to $\mathrm{Mn}(\mathrm{III})$ and subsequent precipitation as a $\mathrm{Mn}$ oxyhydroxide. For example:

$$
\mathrm{Mn}^{2+}+3 / 2 \mathrm{H}_{2} \mathrm{O}+1 / 2 \mathrm{O}_{2} \rightarrow \mathrm{MnOOH}+2 \mathrm{H}^{+}
$$

Table 4.2. Contact Times and Average $\mathrm{pH}$ Values, Oxalate and Mn Concentrations, and Oxalate/Mn Ratios, for the Periodic Replenishment Test on Tank C-106 Sludge Samples

\begin{tabular}{|c|c|c|c|c|c|}
\hline Contact Stage & $\begin{array}{c}\text { Contact } \\
\text { Duration } \\
\text { (days) }\end{array}$ & pH & $\underset{(\mathrm{mol} / \mathrm{L})}{\mathbf{O x}}$ & $\begin{array}{c}\mathrm{Mn} \\
(\mathrm{mol} / \mathrm{L})\end{array}$ & $\mathbf{O x} / \mathbf{M n}$ \\
\hline \multicolumn{6}{|c|}{ Sample 404} \\
\hline 1 & 1 & 7.03 & $2.33 \mathrm{E}-03$ & $1.40 \mathrm{E}-03$ & 1.66 \\
\hline 2 & 1 & 6.87 & $1.54 \mathrm{E}-03$ & $1.30 \mathrm{E}-03$ & 1.18 \\
\hline 3 & 4 & 6.93 & $1.11 \mathrm{E}-03$ & $9.22 \mathrm{E}-04$ & 1.21 \\
\hline 4 & 1 & 6.75 & $6.13 \mathrm{E}-04$ & $4.74 \mathrm{E}-04$ & 1.30 \\
\hline 5 & 1 & 6.73 & $3.61 \mathrm{E}-04$ & $2.66 \mathrm{E}-04$ & 1.37 \\
\hline $6 a$ & 43 & 7.43 & 3.49E-04 & $2.15 \mathrm{E}-04$ & 1.63 \\
\hline $6 \mathrm{~b}$ & 82 & 7.62 & $4.06 \mathrm{E}-04$ & $2.69 \mathrm{E}-04$ & 1.51 \\
\hline \multicolumn{6}{|c|}{ Sample 405} \\
\hline 1 & 1 & 6.71 & $2.14 \mathrm{E}-03$ & $1.36 \mathrm{E}-03$ & 1.57 \\
\hline 2 & 1 & 6.70 & $1.26 \mathrm{E}-03$ & $1.02 \mathrm{E}-03$ & 1.23 \\
\hline 3 & 4 & 6.79 & $8.14 \mathrm{E}-04$ & $6.51 \mathrm{E}-04$ & 1.25 \\
\hline 4 & 1 & 6.68 & $3.35 \mathrm{E}-04$ & $2.37 \mathrm{E}-04$ & 1.41 \\
\hline 5 & 1 & 6.70 & $1.42 \mathrm{E}-04$ & $1.21 \mathrm{E}-04$ & 1.18 \\
\hline $6 a$ & 43 & 7.50 & $2.09 \mathrm{E}-04$ & $8.59 \mathrm{E}-05$ & 2.43 \\
\hline $6 \mathrm{~b}$ & 82 & 7.70 & $1.83 \mathrm{E}-04$ & $1.12 \mathrm{E}-04$ & 1.63 \\
\hline
\end{tabular}

This reaction could take place during the contact period subsequent to dissolution of Mn-oxalate from the residual sludge. After dissolution, the relatively low oxalate concentrations and high $\mathrm{pH}$ combine to create conditions that are favorable for the oxidation and subsequent precipitation of the $\mathrm{Mn}$ as $\mathrm{MnOOH}$ (or other Mn oxyhydroxide phase) and concomitant release of protons. Note that the $\mathrm{Ox} / \mathrm{Mn}$ ratio is significantly greater than 1, suggesting that as Mn-oxalate dissolves and $\mathrm{Mn}$ is oxidized and precipitated, free oxalate in solution becomes elevated relative to $\mathrm{Mn}$. The implication of this process for the release model is that, as Mn-oxalate dissolves and releases $\mathrm{Mn}$ (II), the Mn oxidizes and precipitates. This would tend to increase the overall dissolution rate of Mn-oxalate and precipitation of $\mathrm{Mn}$ oxyhydroxides. It is 
likely, that this process will be limited by the amount of dissolved oxygen in the infiltrating water. For example, at $8 \mathrm{mg} / \mathrm{L}$ the dissolved oxygen concentration is $2.5 \times 10^{-4} \mathrm{~mol} / \mathrm{L}$. From equation 4.1 , it is apparent that this could potentially oxidize $5 \times 10^{-4} \mathrm{~mol} / \mathrm{L}$ of $\mathrm{Mn}(\mathrm{II})$.

Chemical equilibrium modeling indicates (Appendix G), that the first sequential contact (sample 404) is significantly oversaturated with respect to rhodochrosite $(\mathrm{SI}=1.4)$. Two explanations are possible to account for this apparent oversaturation with respect to rhodochrosite. The first is that Mn oxyhydroxide colloids formed during the oxidation of $\mathrm{Mn}$ (II) and that these colloids escaped filtration and were analyzed as part of the total Mn. An alternative explanation is that Mn(III) was stabilized to some degree as oxalate complexes. The stability constants for $\mathrm{Mn}$ (III) oxalate complexes appear to be quite large. For example, an available published value for $\log \mathrm{K}_{1}$ for the formation of the first oxalate complex:

$$
\mathrm{Mn}^{3+}+\mathrm{C}_{2} \mathrm{O}_{4}{ }^{2-} \leftrightarrow \mathrm{MnC}_{2} \mathrm{O}_{4}^{+}
$$

is 10.0, determined in $2 \mathrm{~mol} / \mathrm{L}$ perchloric acid by Taube (1948). The Mn(III) oxalate stability constants of Taube (1948) were determined by an indirect method and are apparently the only published values available. As a result, these constants must be considered as relatively uncertain. The formation of strong $\mathrm{Mn}$ (III) oxalate complexes may act to promote the oxidation of $\mathrm{Mn}$ (II) to $\mathrm{Mn}$ (III) in solution at high $\mathrm{pH} / \mathrm{pe}$ values.

Although it is conceivable that an equilibrium geochemical model could be constructed to estimate the release of contaminants contained within the Mn phases (Mn-oxalate, rhodochrosite, and Mn oxyhydroxides) of the sludge, several factors arise that put significant constraints on the reliability of this approach. The most important factor is that the distribution of the contaminants of concern among the various phases is still very uncertain at this point. In addition, it is not clear what would happen to the contaminants of concern released during the dissolution of Mn oxalate. For example, available evidence suggests that, as Mn oxalate dissolves, Mn oxyhydroxides or rhodochrosite could precipitate. Contaminants released along with the Mn oxalate could be free to migrate or they might adsorb to the newly formed Mn oxyhydroxides or co-precipitate with rhodochrosite.

As a result of these uncertainties, a release model has been developed from empirical solubility data for the contaminants of concern determined from concentration measurements obtained from the water leach tests. This model is described in Section 4.2.

\subsection{Technetium, Uranium, Iodine, and Chromium Release Models}

Because of the highly complex chemical nature of tank C-106 residual sludge, clear and quantitative phase associations of the contaminants of concern with the phases known to exist in the residual sludge are difficult to specify. Although the various characterization methods employed in this study have revealed a number of important observations and have provided valuable data for constructing a scientifically defensible release model, many questions remain. Because a thorough understanding of all the important phase associations for the contaminants of concern cannot be developed at this time, an empirically based release model has been developed. Although less satisfying from a mechanistic point of view, this provides a release model that can be used now and is conservative in nature. Later work 
may provide a better understanding of the phase associations with the contaminants of concern and the release mechanisms from these phases. In this case, a less conservative, but more scientifically defensible release model could be developed.

To estimate the maximum release rates for ${ }^{99} \mathrm{Tc},{ }^{238} \mathrm{U}$, and $\mathrm{Cr}$, their concentrations measured in the single-contact and sequential water extractions were evaluated. The highest concentrations in water correlate with the highest release rates from the sludge. In general, the single-contact water extracts contained the highest concentrations of the contaminants of concern. It was then determined which time period (1 day, 2 week, or 1 month) for the single-contact water-leach tests had the highest concentrations. Once the appropriate time period was selected, the maximum solution concentrations of the 404 and 405 samples were averaged to determine a release concentration. In the case of ${ }^{129} \mathrm{I}$, data are only available for the 1-day single-contact water extract. These values are provided in Table 4.3 (column 3). The total concentrations were determined from either the fusion or EPA acid digestions of the sludge, whichever had the highest concentration. These totals were then averaged for the primary and duplicate samples for both samples 404 and 405. The results are provided in Table 4.3, column 2.

Table 4.3. Summary of Contaminant Release Model Data for C-106

\begin{tabular}{||c|c|c|c||}
\hline \hline Contaminant & Sludge Concentration & Release Concentration & Release Control \\
\hline \hline${ }^{99} \mathbf{T c}$ & $\begin{array}{c}1.2 \mu \mathrm{g}{ }^{99} \mathrm{Tc} / \mathrm{g} \text {-sludge } \\
\left(20,000 \mu \mathrm{pCi}{ }^{99} \mathrm{Tc} / \mathrm{g} \text {-sludge }\right)\end{array}$ & $\begin{array}{c}0.21 \mu \mathrm{g} / \mathrm{L} \\
(3,600 \mathrm{pCi} / \mathrm{L})\end{array}$ & solubility \\
\hline${ }^{238} \mathbf{U}$ & $310 \mu \mathrm{g}{ }^{238} \mathrm{U} / \mathrm{g}$-sludge & $46 \mu \mathrm{g} / \mathrm{L}$ & solubility \\
\hline${ }^{129} \mathbf{I}$ & $\begin{array}{c}0.62 \mu \mathrm{g}{ }^{129} \mathrm{I} / \mathrm{g} \text {-sludge } \\
\left(110 \mathrm{pCi}{ }^{129} \mathrm{I} / \mathrm{g} \text {-sludge }\right)\end{array}$ & $\begin{array}{c}0.059 \mu \mathrm{g} / \mathrm{L} \\
(10 \mathrm{pCi} / \mathrm{L})\end{array}$ & solubility \\
\hline $\mathbf{C r}$ & $897 \mu \mathrm{g} \mathrm{Cr} / \mathrm{g}$-sludge & $19 \mu \mathrm{g} / \mathrm{L}$ & solubility \\
\hline
\end{tabular}

These suggested empirical constant concentration (solubility) limits are based on contact with water that resembles natural infiltration (i.e., rainwater). Water modified by grout placed in the tank or any other amendments could significantly alter the solubilities (hence release concentrations) of these contaminants. 


\subsection{Conclusions}

This report provides the results of laboratory tests on residual sludge and liquid samples from Hanford tank C-106 and describes the development of source term release models for the primary contaminants of concern. The major conclusions from this work are discussed in this section.

- The geochemistry of the residual sludge in tank C-106 is complex because of the variety of minerals present and the impact of chemical treatment of the waste with oxalic acid and $\mathrm{NaOH}$ during retrieval. The initial (pre-retrieved) waste consisted of the minerals gibbsite $\left[\mathrm{Al}(\mathrm{OH})_{3}\right]$, hematite $\left[\mathrm{Fe}_{2} \mathrm{O}_{3}\right]$, dawsonite $\left[\mathrm{NaAlCO}_{3}(\mathrm{OH})_{2}\right]$, cancrinite $\left[\mathrm{Na}_{6} \mathrm{Ca}_{1.5} \mathrm{Al}_{6} \mathrm{Si}_{6} \mathrm{O}_{24}\left(\mathrm{CO}_{3}\right)_{1.6}\right]$, and sidorenkite $\left(\mathrm{NaMnPO}_{4} \mathrm{CO}_{3}\right]$ (Bechtold et al. 2003). It is possible that poorly crystalline $\mathrm{Mn}$ and $\mathrm{Fe}$ oxyhydroxides also occurred in the sludge. ${ }^{99} \mathrm{Tc},{ }^{238} \mathrm{U},{ }^{129} \mathrm{I}$, and $\mathrm{Cr}$ occurred as trace constituents in these minerals. The oxalic acid treatment method for retrieval dissolved and removed approximately $75 \%$ of the sludge, but preferentially removed $\mathrm{Fe}$ (and $\mathrm{Cr}$ ) from the sludge while concentrating $\mathrm{Al}$ and $\mathrm{Mn}$ in the residual solid. It appears that $\mathrm{Al}$ was concentrated in the sludge because gibbsite was not very soluble in the oxalic acid solution. Mn was concentrated in the residual sludge because most of the $\mathrm{Mn}$ that entered the solution from the dissolution of Mn minerals reprecipitated as Mn oxalate and, perhaps, Mn oxyhydroxide minerals. Some of the primary contaminants remained in the undissolved sludge probably associated with the Fe minerals. Some of the contaminants released to solution became associated, and immobilized, with the precipitating Mn minerals. Furthermore, the precipitating Mn minerals appear to have coated many of the original sludge particles, thereby affecting their future interaction with water that might come into contact with the residual sludge. The result is a complicated geochemical system in which the contaminants are present at trace levels associated with a variety of minerals with solubilities that are dependent on each other.

- Mechanistic release models of contaminants from the sludge could not be developed from the available data because of the complexity of the geochemical system. However, empirical release models based on measured total sludge concentrations and maximum solution concentrations in water-leaching tests can be used for performance/risk assessment modeling. These empirical release models are expected to provide conservatively high release rates into recharge water percolating through the residual waste. The values for the chosen empirical constant concentration release models are:

\begin{tabular}{|c|c|c|c|}
\hline \hline Contaminant & Sludge Concentration & Release Concentration & Release Control \\
\hline \hline${ }^{99} \mathbf{T c}$ & $\begin{array}{c}1.2 \mu \mathrm{g}{ }^{99} \mathrm{Tc} / \mathrm{g} \text {-sludge } \\
\left(20,000 \mu \mathrm{pCi}{ }^{99} \mathrm{Tc} / \mathrm{g} \text {-sludge }\right)\end{array}$ & $\begin{array}{c}0.21 \mu \mathrm{g} / \mathrm{L} \\
(3,600 \mathrm{pCi} / \mathrm{L})\end{array}$ & solubility \\
\hline${ }^{238} \mathbf{U}$ & $310 \mu \mathrm{g}{ }^{238} \mathrm{U} / \mathrm{g}$-sludge & $46 \mu \mathrm{g} / \mathrm{L}$ & solubility \\
\hline${ }^{129} \mathbf{I}$ & $\begin{array}{c}0.62 \mu \mathrm{g}{ }^{129} \mathrm{I} / \mathrm{g} \text {-sludge } \\
\left(110 \mathrm{pCi}{ }^{129} \mathrm{I} / \mathrm{g} \text {-sludge }\right)\end{array}$ & $\begin{array}{c}0.059 \mu \mathrm{g} / \mathrm{L} \\
(10 \mathrm{pCi} / \mathrm{L})\end{array}$ & solubility \\
\hline $\mathbf{C r}$ & $897 \mu \mathrm{g} \mathrm{Cr} / \mathrm{g}$-sludge & $19 \mu \mathrm{g} / \mathrm{L}$ & solubility \\
\hline
\end{tabular}


- The concentrations of major metals and anions in the residual sludge were:

$\begin{array}{ll}\circ & \mathrm{Al}-131,483 \mu \mathrm{g} / \mathrm{g} \\ \circ & \mathrm{Mn}-117,767 \mu \mathrm{g} / \mathrm{g} \\ \circ & \mathrm{Fe}-43,777 \mu \mathrm{g} / \mathrm{g} \\ \circ & \mathrm{Ca}-38,221 \mu \mathrm{g} / \mathrm{g} \\ \circ & \mathrm{Na}-60,400 \mu \mathrm{g} / \mathrm{g} \\ \circ & \text { oxalate }-63,900 \mu \mathrm{g} / \mathrm{g} \\ \circ & \text { carbonate }-39,500 \mu \mathrm{g} / \mathrm{g}\end{array}$

- The sum of the TRU constituents $\left({ }^{239} \mathrm{Pu},{ }^{237} \mathrm{~Np}\right.$, and $\left.{ }^{241} \mathrm{Am}\right)$ measured in the tank $\mathrm{C}-106$ residual sludge samples by the fusion method was $8,057 \mathrm{nCi} / \mathrm{g}$; by the EPA acid digestion method it was $8,670 \mathrm{nCi} / \mathrm{g}$. Based on the DOE definition of TRU waste $(>100 \mathrm{nCi} / \mathrm{g})$, this would classify the residual sludge itself in tank C-106 as TRU waste. However, the final waste form in the tank will be a mixture of residual sludge and a tank filling grout, which will lower the concentrations of contaminants an amount dependent on the mixing ratio of the sludge and grout.

- The oxalic acid treatment of the sludge in the tank during retrieval removed much of the waterleachable constituents from the solid. Less than $3 \%$ of the ${ }^{129} \mathrm{I}$ and less than $5 \%$ of the ${ }^{99} \mathrm{Tc}$ and ${ }^{238} \mathrm{U}$ in the sludge were water leachable during a variety of single and multiple-contact water leach tests. Leachable amounts of $\mathrm{Al}$ and $\mathrm{Fe}$ were barely detectable, while about $6 \%$ of the $\mathrm{Ca}, 40 \%$ of the $\mathrm{Mn}$ and $50 \%$ of the $\mathrm{Na}$ were water leachable. The primary water leachable anions were oxalate and carbonate.

- Several of the metals and contaminants could not be leached from the sludge even under the condition of aggressive leaching with concentrated nitric acid. It was found that $56 \%$ of the ${ }^{99} \mathrm{Tc}$ could not be leached under these conditions and $40 \%$ of the $\mathrm{Cr}$ was recalcitrant. Fe and $\mathrm{Al}$ were also relatively immobile with $65 \%$ and $41 \%$ not leachable, respectively.

- The XRD results indicate that the unleached sludge samples contain detectable quantities of the following crystalline phases:

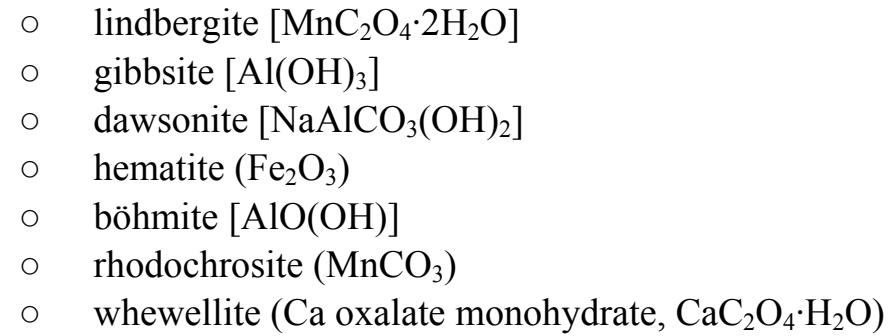

Except for lindbergite, these minerals were also present in the 1-month and 82-day water-leached and HF-extracted sludge samples. A possible Ag-Hg phase was identified by a single reflection, and may be present in the sludge. Amorphous phases of $\mathrm{Al}$ and $\mathrm{Fe}$ that are not detectable by XRD are also likely to be present in the sludge. 
SEM/EDS analysis of the sludge showed a wide variety of morphologies, sizes, surface textures and compositions. Particles range in size from submicrometer to a few hundred micrometers. An Al-rich, multi-faceted, blocky shaped particle is common to all of the samples and may represent gibbsite.

Another common occurrence is particles containing $\mathrm{Mn}, \mathrm{Al}, \mathrm{Fe}, \mathrm{Na}, \mathrm{P}, \mathrm{Si}, \mathrm{Ca}, \mathrm{O}$, and possibly $\mathrm{C}$ and $\mathrm{H}$ that have distinctive shapes (oblong and rounded, orthorhombic, rhombohedral, and pyramidal rhombic). Coatings are present on many of these particles, and may account for some of the elements present in the analysis. The surfaces of the Fe oxide particles contain pits and solution cavities. $\mathrm{Cr}$ and other contaminants are likely associated with this phase. These contaminants will only be released as the host Fe oxide solid(s) dissolves. Other solids analyzed by EDS have the following compositions:

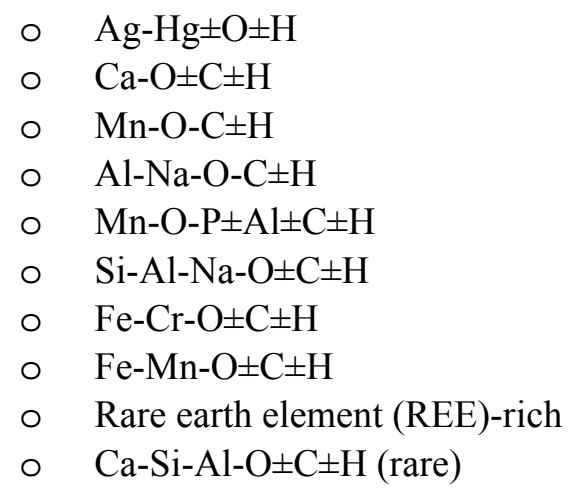




\subsection{References}

10 CFR 830.120. “Quality Assurance.” Code of Federal Regulations, U.S. Department of Energy.

Agnew SF, P Baca, R Corbin, T Duran, and K Jurgensen. 1995. Waste Status and Transaction Record

Summary for the Northeast Quadrant. WHC-SD-WM-TI-615 Rev. 2, Los Alamos National Laboratory, Los Alamos, New Mexico.

Anderson JD. 1990. A History of the 200 Area Tank Farms. WHC-MR-1032, Westinghouse Hanford Company, Richland, Washington.

ASTM. 1998. D2216-98 Standard Test Method for Laboratory Determination of Water (Moisture) Content of Soil and Rock by Mass. American Society for Testing and Materials, West Conshohocken, Pennsylvania.

ASTM. 1999. D3987-85 Standard Test Method for Shake Extraction of Solid Waste with Water. American Society for Testing and Materials, West Conshohocken, Pennsylvania.

Bartrakov VV, IG Gorichev, SV Prigzhaya, AD Izotov, and AV Kuznetsov. 1998. "Kinetics of Manganese Dioxide Dissolution in Sulfuric Acid Solutions Containing Oxalic Acid." Inorganic Materials 34(2):149-156.

Bechtold DB, GA Cooke, DL Herting, JC Person, RS Viswanath, and RW Warrant. 2003. Laboratory Testing of Oxalic Acid Dissolution of Tank 241-C-106 Sludge. RPP-17158, Rev. 0, Fluor Hanford, Inc. Richland, Washington.

Bickmore BR, KL Nagy, JS Young, and JW Drexler. 2001. "Nitrate-Cancrinite Precipitation on Quartz Sand in Simulated Hanford Tank Solutions." Environmental Science and Technology 35(22):4481-4486.

Bredt PR, BK McNamara, BW Arey, AP Poloski, EC Buck, RG Swoboda, and ED Jenson. 2003. Rheological and Physical Properties of AP-101 LAW Pretreated Waste and Melter Feed. PNWD-3279 (WTP-RPT-064, Rev. 0), prepared by Battelle - Pacific Northwest Division for Bechtel National, Inc., Richland, Washington.

Brown CF, KN Geiszler, MJ Lindberg, and WJ Deutsch. 2004. “Analysis of ${ }^{129}$ I by Inductively Coupled Plasma Mass Spectrometry: A Comparison of Analytical Methods and Techniques." Presented at the 227th National Meeting of the American Chemical Society, Anaheim, CA. PNNL-SA-41108, Pacific Northwest National Laboratory, Richland, Washington.

Buck EC and BK McNamara. 2004. "Precipitation of nitrate-cancrinite in Hanford tank sludge." Environ. Sci. Technolol. 38:4432-4438.

Cantrell KJ, RJ Serne, and GV Last. 2003. Hanford Contaminant Distribution Coefficient Database and Users Guide. PNNL-13895, Rev. 1, Pacific Northwest National Laboratory, Richland, Washington. 
Clesceri LS, AE Greenberg, and AD Eaton. 1998. Standard Methods for the Examination of Water and Wastewater, 20th Edition. American Public Health Association, American Water Works Association, and Water Environment Federation, Washington, D.C.

Conner JM. 1996. Tank Characterization Report for Single-Shell Tank 241-C-204.

WHC-SD-WM-ER-479 Rev. 0, Westinghouse Hanford Company, Richland, Washington.

De Lorenzo DS, AT DiCenso, DB Hiller, KW Johnson, JH Rutherford, DJ Smith, and BC Simpson. 1994. Tank Characterization Reference Guide. WHC-SD-WM-TI-648, Rev. 0, prepared by Los Alamos Technical Associates for Westinghouse Hanford Company, Richland, Washington.

Deutsch WJ, KM Krupka, MJ Lindberg, KJ Cantrell, CF Brown, and HT Schaef. 2004. Hanford Tanks 241-C-203 and 241-C-204: Residual Waste Contaminant Release Model and Supporting Data. PNNL14903, Pacific Northwest National Laboratory, Richland, Washington.

DOE. 1995. Environmental Assessment - Tank 241-C-106 Past-Practice Sluicing Waste Retrieval, Hanford Site, Richland, Washington. DOE/EA-0933, U.S. Department of Energy, Washington, D.C.

DOE. 1998. Hanford Analytical Services Quality Assurance Requirements Documents. HASQARD, DOE/RL-96-68, Volumes 1, 2, 3, and 4, U.S. Department of Energy, Richland, Washington.

DOE Order 414.1A. Management Assessment and Independent Assessment Guide. U.S. Department of Energy, Washington, D.C. Available online at http://www.directives.doe.gov/pdfs/doe/doetext/neword/414/g4141-1a.html.

DOE Order 435.1. 2001. Radioactive Waste Management. U.S. Department of Energy, Washington, D.C. Available on the internet at http://directives.doe.gov/pdfs/doe/doetext/neword/435/g4351-1.pdf

EPA. 1994a. "Method 6020. Inductively Coupled Plasma-Mass Spectrometry." In Test Methods for Evaluating Solid Wastes: Physical/Chemical Methods, EPA SW-846, Third Ed., Vol. I, Section A, Chapter 3 (Inorganic Analytes), pp. 6020-1 to 6020-18, U.S. Environmental Protection Agency, Office of Solid Waste and Emergency Response, Washington, D.C. Available at:

http://www.epa.gov/epaoswer/hazwaste/test/pdfs/6020.pdf

EPA. 1994b. "Method 9056. Determination of Inorganic Anions by Ion Chromatography." In Test Methods for Evaluating Solid Wastes: Physical/Chemical Methods, EPA SW-846, Third Ed., Vol. I, Section C, Chapter 5 (Miscellaneous Test Methods), pp. 9056-1 to 9056-16, U.S. Environmental Protection Agency, Office of Solid Waste and Emergency Response, Washington, D.C. Available at: http://www.epa.gov/epaoswer/hazwaste/test/pdfs/9056.pdf

EPA. 1995. "Method 9040B. pH Electrometric Measurement." In Test Methods for Evaluating Solid Wastes: Physical/Chemical Methods, EPA SW-846, Third Ed., Vol. I, Section C, Chapter 8 (Methods for Determining Characteristics), pp. 9040B-1 to 9040B-5, U.S. Environmental Protection Agency, Office of Solid Waste and Emergency Response, Washington, D.C. Available at: http://www.epa.gov/epaoswer/hazwaste/test/pdfs/9040b.pdf 
EPA. 1996a. "Method 3050B. Acid Digestion of Sediments, Sludges, and Soils." Revision 2 (December 1996)." In Test Methods for Evaluating Solid Wastes: Physical/Chemical Methods, EPA SW-846, Third Ed., Vol. I, Section A, Chapter 3 (Inorganic Analytes), pp. 3050B-1-3050B-12, U.S. Environmental Protection Agency, Office of Solid Waste and Emergency Response, Washington, D.C. Available at: http://www.epa.gov/epaoswer/hazwaste/test/pdfs/3050b.pdf.

EPA. 1996b. "Method 6010B. Inductively Coupled Plasma-Atomic Emission Spectrometry." In Test Methods for Evaluating Solid Wastes: Physical/Chemical Methods, EPA SW-846, Third Ed., Vol. I, Section A, Chapter 3 (Inorganic Analytes), pp. 6010B-1 to 6010B-25, U.S. Environmental Protection Agency, Office of Solid Waste and Emergency Response, Washington, D.C. Available at: http://www.epa.gov/epaoswer/hazwaste/test/pdfs/6010b.pdf

Fiskum SK, CJ Barinaga, JP Bramson, KJ Carson, and JR DesChane. 2000. Inorganic and Radiochemical Analysis of 241-C-104 Tank Waste. PNNL-13364 (WTP-RPT-007-Rev. 0) (formerly BNFL-RPT-043), Pacific Northwest National Laboratory, Richland, Washington.

Grenthe I, J Fuger, RJM Konings, RJ Lemire, AB Muller, C Ngyen-Trung Cregu, and H Wanner. 1992. Chemical Thermodynamics of Uranium. North-Holland, New York.

Hanlon BM. 1996. Waste Tank Summary Report for Month Ending May 31, 1996. WHC-EP-0182-98, Westinghouse Hanford Company, Richland, Washington.

Krupka KM, WJ Deutsch, MJ Lindberg, KJ Cantrell, NJ Hess, HT Schaef, and BW Arey. 2004. Hanford Tanks 241-AY-102 and 241-BX-101: Sludge Composition and Contaminant Release Data. PNNL-14614, Pacific Northwest National Laboratory, Richland, Washington.

Lindberg MJ and WJ Deutsch. 2003. Tank 241-AY-102 Data Report. PNNL-14344, Pacific Northwest National Laboratory, Richland, Washington.

Lumetta GJ, MJ Wagner, FV Hoopes, and RT Steele. 1996. Washing and Caustic Leaching of Hanford Tank C-106 Sludge. PNNL-11381, Pacific Northwest National Laboratory, Richland, Washington.

McKinney SG. 2004. Analytical Results for Tank 241-C-106 Solid Clam Shell Samples Supporting Closure Action. RPP-20264, Rev. 0, CH2M HILL Hanford Group, Inc., Richland, Washington.

Schreiber RD, JG Douglas, RD Cromar, TL Welsh, BC Simpson, LF Fergestrom, and RM Ozanich. 1996. Tank Characterization Report for Single-Shell Tank 241-C-106. WHC-SD-WM-ER-616, Rev 0, Westinghouse Hanford Company, Richland, Washington.

Simpson BC. 1994. Tank 241-T-111 Characterization Report. WHC-EP-0806, Westinghouse Hanford Company, Richland, Washington.

Smith GL, DJ Bates, RW Goles, LR Greenwood, RC Lettau, GF Piepel, MJ Schweiger, HD Smith, MW Urie, and JJ Wagner. 2001. Vitrification and Product Testing of C-104 and AZ-102 Pretreated Sludge Mixed with Flowsheet Quantities of Secondary Wastes. PNNL-13452, Pacific Northwest National Laboratory, Richland, Washington. 
Strachan DM, HT Schaef, MJ Schweiger, KL Simmons, LJ Woodcock, and MK Krouse. 2003. "A Versatile and Inexpensive XRD Specimen Holder for Highly Radioactive or Hazardous Specimens." Powder Diffraction 18(1):23-28.

Suter D, S Banwart, and W Stumm. 1991. "Dissolution of Hydrous Iron(III) Oxides by Reductive Mechanism." Langmuir 7:809-813.

Taube H. 1948. "Catalysis of Manganic Ion of the Reaction of Bromine and Oxalic Acid. Stability of Manganic Ion Complexes.” J. Am. Chem. Soc. 70:3928-3935.

Um W, RJ Serne, and KM Krupka. 2004. "Linearity and Reversibility of Iodide Adsorption on Sediments from Hanford, Washington, Under Water-Saturated Conditions.” Water Research 38:20092016.

Zhang Y, N Kallay, and E Matijevic. 1985. "Interactions of Metal Hydrous Oxides with Chelating Agents. 7. Hematite-Oxalic Acid and-Citric Acid Systems.” Langmuir 1:201-206. 


\section{Appendix A}

\section{X-Ray Diffraction Patterns for Unleached, Water-Leached, and HF-Extracted Sludge Samples from Tank C-106}




\section{Appendix A}

\section{X-Ray Diffraction Patterns for Unleached, Water-Leached, and HF-Extracted Sludge Samples from Tank C-106}

This appendix presents the as-measured and background-subtracted X-ray powder diffraction (XRD) patterns for the unleached (as-received), 1-month and 82-day water-leached, and hydrofluoric (HF)extracted sludge samples 404 and 405 from tank 241-C-106 (C-106). The instrumentation and procedures used for measuring, subtracting background, and interpreting the XRD patterns for these materials are described in the main report. The vertical axis in each of the following patterns represents the intensity in counts per second (cps) of the XRD peaks. The horizontal axis is in terms of degrees $2 \theta$ based on $\mathrm{Cu}_{\mathrm{K} a}$ radiation ( $\lambda=1.5406 \AA$ ), and is related to $d$ spacing according to the Bragg law (Cullity 1956). ${ }^{\text {(a) }}$ For comparison to the background signal in the as-measured XRD patterns included in this appendix, Figure A.1 shows the XRD pattern for collodion film measured in the absence of any sludge material and reported by Krupka et al. (2004). ${ }^{(b)}$

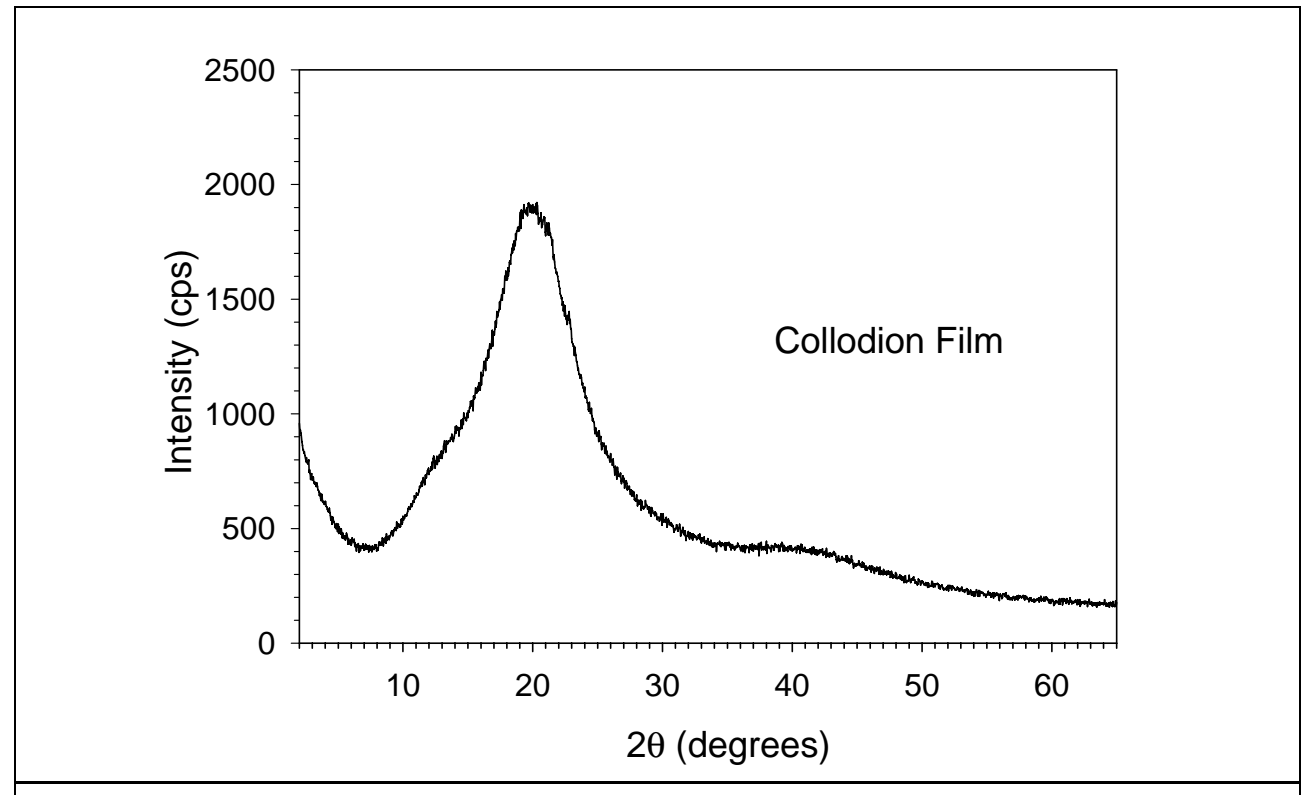

Figure A.1. XRD Pattern for Collodion-Solution Film (from Krupka et al. 2004)

(a) Cullity BD. 1967. Elements of X-Ray Diffraction. Addison-Wesley Publishing Company, Inc., Reading, Massachusetts.

(b) Krupka KM, WJ Deutsch, MJ Lindberg, KJ Cantrell, NJ Hess, HT Schaef, and BW Arey. 2004. Hanford Tanks 241-AY-102 and 241-BX-101: Sludge Composition and Contaminant Release Data. PNNL-14614, Pacific Northwest National Laboratory, Richland, Washington. 


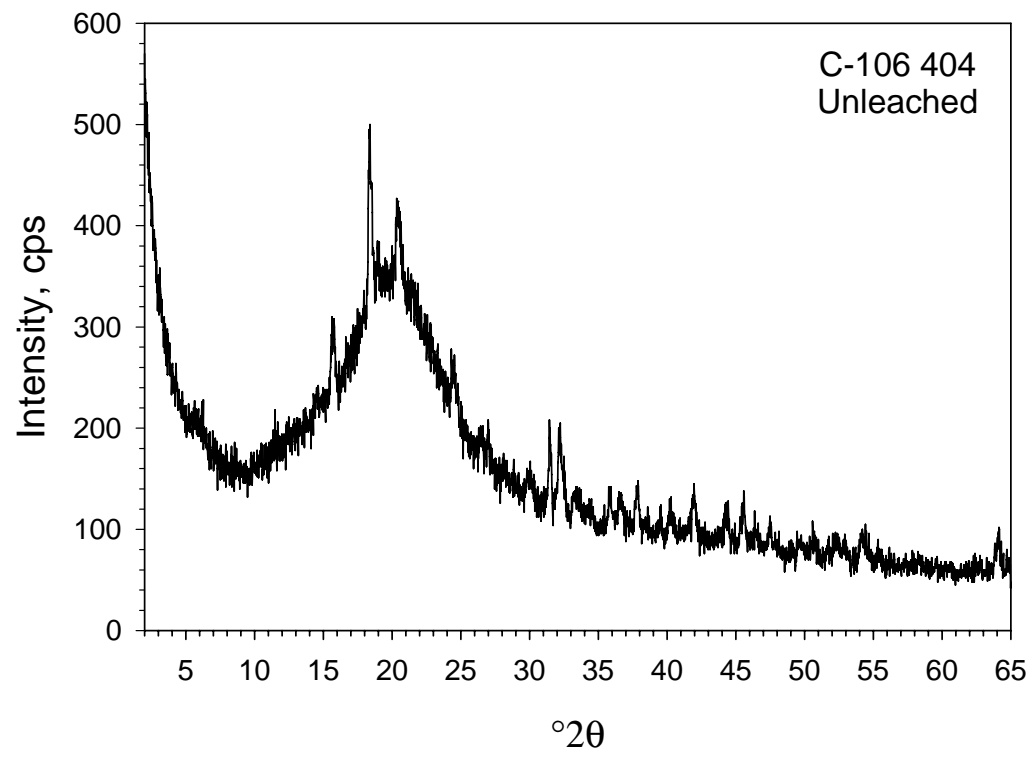

Figure A.2. $\quad$ As-Measured XRD Pattern (without background subtraction) for Unleached (as-received) Sludge Sample 404

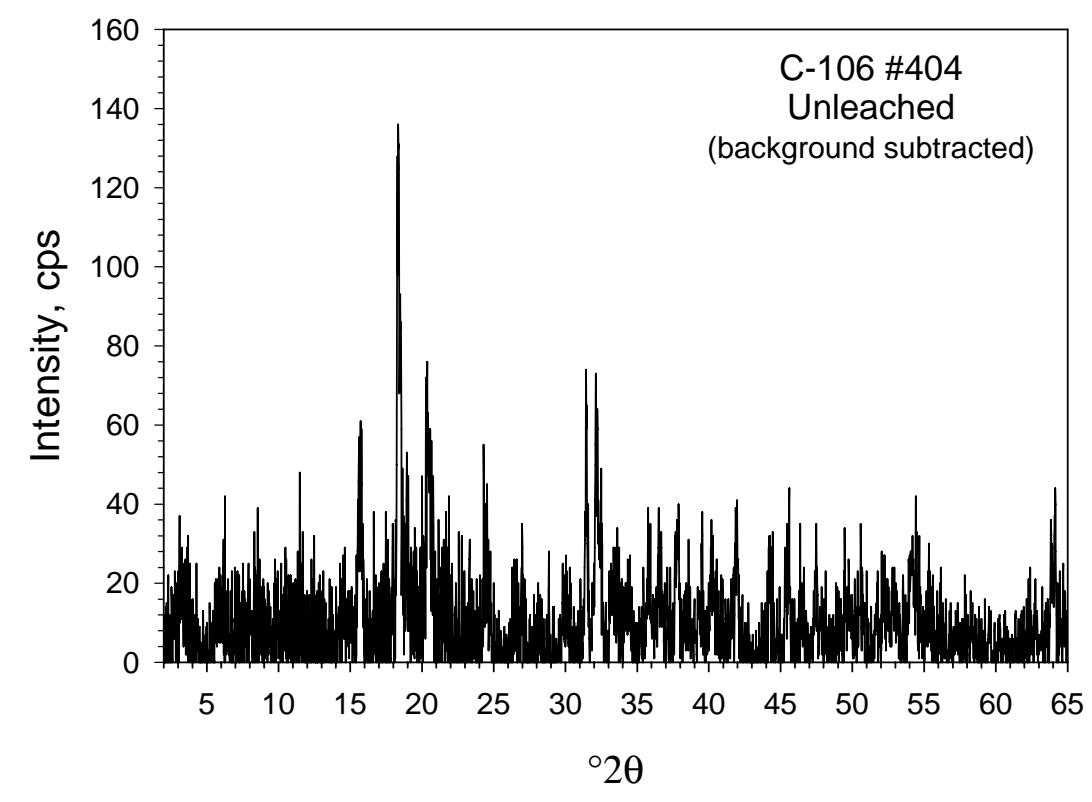

Figure A.3. Background-Subtracted XRD Pattern for Unleached (as-received) Sludge Sample 404 


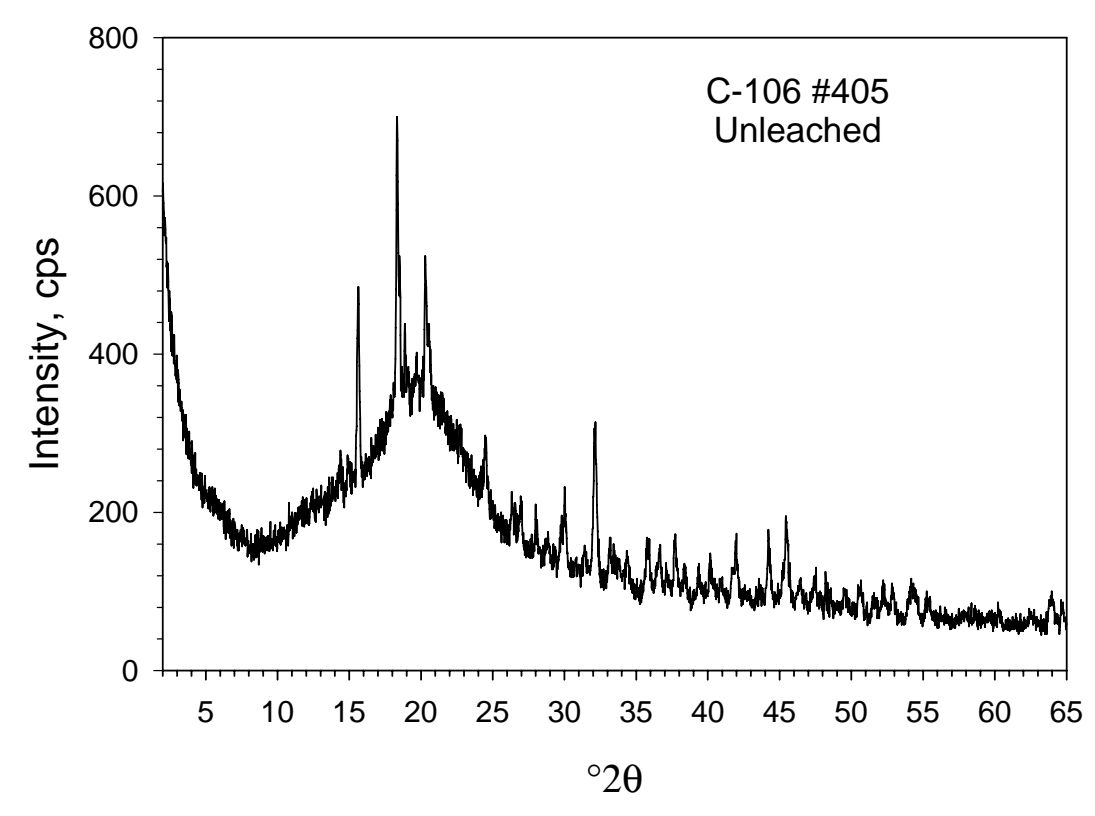

Figure A.4. As-Measured XRD Pattern (without background subtraction) for Unleached (as-received) Sludge Sample 405

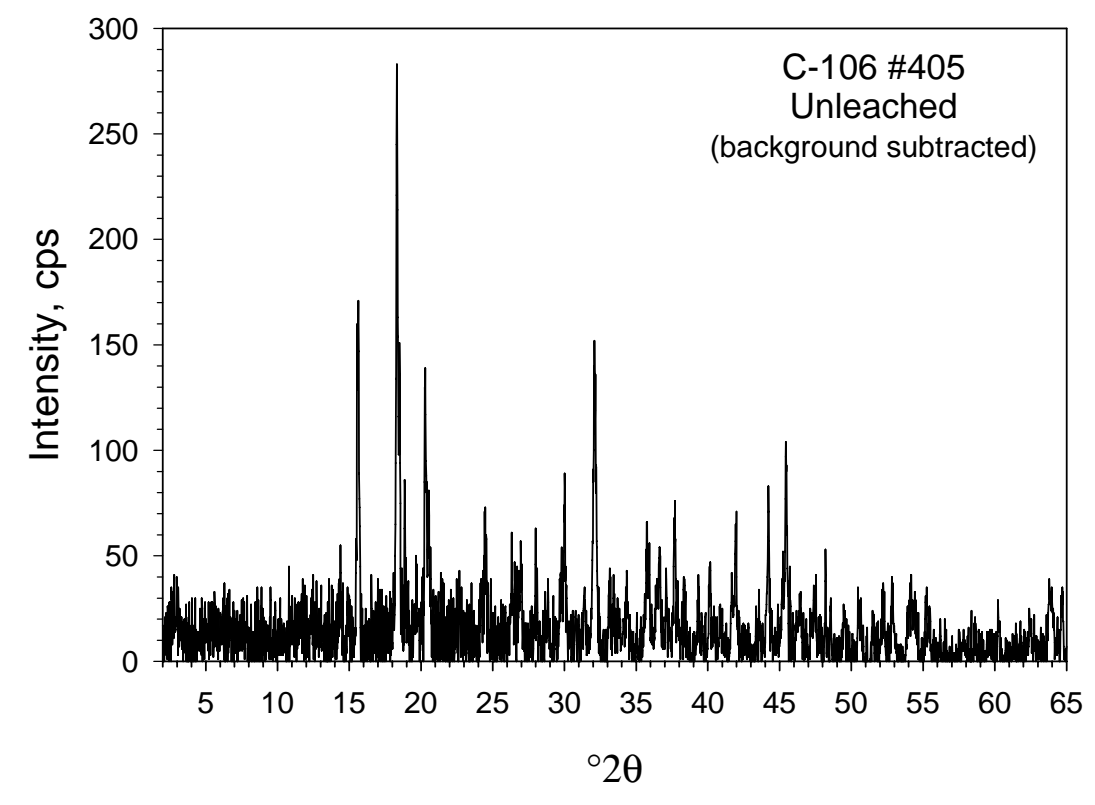

Figure A.5. Background-Subtracted XRD Pattern for Unleached (as-received) Sludge Sample 405 


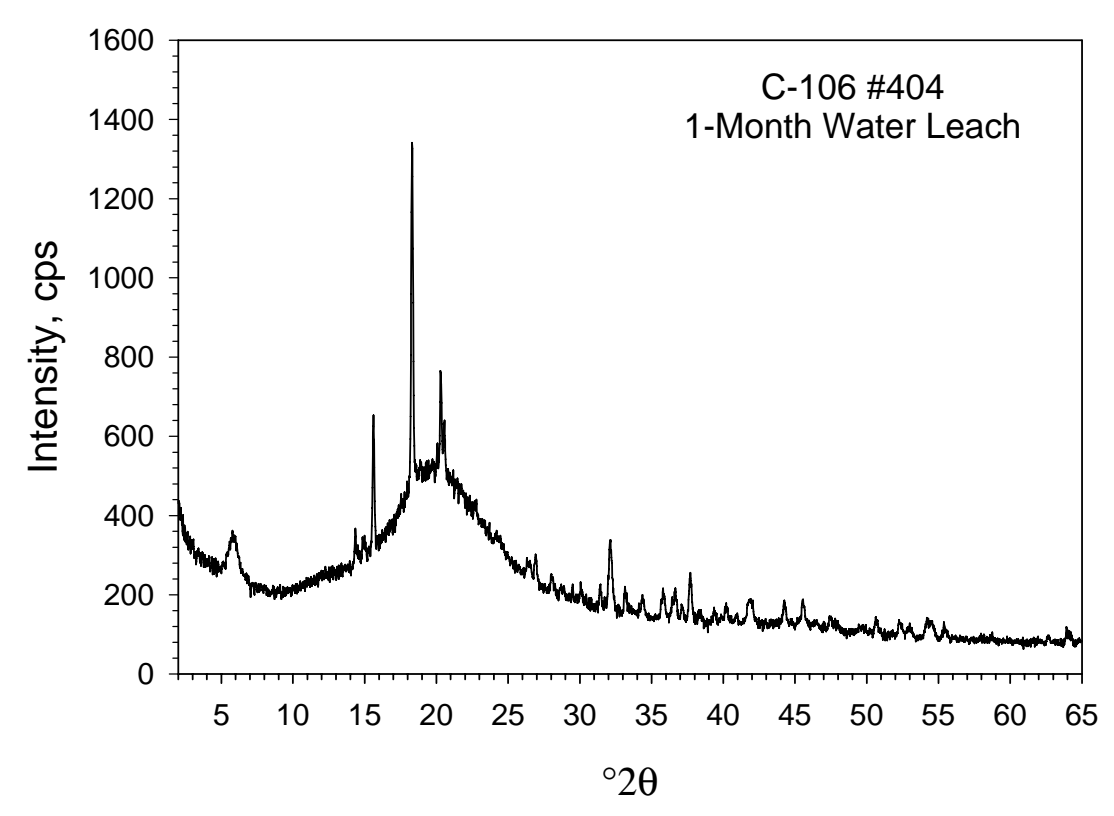

Figure A.6. As-Measured XRD Pattern (without background subtraction) for 1-Month Water-Leached Sludge Sample 404

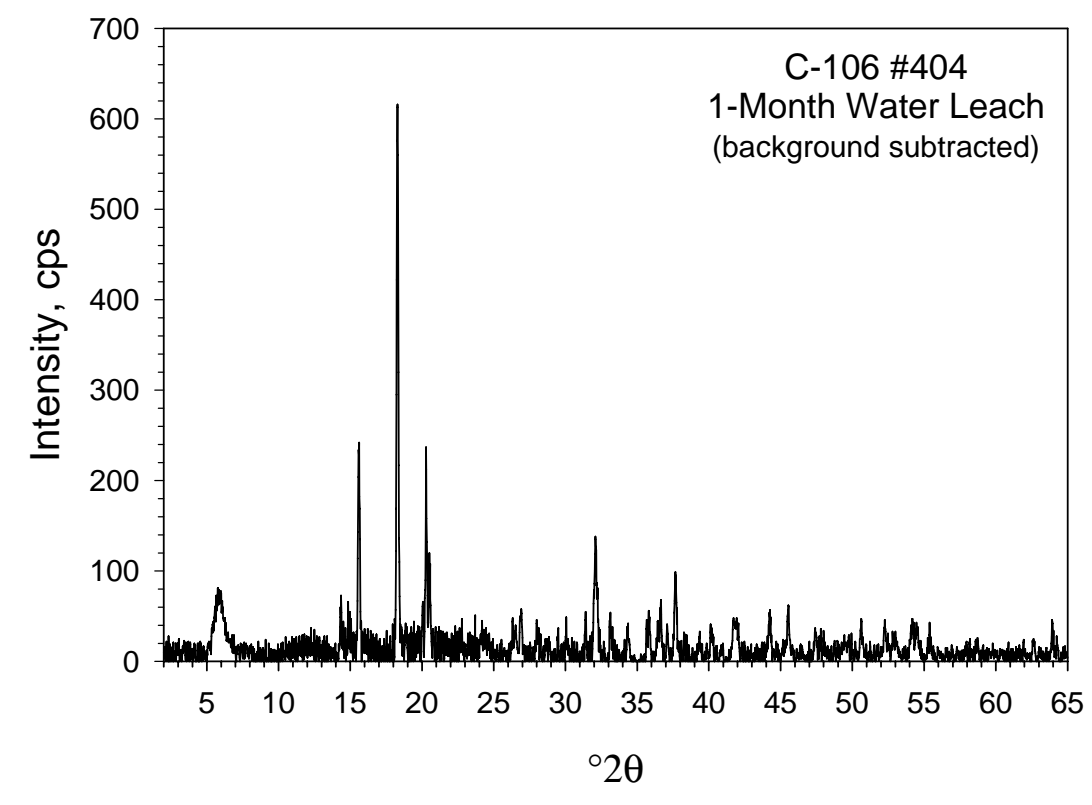

Figure A.7. Background-Subtracted XRD Pattern for 1-Month WaterLeached Sludge Sample 404 


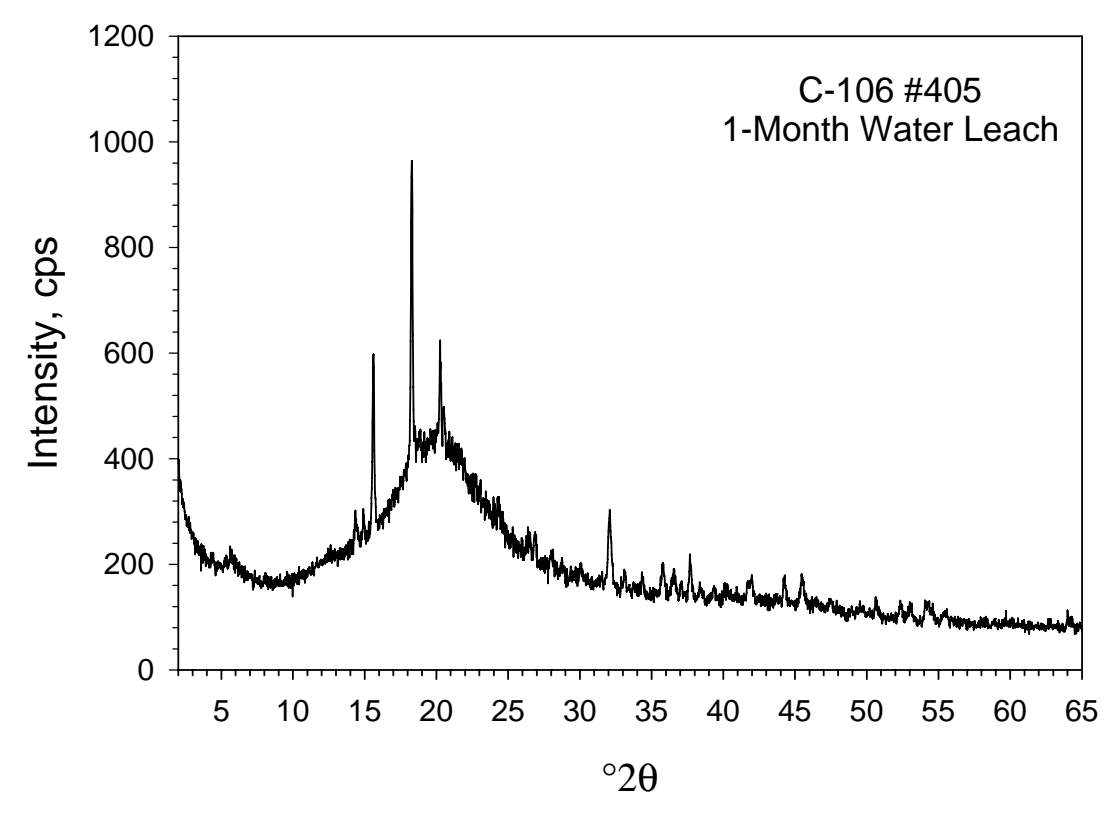

Figure A.8. As-Measured XRD Pattern (without background subtraction) for 1-Month Water-Leached Sludge Sample 405

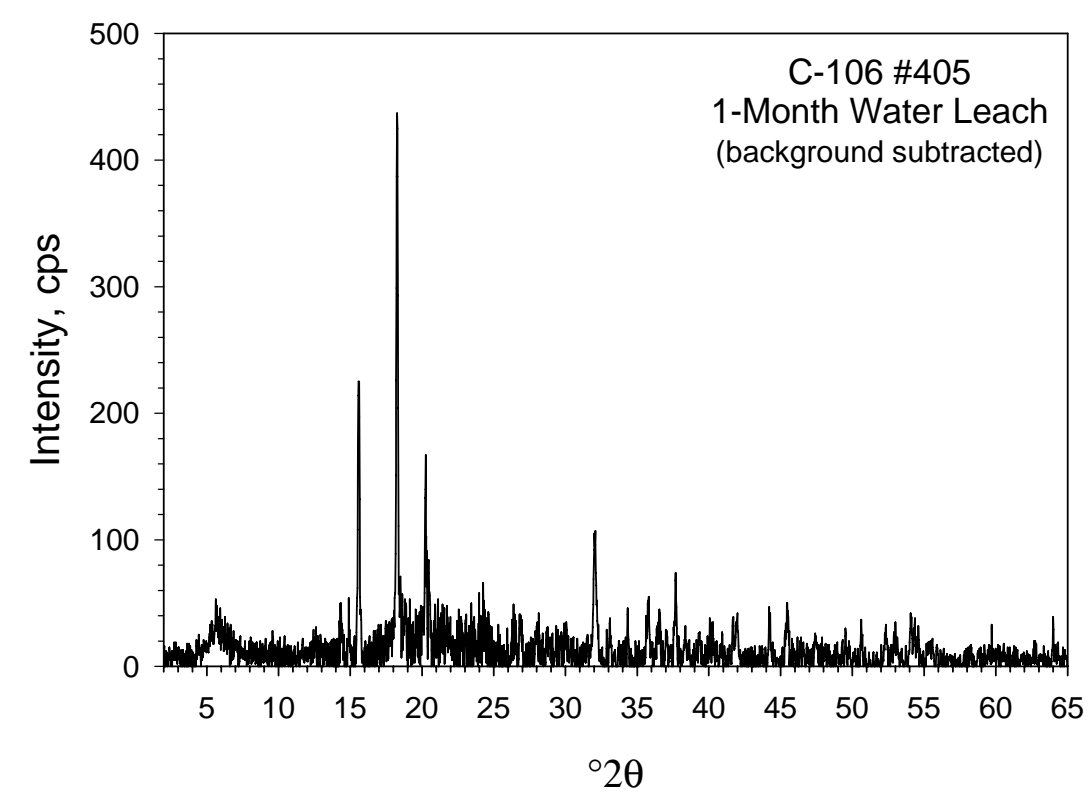

Figure A.9. Background-Subtracted XRD Pattern for 1-Month WaterLeached Sludge Sample 405 


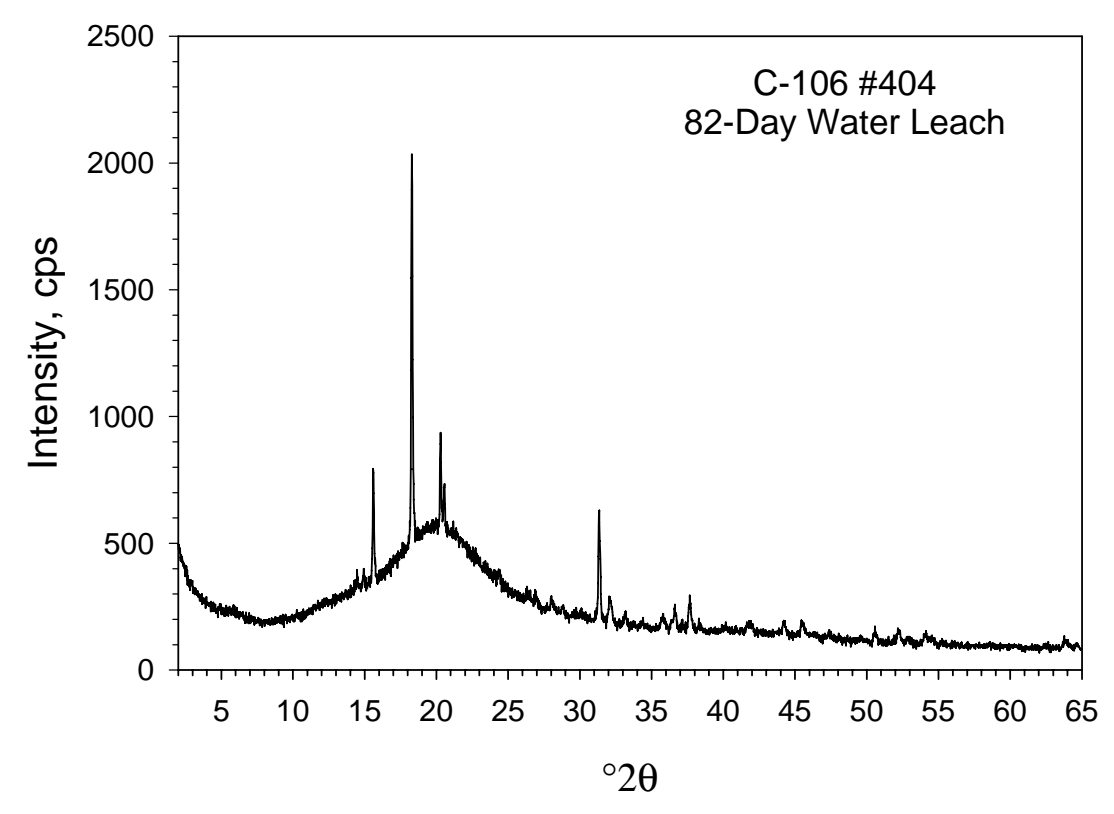

Figure A.10. As-Measured XRD Pattern (without background subtraction) for 82-day Water-Leached Sludge Sample 404

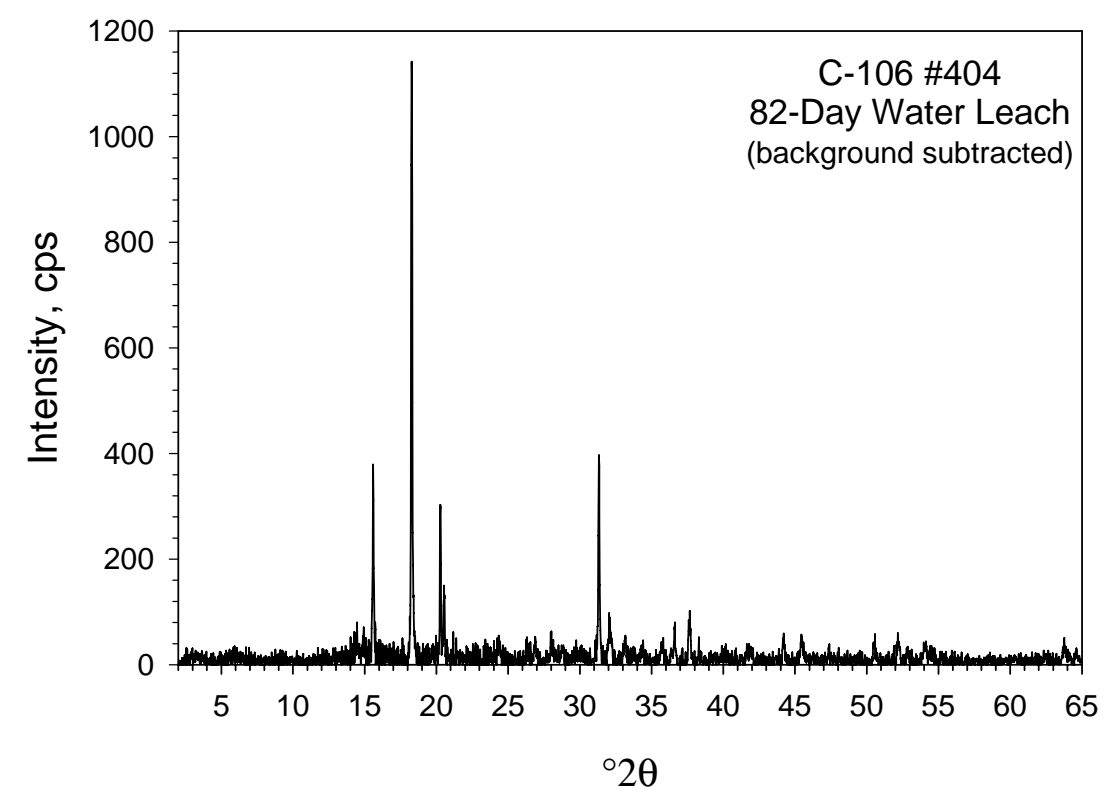

Figure A.11. Background-Subtracted XRD Pattern for 82-day WaterLeached Sludge Sample 404 


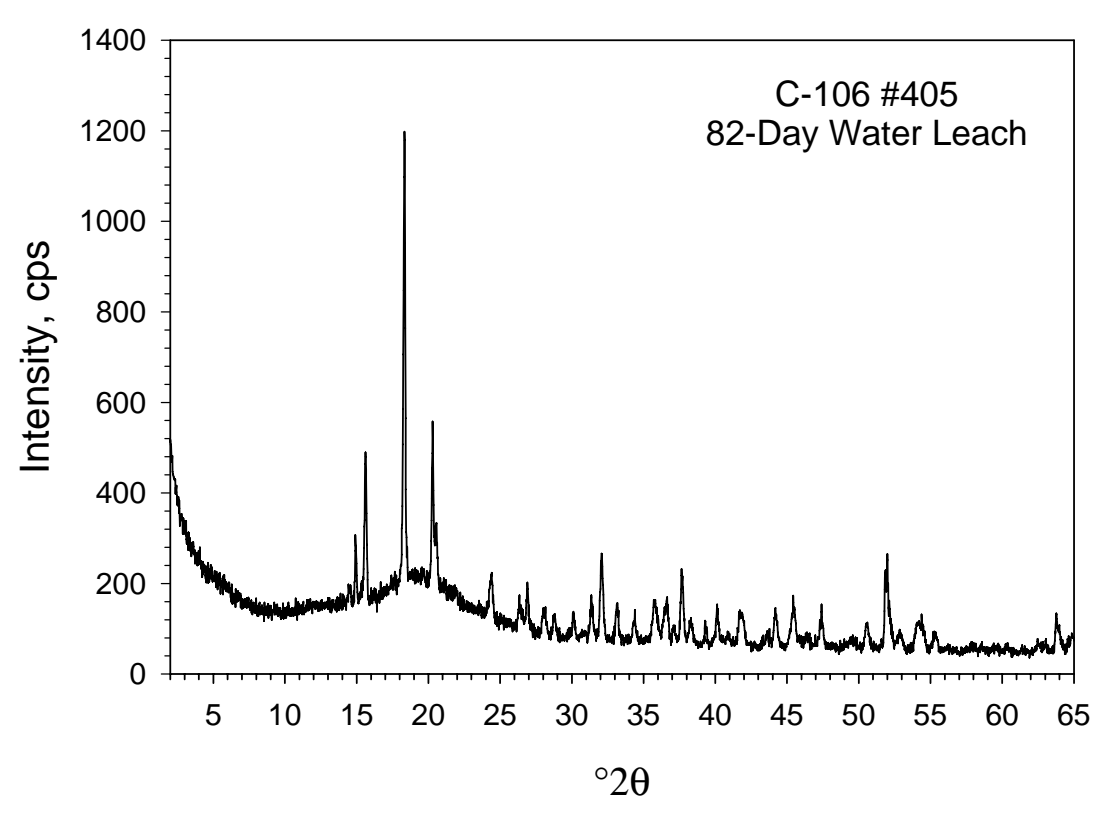

Figure A.12. As-Measured XRD Pattern (without background subtraction) for 82-day Water-Leached Sludge Sample 405

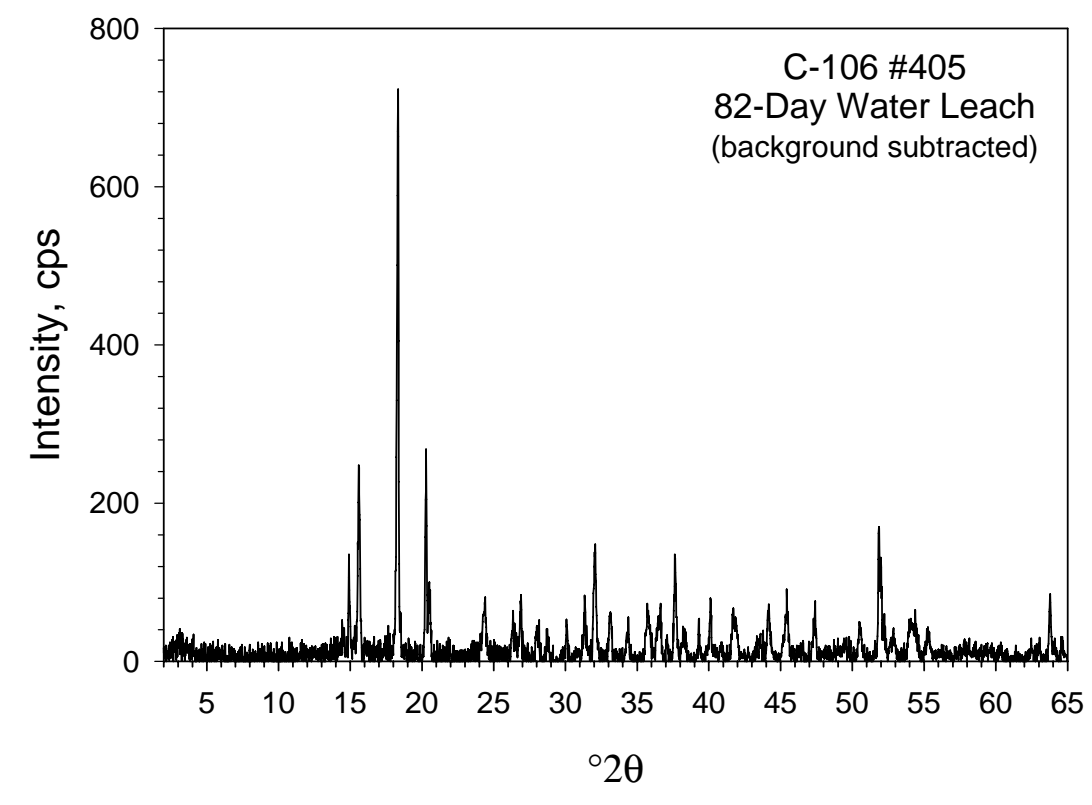

Figure A.13. Background-Subtracted XRD Pattern for 82-day WaterLeached Sludge Sample 405 


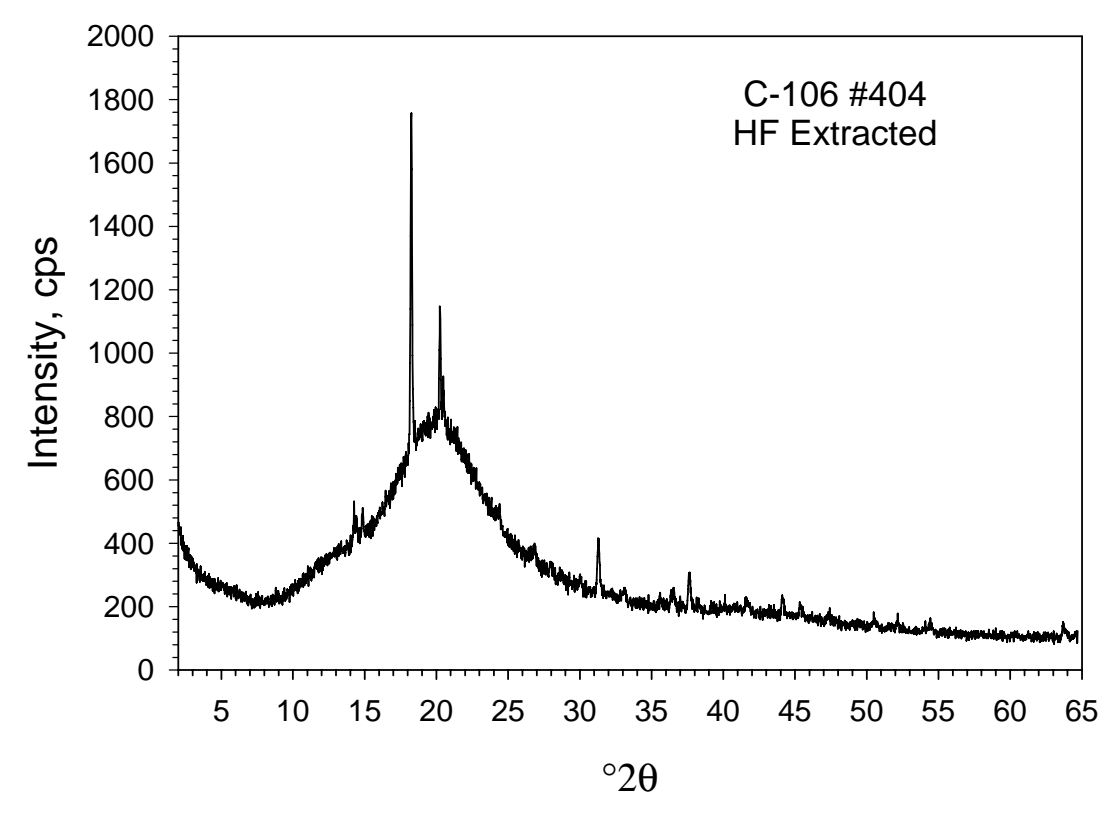

Figure A.14. As-Measured XRD Pattern (without background subtraction) for HF-Extracted Sludge Sample 404

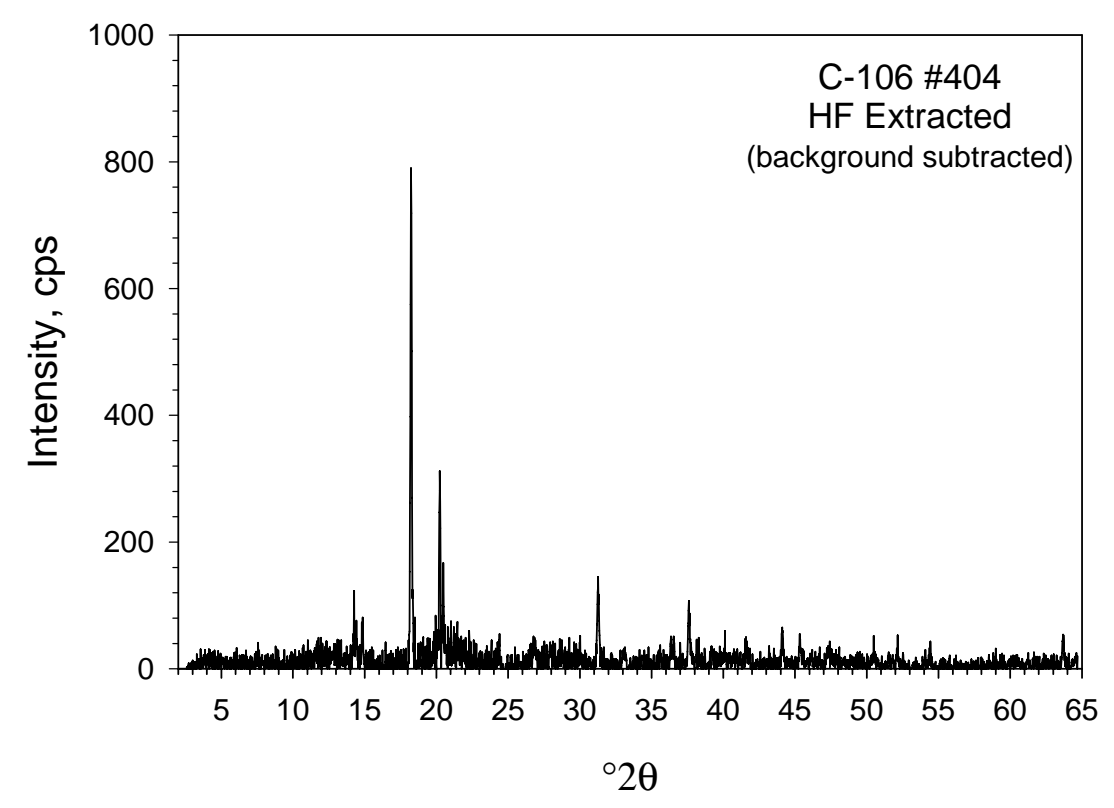

Figure A.15. Background-Subtracted XRD Pattern for HF-Extracted Sludge Sample 404 


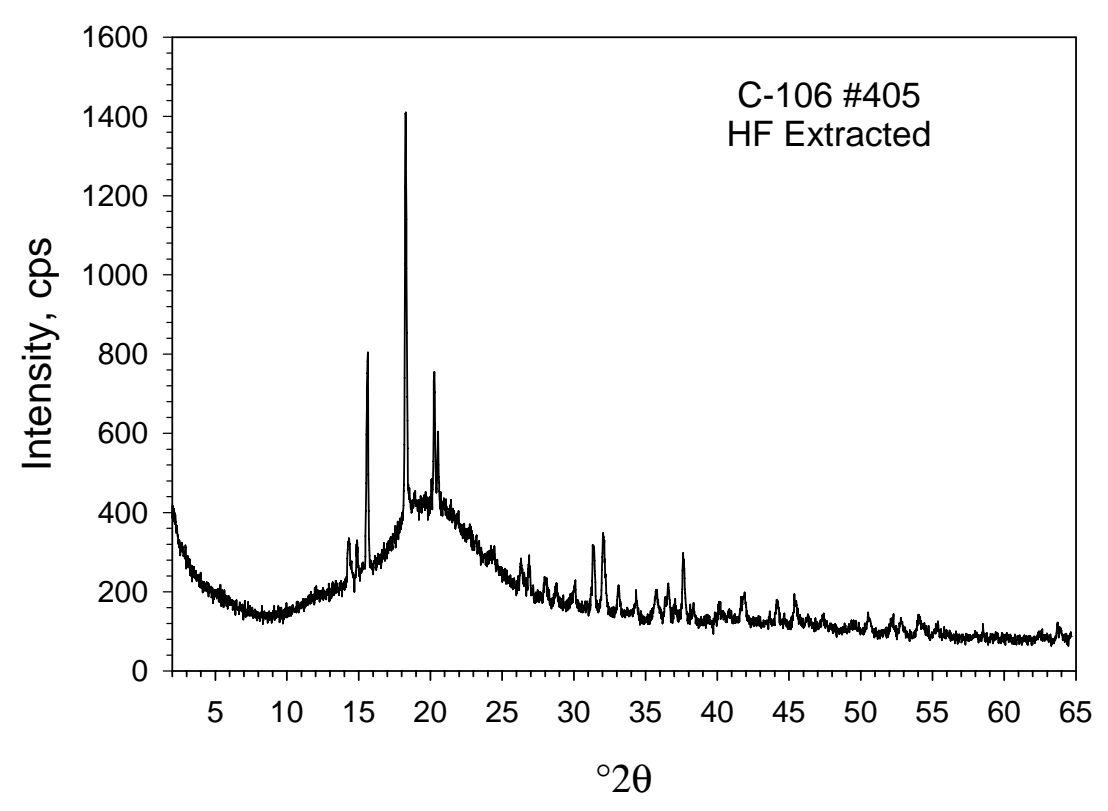

Figure A.16. As-Measured XRD Pattern (without background subtraction) for HF-Extracted Sludge Sample 405

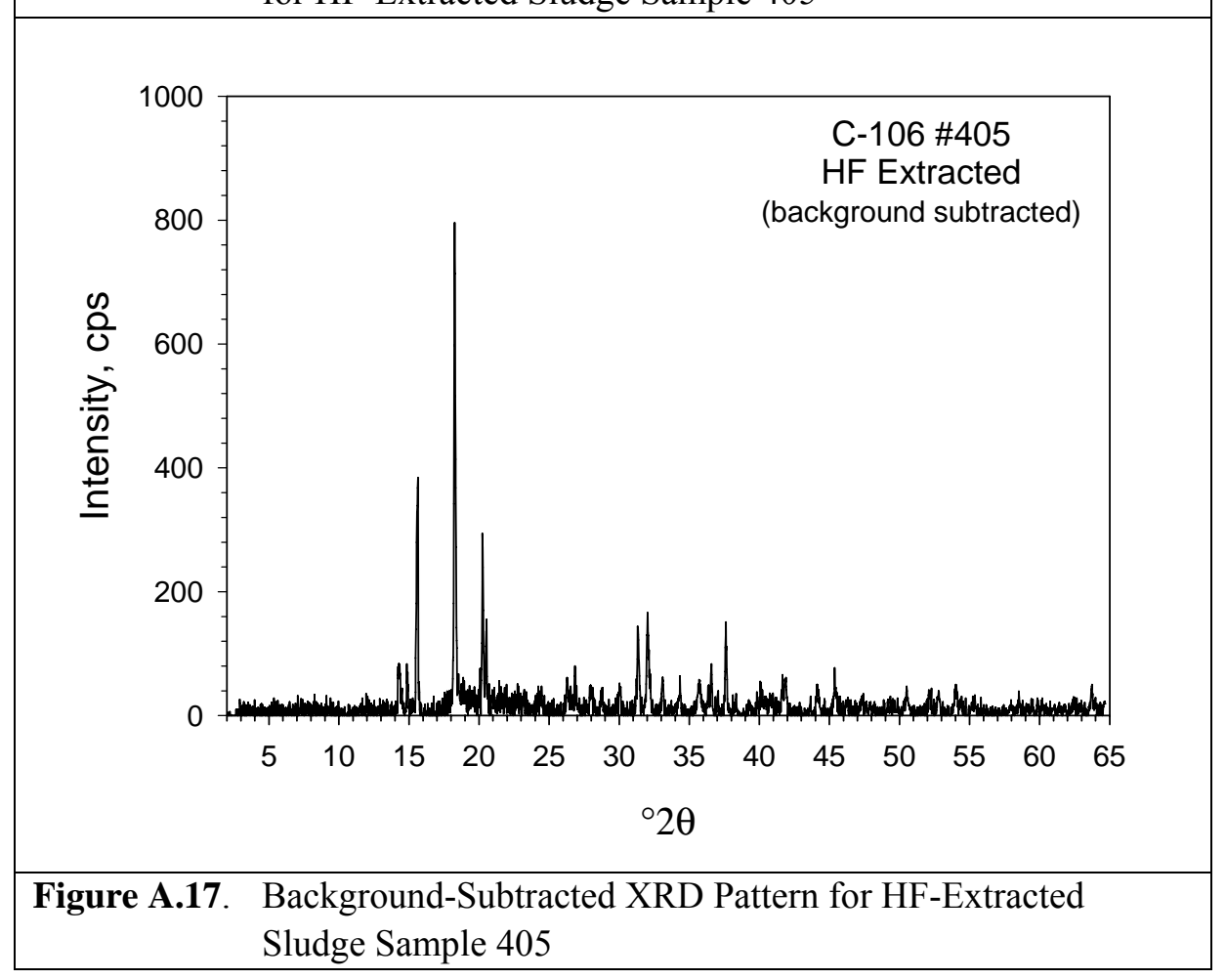




\section{Appendix B}

SEM Micrographs and EDS Spectra for Unleached Tank C-106 Sludge 


\section{Appendix B}

\section{SEM Micrographs and EDS Spectra for Unleached Tank C-106 Sludge}

This appendix includes the scanning electron microscope (SEM) micrographs and the energydispersive X-ray spectrometry (EDS) spectra for samples of unleached residual tank waste from tank 241C-106 (C-106) (primary 404 and duplicate 405). The operating conditions for the SEM and procedures used for mounting the SEM samples are described in Section 2.4 of the main report.

The name of each SEM digital image file, sample identification number, and a size scale bar are given, respectively, at the bottom left, center, and right of each SEM micrograph in this appendix. Micrographs labeled by "BSE" to the immediate right of the digital image file name indicate that the micrograph was collected with backscattered electrons. Areas identified by a letter and/or outlined by a dashed-line square in a micrograph designate sample material that was imaged at higher magnification, which is typically shown in figure(s) that immediately follow in the series for that sample.

Areas labeled by "eds" in the following SEM micrographs in this appendix identify locations of particles for which EDS spectra were recorded. The "eds” label given with each EDS spectrum correspond to the same "EDS" label used in the SEM micrographs for this sample.

\section{B.1 Unleached Sludge Sample 404}

Two mounts of unleached (raw) residual sludge from tank C-106 (primary 404) were analyzed by SEM/EDS. The SEM micrographs for the first mount (1) of sample 404 sludge are shown in Figures B.1 through B.22. The EDS spectra for this mount are given in Figures B.23 through B.27. The SEM micrographs for the second mount (3) of sample 404 sludge are shown in Figures B.28 through B.35, and the EDS spectra for the second mount are given in Figures B.36 through B.38. 


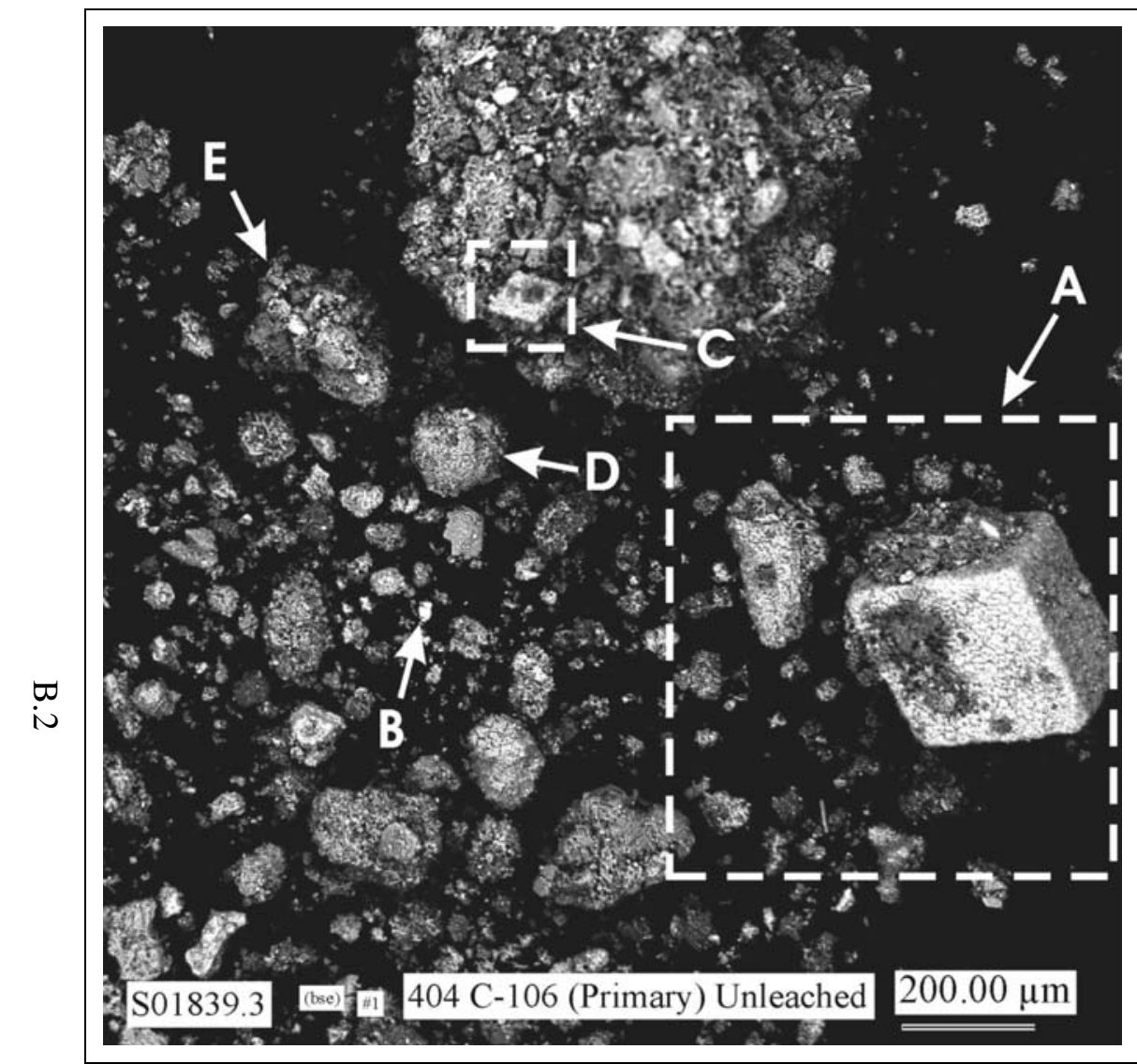

Figure B.1. $\quad$ Low Magnification SEM Micrograph Showing General Morphologies of Particles in SEM Mount 1 of Unleached Sludge Sample 404

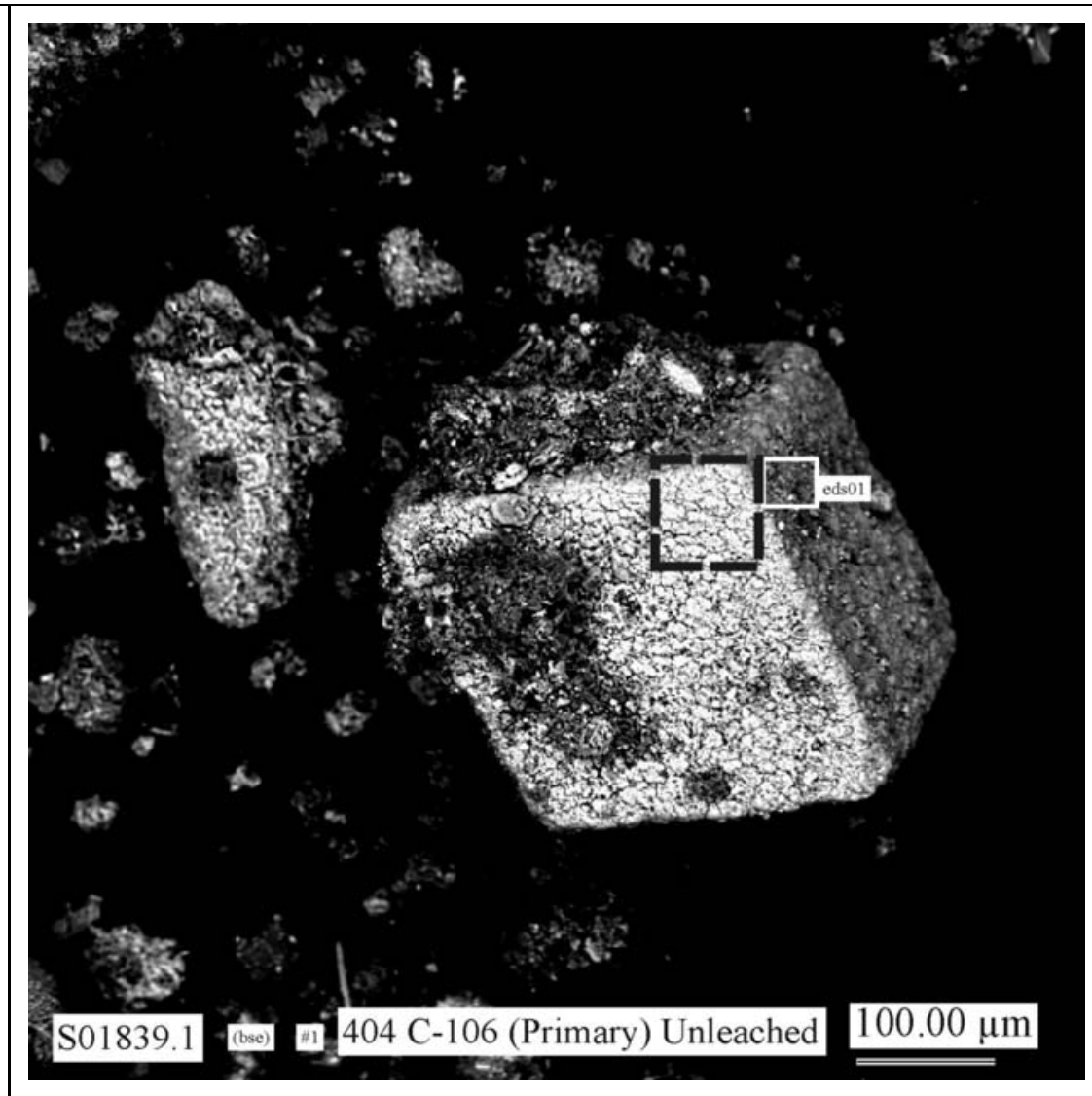

Figure B.2. $\quad$ Micrograph Showing at Higher Magnification the Area Indicated by the White Dashed-line Square Labeled A in Figure B.1 


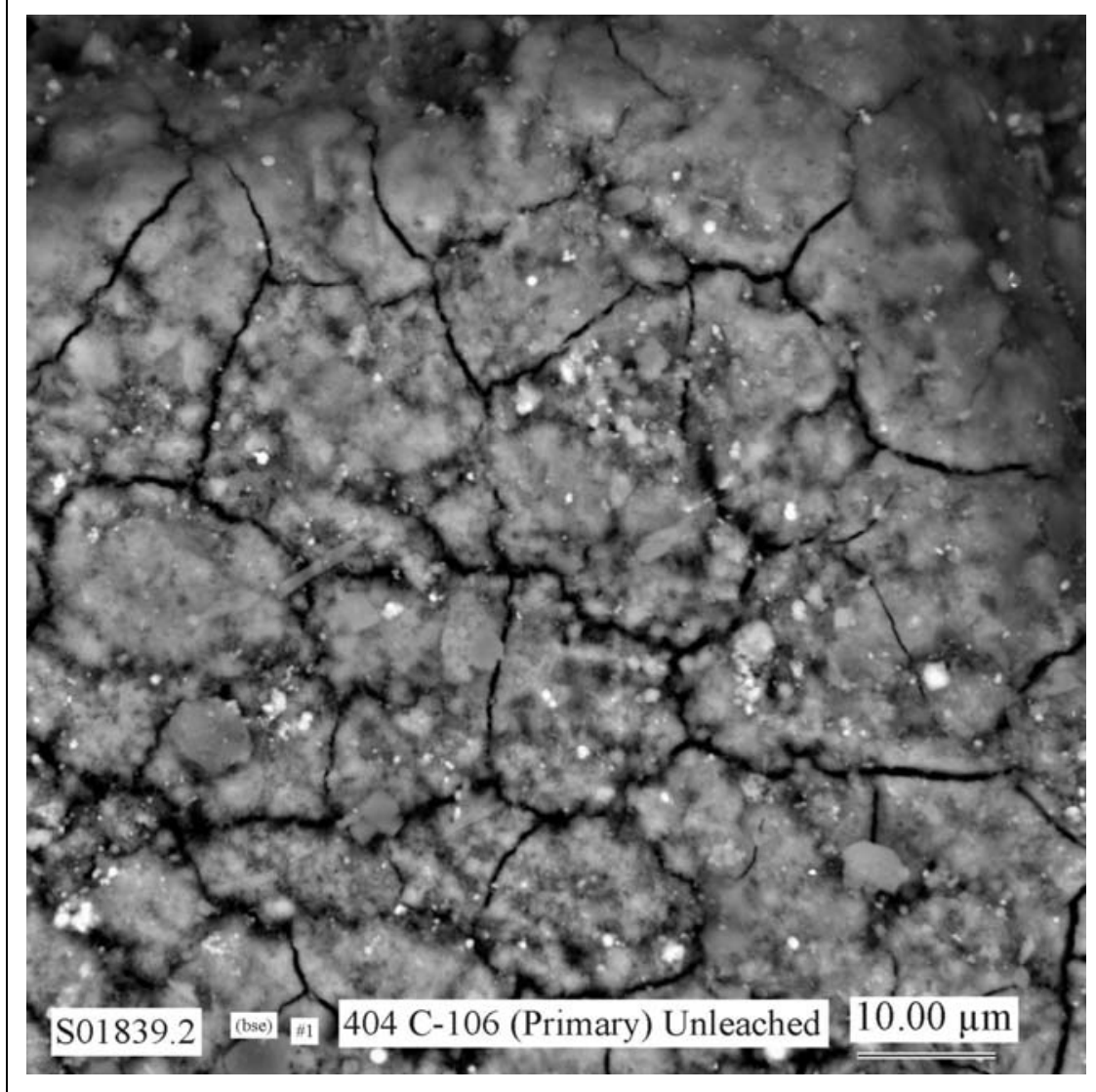

Figure B.3. $\quad$ Micrograph Showing at Higher Magnification the Area Indicated by the Black Dashed-Line Square in Figure B.2

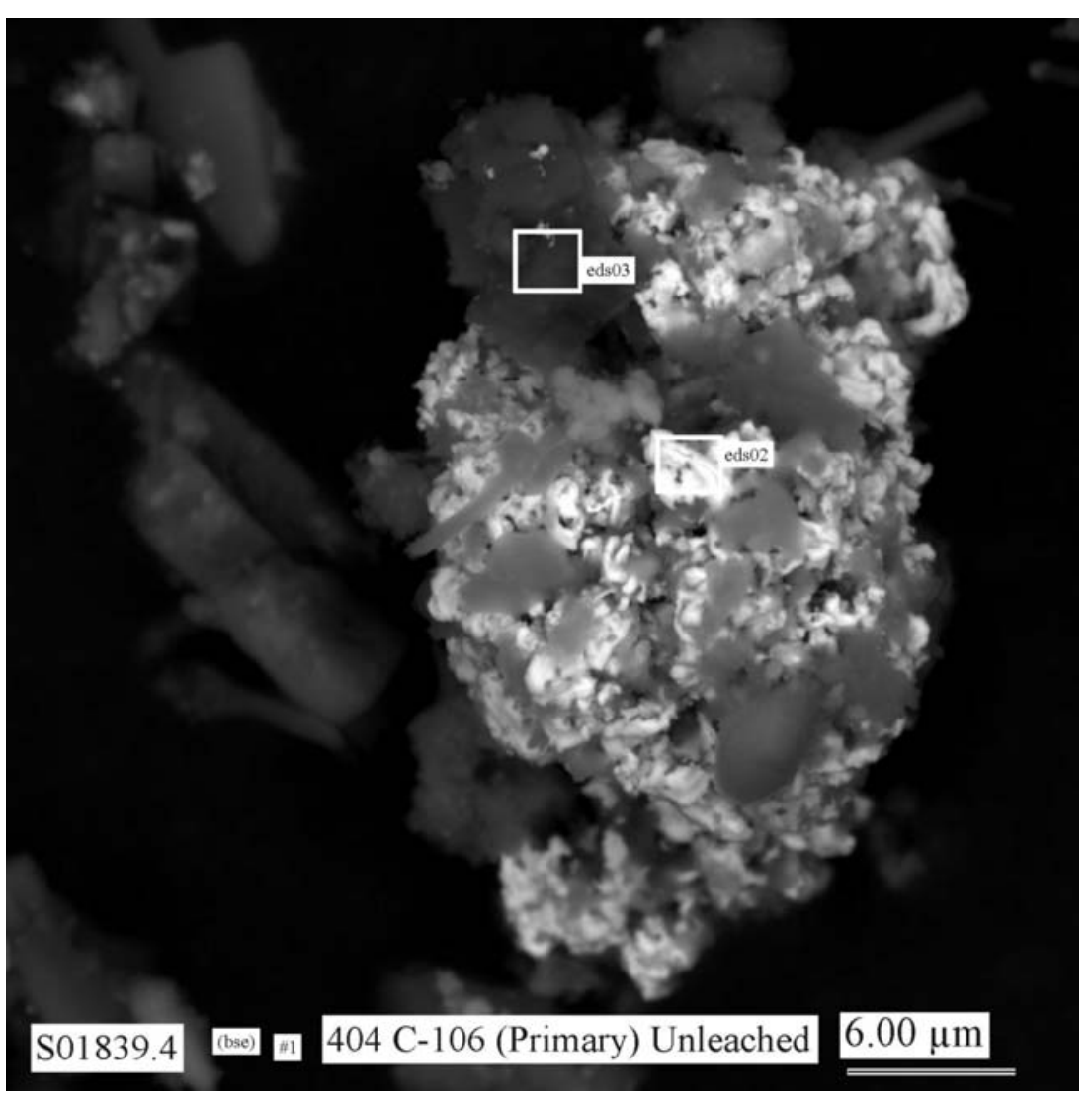

Figure B.4. Micrograph Showing at Higher Magnification the Particle Labeled B in Figure B.1 

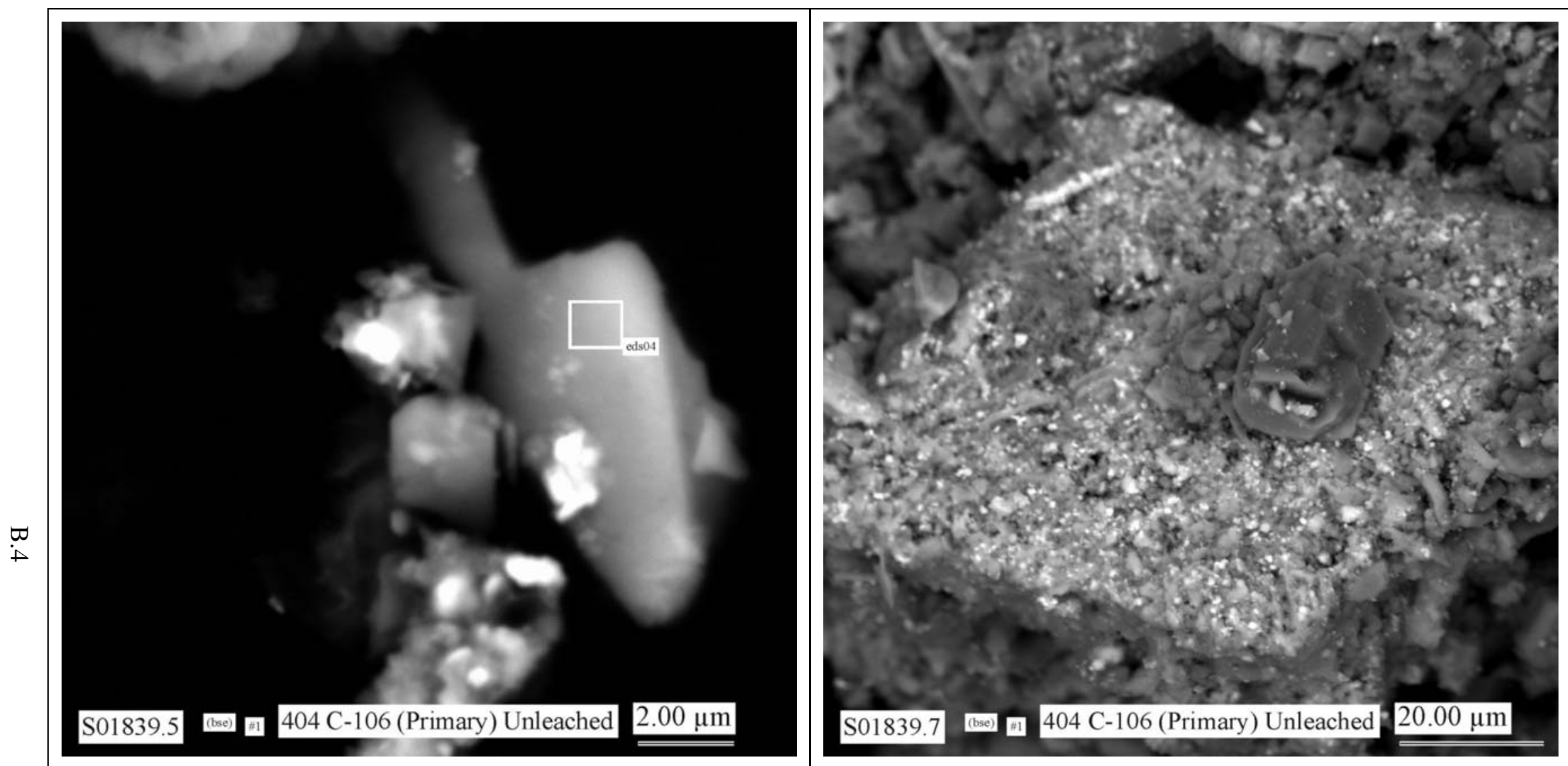

Figure B.5. Micrograph Showing at Higher Magnification a "Bright" Particle Present in the Field of View in Figure B.1

Figure B.6. $\quad$ Micrograph Showing at Higher Magnification the Area Indicated by the White Dashed-Line Square Labeled C in Figure B.1 


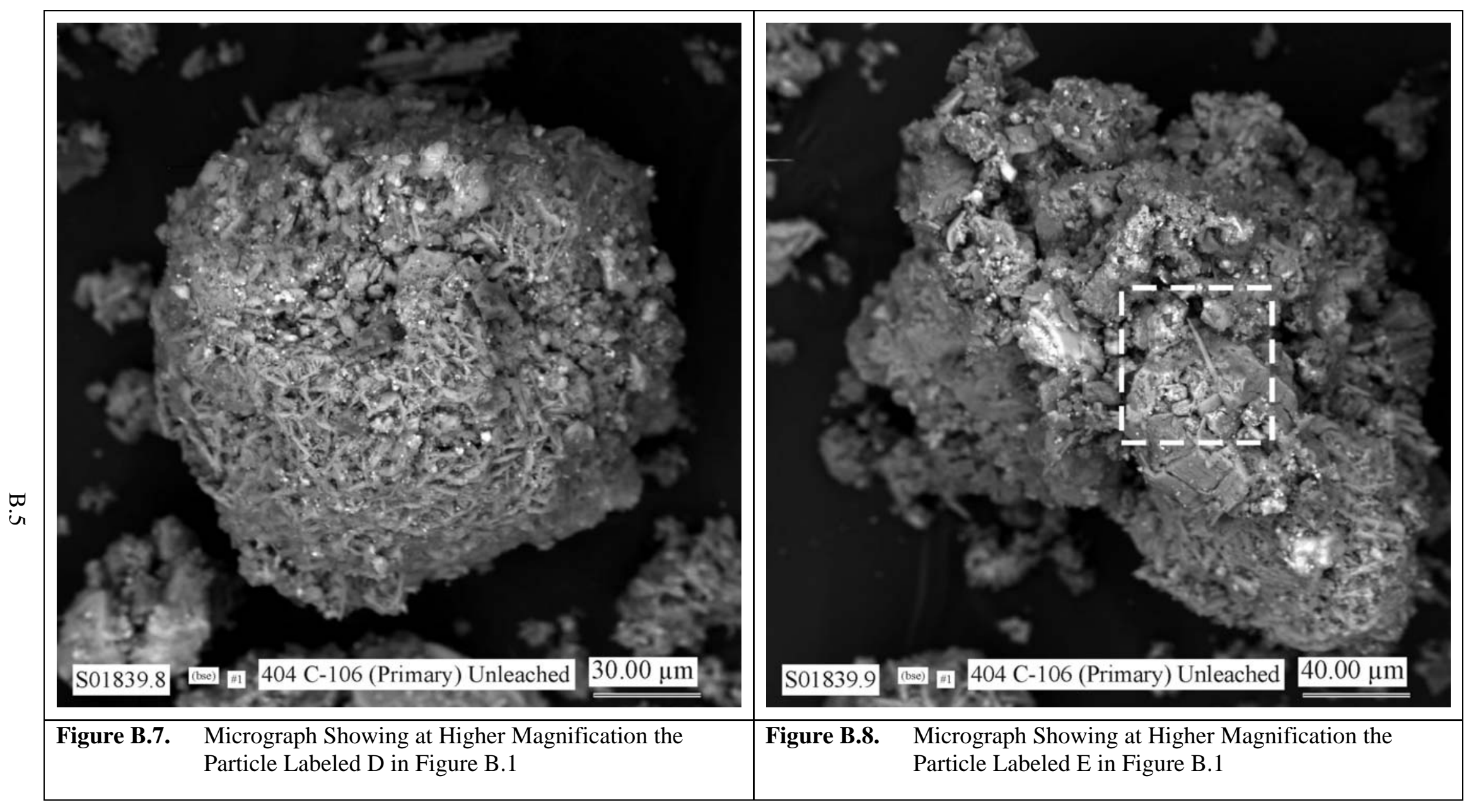




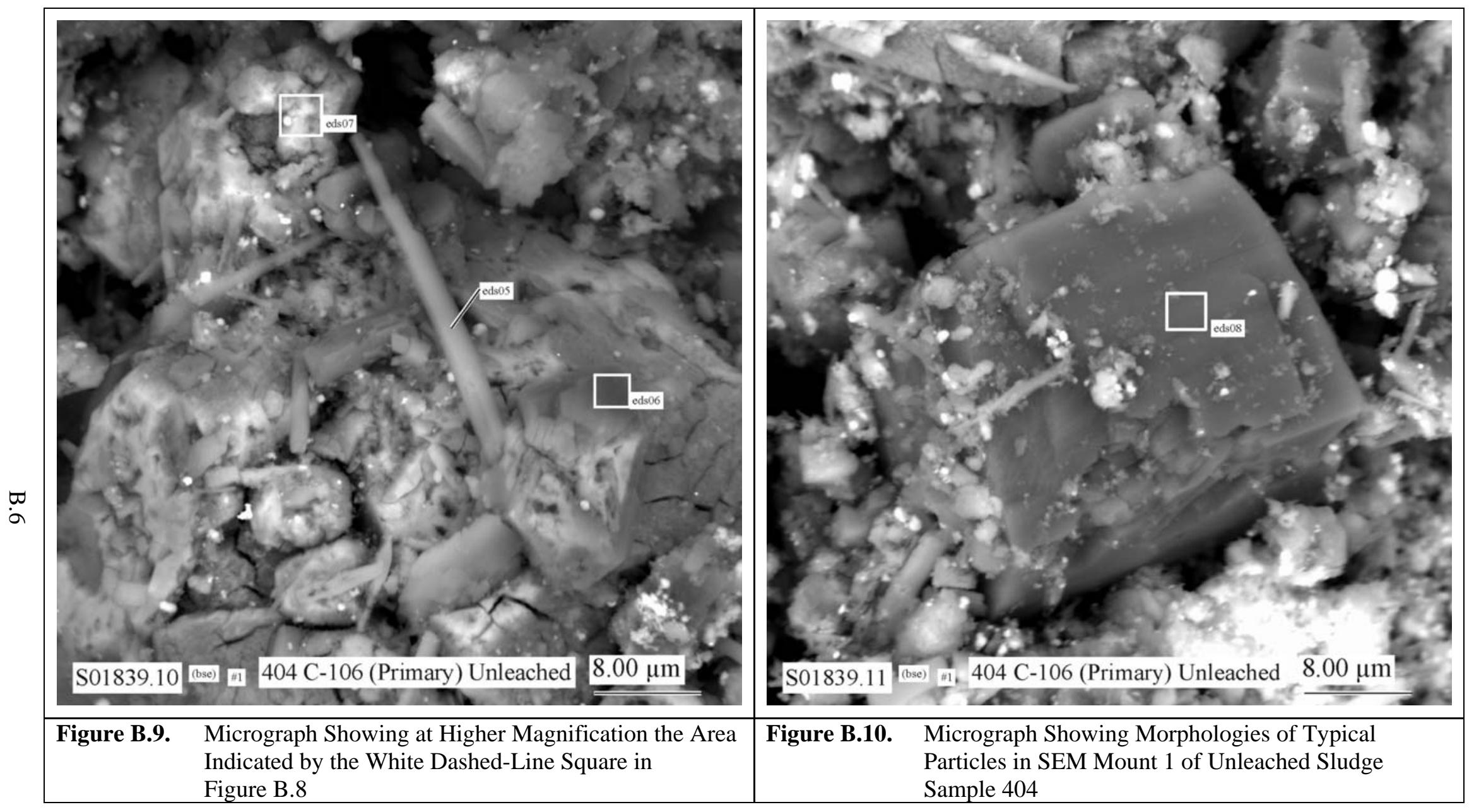




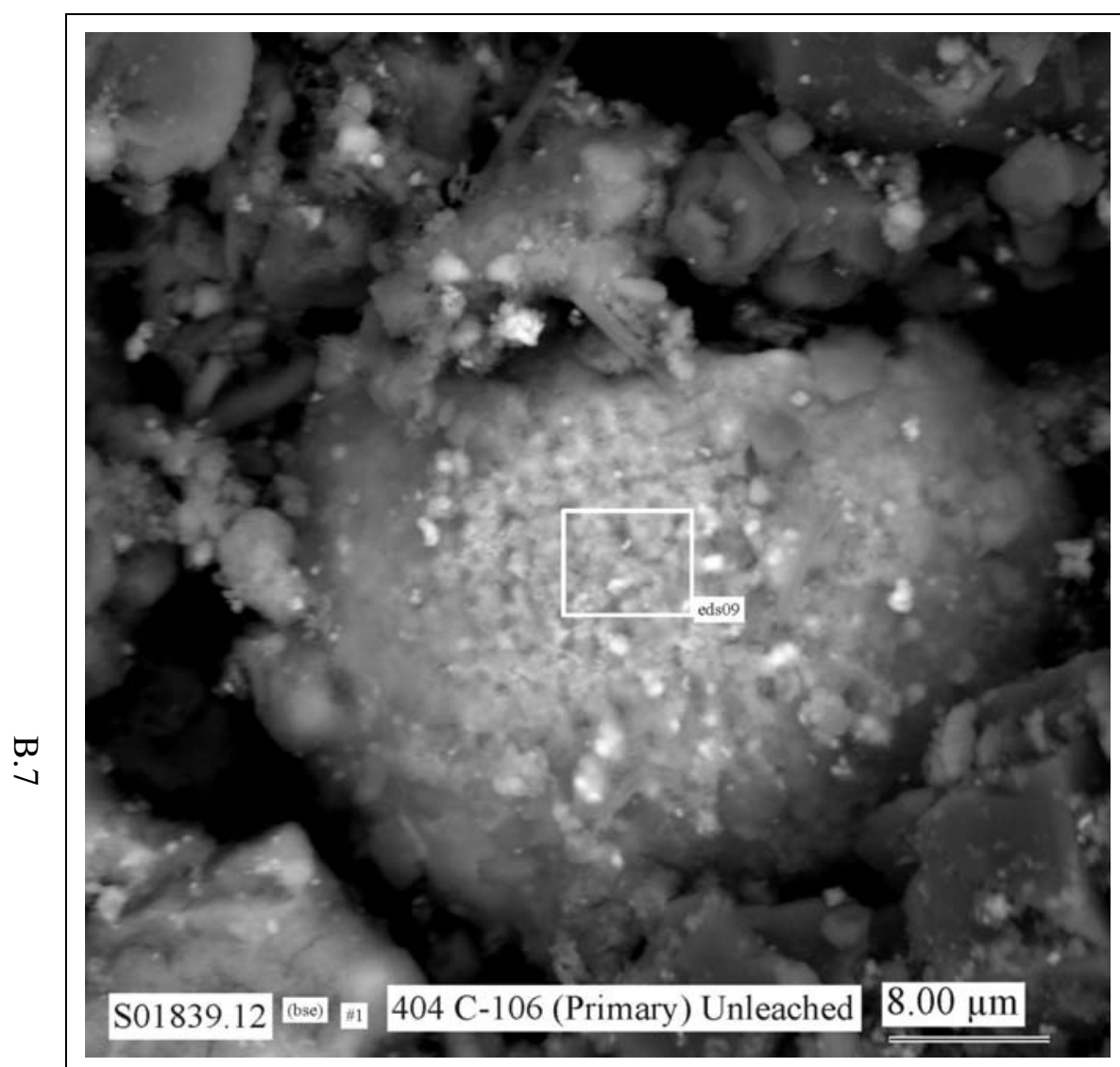

Figure B.11. $\quad$ Micrograph Showing Morphologies of Typical Particles in SEM Mount 1 of Unleached Sludge Sample 404

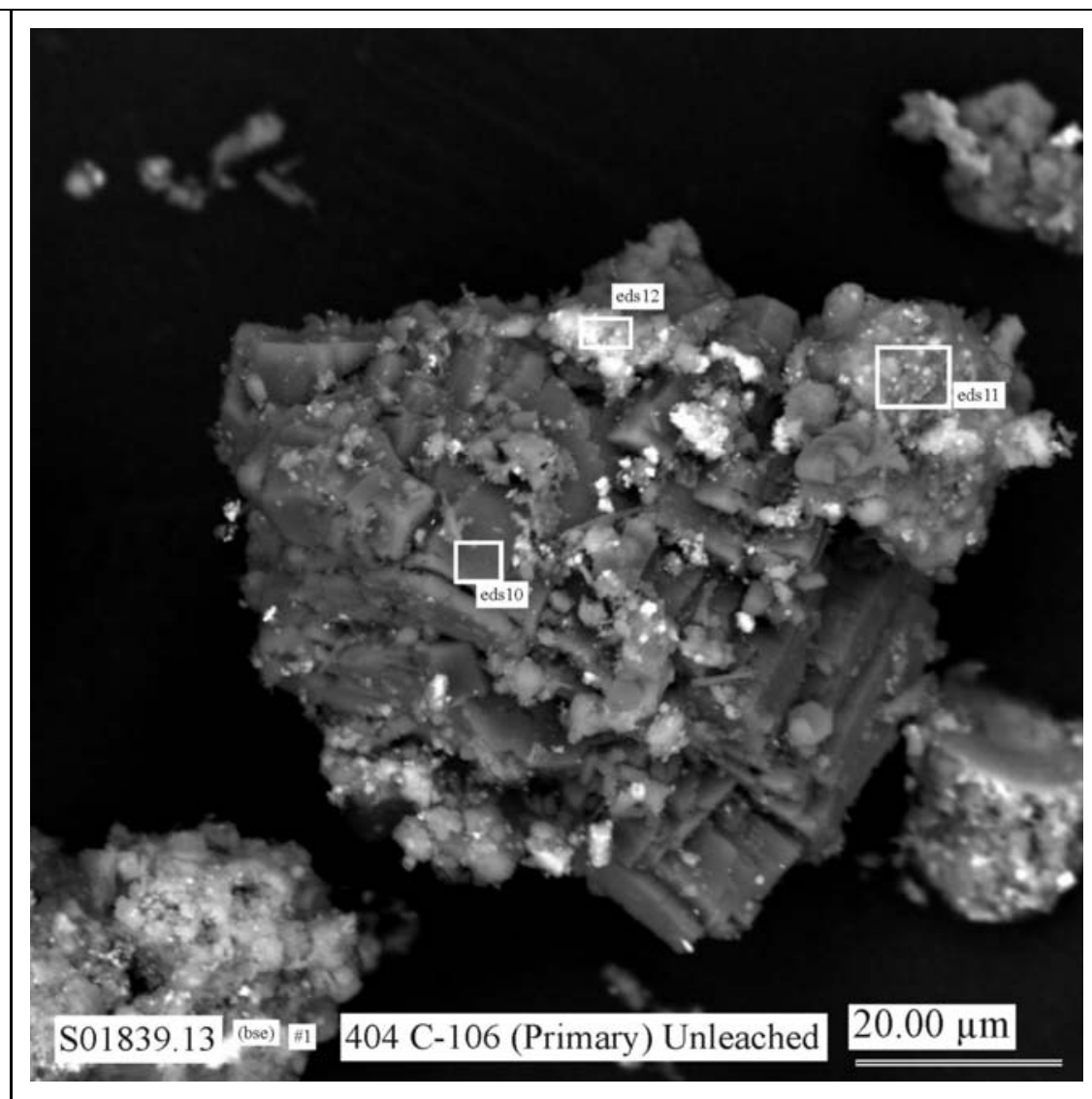

Figure B.12. Micrograph Showing Morphologies of Typical Particles in SEM Mount 1 of Unleached Sludge Sample 404 


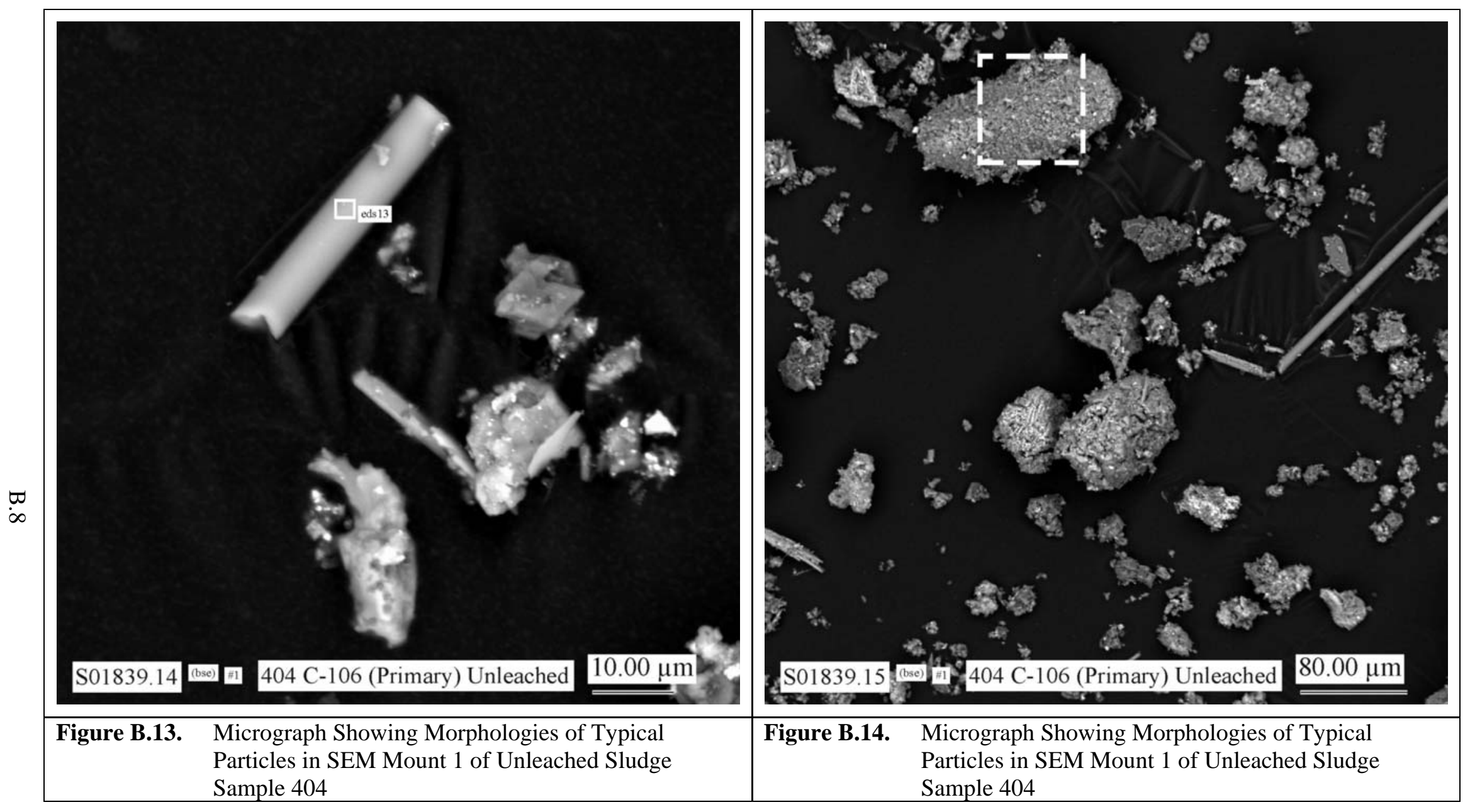




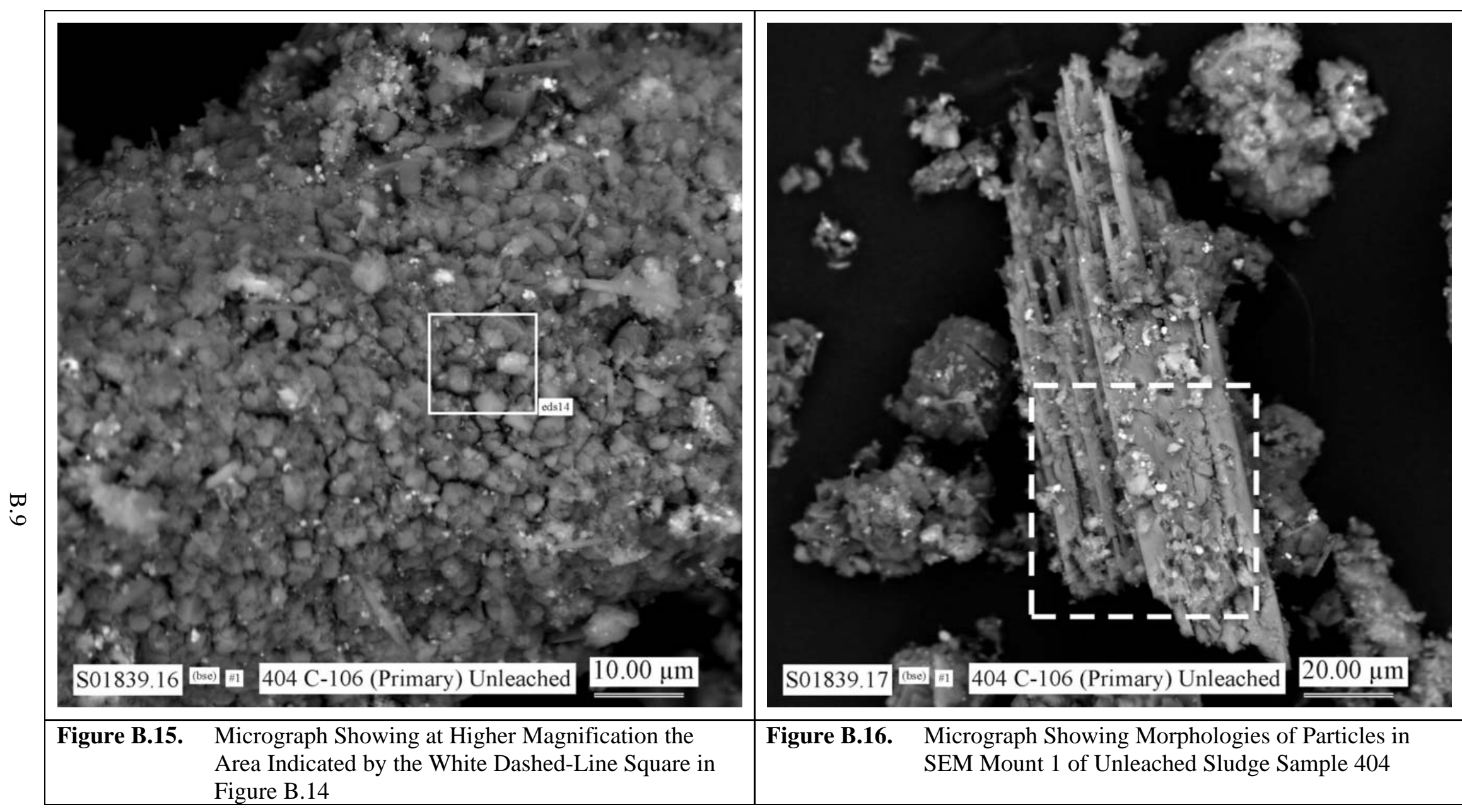



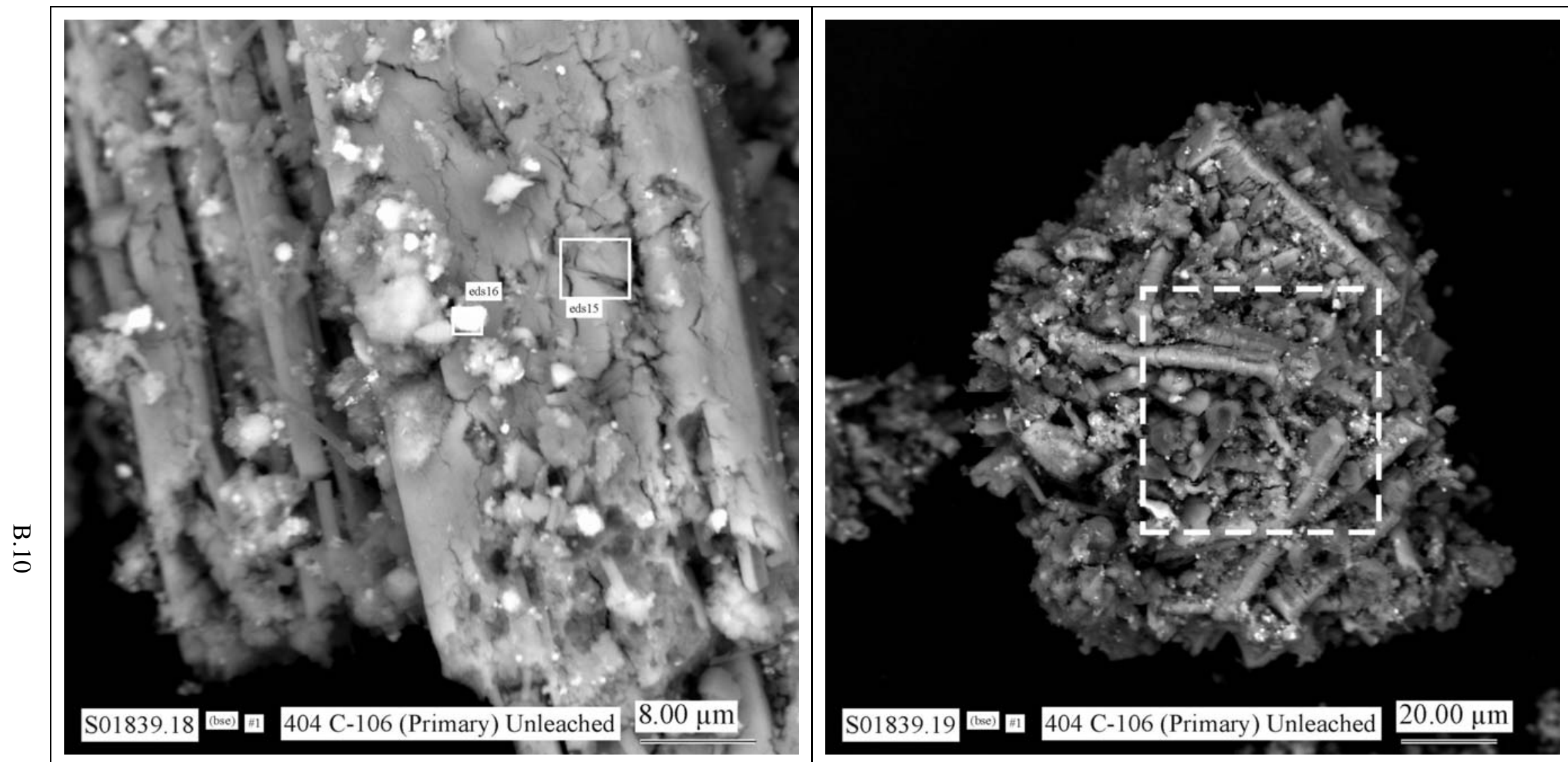

Figure B.17. Micrograph Showing at Higher Magnification the Area Indicated by the White Dashed-Line Square in Figure B.16

Figure B.18. $\quad$ Micrograph Showing Morphologies of Typical Particles in SEM Mount 1 of Unleached Sludge Sample 404 


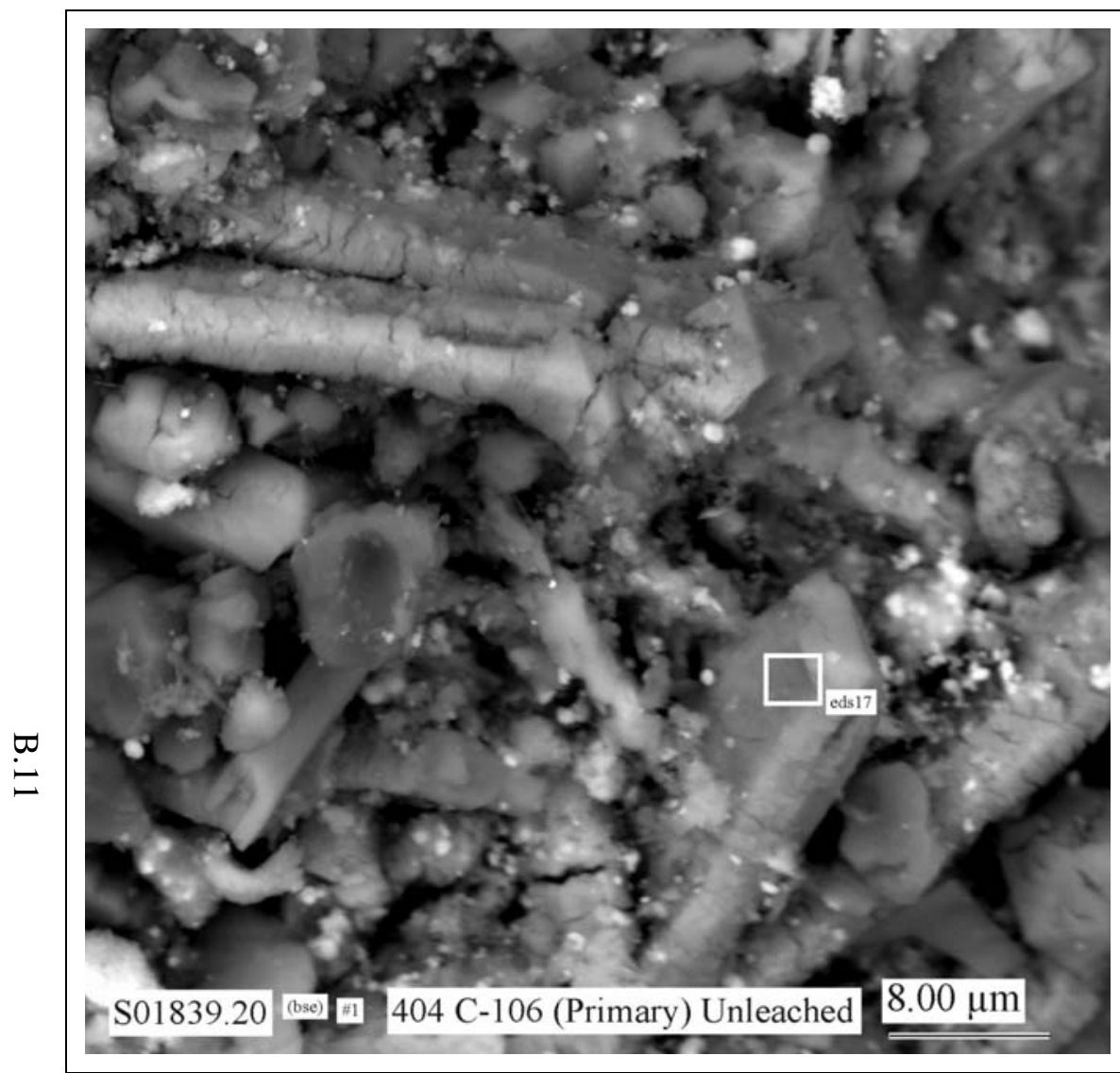

Figure B.19. Micrograph Showing at Higher Magnification the Area Indicated by the White Dashed-Line Square in Figure B.18

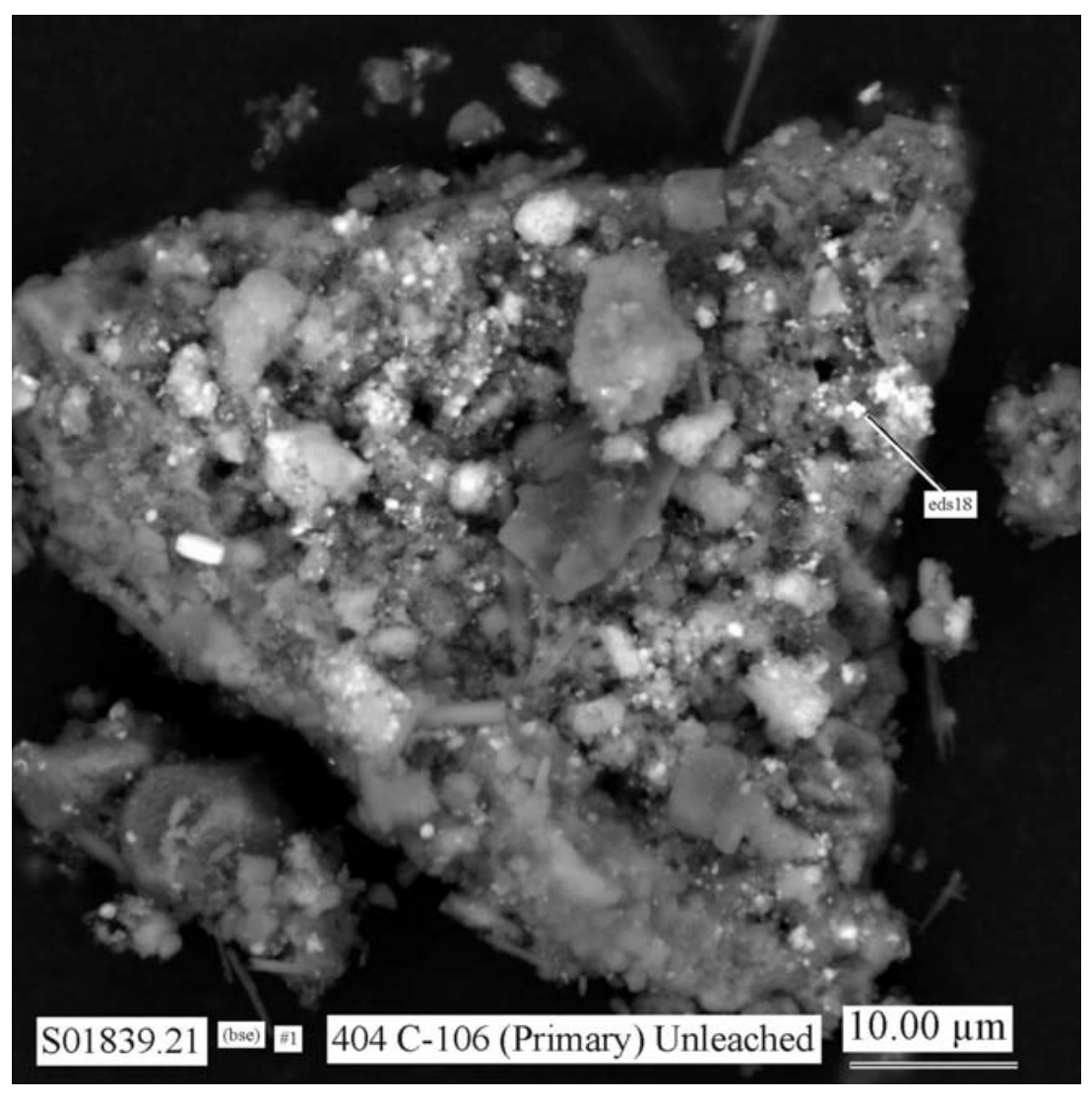

Figure B.20. $\quad$ Micrograph Showing Morphologies of Typical Particles in SEM Mount 1 of Unleached Sludge Sample 404 


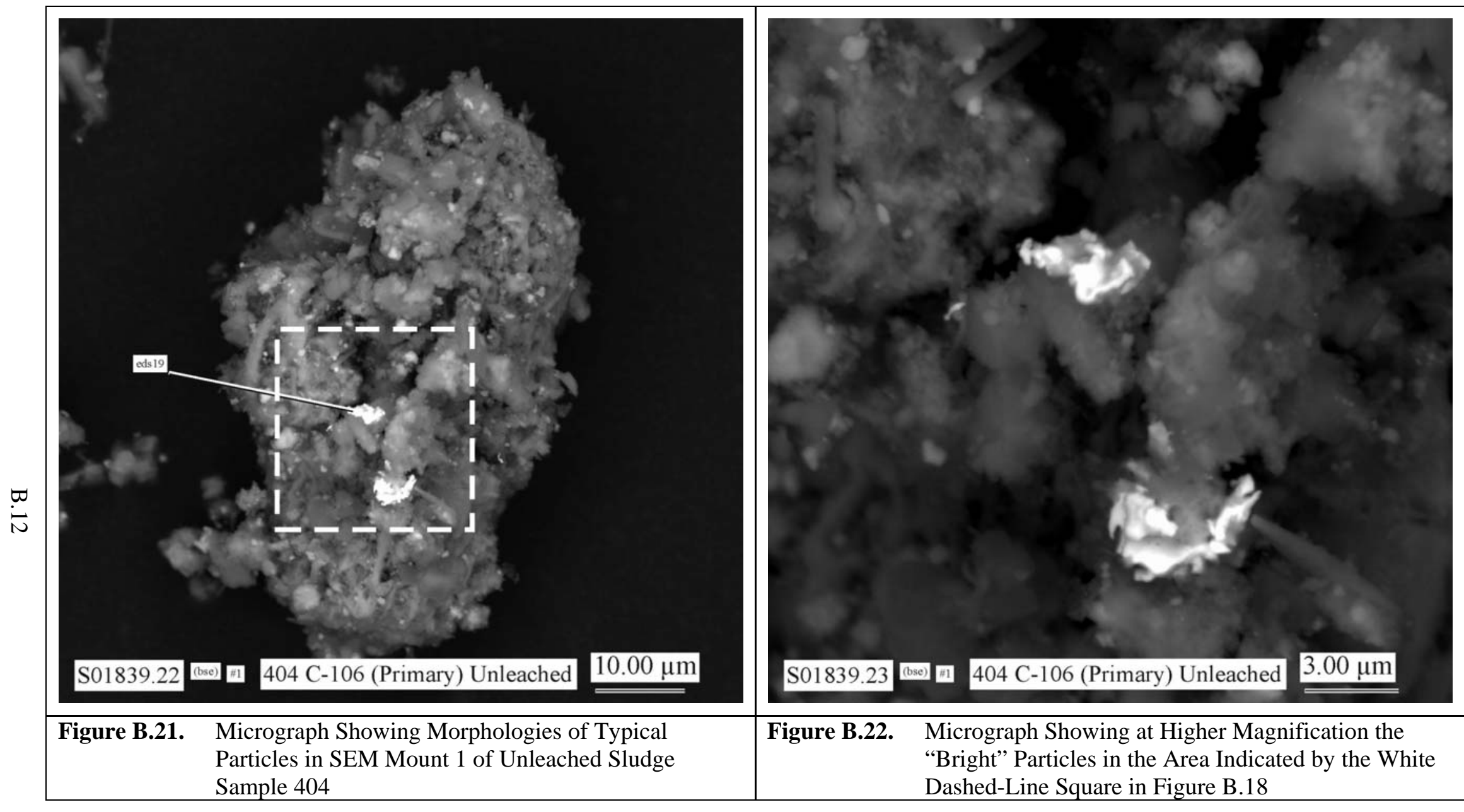




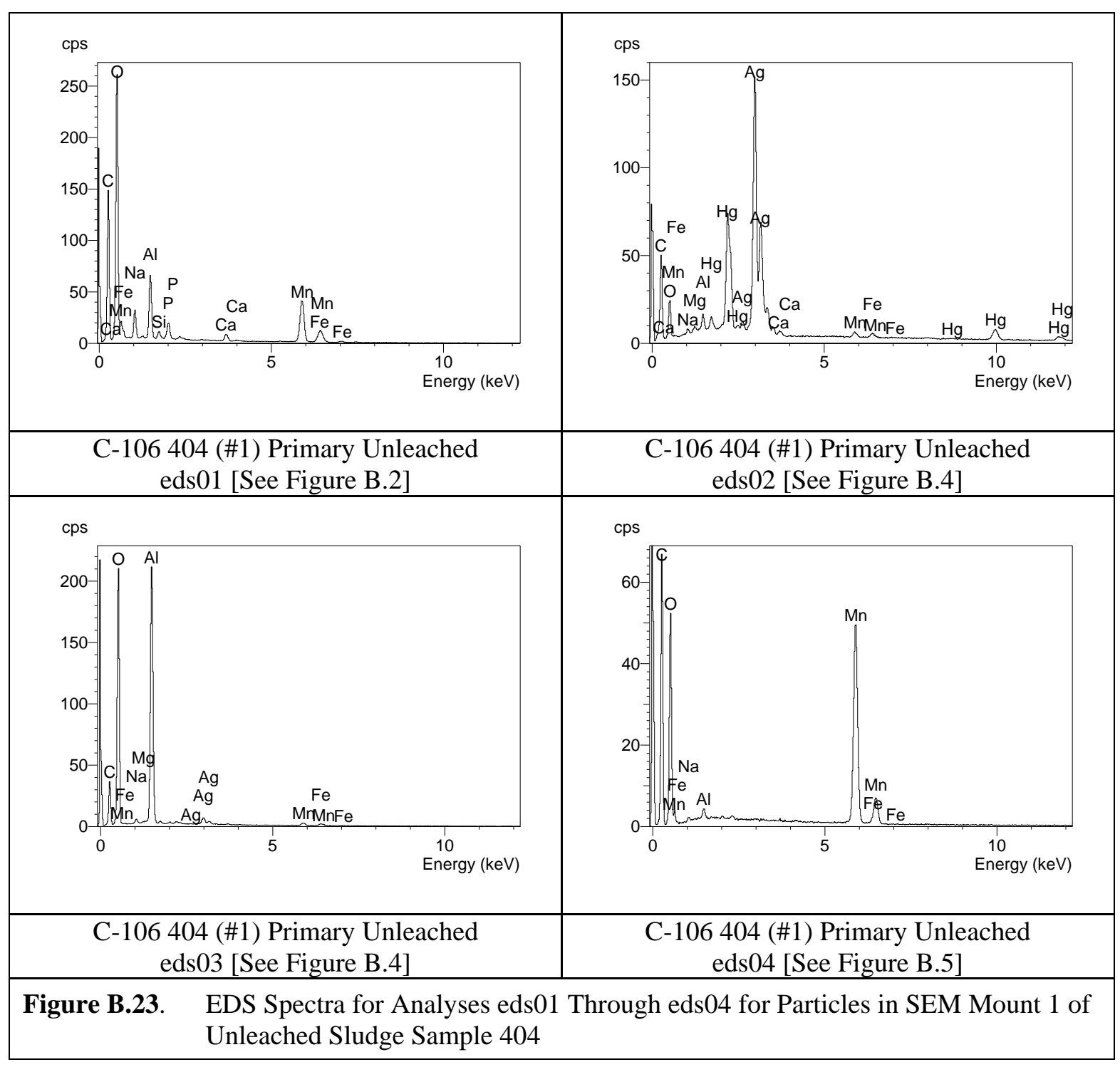




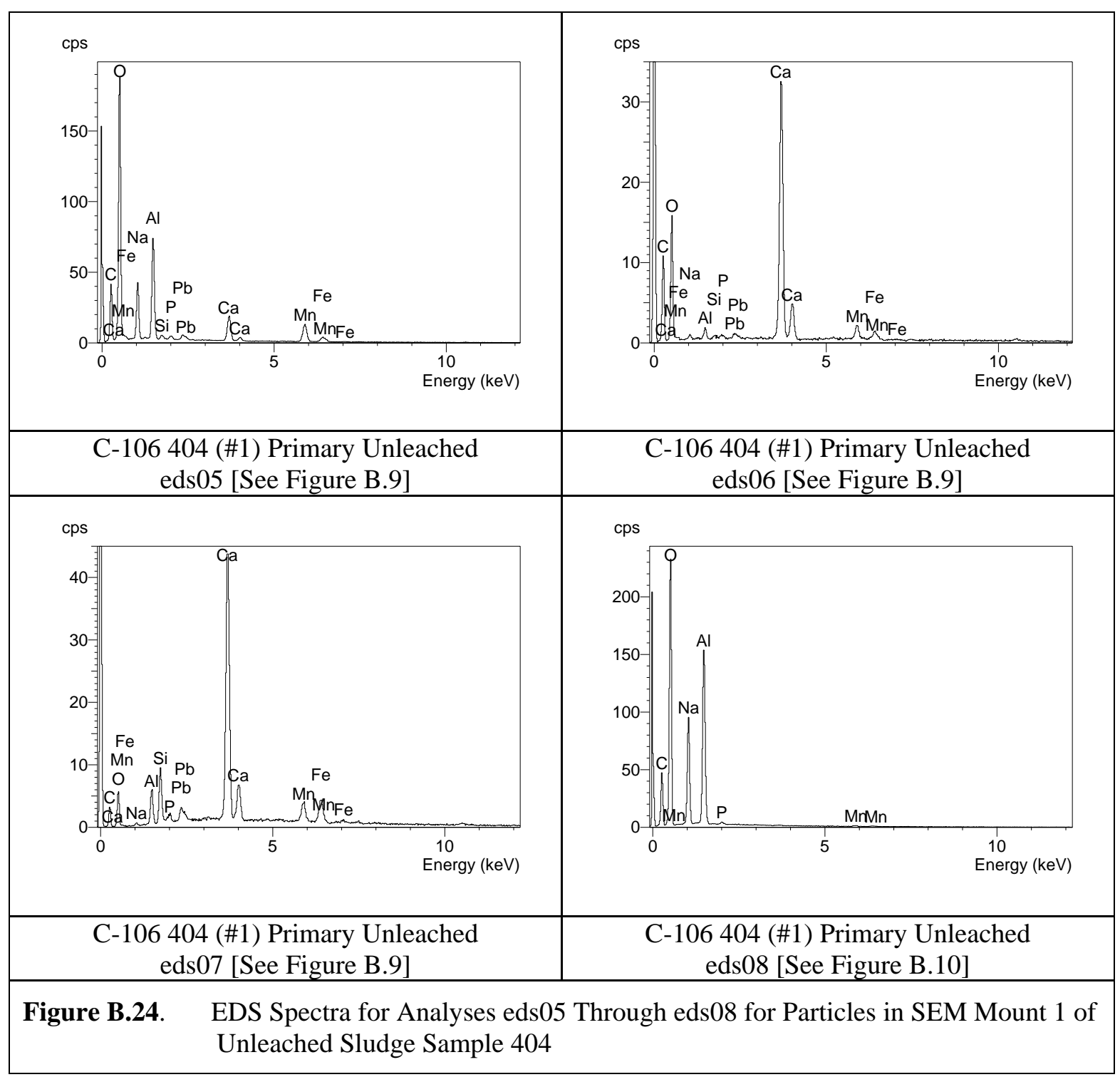




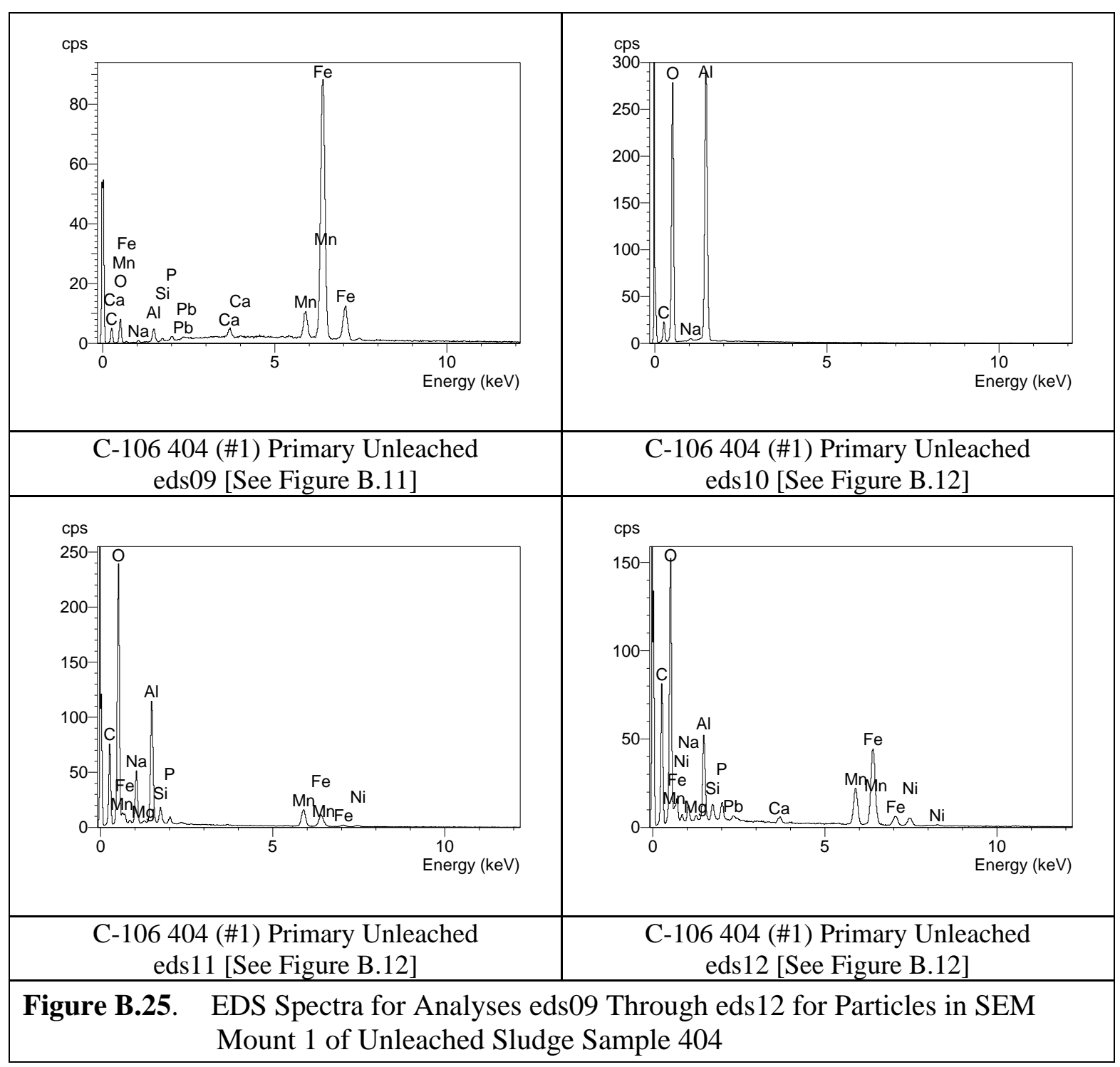

B.15 


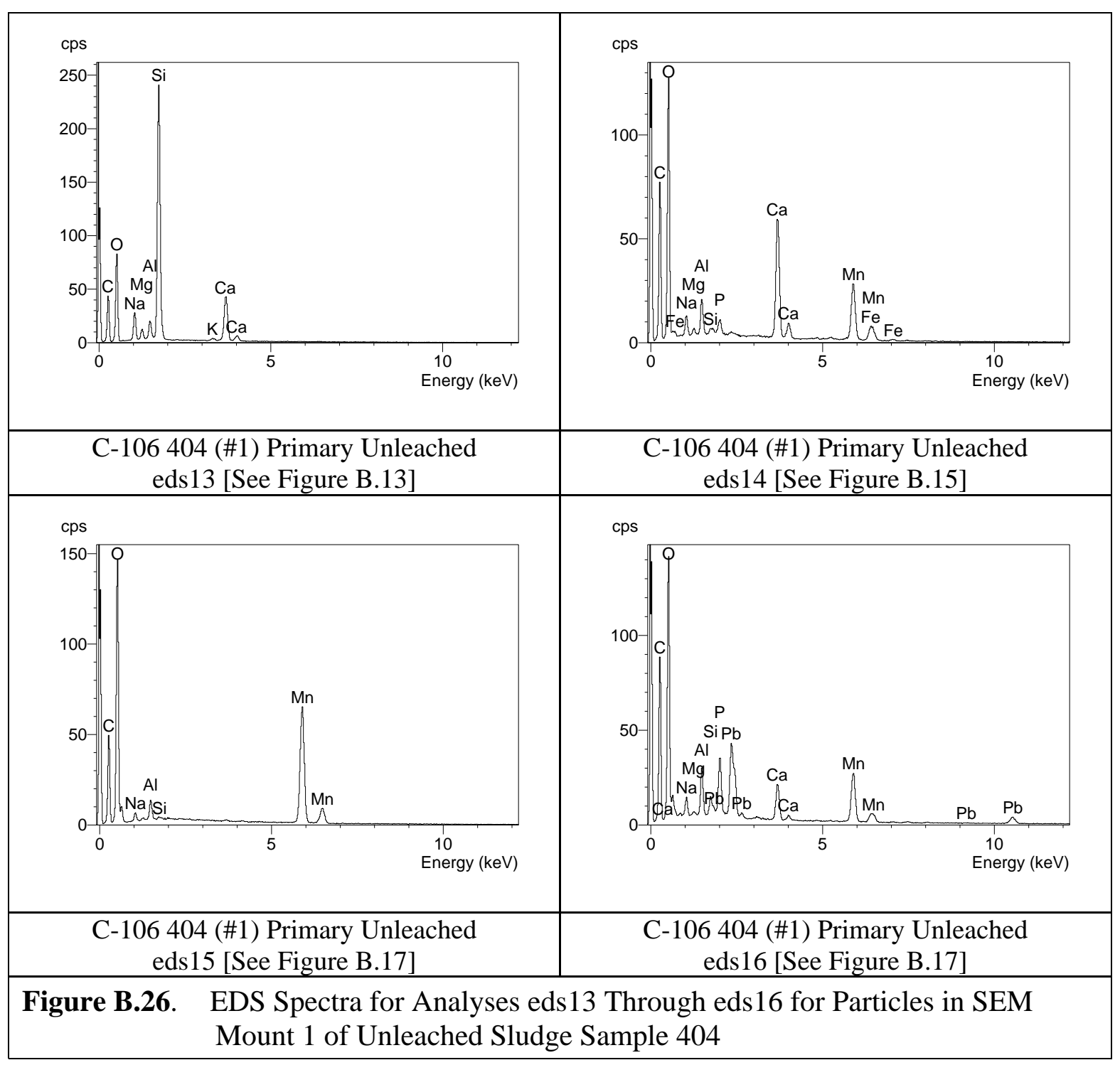

B.16 


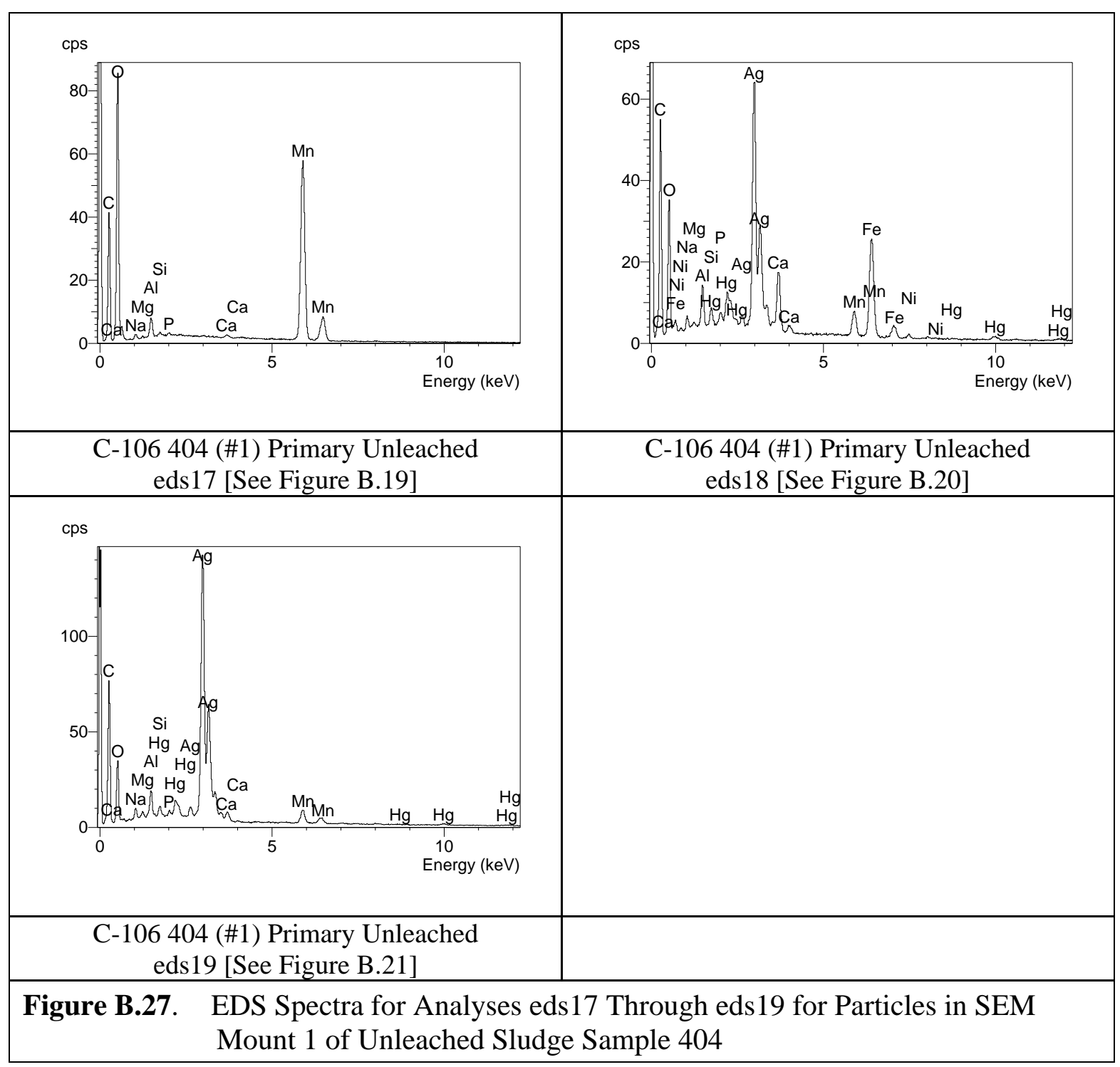




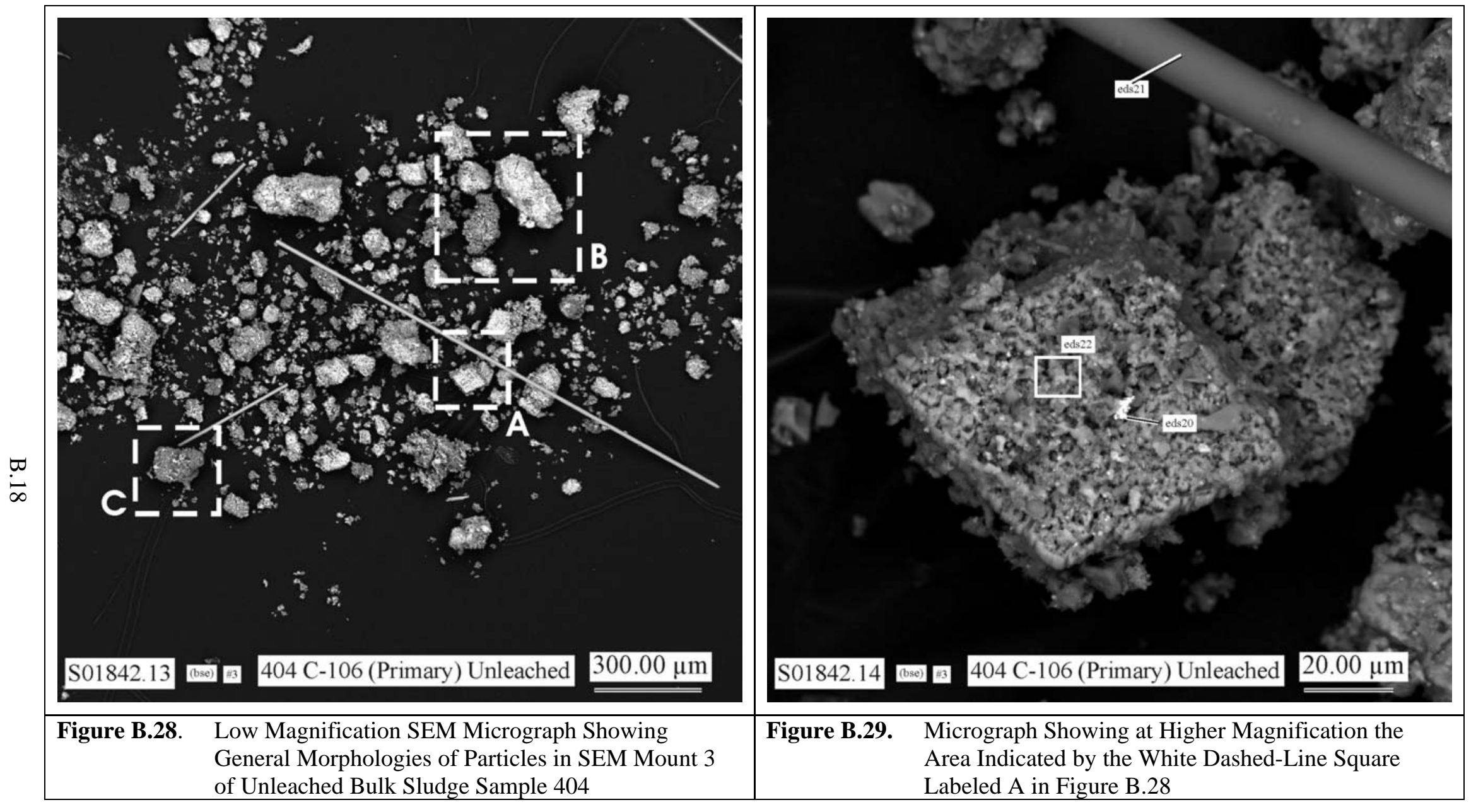




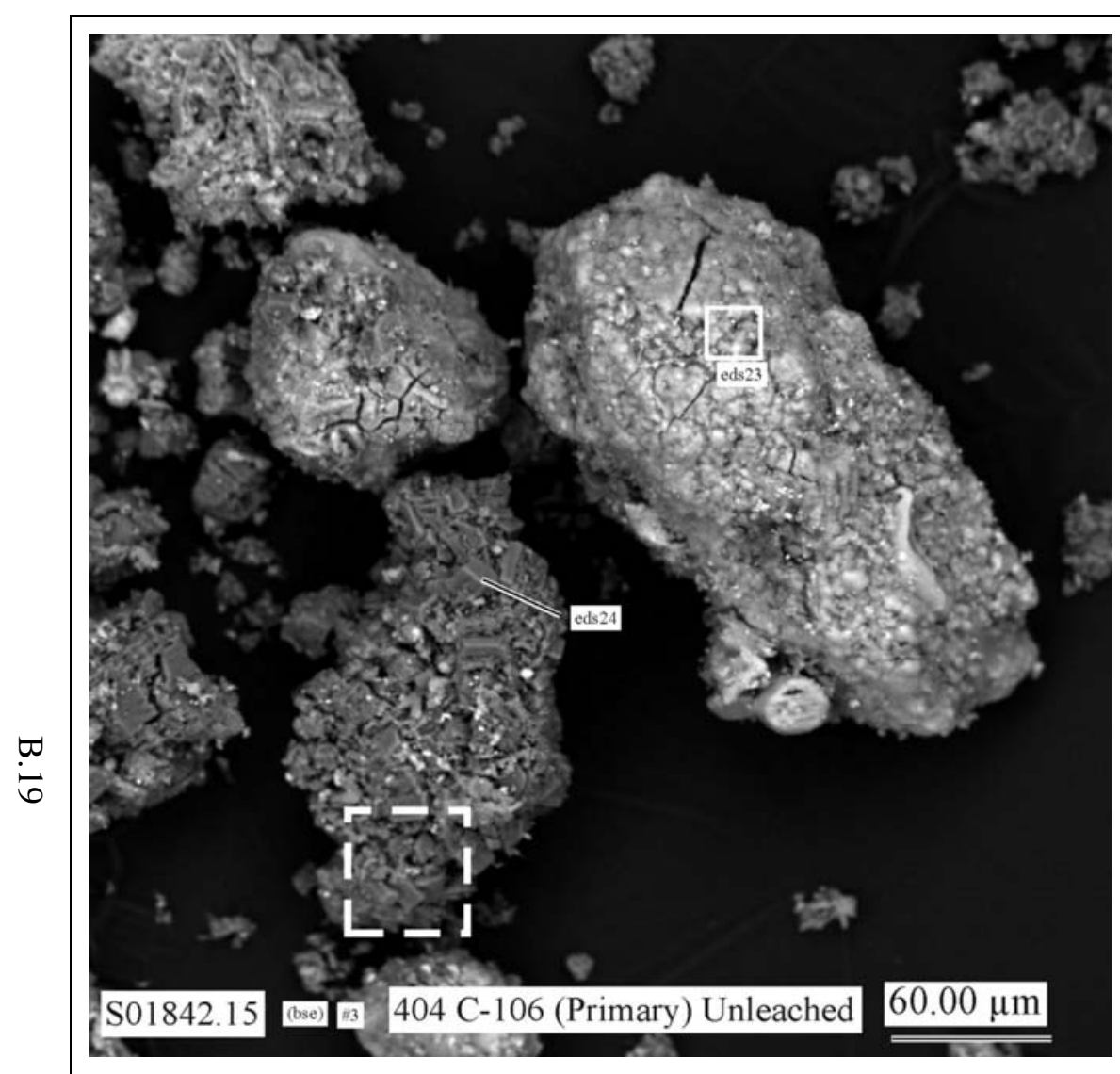

Figure B.30. Micrograph Showing at Higher Magnification the Area Indicated by the White Dashed-Line Square Labeled B in Figure B.28

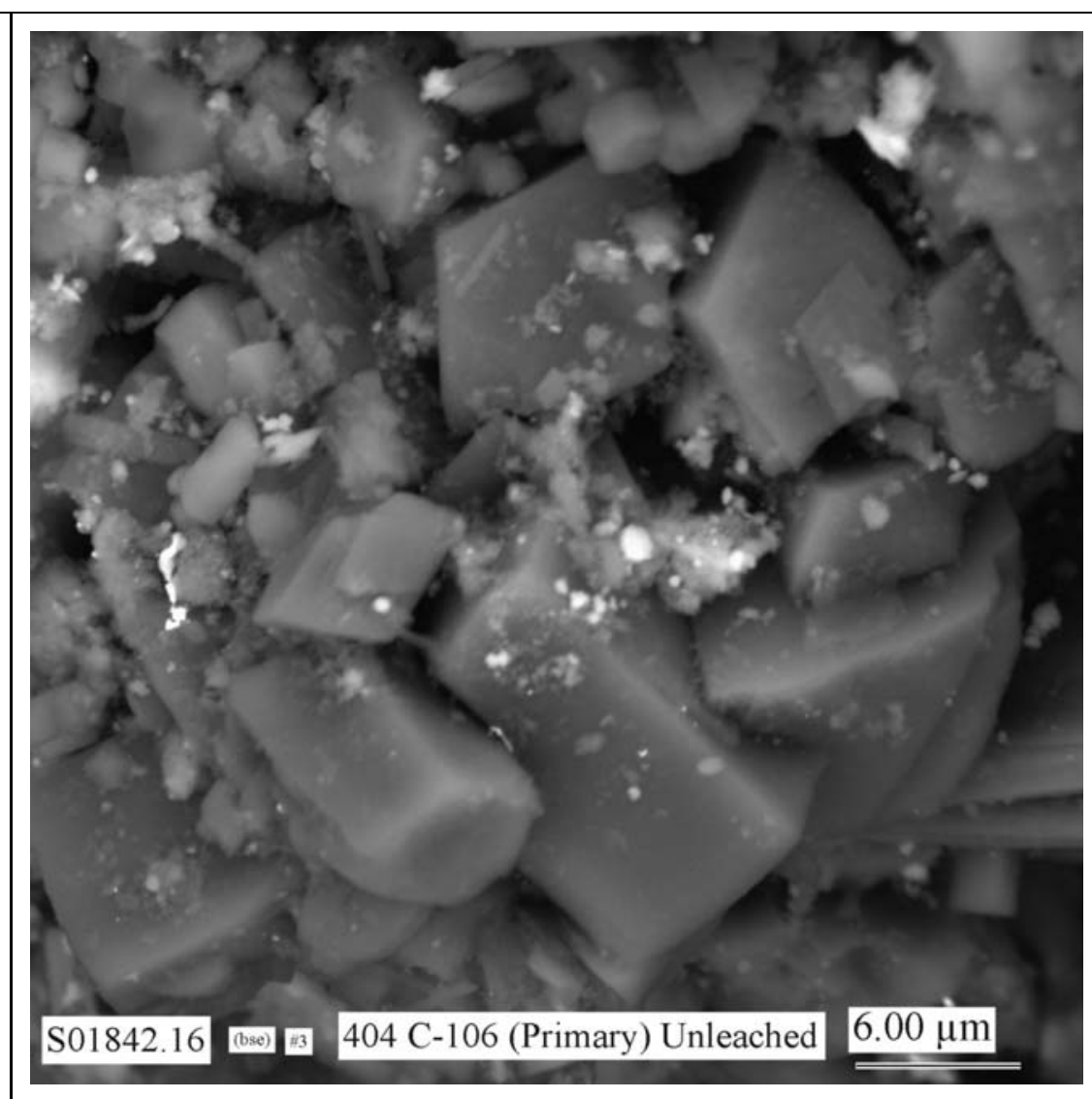

Figure B.31. Micrograph Showing at Higher Magnification the Area Indicated by the White Dashed-Line Square in Figure B.30 

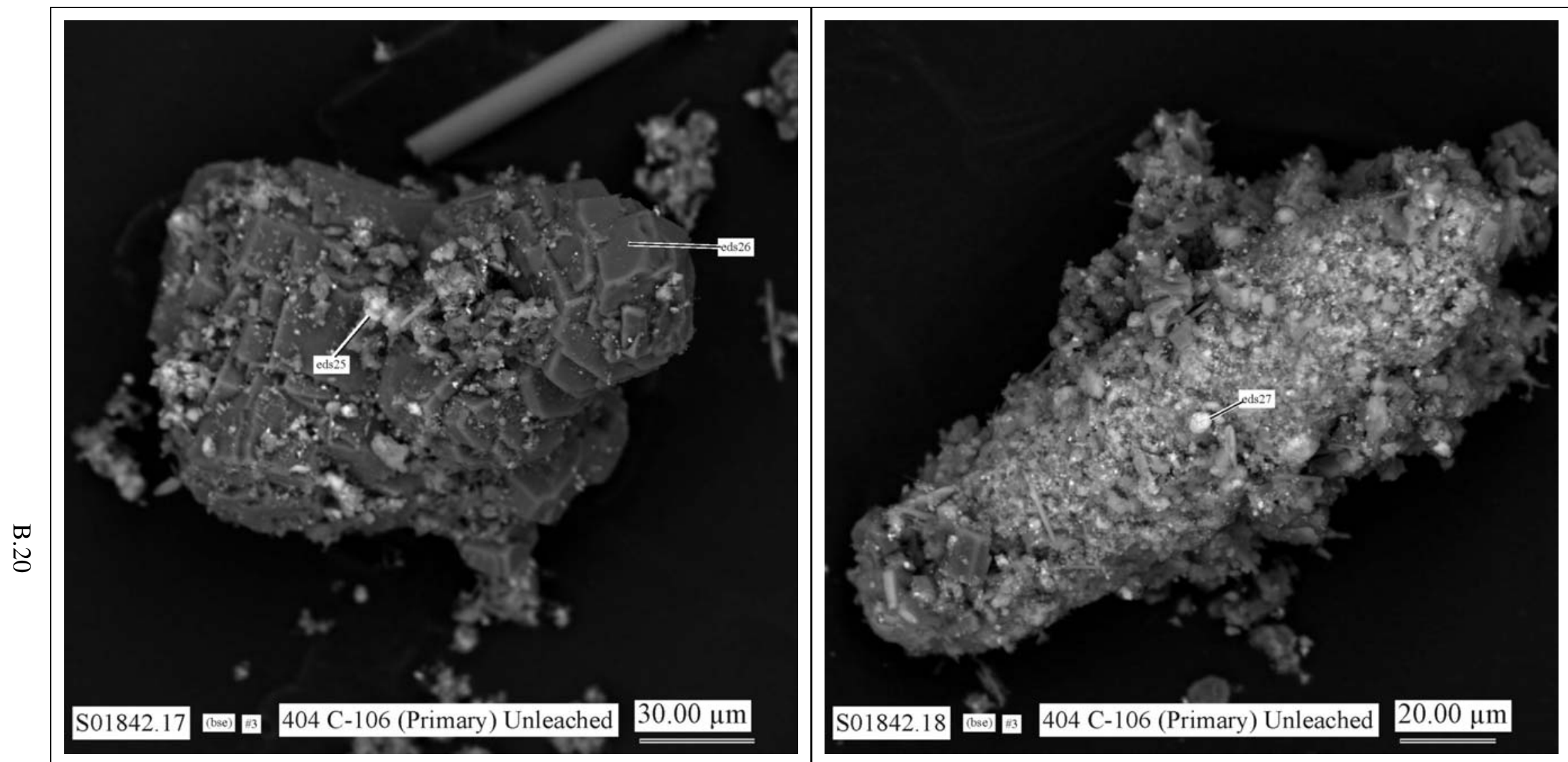

Figure B.32. Micrograph Showing at Higher Magnification the Area Indicated by the White Dashed-Line Square Labeled C in Figure B.28

Figure B.33. Micrograph Showing Morphologies of Typical Particles in SEM Mount 3 of Unleached Sludge Sample 404 


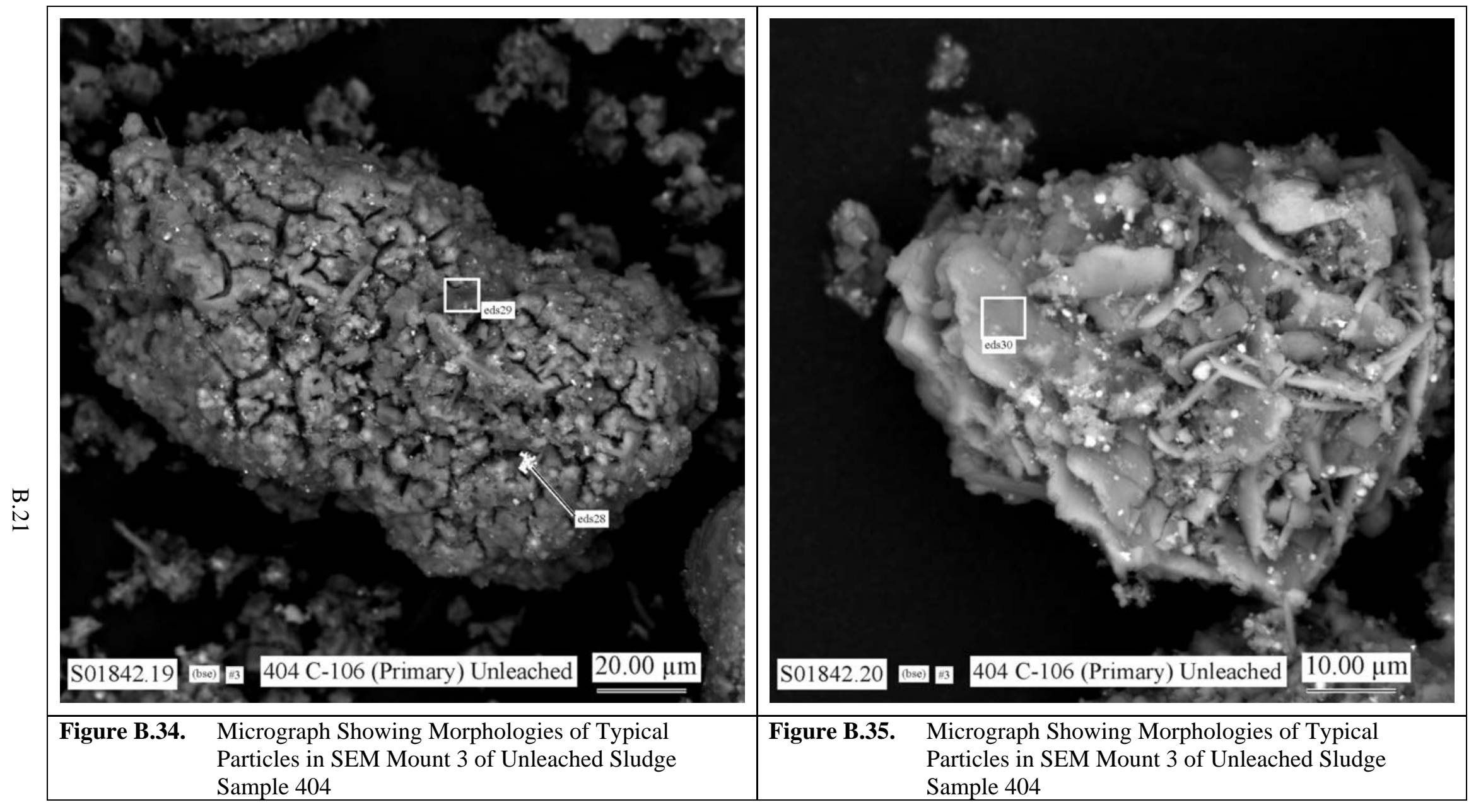




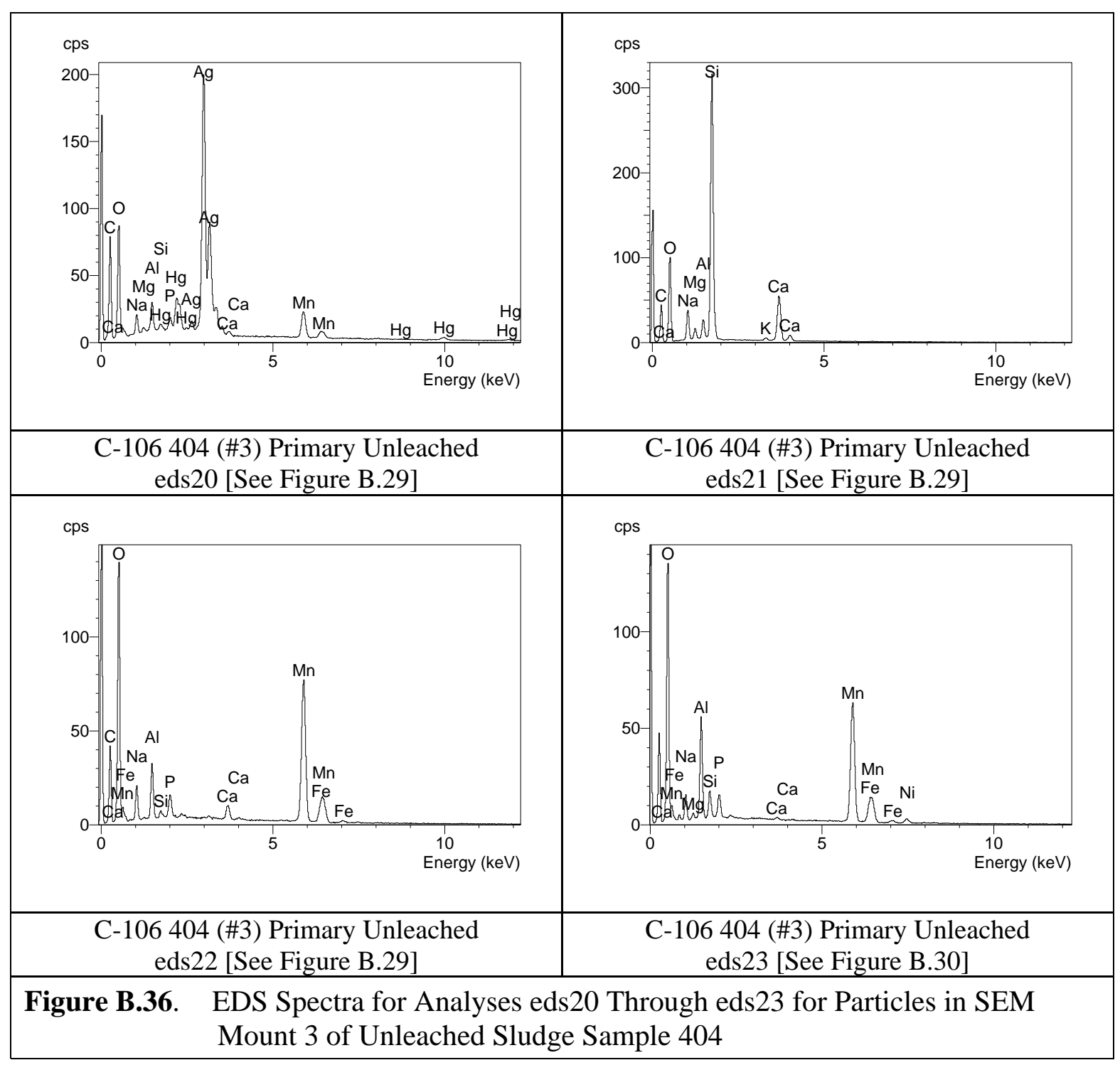




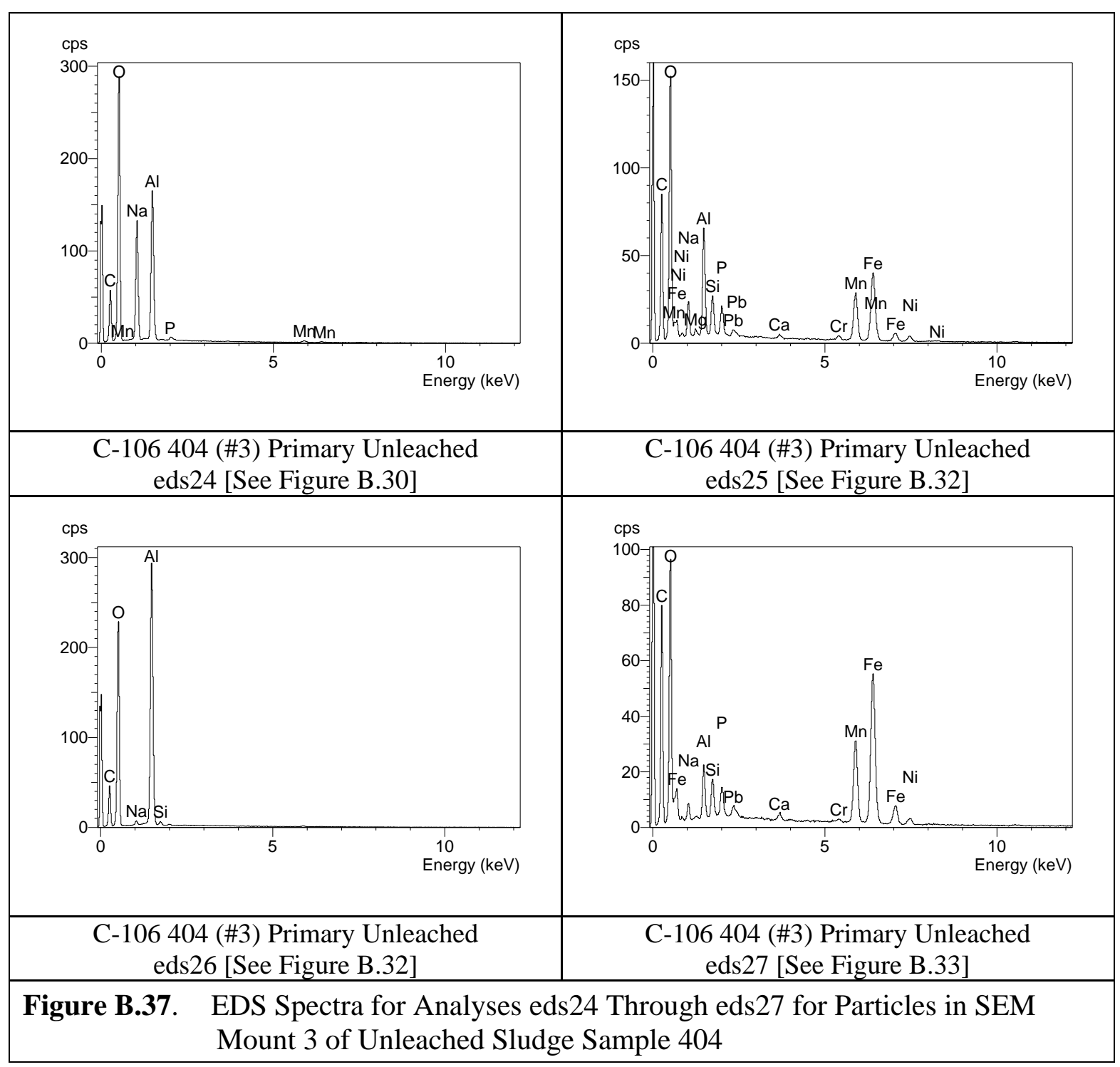




\begin{tabular}{|c|c|c|c|c|}
\hline & \\
\hline
\end{tabular}




\section{B.2 Unleached Sludge Sample 405}

Two mounts of unleached (raw) residual sludge from tank C-106 (duplicate 405) were analyzed by SEM/EDS. The SEM micrographs for the first mount (4) of sample 405 sludge are shown in Figures B.39 through B.52. The EDS spectra for this mount are given in Figures B.53 through B.57. The SEM micrographs for the second mount (6) of sample 405 sludge are shown in Figures B.58 through B.67, and the EDS spectra for the second mount are given in Figures B.68 through B.70. 


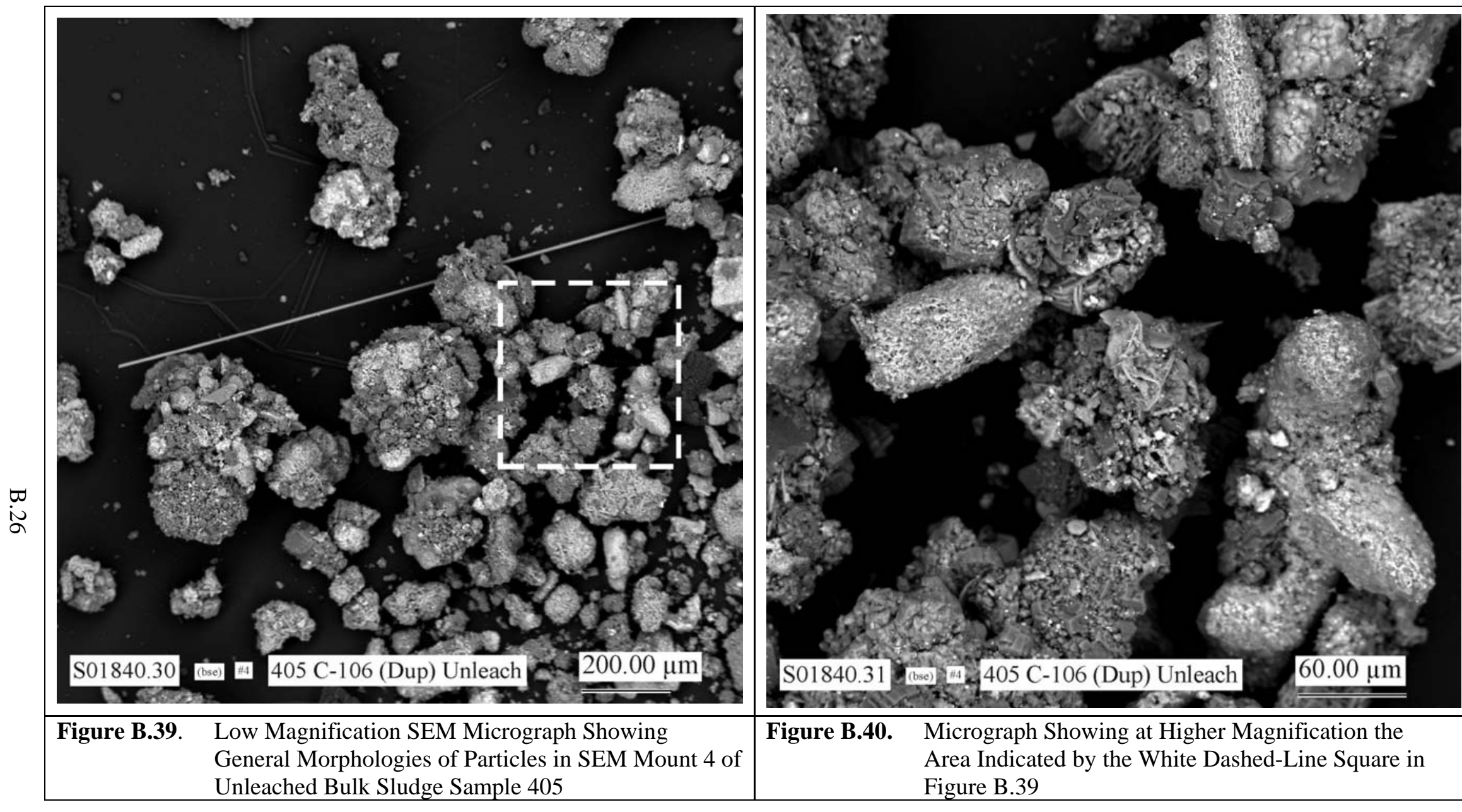




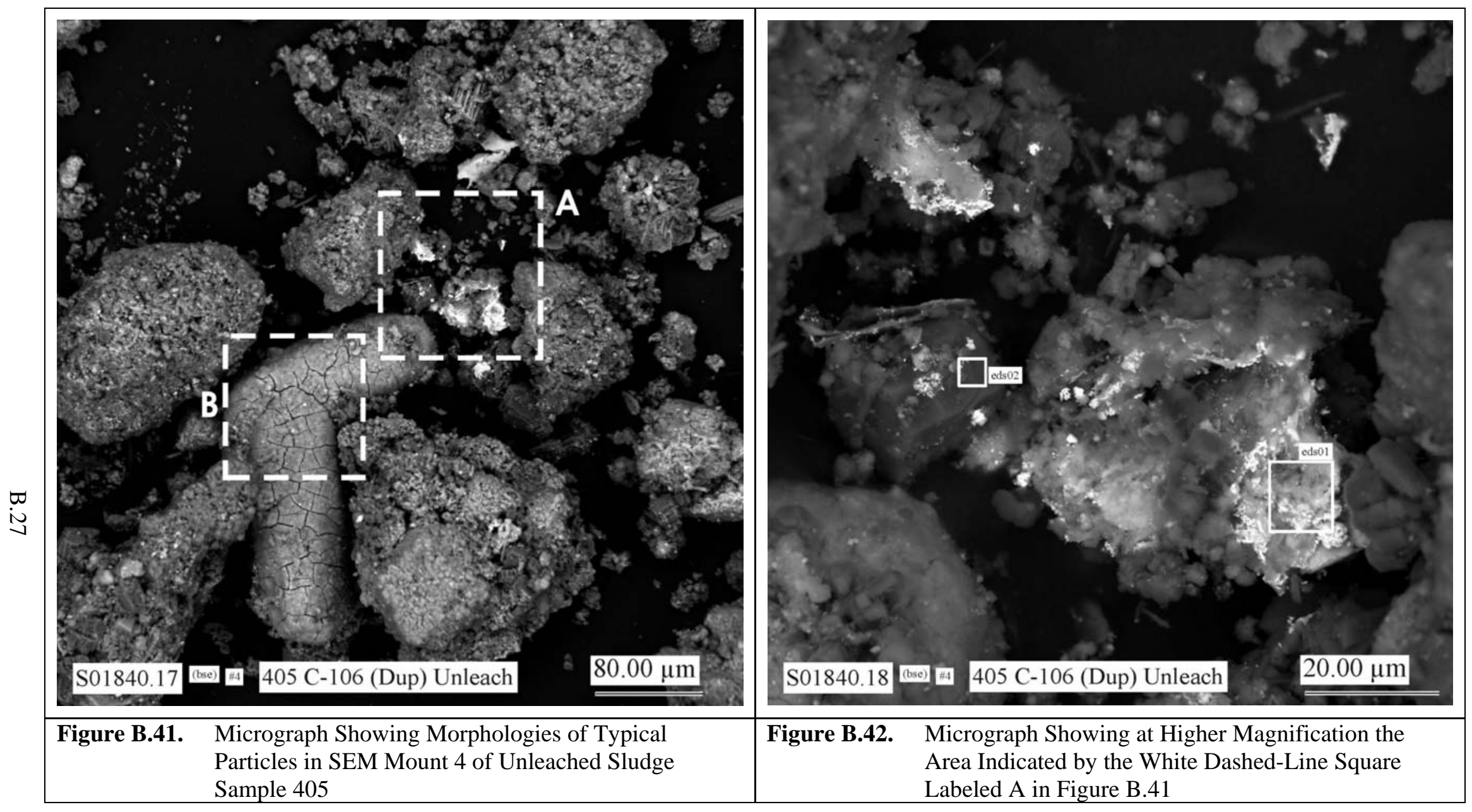




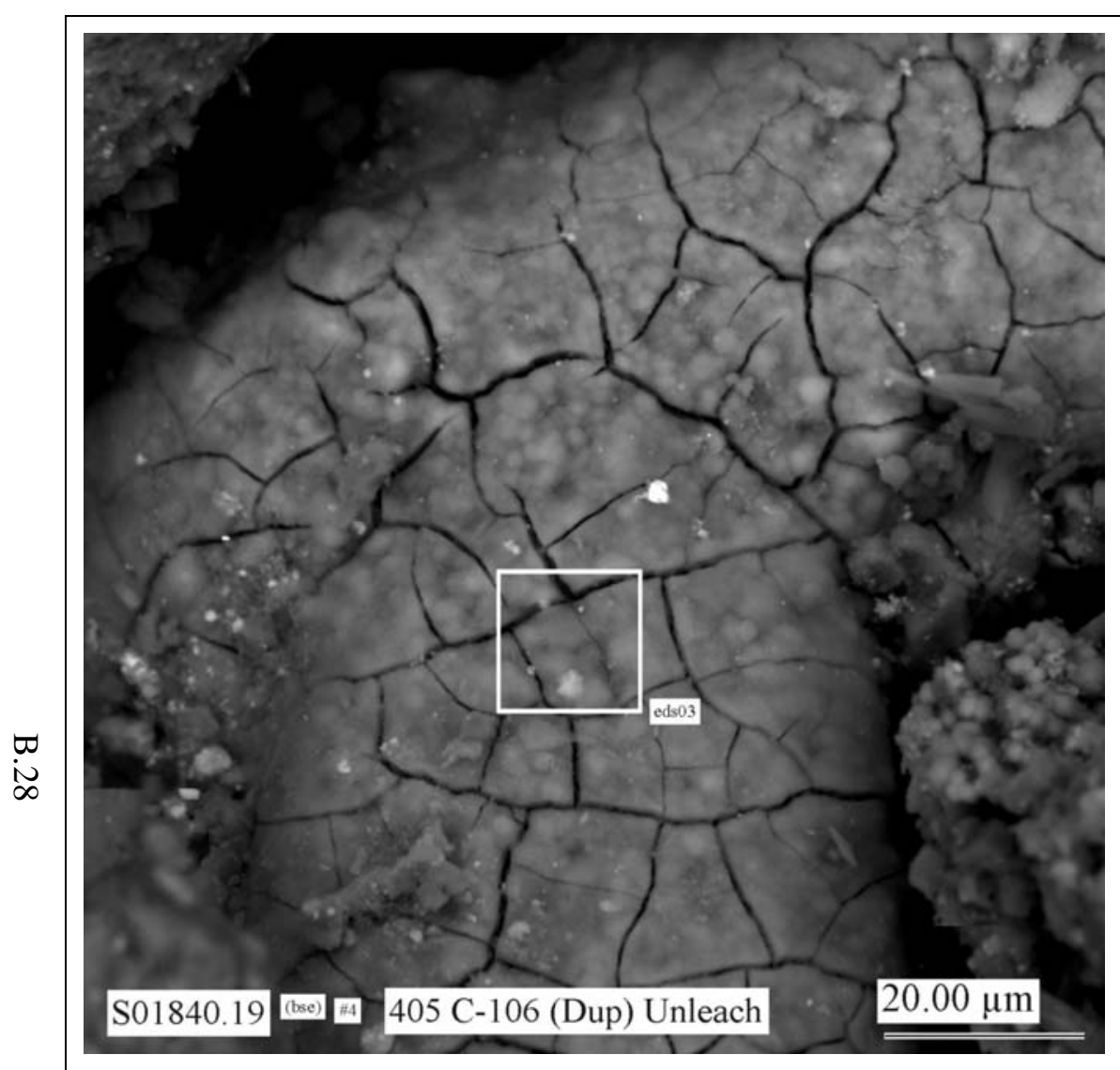

Figure B.43. Micrograph Showing at Higher Magnification the Area Indicated by the White Dashed-Line Square Labeled B in Figure B.41

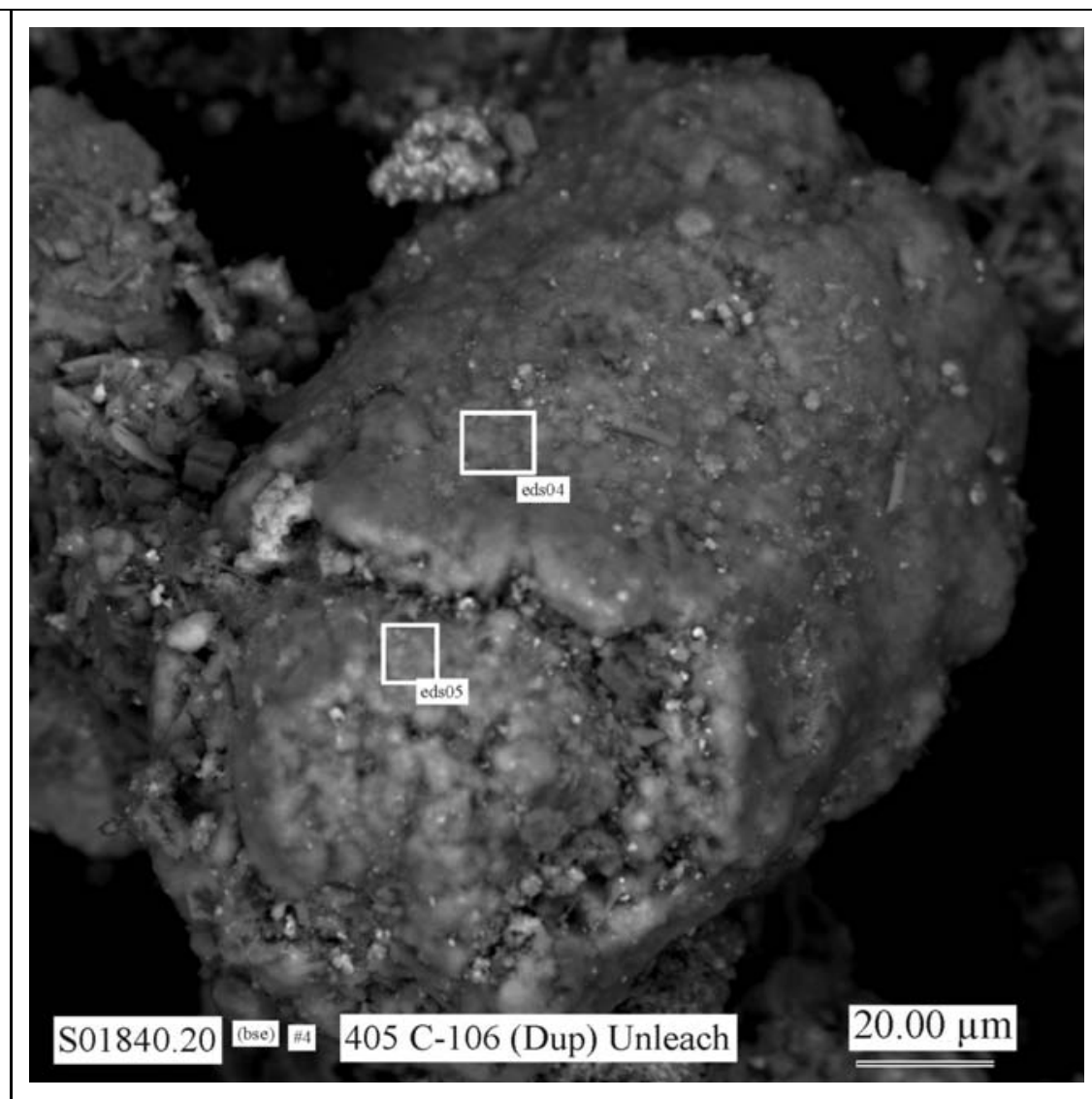

Figure B.44. Micrograph Showing Morphologies of Typical Particles in SEM Mount 4 of Unleached Sludge Sample 405 


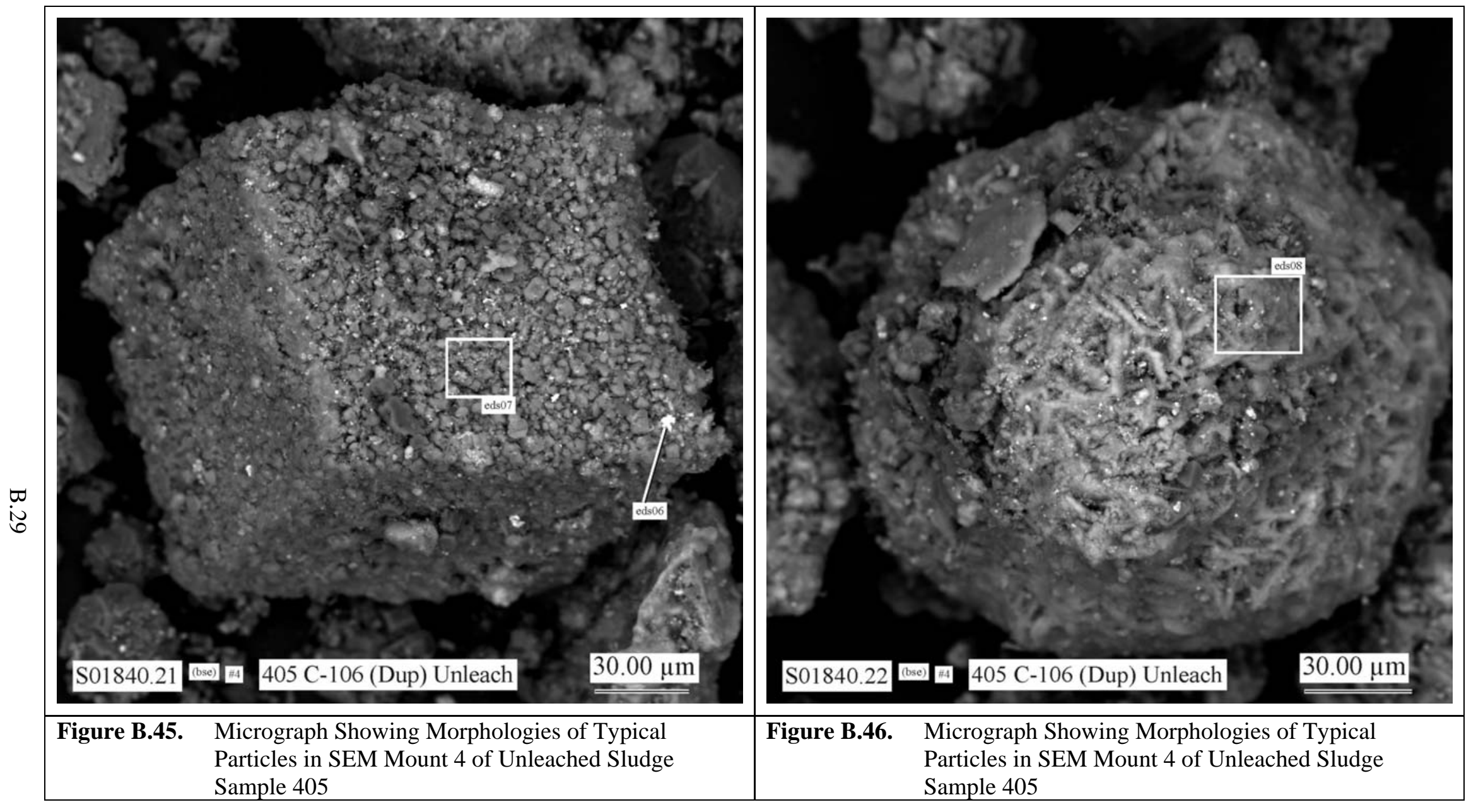




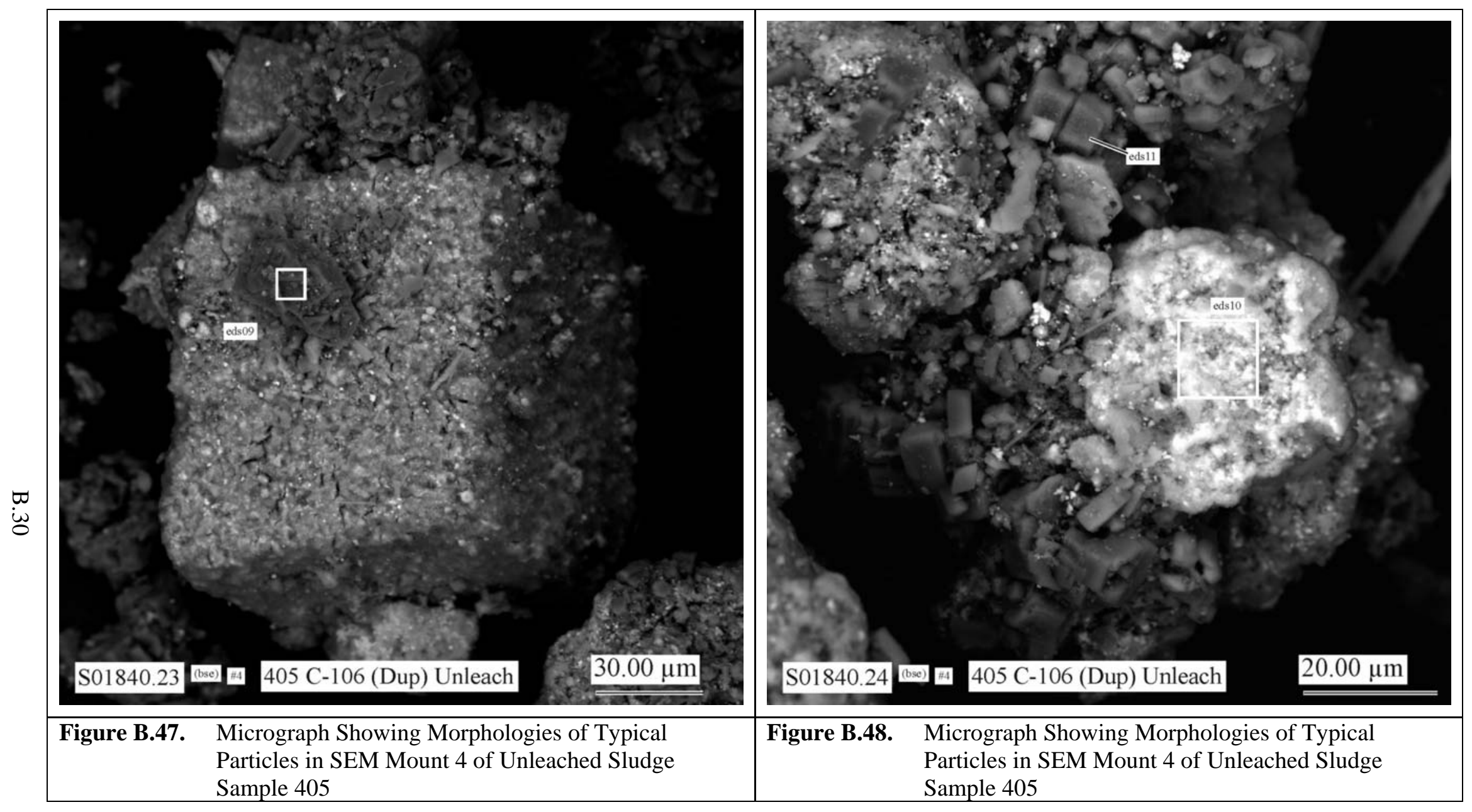



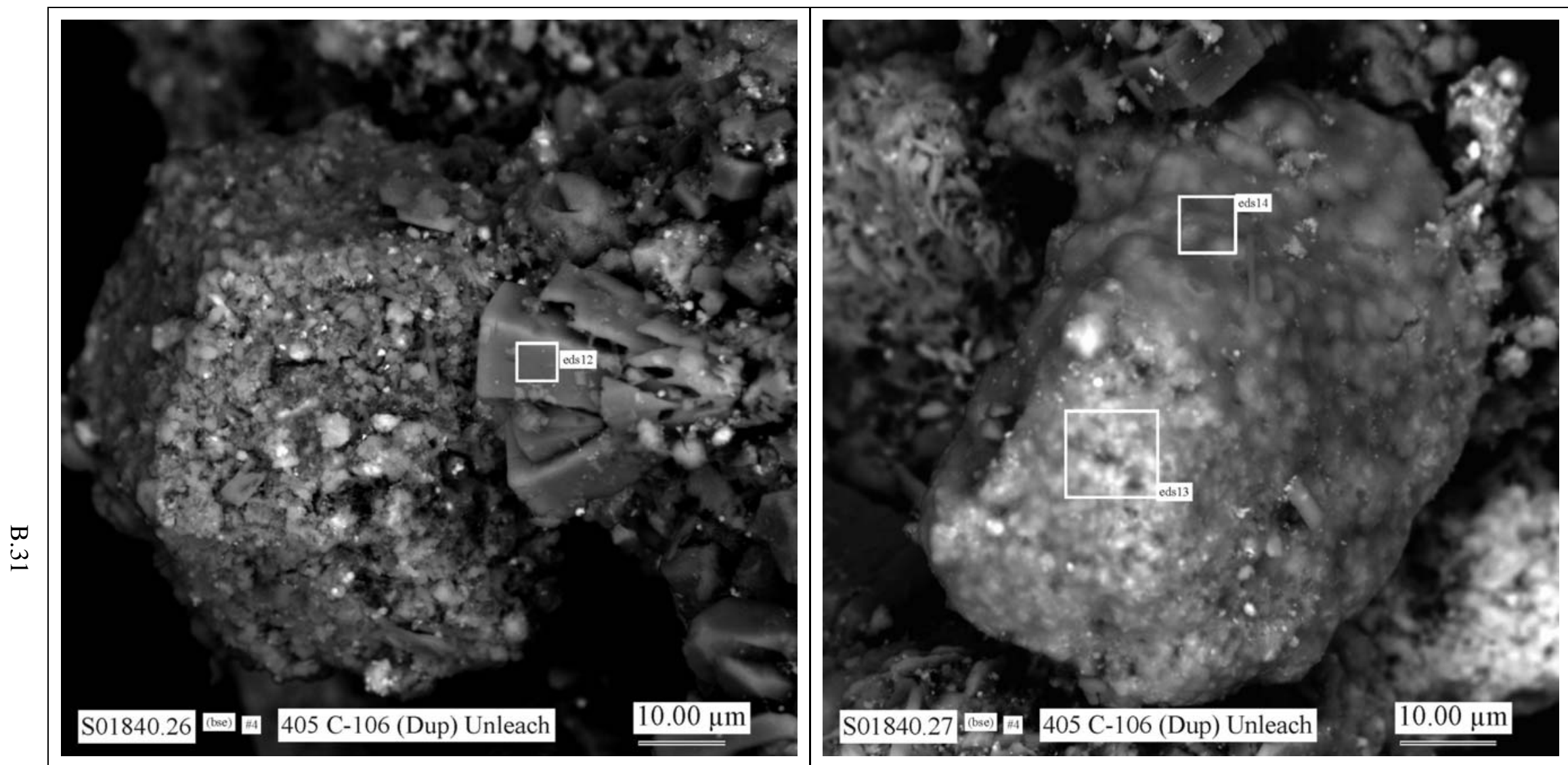

Figure B.49. Micrograph Showing Morphologies of Typical Particles in SEM Mount 4 of Unleached Sludge Sample 405

Figure B.50. Micrograph Showing Morphologies of Typical Particles in SEM Mount 4 of Unleached Sludge Sample 405 


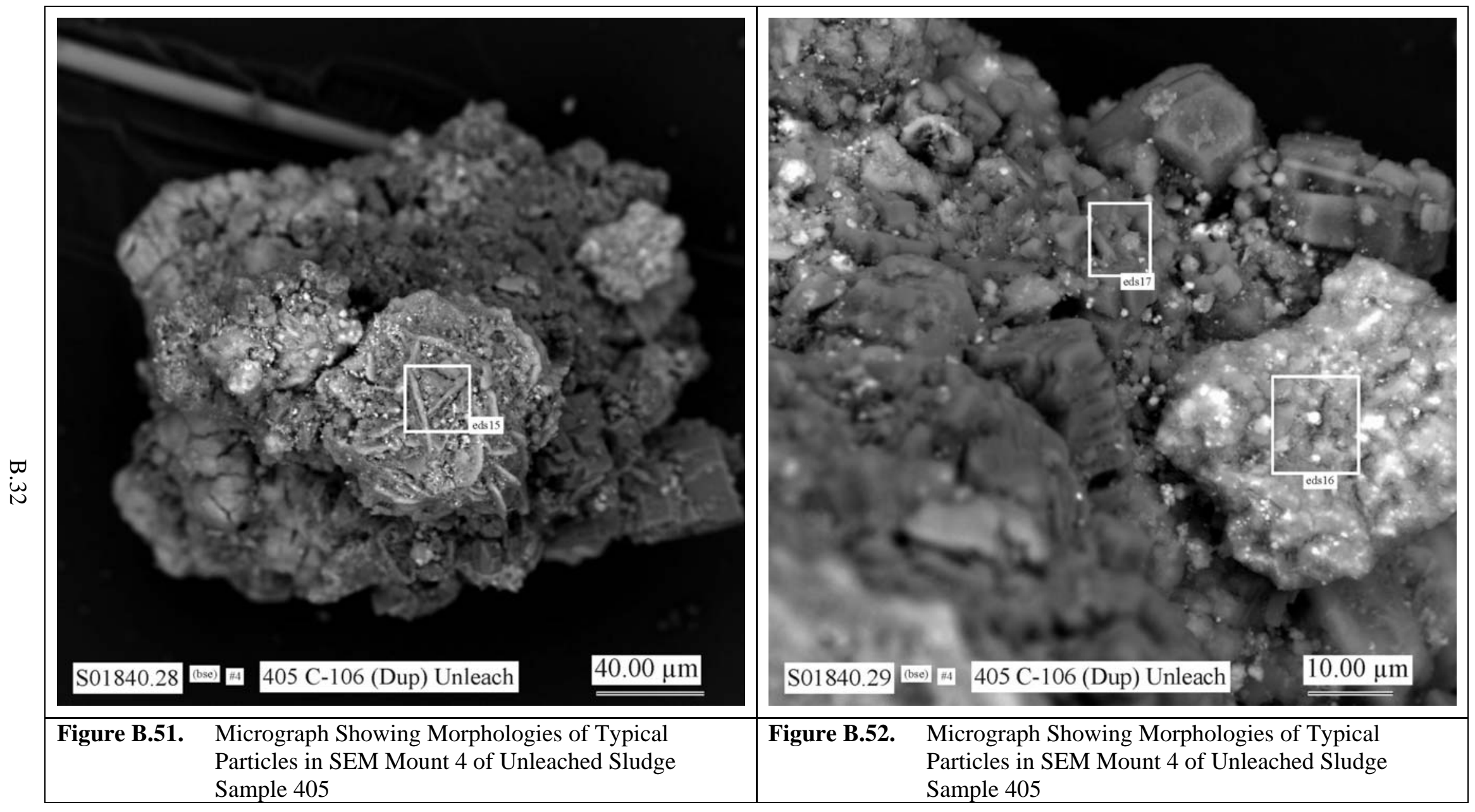




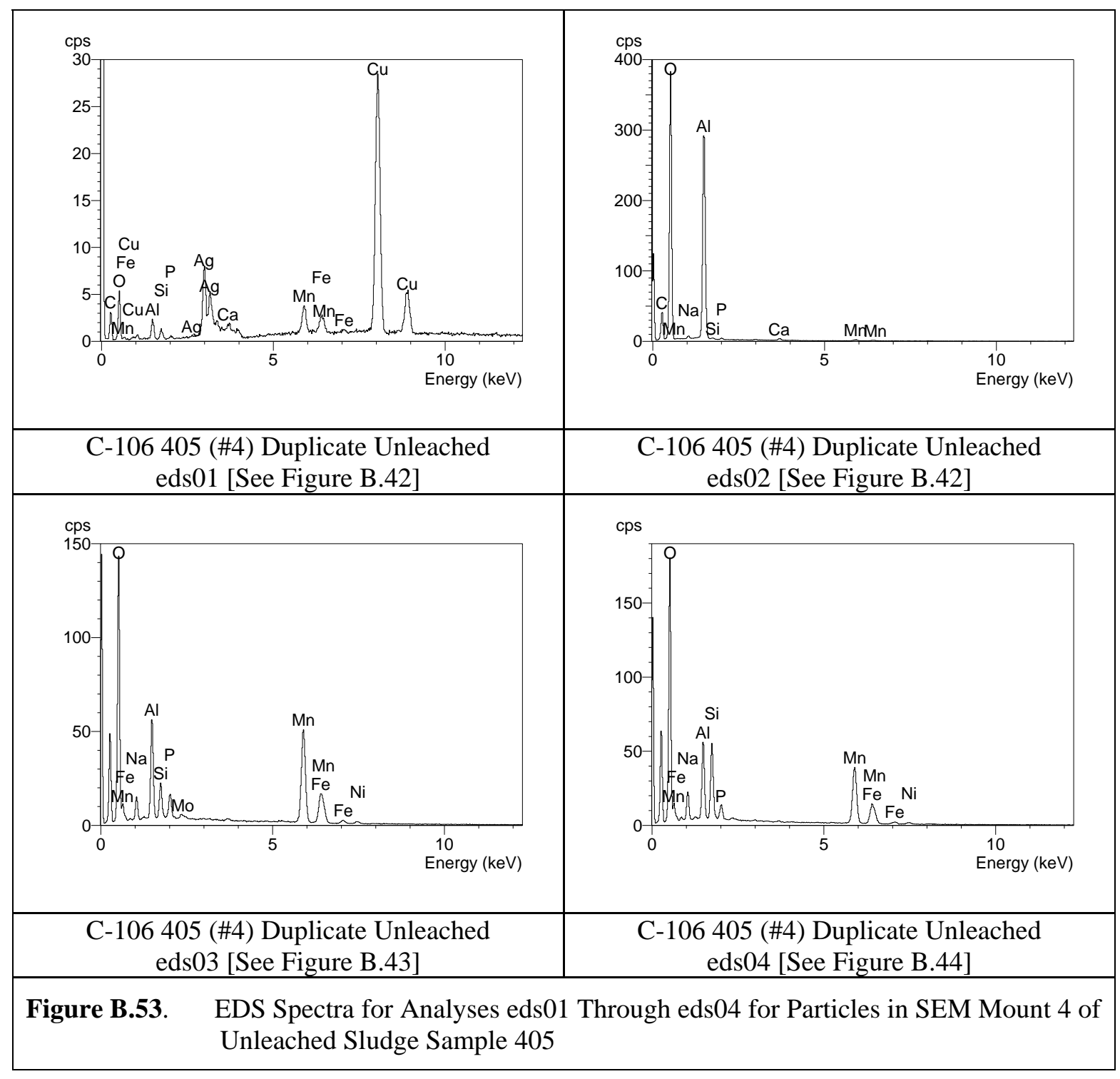




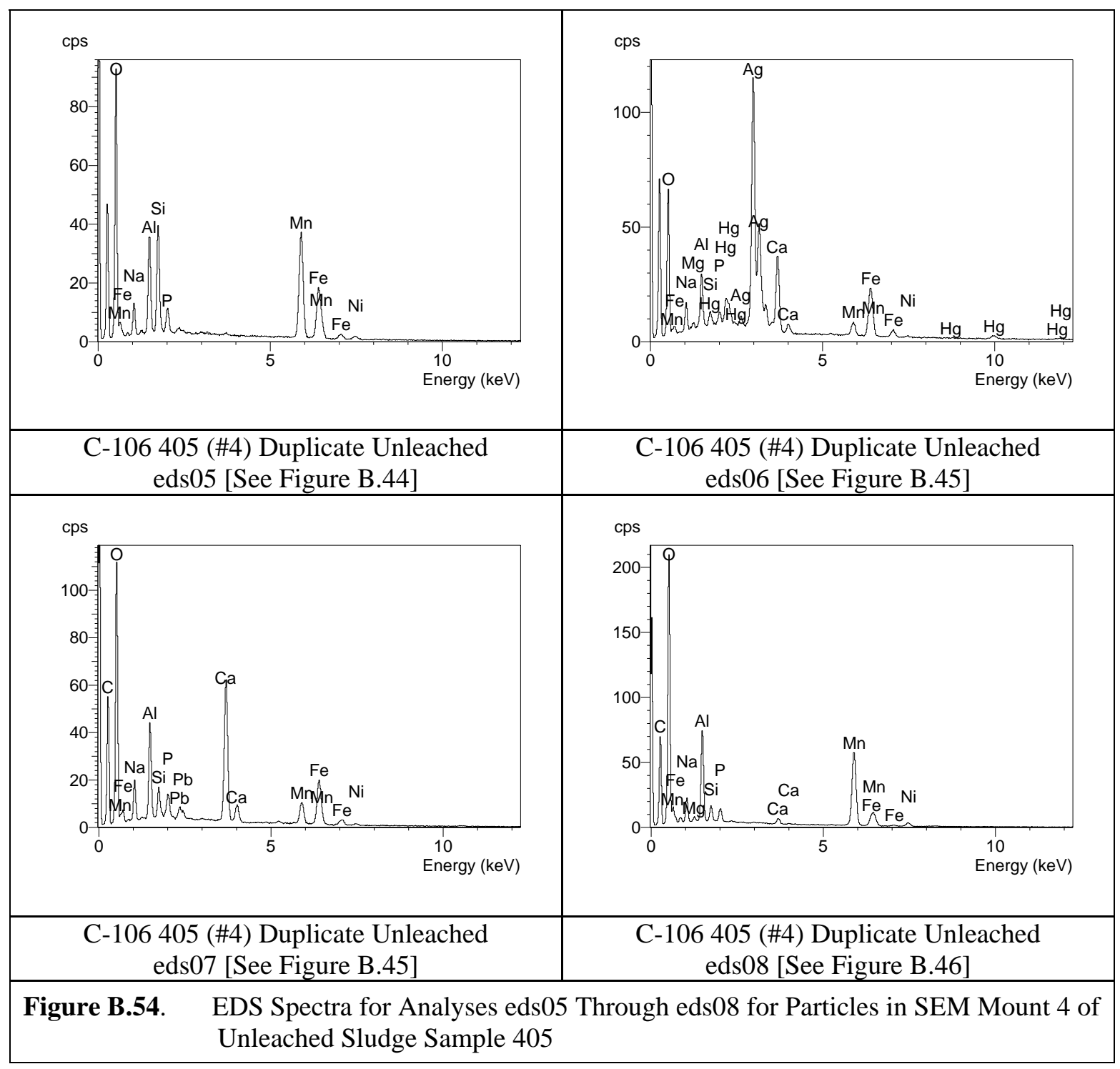




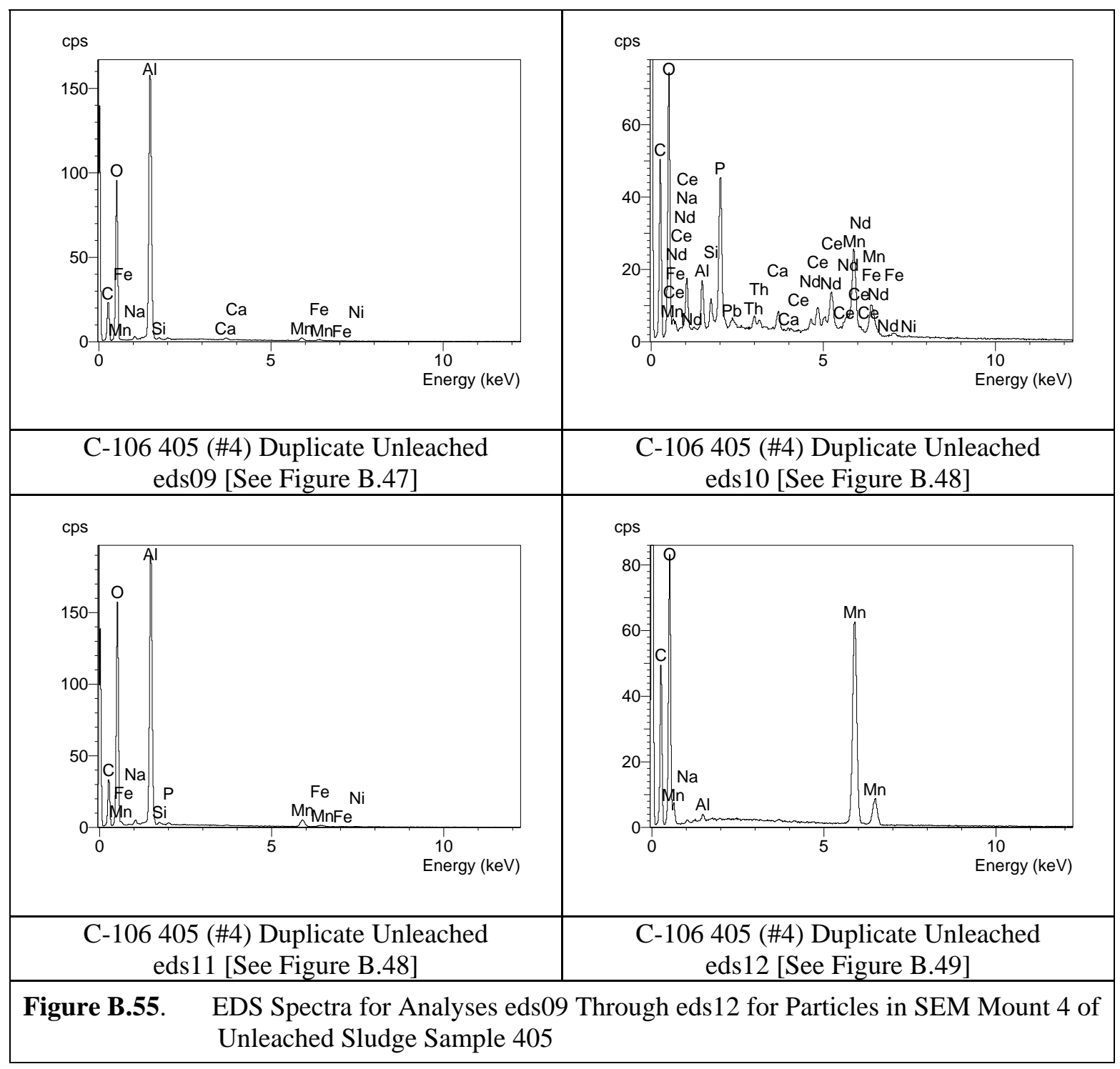




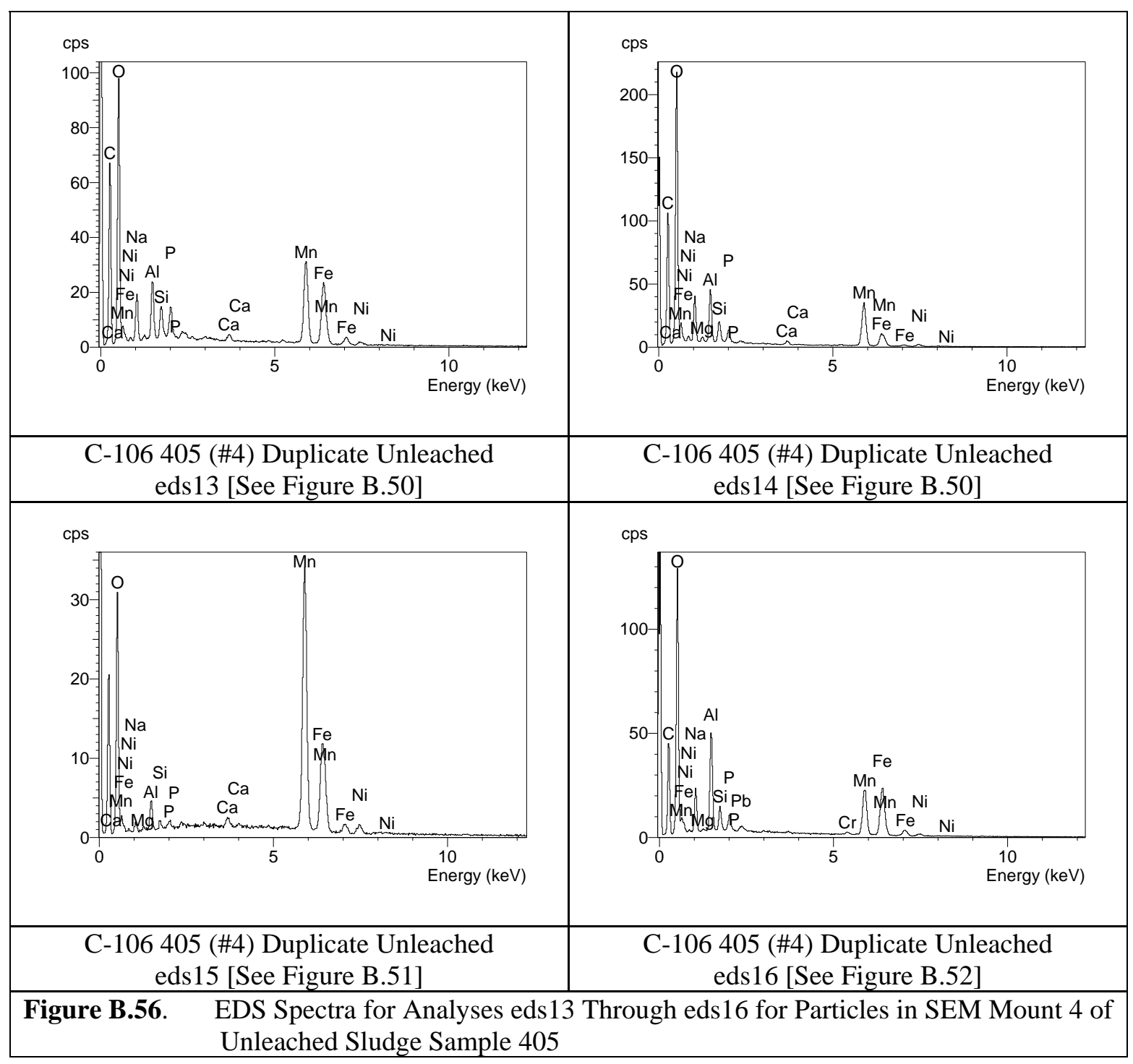




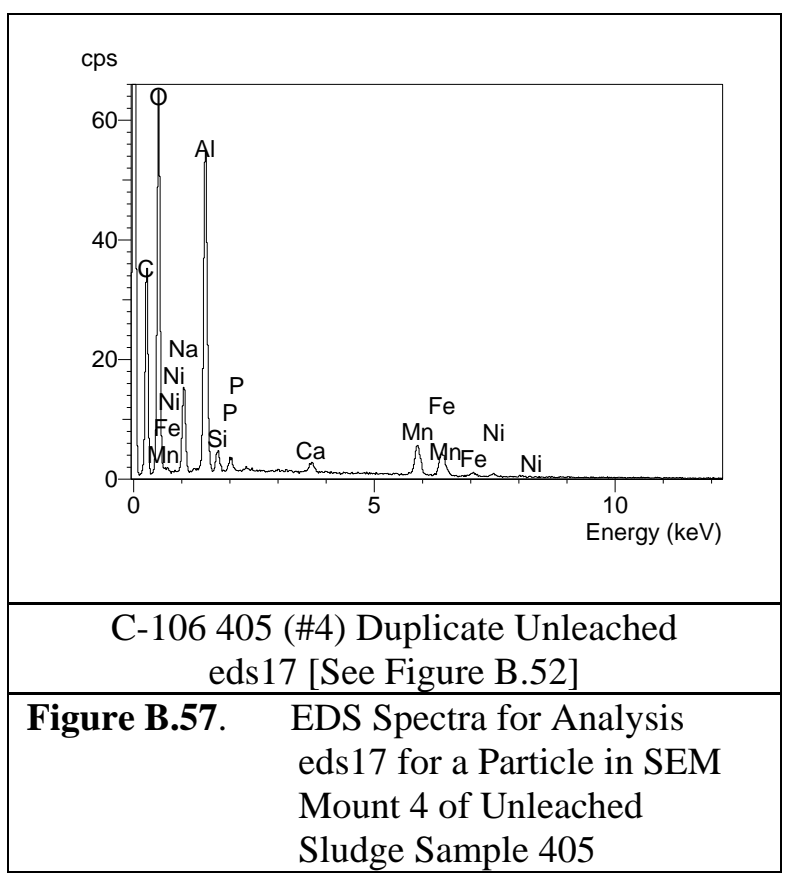

B.37 


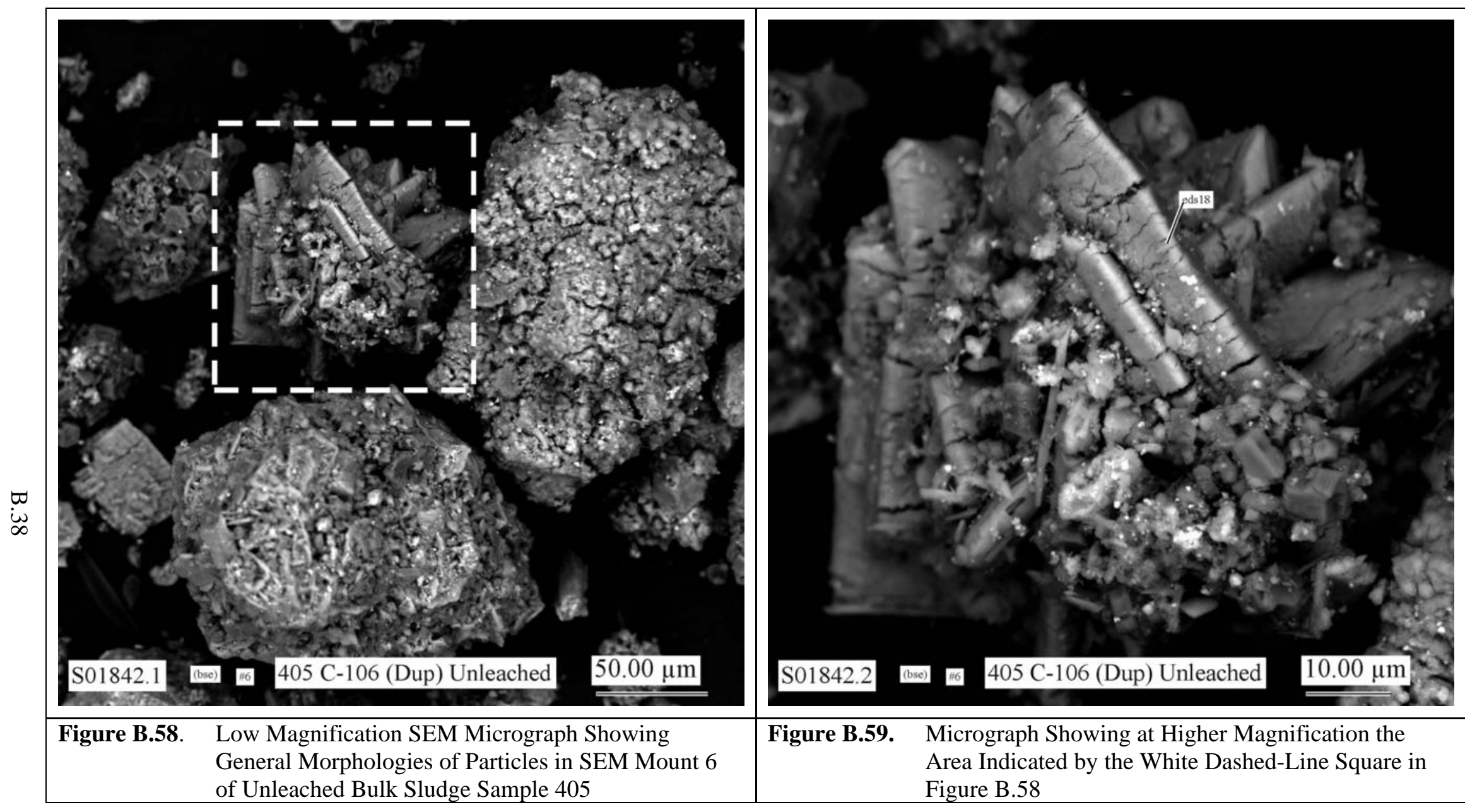




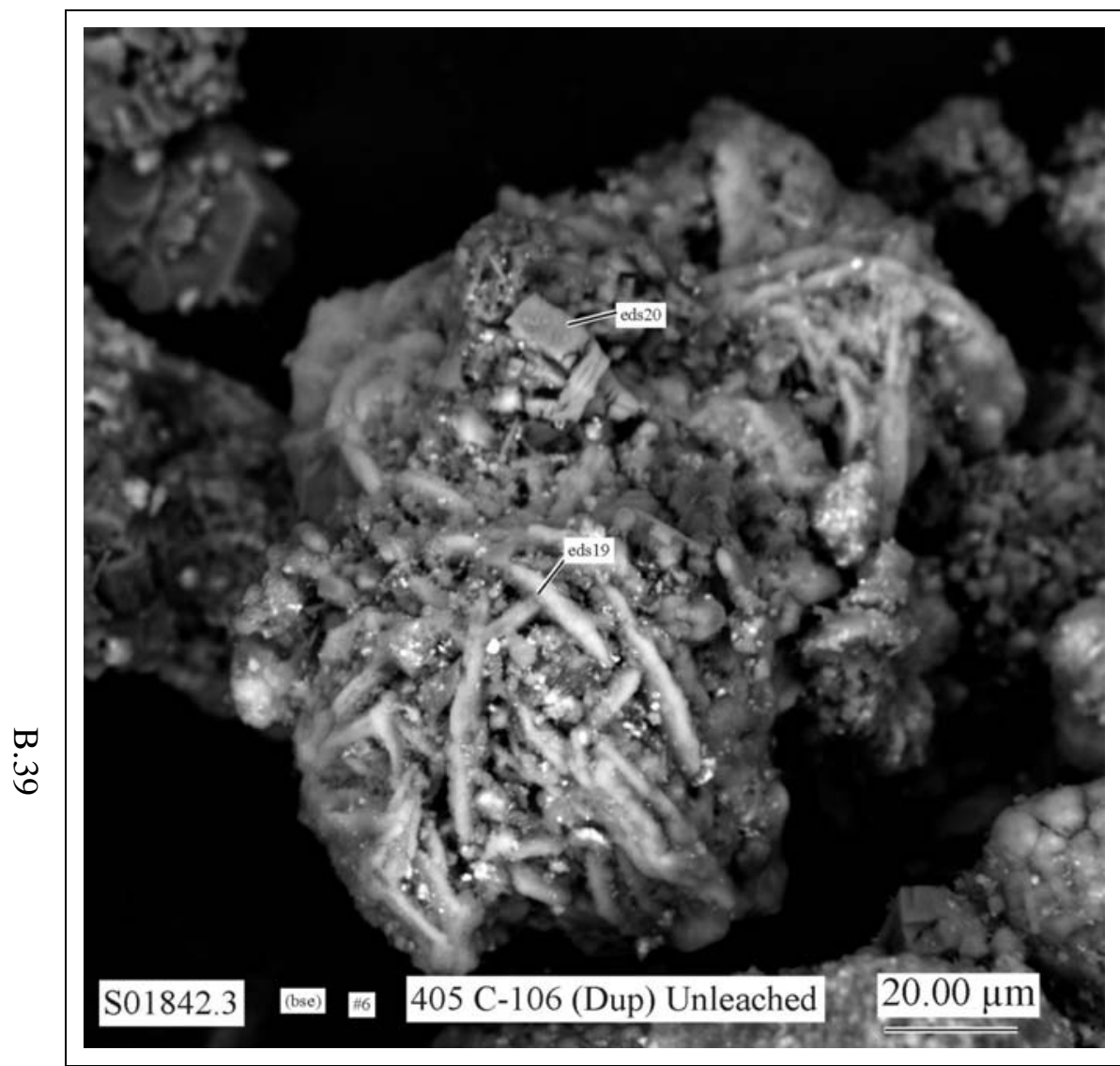

Figure B.60. $\quad$ Micrograph Showing Morphologies of Typical Particles in SEM Mount 6 of Unleached Sludge Sample 405

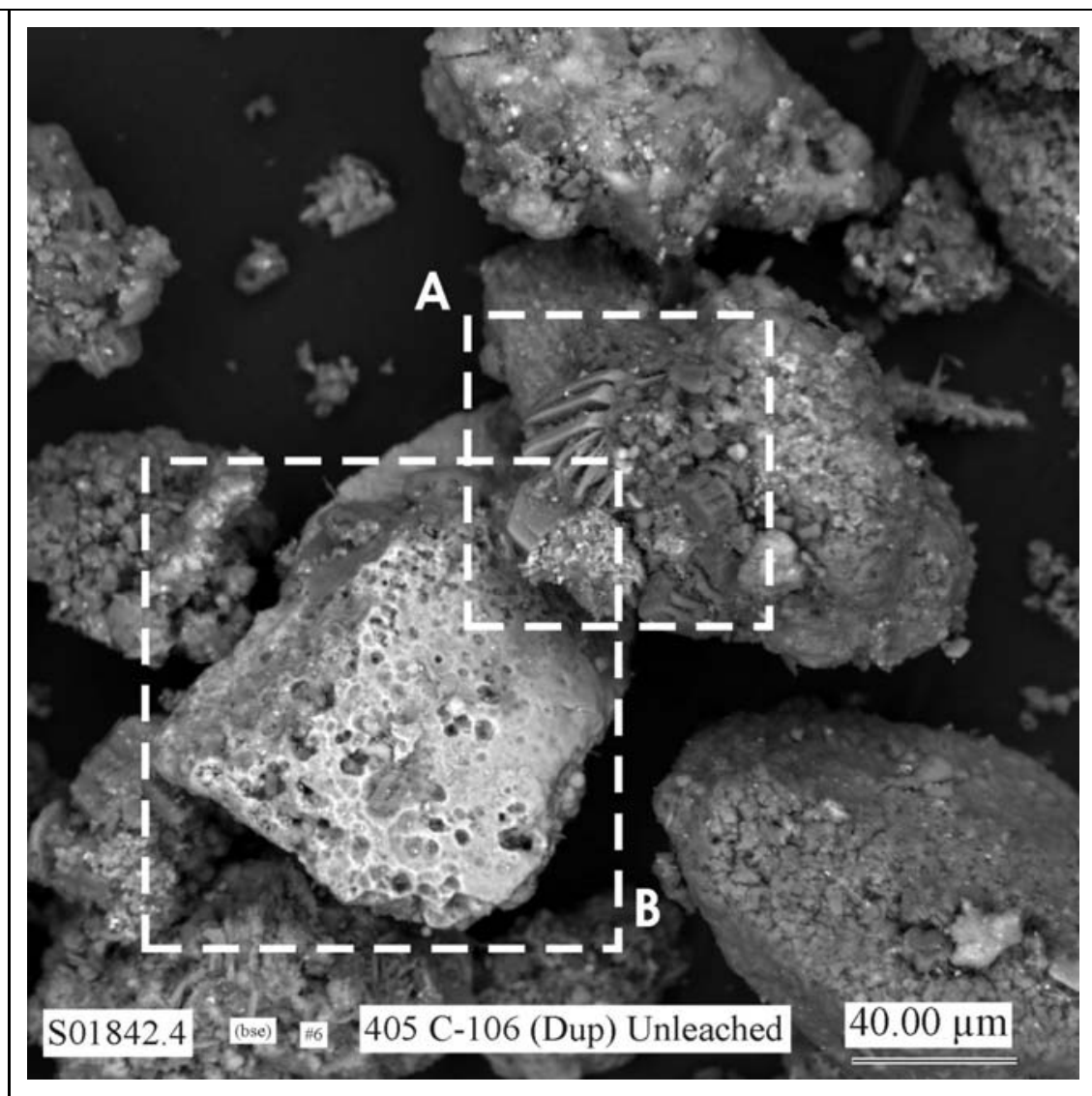

Figure B.61. Micrograph Showing Morphologies of Typical Particles in SEM Mount 6 of Unleached Sludge Sample 405 


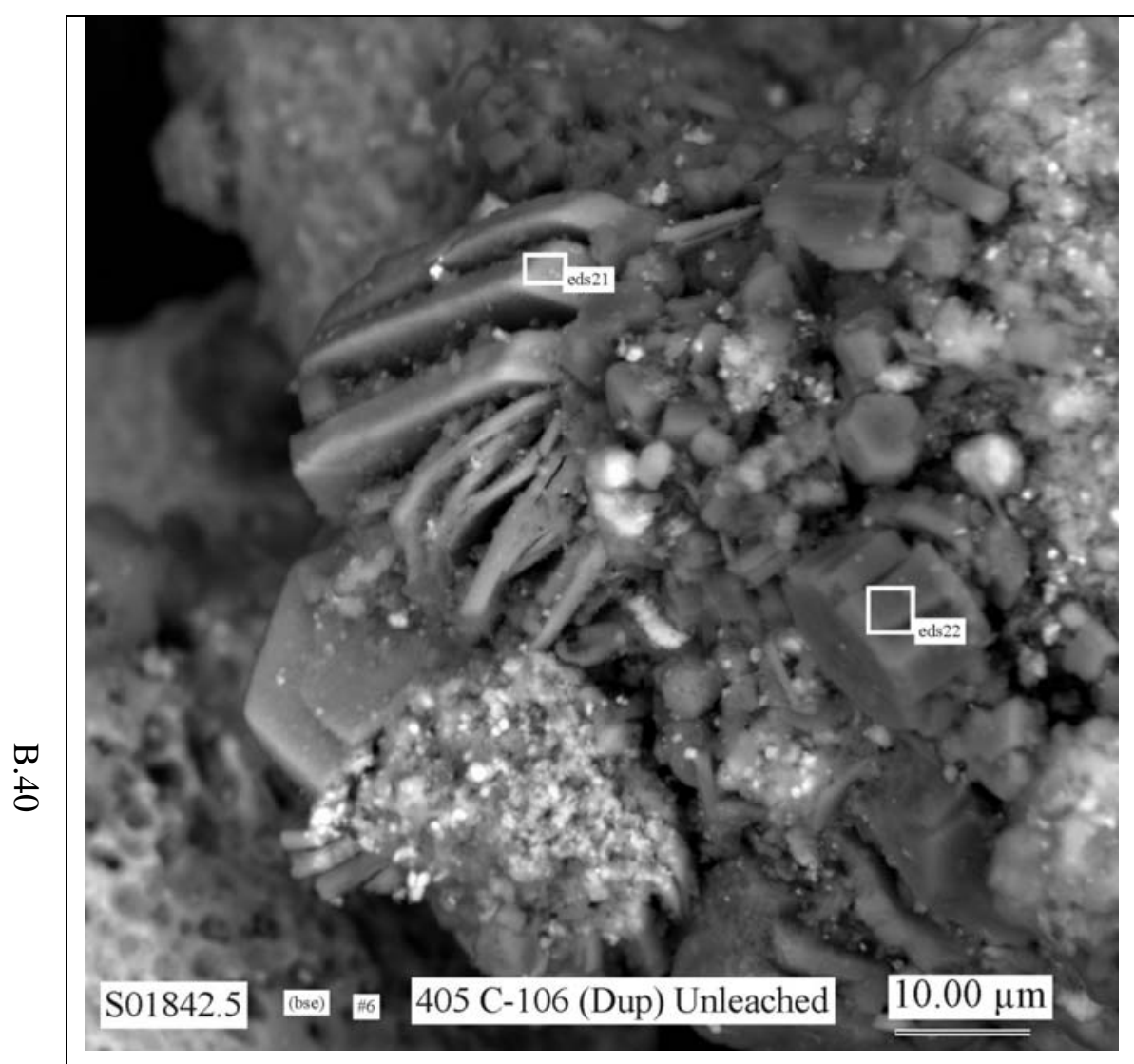

Figure B.62. Micrograph Showing at Higher Magnification the Area Indicated by the White Dashed-Line Square Labeled A in Figure B.61

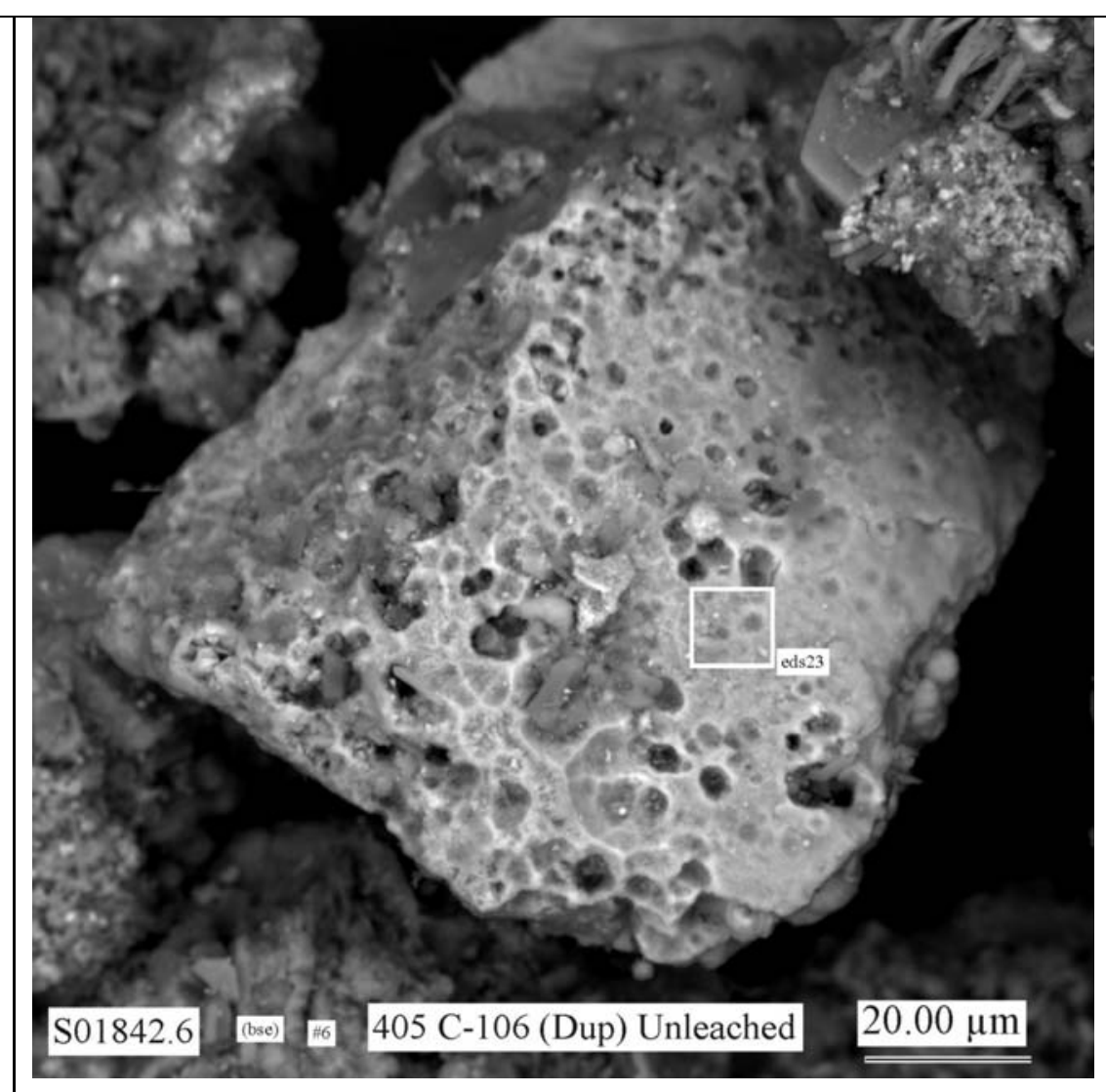

Figure B.63. Micrograph Showing at Higher Magnification the Area Indicated by the White Dashed-Line Square Labeled b in Figure B.61 


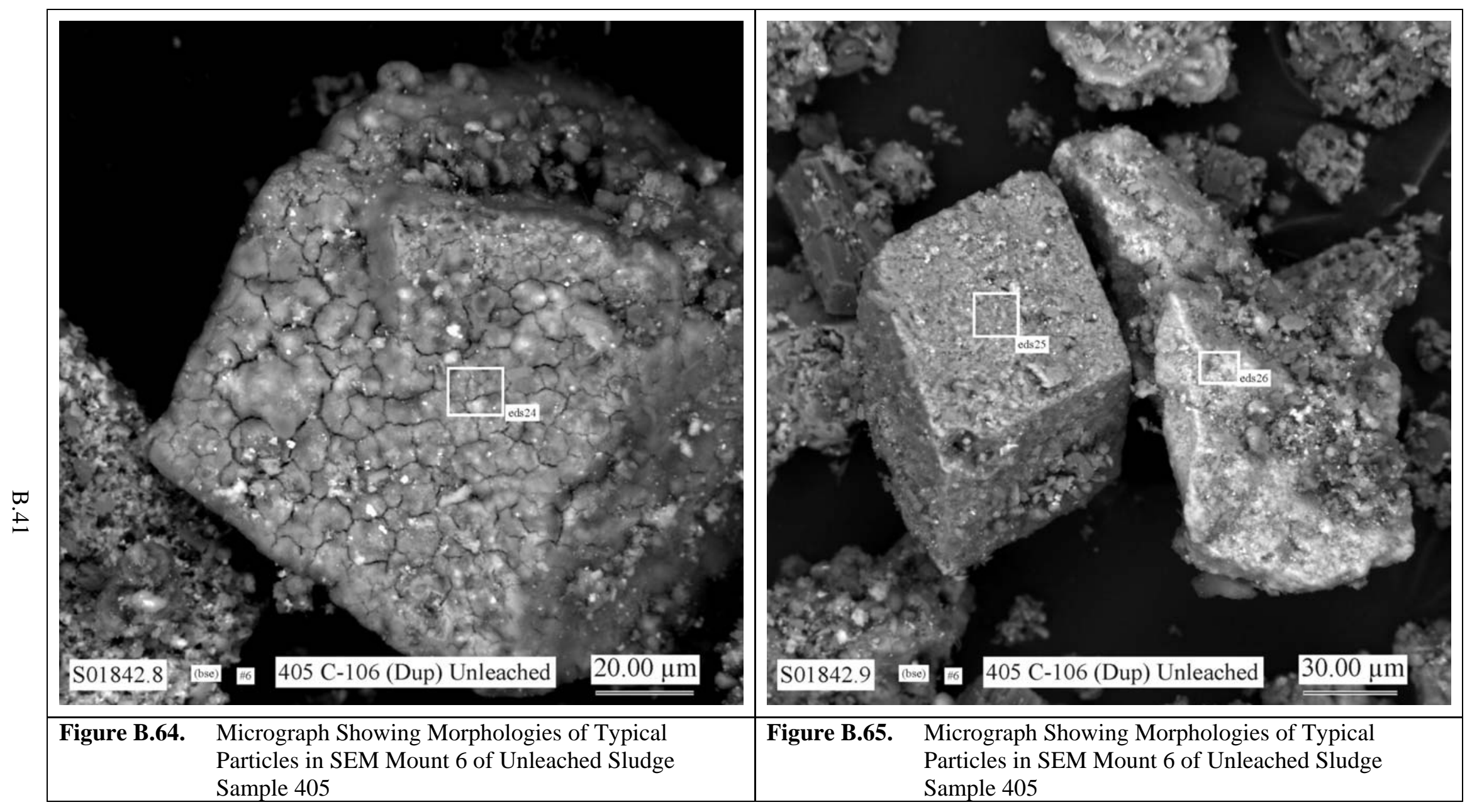




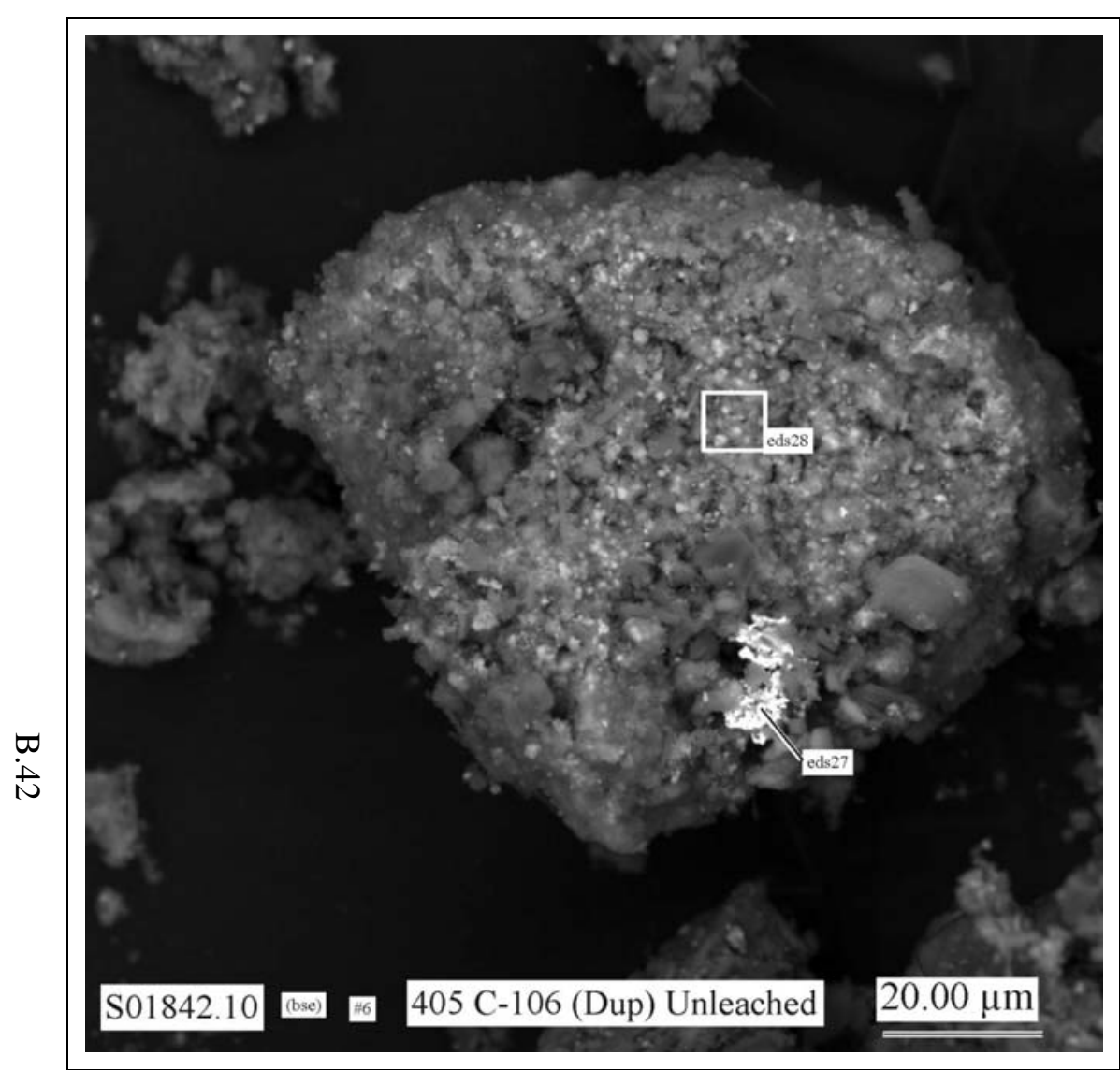

Figure B.66. $\quad$ Micrograph Showing Morphologies of Typical Particles in SEM Mount 6 of Unleached Sludge Sample 405

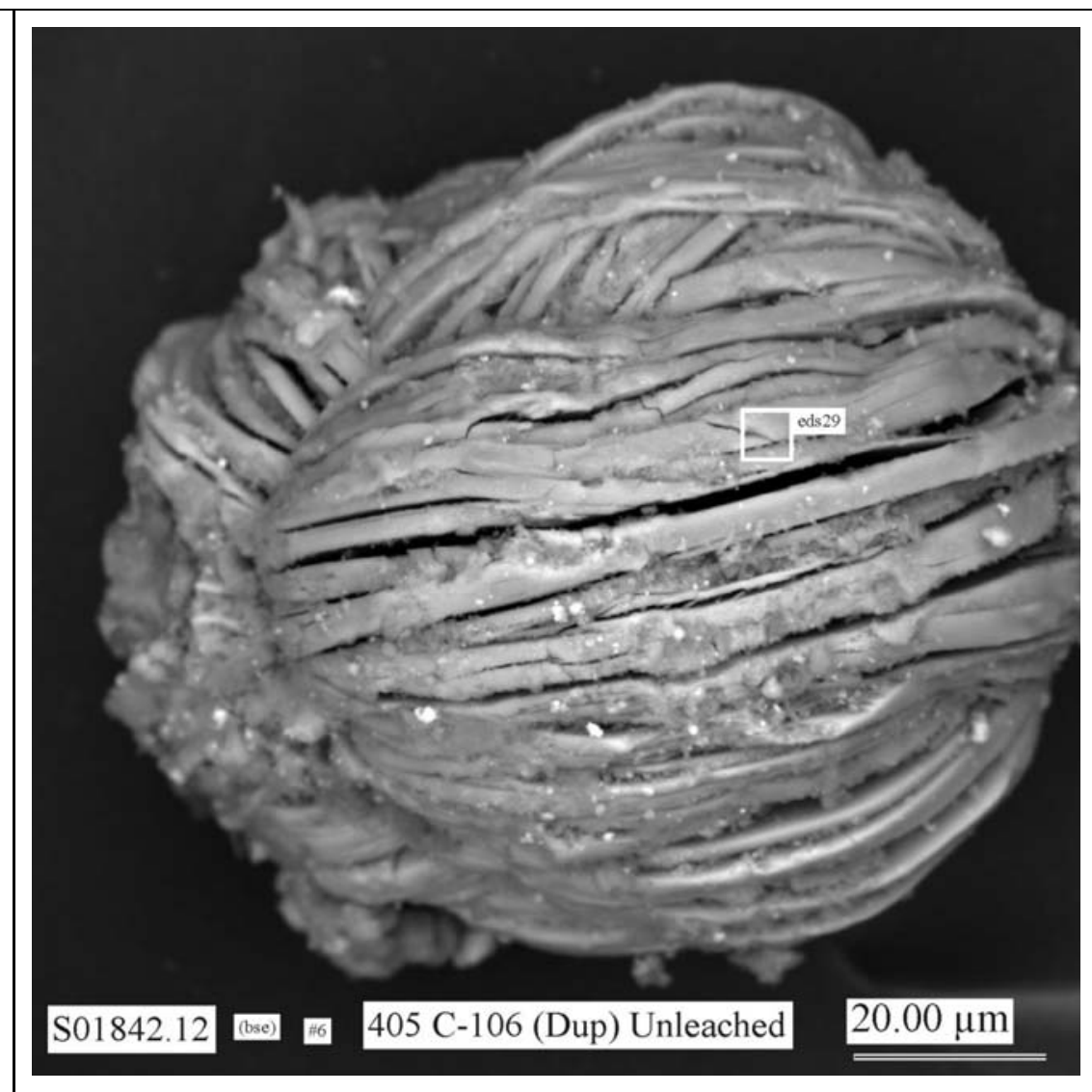

Figure B.67. Micrograph Showing Morphology of Unusual Particle (composed primarily of Mn, P, O, and possibly C) in SEM Mount 6 of Unleached Sludge Sample 405 


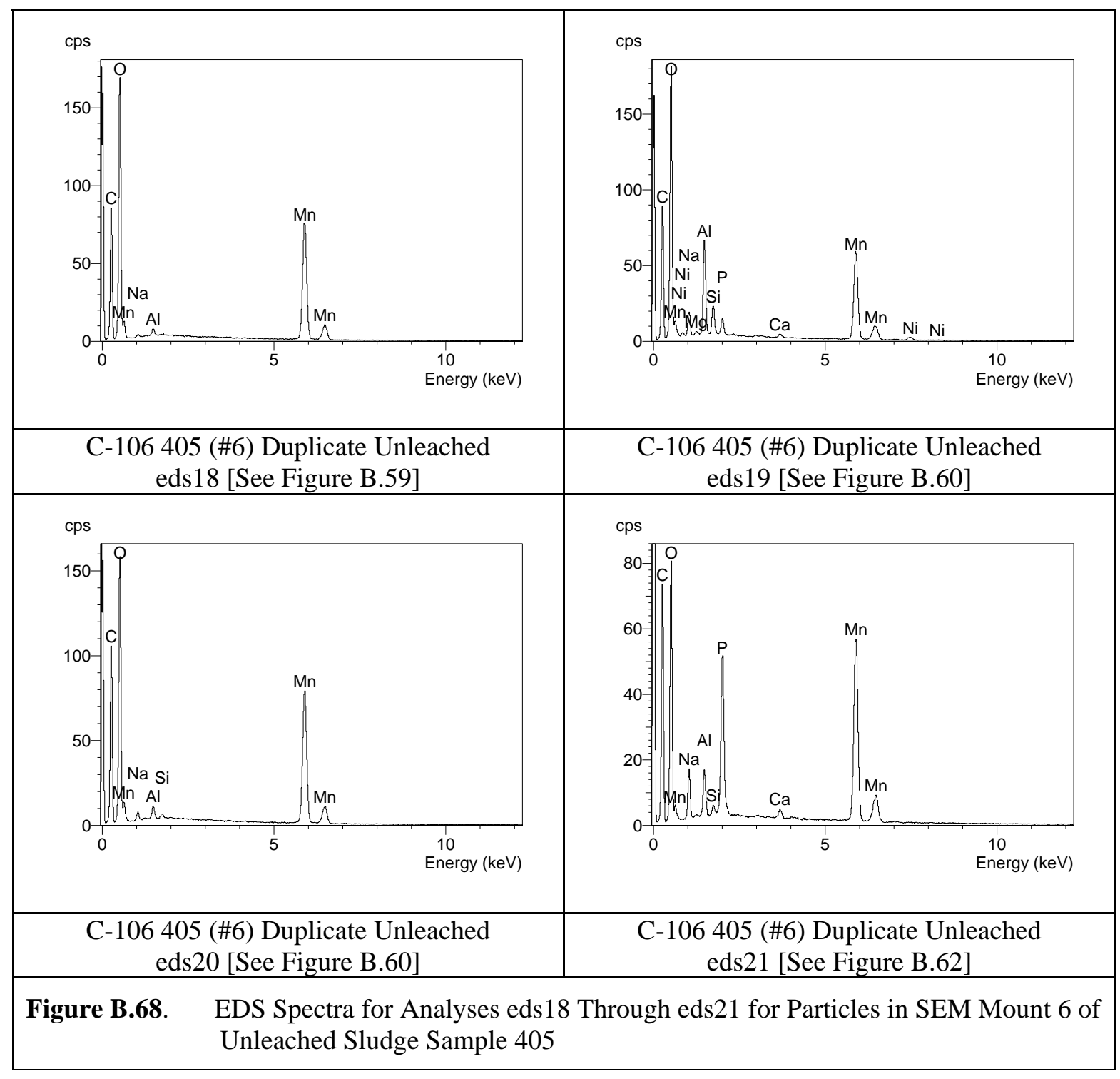




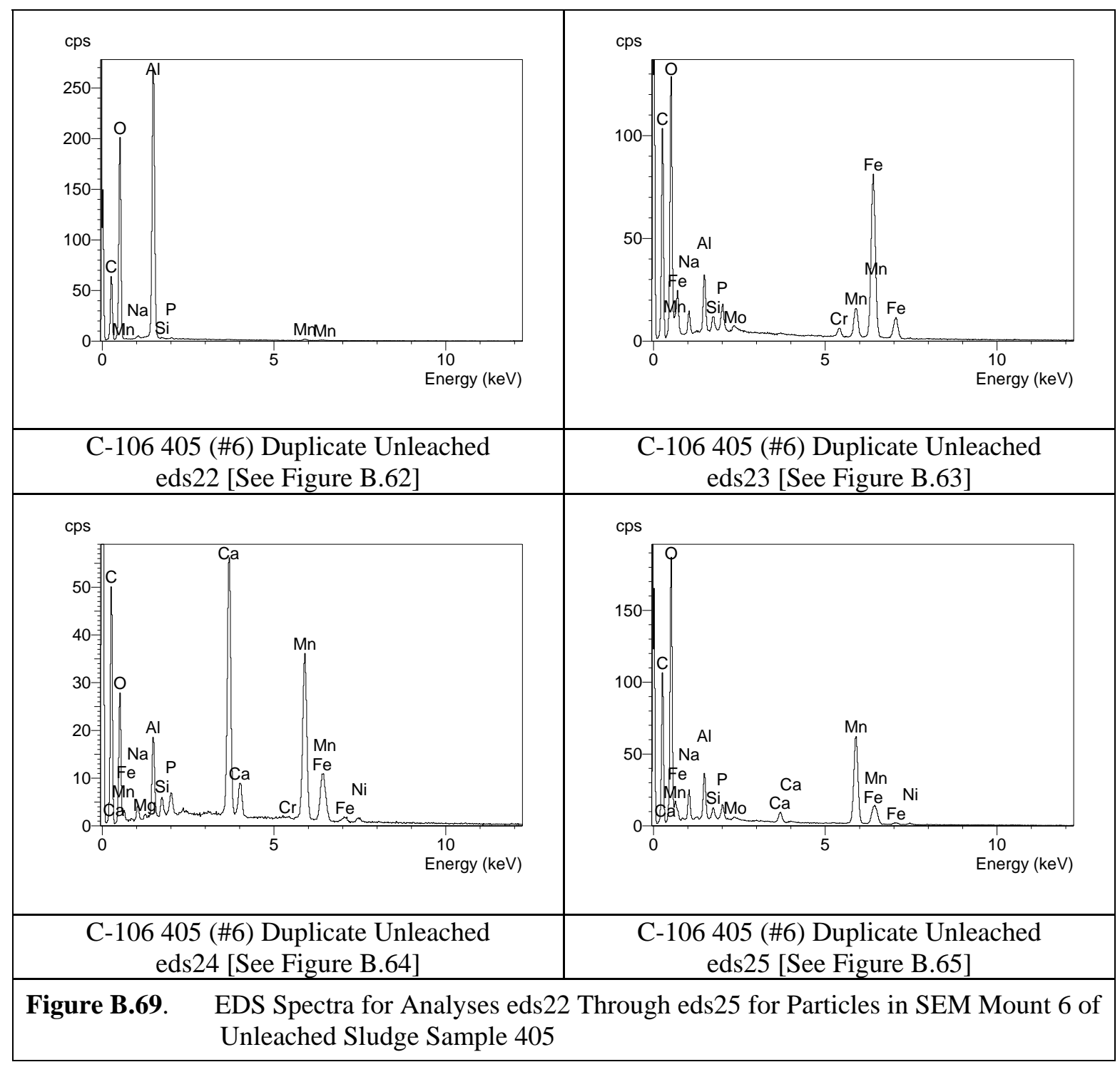




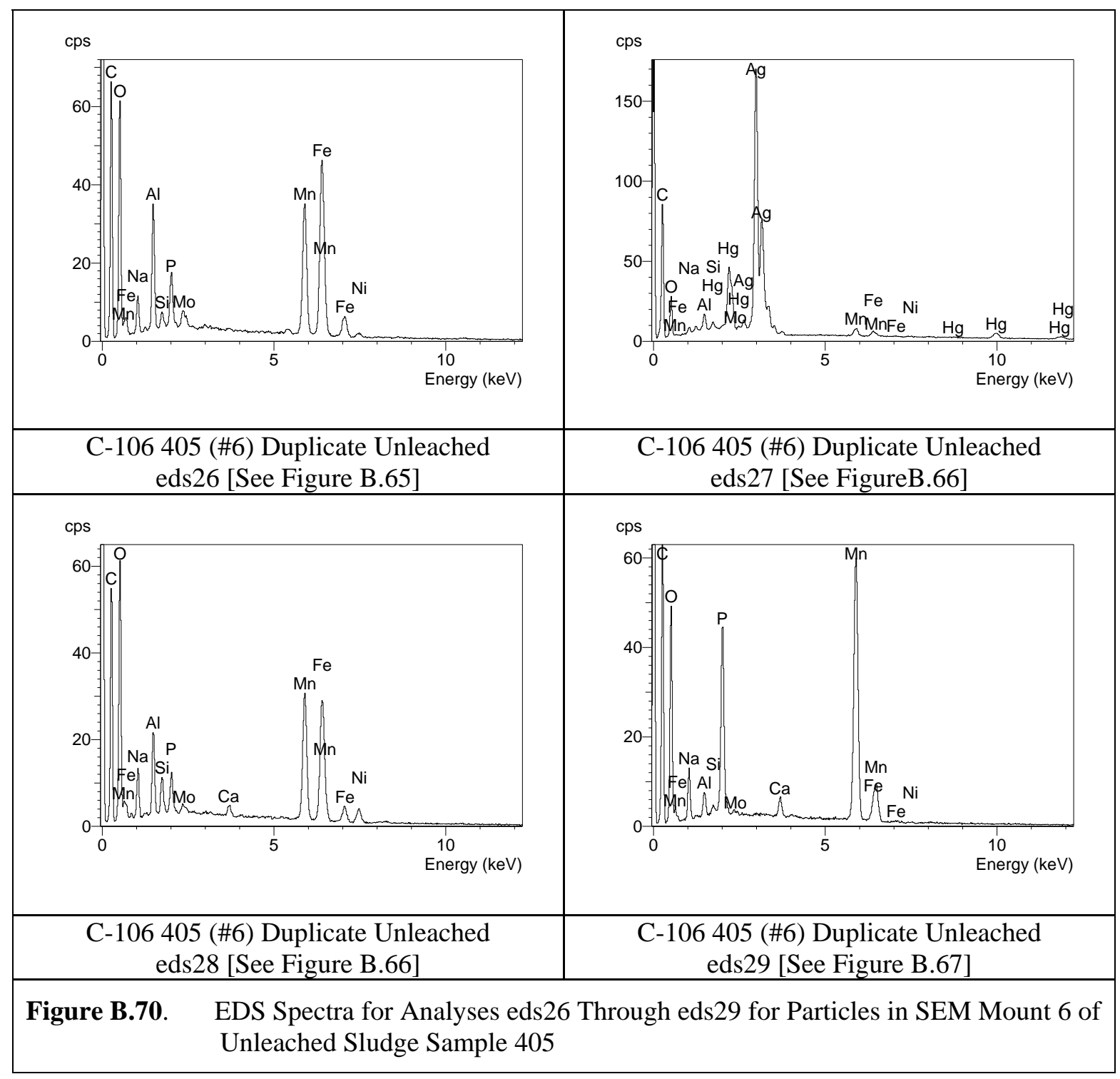




\section{Appendix C}

SEM Micrographs and EDS Spectra for Water-Leached Sludge from Tank C-106 


\section{Appendix C}

\section{SEM Micrographs and EDS Spectra for Water-Leached Sludge from Tank C-106}

This appendix includes the scanning electron microscope (SEM) micrographs and the energydispersive X-ray spectrometry (EDS) spectra for samples of tank 241-C-106 (C-106) (primary 404 and duplicate 405) 1-month and 82-day water-leached residual tank waste. The operating conditions for the SEM and procedures used for mounting the SEM samples are described in Section 2.4 of the main report.

The name of each SEM digital image file, sample identification number, and a size scale bar are given, respectively, at the bottom left, center, and right of each SEM micrograph in this appendix. Micrographs labeled by "BSE" to the immediate right of the digital image file name indicate that the micrograph was collected with backscattered electrons. Areas identified by a letter and/or outlined by a dashed-line square in a micrograph designate sample material that was imaged at higher magnification, which is typically shown in figure(s) that immediately follow in the series for that sample.

Areas labeled by “eds” in the following SEM micrographs in this appendix identify locations of particles for which EDS spectra were recorded. The "eds" label given with each EDS spectrum correspond to the same "EDS" label used in the SEM micrographs for this sample.

\section{C.1 Water-Leached (1-Month) Sample 404}

Two mounts of 1-month water-leached residual sludge from tank C-106 (primary 404) were analyzed by SEM/EDS. The SEM micrographs for the first mount (7) of the 1-month water-leached sample 404 are shown in Figures C.1 through C.15. The EDS spectra for this mount are given in Figures C.16 through C.19. The SEM micrographs for the second mount (8) of the 1-month water-leached sample 404 are shown in Figures C.20 through C.27, and the EDS spectra for the second mount are given in Figures C.28 through C.30. 


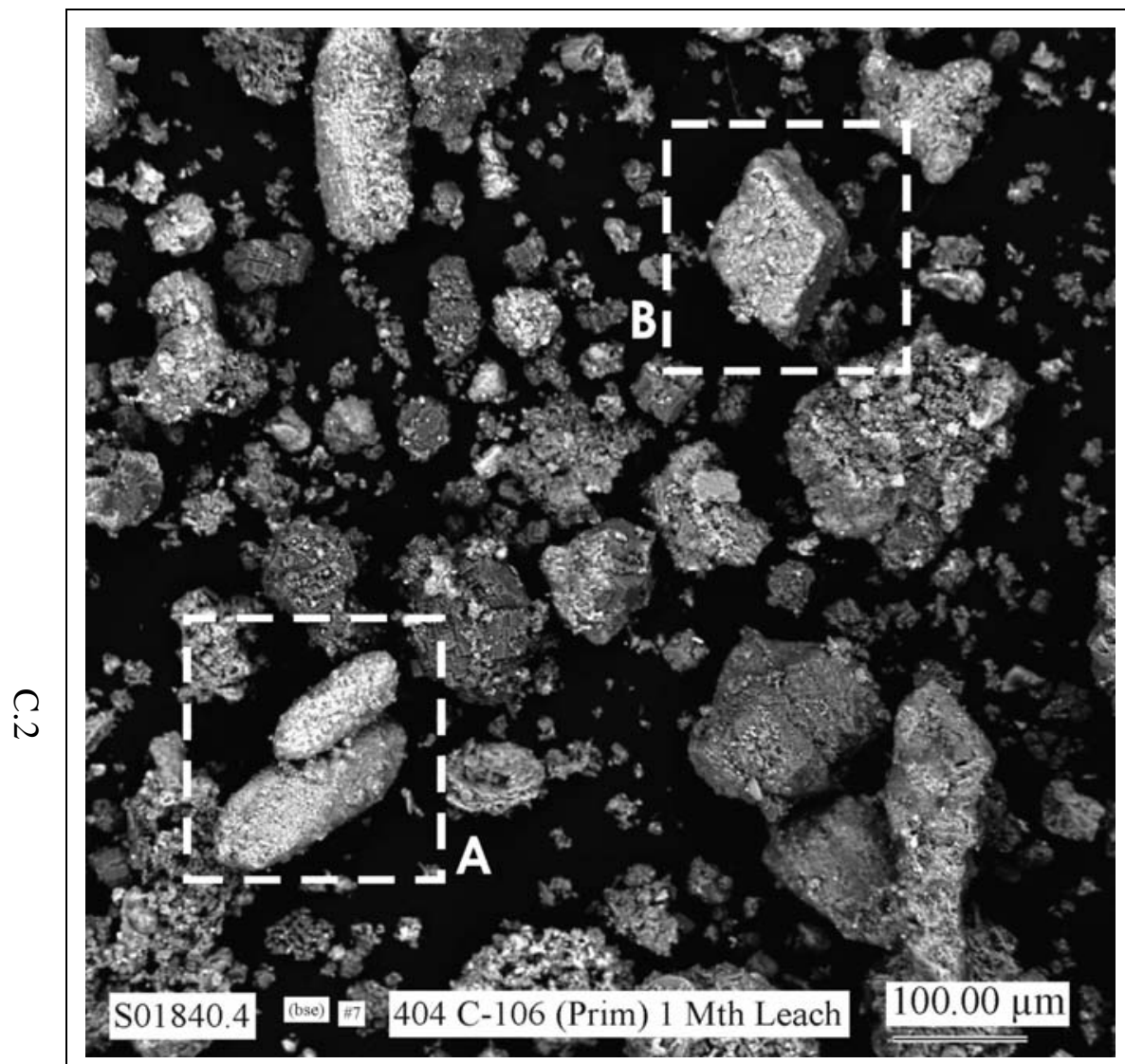

Figure C.1. Low Magnification SEM Micrograph Showing General Morphologies of Particles in SEM Mount 7 of 1-Month Water-Leached Sludge Sample 404

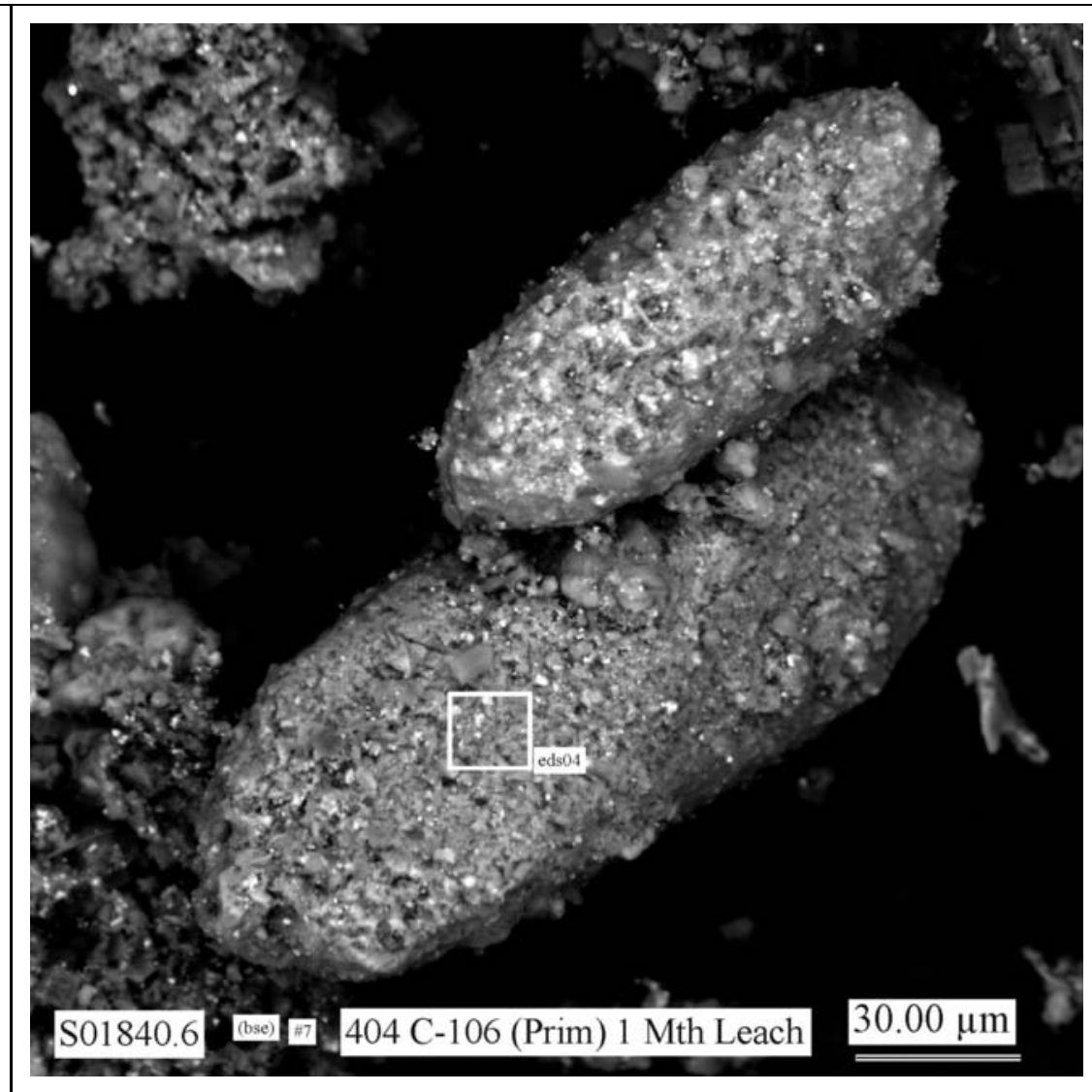

Figure C.2. $\quad$ Micrograph Showing at Higher Magnification the Area Indicated by the White Dashed-Line Square Labeled A in Figure C.1 


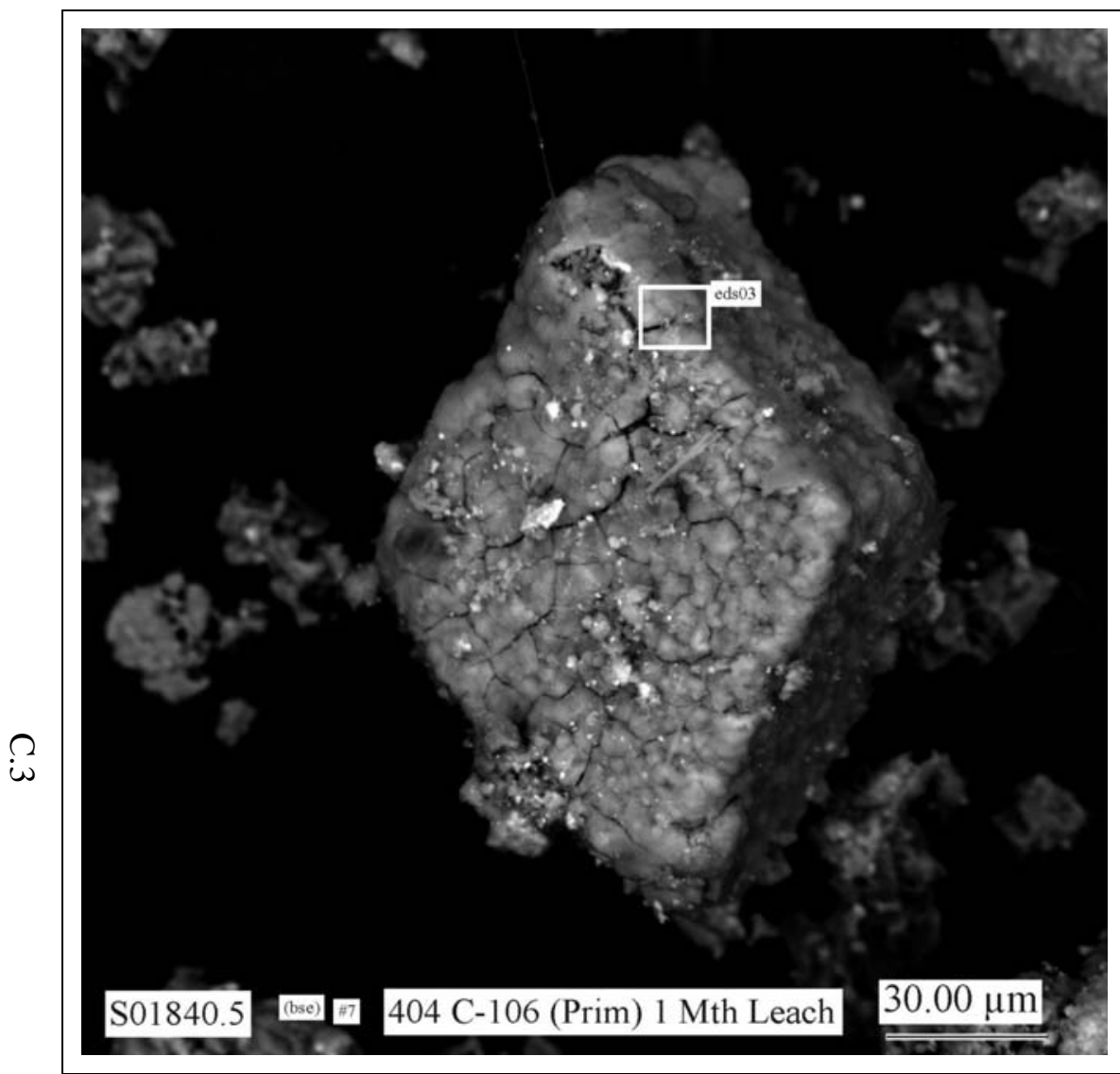

Figure C.3. Micrograph Showing at Higher Magnification the Area Indicated by the White Dashed-Line Square Labeled B in Figure C.1

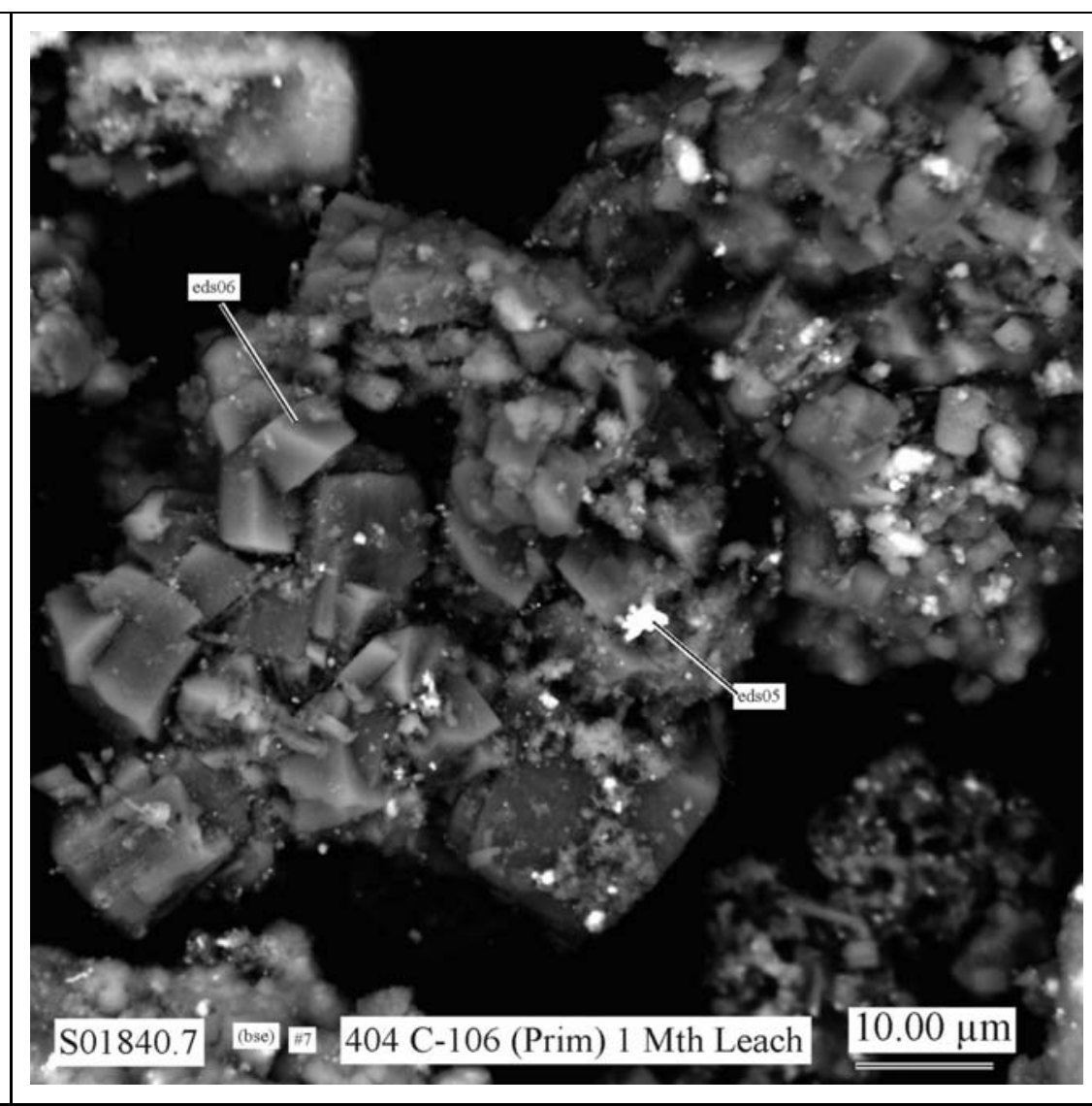

Figure C.4. Micrograph Showing Morphologies of Typical Particles in SEM Mount 7 of 1-Month Water-Leached Sludge Sample 404 


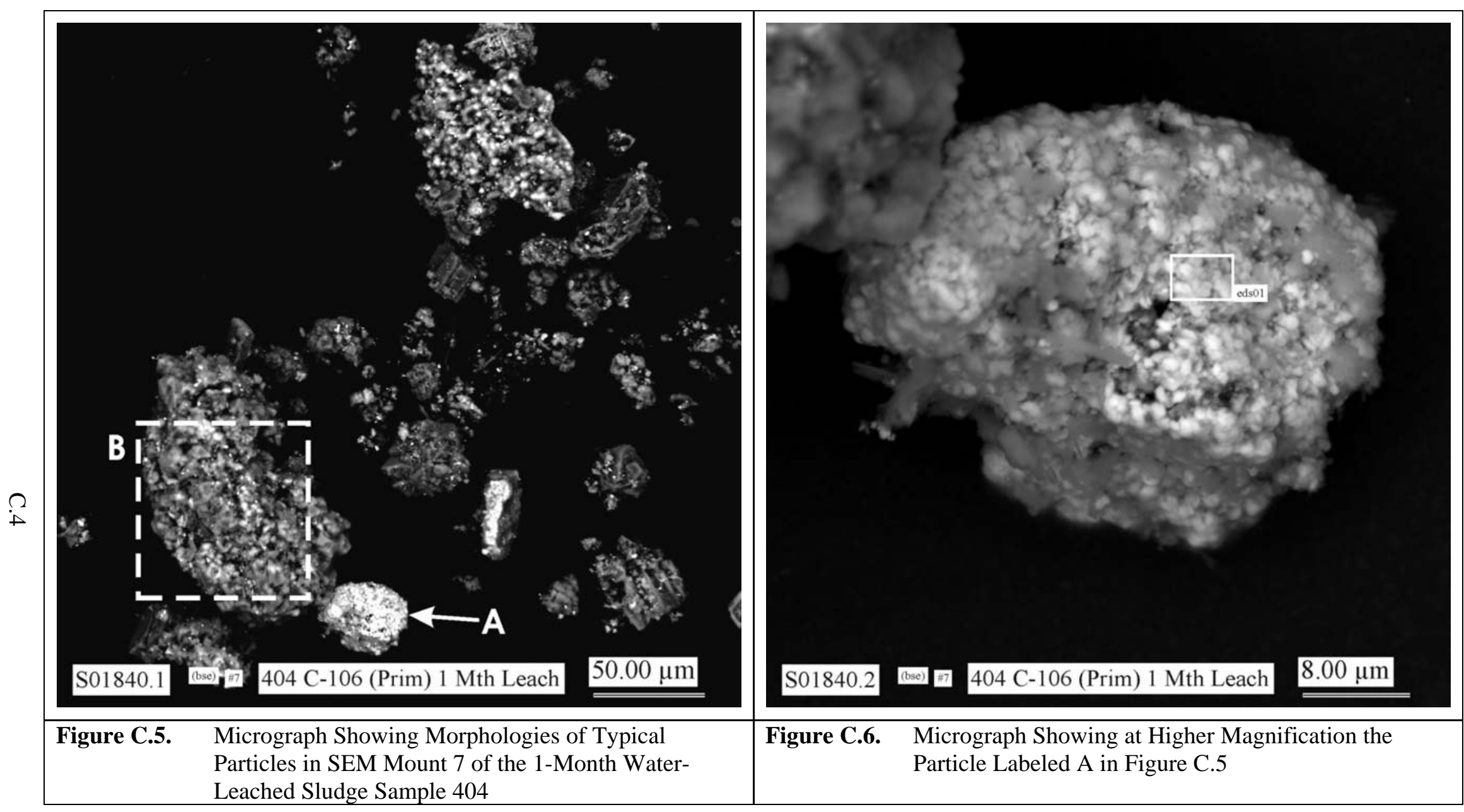



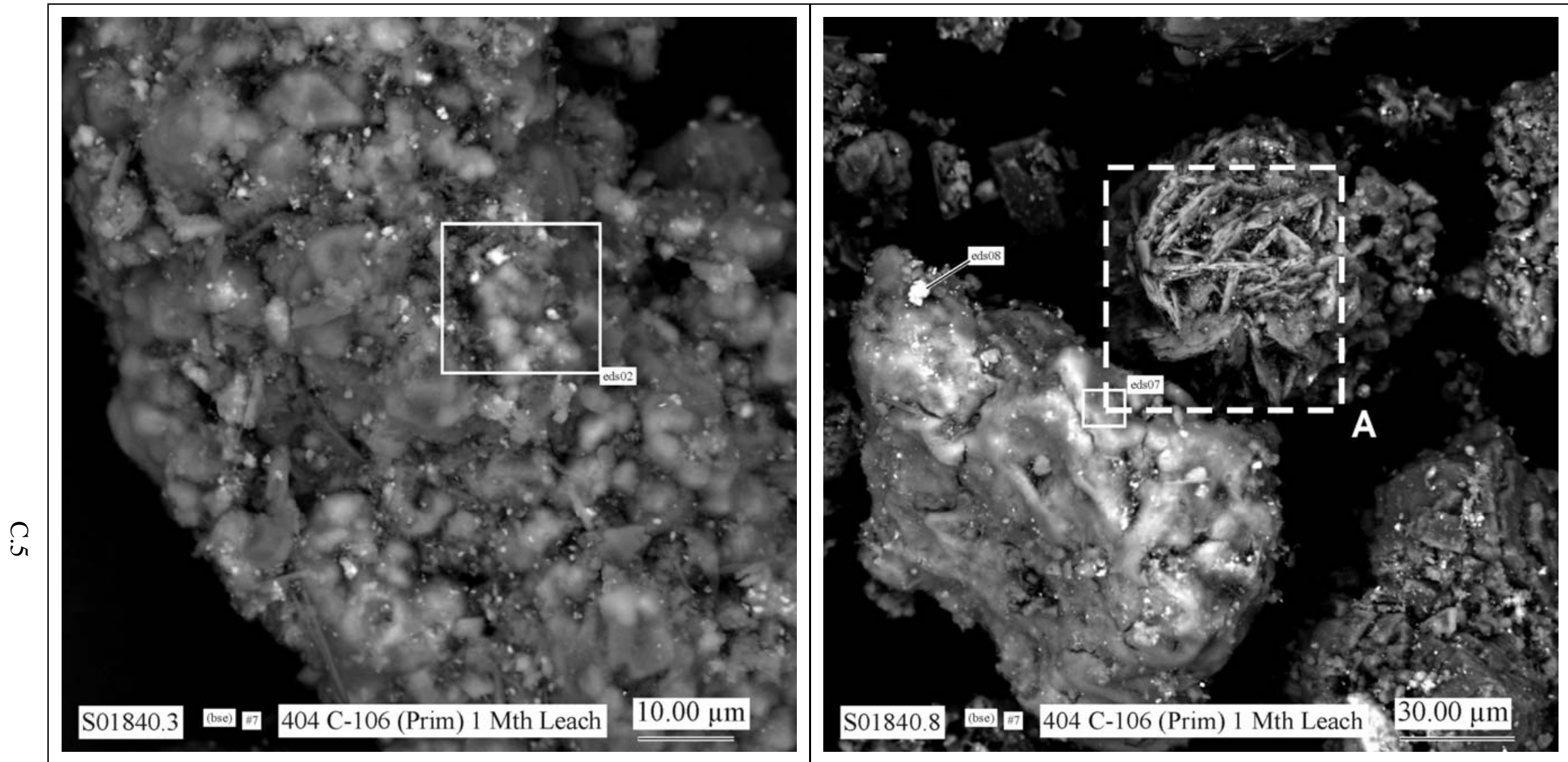

Figure C.7. Micrograph Showing at Higher Magnification the Area Indicated by the White Dashed-Line Square Labeled B

Figure C.8. Micrograph Showing Morphologies of Typical in Figure C.5 Particles in SEM Mount 7 of the 1-Month WaterLeached Sludge Sample 404 

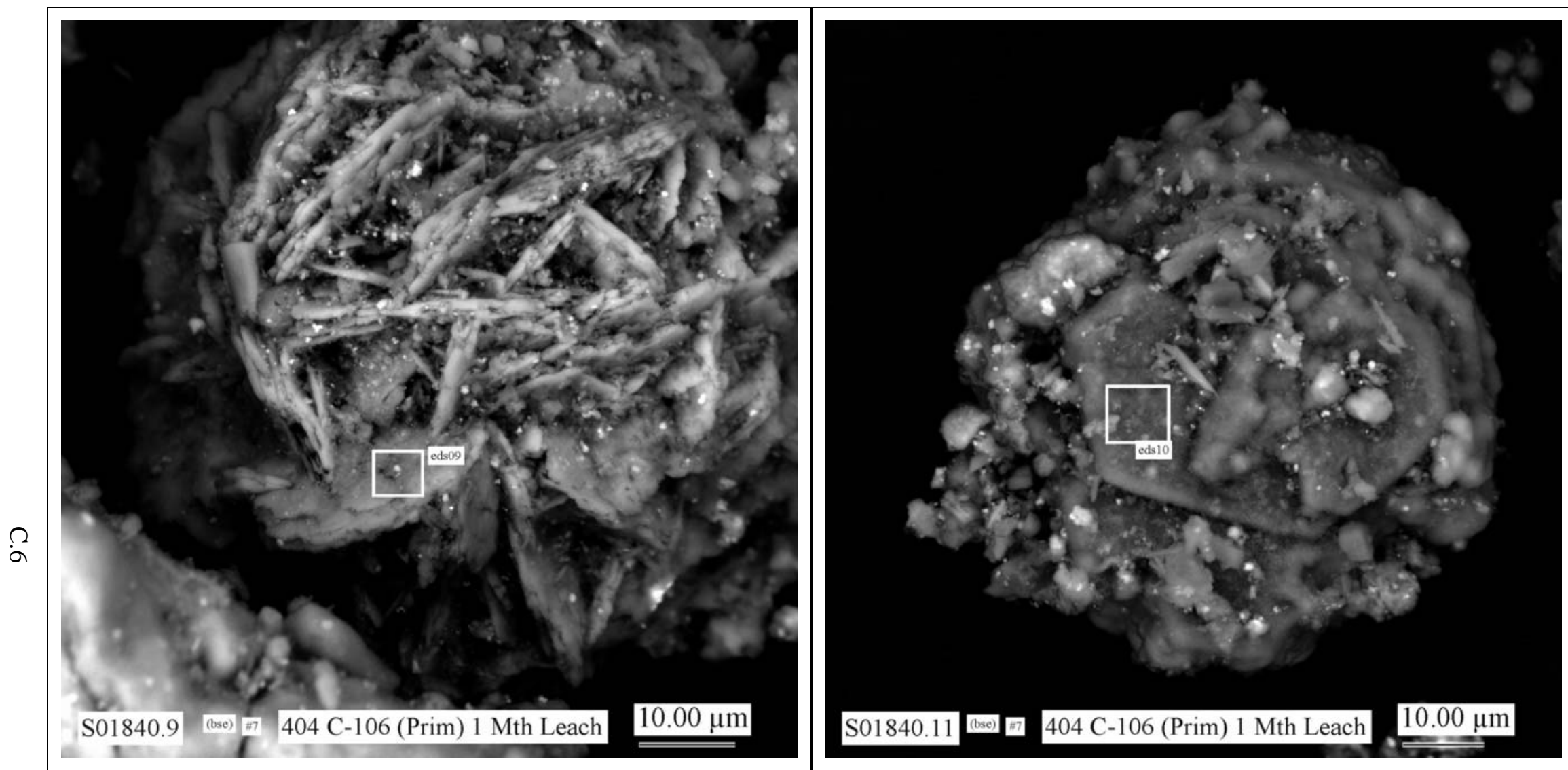

Figure C.9. Micrograph Showing at Higher Magnification the Particle Labeled A in Figure C.8

Figure C.10. $\quad$ Micrograph Showing Morphologies of Typical Particles in SEM Mount 7 of the 1-Month WaterLeached Sludge Sample 404 


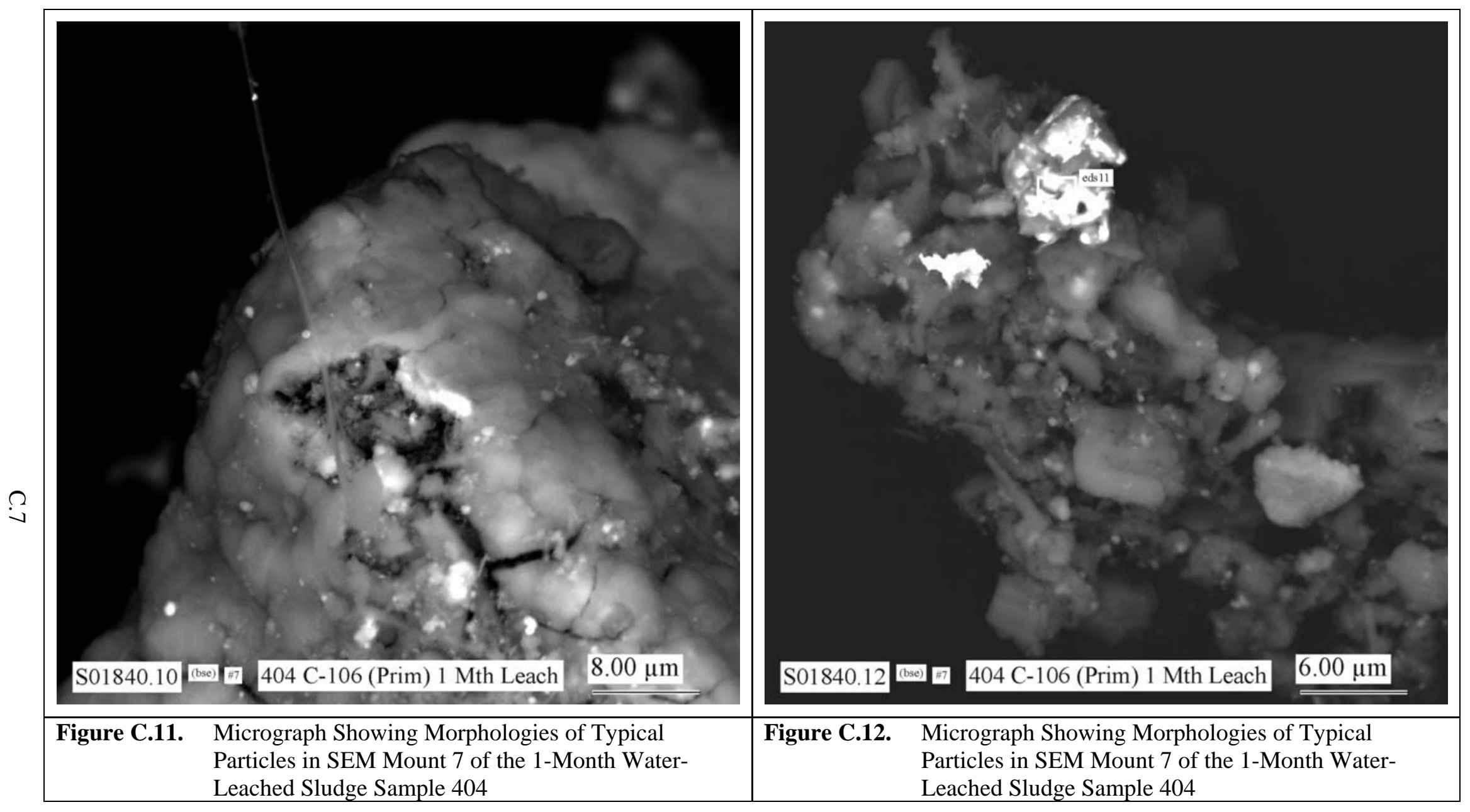




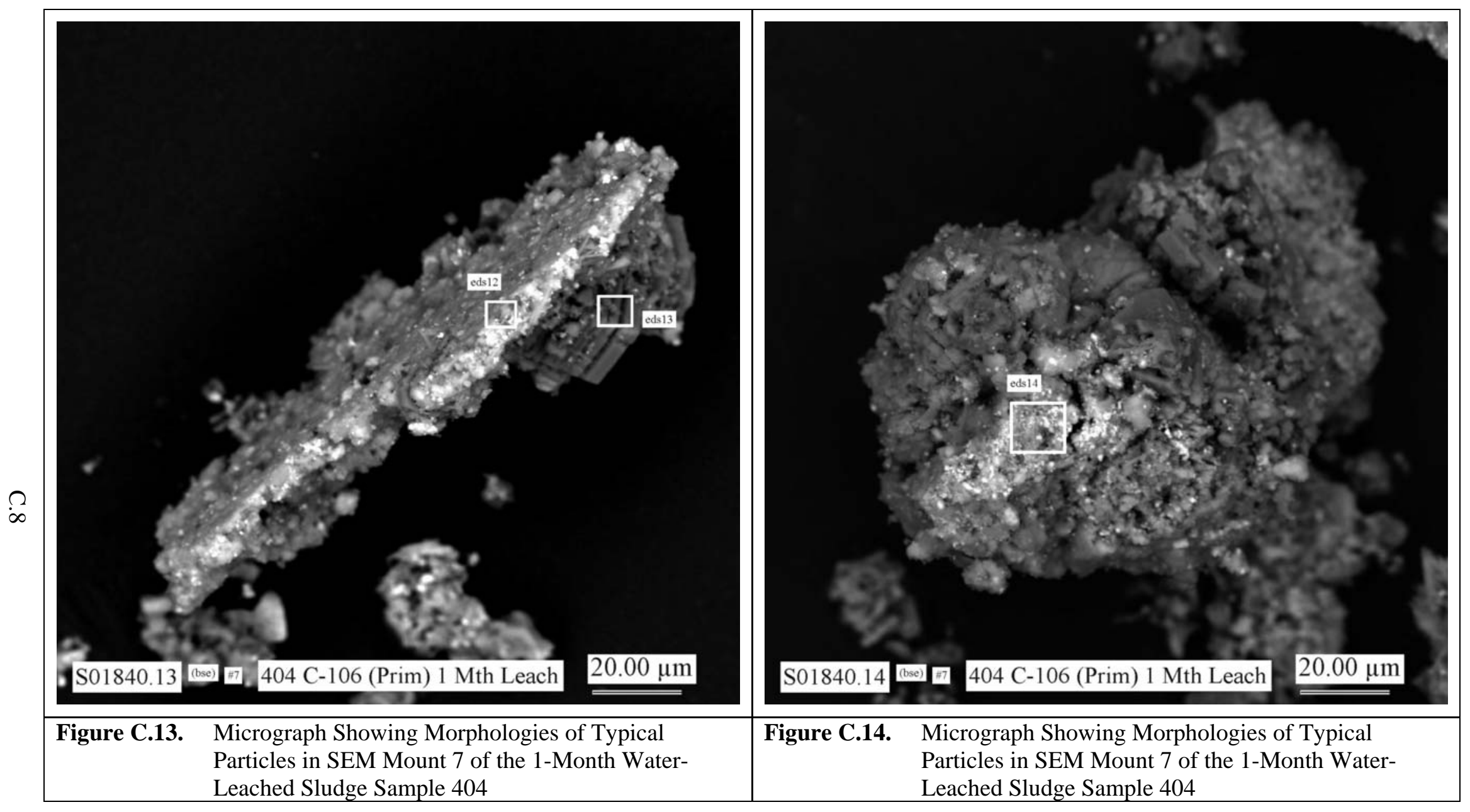




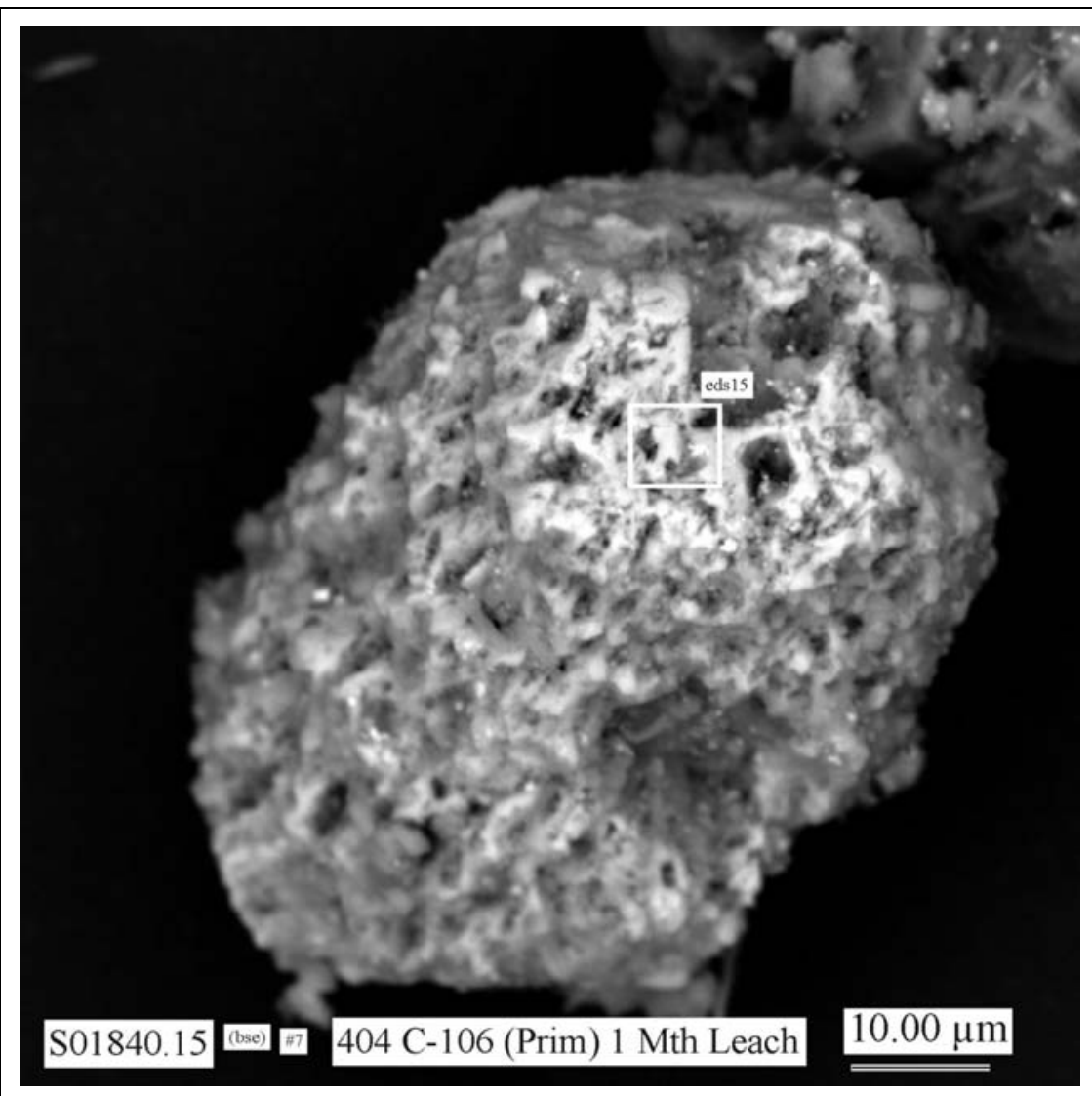

Figure C.15. Micrograph Showing Morphologies of Typical Particles in SEM Mount 7 of the 1-Month WaterLeached Sludge Sample 404 


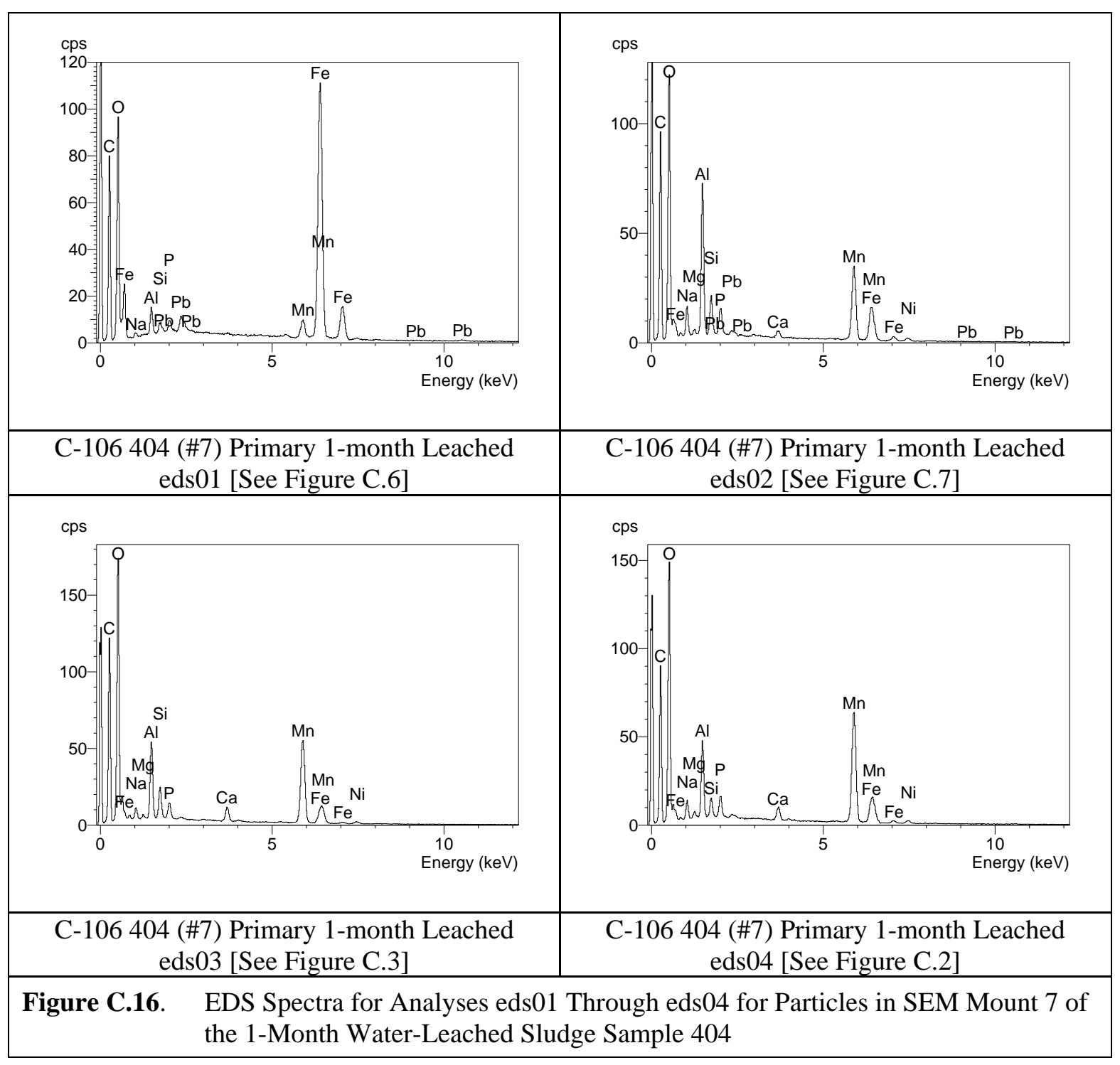




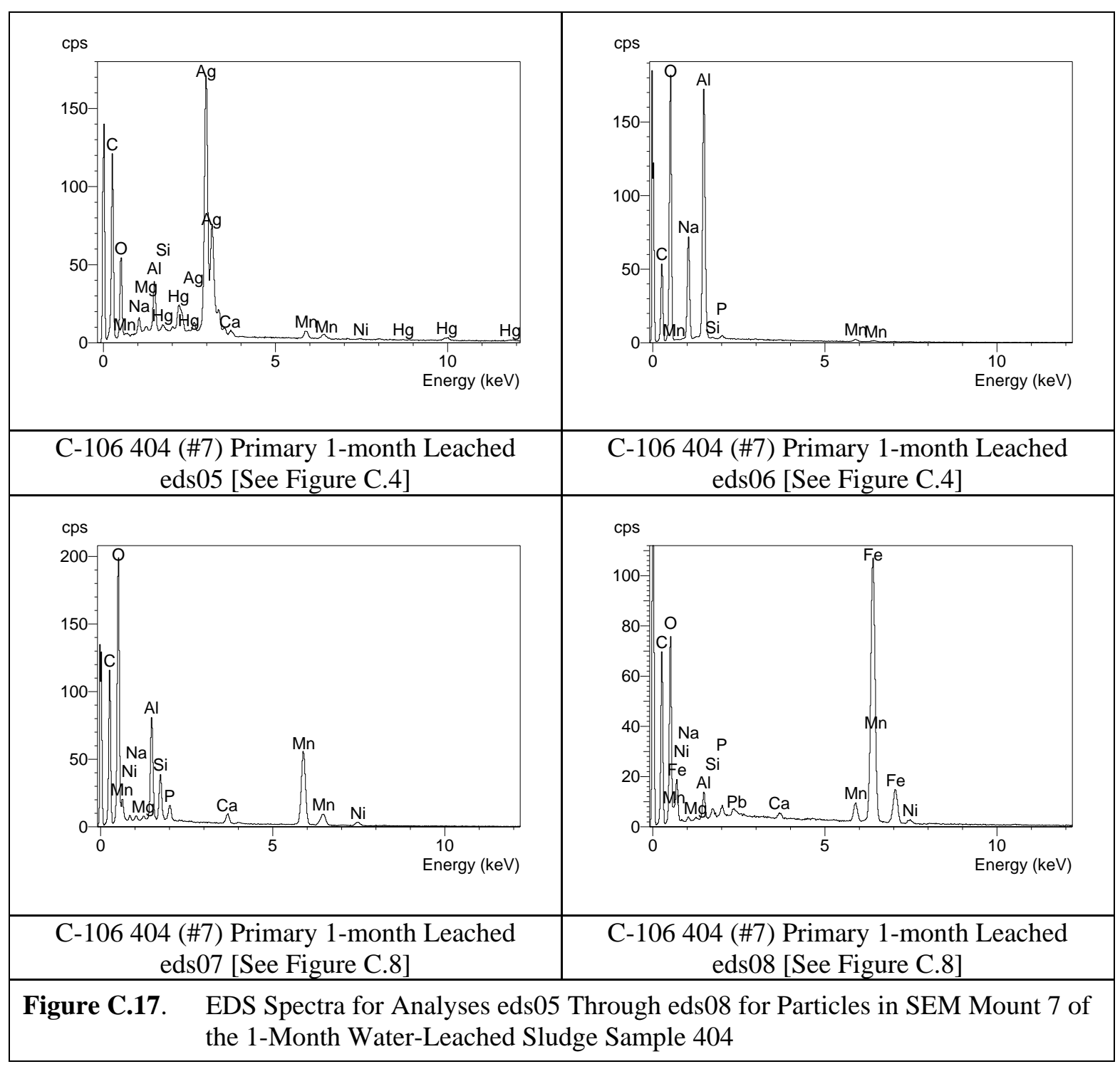




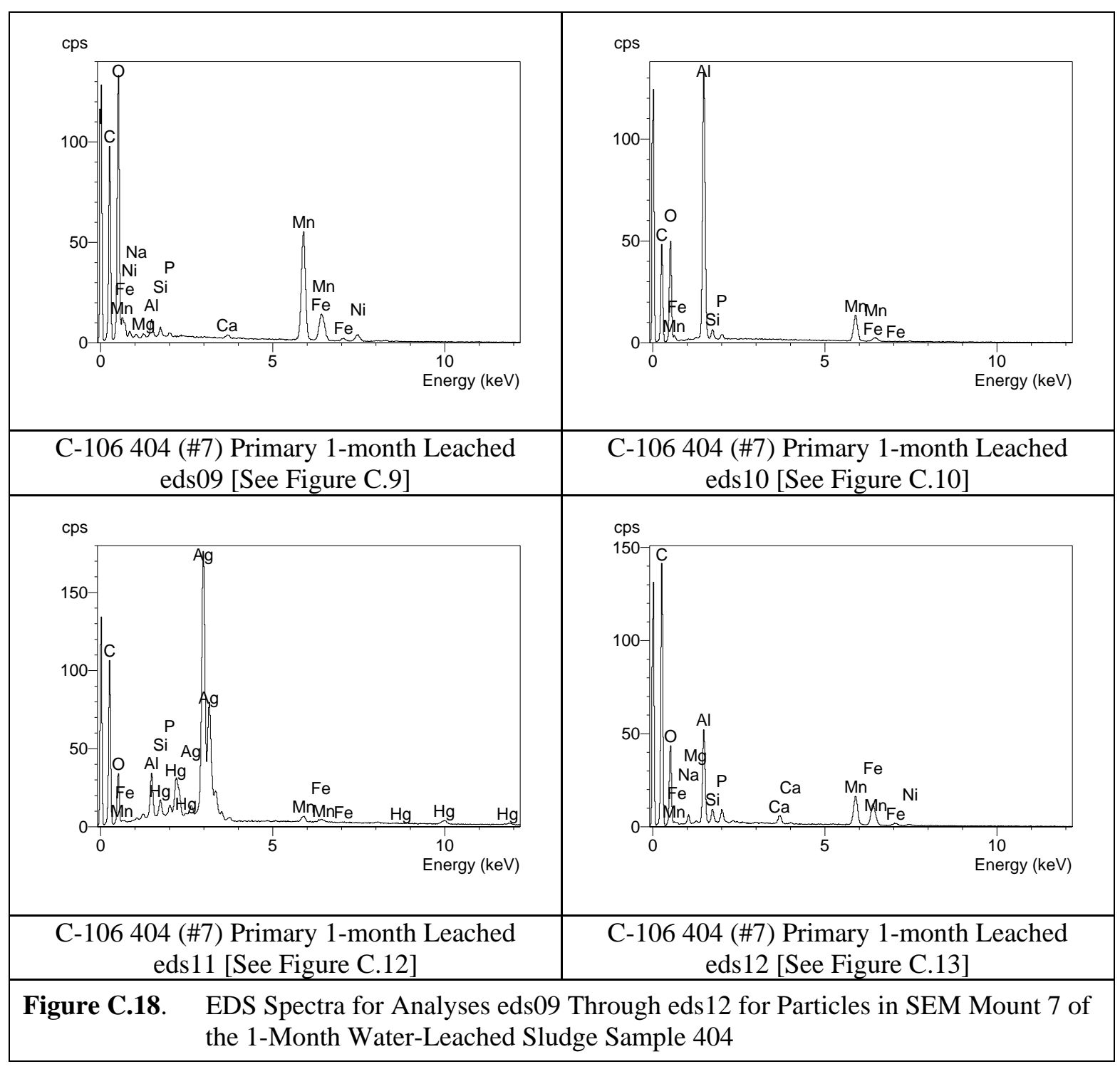




\begin{tabular}{|c|c|c|}
\hline & \\
\hline
\end{tabular}



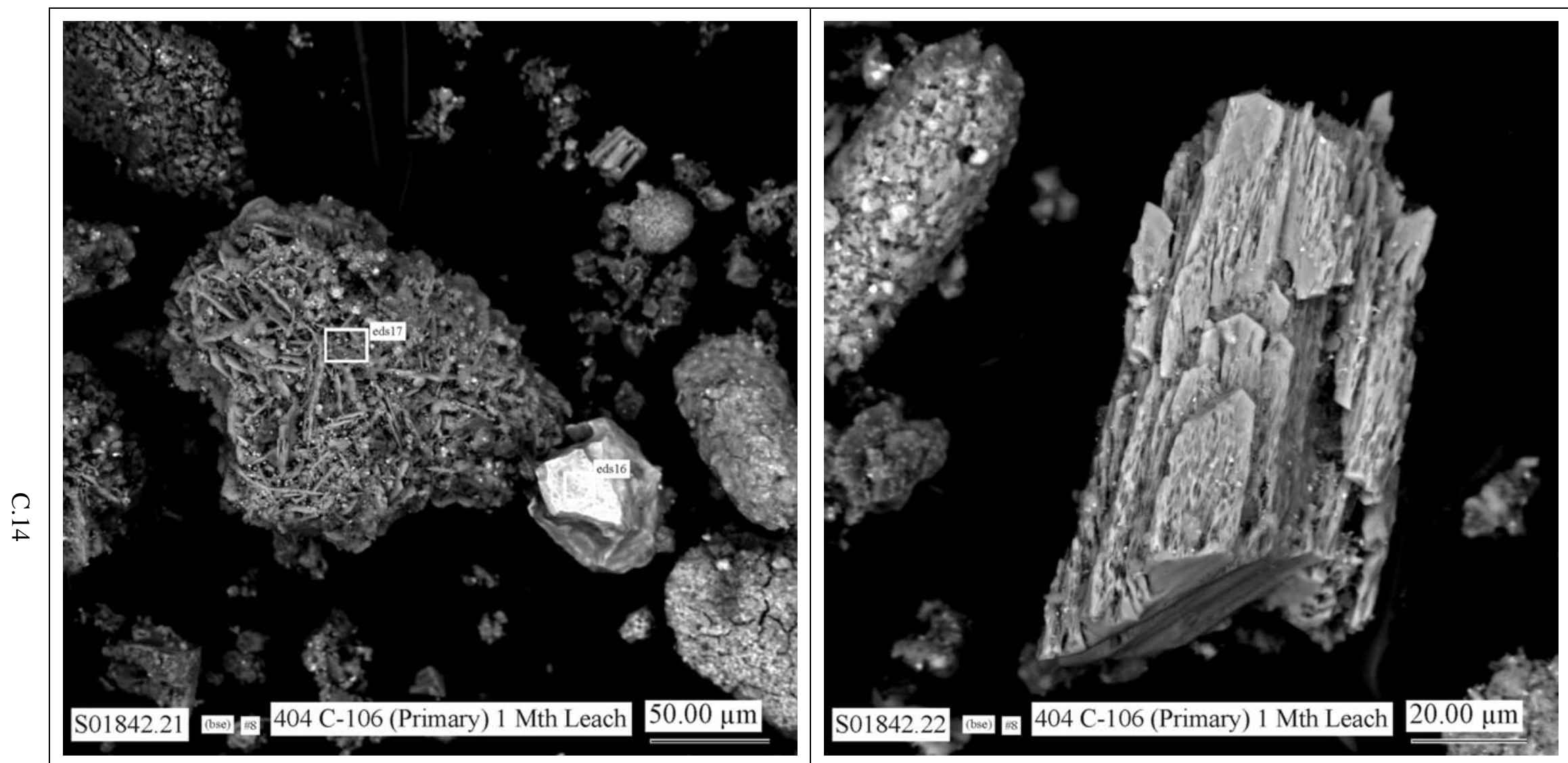

$\begin{array}{ll}\text { Figure C.20. } & \text { Micrograph Showing Morphologies of Typical }\end{array}$ Particles in SEM Mount 8 of the 1-Month WaterLeached Sludge Sample 404

Figure C.21. $\quad$ Micrograph Showing Morphologies of Typical Particles in SEM Mount 8 of the 1-Month WaterLeached Sludge Sample 404 


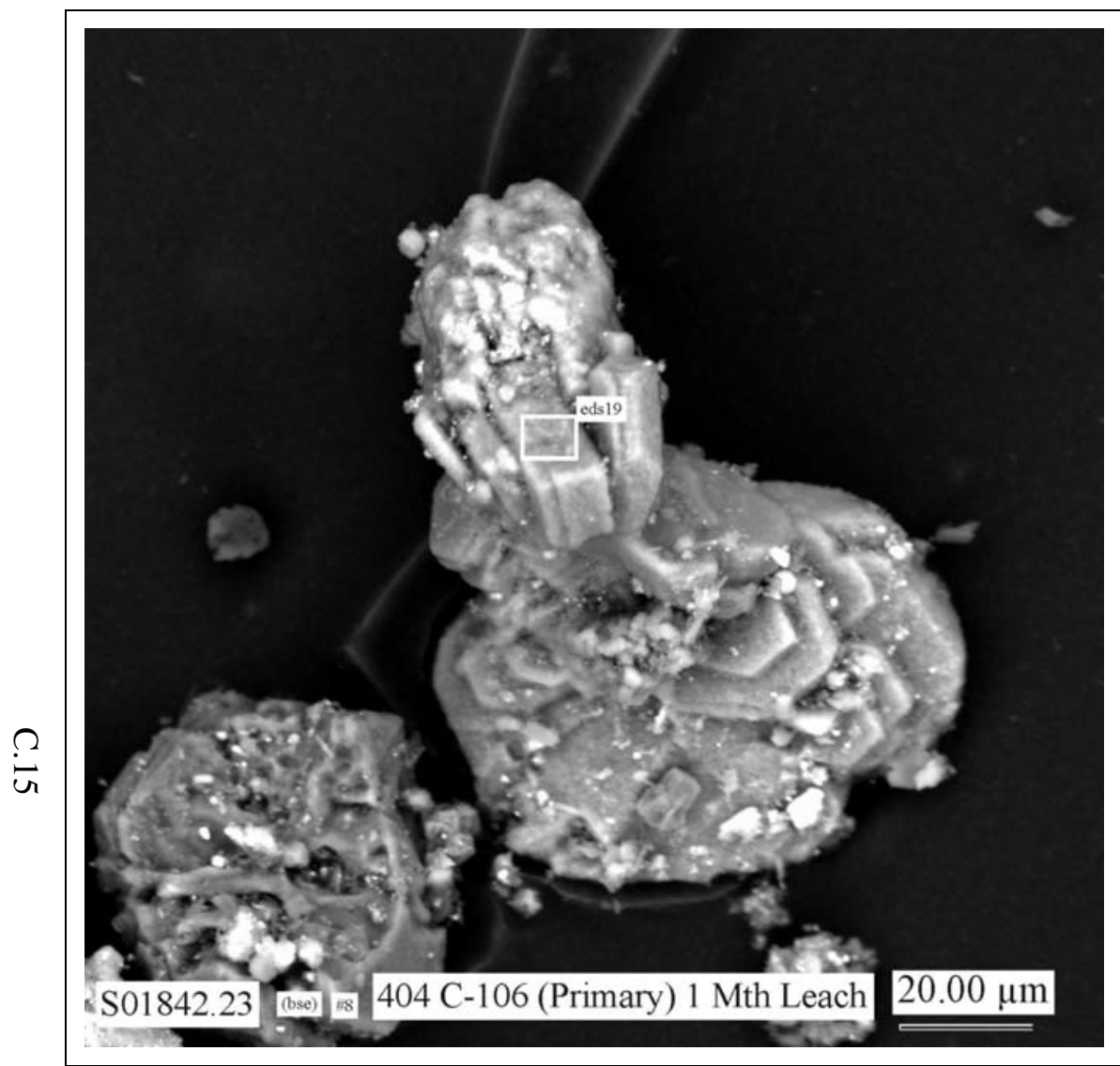

Figure C.22. $\quad$ Micrograph Showing Morphologies of Typical Particles in SEM Mount 8 of the 1-Month WaterLeached Sludge Sample 404

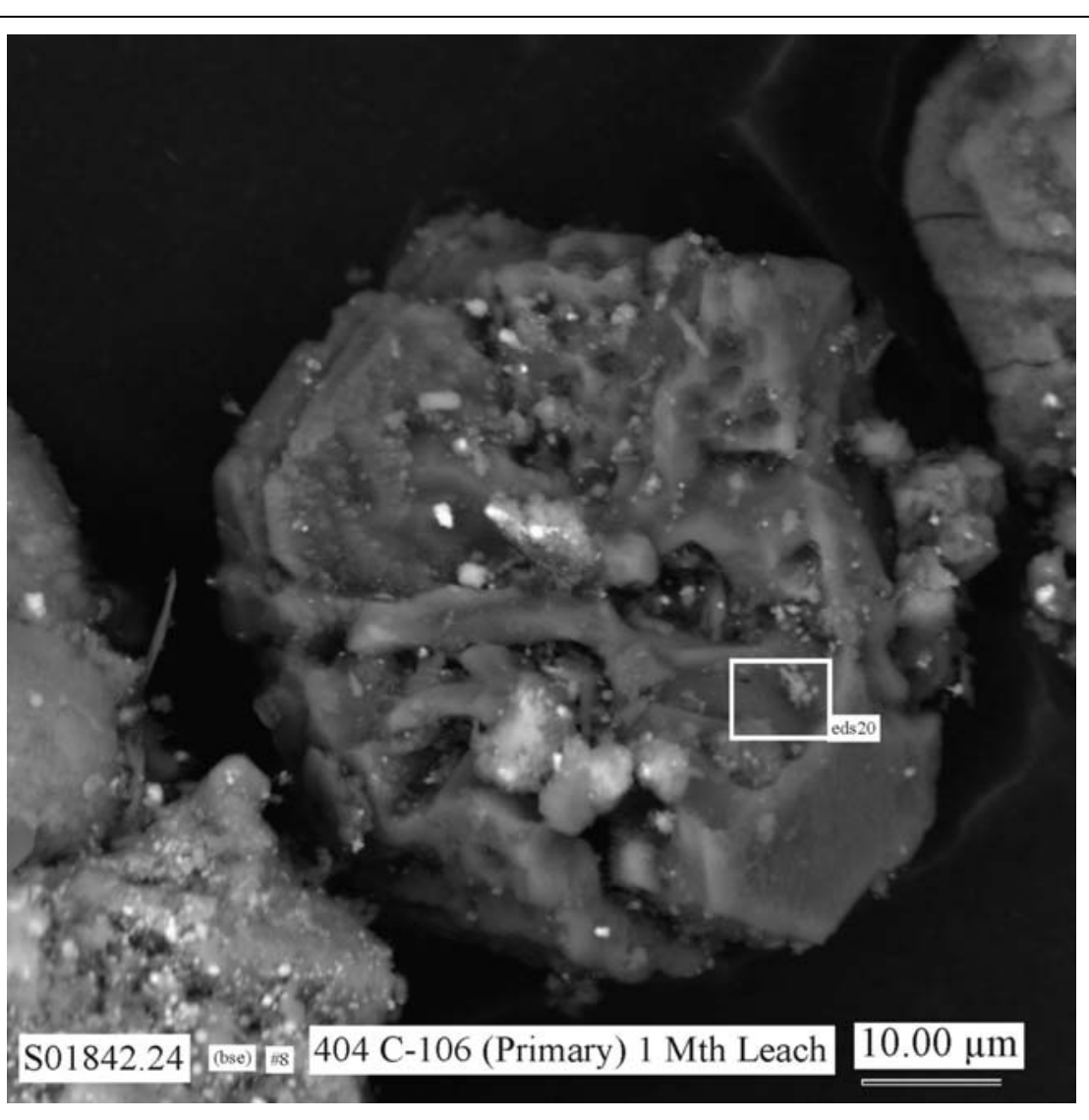

Figure C.23. Micrograph Showing Morphologies of Typical Particles in SEM Mount 8 of the 1-Month WaterLeached Sludge Sample 404 


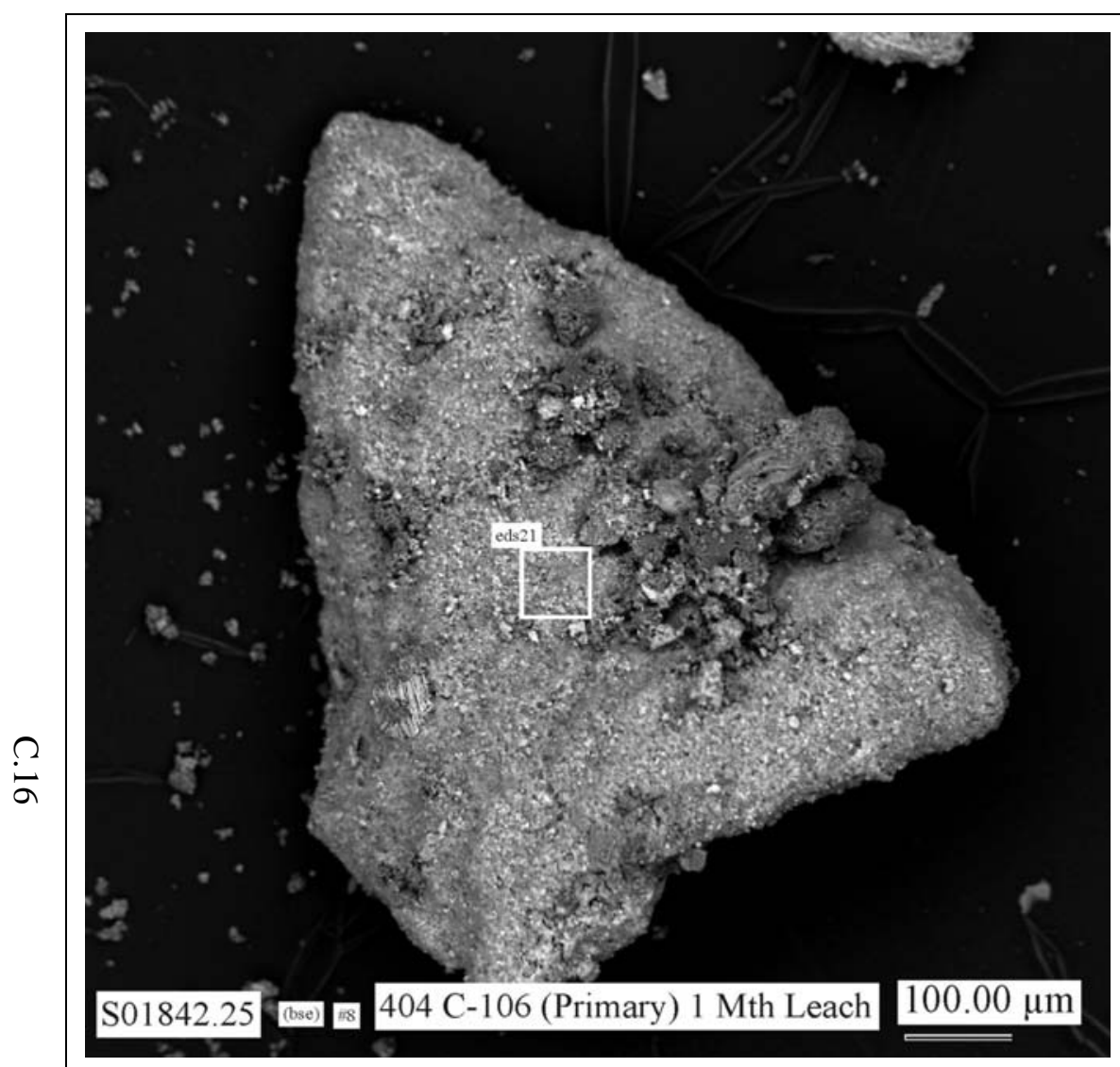

Figure C.24. Micrograph Showing Morphologies of Typical Particles in SEM Mount 8 of the 1-Month WaterLeached Sludge Sample 404

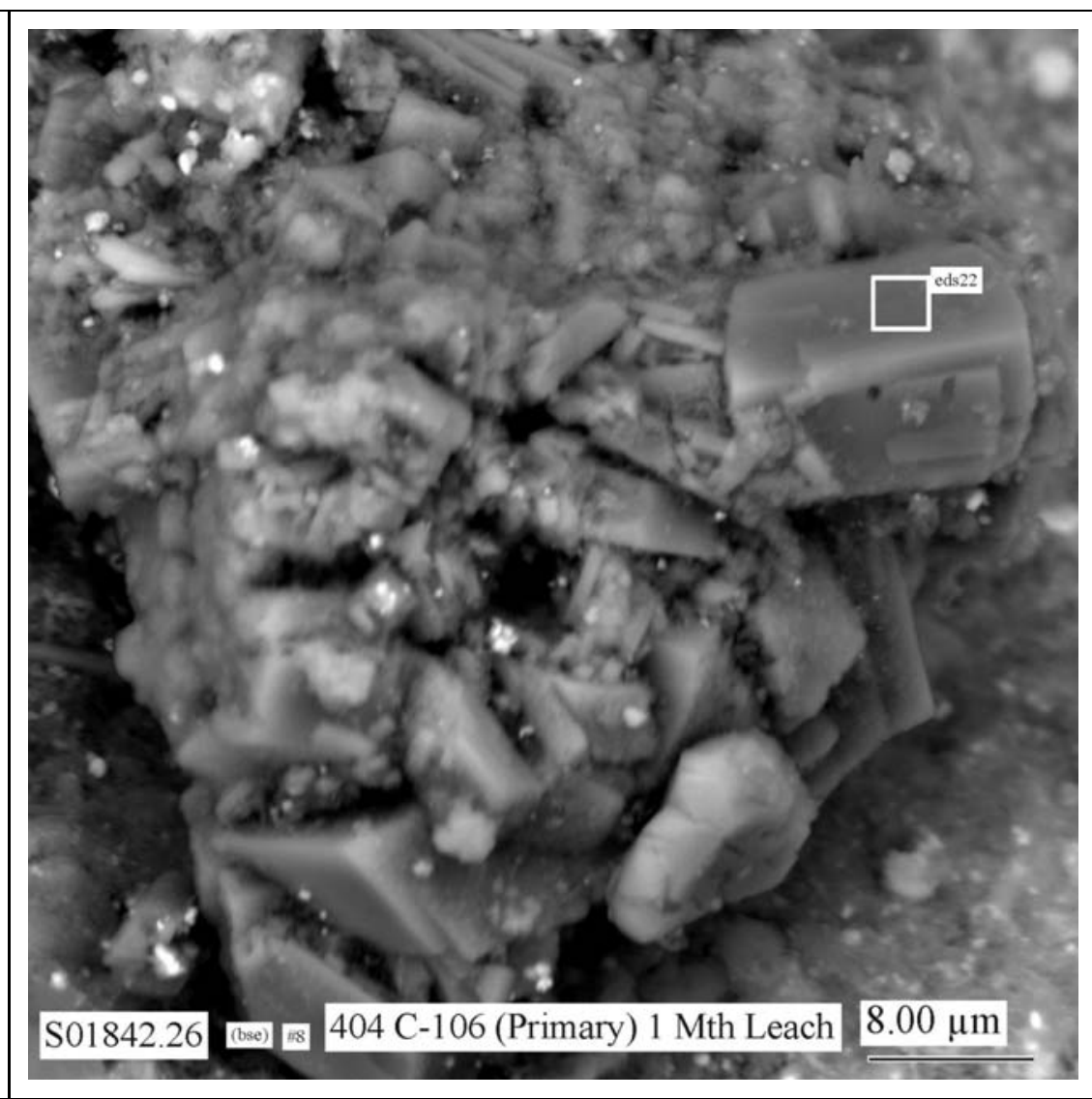

Figure C.25. Micrograph Showing Morphologies of Typical Particles in SEM Mount 8 of the 1-Month WaterLeached Sludge Sample 404 

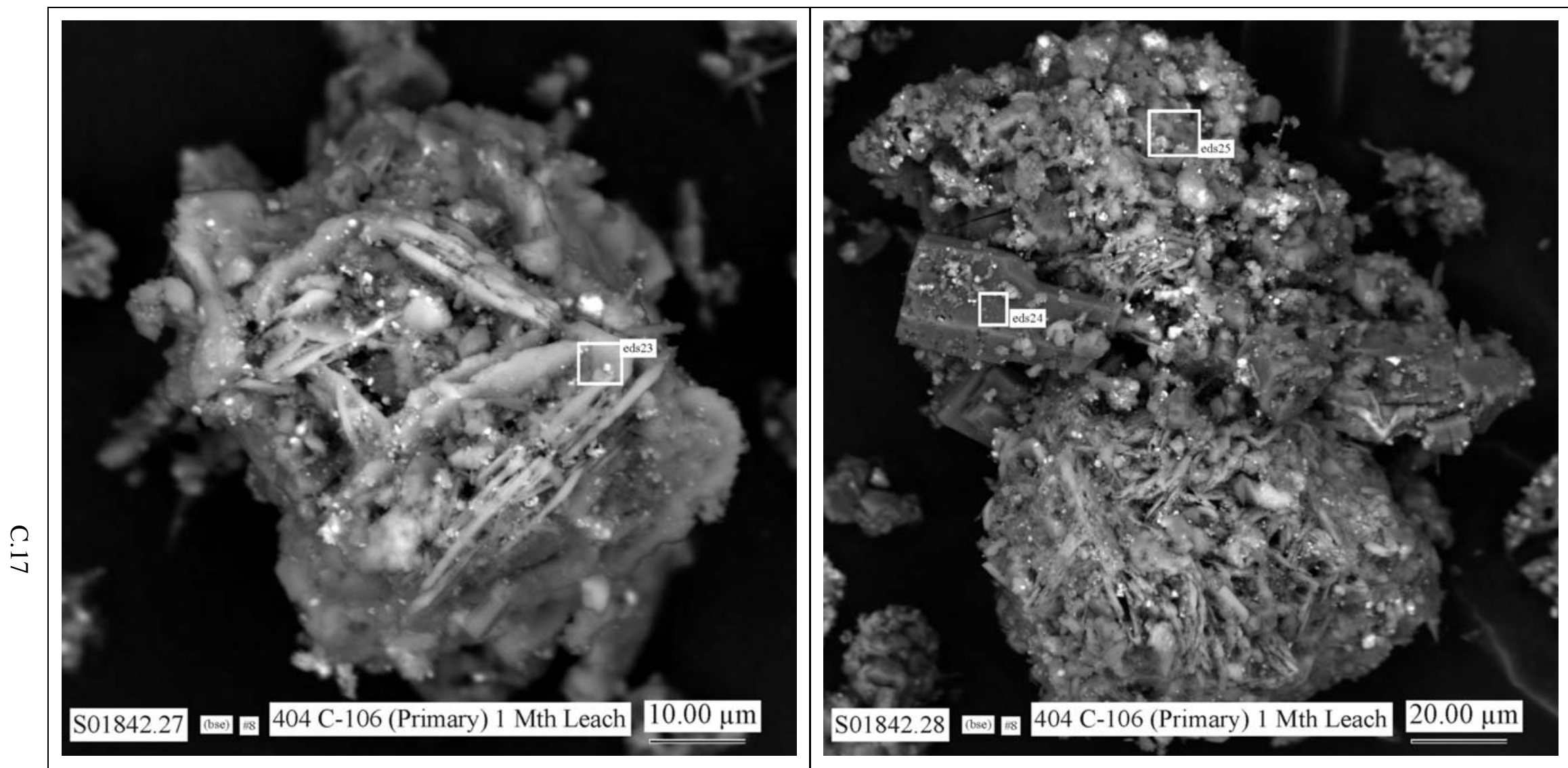

Figure C.26. $\quad$ Micrograph Showing Morphologies of Typical Particles in SEM Mount 8 of the 1-Month WaterLeached Sludge Sample 404

Figure C.27. Micrograph Showing Morphologies of Typical Particles in SEM Mount 8 of the 1-Month WaterLeached Sludge Sample 404 


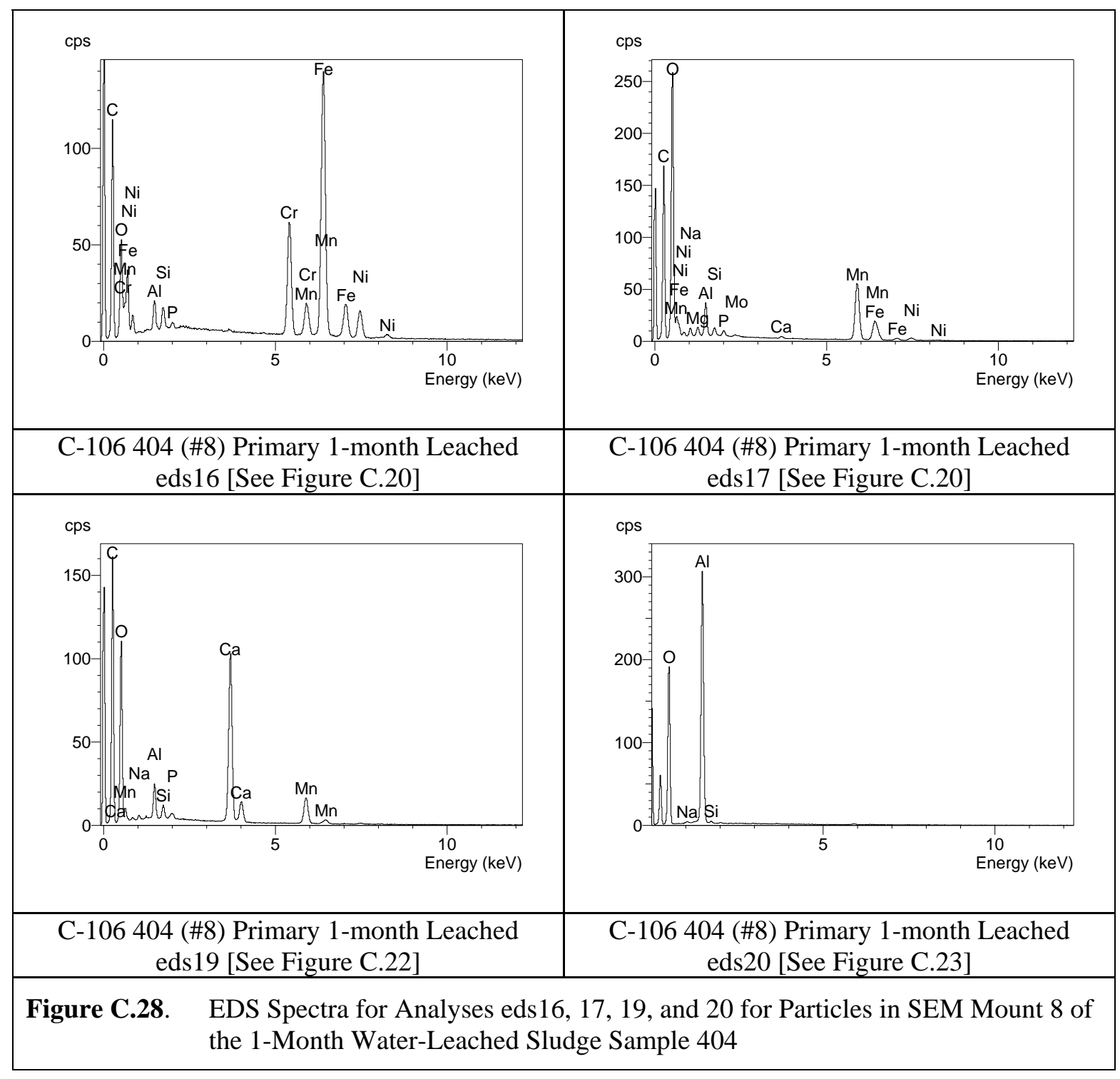




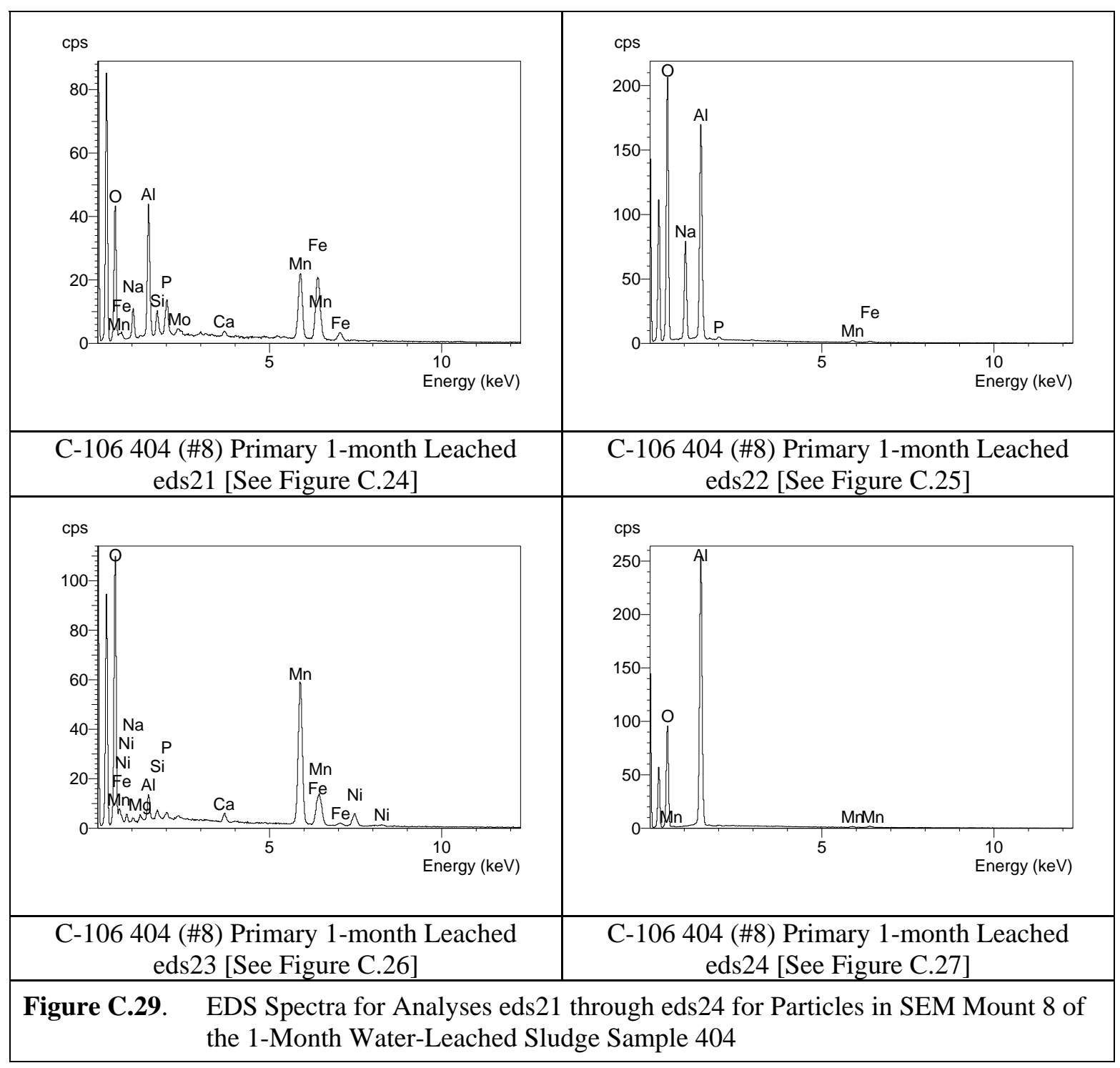




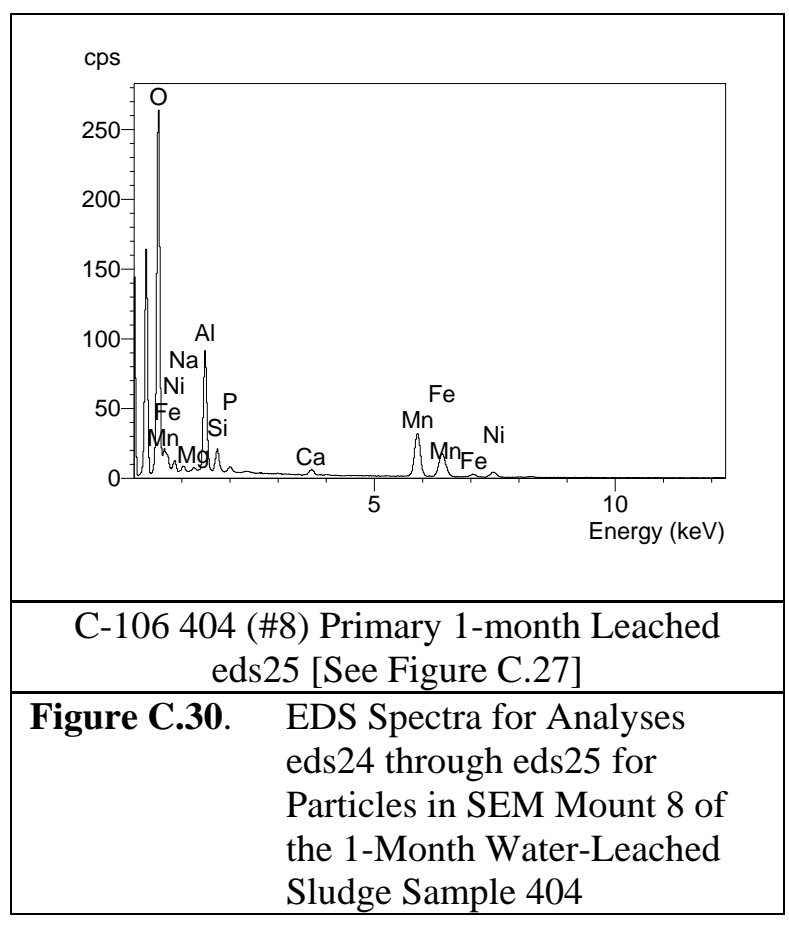




\section{C.2 Water-Leached (1-Month) Sample 405}

Two mounts of the 1-month water-leached residual sludge from tank C-106 (duplicate 405) were analyzed by SEM/EDS. The SEM micrographs for the first mount (10) of the 1-month water-leached sample 405 sludge are shown in Figures C.31 through C.44 The EDS spectra for this mount are given in Figures C.45 through C.48. The SEM micrographs for the second mount (11) of sample 405 are shown in Figures C.49 through C.54, and the EDS spectra for the second mount are given in Figures C.55 through C.56. 


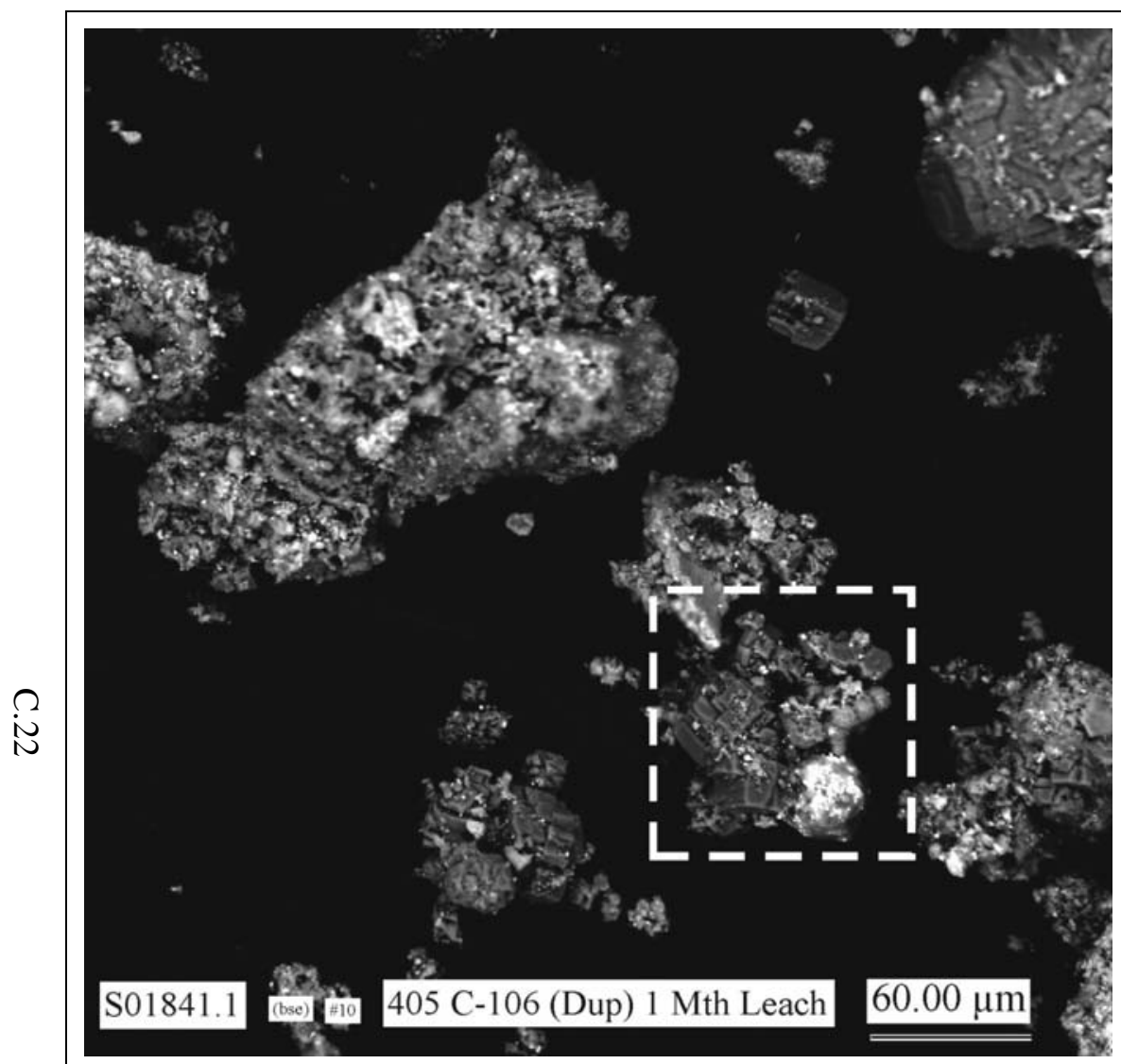

Figure C.31. Low Magnification SEM Micrograph Showing General Morphologies of Particles in SEM Mount 10 of the 1-Month Water-Leached Sludge Sample 405

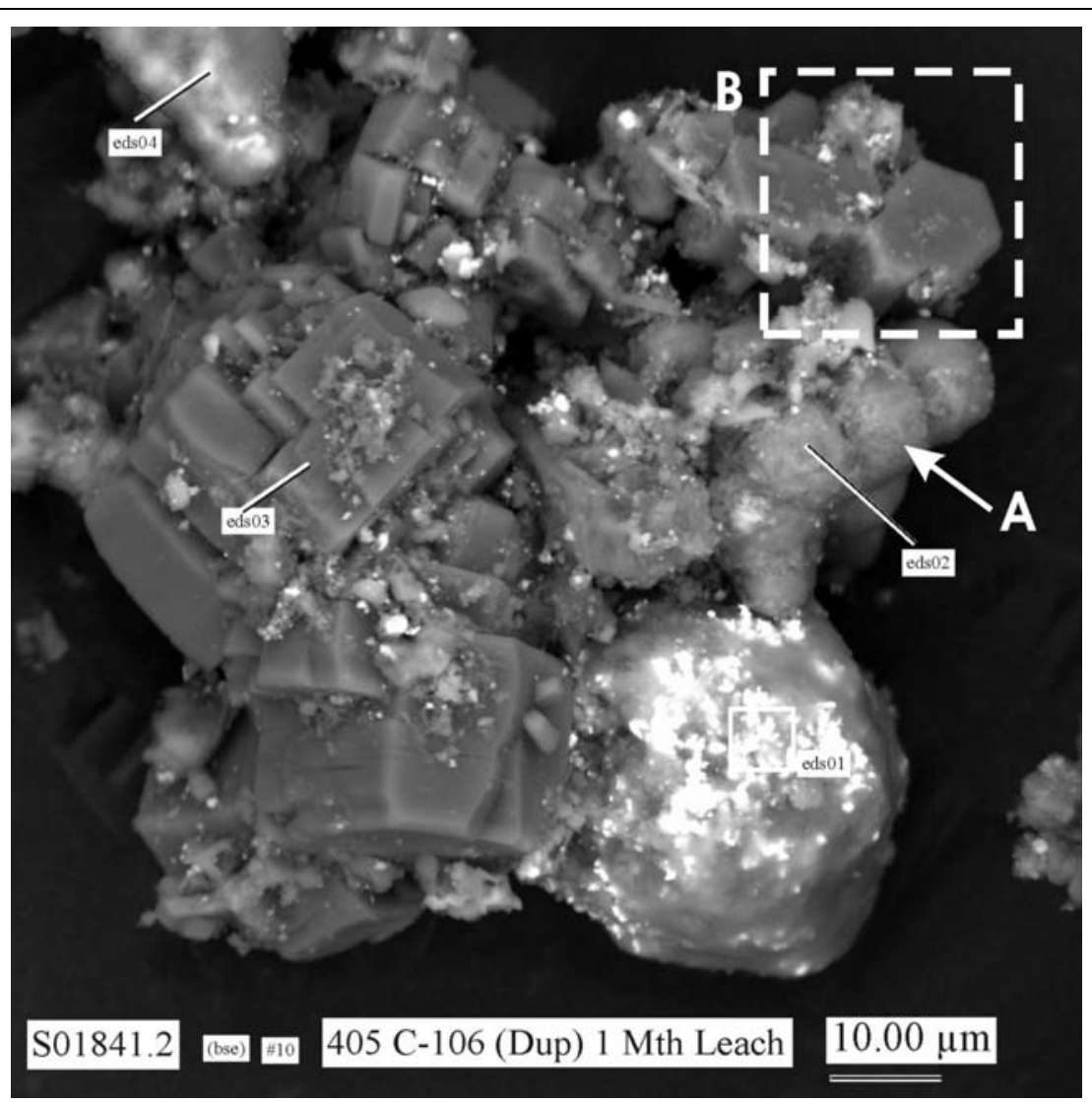

Figure C.32. Micrograph Showing at Higher Magnification the Area Indicated by the White Dashed-Line Square in Figure C.31 


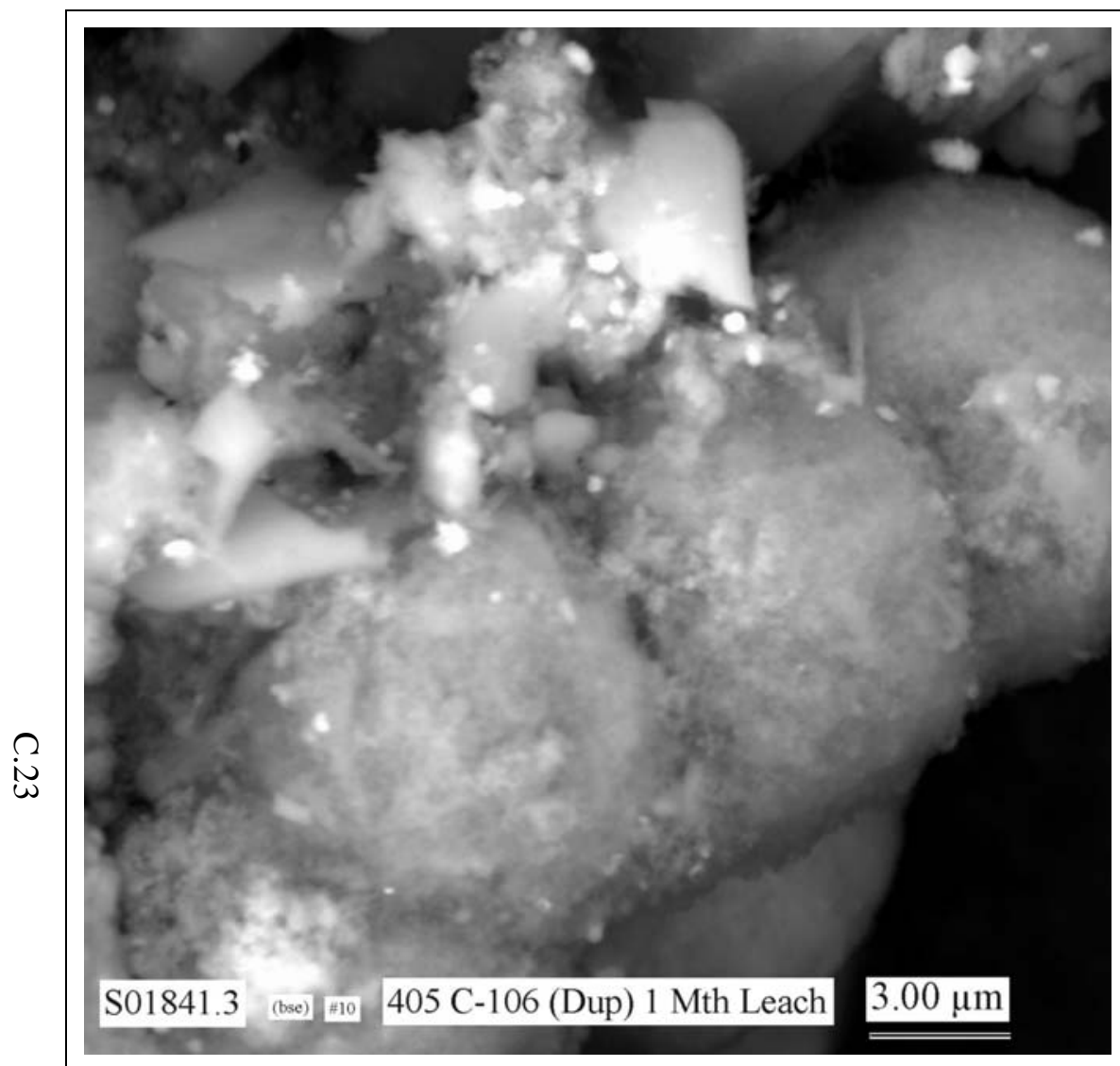

Figure C.33. Micrograph Showing at Higher Magnification the Spherical Particles Labeled A in Figure C.32

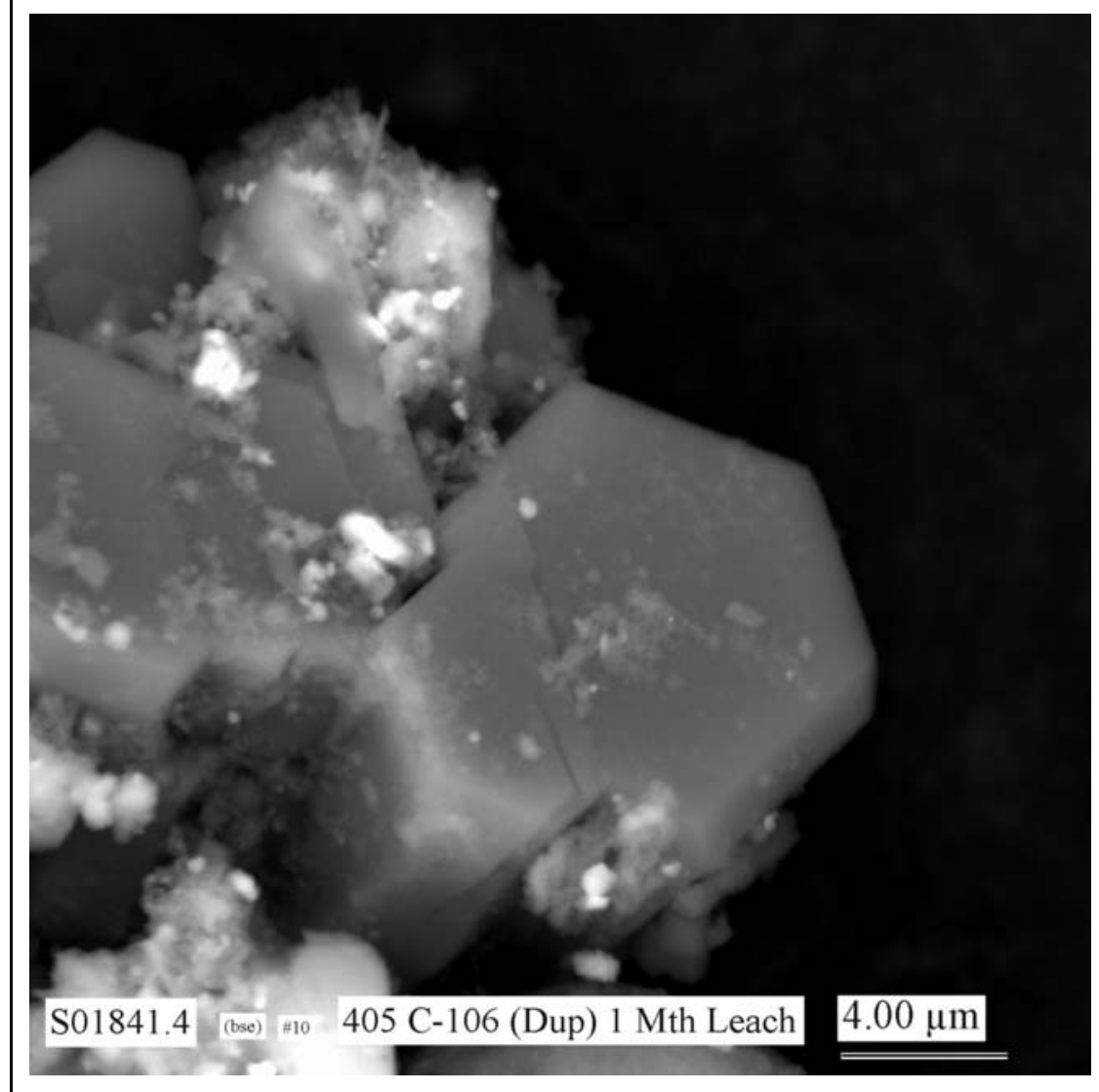

Figure C.34. Micrograph Showing at Higher Magnification the Area Indicated by the White Dashed-Line Square Labeled B in Figure C.32 


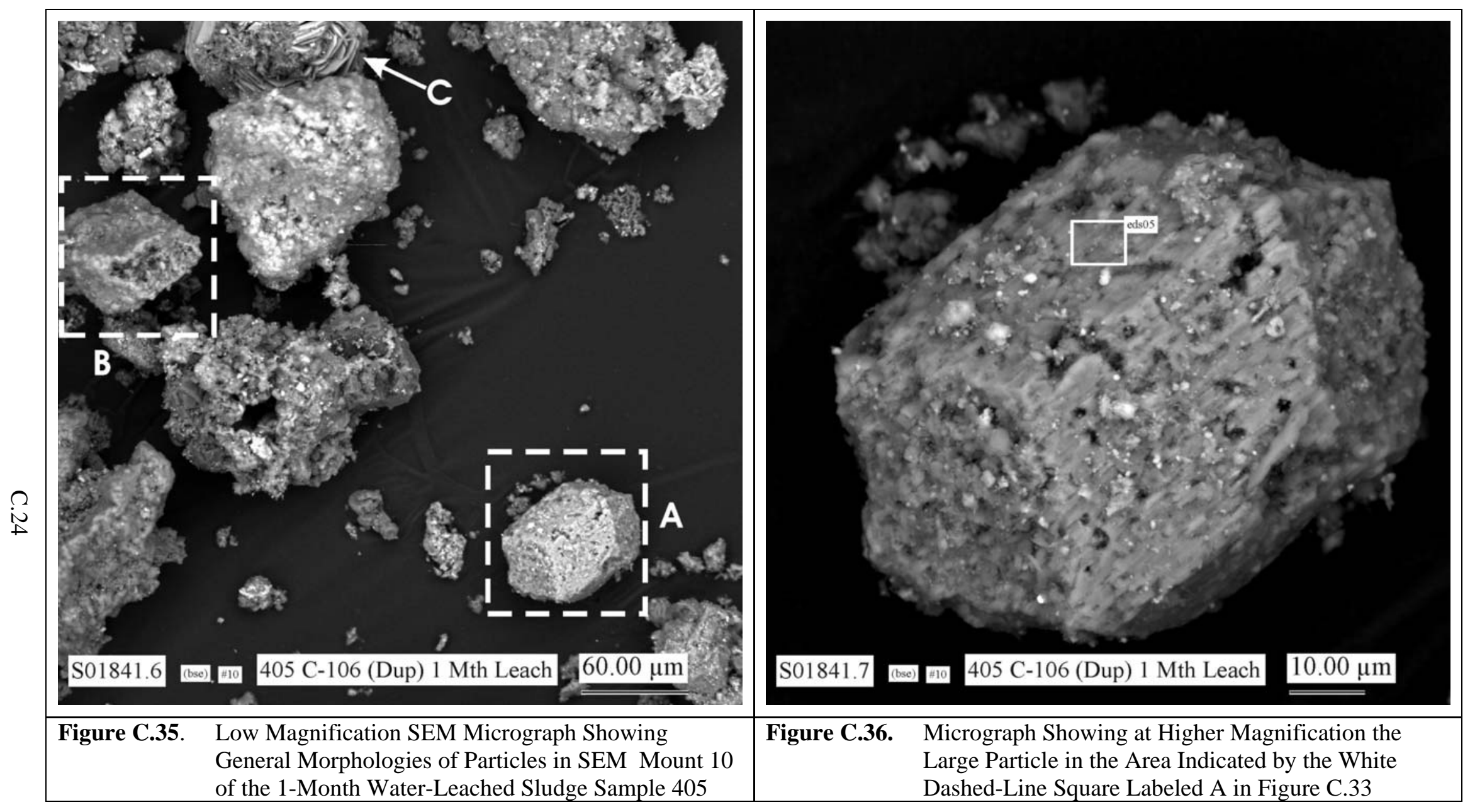




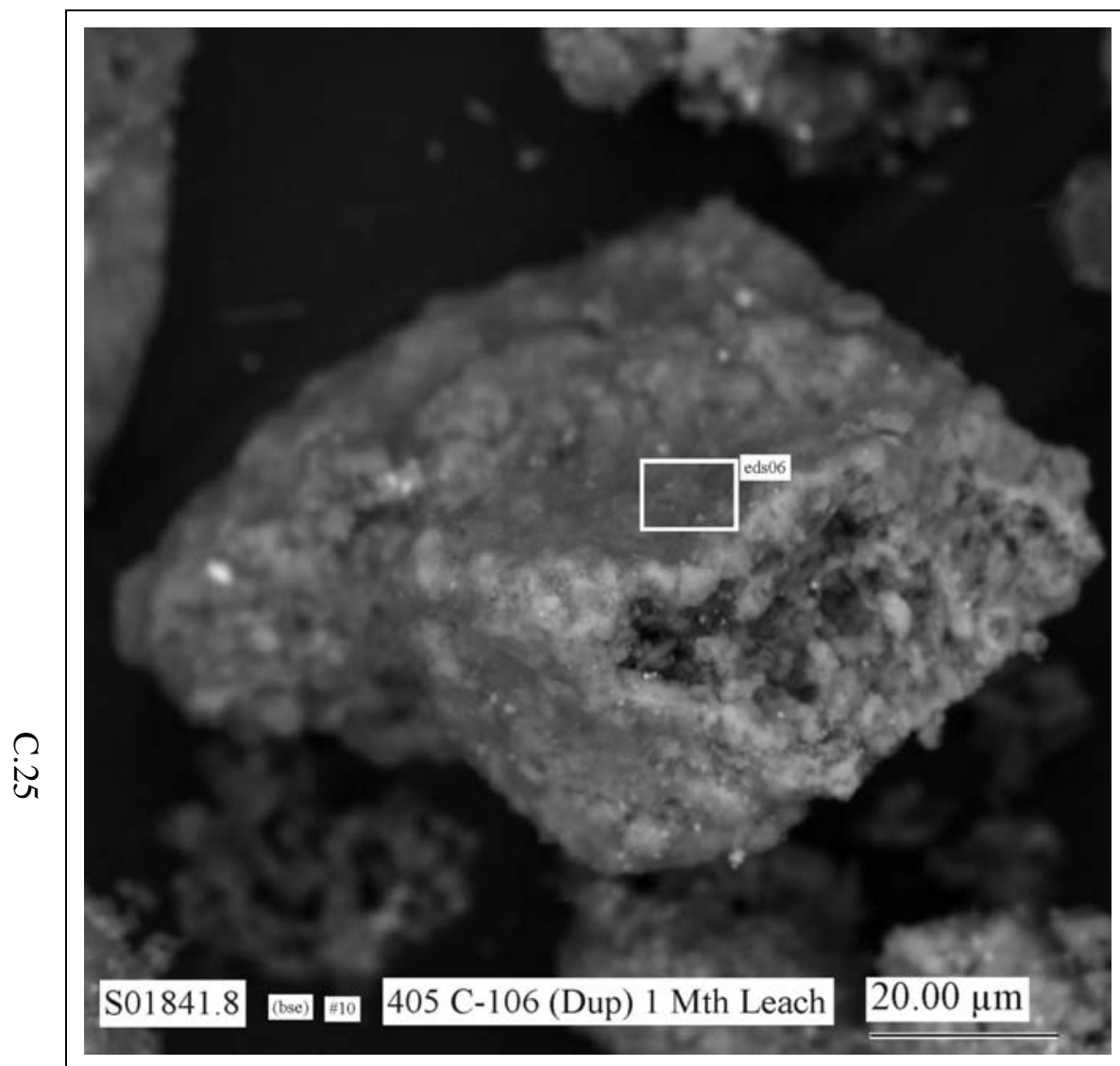

Figure C.37. Micrograph Showing at Higher Magnification the Area Indicated by the White Dashed-Line Square Labeled B in Figure C.33

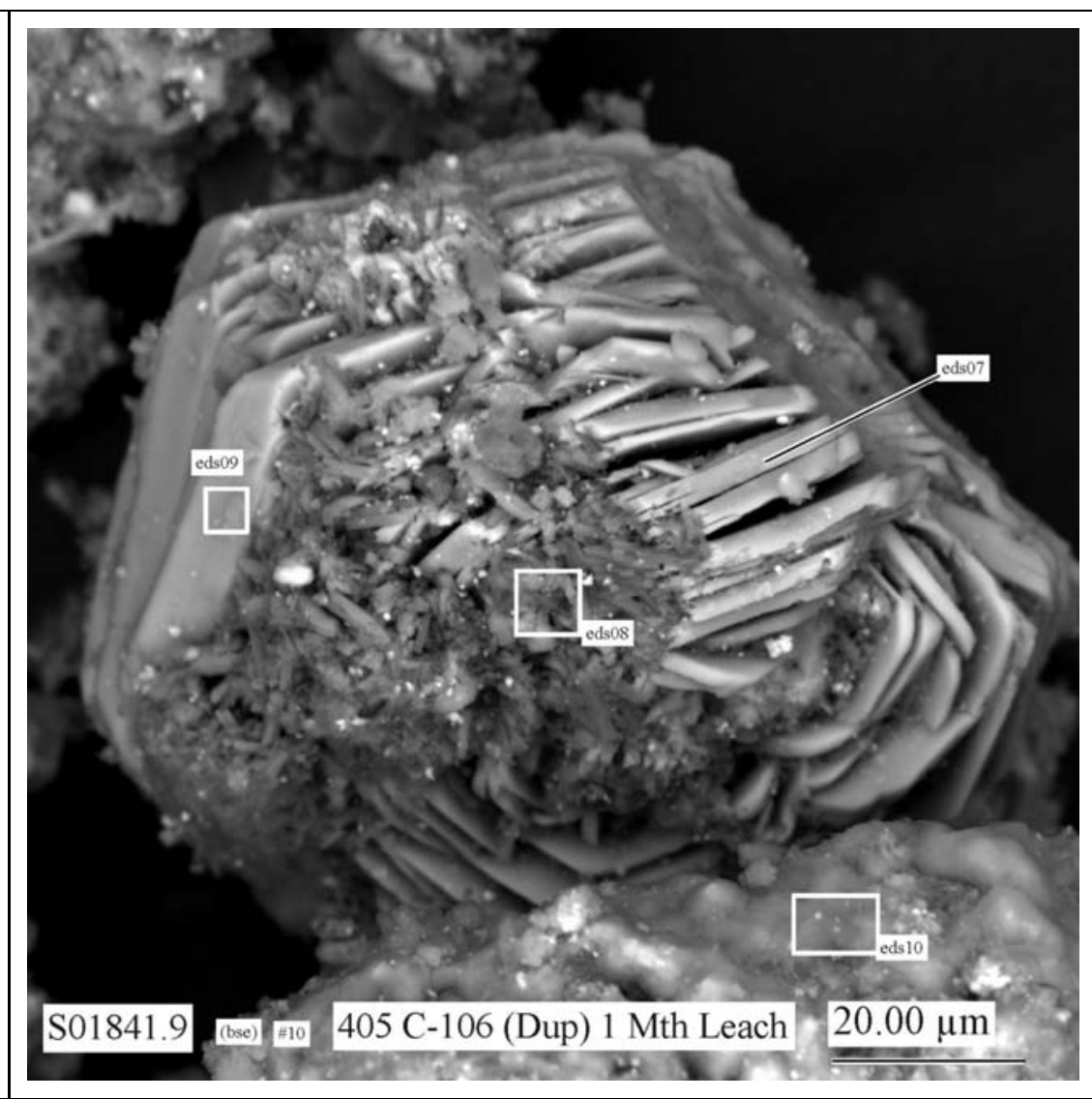

Figure C.38. Micrograph Showing at Higher Magnification the Large Particle Labeled C in Figure C.33 


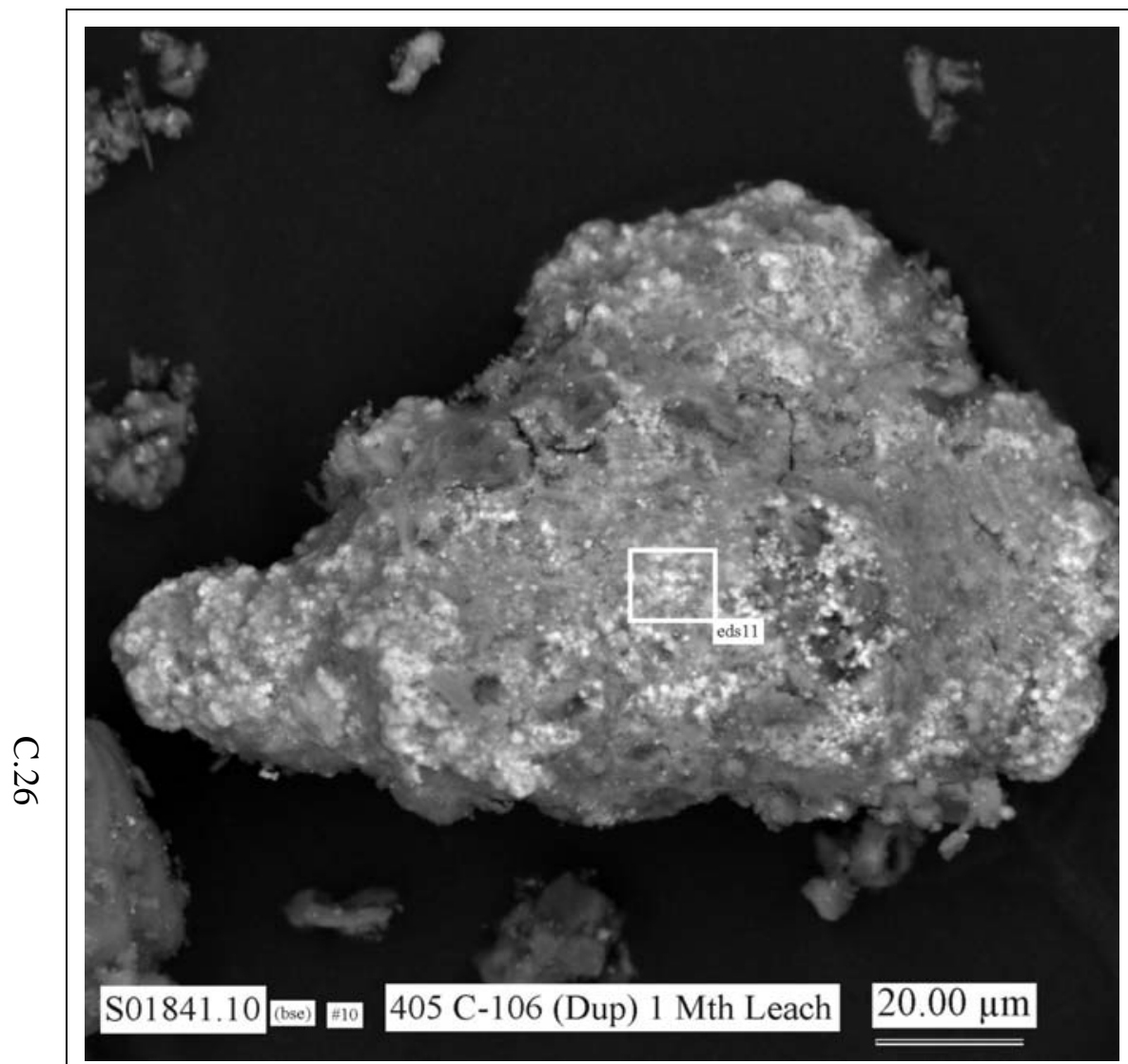

Figure C.39. $\quad$ Micrograph Showing Morphologies of Typical Particles in SEM Mount 10 of the 1-Month WaterLeached Sludge Sample 405

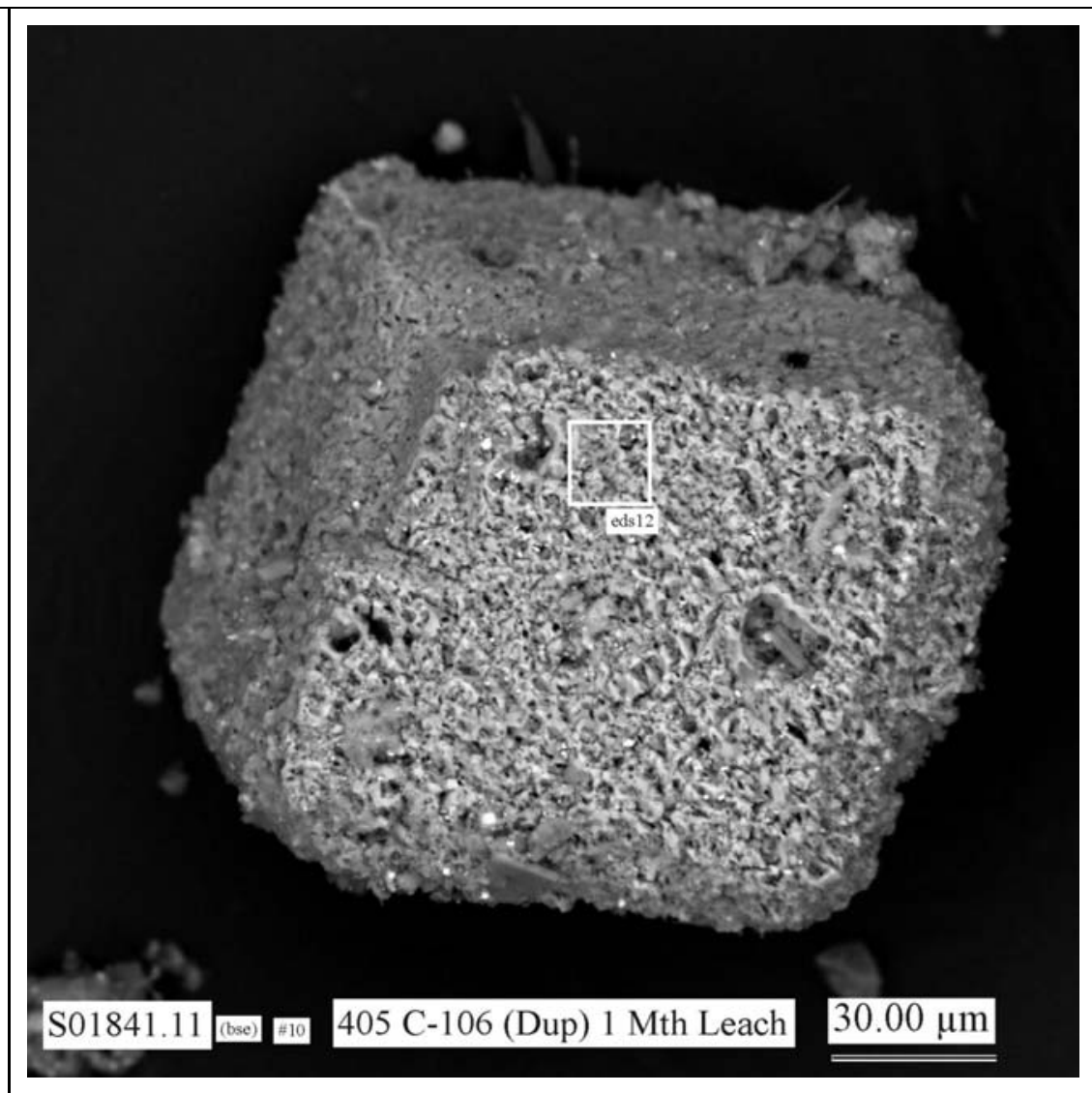

Figure C.40. Micrograph Showing Morphologies of Typical Particles in SEM Mount 10 of the 1-Month WaterLeached Sludge Sample 405 

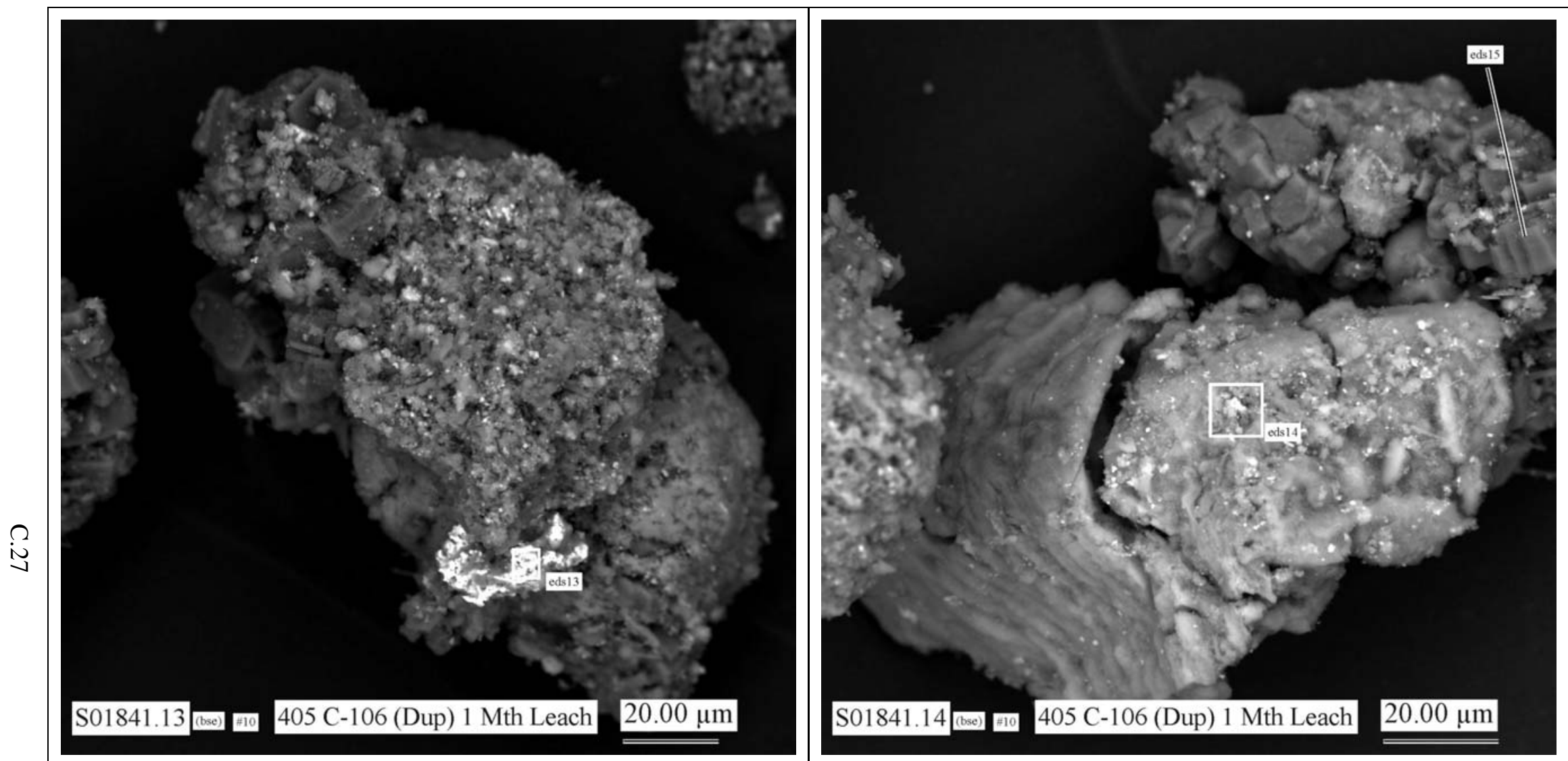

Figure C.41. Micrograph Showing Morphologies of Typical Particles in SEM Mount 10 of the 1-Month WaterLeached Sludge Sample 405

Figure C.42. Micrograph Showing Morphologies of Typical Particles in SEM Mount 10 of the 1-Month WaterLeached Sludge Sample 405 


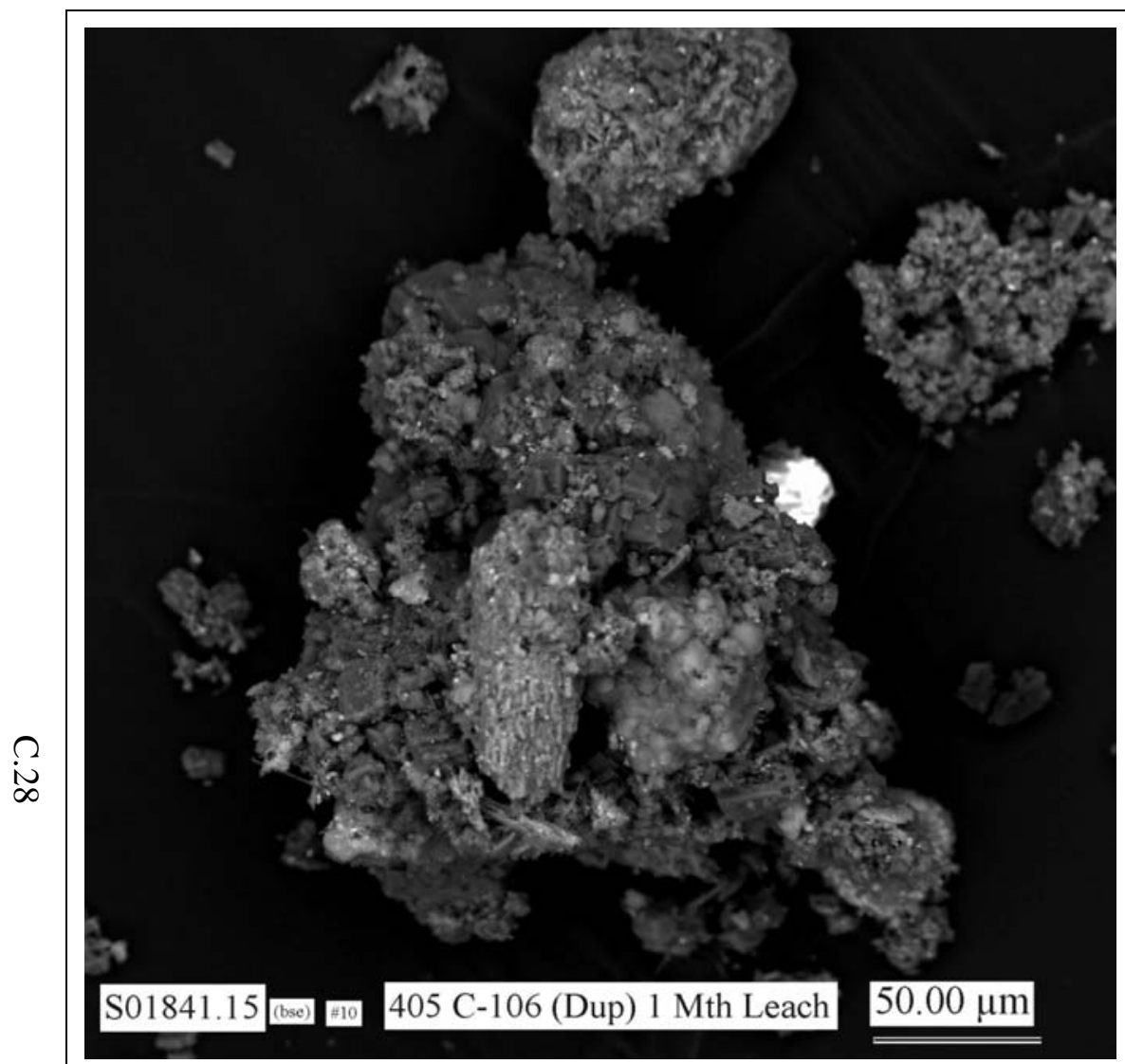

Figure C.43. Micrograph Showing Morphologies of Typical Particles in SEM Mount 10 of the 1-Month WaterLeached Sludge Sample 405

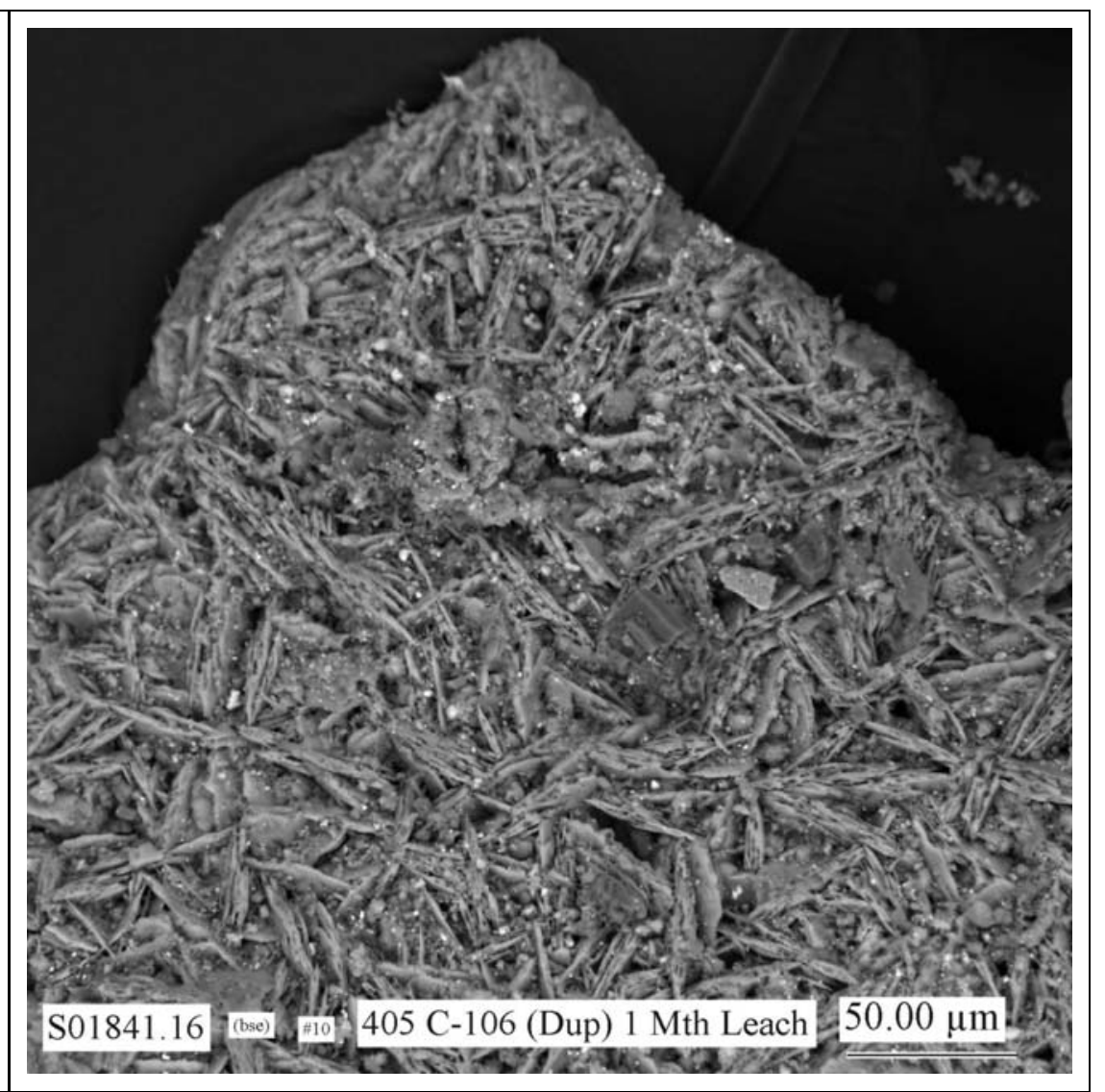

Figure C.44. Micrograph Showing Morphologies of Typical Particles in SEM Mount 10 of the 1-Month WaterLeached Sludge Sample 405 


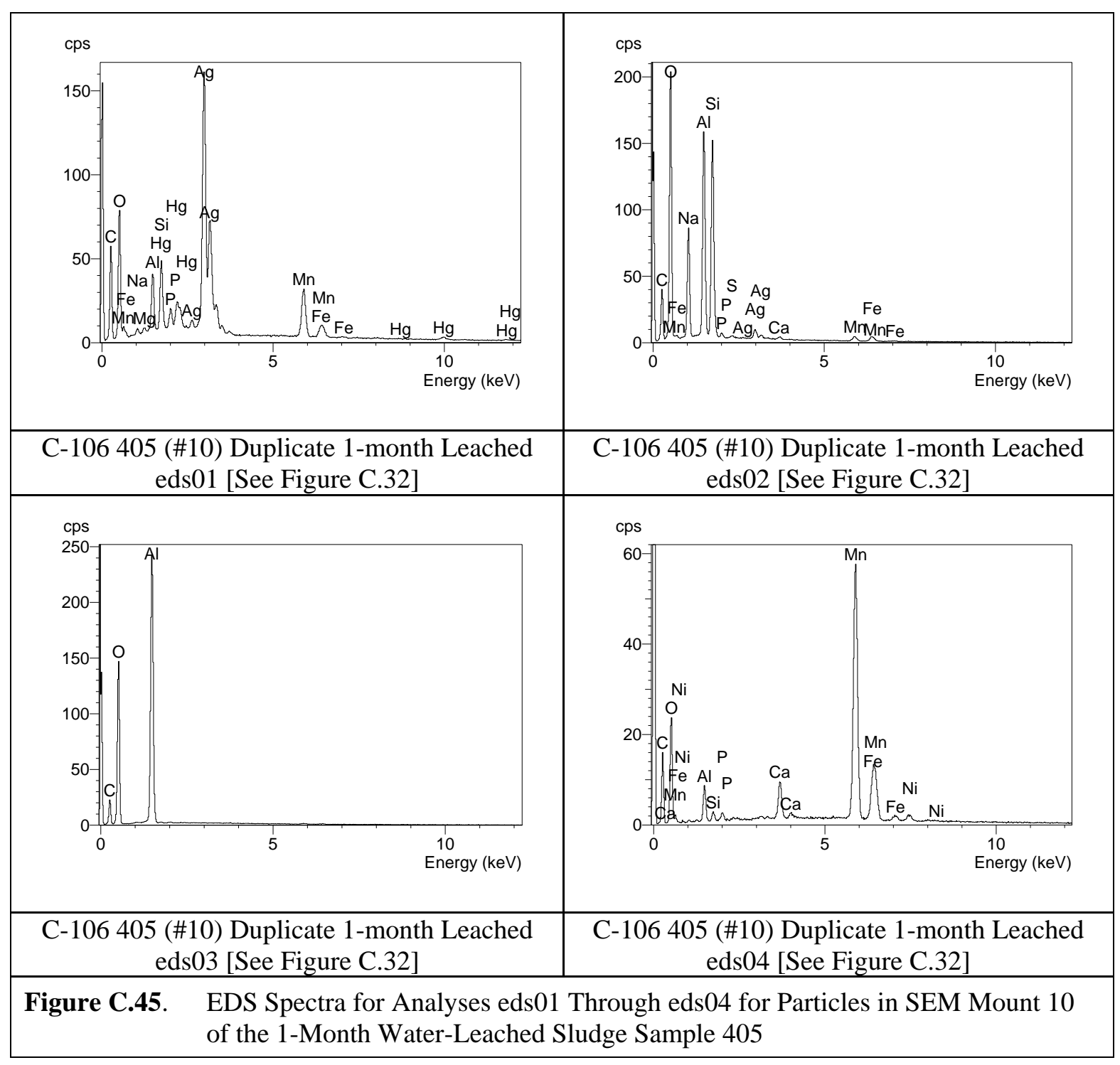




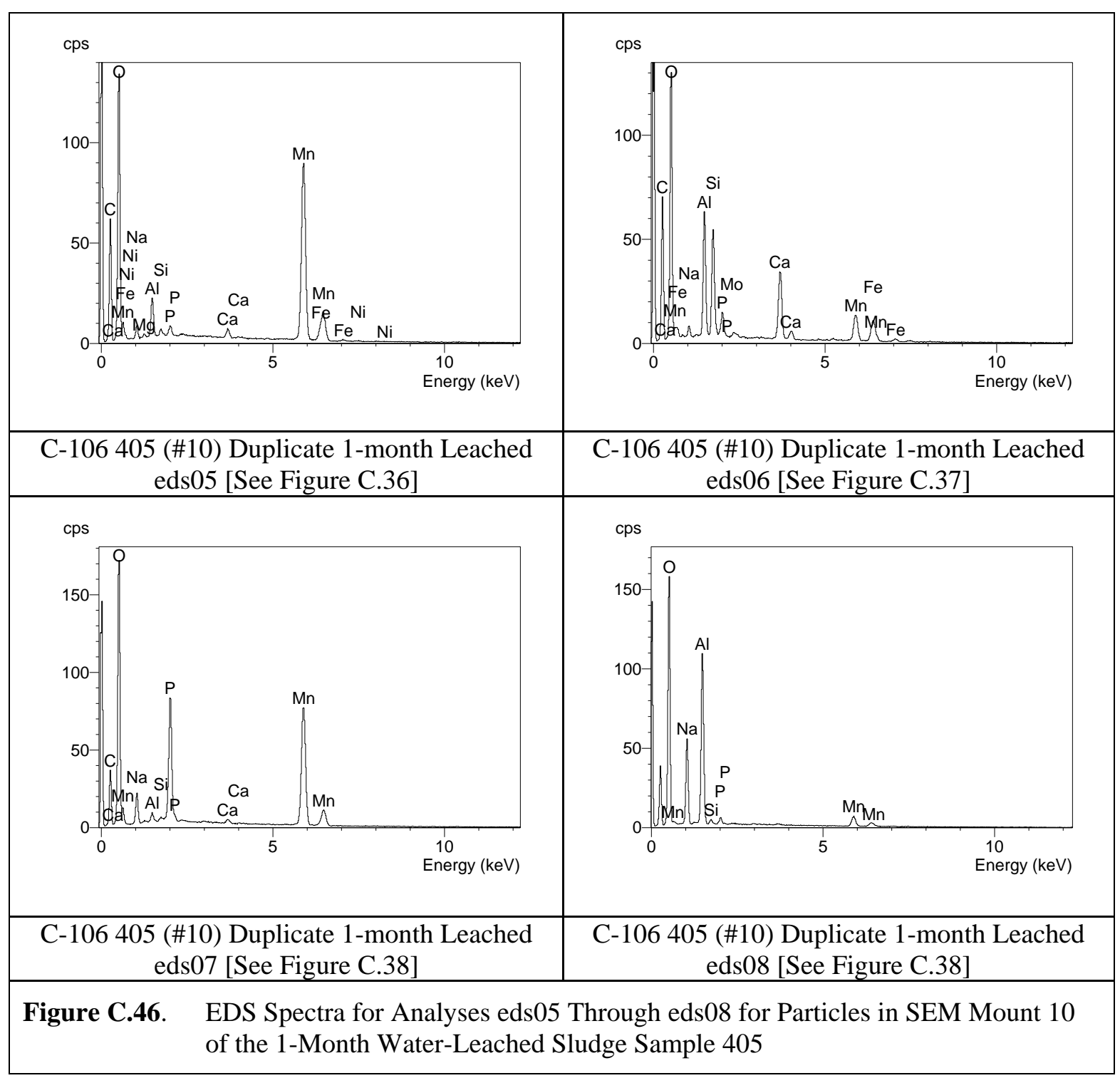




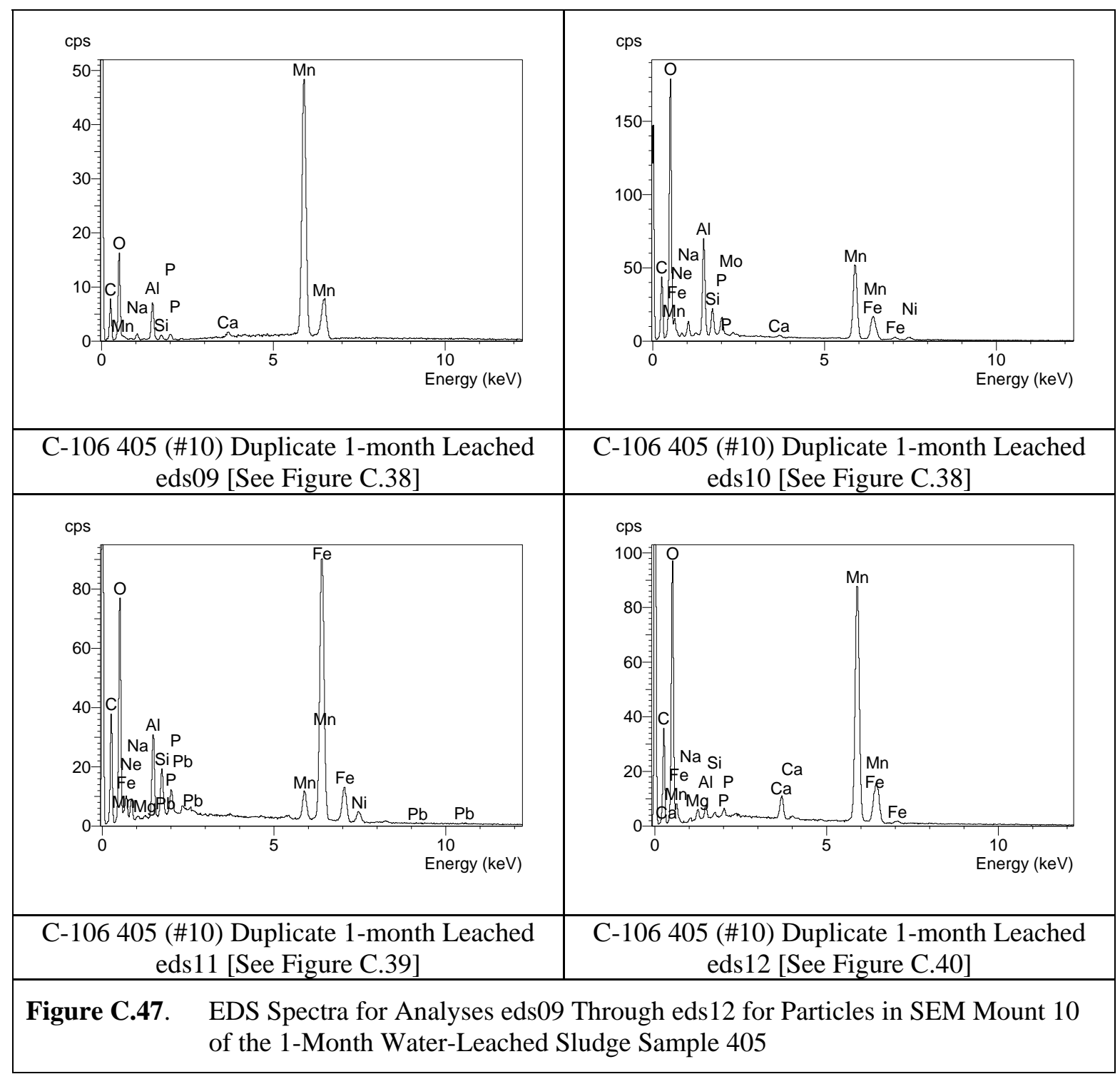




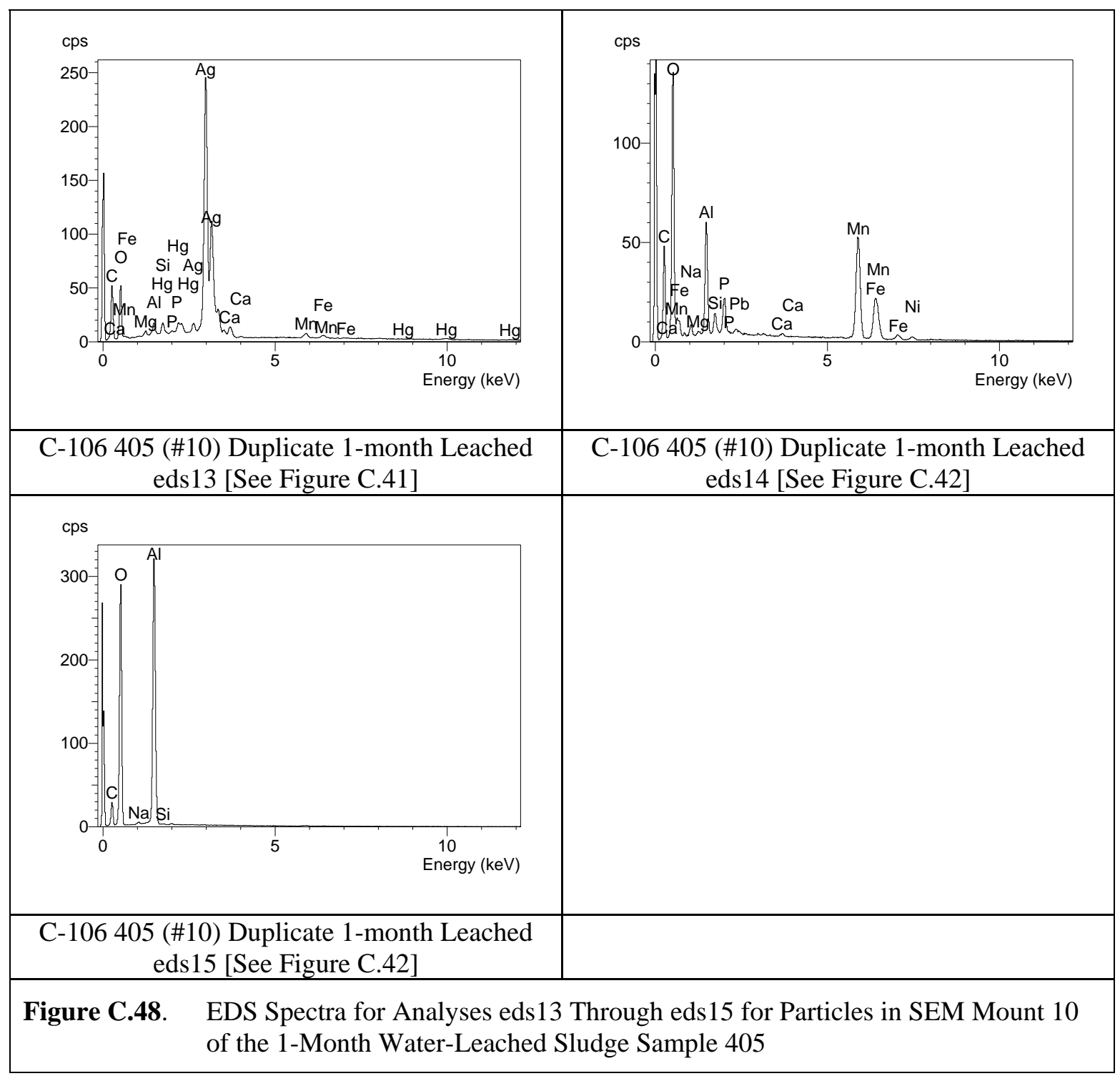




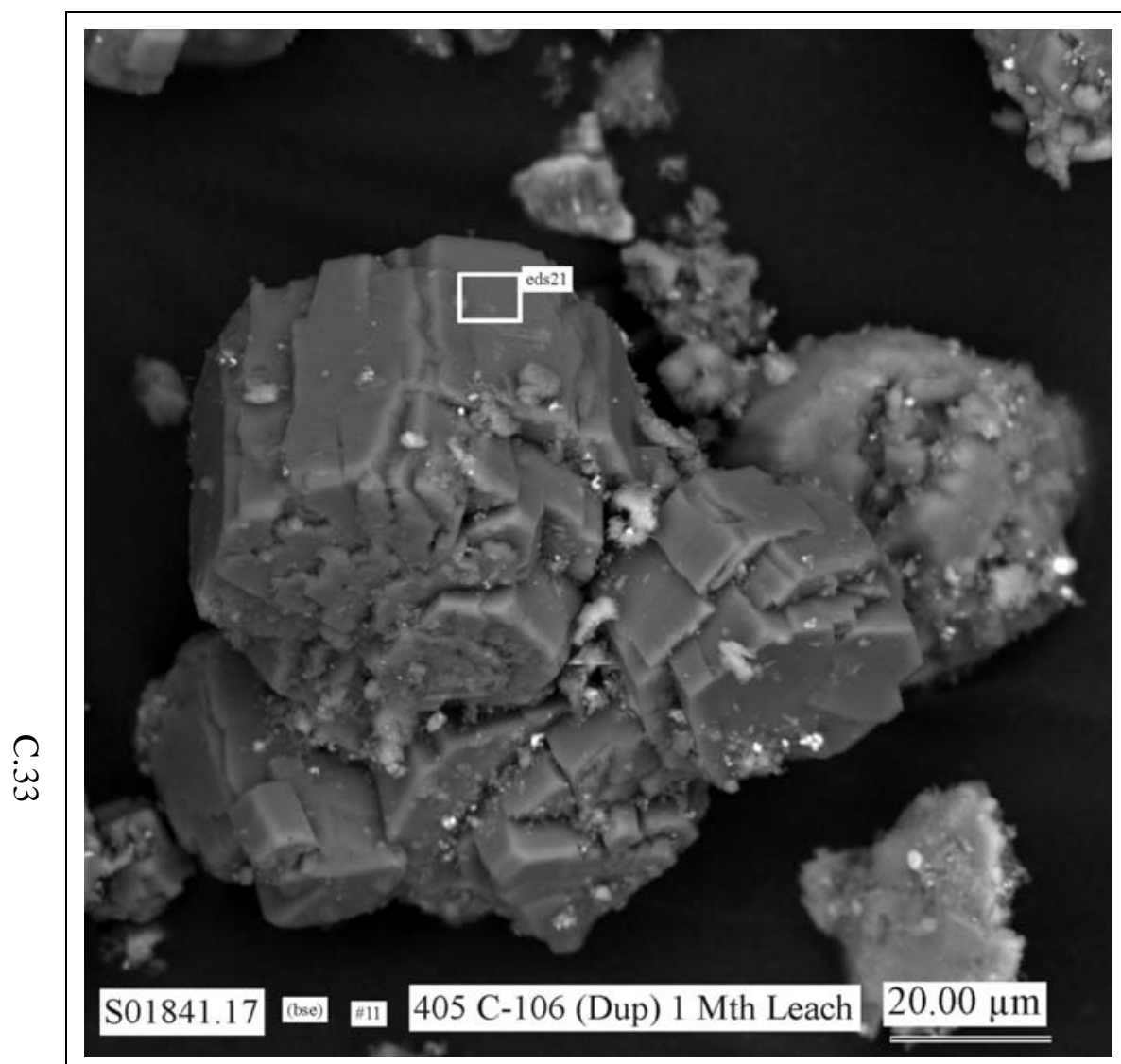

Figure C.49. Micrograph Showing Morphologies of Typical Particles in SEM Mount 11 of the 1-Month WaterLeached Sludge Sample 405

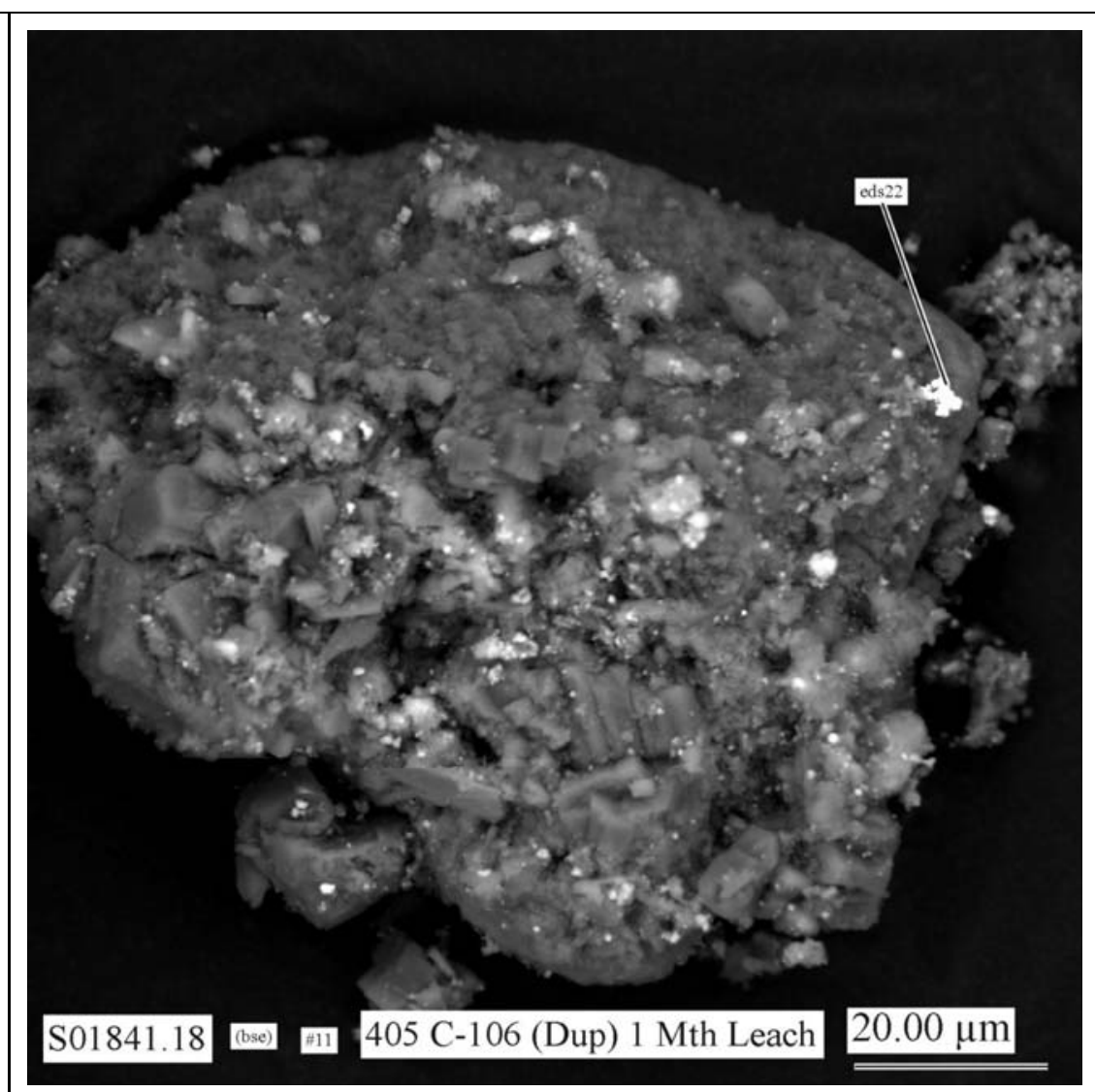

Figure C.50. Micrograph Showing Morphologies of Typical Particles in SEM Mount 11 of the 1-Month WaterLeached Sludge Sample 405 


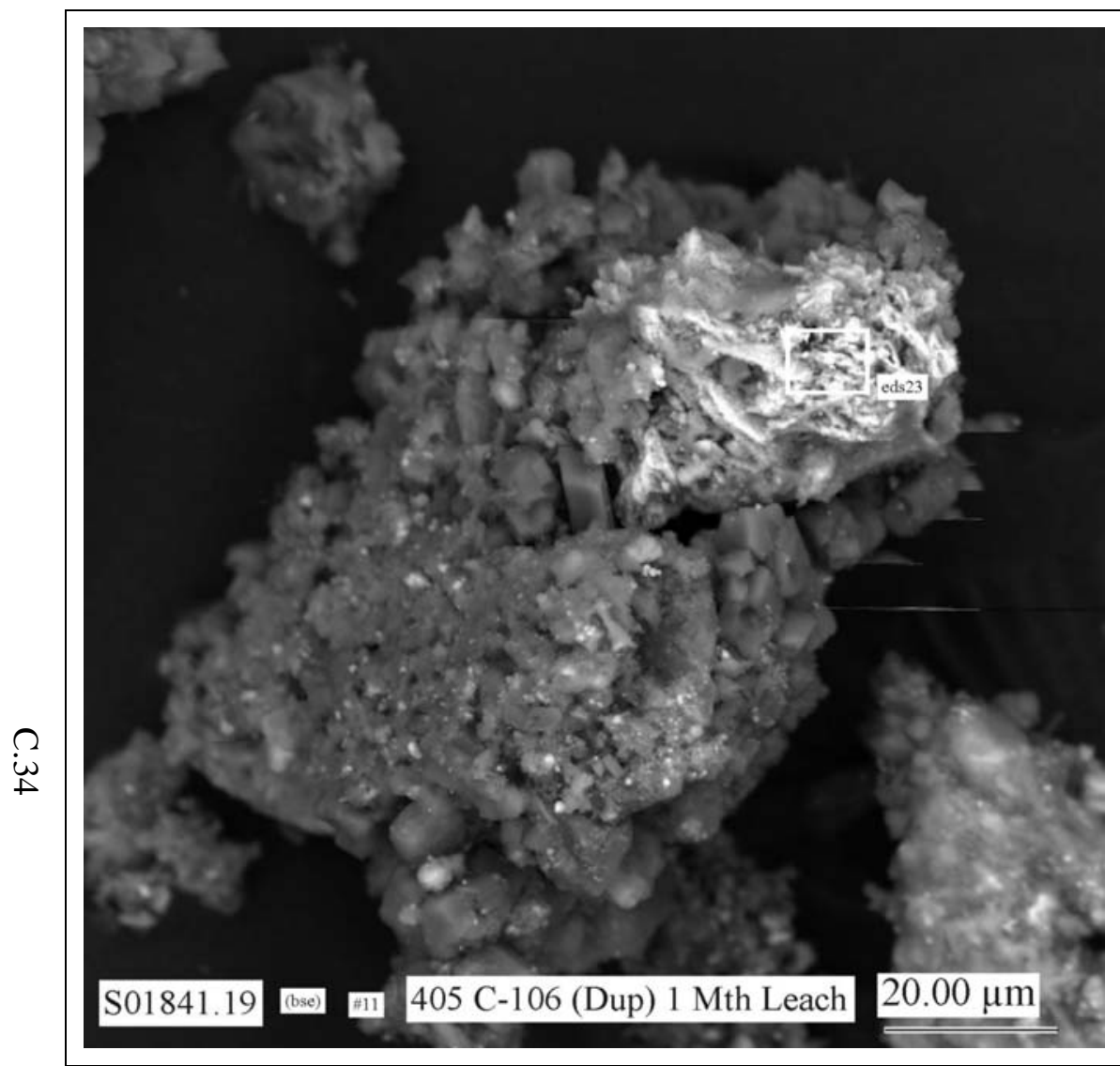

Figure C.51. Micrograph Showing Morphologies of Typical Particles in SEM Mount 11 of the 1-Month WaterLeached Sludge Sample 405

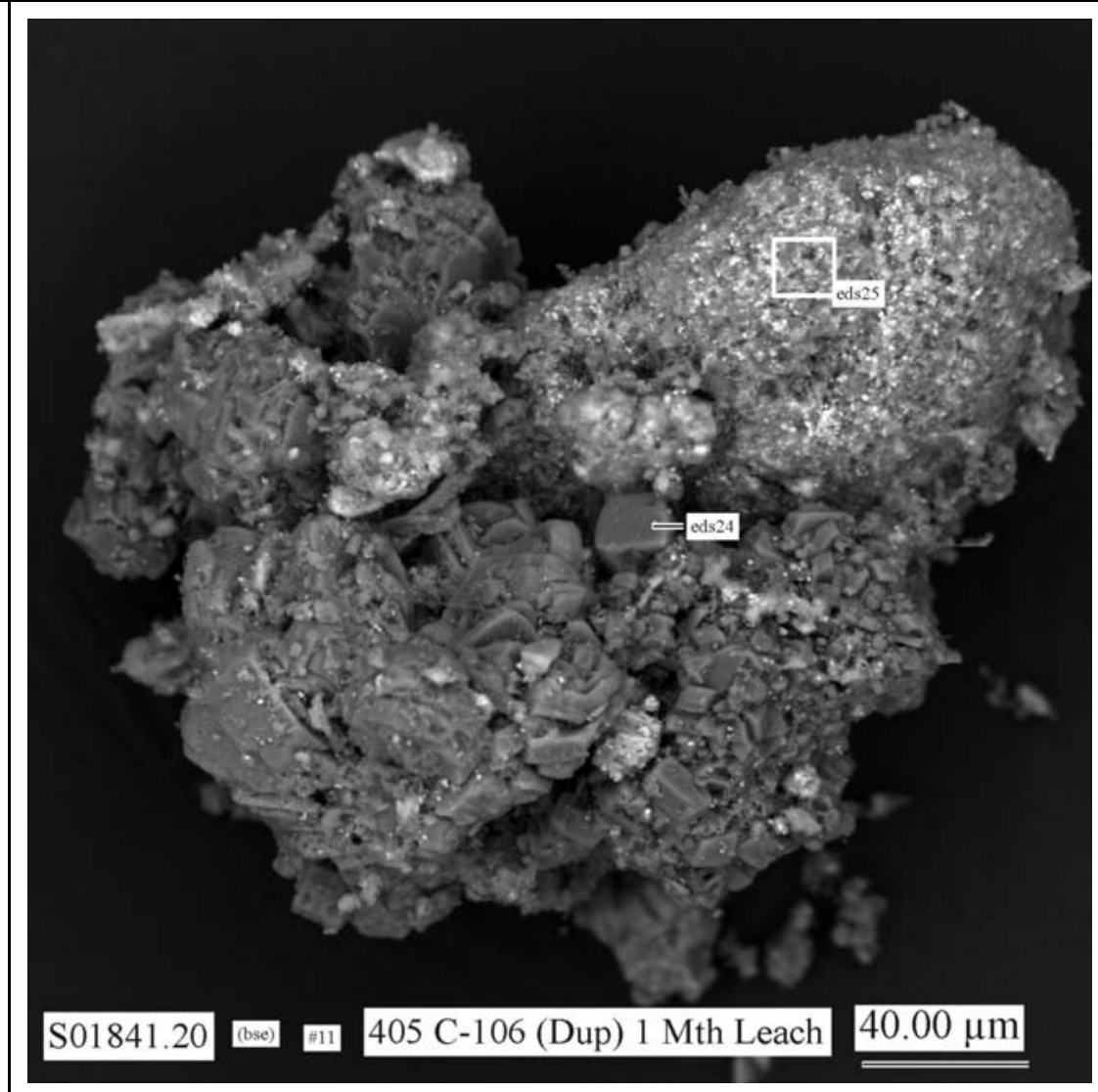

Figure C.52. Micrograph Showing Morphologies of Typical Particles in SEM Mount 11 of the 1-Month WaterLeached Sludge Sample 405 

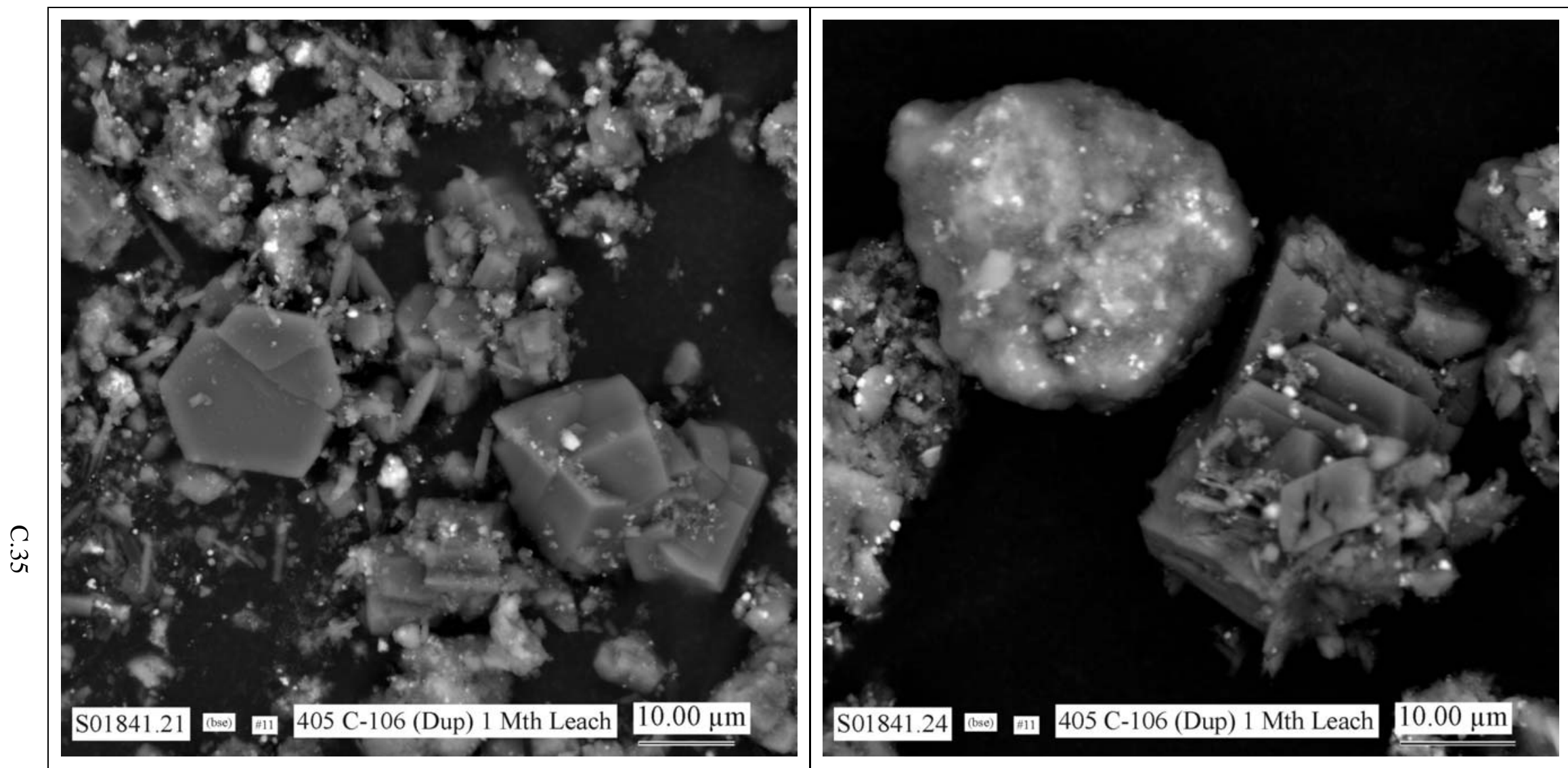

Figure C.53. Micrograph Showing Morphologies of Typical Particles in SEM Mount 11 of the 1-Month WaterLeached Sludge Sample 405

Figure C.54. Micrograph Showing Morphologies of Typical Particles in SEM Mount 11 of the 1-Month WaterLeached Sludge Sample 405 


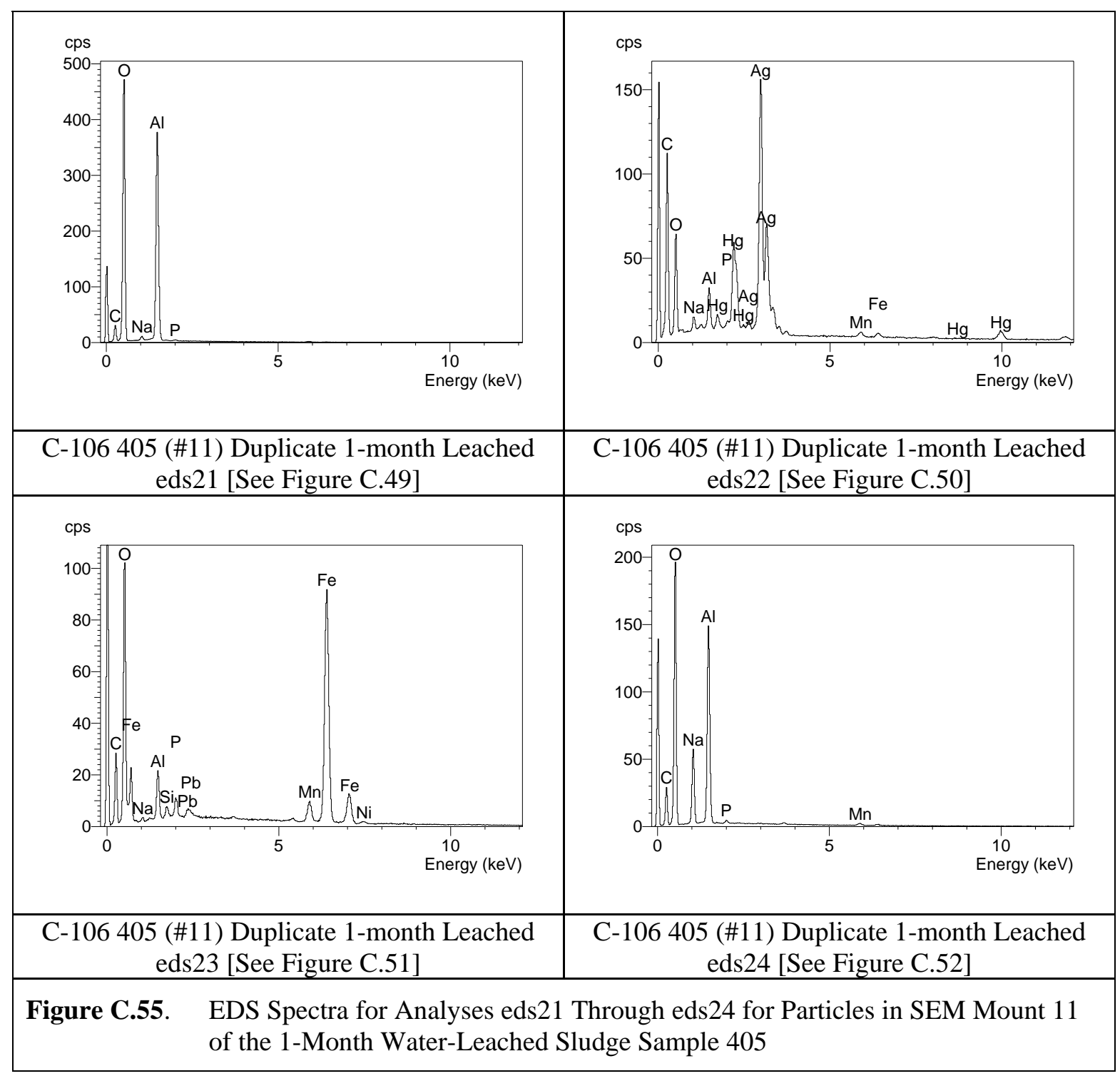




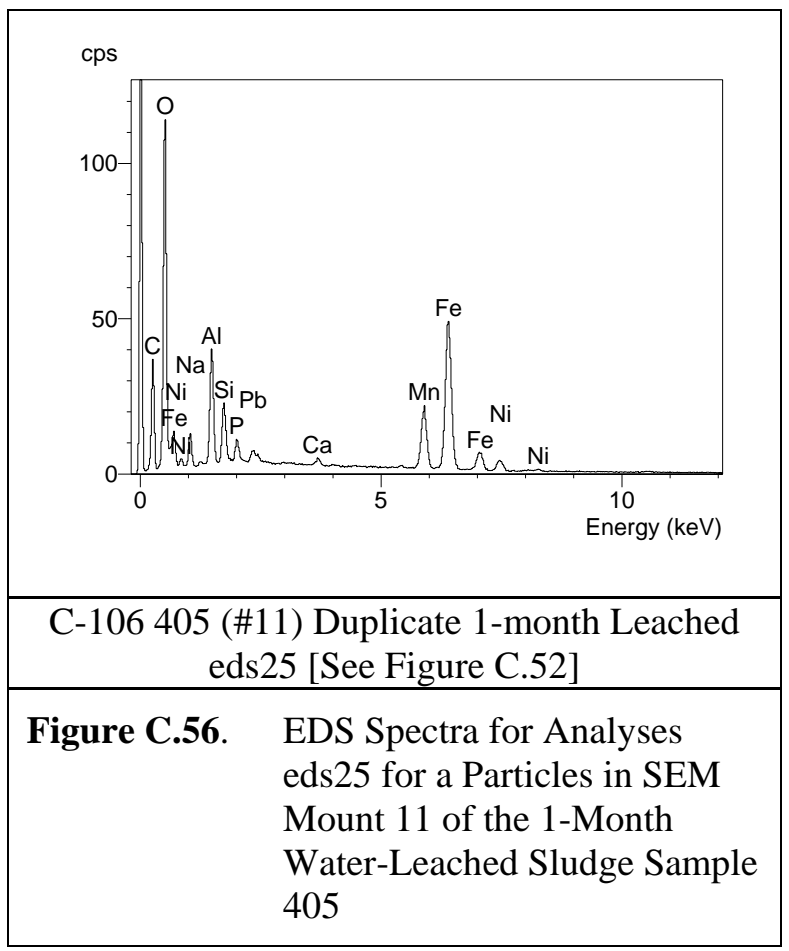

\section{C.3 Water-Leached (82-Day) Sample 404}

One mount of sample from the 82-day water-leached residual sludge from tank C-106 (primary 404) was analyzed by SEM/EDS. The SEM micrographs for this sample are shown in Figures C.57 through C.72, and the EDS spectra for this mount are given in Figures C.73 through C.76. 


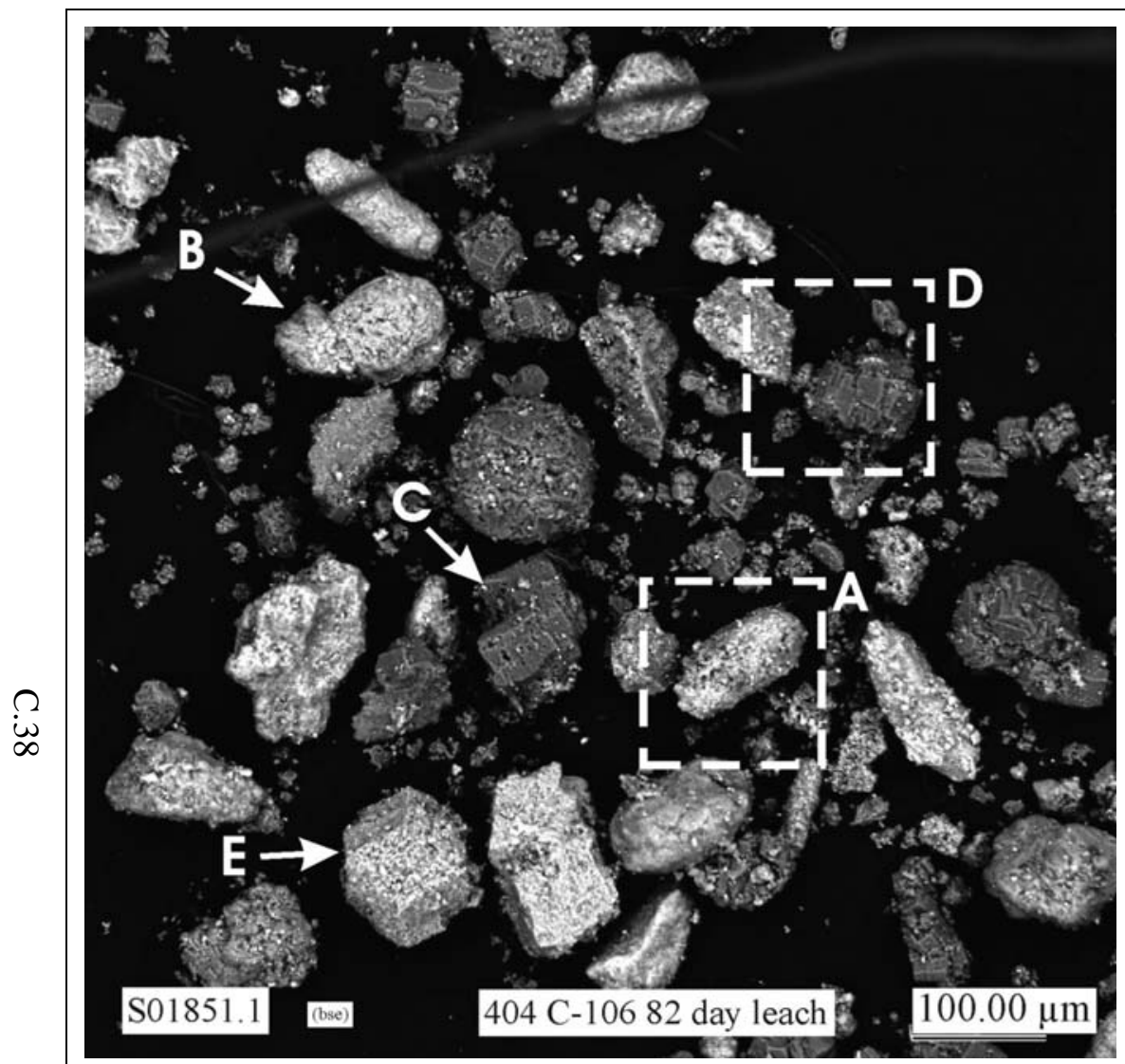

Figure C.57. Low Magnification SEM Micrograph Showing General Morphologies of Particles in Sample 404 of 82-Day Water-Leached Sludge

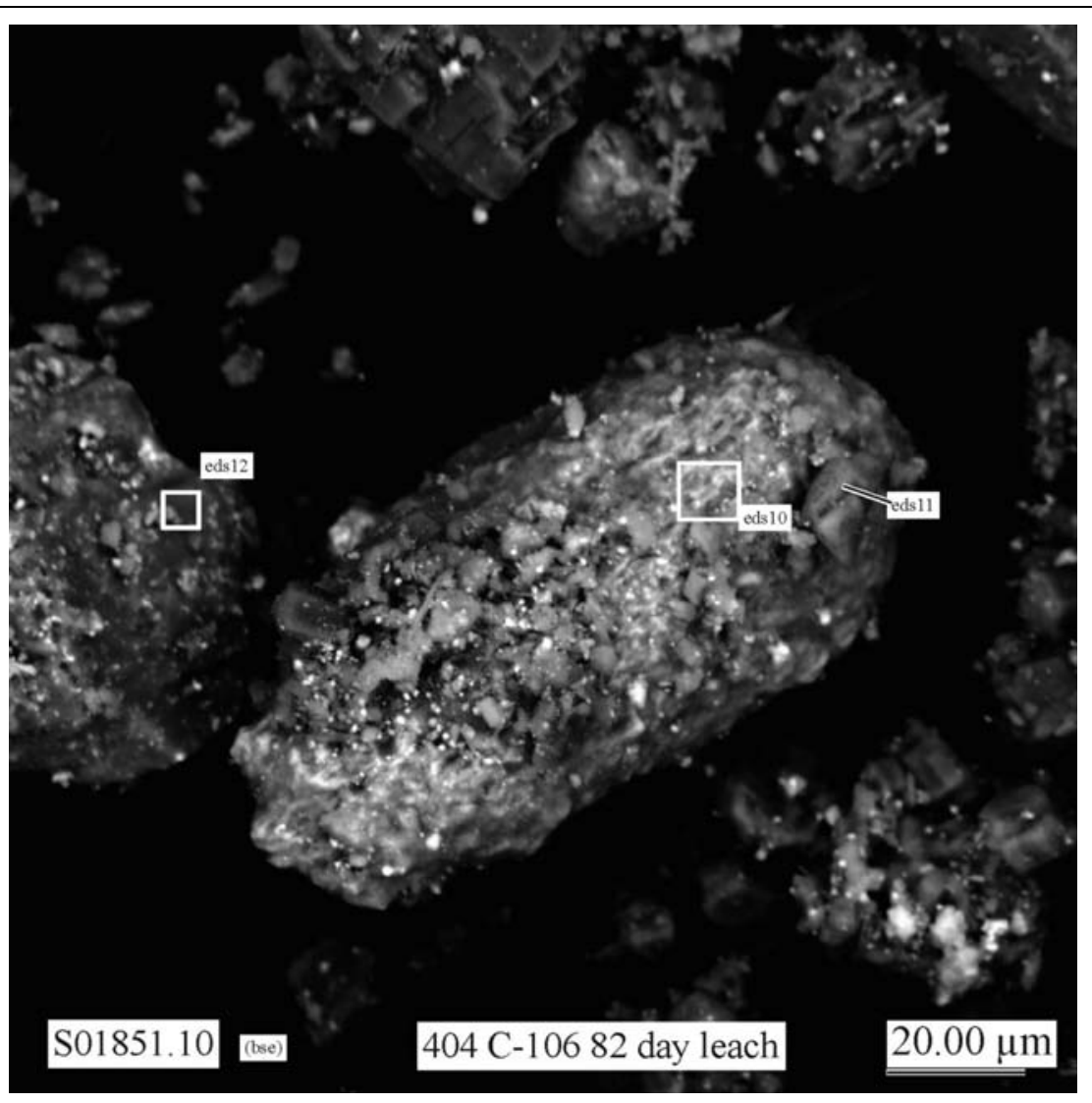

Figure C.58. Micrograph Showing at Higher Magnification the Area Indicated by the White Dashed-Line Square Labeled A in Figure C.57 


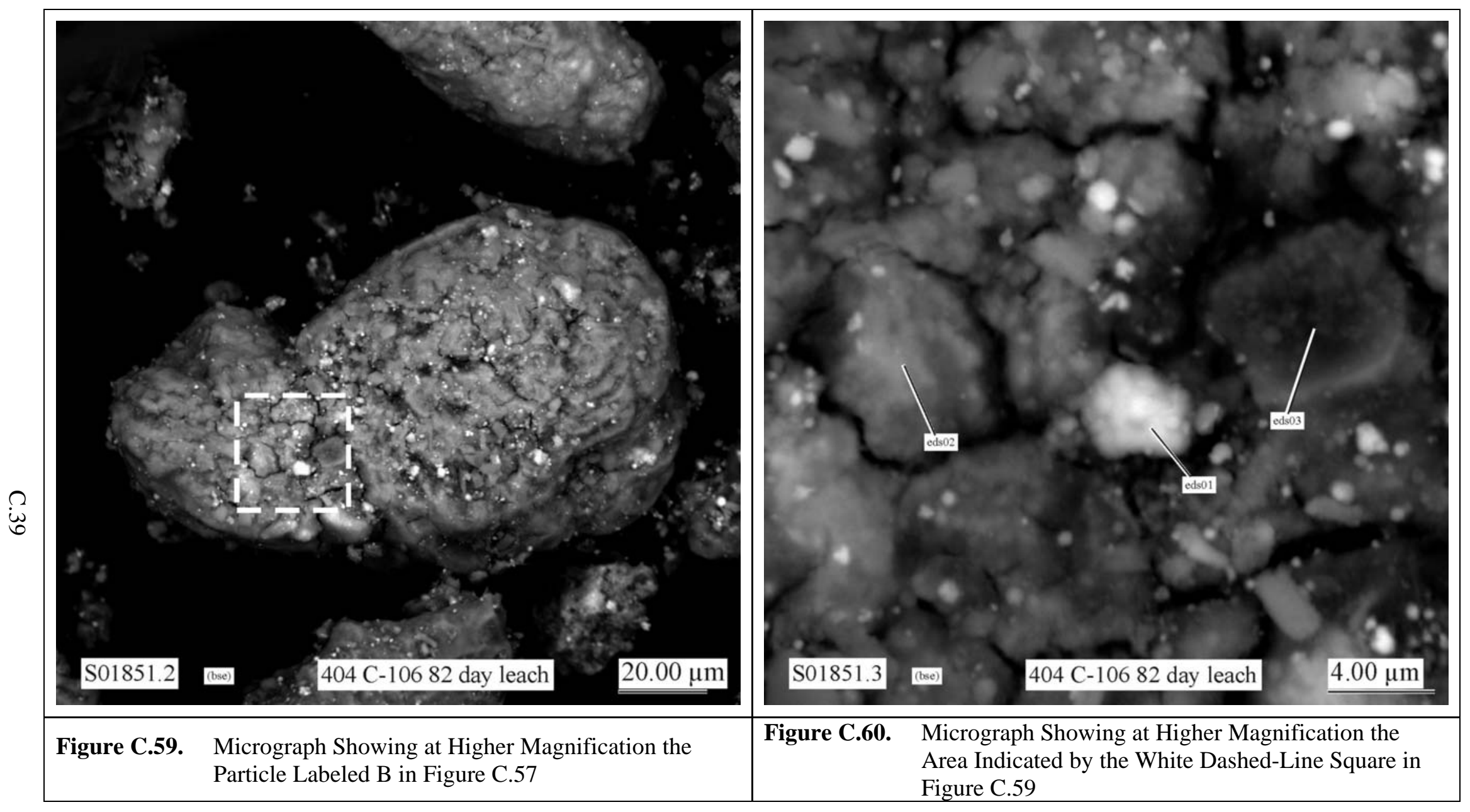




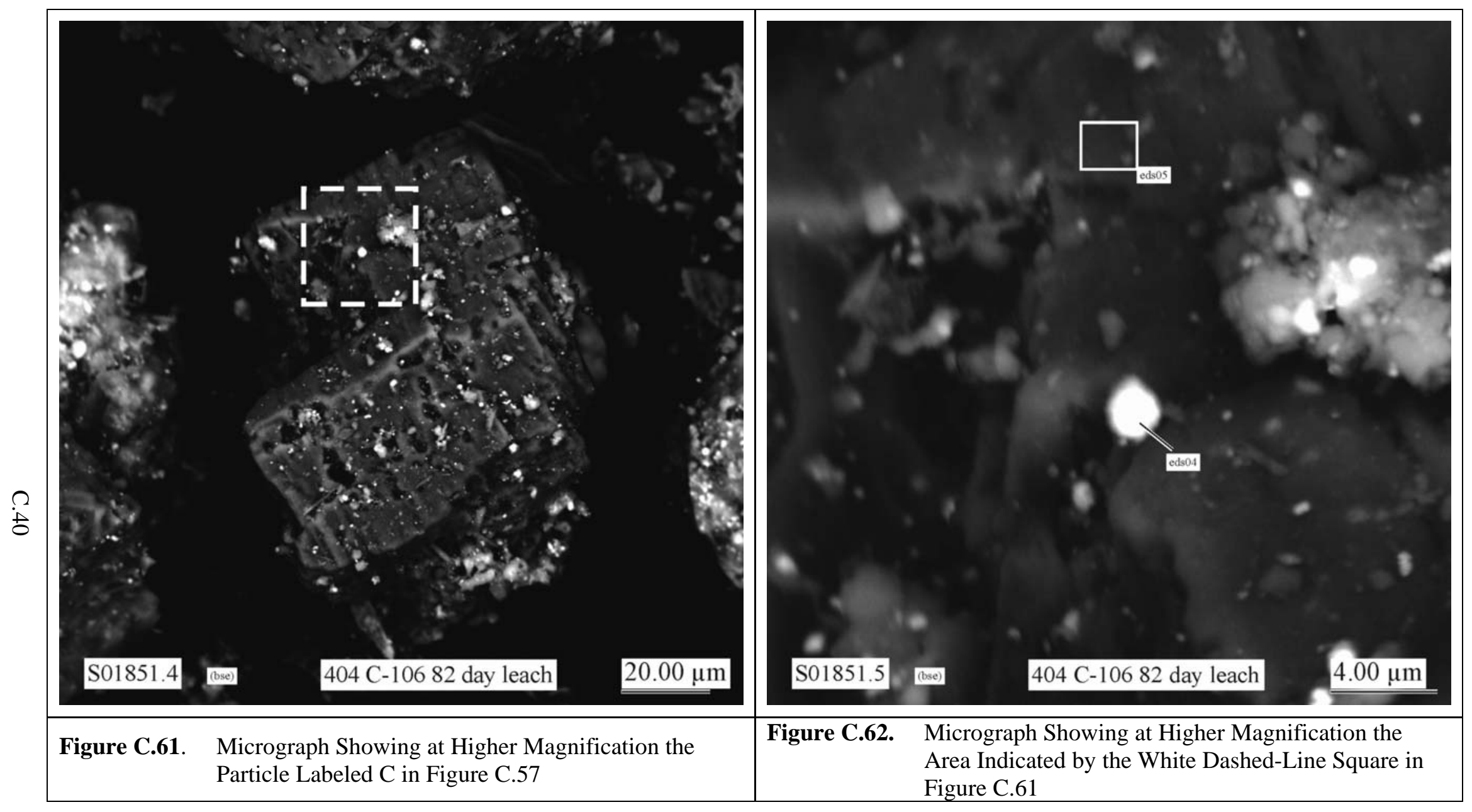




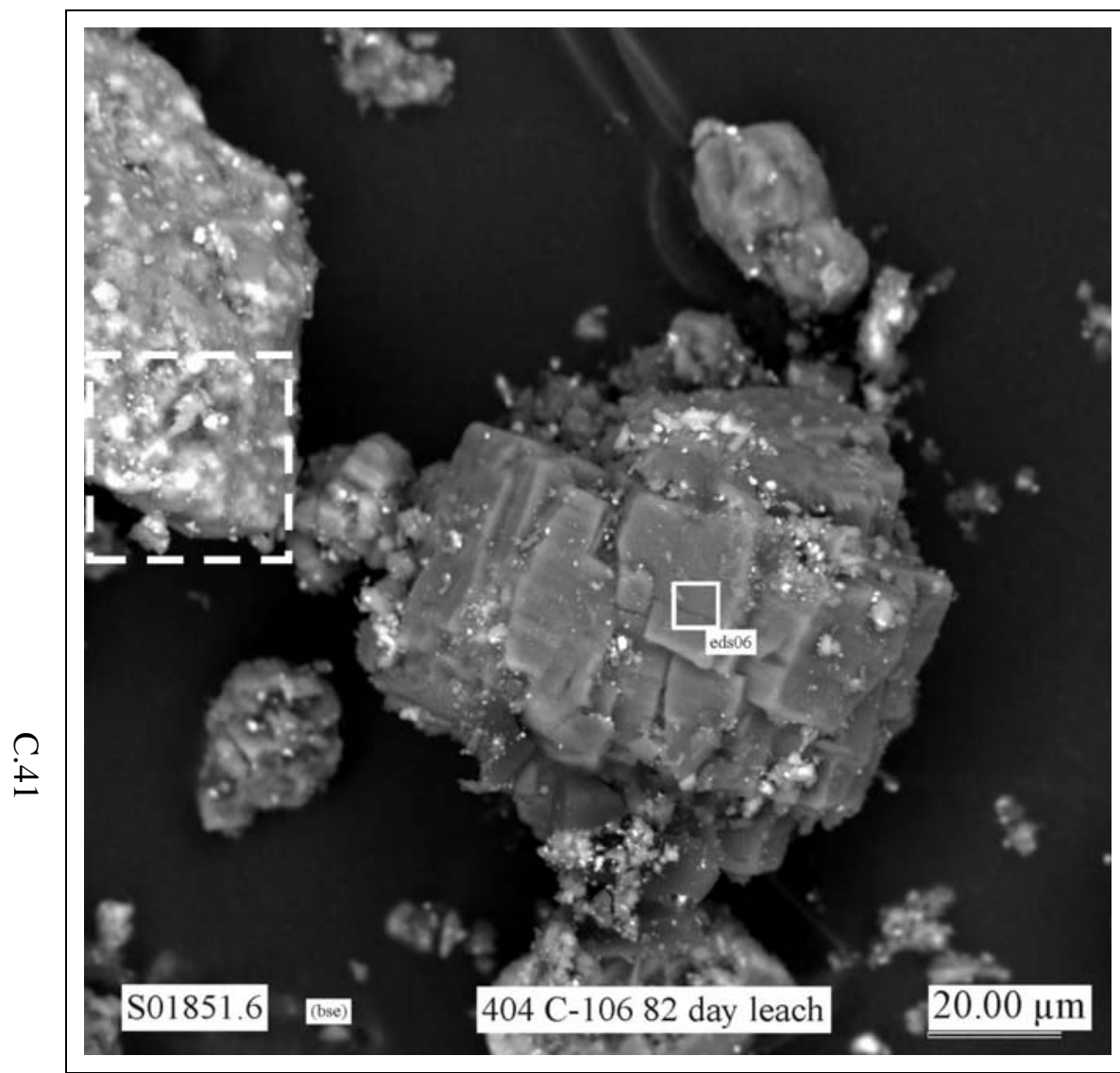

Figure C.63. Micrograph Showing at Higher Magnification the Area Indicated by the White Dashed-Line Square Labeled D in Figure C.57

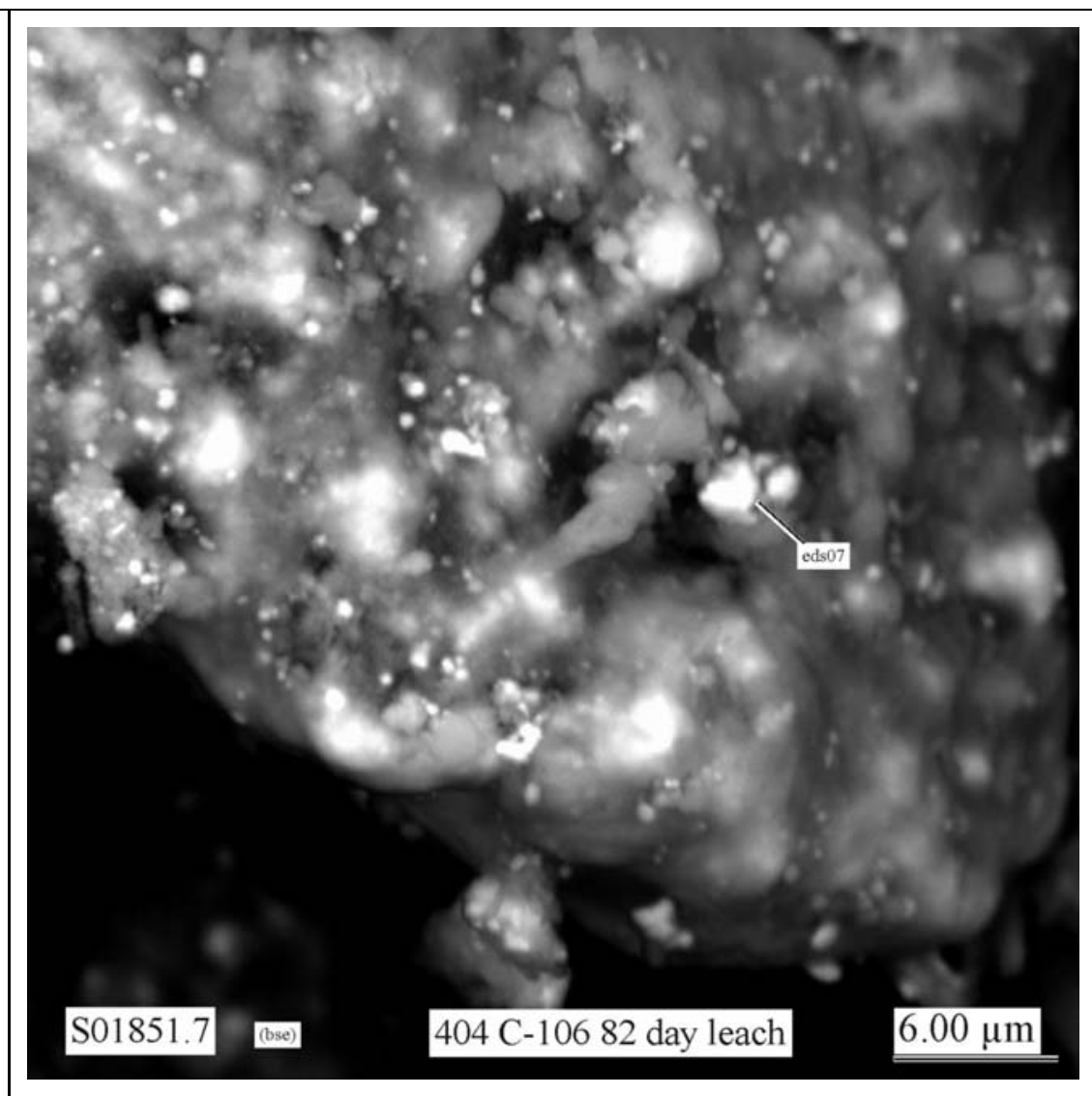

Figure C.64. Micrograph Showing at Higher Magnification the Area Indicated by the White Dashed-Line Square in Figure C.63 


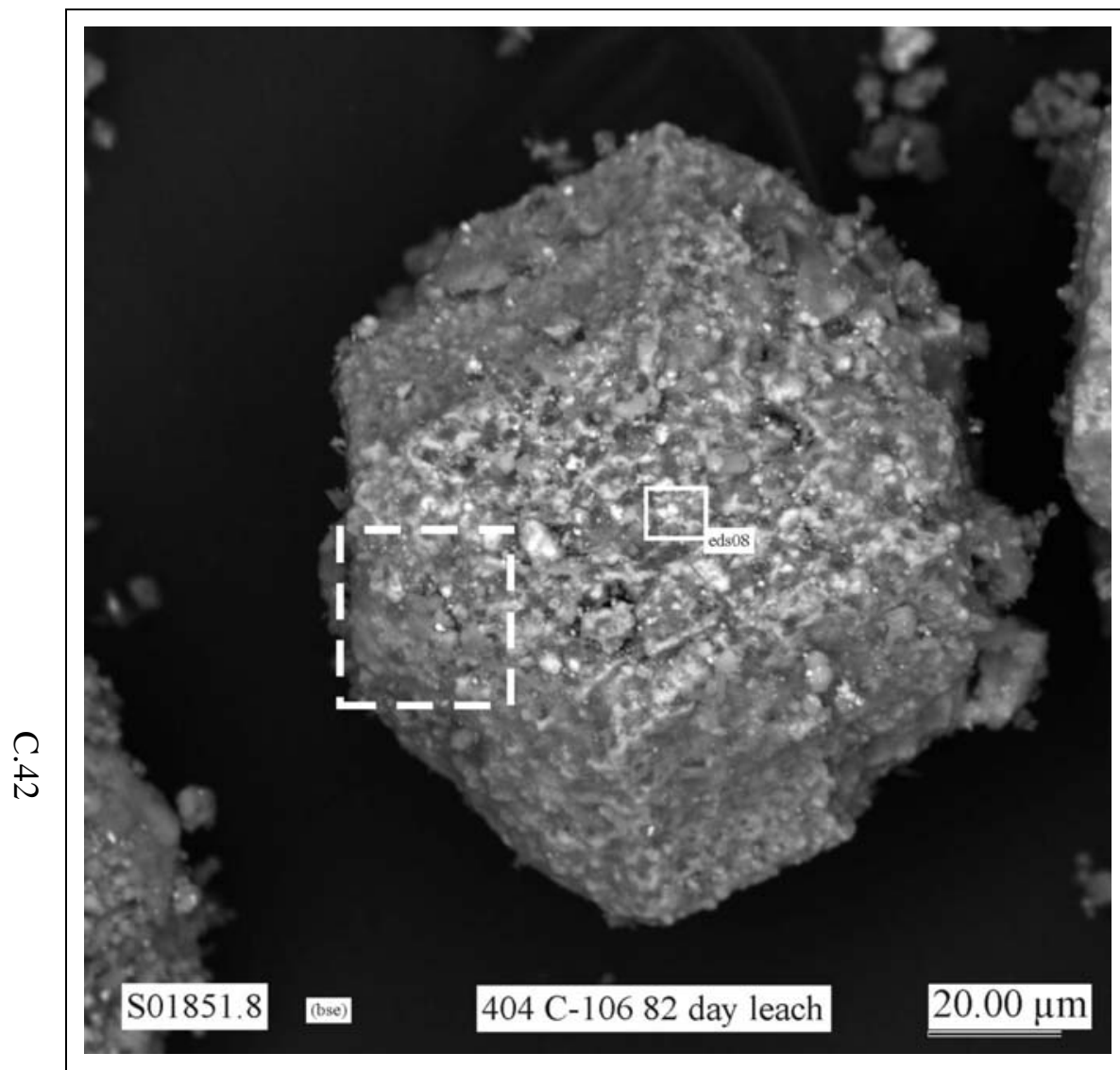

Figure C.65. Micrograph Showing at Higher Magnification the Particle Labeled E in Figure C.57

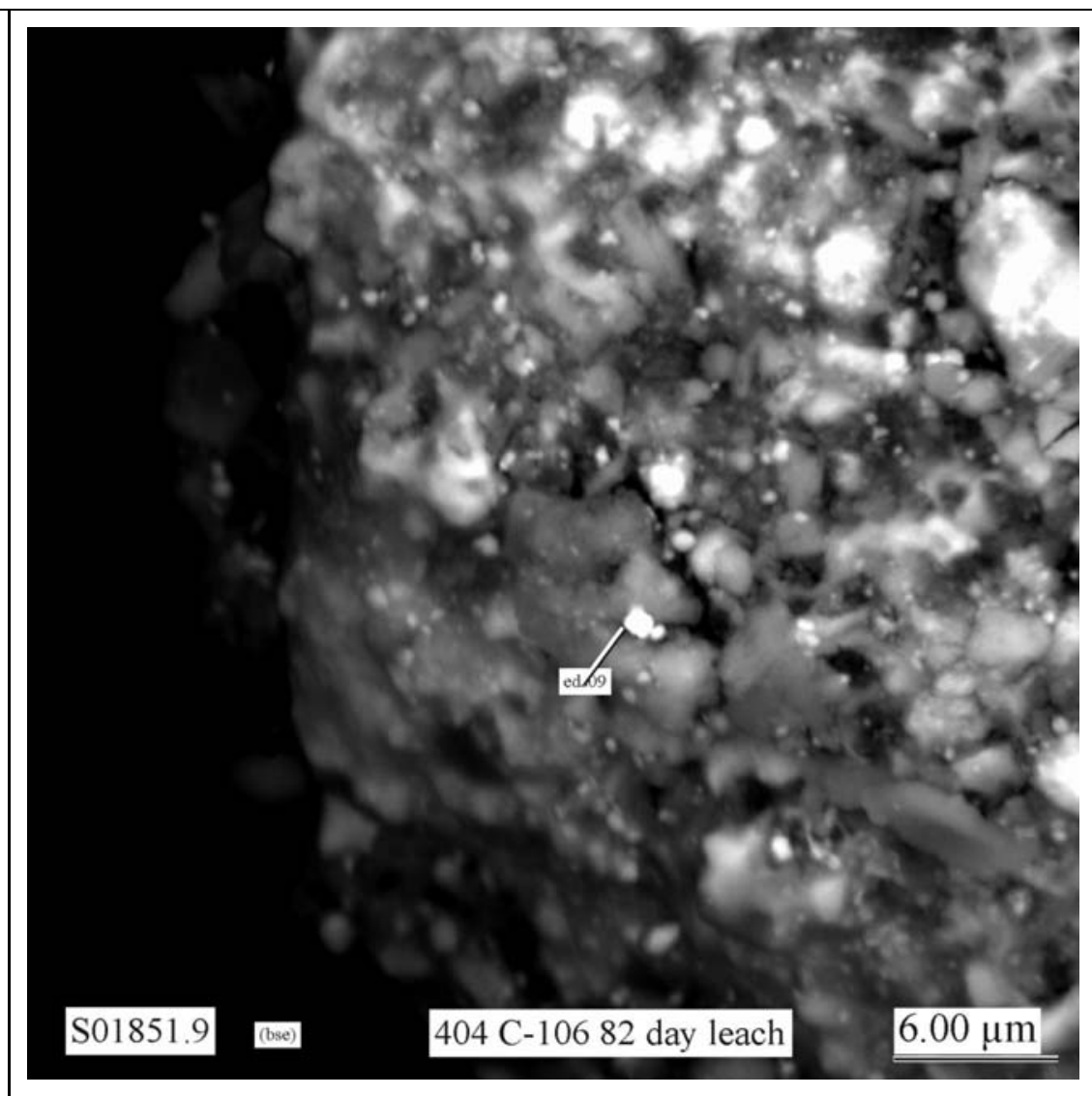

Figure C.66. Micrograph Showing at Higher Magnification the Area Indicated by the White Dashed-Line Square in Figure C.65 


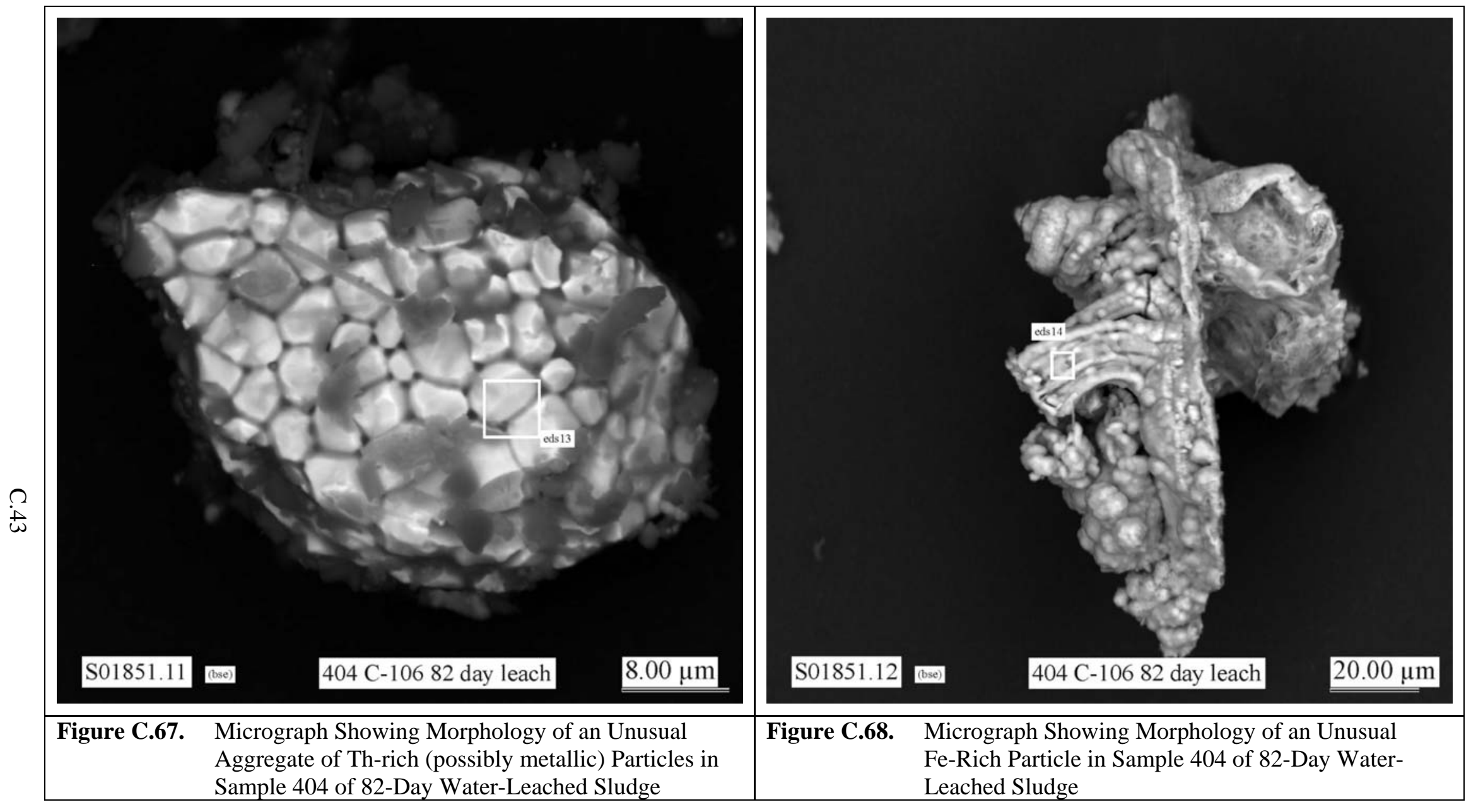




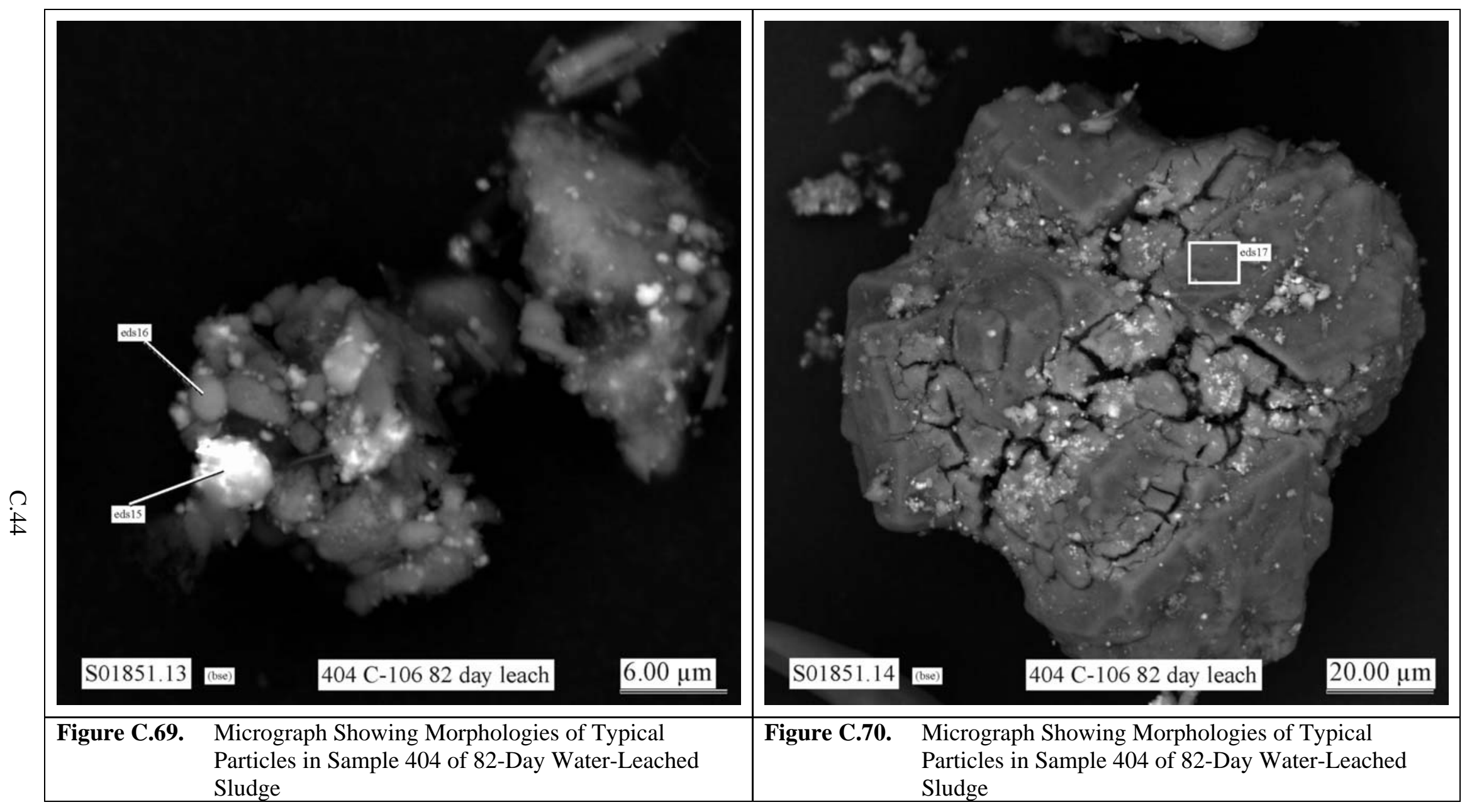




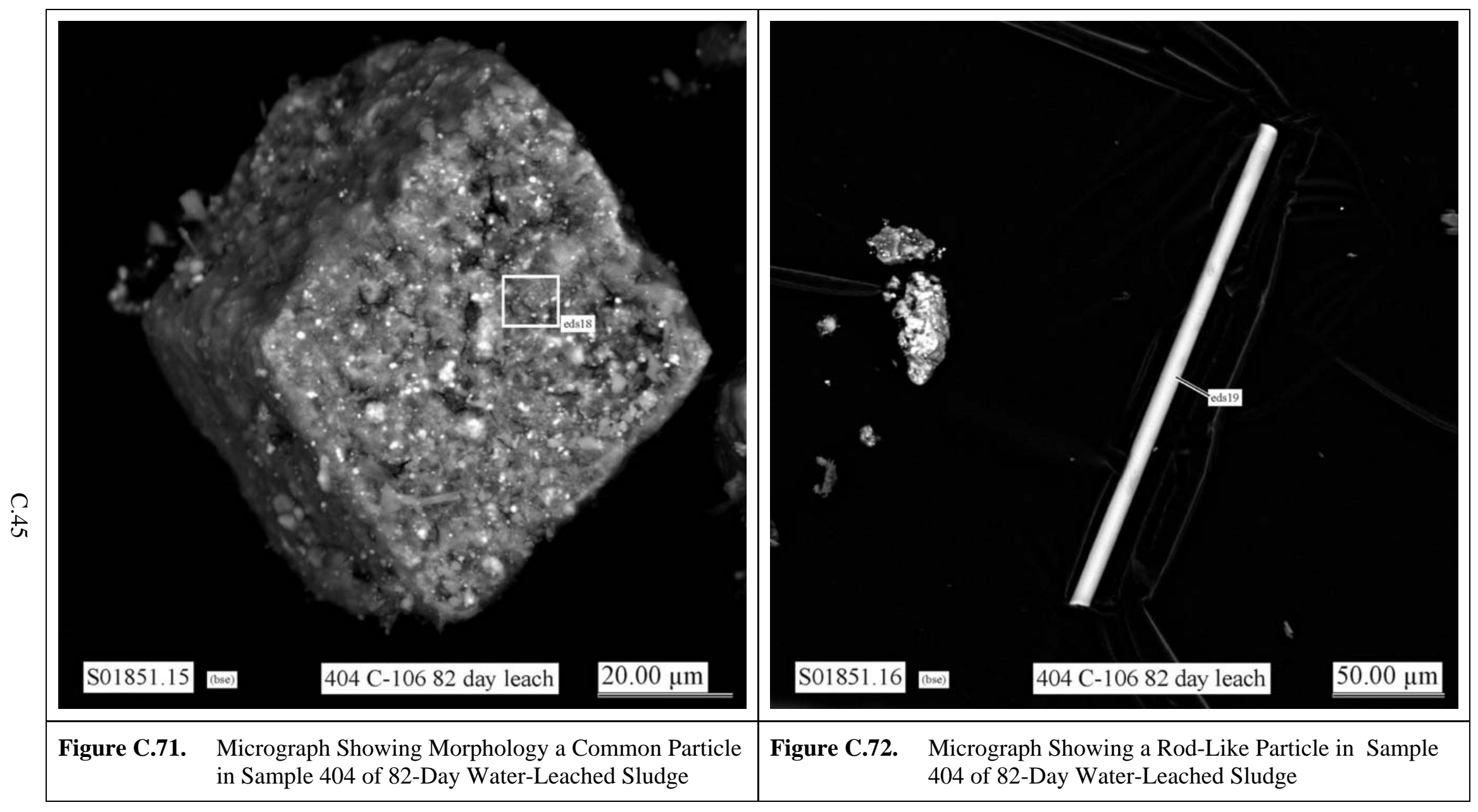




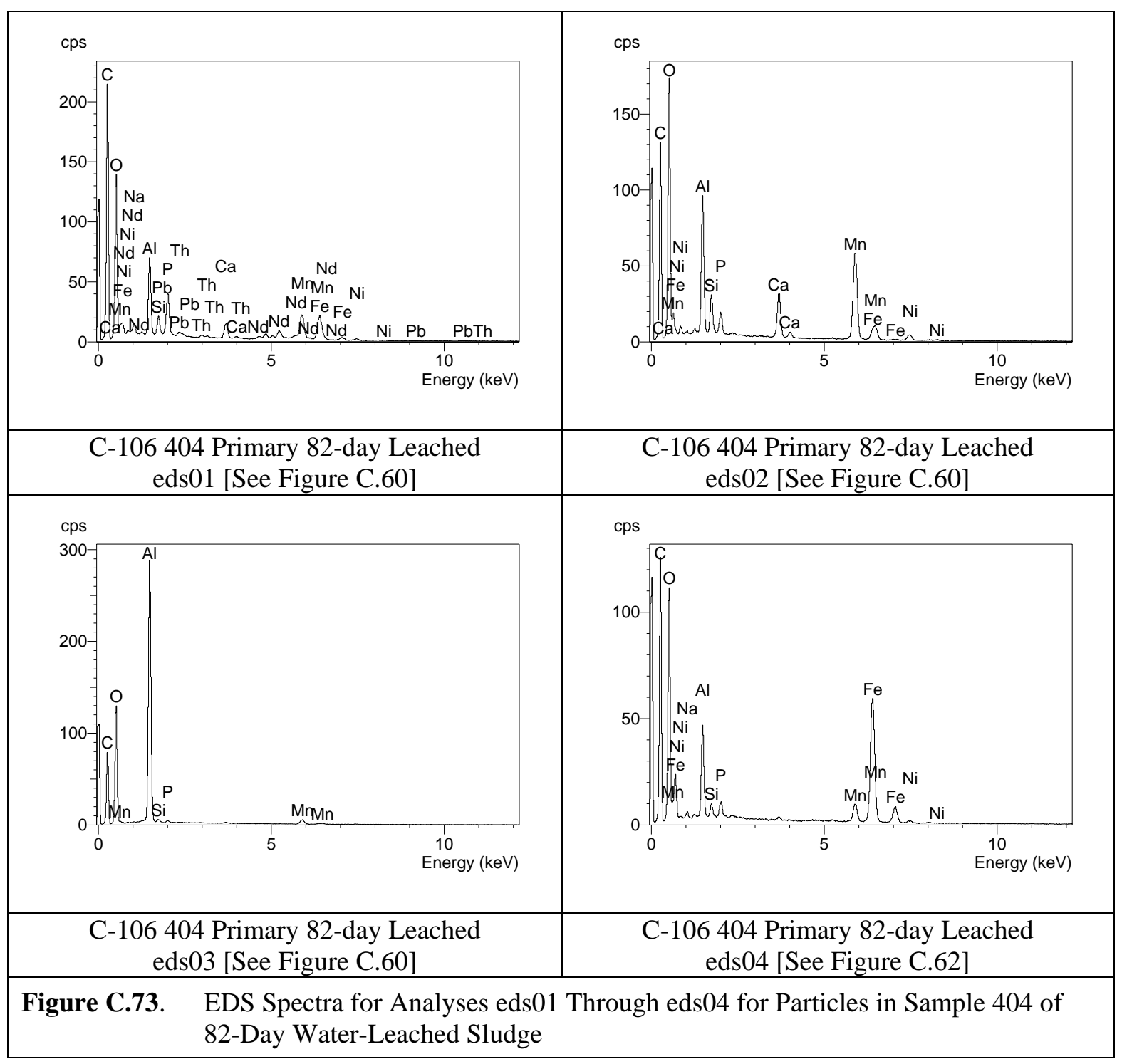




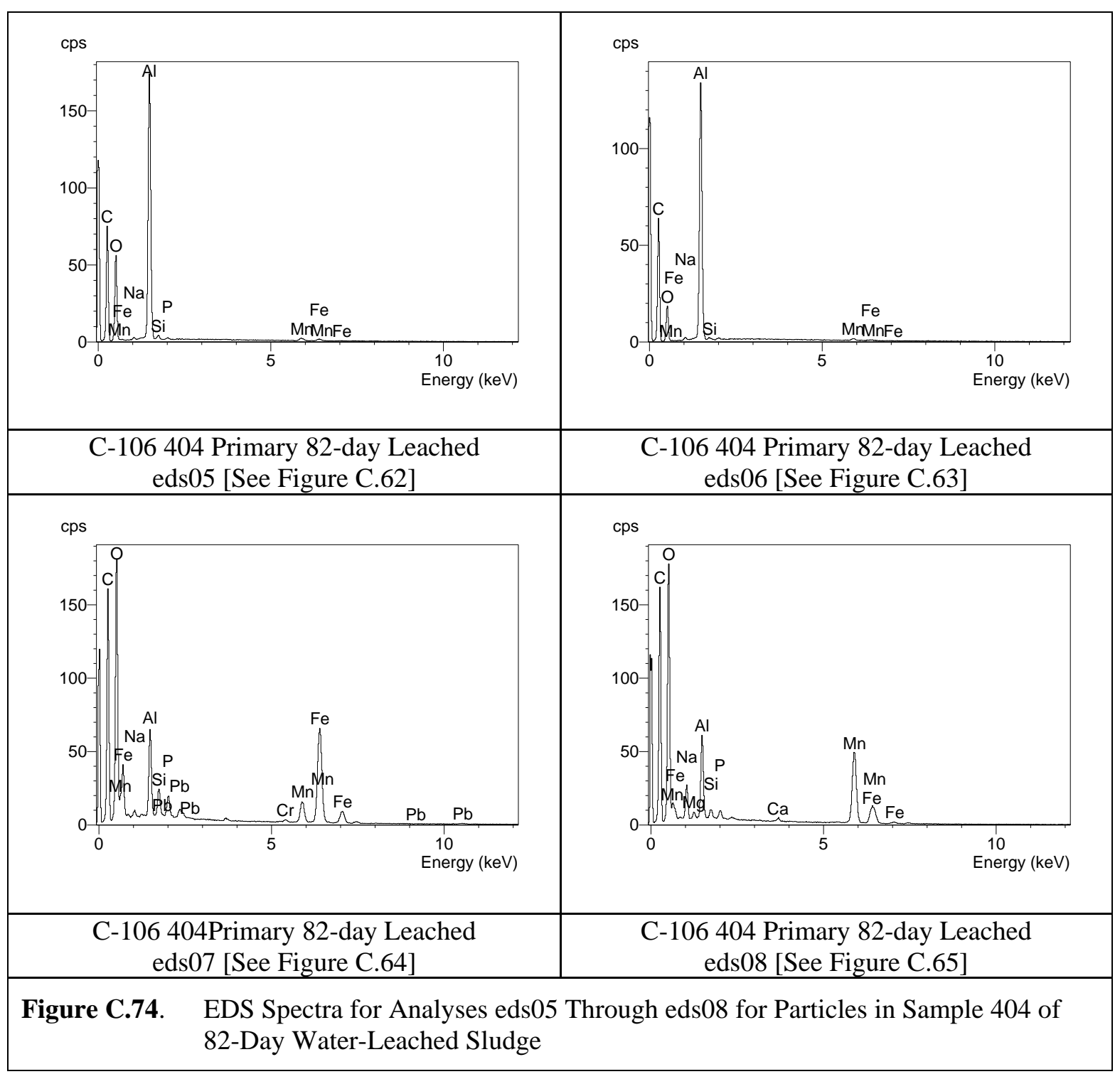




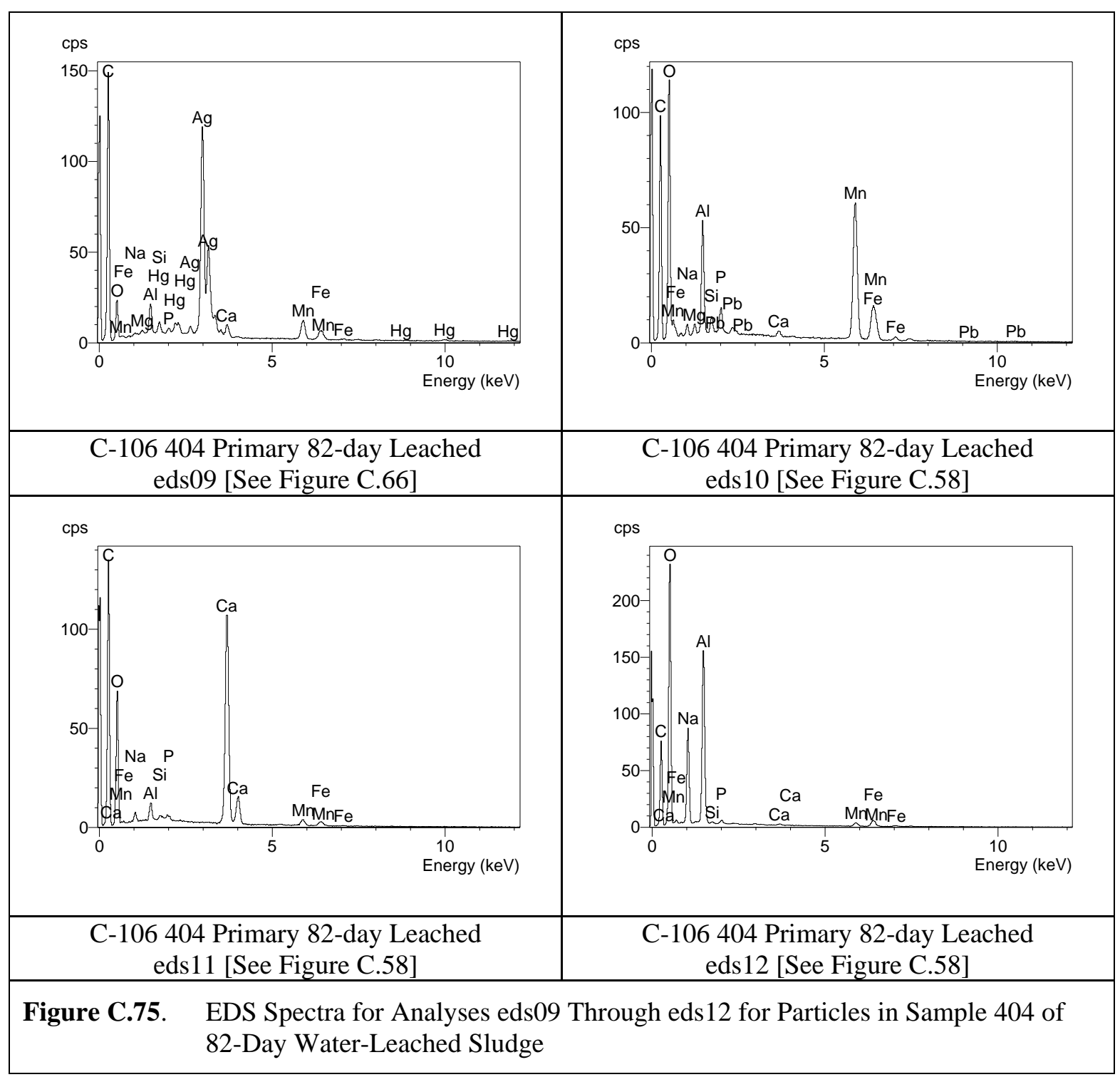




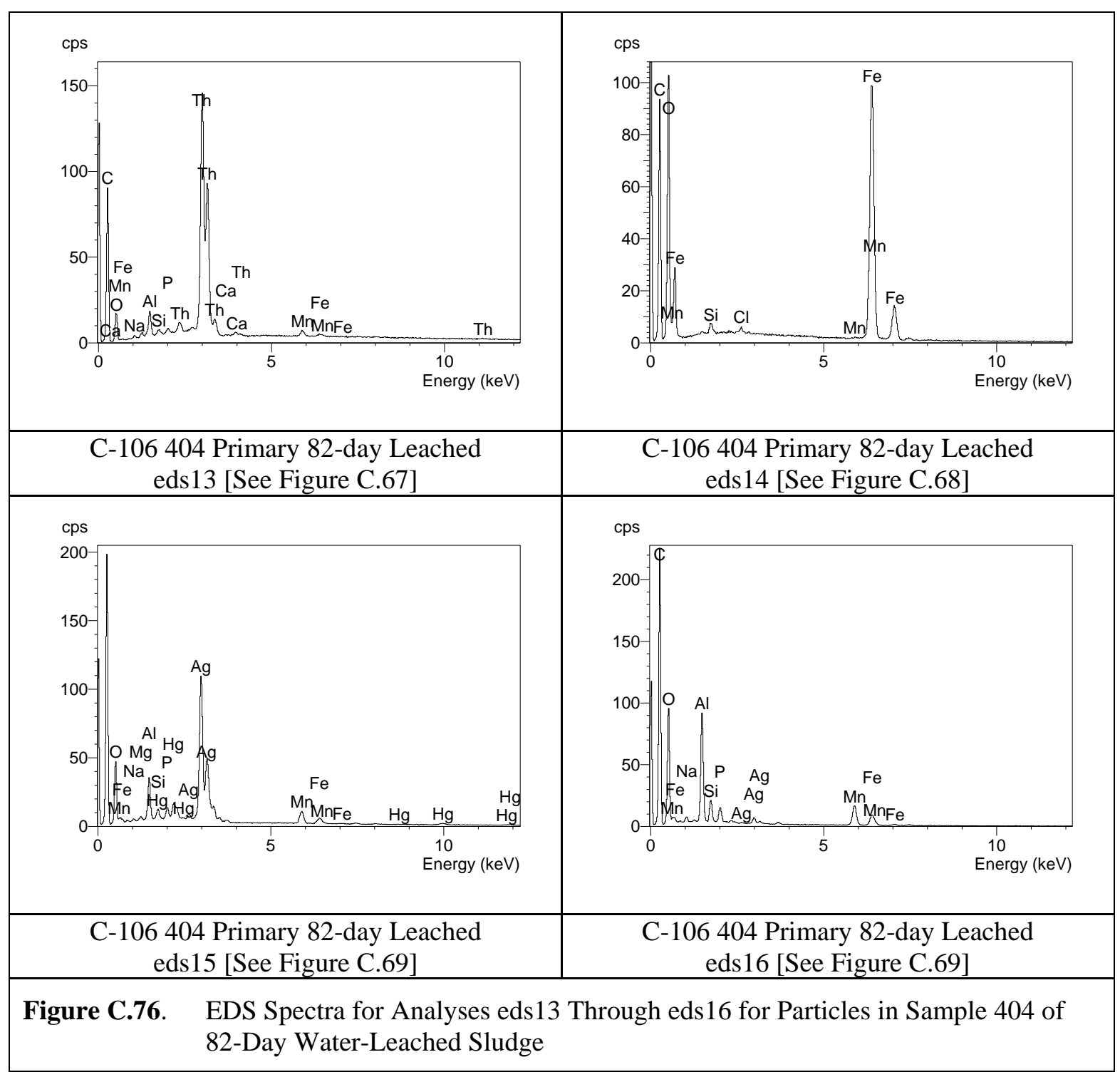




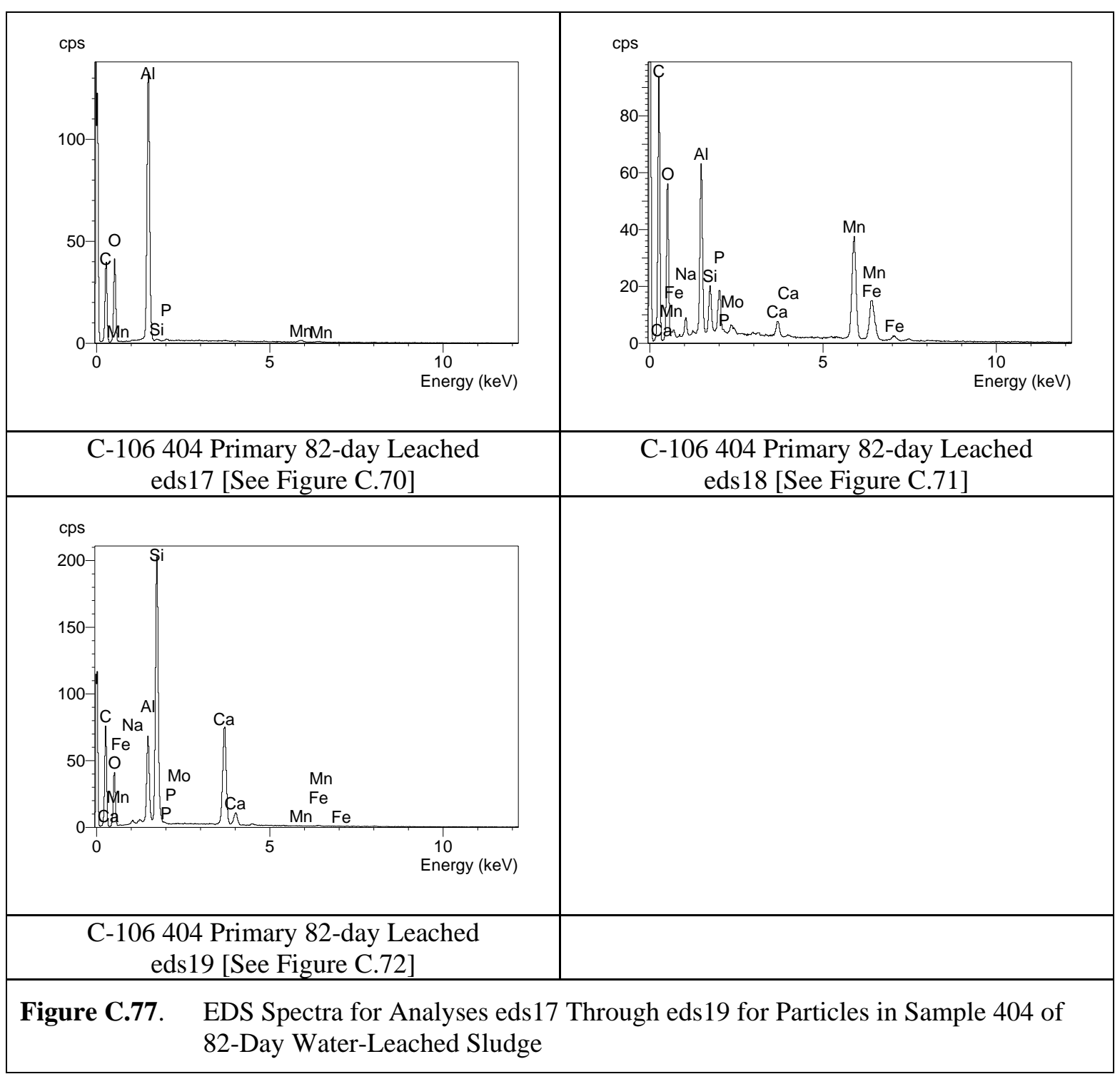

\section{C.4 Water-Leached (82-Day) Sample 405}

One mount of sample from the 82-day water-leached residual sludge from tank C-106 (primary 405) was analyzed by SEM/EDS. The SEM micrographs for this sample are shown in Figures C.78 through C.93, and the EDS spectra for this mount are given in Figures C.94 through C.98. 


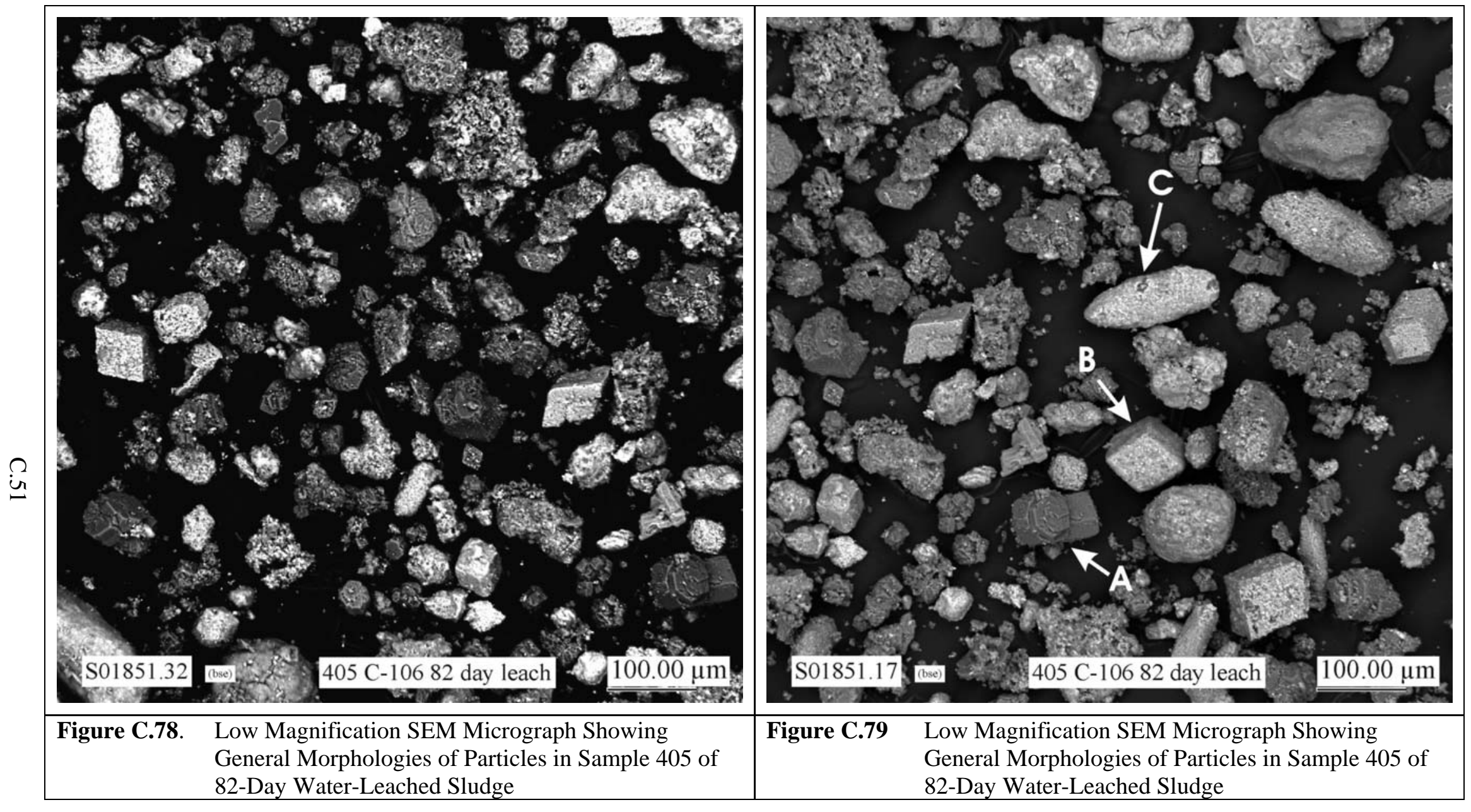




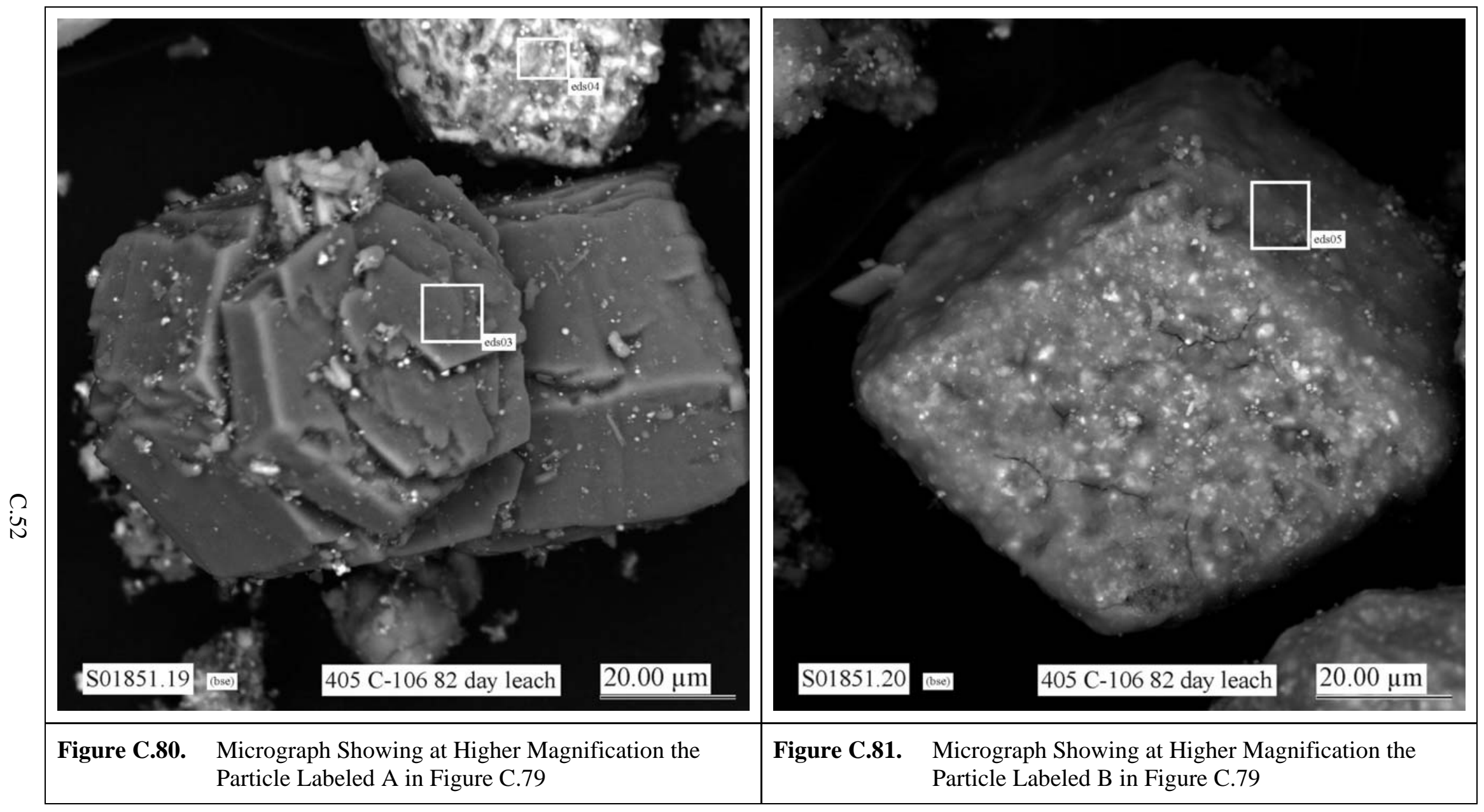




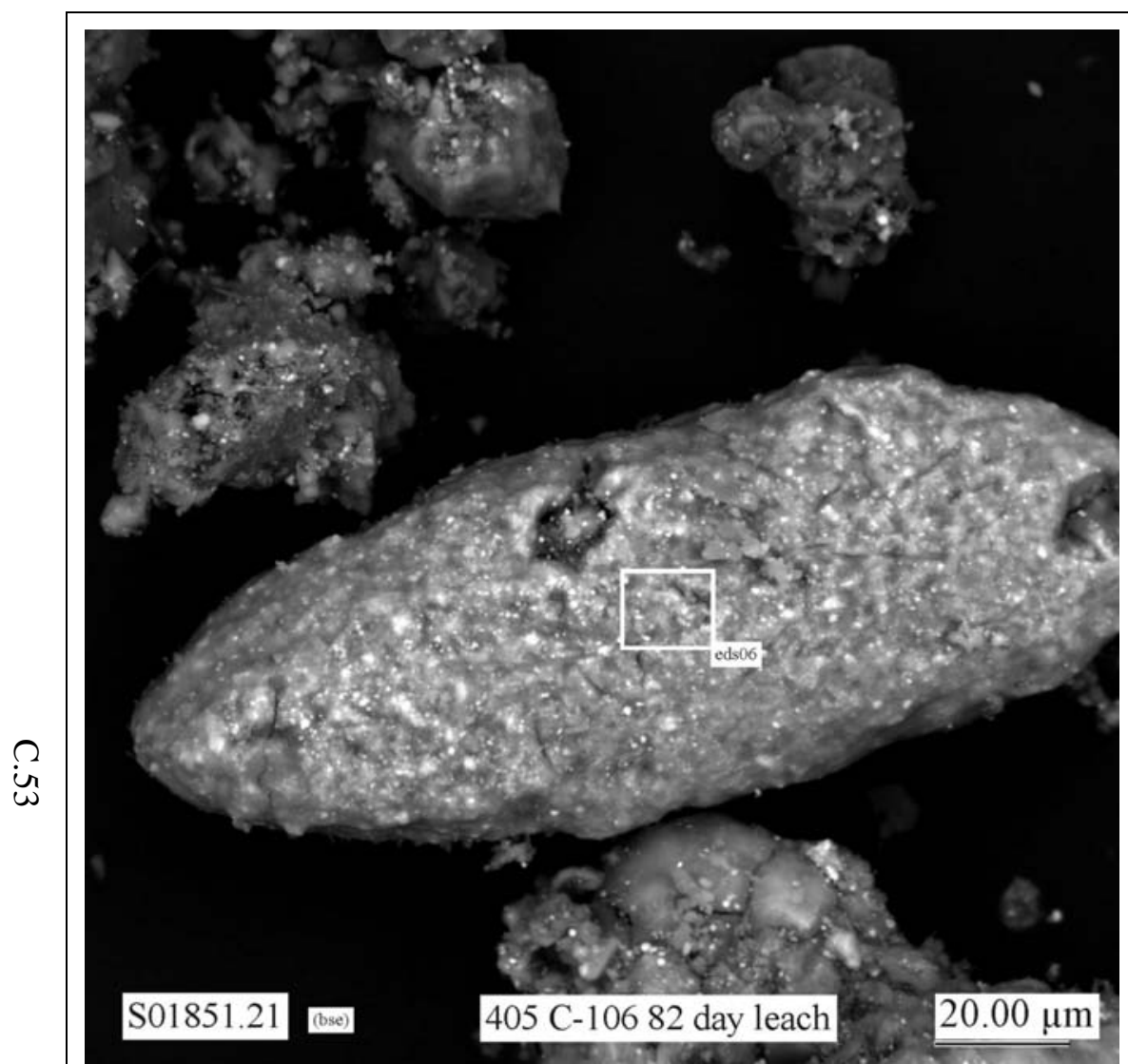

Figure C.82. Micrograph Showing at Higher Magnification the Particle Labeled B in Figure C.79

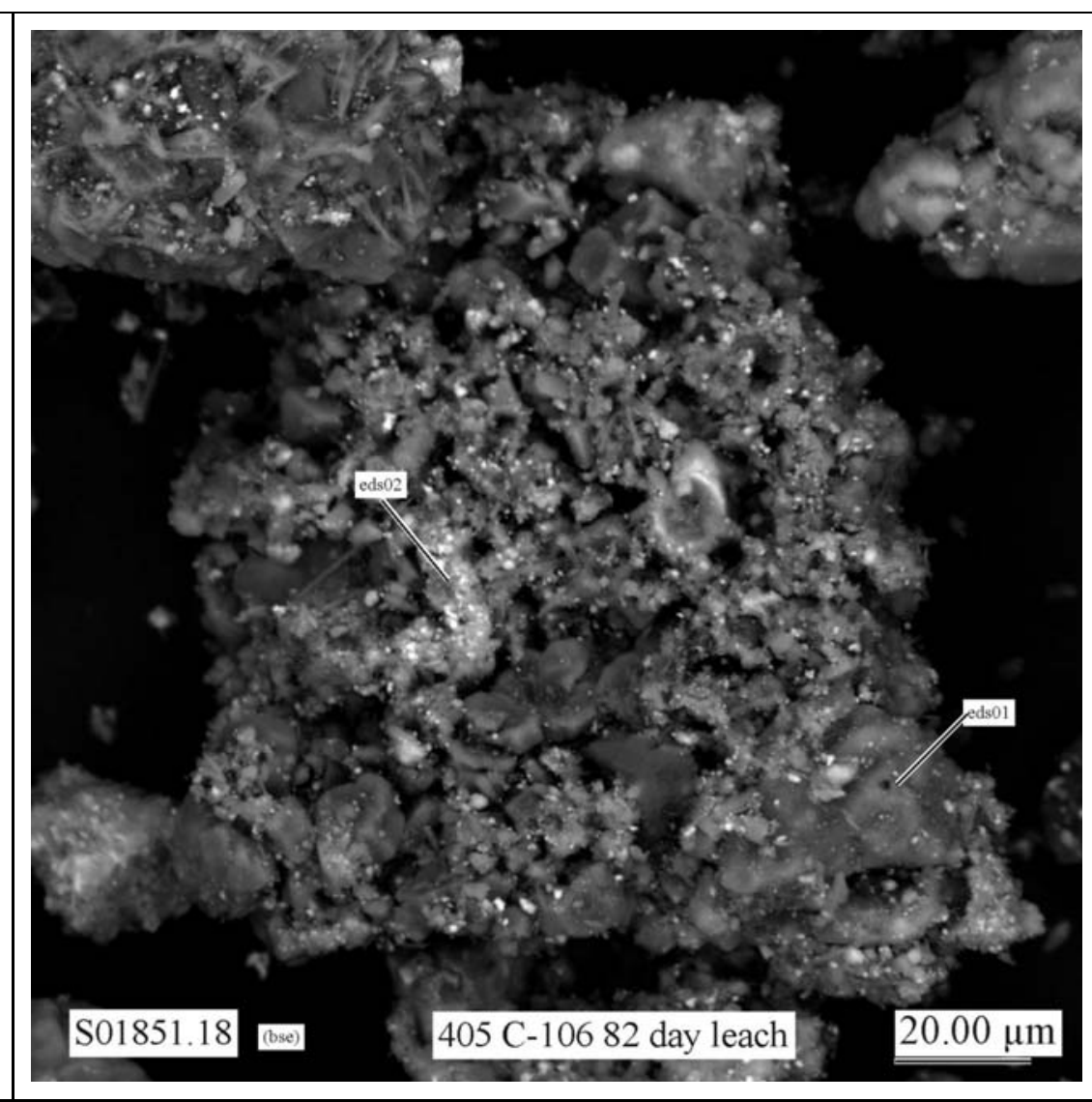

Figure C.82. Micrograph Showing Morphologies of Typical Particles in Sample 405 of 82-Day Water-Leached Sludge 


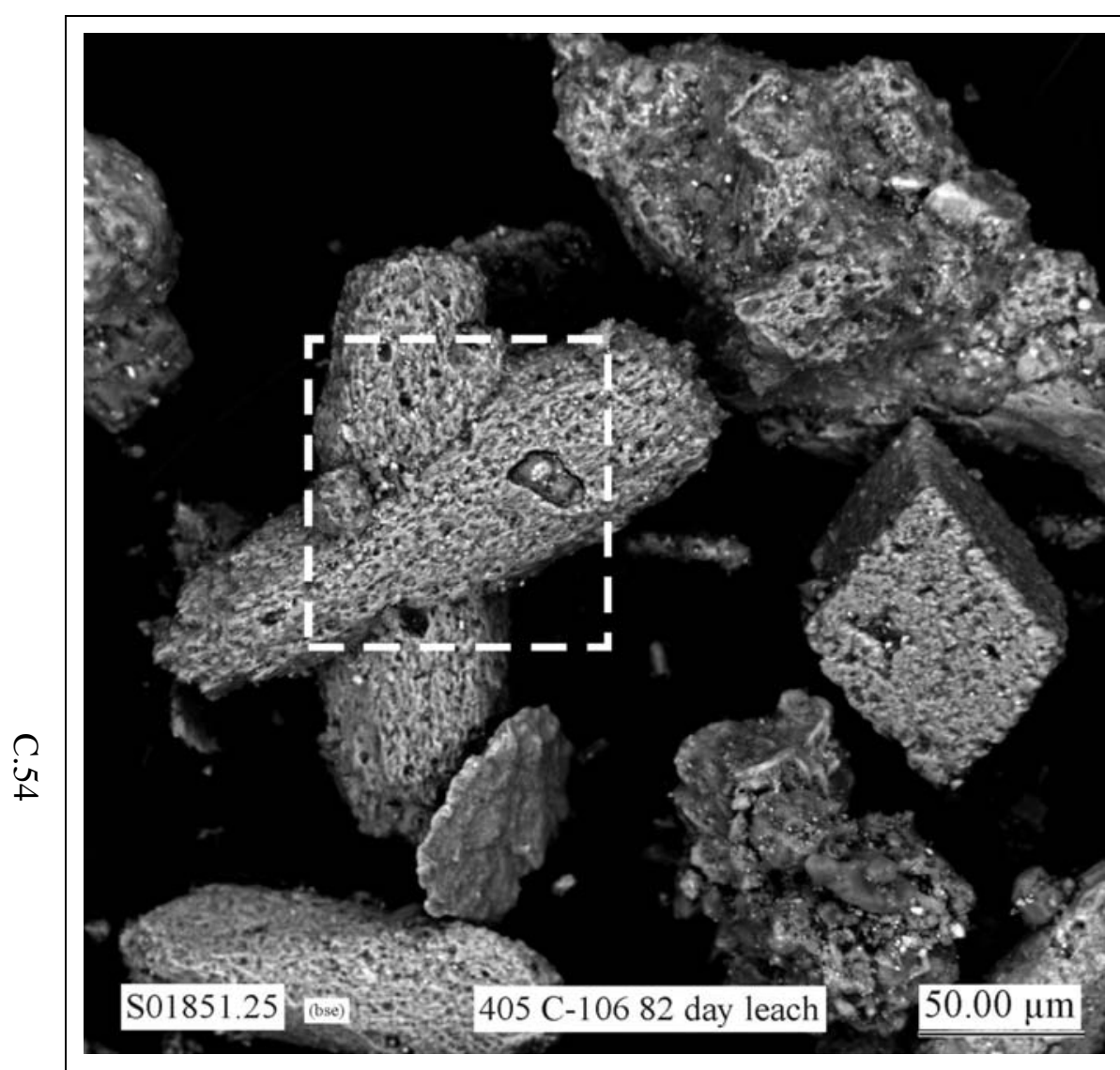

Figure C.84. Micrograph Showing Morphologies of Typical Particles in Sample 405 of 82-Day Water-Leached Sludge

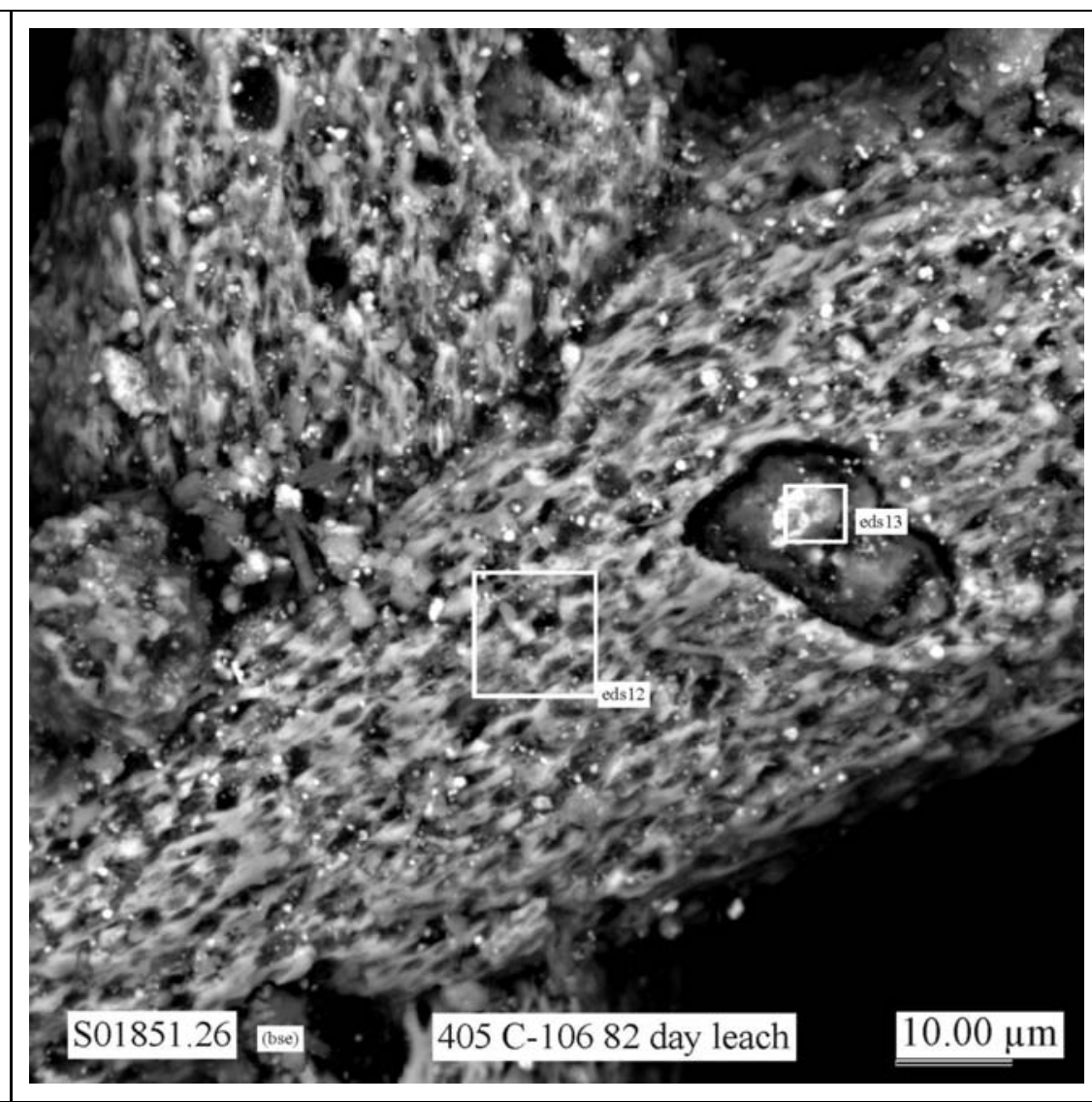

Figure C.85. Micrograph Showing at Higher Magnification the Area Indicated by the White Dashed-Line Square in Figure C.84 


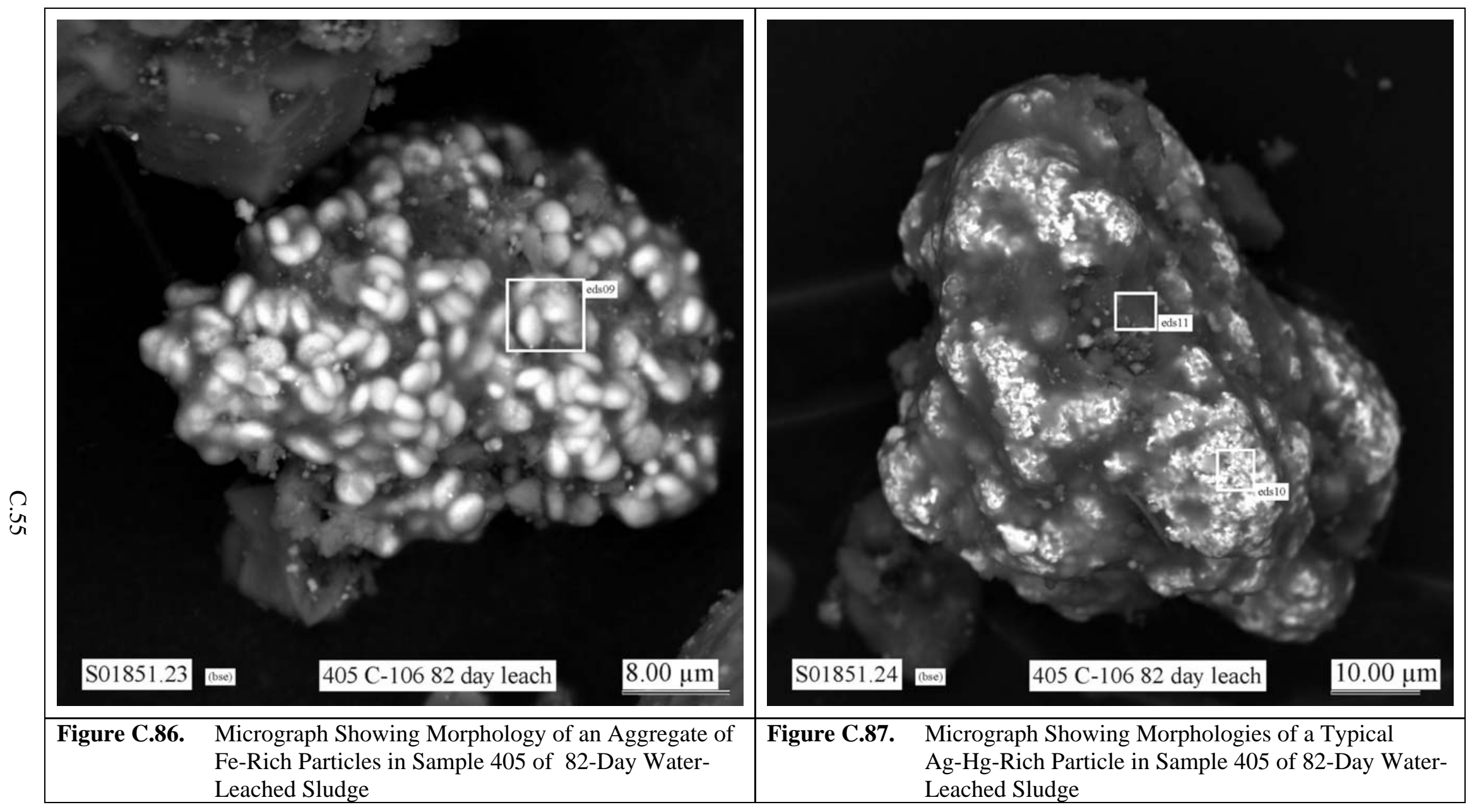




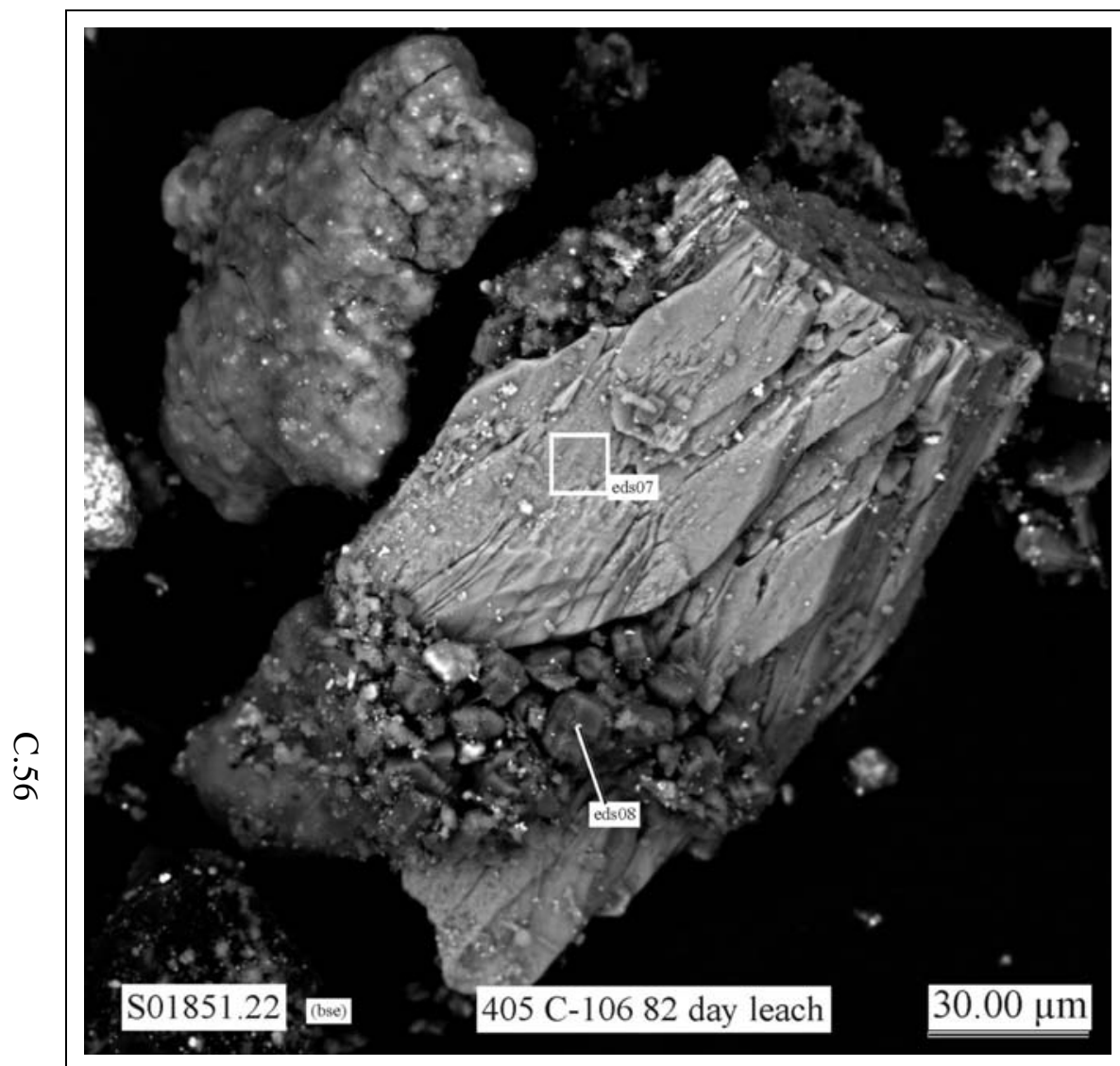

Figure C.88. Micrograph Showing Morphologies of Typical Particles in Sample 405 of 82-Day Water-Leached Sludge

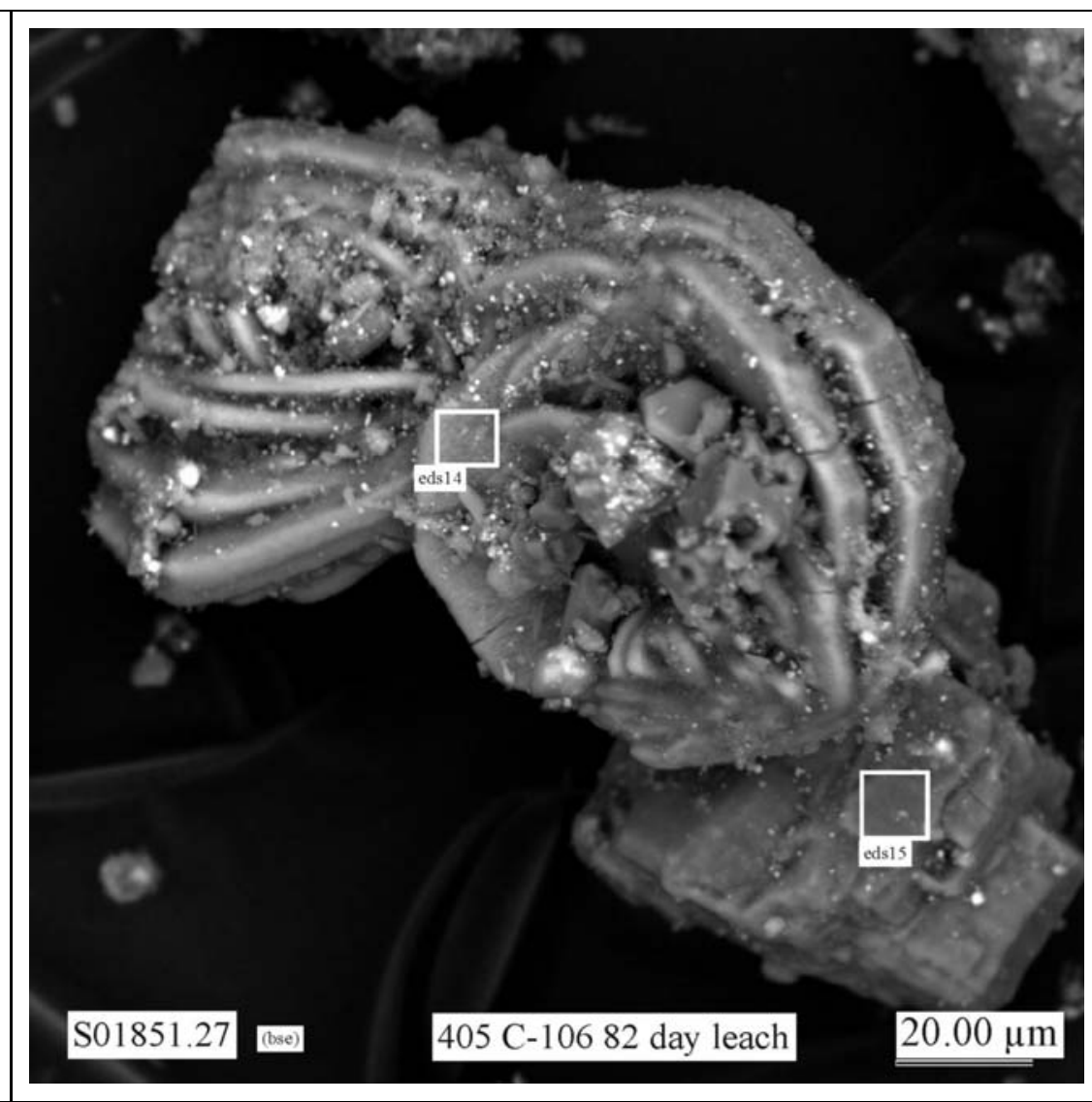

Figure C.89. $\quad$ Micrograph Showing Morphologies of Typical Particles in Sample 405 of 82-Day Water-Leached Sludge 


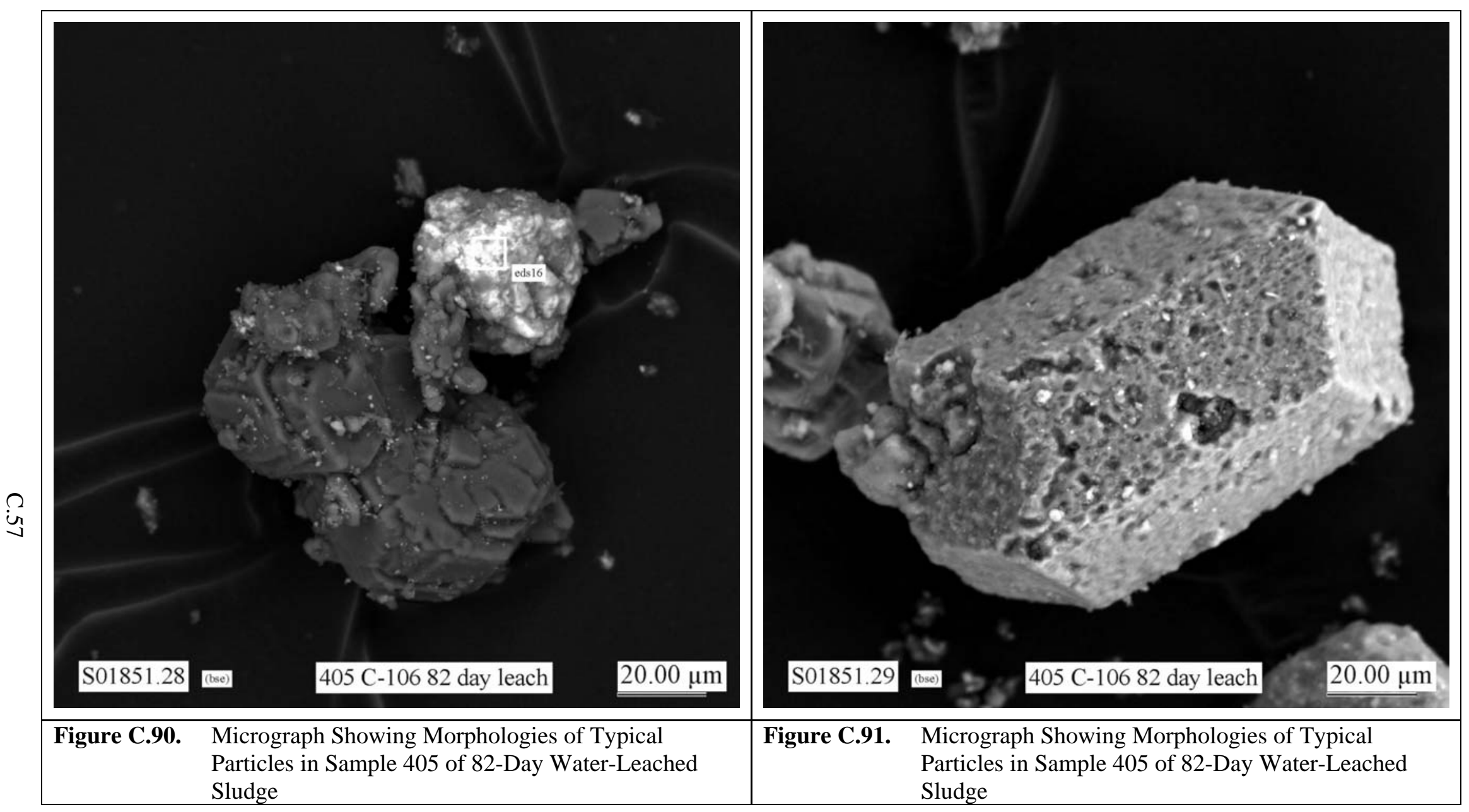




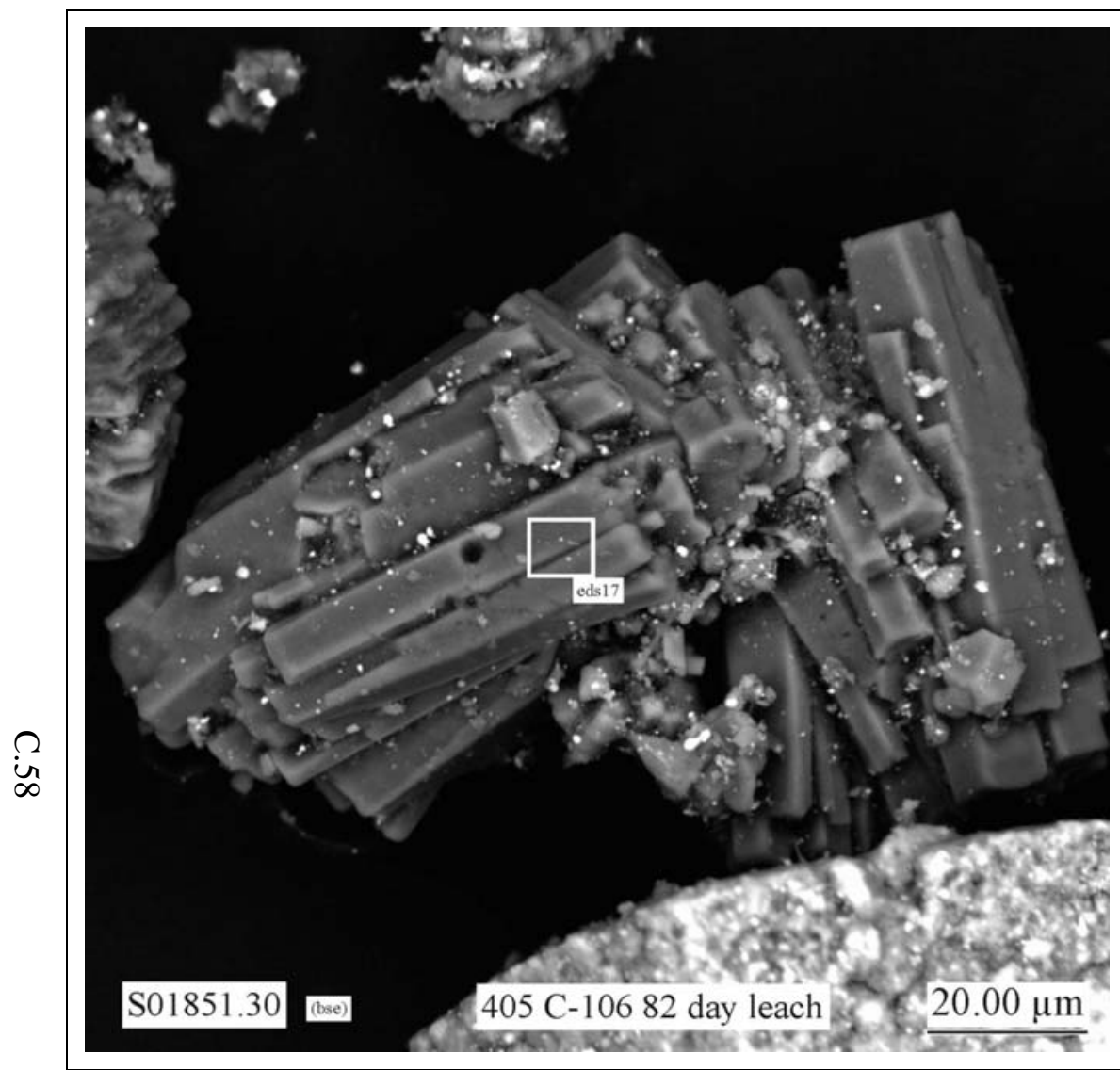

Figure C.92. Micrograph Showing Morphologies of Typical Particles in Sample 405 of 82-Day Water-Leached Sludge

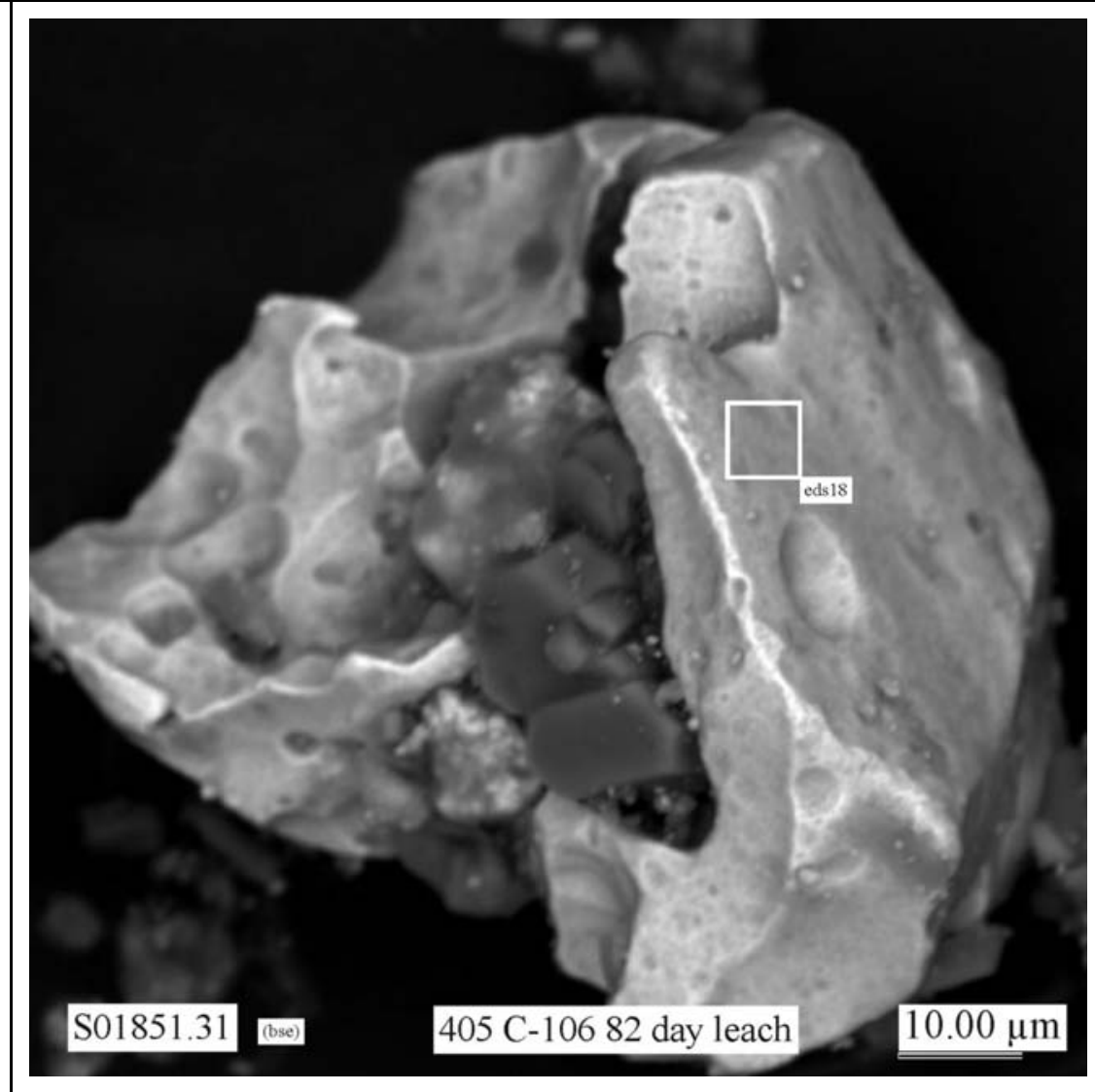

Figure C.93. Micrograph Showing Morphology of an Fe-Cr-Rich Particle in Sample 405 of 82-Day Water-Leached Sludge 


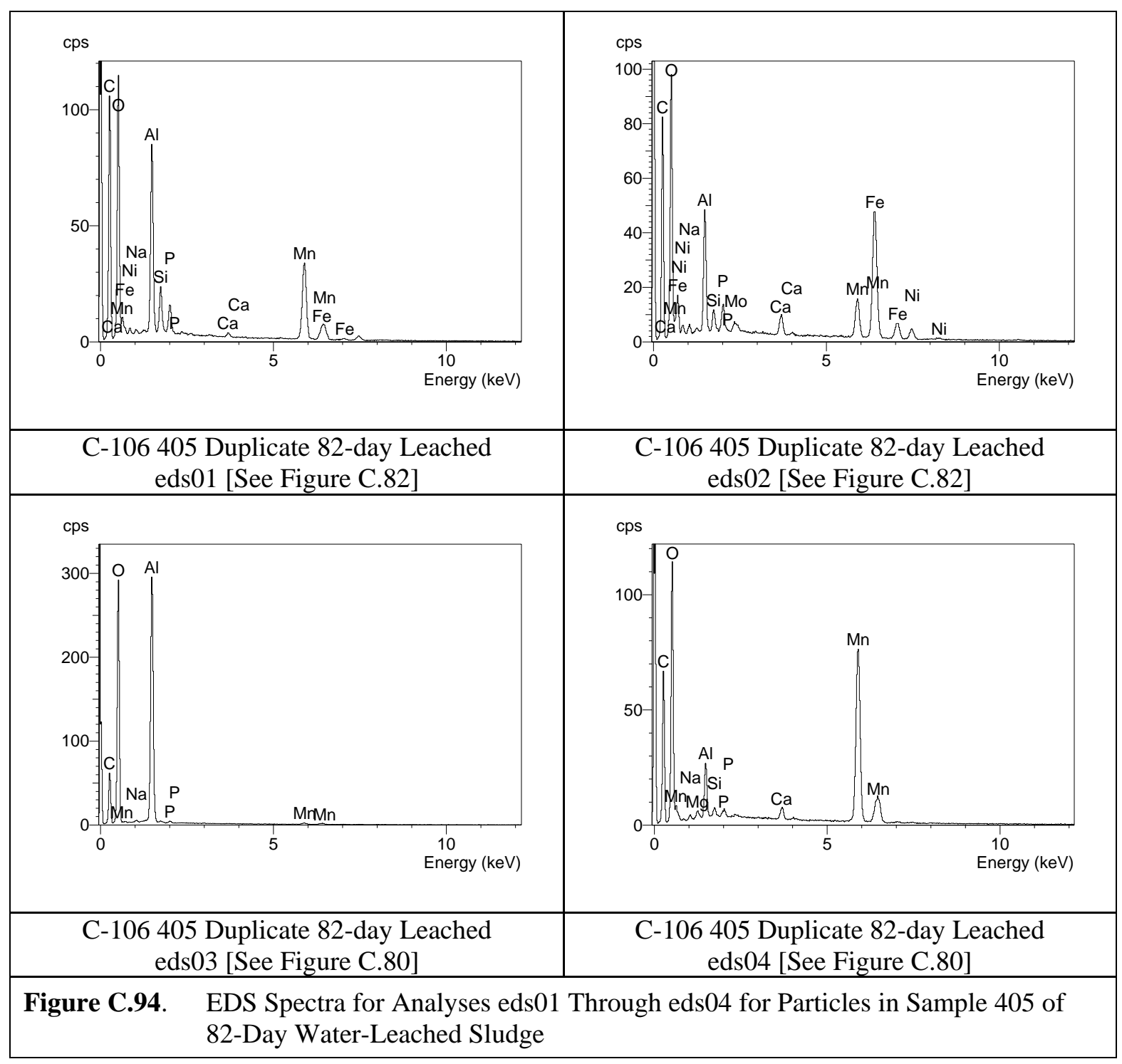




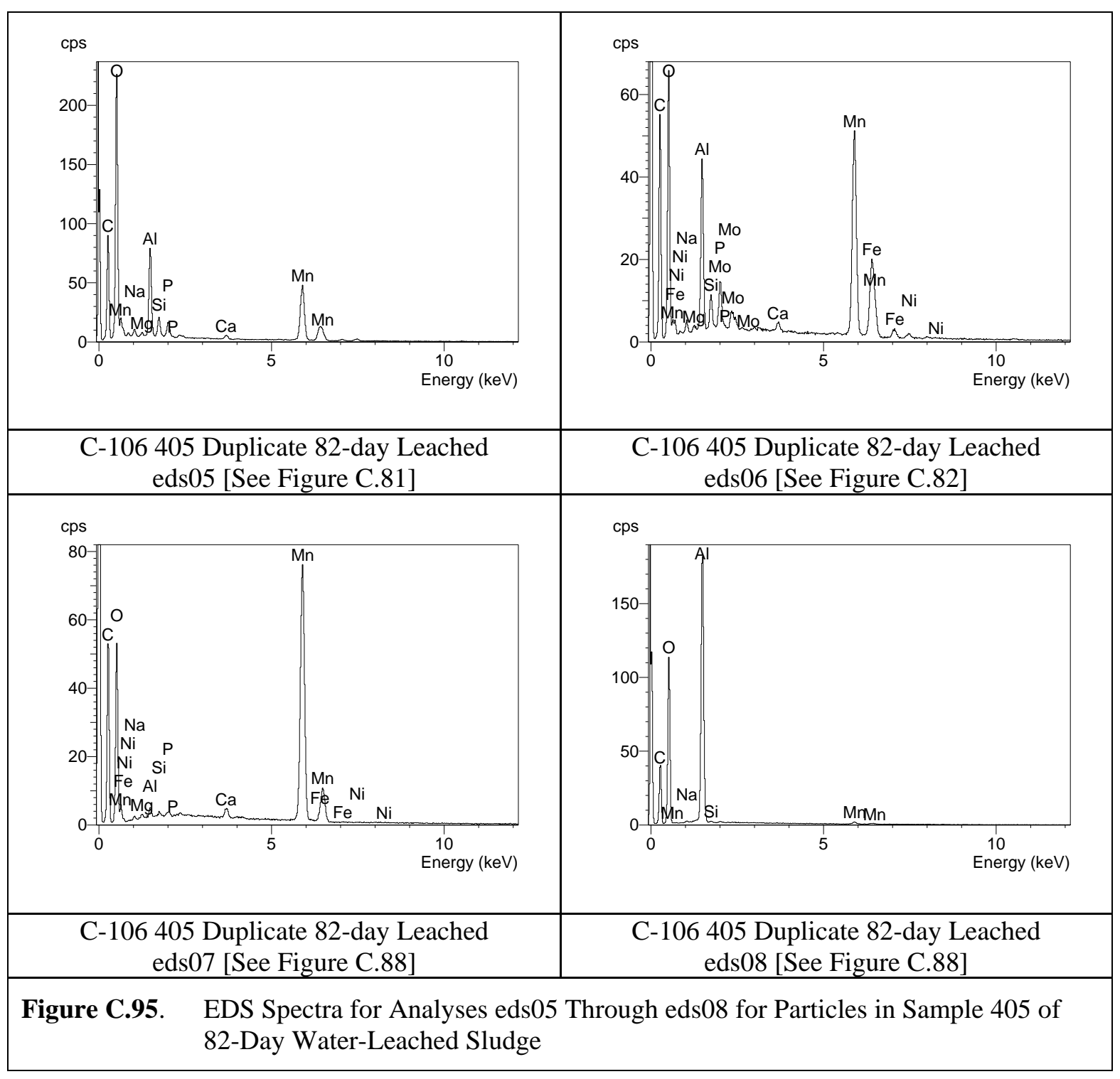




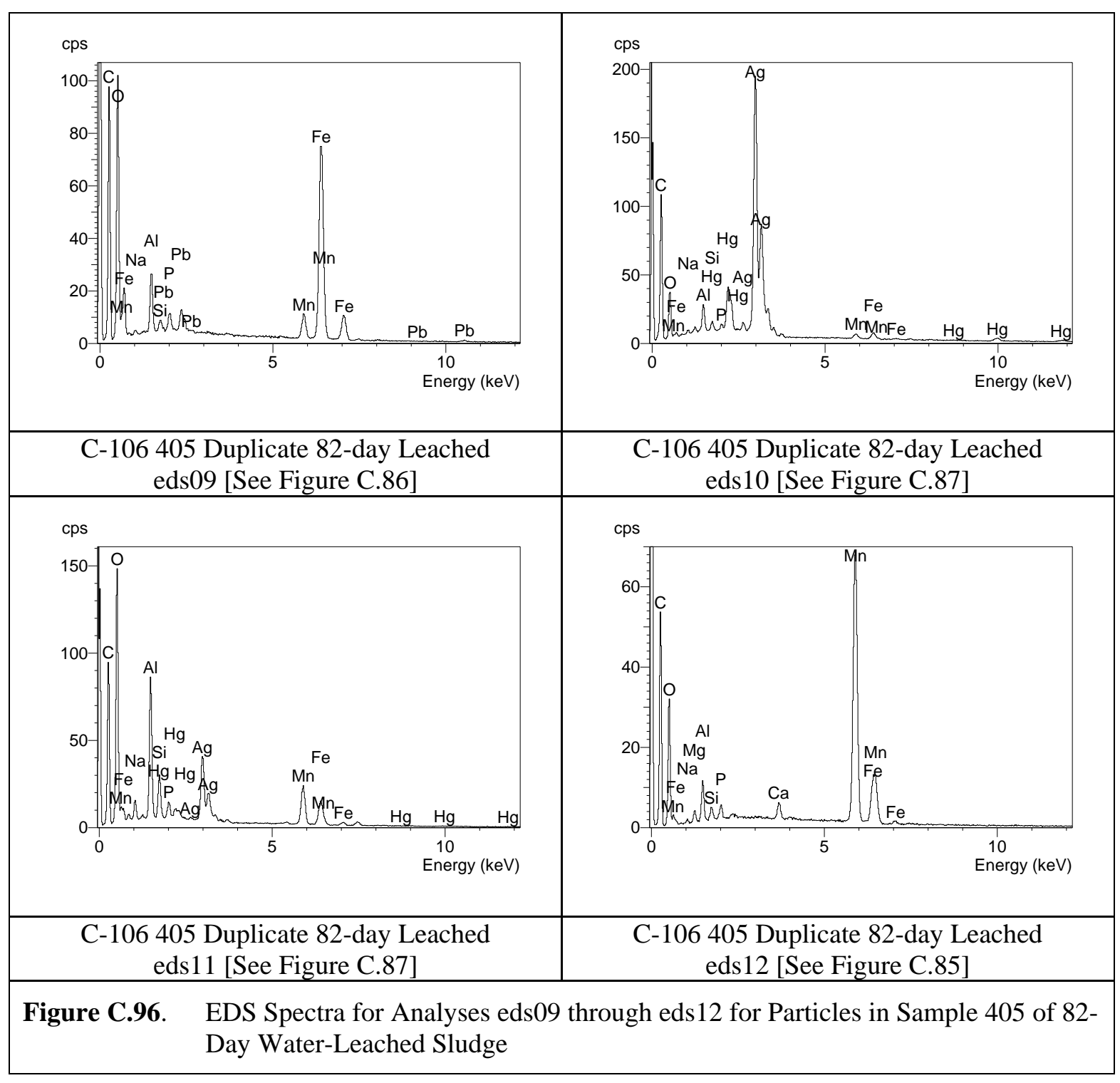




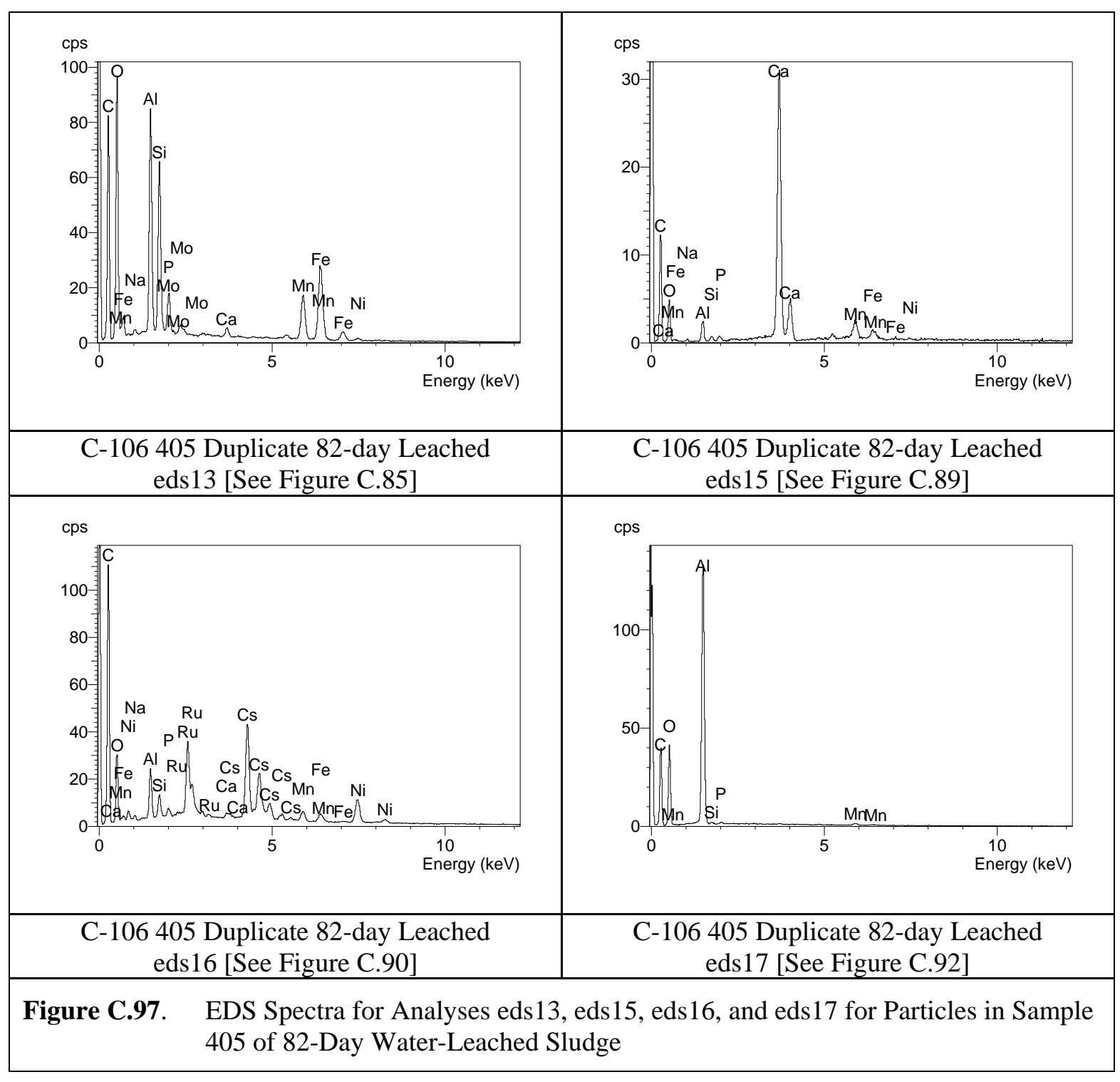




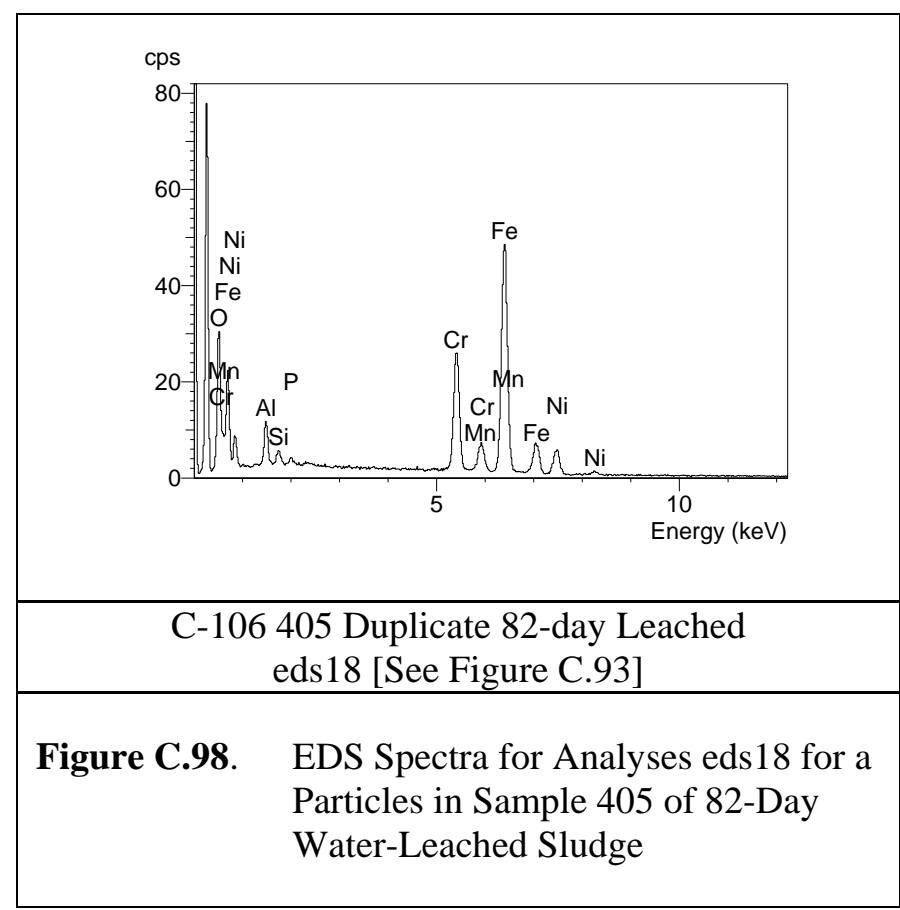




\section{Appendix D}

SEM Micrographs and EDS Spectra for HF Sequential Extract of Tank C-106 Sludge 


\section{Appendix D}

\section{SEM Micrographs and EDS Spectra for HF Sequential Extract of Tank C-106 Sludge}

This appendix includes the scanning electron microscope (SEM) micrographs and the energydispersive X-ray spectrometry (EDS) spectra for samples of residual sludge from tank 241-C-106 (C-106) (primary 404 and duplicate 405) remaining after the hydrofluoric (HF) (Stage 1) sequential extraction. The operating conditions for the SEM and procedures used for mounting SEM samples are described in Section 2.4 of the main report.

The name of each SEM digital image file, sample identification number, and a size scale bar are given, respectively, at the bottom left, center, and right of each SEM micrograph in this appendix. Micrographs labeled by "BSE" to the immediate right of the digital image file name indicate that the micrograph was collected with backscattered electrons. Areas identified by a letter and/or outlined by a dashed-line square in a micrograph designate sample material that was imaged at higher magnification, which is typically shown in figure(s) that immediately follow in the series for that sample.

Areas labeled by "eds" in the following SEM micrographs in this appendix identify locations of particles for which EDS spectra were recorded. The "eds" label given with each EDS spectrum correspond to the same "EDS" label used in the SEM micrographs for this sample.

One mount each of HF-extracted sludge from tank C-106 (primary 404 and duplicate 405) were analyzed by SEM/EDS. The SEM micrographs for the mount of HF-extracted sample 404 are shown in Figures D.1 through D.20. The EDS spectra for this mount are given in Figures D.21 through D.25. The SEM micrographs for the mount of HF-extracted sample 405 are shown in Figures D.26 through D.45, and the EDS spectra for this sample are given in Figures D.46 through D.51. 


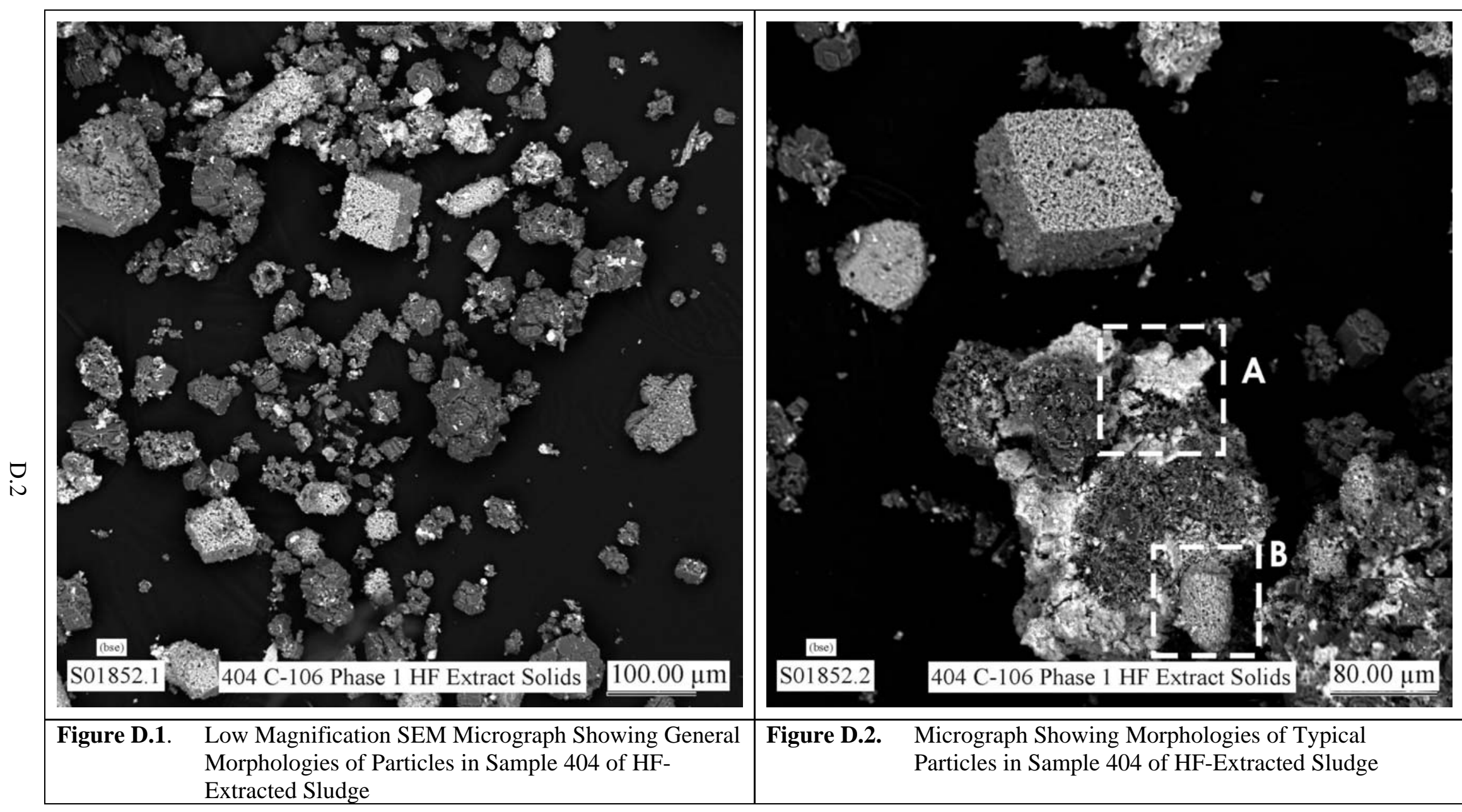




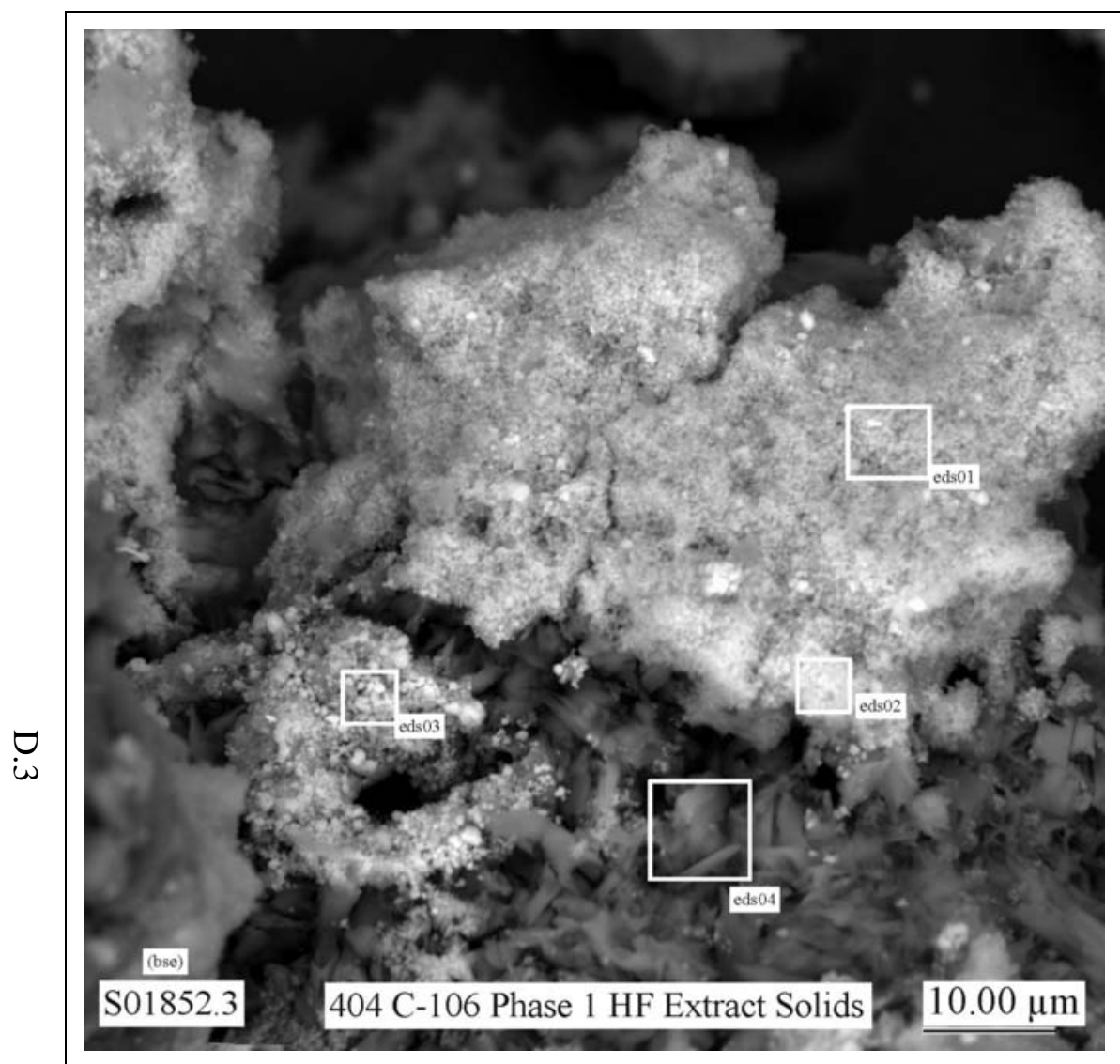

Figure D.3. Micrograph Showing at Higher Magnification the Area Indicated by the White Dashed-Line Square Labeled A in Figure D.2

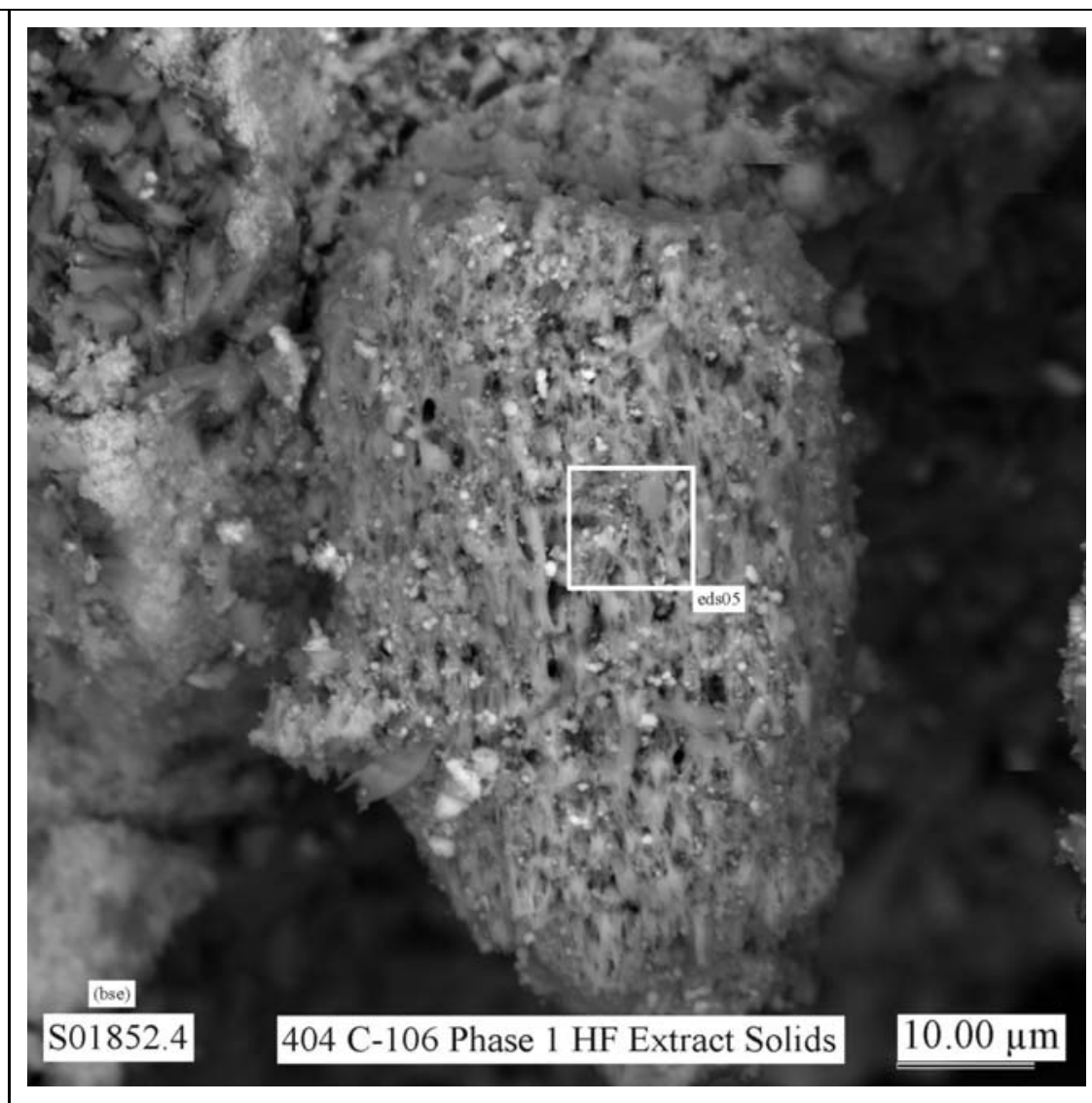

Figure D.4. Micrograph Showing at Higher Magnification the Area Indicated by the White Dashed-Line Square Labeled B in Figure D.2 


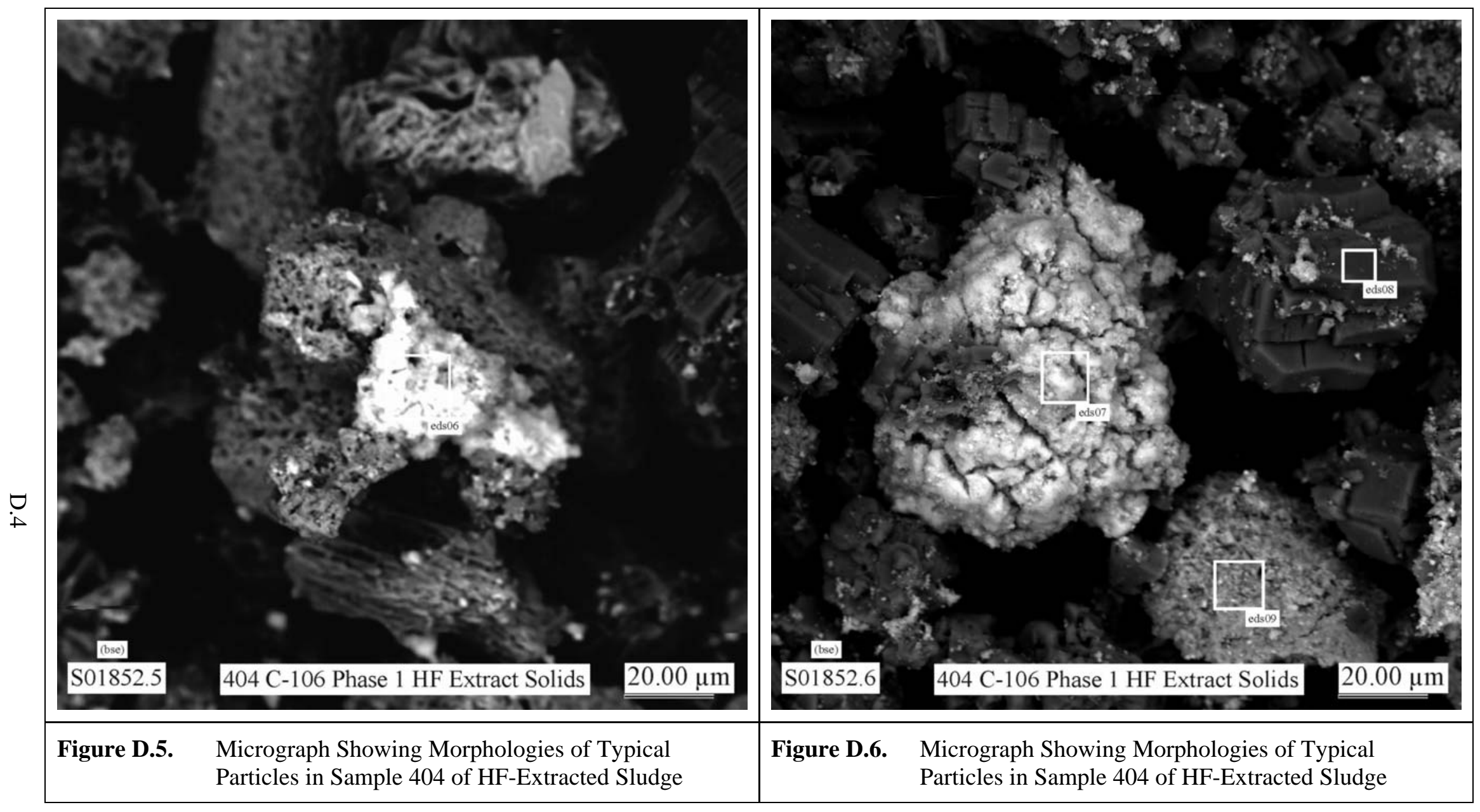




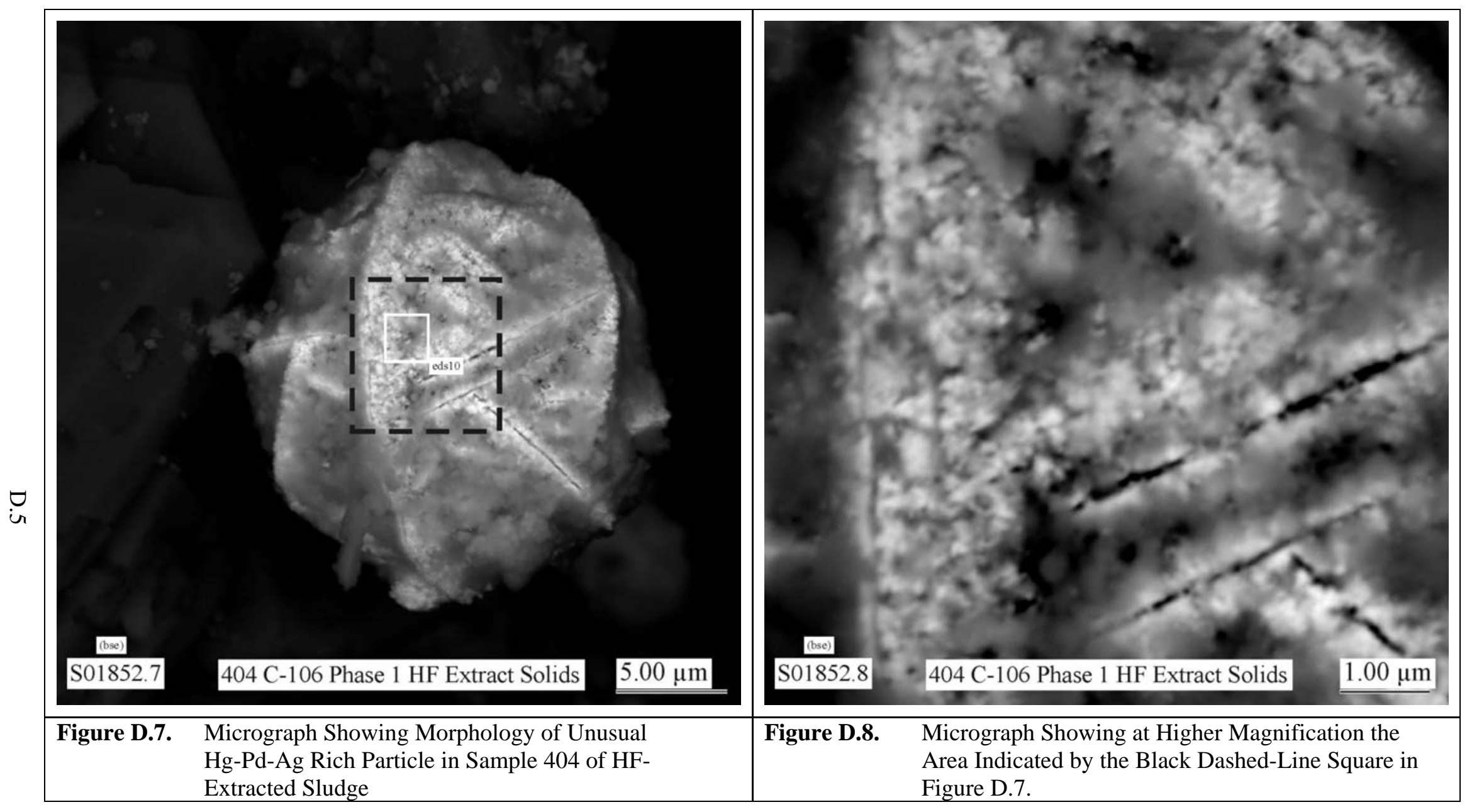




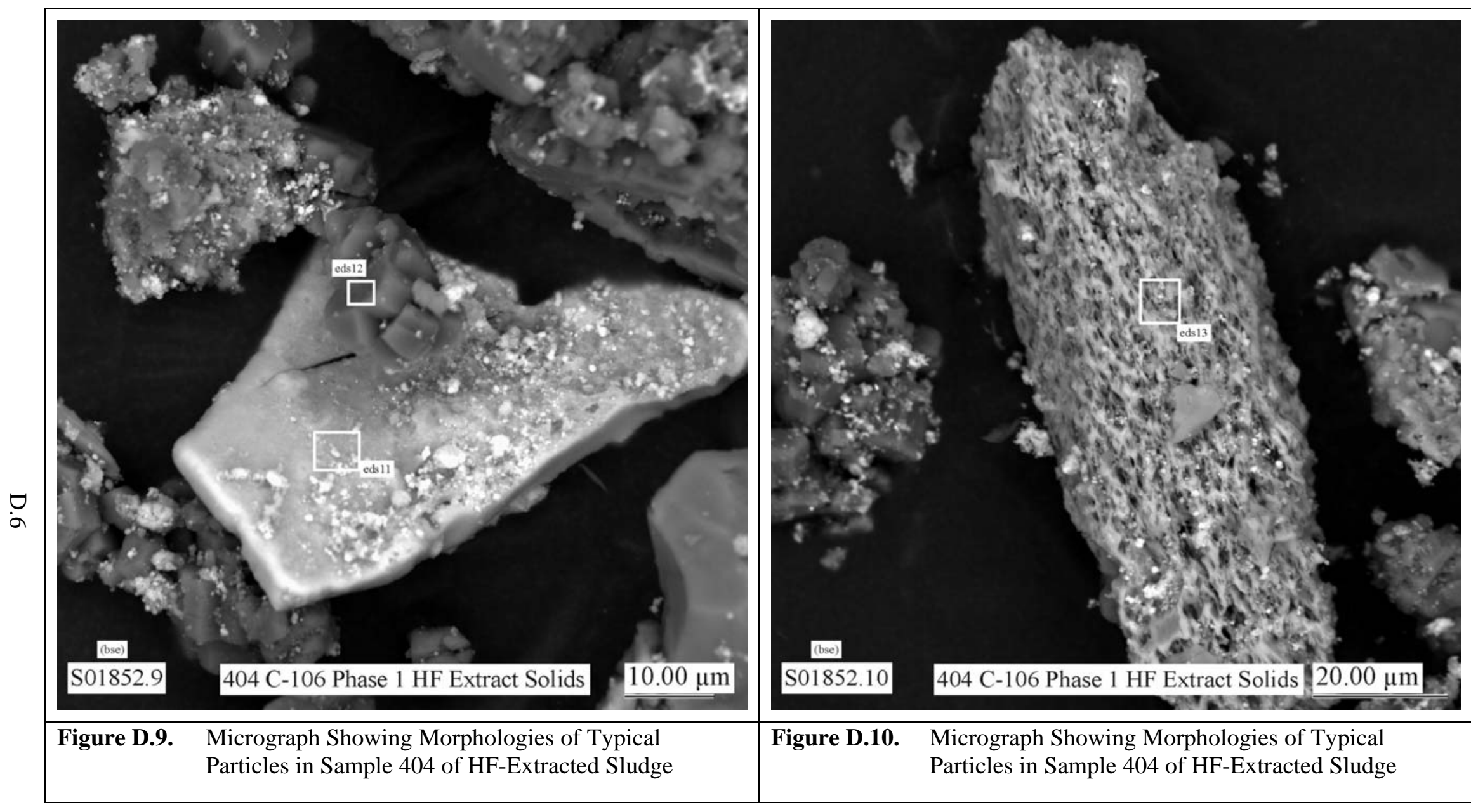




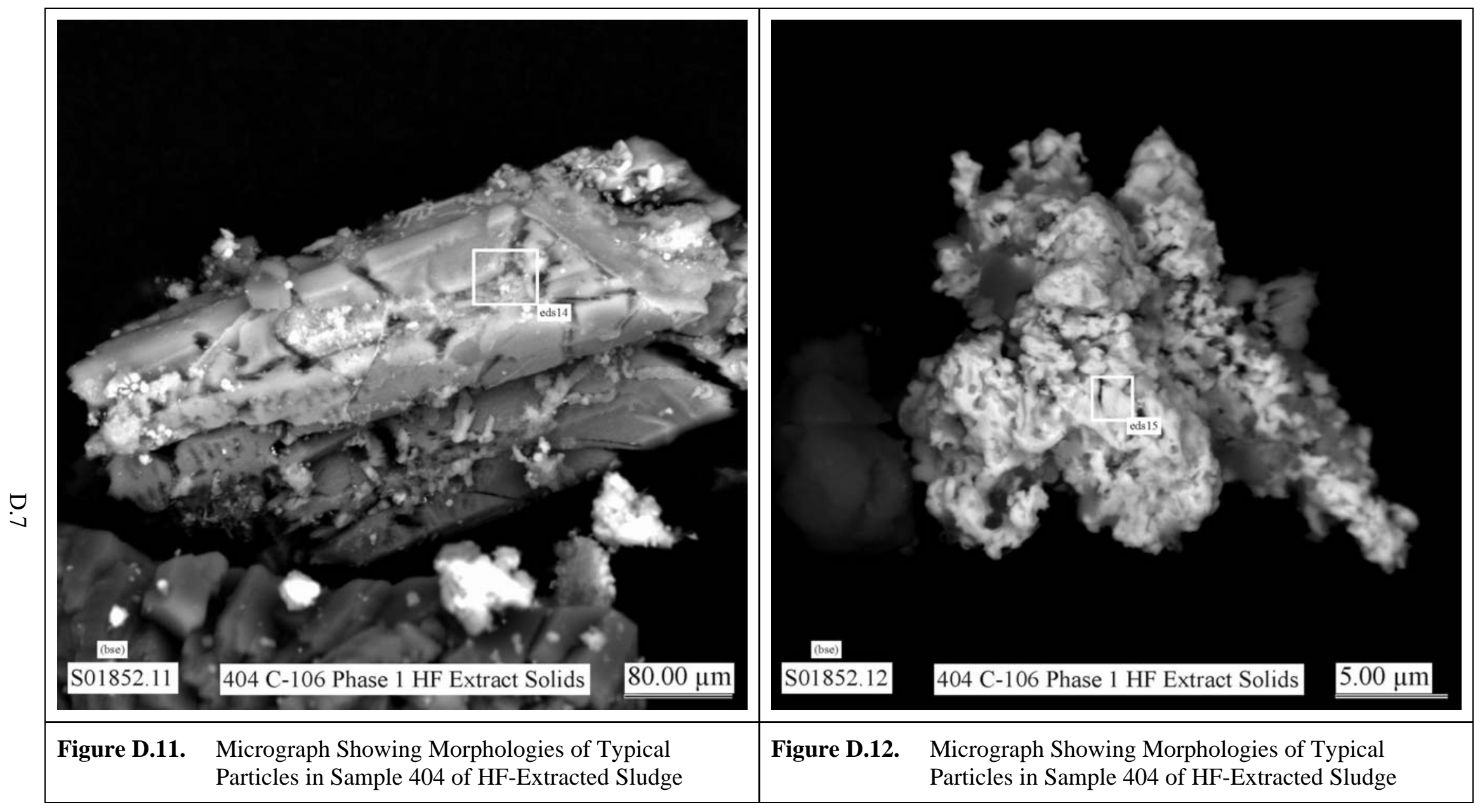




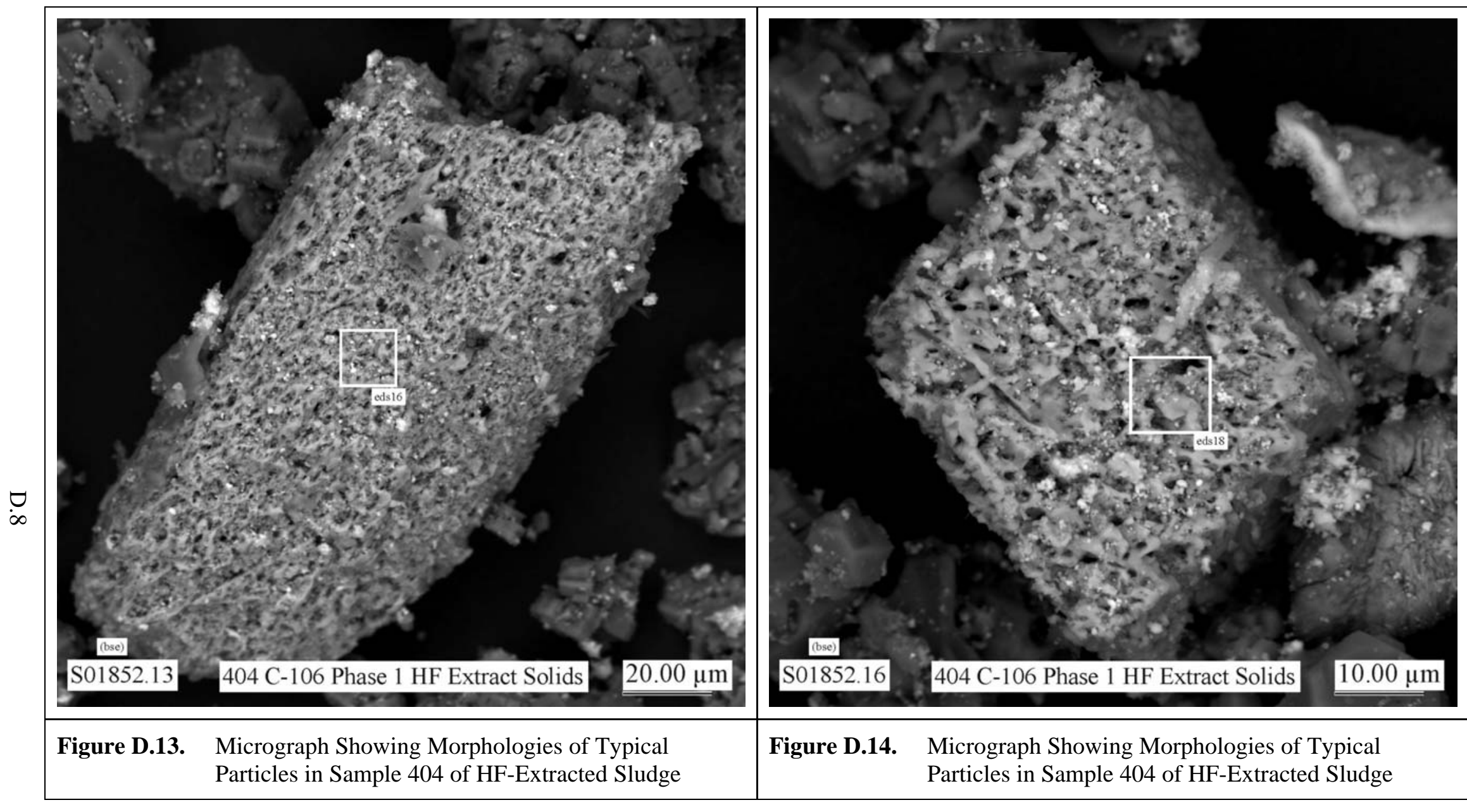




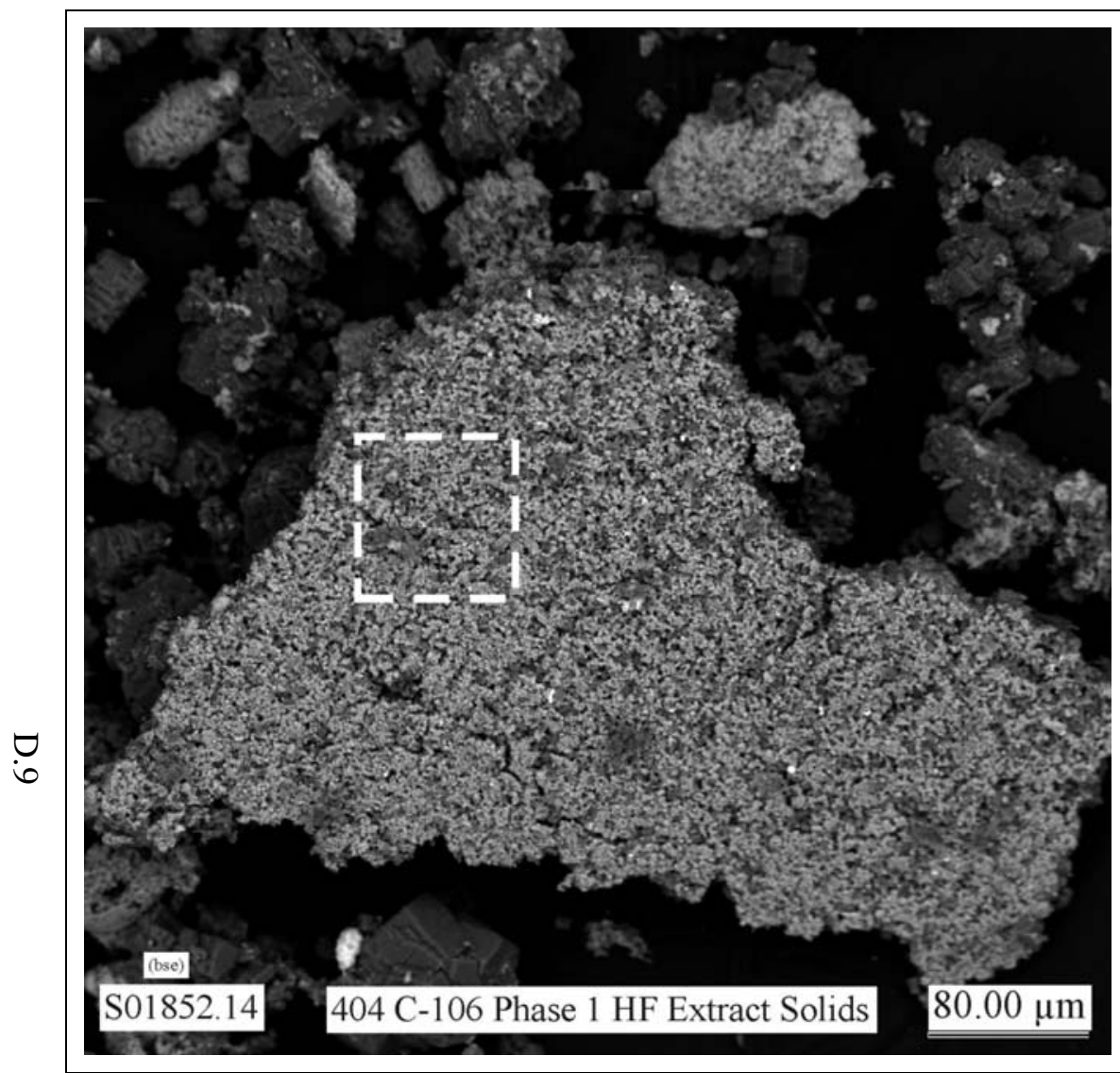

Figure D.15. $\quad$ Micrograph Showing Morphologies of Typical Particles in Sample 404 of HF-Extracted Sludge

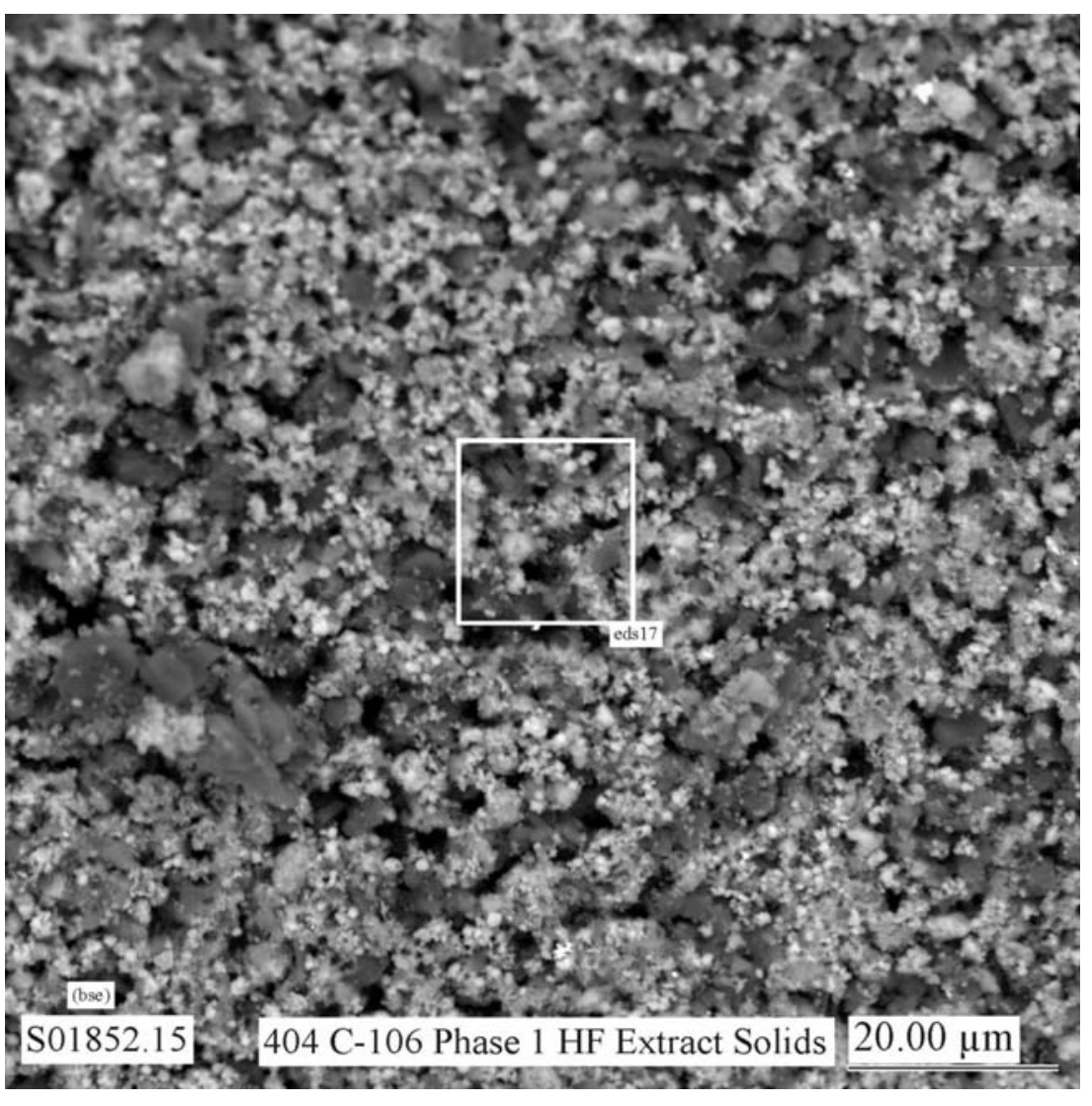

Figure D.16. Micrograph Showing at Higher Magnification the Area Indicated by the White Dashed-Line Square in Figure D.15. 


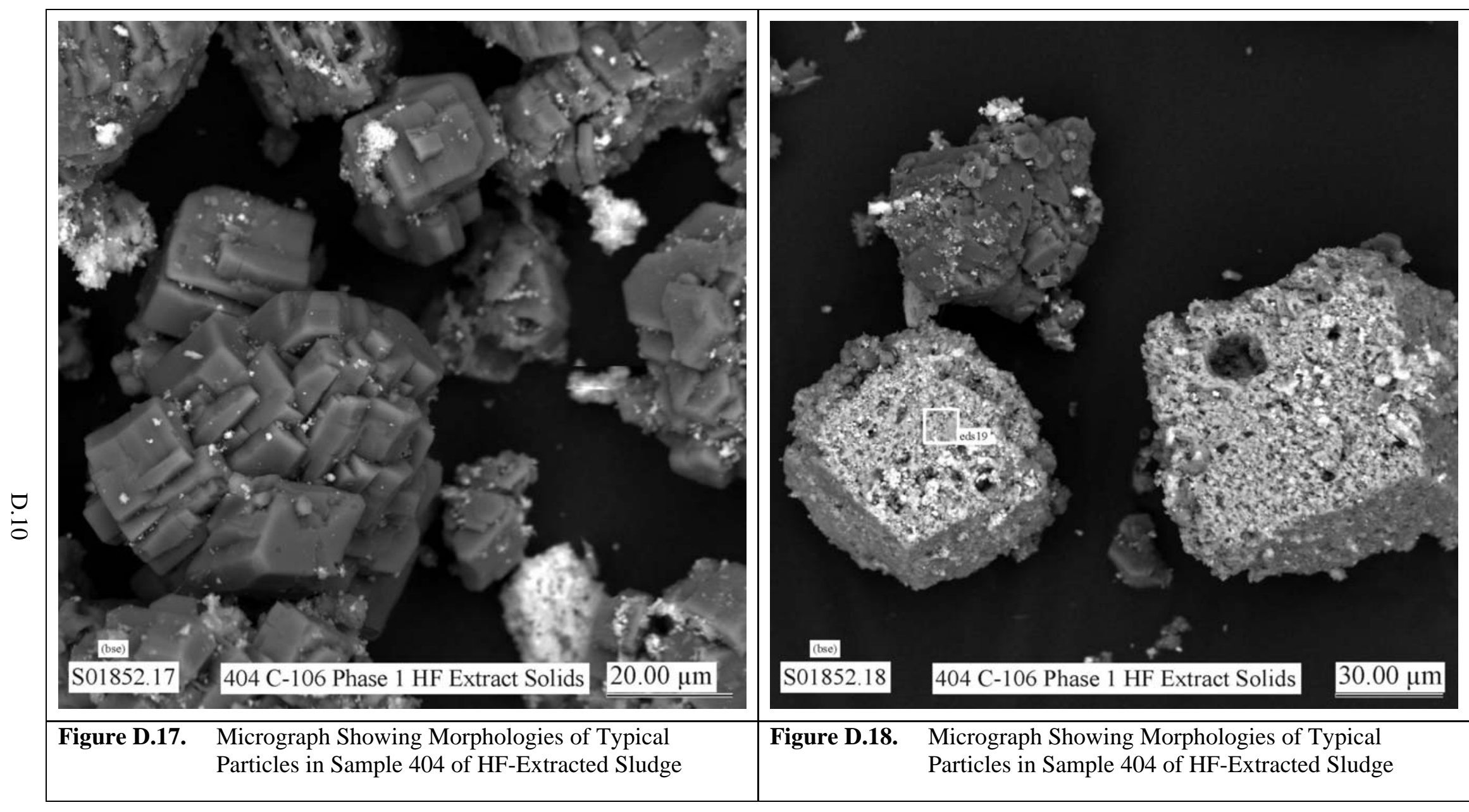




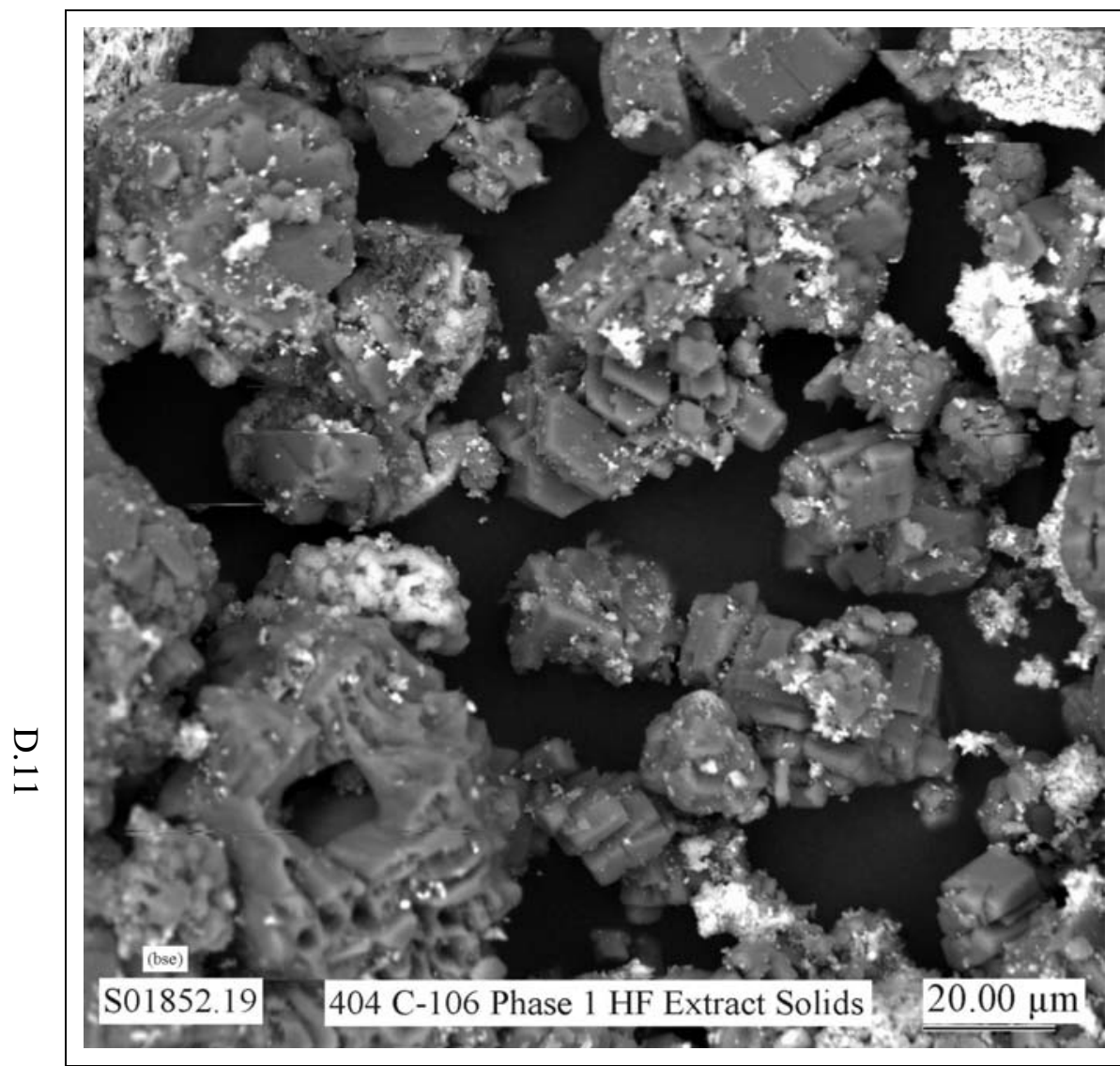

Figure D.19. Micrograph Showing Morphologies of Typical Particles in Sample 404 of HF-Extracted Sludge

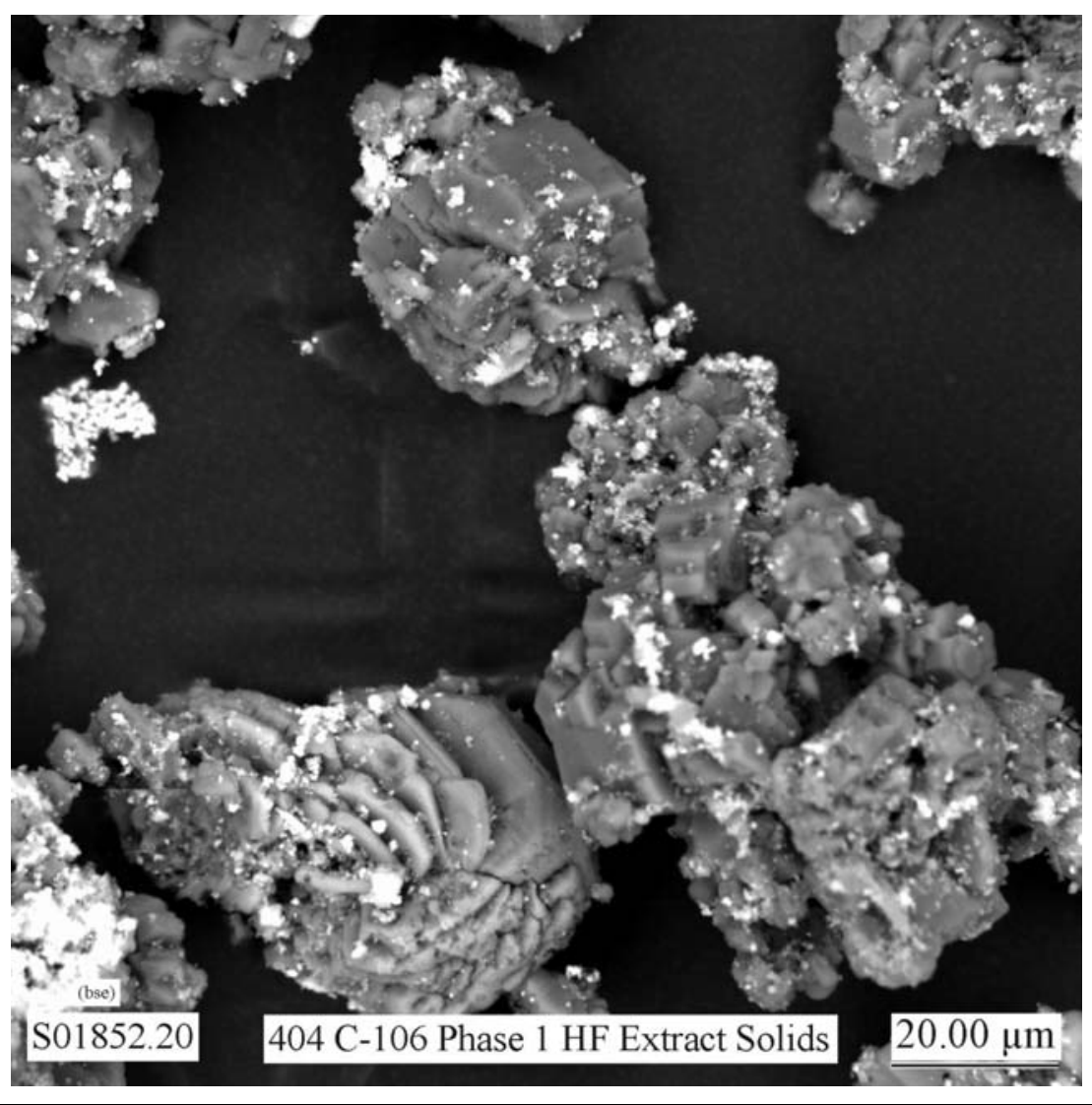

Figure D.20. $\quad$ Micrograph Showing Morphologies of Typical Particles in Sample 404 of HF-Extracted Sludge 


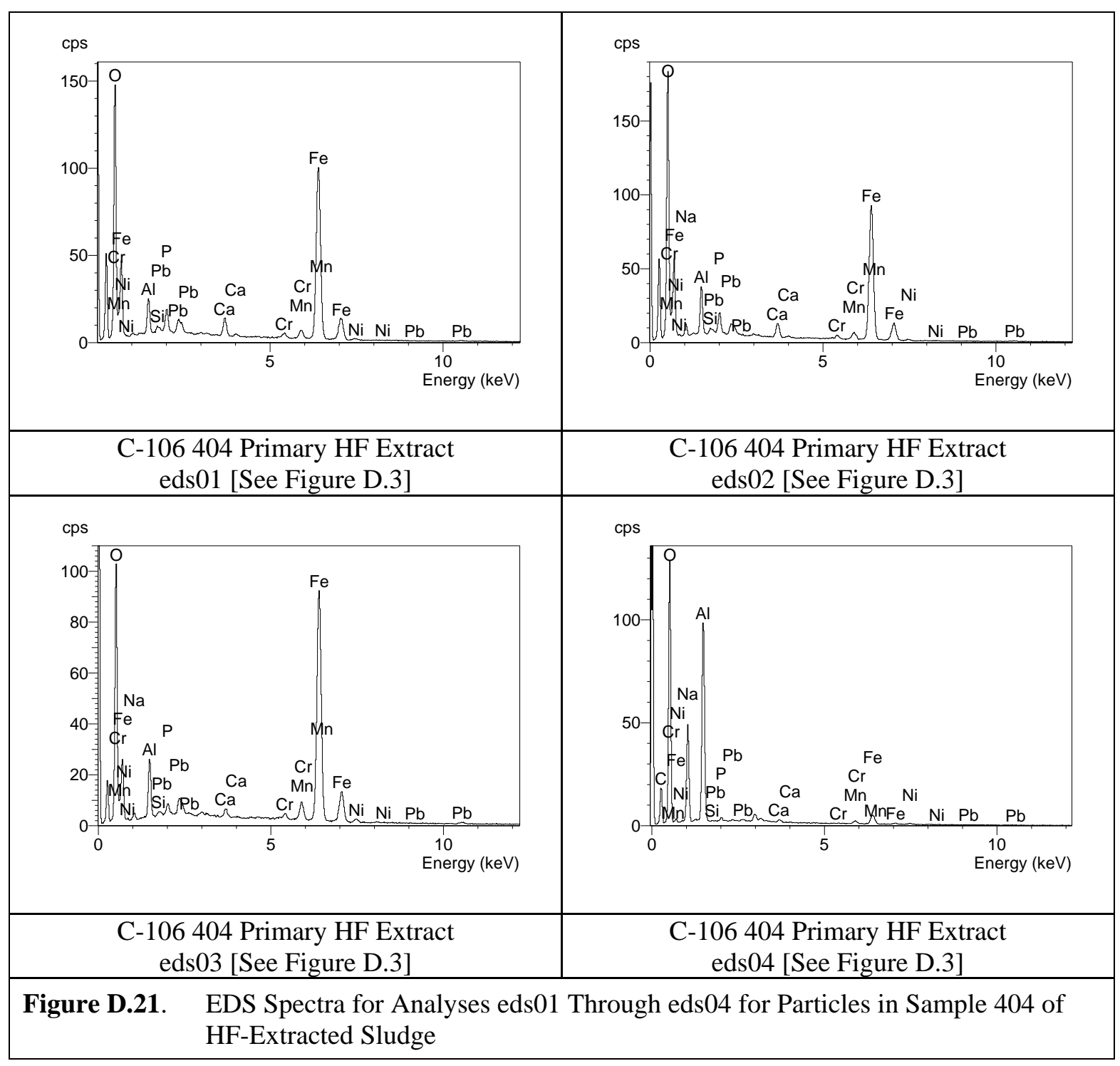

D.12 


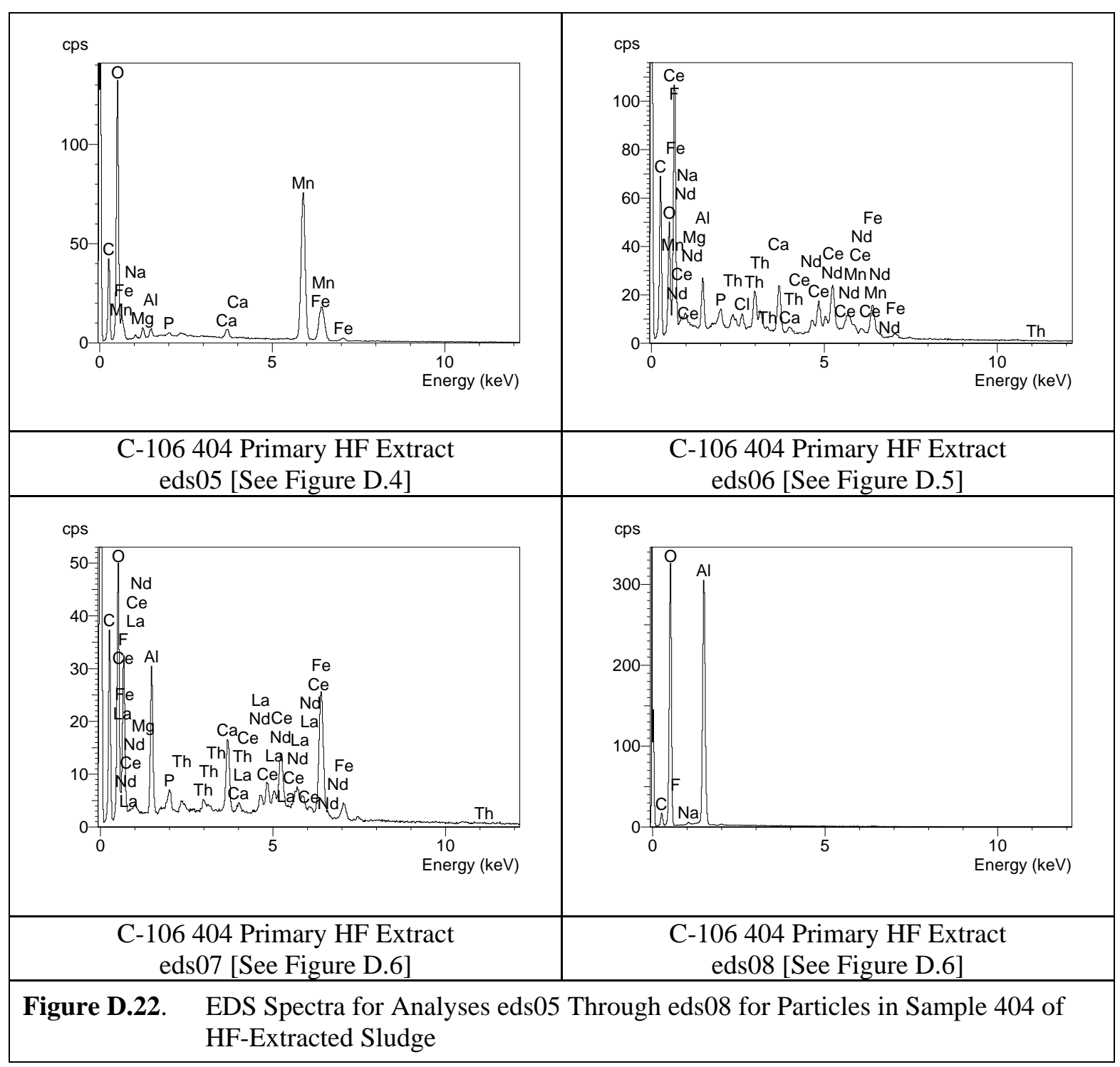

D.13 


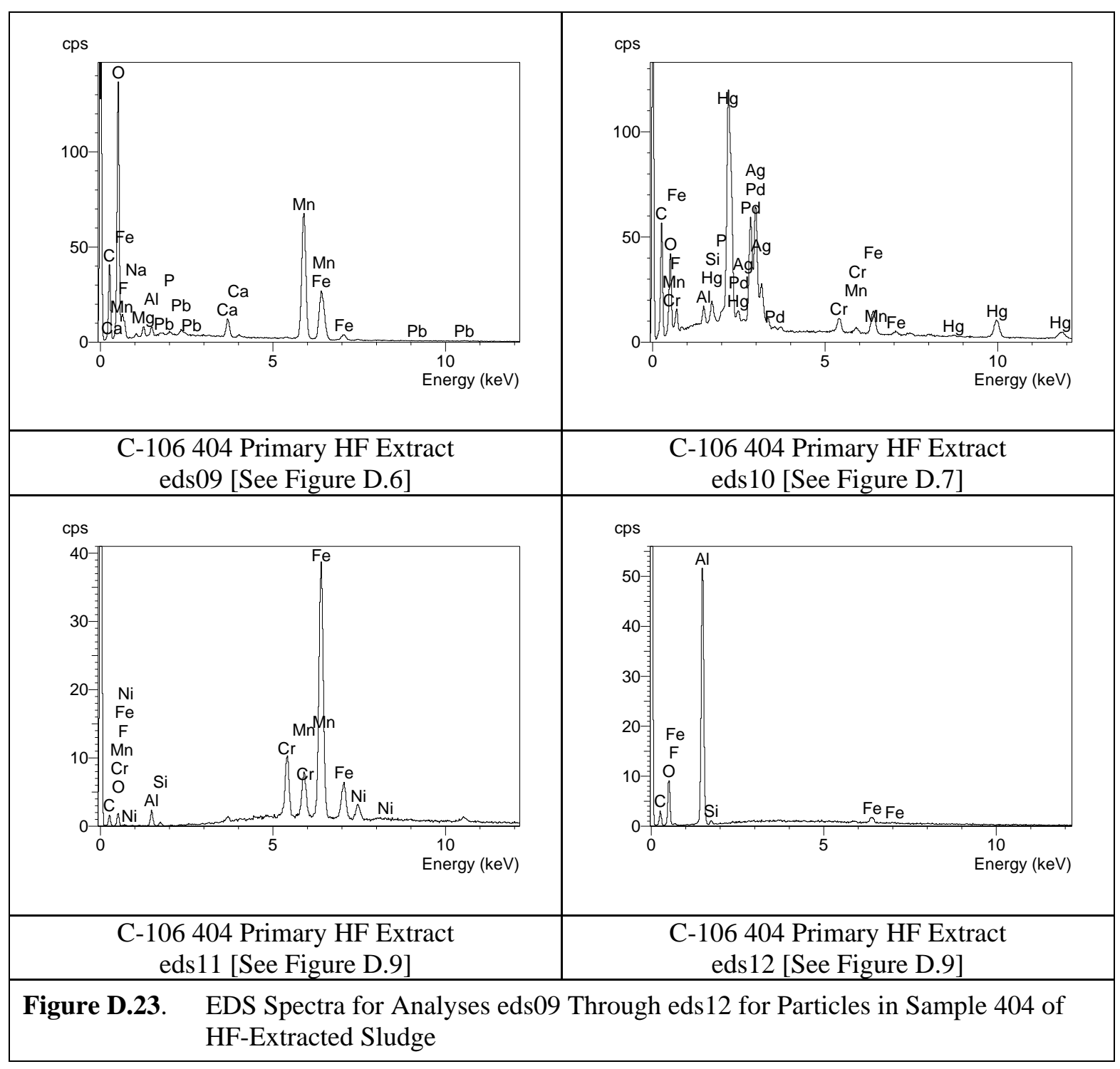




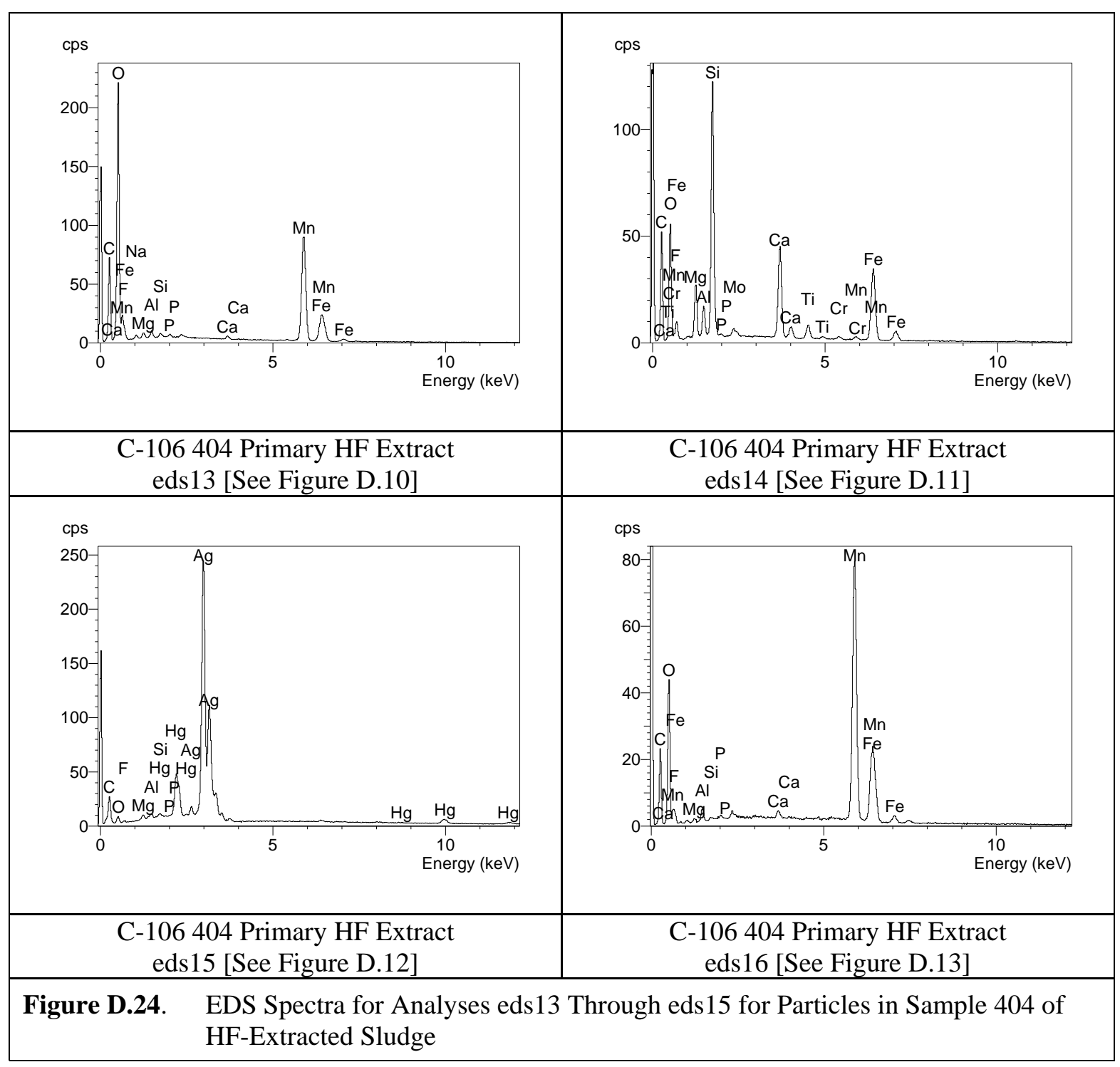

D.15 


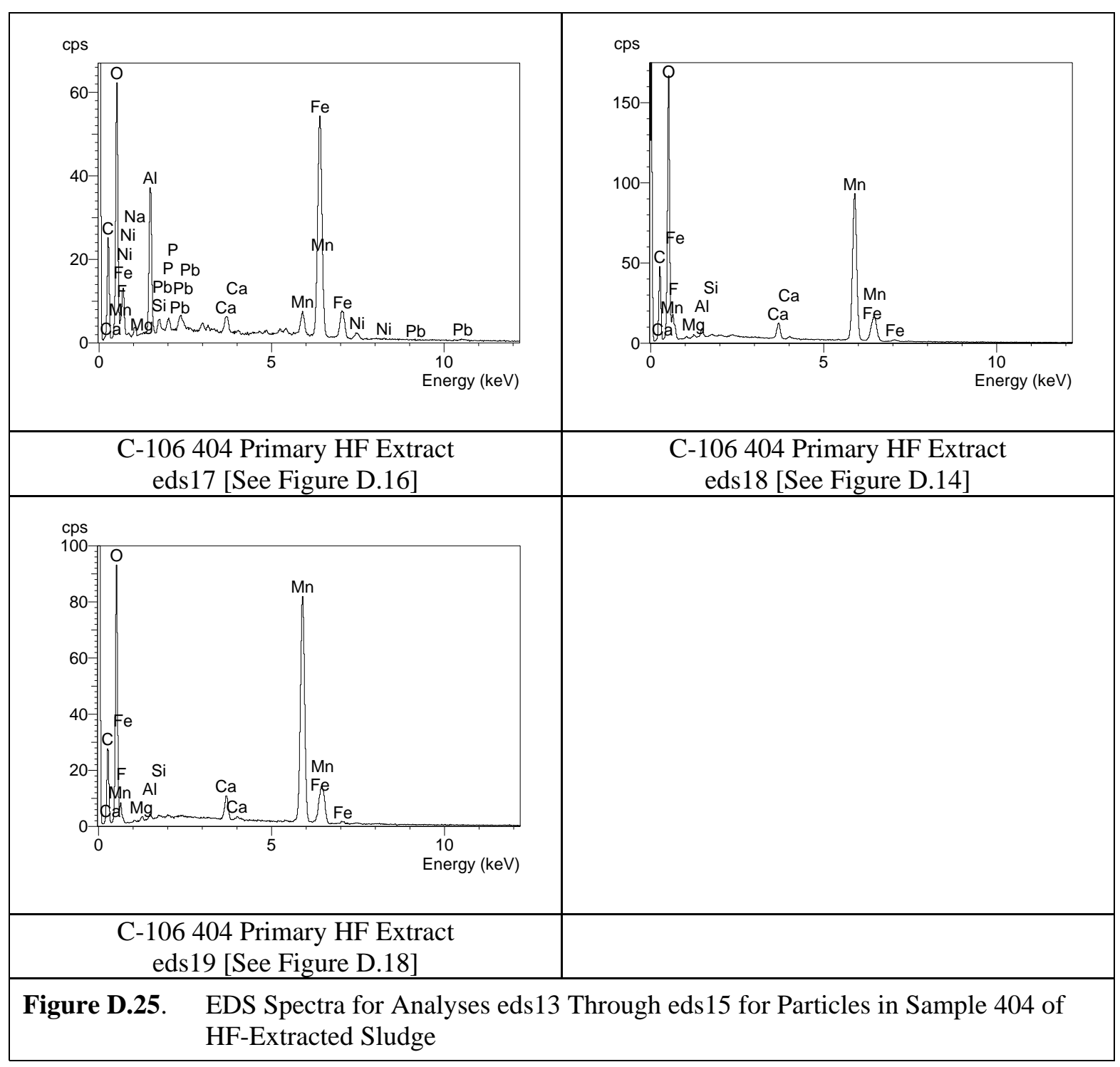




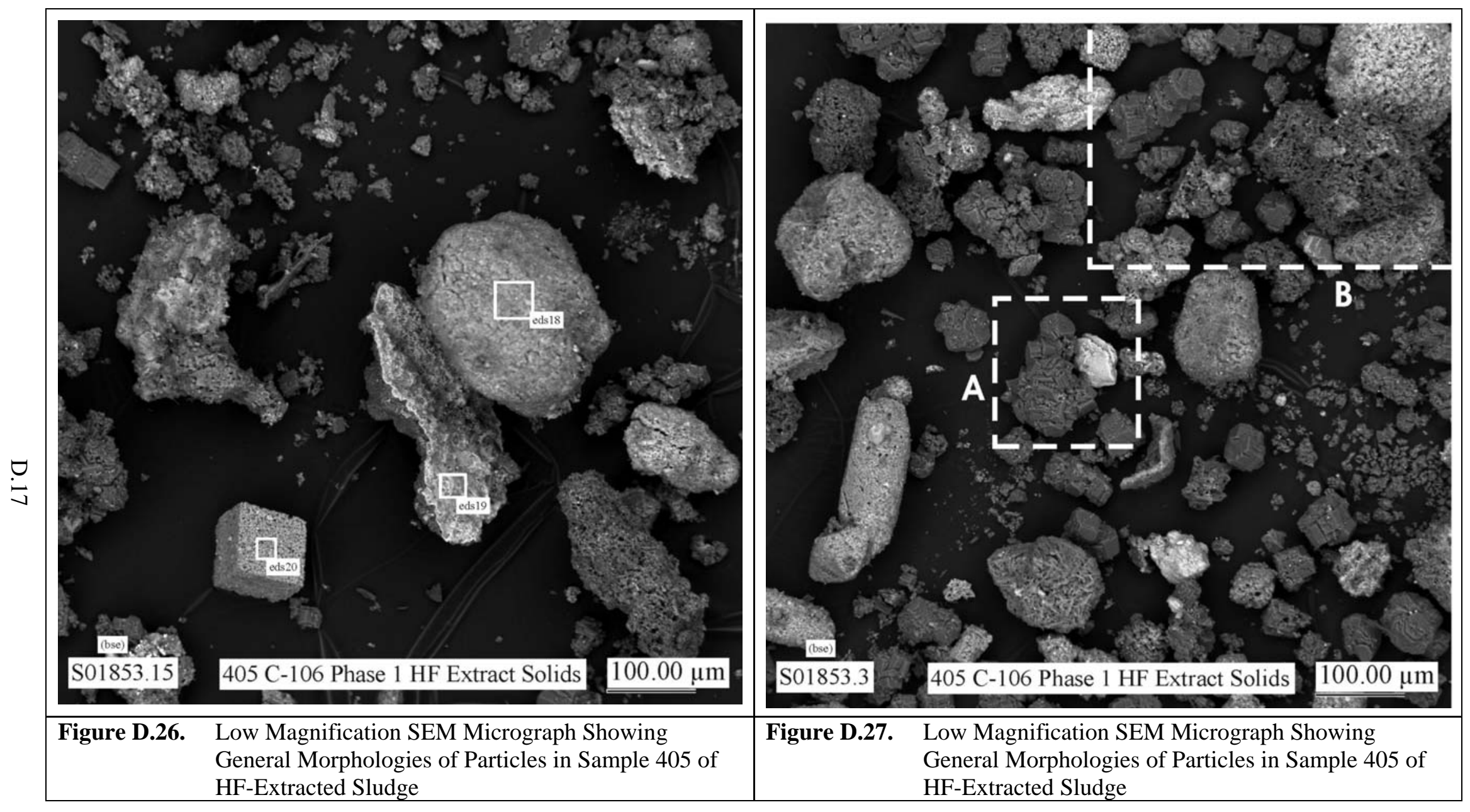




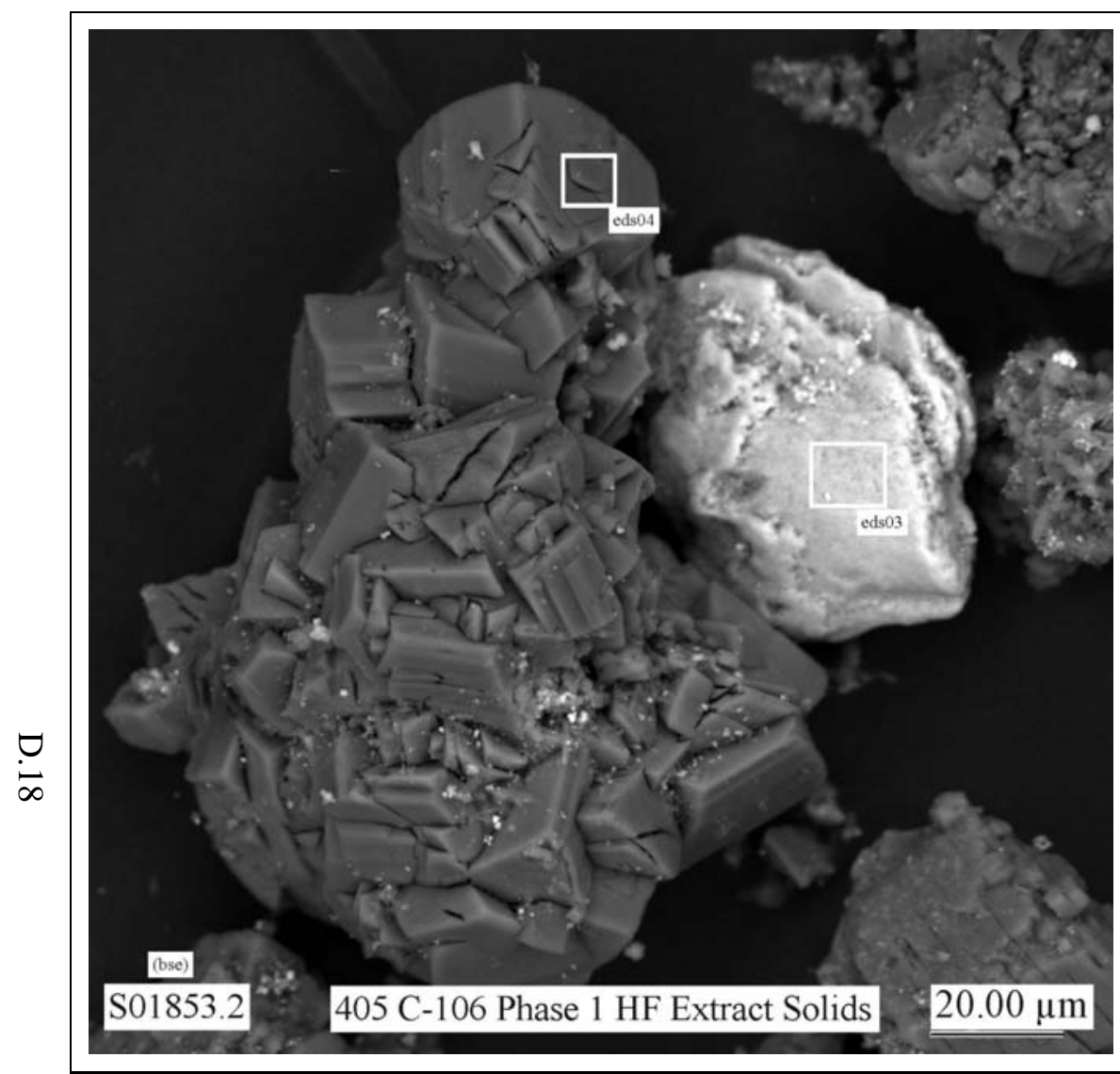

Figure D.28. Micrograph Showing at Higher Magnification the Area Indicated by the White Dashed-Line Square Labeled A in Figure D.27

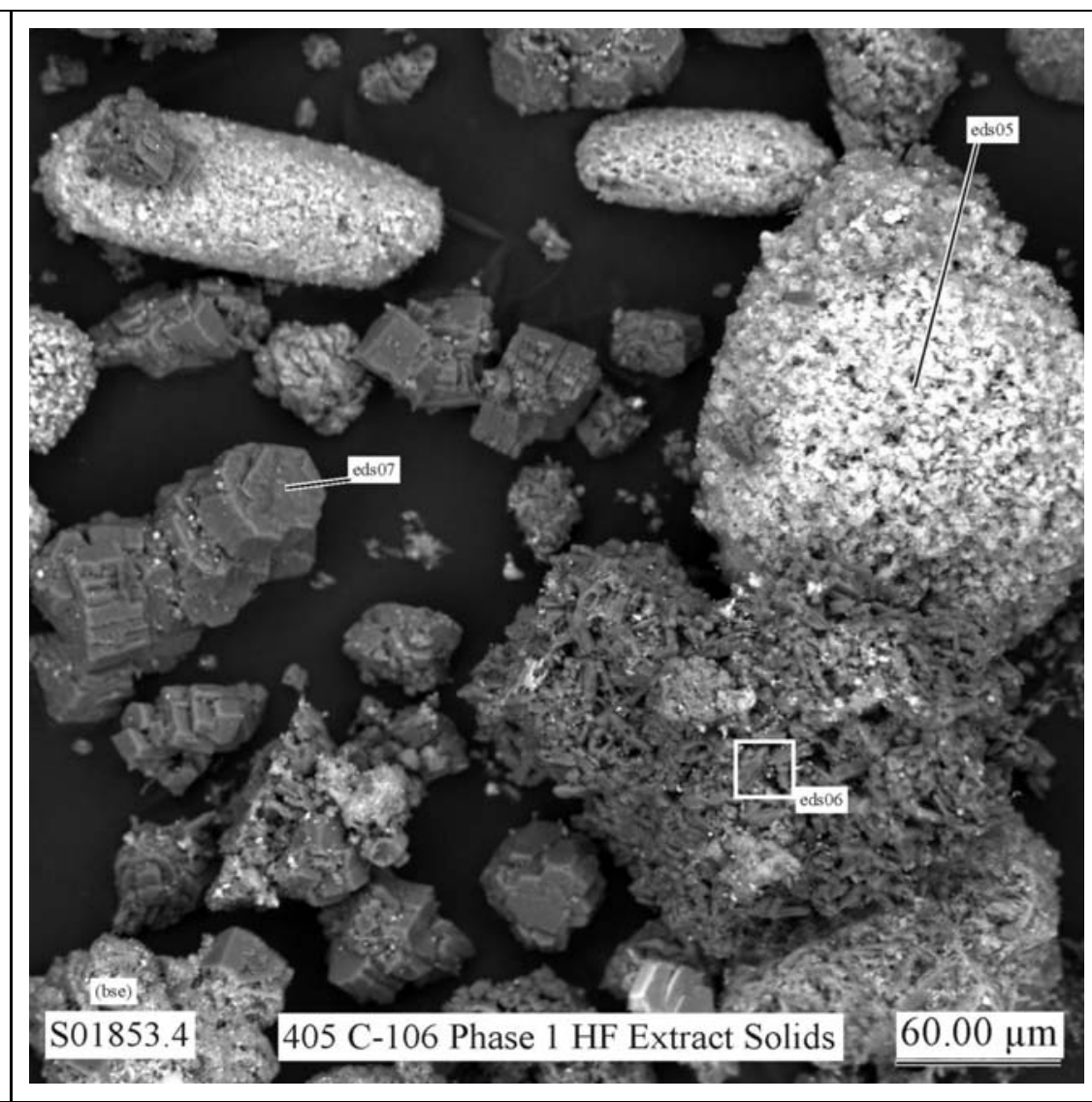

Figure D.29. Micrograph Showing at Higher Magnification the Area Indicated by the White Dashed-Line Square Labeled B in Figure D.27 


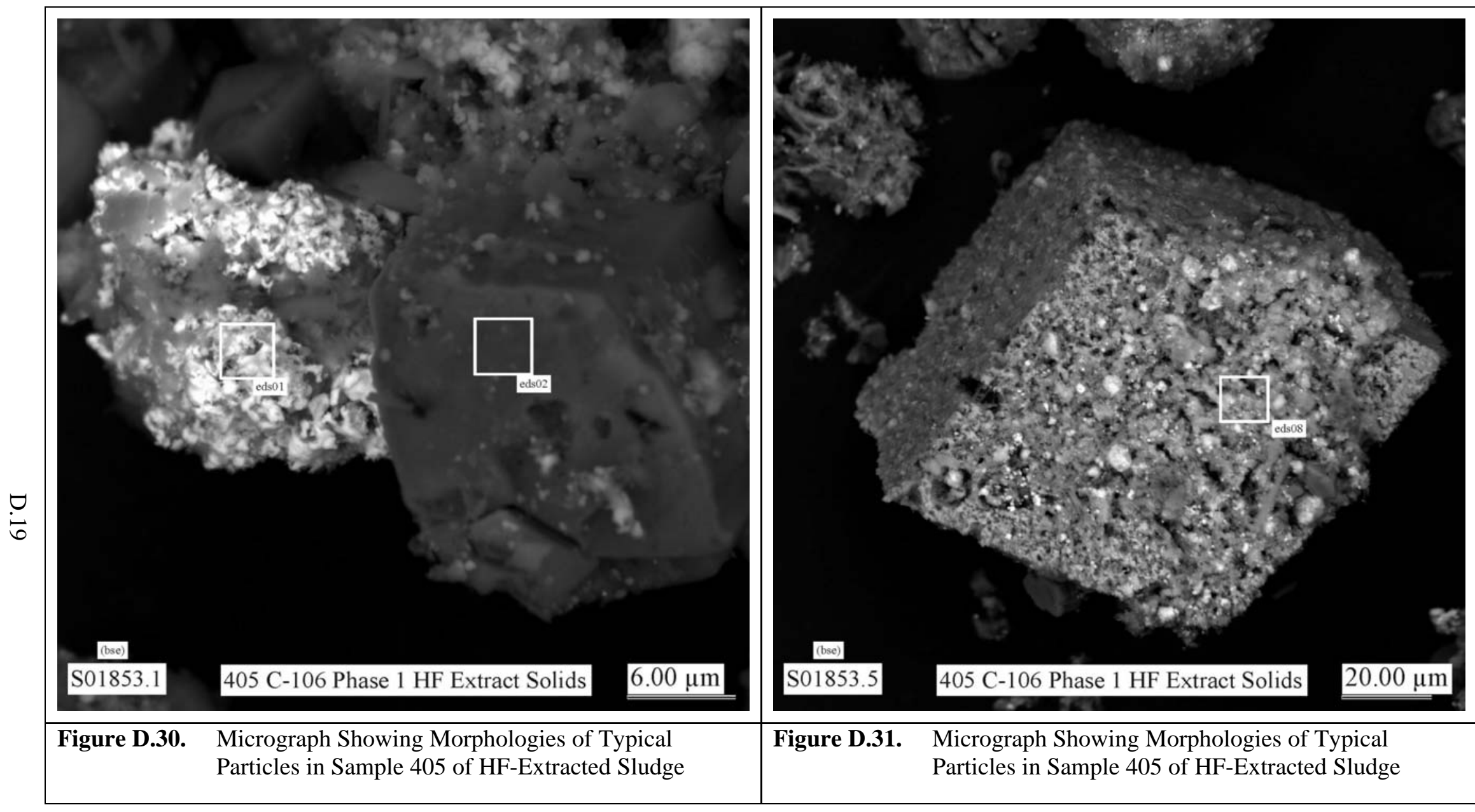




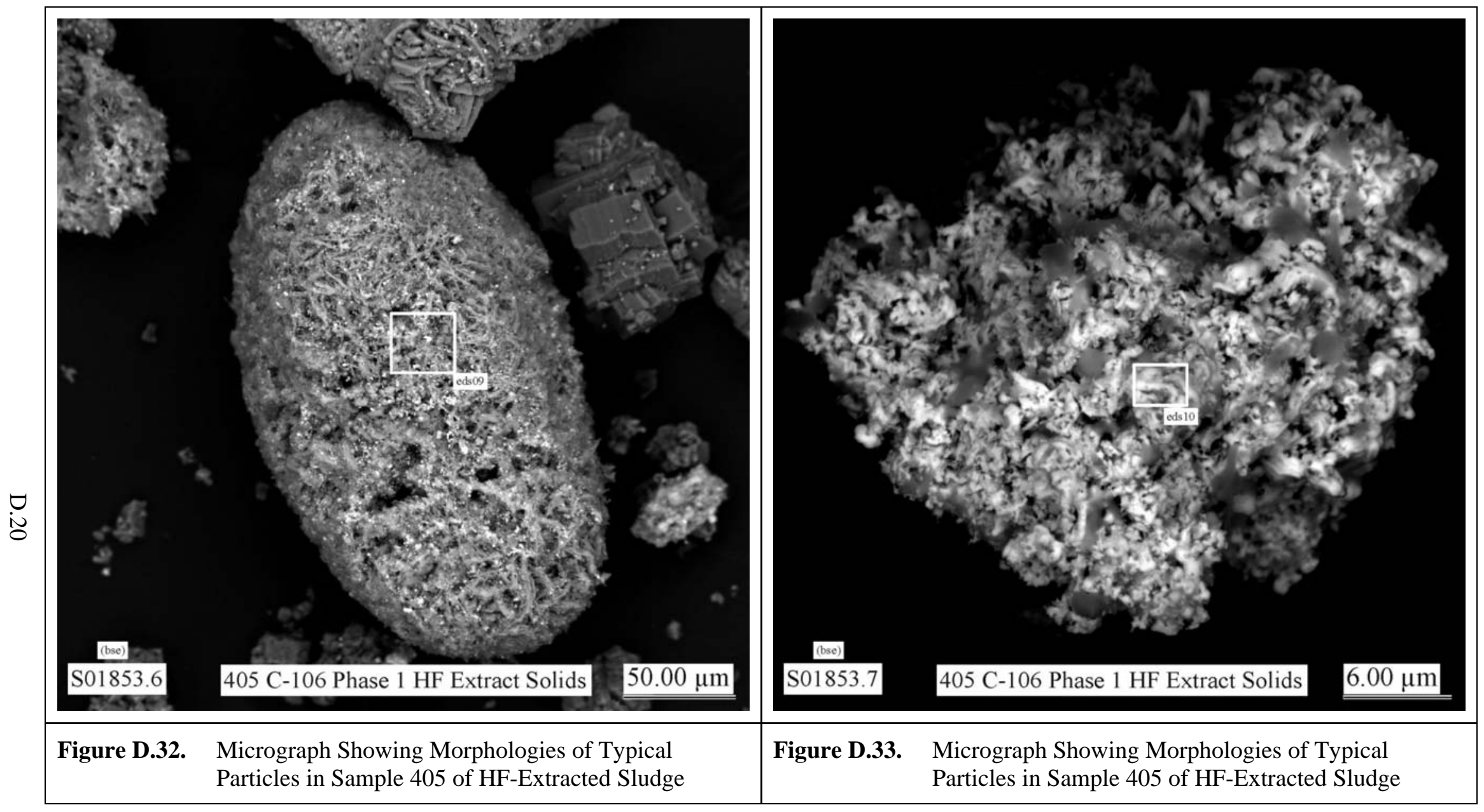




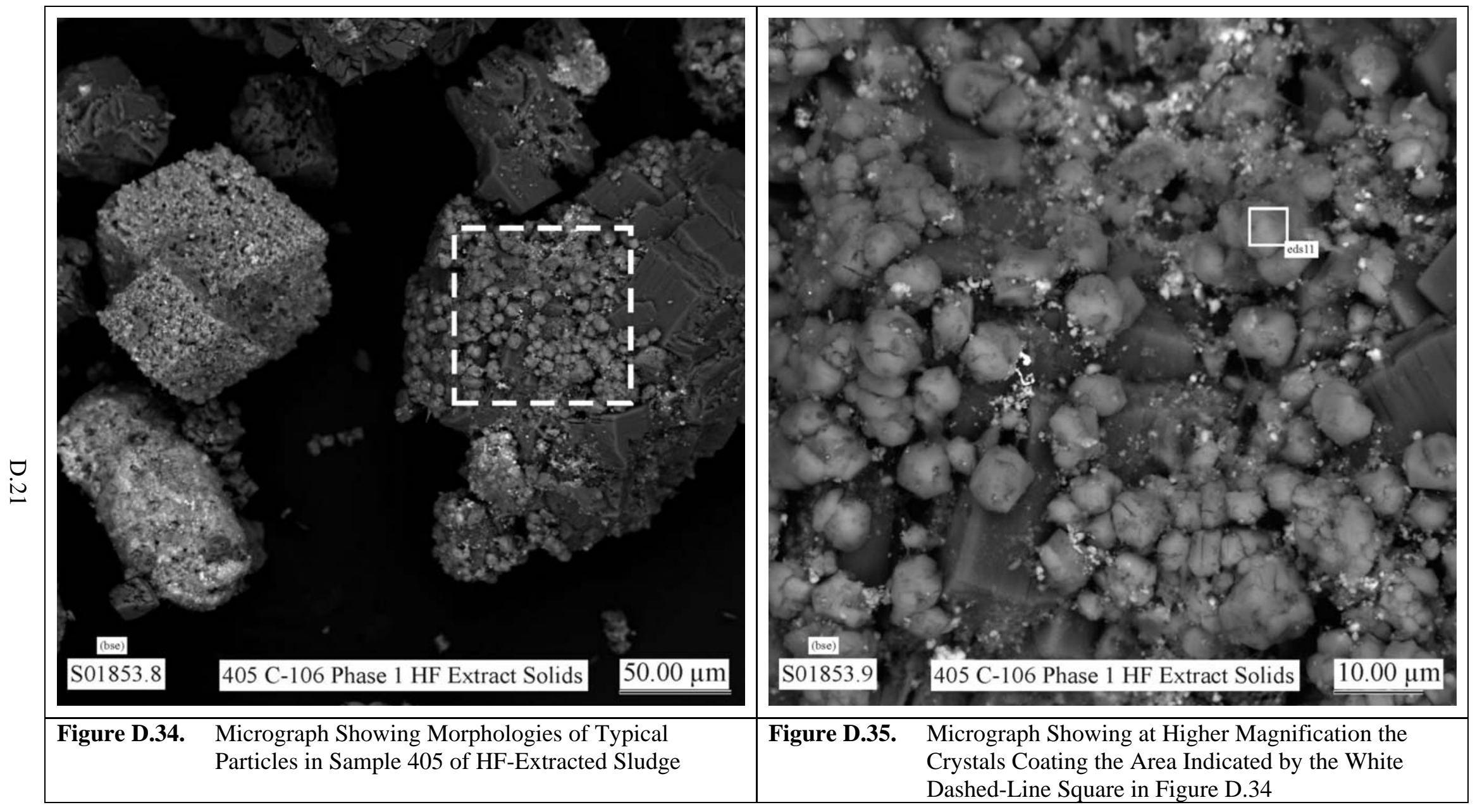




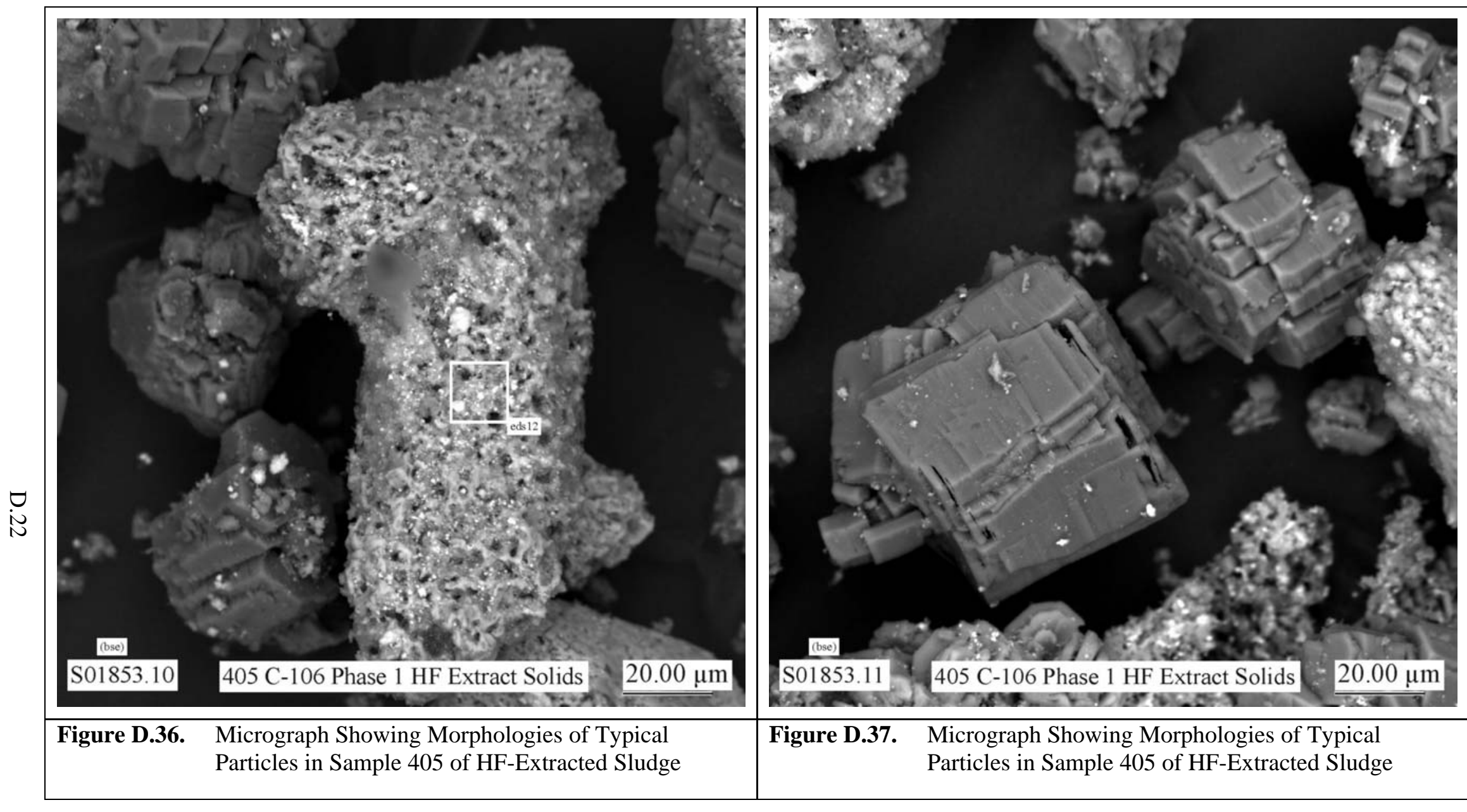




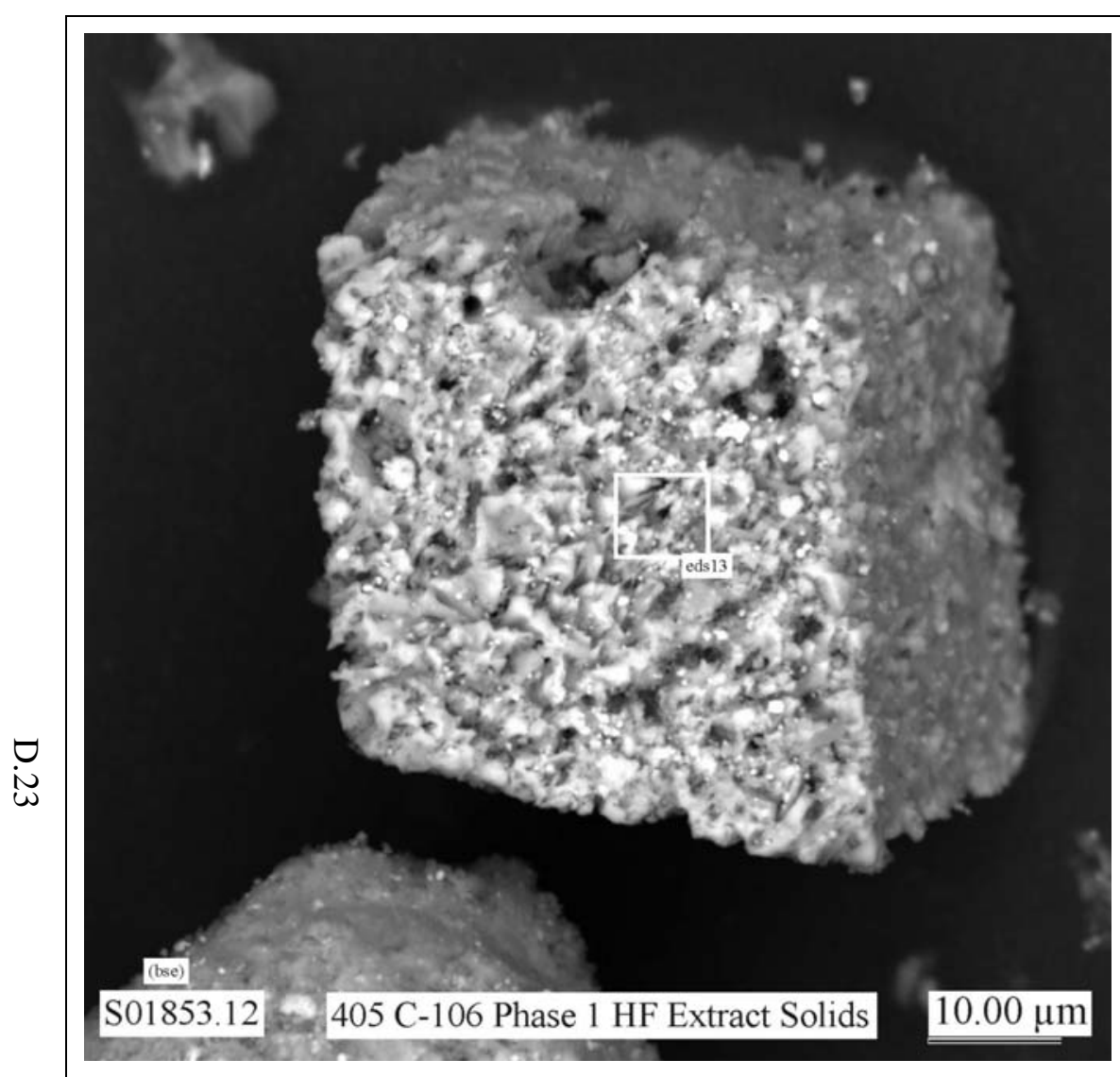

Figure D.38. $\quad$ Micrograph Showing Morphologies of Typical Particles in Sample 405 of HF-Extracted Sludge

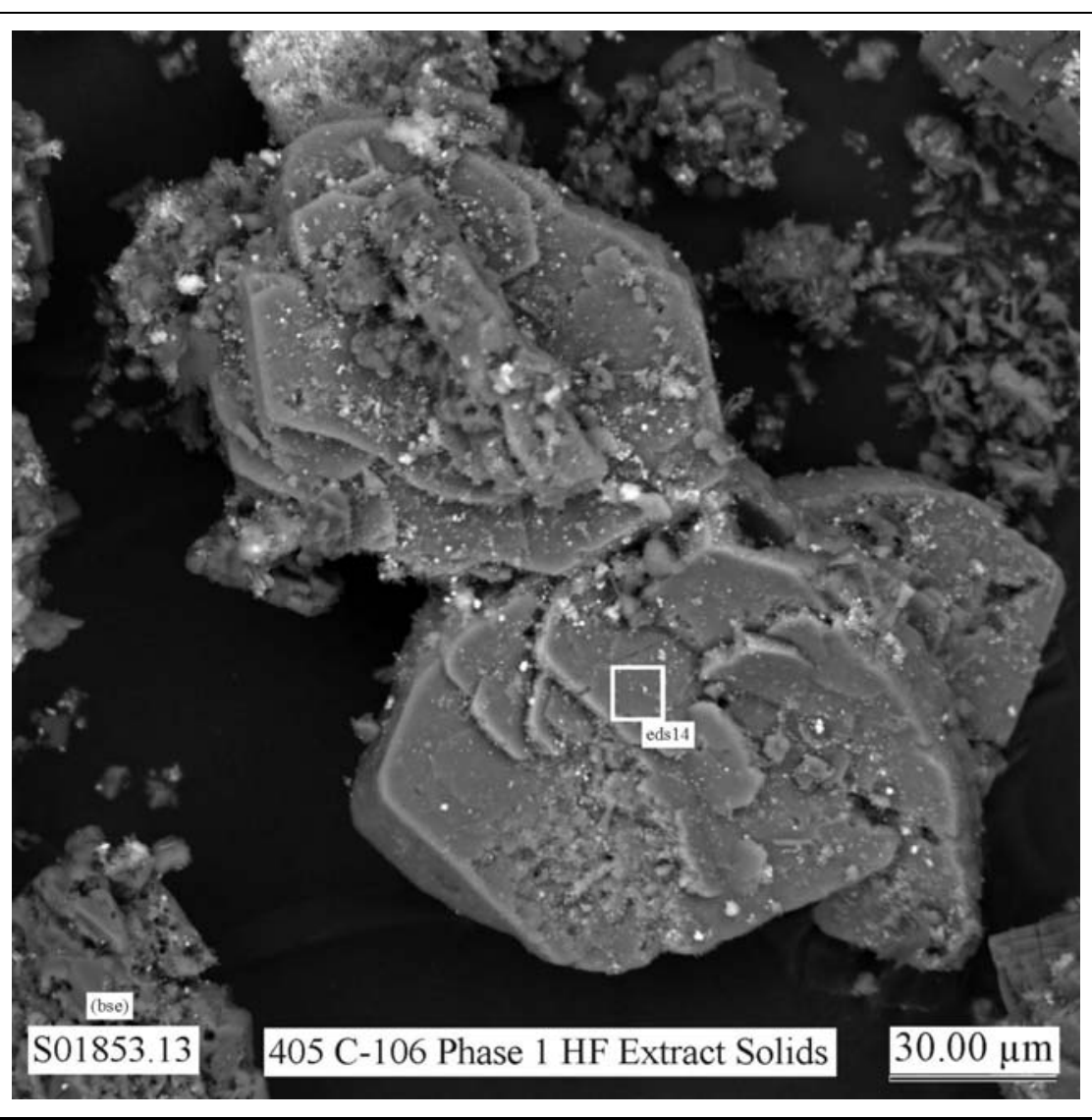

Figure D.39. Micrograph Showing Morphologies of Typical Particles in Sample 405 of HF-Extracted Sludge 


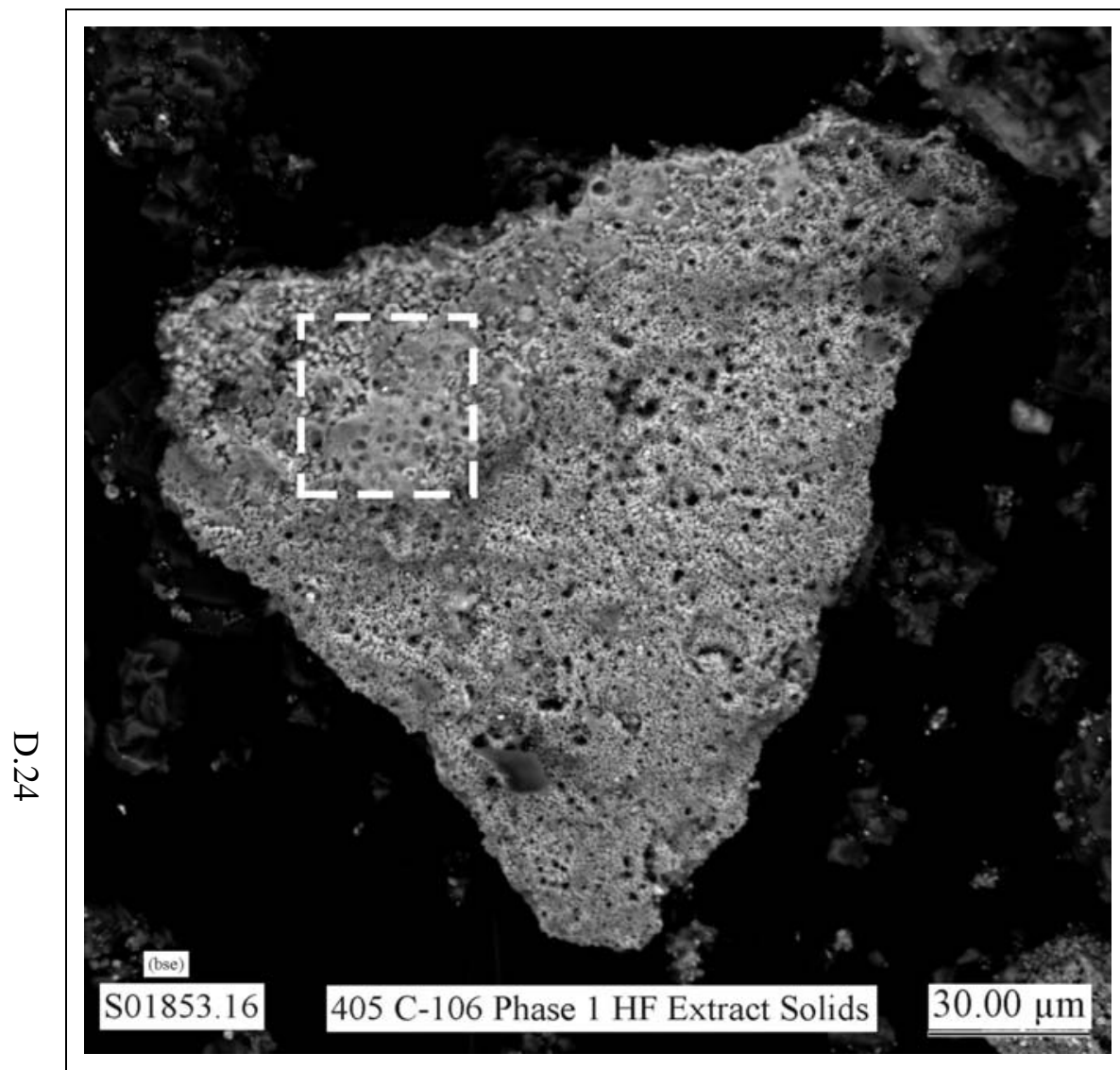

Figure D.40. Micrograph Showing Surface Morphology of a Fe-Rich Particle in Sample 405 of HF-Extracted Sludge

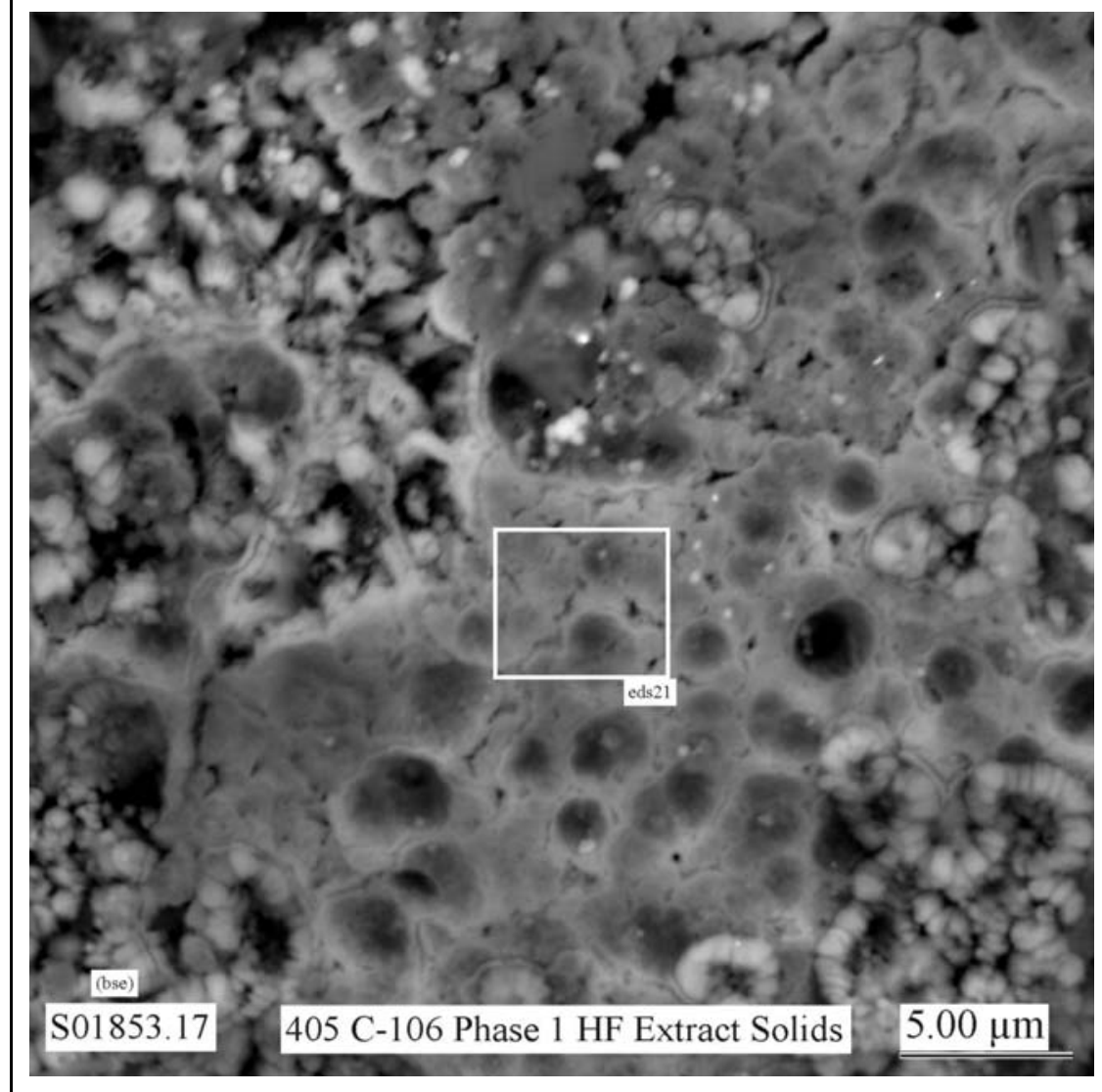

Figure D.41. Micrograph Showing at Higher Magnification the Area Indicated by the White Dashed-Line Square in Figure D.40 


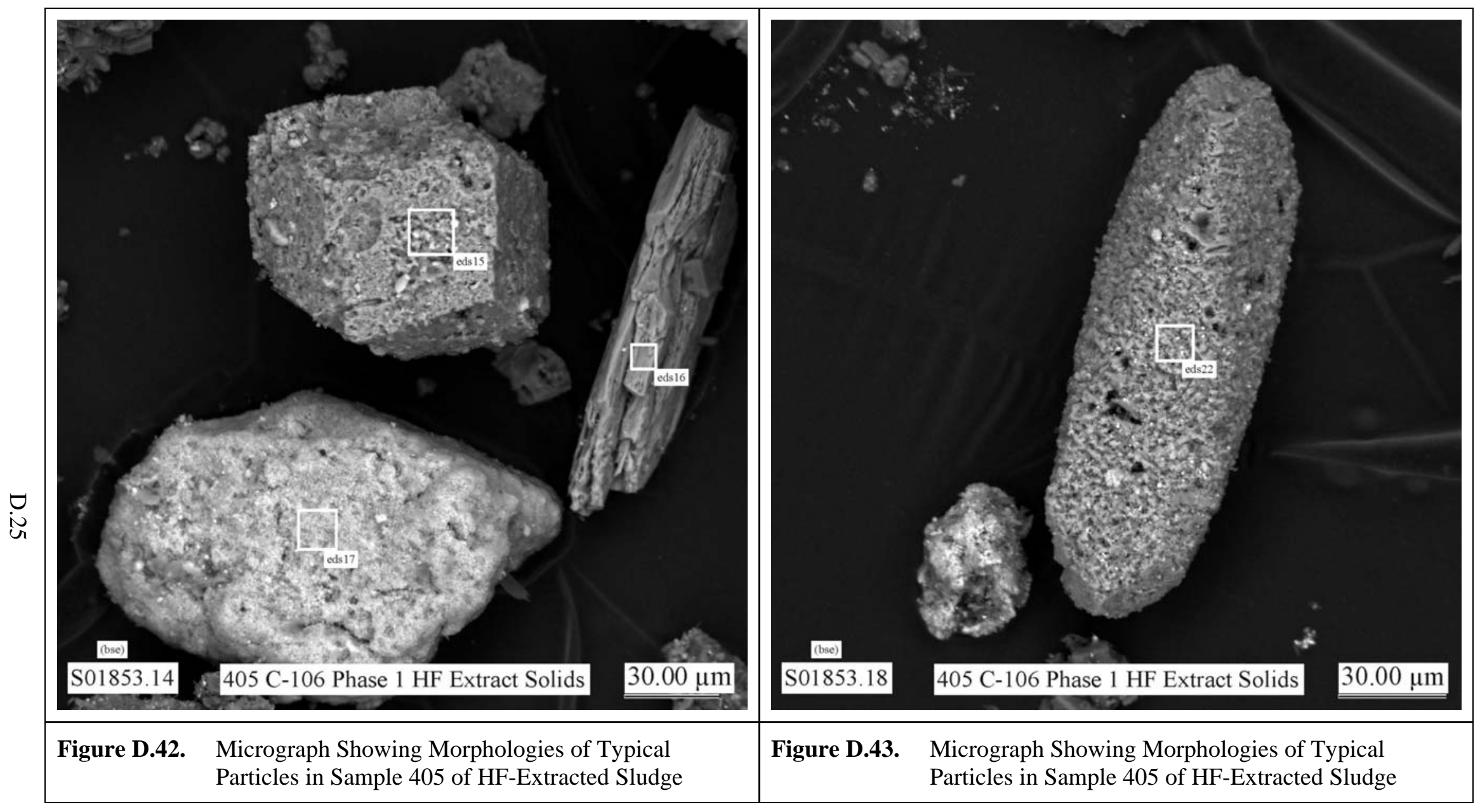




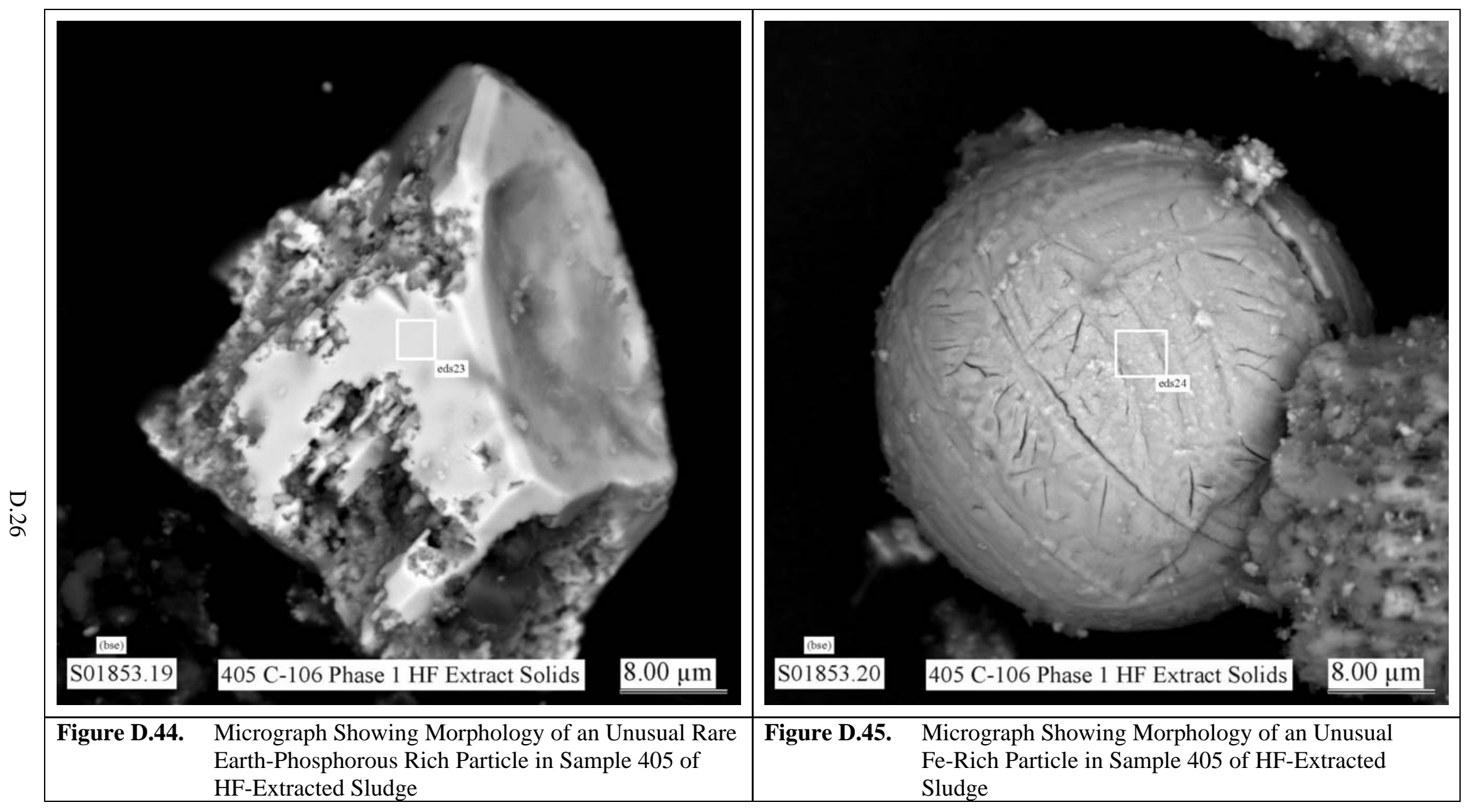




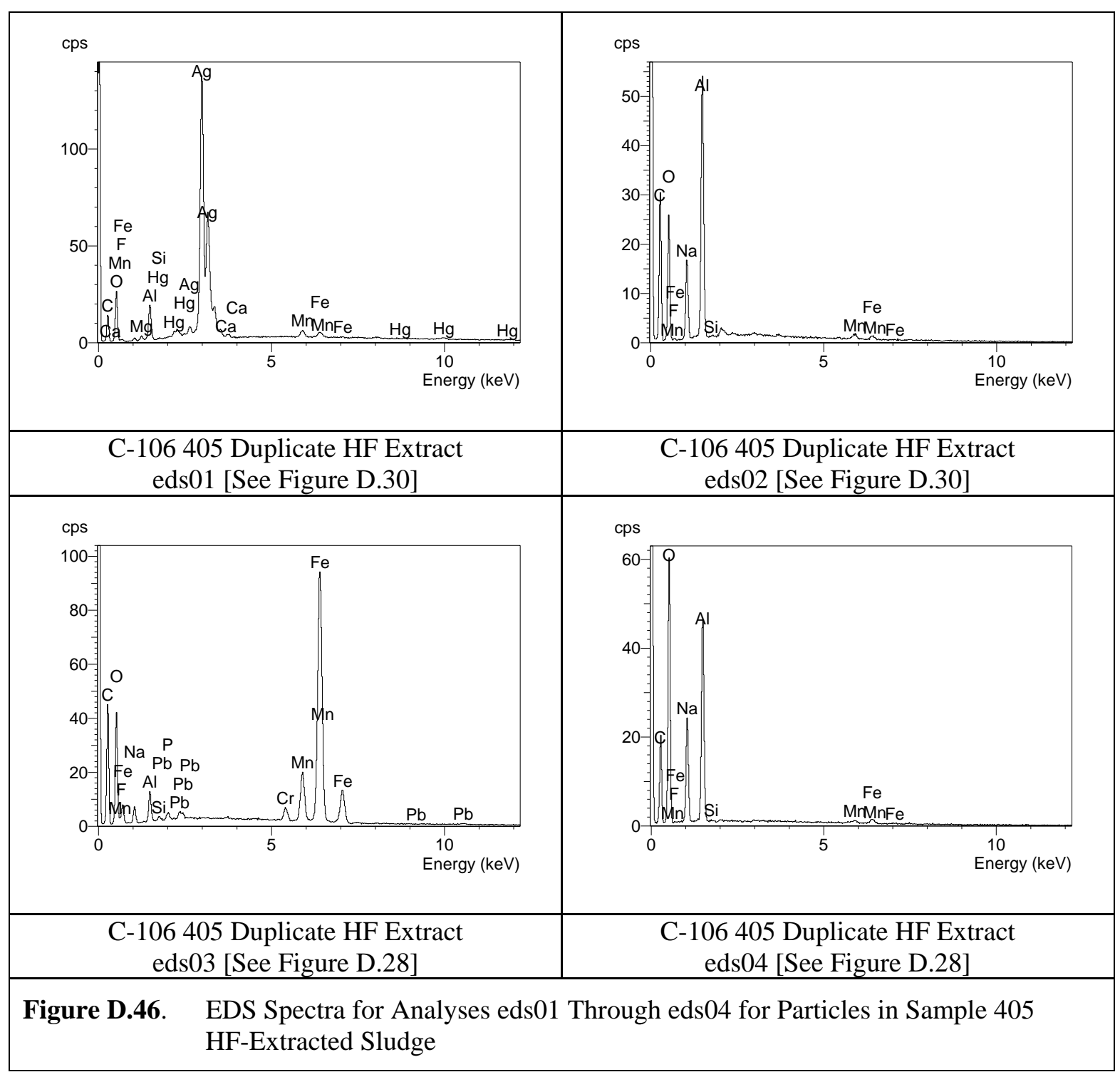




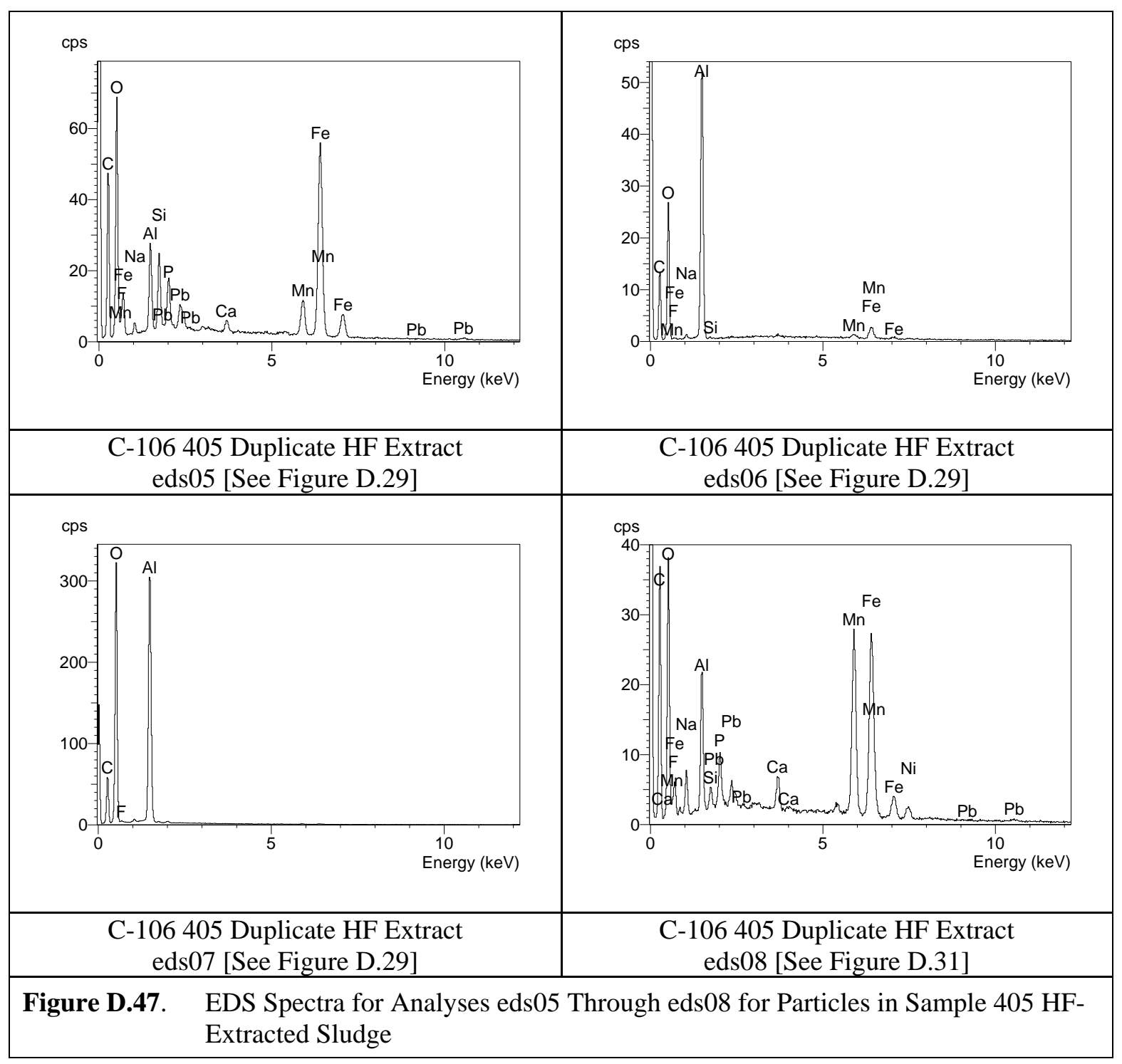




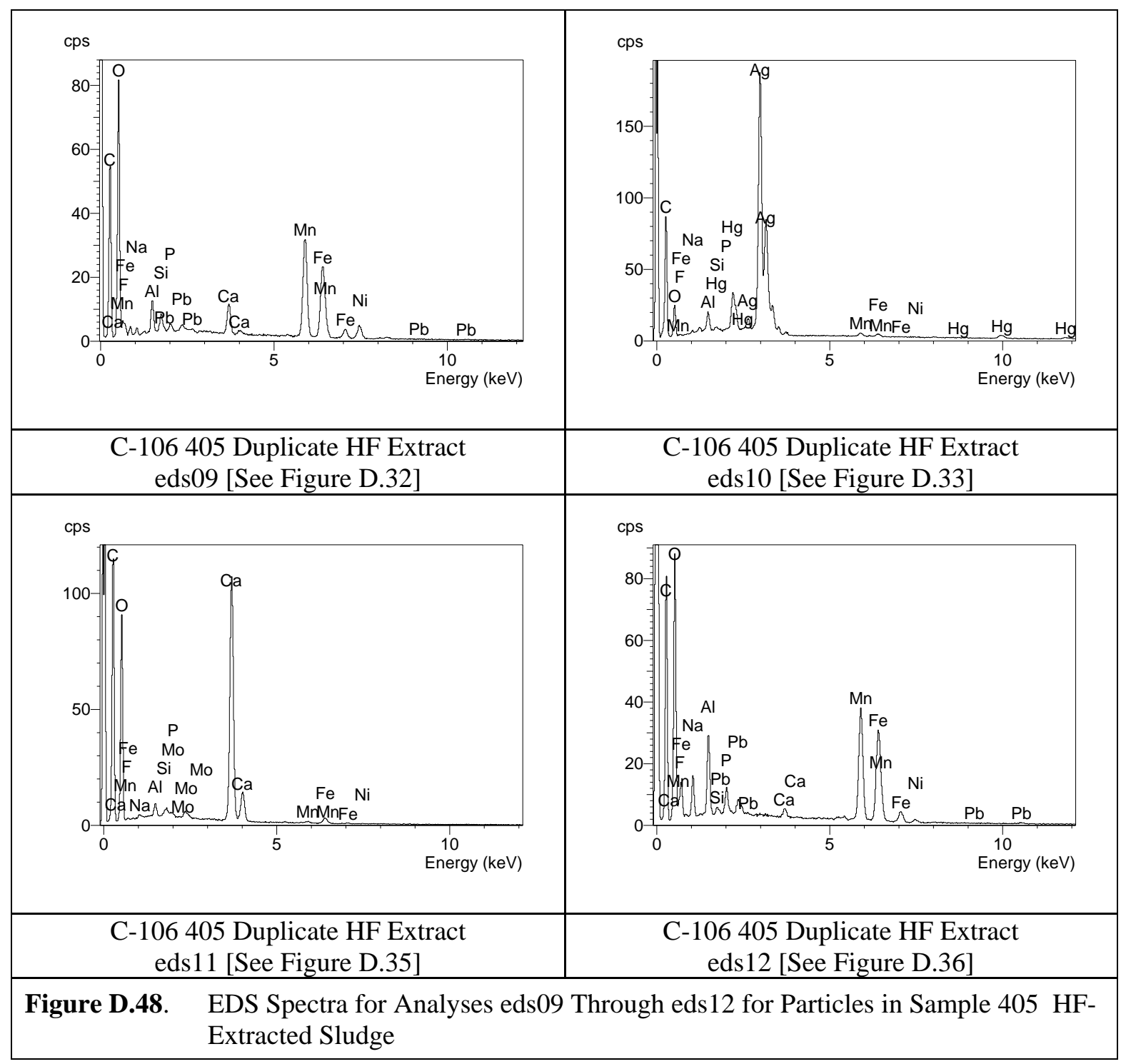




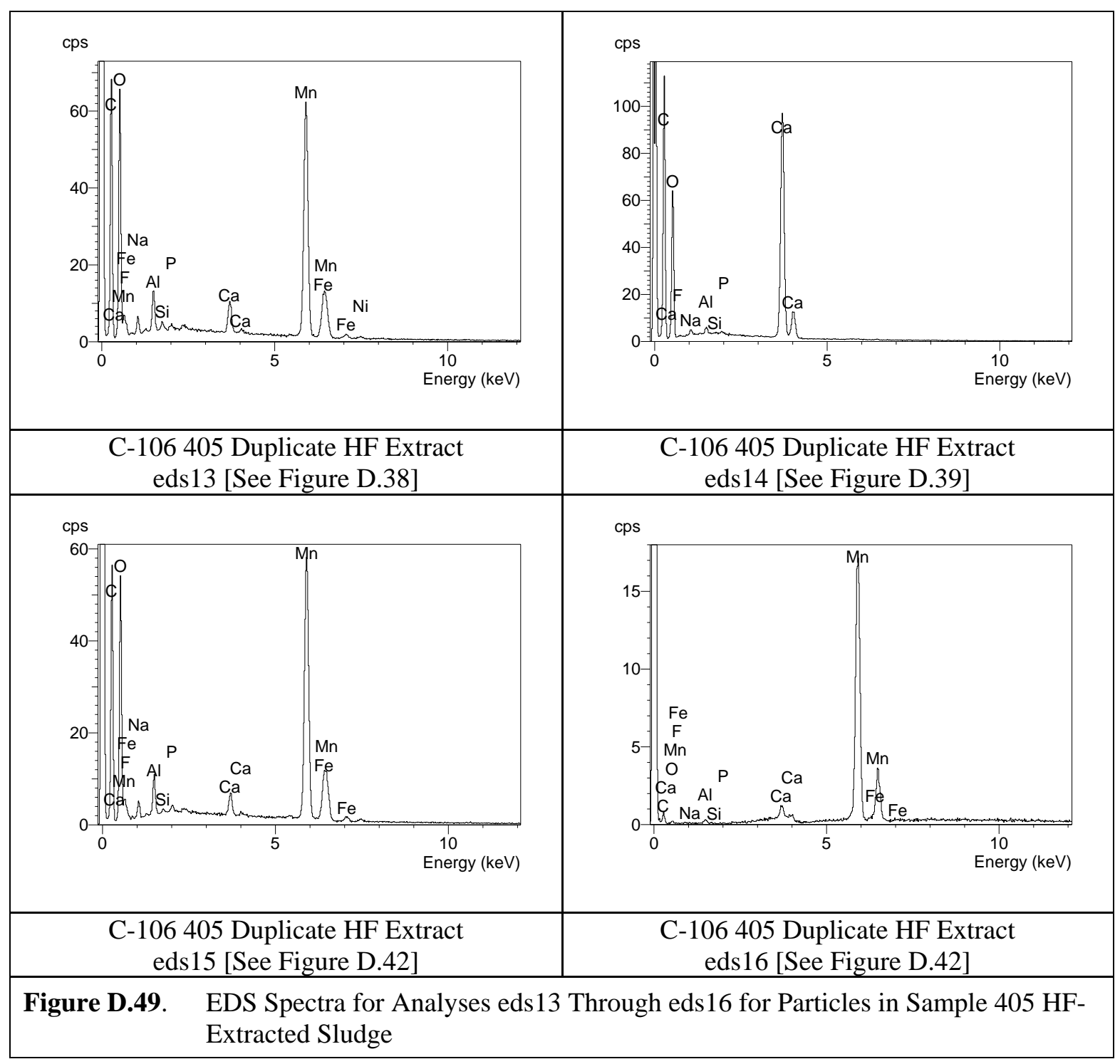




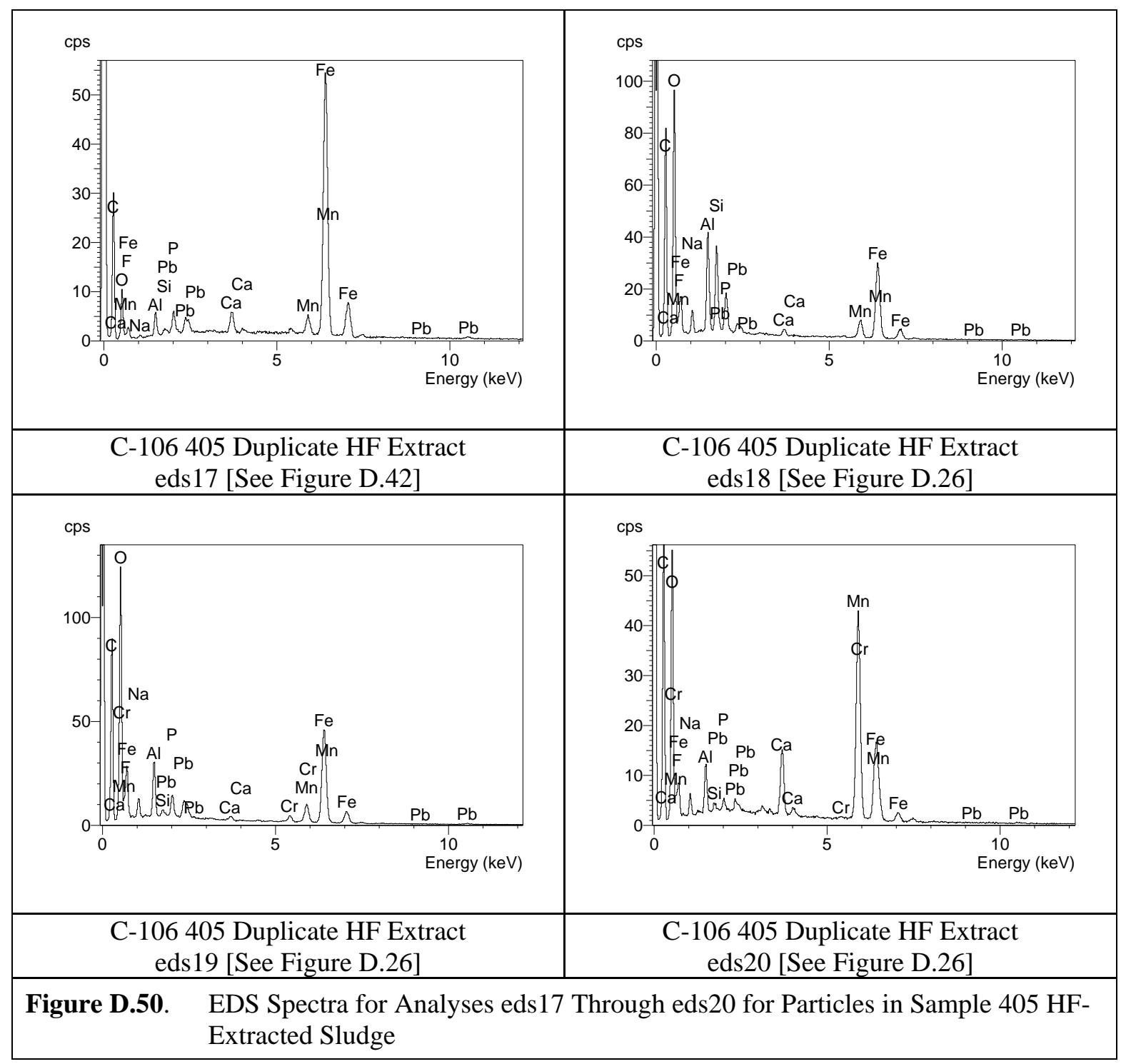




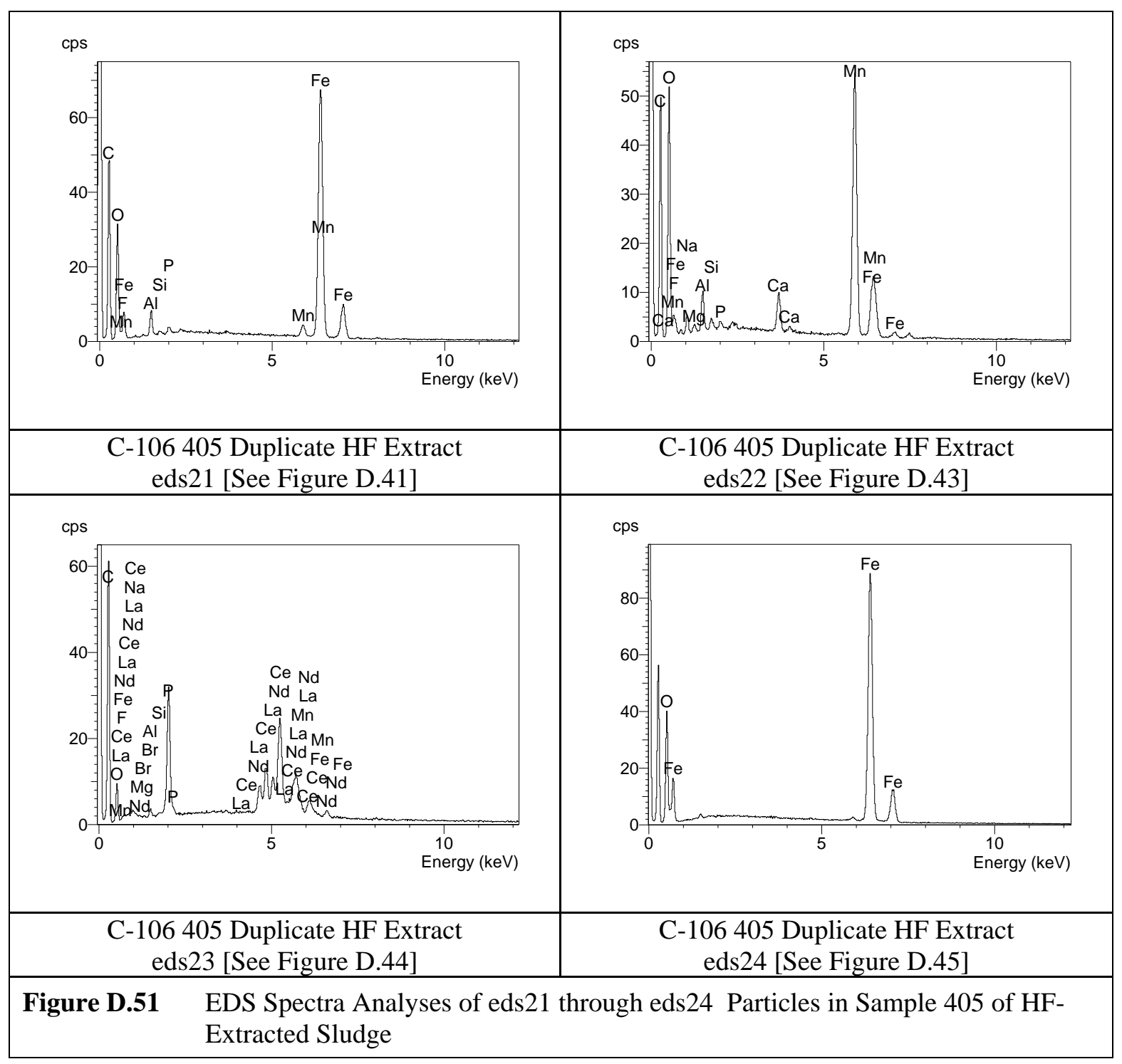

D.32 
Appendix E

SEM/EDS Element Distribution Maps for Samples of Tank C-106 Sludge 


\section{Appendix E}

\section{SEM/EDS Element Distribution Maps for Samples of Tank C-106 Sludge}

This appendix includes the scanning electron microscope (SEM) micrographs and corresponding energy-dispersive X-ray spectrometry (EDS) element distribution maps 9Figures E1 through E.10) for a limited number of imaged areas of SEM mounts of unleached, 82-day water-leached, and hydrofluoric (HF)-extracted sludge samples from tank 241-C-106 (C-106). The operating conditions for the SEM and procedures used for mounting SEM samples are described in Section 2.4 of the main report. After the studies and analyses of tank C-106 sludge had been completed and presented in draft form, the instrument hardware and software for the JEOL JSM-840 SEM used for the SEM/EDS analyses (see micrographs and EDS spectra in Appendices B, C, and D) were upgraded to the INCAEnergy EDS System to automate the collection of EDS spectra over multi-micrometer-sized areas of an SEM-imaged sample. This upgrade permits the mapping of the spatial distributions over user-selected areas of the relative concentrations of any user-specified element detectable by EDS. The installed INCAEnergy EDS System was tested and demonstrated using the sludge samples from tank C-106.

The top of each figure in this appendix shows an SEM micrograph of the area of the sample mount that was scanned by EDS using the INCAEnergy EDS System. Each of the included SEM micrographs was imaged using backscattered electrons (BSE). A series of element distribution maps that show the spatial distributions of the relative concentrations for the indicated elements is included below each micrograph. The concentration of each listed element is directly proportional to the regions of brightness (i.e., brighter the area, the higher the concentration of the selected element) in the corresponding distribution map. 


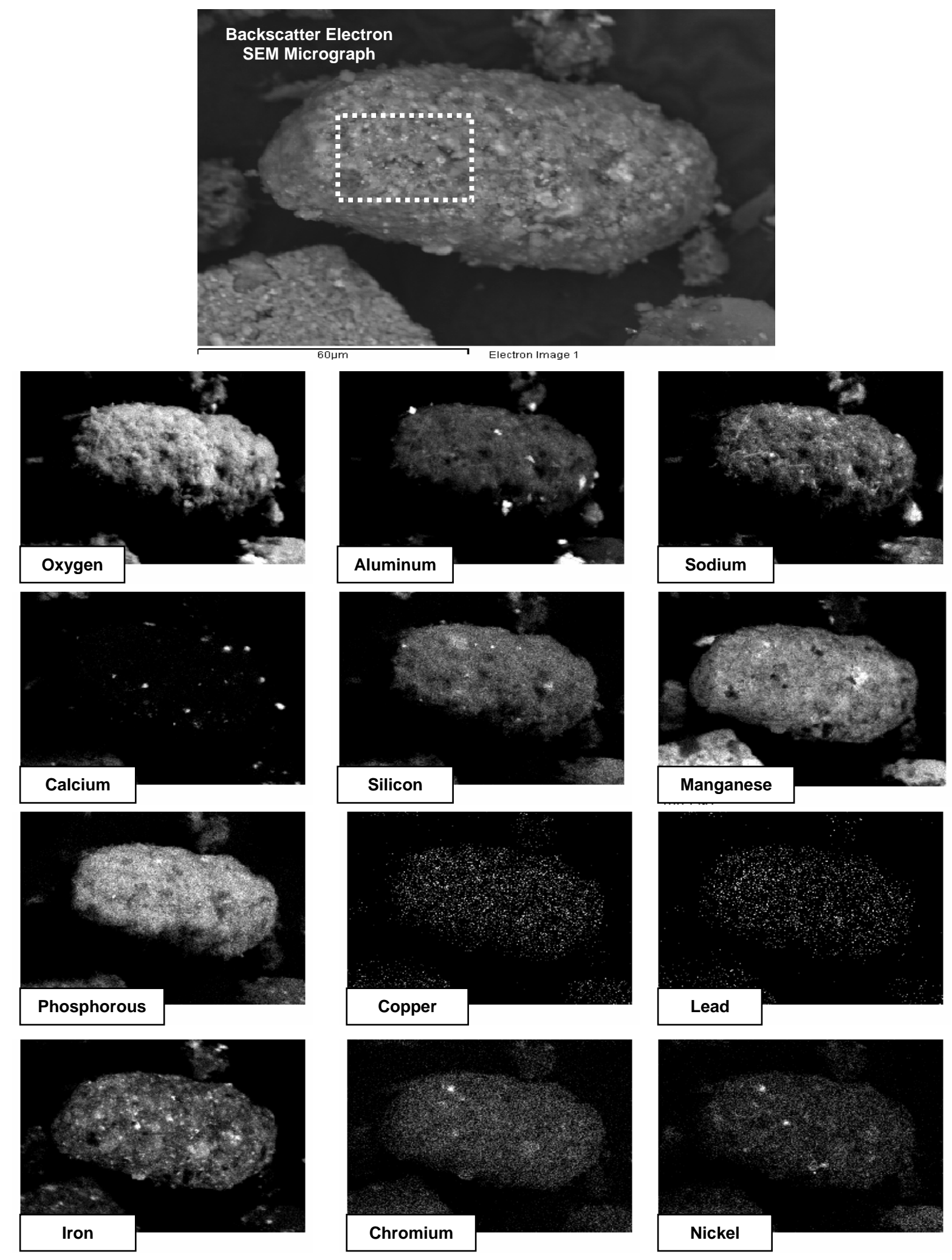

Figure E.1. SEM Micrograph and Element Distribution Maps for Particles in Unleached Sludge from Tank C-106 


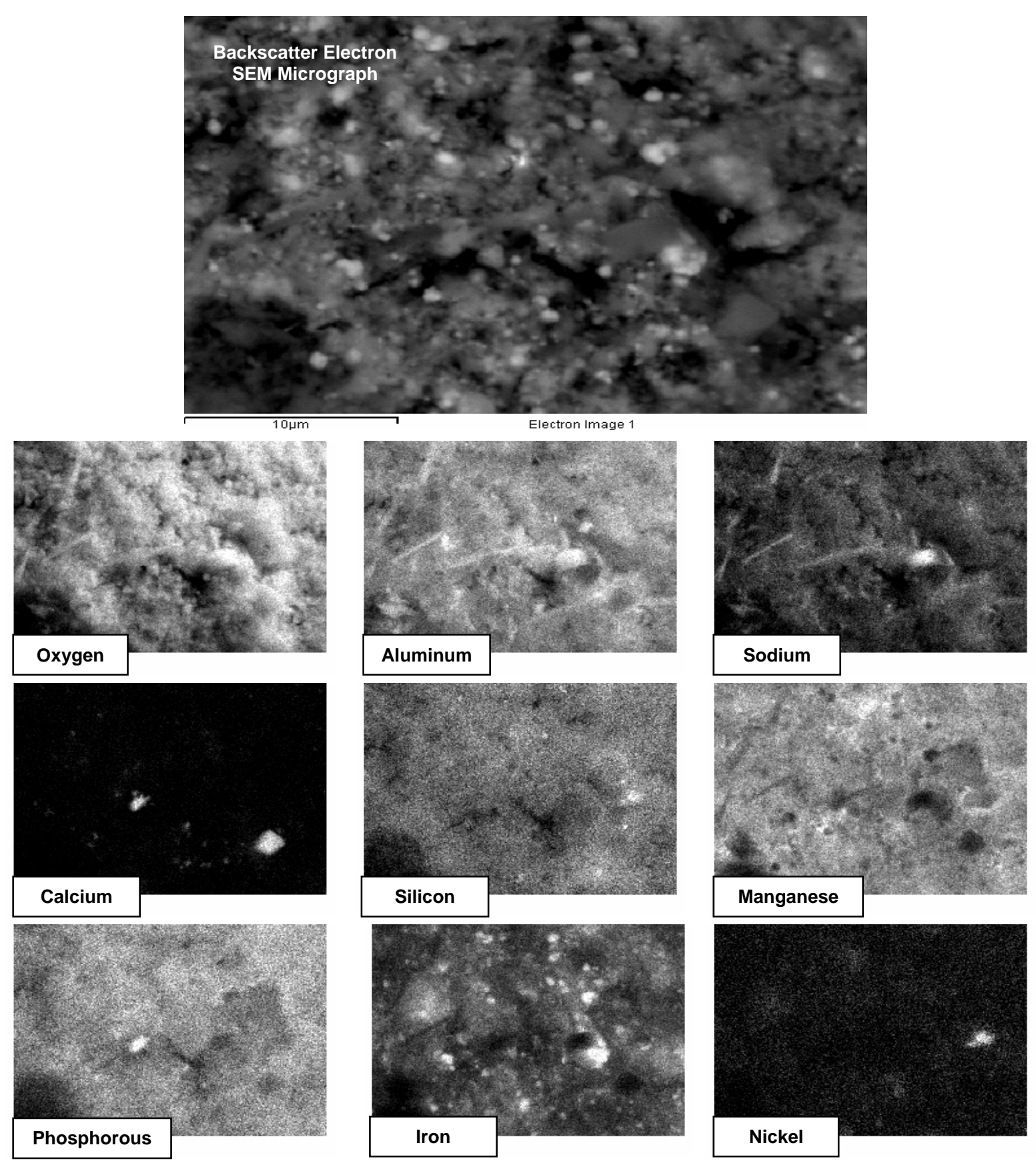

Figure E.2. SEM Micrograph and Element Distribution Maps for the Area of the Particle Indicated by the White Dashed-Line Rectangle in Figure E.1 of Unleached Sludge from Tank C-106 


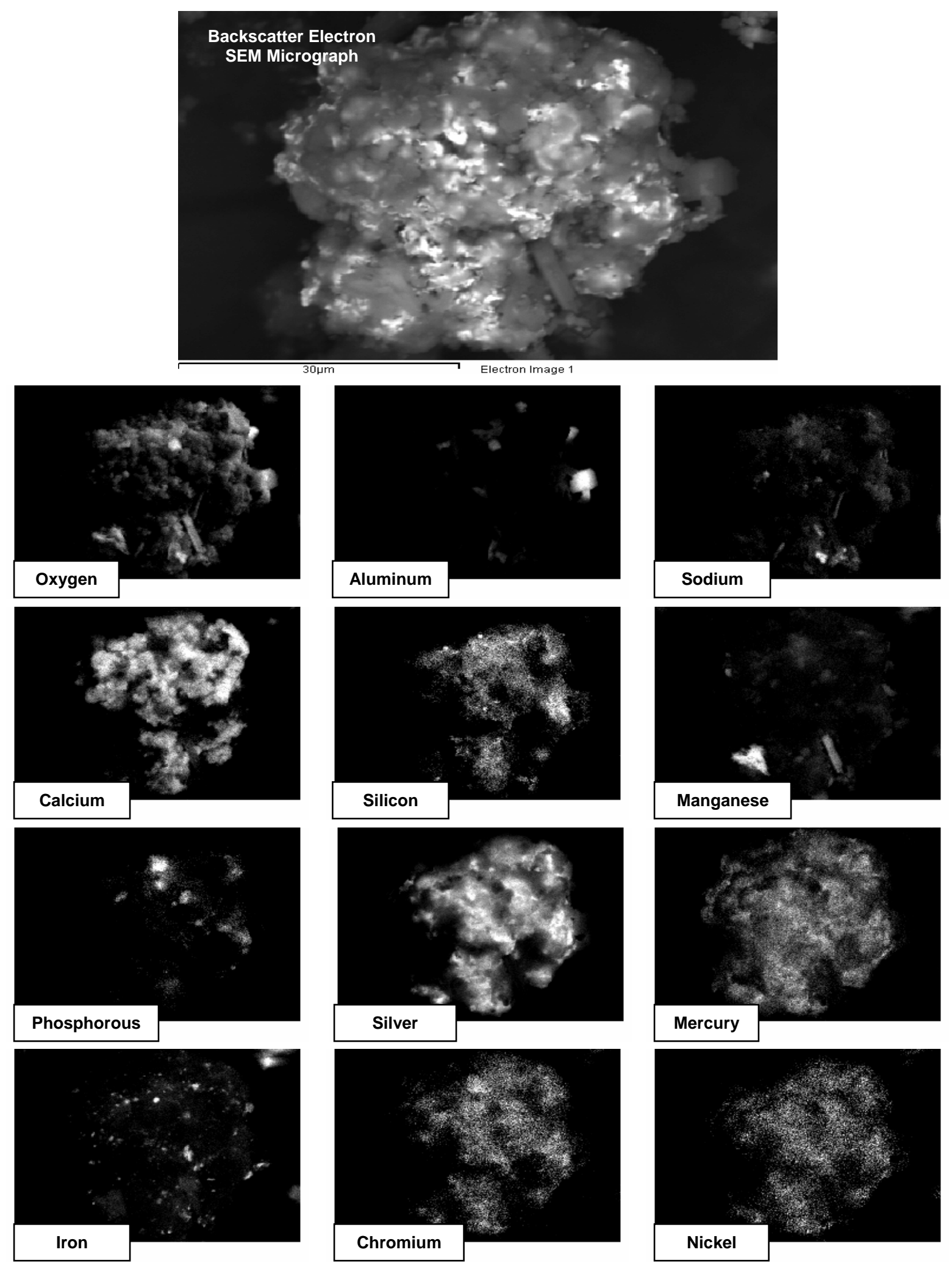

Figure E.3. SEM Micrograph and Element Distribution Maps for a Particle Aggregate in Unleached Sludge from Tank C-106 


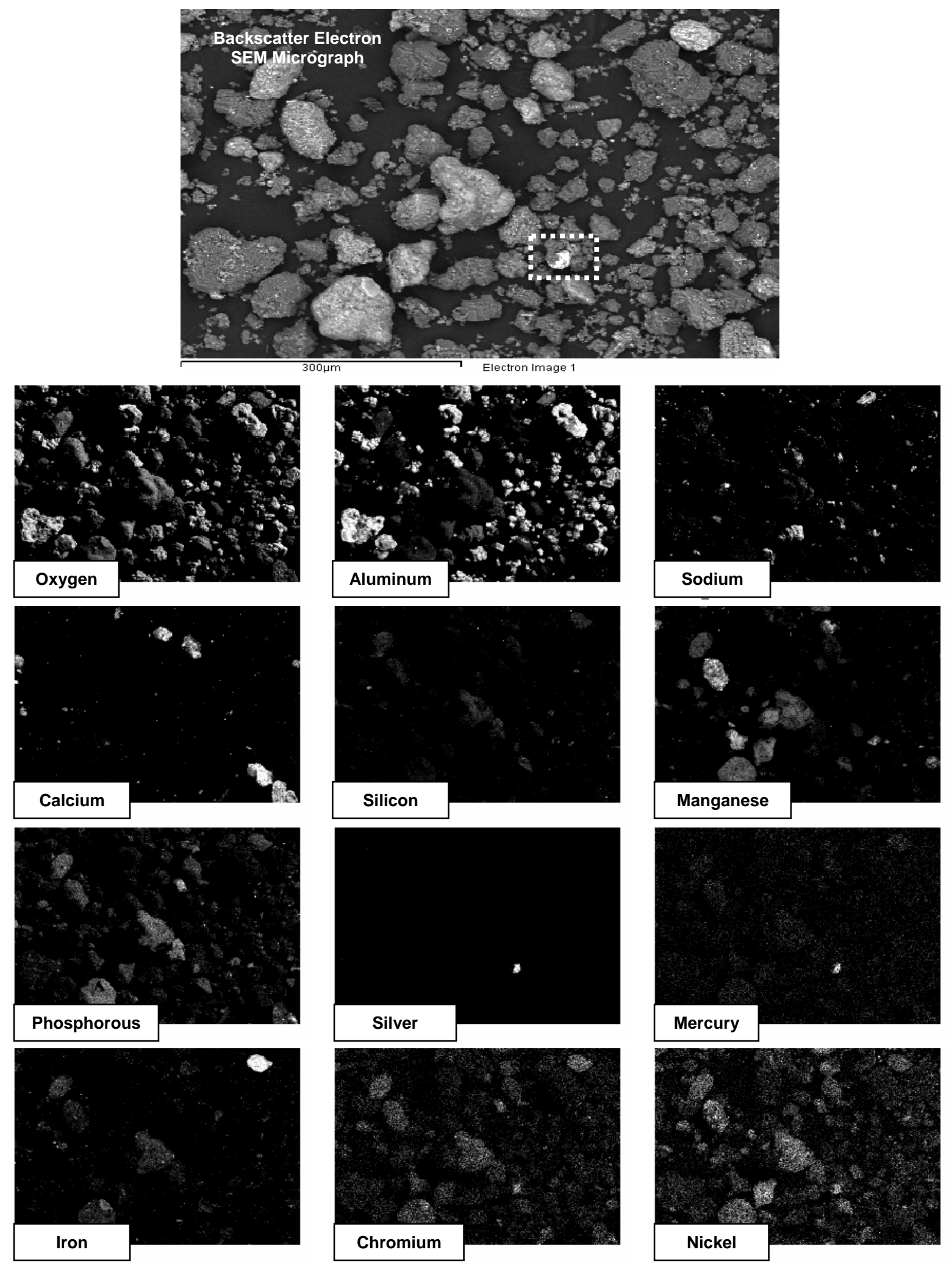

Figure E.4. Low Magnification SEM Micrograph and Element Distribution Maps for Particles in 82-Day Water-Leached Sludge from Tank C-106 


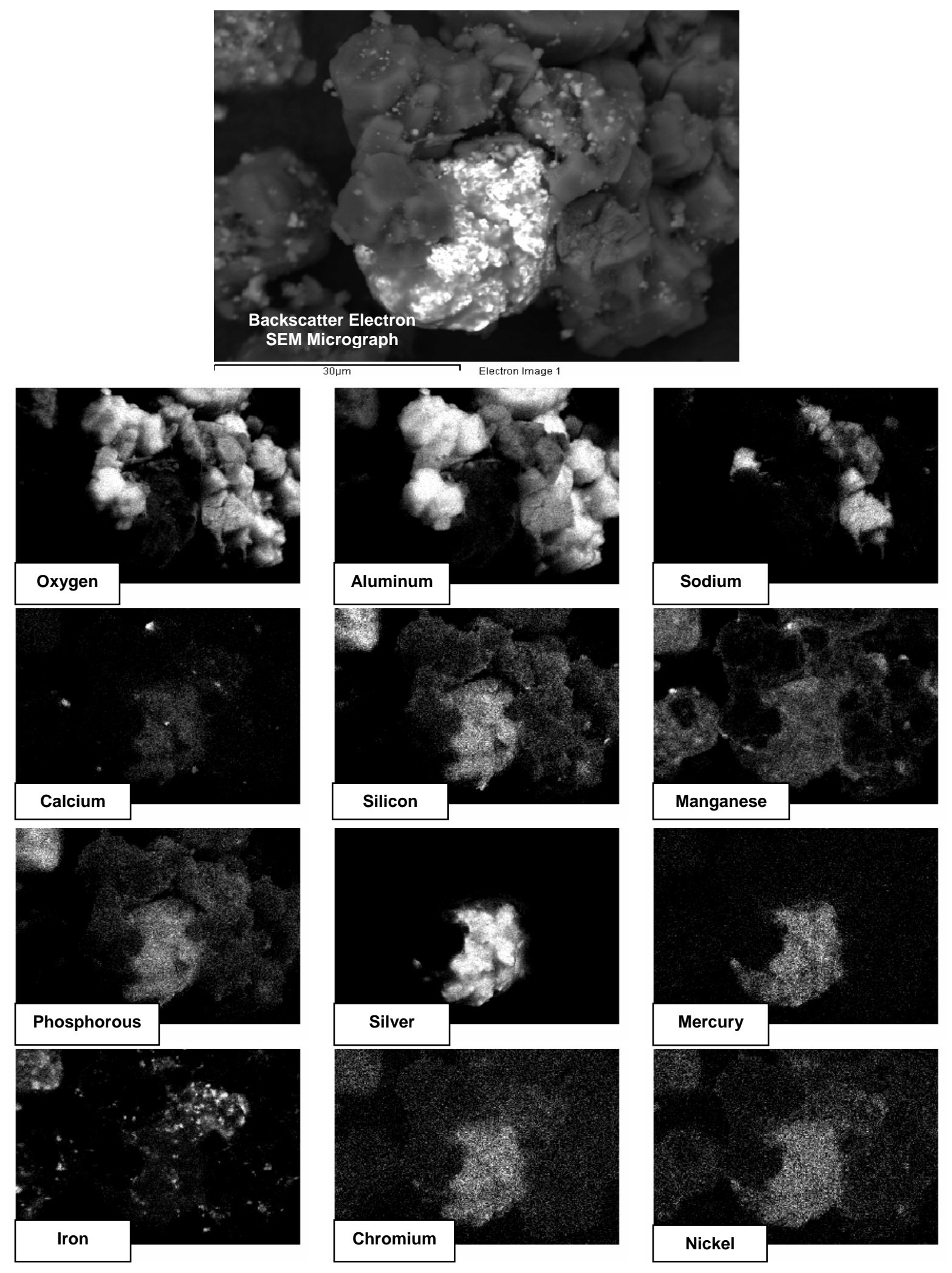

Figure E.5. SEM Micrograph and Element Distribution Maps for Particles in the Area Indicated by the White Dashed-Line Rectangle in Figure E.4 for 82-Day Water-Leached Sludge from Tank C-106 


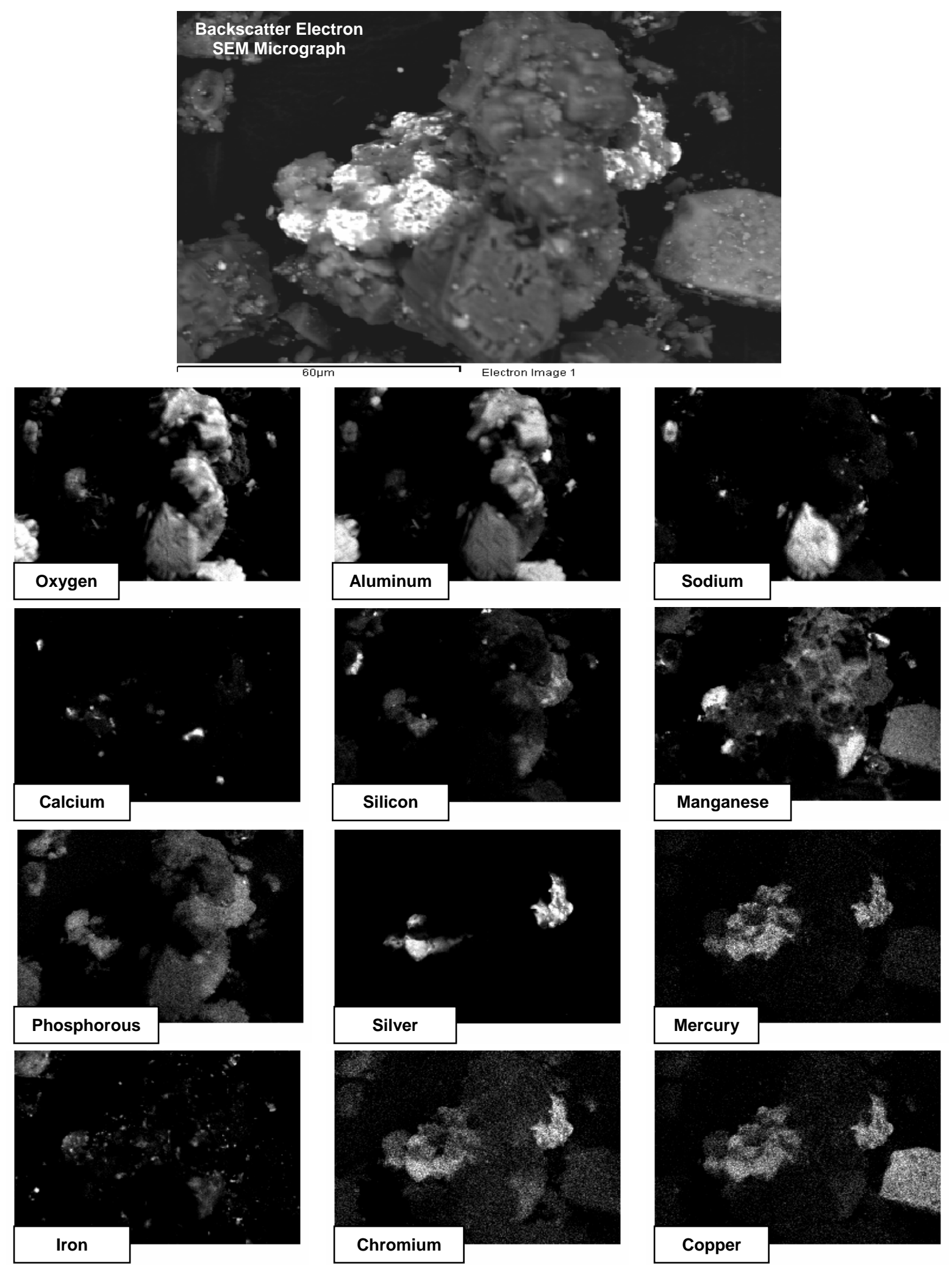

Figure E.6. SEM Micrograph and Element Distribution Maps for a Particle Aggregate in 82-Day WaterLeached Sludge from Tank C-106 


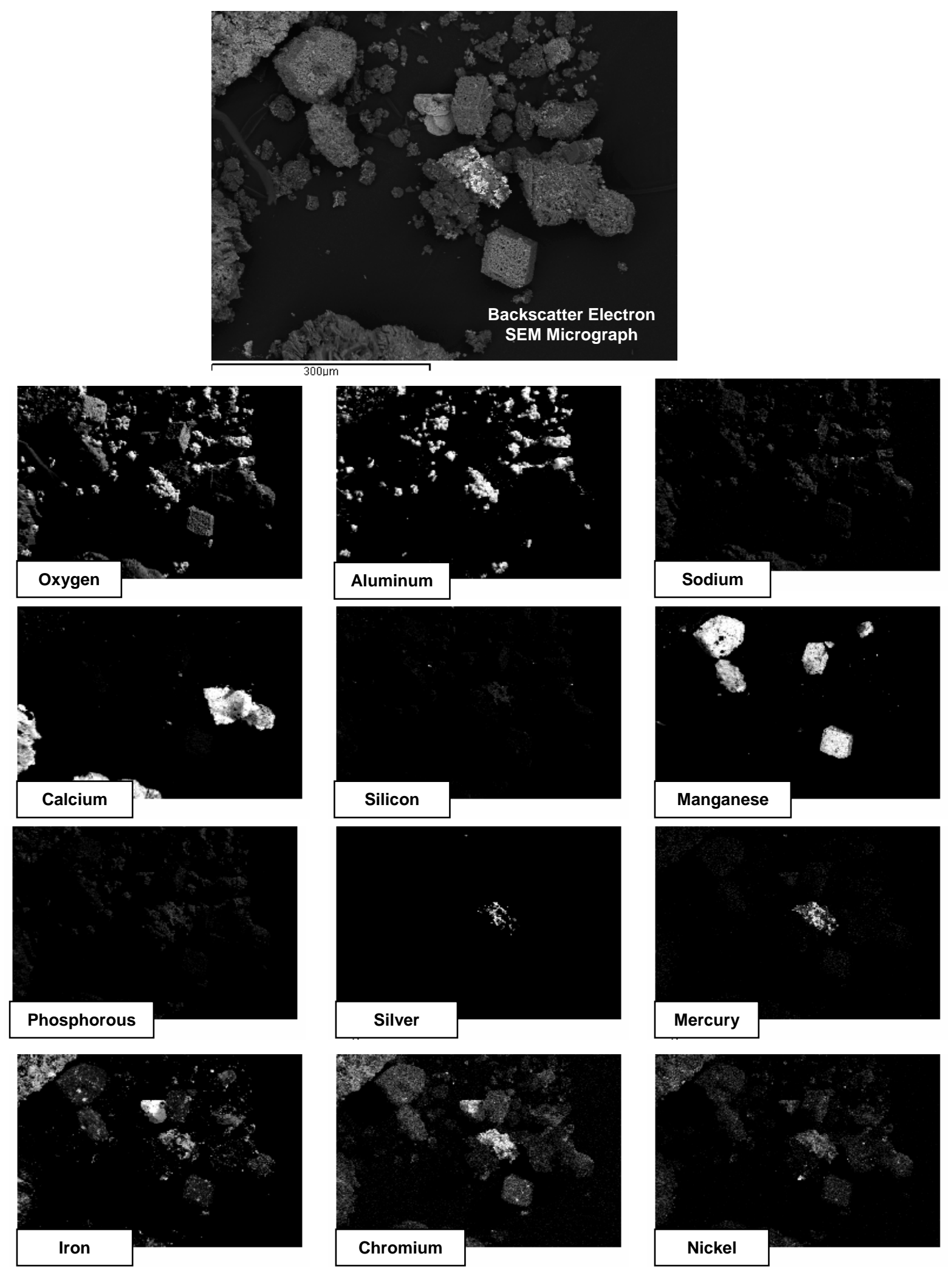

Figure E.7. Low Magnification SEM Micrograph and Element Distribution Maps for Particles in HFExtracted Sludge from Tank C-106 


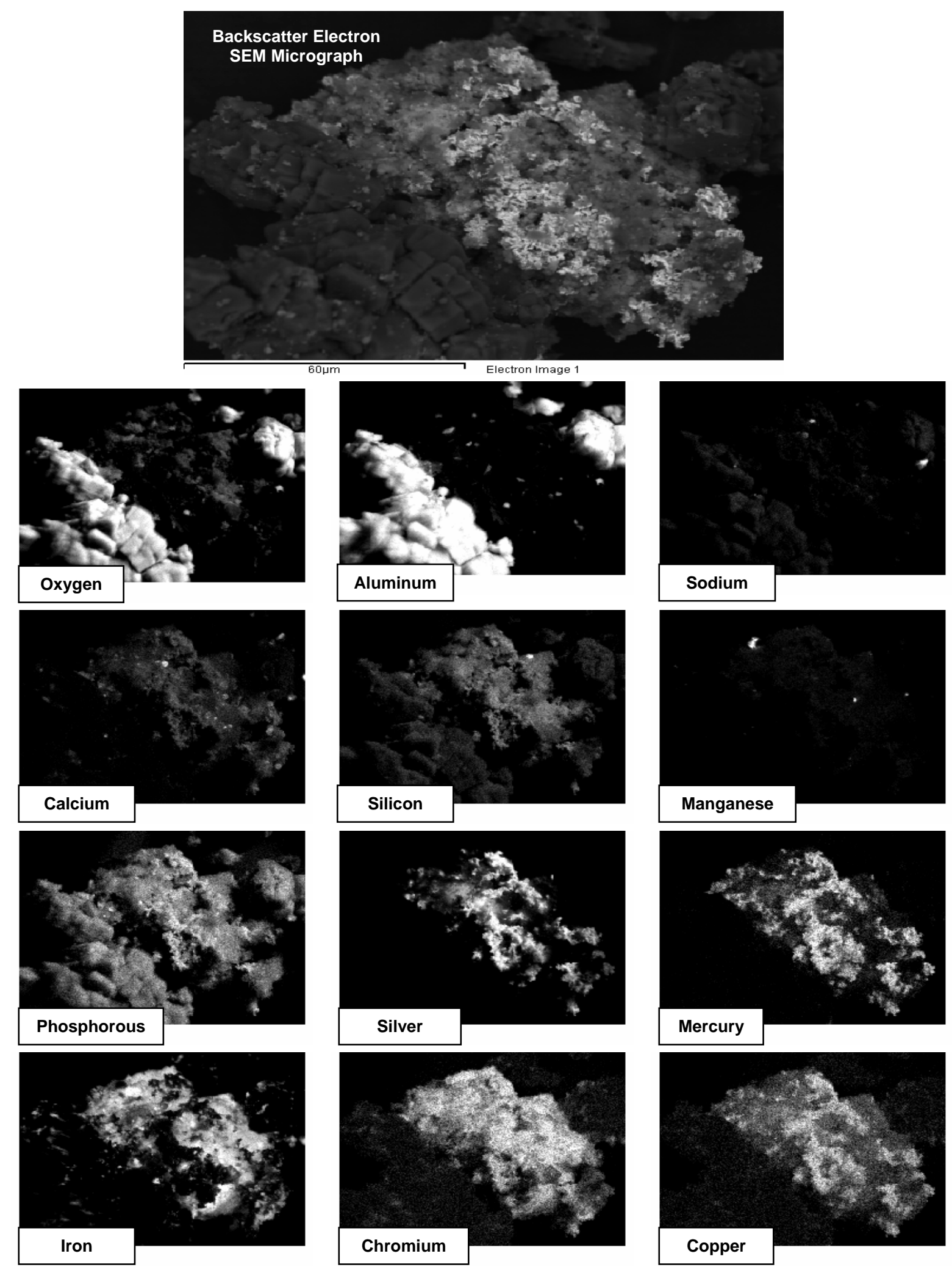

Figure E.8. SEM Micrograph and Element Distribution Maps for a Particle Aggregate in HF-Extracted Sludge from Tank C-106 


\section{Appendix F}

Solution Concentrations of Tank C-106 Residual Liquid and Water Contact Tests with Residual Sludge 


\section{Appendix F}

\section{Solution Concentrations of Tank C-106 Residual Liquid and Water Contact Tests with Residual Sludge}

This appendix provides the data (Tables F.1 through F.5) from water-leaching tests on sludge samples 404 and 405 from single-shell tank 241-C-106 (C-106) at the Hanford Site. These include results from the single-contact and periodic replenishment tests. Dissolved concentrations in extracts from these tests are provided on a per liter of solution basis.

Table F.1 Analysis of the Residual Liquid (Supernatant) Sample

\begin{tabular}{|c|c|c|}
\hline & $\begin{array}{c}403 \text { Liquid } \\
\text { Avg }\end{array}$ & $\begin{array}{c}\text { Sample } \\
\text { Units }\end{array}$ \\
\hline $\mathrm{Al}$ & 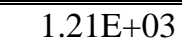 & 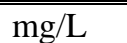 \\
\hline $\mathrm{Ba}$ & $(1.80)$ & $\mathrm{mg} / \mathrm{L}$ \\
\hline $\mathrm{Ca}$ & $(1.30 \mathrm{E}+01)$ & $\mathrm{mg} / \mathrm{L}$ \\
\hline $\mathrm{Fe}$ & $(1.27)$ & $\mathrm{mg} / \mathrm{L}$ \\
\hline $\mathrm{Mg}$ & (5.75E-01) & $\mathrm{mg} / \mathrm{L}$ \\
\hline $\mathrm{Mn}$ & (1.3E-01) & $\mathrm{mg} / \mathrm{L}$ \\
\hline $\mathrm{Ni}$ & 3.48 & $\mathrm{mg} / \mathrm{L}$ \\
\hline $\mathrm{Pb}$ & (1.44) & $\mathrm{mg} / \mathrm{L}$ \\
\hline $\mathrm{Na}$ & $1.02 \mathrm{E}+04$ & $\mathrm{mg} / \mathrm{L}$ \\
\hline Fluoride & $<1.28$ & $\mathrm{mg} / \mathrm{L}$ \\
\hline Chloride & $2.05 \mathrm{E}+01$ & $\mathrm{mg} / \mathrm{L}$ \\
\hline $\mathrm{N}$ as Nitrate & 9.42 & $\mathrm{mg} / \mathrm{L}$ \\
\hline Carbonate & $6.55 E+03$ & $\mathrm{mg} / \mathrm{L}$ \\
\hline Sulfate & $2.76 \mathrm{E}+01$ & $\mathrm{mg} / \mathrm{L}$ \\
\hline Oxalate & $1.34 \mathrm{E}+03$ & $\mathrm{mg} / \mathrm{L}$ \\
\hline P as phosphate & $7.49 \mathrm{E}+01$ & $\mathrm{mg} / \mathrm{L}$ \\
\hline${ }^{90} \mathrm{Sr}$ & $7.23 \mathrm{E}-02$ & $\mu \mathrm{Ci} / \mathrm{L}$ \\
\hline${ }^{239} \mathrm{Pu}$ & $(4.7 \mathrm{E}-02)$ & $\mu \mathrm{Ci} / \mathrm{L}$ \\
\hline${ }^{237} \mathrm{~Np}$ & $<5.00 \mathrm{E}-02$ & $\mu \mathrm{Ci} / \mathrm{L}$ \\
\hline${ }^{241} \mathrm{Am}$ & $<5.00 \mathrm{E}-02$ & $\mu \mathrm{Ci} / \mathrm{L}$ \\
\hline${ }^{129} \mathrm{I}$ & $1.64 \mathrm{E}-04$ & $\mu \mathrm{Ci} / \mathrm{L}$ \\
\hline${ }^{60} \mathrm{CO}$ & $<1.40 \mathrm{E}-01$ & $\mu \mathrm{Ci} / \mathrm{L}$ \\
\hline${ }^{137} \mathrm{CS}$ & $4.74 \mathrm{E}+02$ & $\mu \mathrm{Ci} / \mathrm{L}$ \\
\hline TIC & $2.80 \mathrm{E}+02$ & $\mathrm{mg} \mathrm{C} / \mathrm{L}$ \\
\hline TOC & $3.35 \mathrm{E}+02$ & $\mathrm{mg} \mathrm{C} / \mathrm{L}$ \\
\hline \multicolumn{3}{|c|}{$\begin{array}{l}\text { Avg = Average } . \\
\text { TIC = Total inorganic carbon. } \\
\text { TOC = Total organic carbon. }\end{array}$} \\
\hline
\end{tabular}


Table F.2. Solution Concentrations for Water-Leach Tests of Sample 404

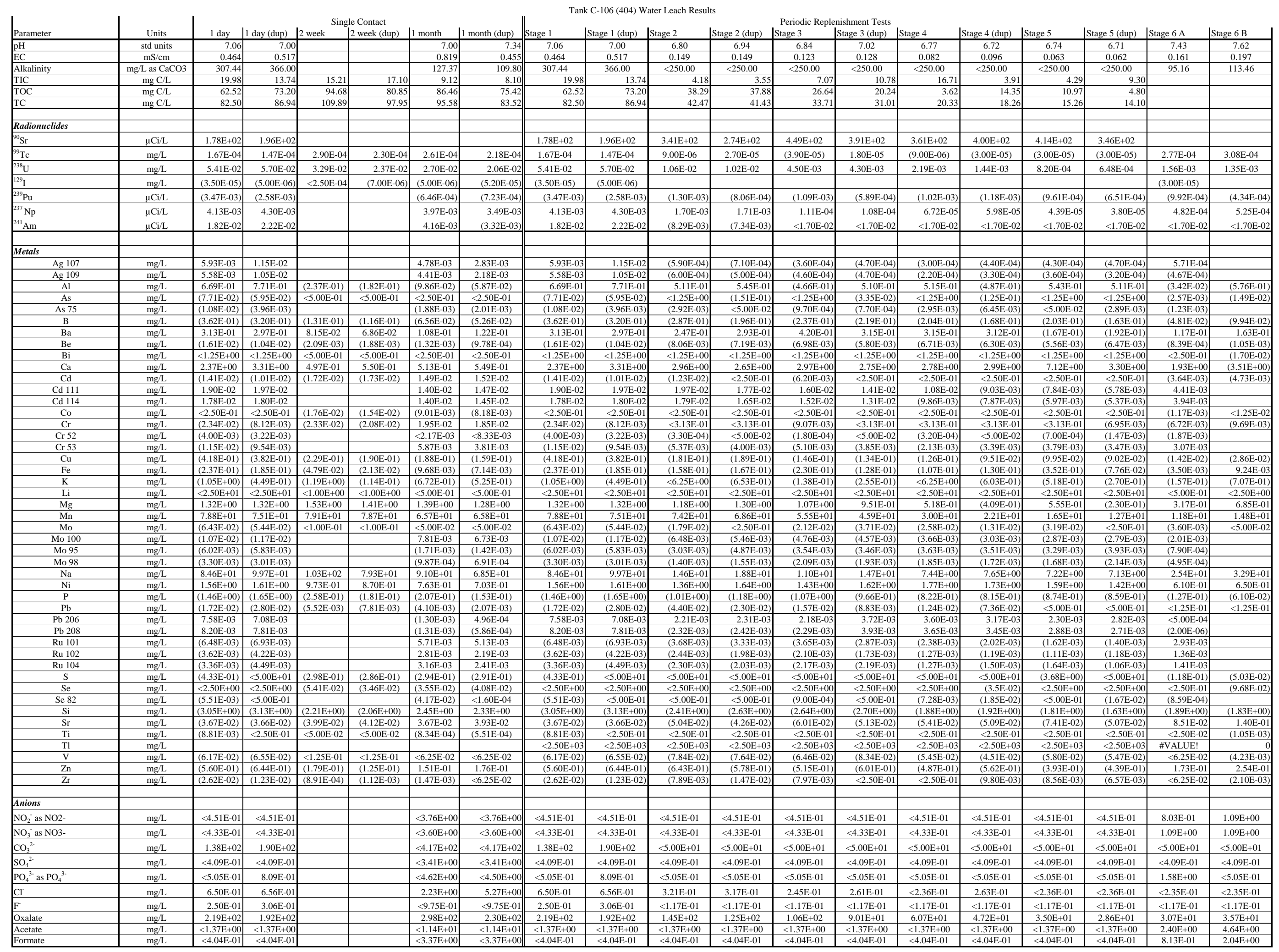


Table F.3. Molar Concentrations for Water-Leach Tests of Sample 404

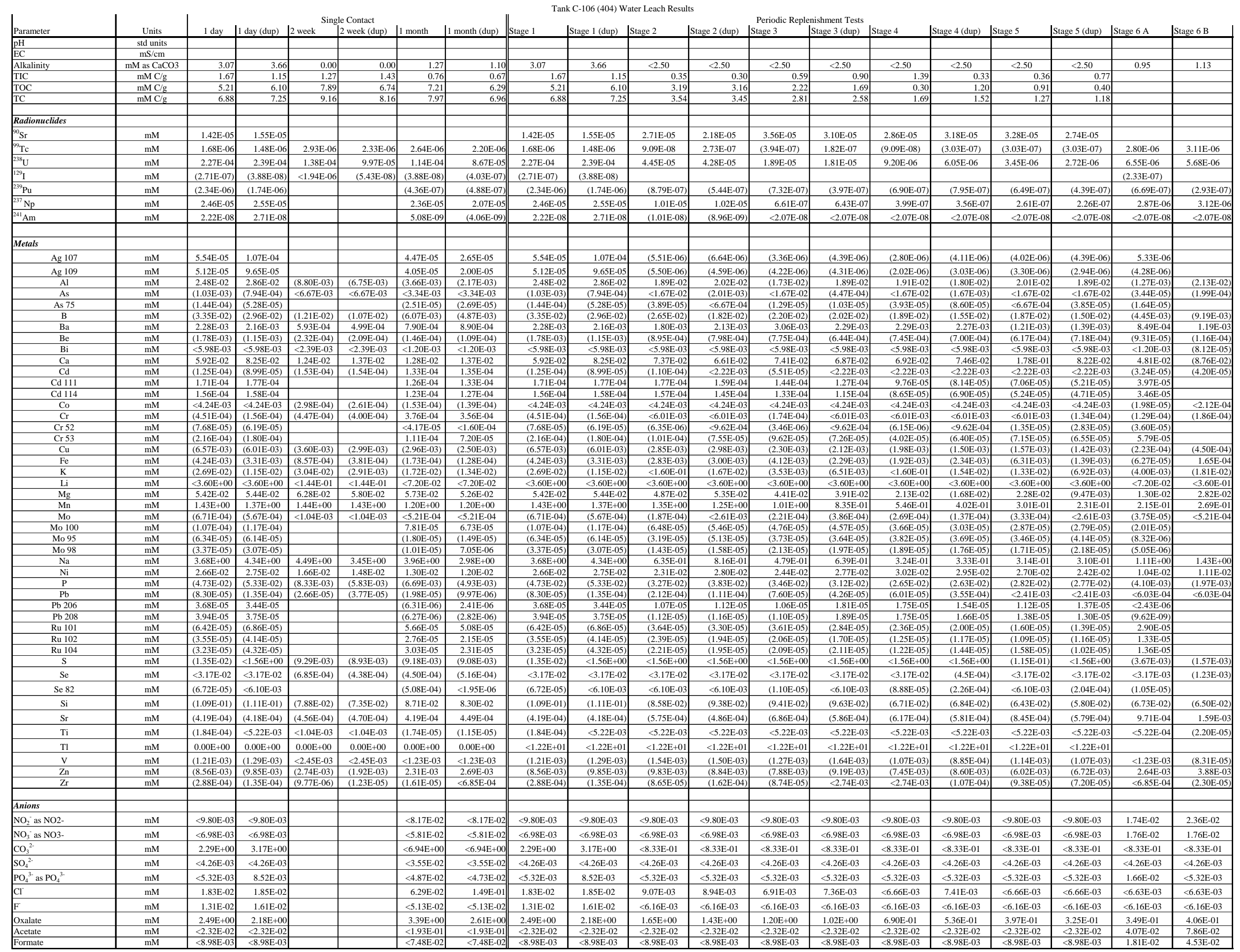


Table F.4. Solution Concentrations for Water-Leach Tests of Sample 405

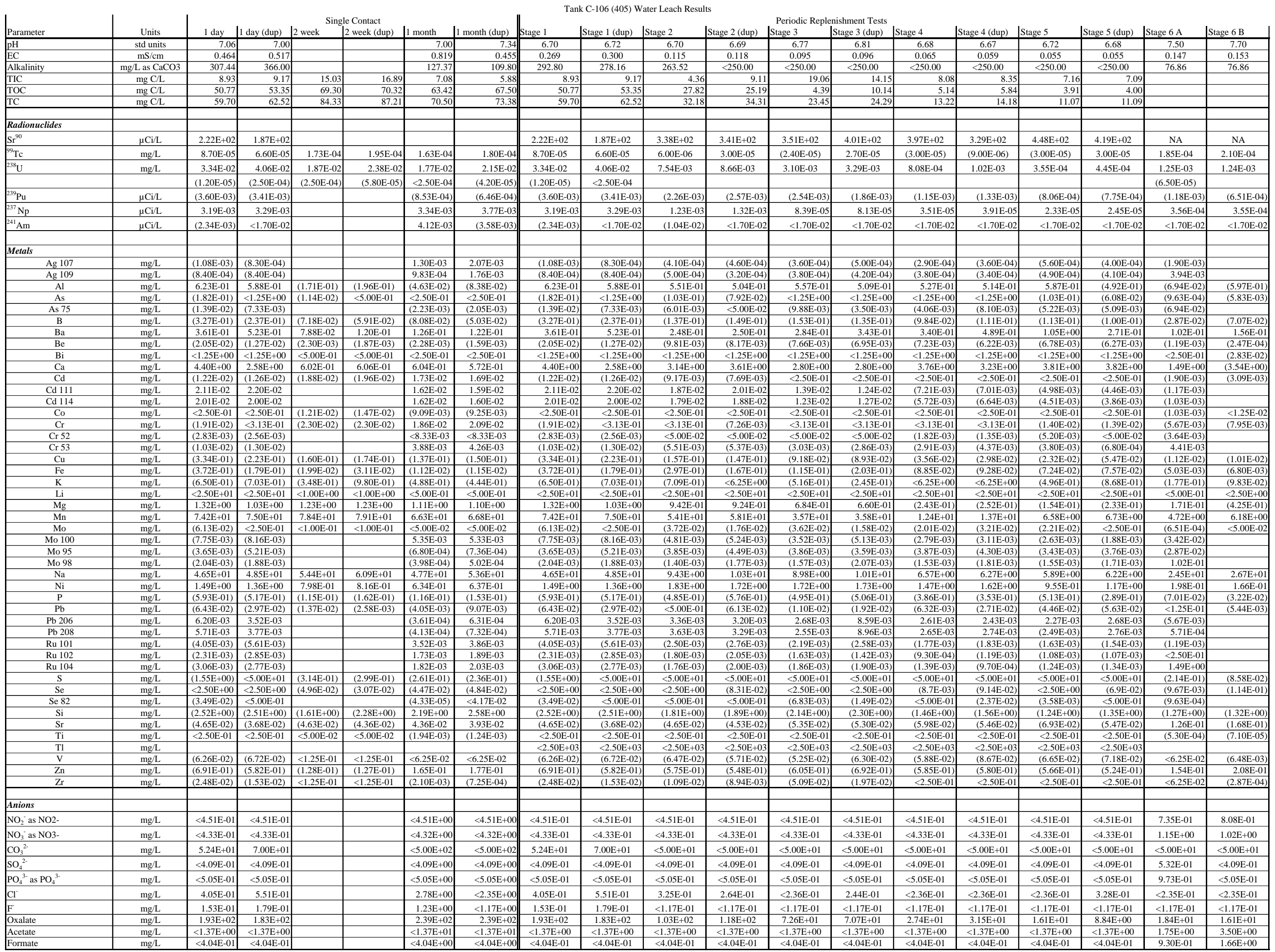


Table F.5. Molar Concentrations for Water-Leach Tests of Sample 405

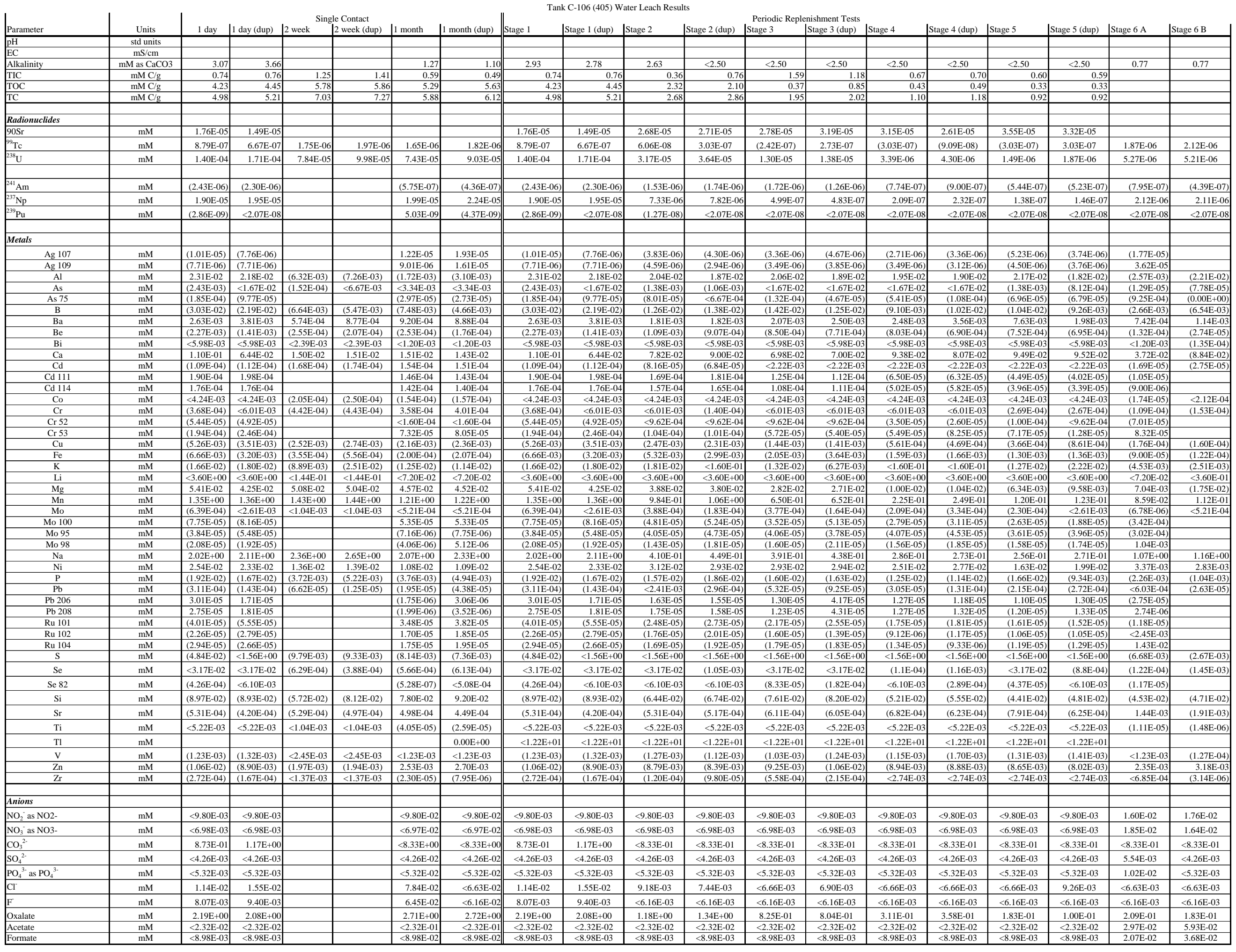




\section{Appendix G}

Chemical Equilibrium Modeling Calculations 


\section{Appendix G}

\section{Chemical Equilibrium Modeling Calculations}

Chemical equilibrium calculations were made to estimate the saturation condition of the 1-day waterleach solution (sample 404) with respect to phases expected to be present in the sludge. MINEQL+ Version 4.0 for Windows (Schecher and McAvoy 1998) was used to make these calculations. The thermodynamic database used in MINEQL + derives much of its data from the U.S. Environmental Protection Agency's MINTEQA2 database, which originally came from the U.S. Geological Survey WATEQ4F program. Additional thermodynamic data for components not included in MINTEQA2 database were taken from the original MINEQL program. Formation constants for manganese oxalate $\left(\mathrm{MnC}_{2} \mathrm{O}_{4} \cdot 2 \mathrm{H}_{2} \mathrm{O}\right)$ and dawsonite are not in the MINEQL+ database and were added manually. The formation constant (the inverse of the solubility constant) for $\mathrm{MnC}_{2} \mathrm{O}_{4} \cdot 2 \mathrm{H}_{2} \mathrm{O}$ at $298.2 \mathrm{~K}\left(25^{\circ} \mathrm{C}\right)$ was calculated from thermodynamic data available in the National Bureau of Standards (NBS) Tables of Chemical Thermodynamic Properties (Wagman et al. 1982). The formation constant used for dawsonite at $298.2 \mathrm{~K}\left(25^{\circ} \mathrm{C}\right)$ was reported by Ferrante et al. (1976) and accepted in the compilation of thermodynamic values published by Robie and Hemingway (1995). The input concentrations and conditions used in most of the calculations discussed in the text are shown in Table G.1.

Table G.1. Initial Concentrations and Conditions Used for Most Equilibrium Modeling Calculations

\begin{tabular}{|c|c|}
\hline $\begin{array}{l}\text { Component or } \\
\text { Parameter }\end{array}$ & $\begin{array}{c}\text { Concentration }(\mathrm{mol} / \mathrm{L}) \\
\text { or Condition }\end{array}$ \\
\hline$\overline{\mathrm{pH}}$ & 7.03 \\
\hline pe & 6.76 \\
\hline Ionic Strength & 0.02 (fixed) \\
\hline Precipitation & Not allowed \\
\hline $\begin{array}{l}\text { Alkalinity } \\
\text { (total } \mathrm{CO}_{2} \text { ) }\end{array}$ & $\begin{array}{l}6.73 \times 10^{-3}(\mathrm{eq} / \mathrm{L}) \\
\left(8.13 \times 10^{-3}\right) \\
\end{array}$ \\
\hline $\mathrm{Na}^{+}$ & $4.0 \times 10^{-3}$ \\
\hline Oxalate $\left(\mathrm{C}_{2} \mathrm{O}_{4}{ }^{2-}\right)$ & $2.3 \times 10^{-3}$ \\
\hline $\mathrm{Mn}^{2+}$ & $1.4 \times 10^{-3}$ \\
\hline $\mathrm{Ca}^{2+}$ & $7.1 \times 10^{-5}$ \\
\hline $\mathrm{Mg}^{2+}$ & $5.4 \times 10^{-5}$ \\
\hline $\mathrm{Al}^{3+}$ & $2.7 \times 10^{-5}$ \\
\hline
\end{tabular}

The calculated saturation index (SI), for relevant minerals are shown in Table G.2. SI $=\mathrm{Log}\left(\mathrm{Q} / \mathrm{K}_{\mathrm{sp}}\right)$, $\mathrm{Q}$ is the ion product for the solid, and $\mathrm{K}_{\mathrm{sp}}$ is the solubility constant for the mineral. 
Table G.2. Calculated Saturation Indices of Relevant Minerals for Water-Leached Sample 404

\begin{tabular}{||c|c||}
\hline Mineral & SI \\
\hline \hline $\begin{array}{c}\text { Dawsonite } \\
{\left[\mathrm{AlNaCO}_{3}(\mathrm{OH})_{2}\right]}\end{array}$ & -0.17 \\
\hline $\begin{array}{c}\mathrm{Mn} \text { oxalate } \\
{\left[\mathrm{MnC}_{2} \mathrm{O}_{4} \cdot 2 \mathrm{H}_{2} \mathrm{O}\right]}\end{array}$ & 0.43 \\
\hline $\begin{array}{c}\text { whewellite } \\
{\left[\mathrm{CaC}_{2} \mathrm{O}_{4} \cdot \mathrm{H}_{2} \mathrm{O}\right]}\end{array}$ & 0.12 \\
\hline $\begin{array}{c}\mathrm{Gibbsite} \\
{\left[\mathrm{Al}(\mathrm{OH})_{3}\right]}\end{array}$ & 0.09 \\
\hline $\begin{array}{c}\text { Rhodochrosite } \\
{\left[\mathrm{MnCO}{ }_{3}\right]}\end{array}$ & 1.41 \\
\hline $\left.\begin{array}{c}\text { Calcite } \\
{[\mathrm{CaCO}}\end{array}\right]$ & -2.17 \\
\hline $\begin{array}{c}\text { Manganite } \\
{[\mathrm{MnOOH}]}\end{array}$ & -0.83 \\
\hline $\begin{array}{c}\text { Hausmannite } \\
{\left[\mathrm{Mn}_{3} \mathrm{O}_{4}\right]}\end{array}$ & -2.07 \\
\hline $\begin{array}{c}\text { Pyrolusite } \\
{\left[\mathrm{MnO}_{2}\right]}\end{array}$ & -3.08 \\
\hline SI = standard & \\
\hline
\end{tabular}

Ferrante MJ, JM Stuve, and DW Richardson. 1976. Thermodynamic Data for Synthetic Dawsonite. U.S. Bureau of Mines Report Investigation 8129, U.S. Bureau of Mines, Washington, D.C.

Robie RA and BS Hemingway. 1995. Thermodynamic Properties of Minerals and Related Substances at $298.15 \mathrm{~K}$ and 1 bar (10 5 Pascals) Pressure and at Higher Temperatures. U.S. Geological Survey Bulletin 2131, U.S. Geological Survey, Washington, D.C.

Schecher WD and DC McAvoy. 1998. MINEQL+, A Chemical Equilibrium Modeling System, Version 4.0 for Windows, A User's Manual. Environmental Research Software, Hallowell, Maine.

Wagman DD, WH Evans, VB Parker, RH Schumm, I Halow, SM Bailey, KL Cherney, and RL Nuttall. 1982. "NBS Tables of Chemical Thermodynamic Properties - Selected Values for Inorganic and C-1 and C-2 Organic-Substances in SI Units.” J. Phys. Chem. Ref. Data 11: 1-\& Suppl. 2. 


\section{Distribution*}

No. of

$\underline{\text { Copies }}$

\section{OFFSITE}

Steve Airhart

Freestone Environmental Services

1933 Jadwin Ave.

Richland, WA 99354

Dr. Harry Babad

2540 Cordoba Court

Richland, WA 99352-1609

Pat Brady

Geochemistry Department, 6118

Sandia National Laboratories

P.O. Box 5800

Albuquerque, NM 87185-0750

Charles R. Bryan

Sandia National Laboratories

4100 National Parks Highway

Carlsbad, NM 88220

Susan Carroll

Lawrence Livermore National Laboratory

MS L-219

Livermore, CA 94550

Jon Chorover

Associate Professor - Environmental

Chemistry

Department of Soil, Water, and

Environmental Science

Shantz 429, Building \#38

University of Arizona

Tucson, AZ 85721-0038
No. of

$\underline{\text { Copies }}$

Dave G. Coles

Coles Environmental Consulting

750 South Rosemont Road

West Linn, OR 97068

Mark Conrad

Department of Earth and Planetary Sciences

University of California, Berkeley

Berkeley, CA 94720

Dr. James A. Davis

U.S. Geological Survey

MS 465

345 Middlefield Road

Menlo Park, CA 94025

Donald J. DePaolo

Geology \& Geophysics Dept. MC4767

University of California

Berkeley, CA 94720-4767

Dirk A. Dunning

Oregon Office of Energy

625 Marion Street, N.E.

Salem, OR 97301-3742

Mark Ewanic

MSE Technology Applications, Inc.

200 Technology Way

Butte, MT 59701

Markus Flury

Department of Crop and Soil Sciences

Washington State University

Pullman, WA 99164

*Distribution of this report is made by electronic media via http://www.osti.gov/bridge/. 
No. of

Copies

Amy P. Gamerdinger

2122 E. Hawthorne

Tucson, AZ 85719

Jim Harsh

Department of Crop \& Soil Sciences

Washington State University

Johnson Hall, Room 249

Pullman, WA 99164-6420

Dr. Cliff Johnston

Soil Chemistry and Mineralogy

1150 Lily Hall

Purdue University

West Lafayette, IN 47907-1150

Dr. Daniel I. Kaplan

Westinghouse Savannah River Company

Building 774-43A, Room 215

Aiken, SC 29808

Dr. Jim Krumhansl

Sandia National Laboratory

P.O. Box 5800

Albuquerque, NM 87185-0750

Dr. Christine Langston

Westinghouse Savannah River Co.

Building 774-43A

Aiken, SC 29808

Dr. Peter C. Lichtner

Los Alamos National Laboratory

P.O. Box 1663

Los Alamos, NM 87545

Sandra Lilligren

Nez Perce

P.O. Box 365

Lapwai, ID 83540
No. of

Copies

Kate Maher

The Center for Isotope Geochemistry

301 McCone Hall

University of California, Berkeley

Berkeley, CA 94702-4746

Melaine A. Mayes

Environmental Sciences Division

Oak Ridge National Laboratory

P.O. Box 2008

[Bethel Valley Road for FedEX/UPS

delivery]

Oak Ridge, TN 37831-6038

Dr. Kathryn L. Nagy

Department of Earth and Environmental Sciences

University of Illinois at Chicago (MC-186)

845 West Taylor Street

Chicago, IL 60607-7059

Heino Nitsche

Director, Center for Advanced

Environmental and Nuclear Studies

Lawrence Berkeley National Laboratory

1 Cyclotron Road

MS 70A-1150

Berkeley, CA 94720

Phil Reed

U.S. Nuclear Regulatory Commission

Office of Nuclear Regulatory Research

Division of Systems Analysis and

Regulatory Effectiveness

Radiation Protection, Env. Risk and Waste

Management Branch

MS T9-F31

Washington, D.C. 20555-0001

*Distribution of this report is made by electronic media via http://www.osti.gov/bridge/. 
PNNL-15187

Rev. 1

No. of

$\underline{\text { Copies }}$

Richard J. Reeder

Department of Geosciences

State University of New York at Stony

Brook

Stony Brook, NY 11794-2100

Al Robinson

68705, E 715 PRNE

Richland, WA 99352

Phil Rogers

13 Mountain Oak

Littleton, CO 80127

Dr. Sherry Samson

Dept. EES

University of Illinois at Chicago (MC-186)

845 West Taylor St.

Chicago, IL 60607-7059

David Shafer

Desert Research Institute

University of Nevada

P.O. Box 19040

Las Vegas, NV 89132-0040

Dawn A. Shaughnessy

Glen T. Seaborg Center

Lawrence Berkeley National Laboratory

1 Cyclotron Road

MS 70A-1150

Berkeley, CA 94720

Doug Sherwood

Rivers Edge Environmental

1616 Riverside Drive

West Richland, WA 99353
No. of

Copies

David K. Shuh

Lawrence Berkeley National Laboratory

1 Cyclotron Road

MS 70A-1150

Berkeley, CA 94720

James “Buck" Sisson

Idaho National Engineering and

Environmental Laboratory

P.O. Box 1625, MS 2107

Idaho Falls, ID 83415-2107

Carl I. Steefel

Lawrence Livermore National Laboratory

Earth \& Environmental Sciences Directorate

MS L-204

P.O. Box 808

Livermore, CA 94551-9900

Dr. Samuel J. Traina, Director

Sierra Nevada Research Institute

University of California, Merced

P.O. Box 2039

Merced, CA 95344

Dan Tyler

Freestone Environmental Services

1933 Jadwin Ave.

Richland, WA 99354

Dr. T. T. Chuck Vandergraaf

Atomic Energy of Canada, Limited

Whiteshell Nuclear Research Establishment

Pinawa, Manitoba ROE 1LO

Canada

Dr. Jiamin Wan

Lawrence Berkeley National Laboratory

1 Cyclotron Road, MS 70-0127A

Berkeley, CA 94720

*Distribution of this report is made by electronic media via http://www.osti.gov/bridge/. 
No. of

Copies

Mr. Ronald G. Wilhelm

Office of Radiation and Indoor Air

401 M Street, S.W.

Mail Code 6603J

Washington, D.C. 20460

W. Alexander Williams

U.S. Department of Energy

Office of Environmental Restoration

EM-33

19901 Germantown Road

Germantown, MD 20874-1290

\section{ONSITE}

3 DOE Office of River Protection

P. E. LaMont

H6-60

R. W. Lober

H6-60

R. B. Yasek

8 DOE Richland Operations Office

B. L. Foley

J. P. Hanson

R. D. Hildebrand

K. A. Kapsi

J. G. Morse

K. M. Thompson

DOE Public Reading Room (2)

16 CH2M HILL Hanford Group, Inc.

M. P. Connelly (5)

E6-35

M. E. Johnson

T. E. Jones (2)

F. J. Anderson

A. J. Knepp

F. M. Mann

W. J. McMahon

H6-60

A6-38

A5-13

A6-38

A5-13

A6-38

A6-38

H2-53

E6-35

E6-35
No. of

Copies

C. W. Miller

H9-03

D. A. Myers

E6-35

D. M. Nguyen

R2-12

T. L. Sams

H6-05

3 Duratek Federal Services, Inc., Northwest Operations

M. G. Gardner

H1-11

K. D. Reynolds

H1-11

D. E. Skoglie

H1-11

3 Environmental Protection Agency
N. Ceto
B5-01
D. A. Faulk
B5-01
M. L. Goldstein
B5-01

\section{Fluor Federal Services}
R. Khaleel
E6-17
R. J. Puigh
E6-17

8 Fluor Hanford, Inc.

T. W. Fogwell

E6-35

B. H. Ford

E6-35

J. G. Hogan

H1-11

D. G. Horton

E6-35

V. G. Johnson

E6-35

S. M. Narbutovskih

E6-44

B. A. Williams

E6-44

M. I. Wood

H8-44

Stoller

R. G. McCain

B2-62

*Distribution of this report is made by electronic media via http://www.osti.gov/bridge/. 
No. of

Copies

5 Washington State Department of Ecology

S. Dahl

$\mathrm{H} 0-57$

J. A. Caggiano

H0-57

A. D. Huckaby

H0-57

J. Yokel

H0-57

F. Hodges

$\mathrm{H} 0-57$

54 Pacific Northwest National Laboratory

D. H. Bacon

S. R. Baum

B. N. Bjornstad

C. F. Brown

R. W. Bryce

K. J. Cantrell

R. E. Clayton

W. J. Deutsch (10)

P. E. Dresel

K. N. Geiszler

M. J. Fayer

A. R. Felmy

M. D. Freshley

J. S. Fruchter

N. J. Hess
K9-33

P7-22

K6-81

P7-22

E6-35

K6-81

P7-22

K6-81

K6-96

P7-22

K9-33

K8-96

K9-33

K6-96

P7-50
No. of

Copies

J. P. Icenhower K6-81

C. T. Kincaid

E6-35

K. M. Krupka (5)

K6-81

I. V. Kutnyakov

P7-22

G. V. Last

V. L. LeGore

K6-81

P7-22

P7-22

C. W. Lindenmeier K6-96

W. J. Martin

K6-81

K6-81

K6-81

B. P. McGrail

P. D. Meyer

BPO

C. J. Murray

R. D. Orr

E. M. Pierce

N. Qafoku

R. G. Riley

R. J. Serne

H. T. Schaef

W. Um

M. Valenta

T. S. Vickerman

S. B. Yabusaki

J. M. Zachara

Hanford Technical Library (2)
K6-81

K6-81

K6-81

K3-61

K6-81

P7-22

K6-81

P7-22

P7-22

P7-22

K9-36

K8-96

P8-55

*Distribution of this report is made by electronic media via http://www.osti.gov/bridge/. 